Supporting Information

\title{
Chameleon-Like Activating Nature of the Spirooxindole Group in Donor-Acceptor Cyclopropanes
}

Andrey A. Akaev, Mikhail Ya. Melnikov and Ekaterina M. Budynina

Department of Chemistry, Lomonosov Moscow State University,

Leninskie gory 1-3, Moscow 119991, Russia

E-mail: ekatbud@kinet.chem.msu.ru 


\section{Table of contents}

General information

Two-step synthesis of cyclopropanes $2 \quad 4$

A. General procedure for the synthesis of 3-diazooxindoles S1 4

B. General procedure for the synthesis of cyclopropanes 2

Nucleophilic ring opening of cyclopropanes 2 with the azide ion 12

Nucleophilic ring opening of cyclopropane 1a with the aniline 15

General procedure for the nucleophilic ring opening of cyclopropanes 2 with anilines 17

$\begin{array}{ll}\text { General procedure for the } \gamma \text {-lactamization of } 7 & 27\end{array}$

General procedure for the PMB- $N$-deprotection of lactames 8

General procedure for the synthesis of cyclopropanes 10

\begin{tabular}{l|l} 
General procedure for the synthesis of lactames 11 & 57
\end{tabular}

References

Cartesian coordinates and energies of stationary points 63

\begin{tabular}{ll} 
NMR spectra & 94 \\
\hline
\end{tabular} 


\section{General information}

NMR spectra were acquired on Bruker Avance $600 \mathrm{MHz}$ spectrometers at room temperature; the chemical shifts $\delta$ were measured in ppm with respect to solvent $\left({ }^{1} \mathrm{H}: \mathrm{CDCl}_{3}, \delta=7.27 \mathrm{ppm} ;{ }^{13} \mathrm{C}\right.$ : $\mathrm{CDCl}_{3}, \delta=77.0 \mathrm{ppm}$ ). Splitting patterns are designated as s, singlet; d, doublet; t, triplet; $\mathrm{m}$, multiplet; dd, double doublet. Coupling constants $(J)$ are given in Hertz $(\mathrm{Hz})$. The structures of compounds were elucidated with the aid of 1D NMR $\left({ }^{1} \mathrm{H},{ }^{13} \mathrm{C}\right)$ and $2 \mathrm{D}$ NMR $\left({ }^{1} \mathrm{H}-{ }^{1} \mathrm{H}\right.$ COSY, ${ }^{1} \mathrm{H}-$ ${ }^{1} \mathrm{H}$ NOESY, ${ }^{1} \mathrm{H}_{-}{ }^{13} \mathrm{C}$ HSQC and HMBC) spectroscopy. IR spectroscopic analysis was performed using attenuated total reflection (ATR) sampling technique. IR spectra were registered within the wavenumber range of $400-4000 \mathrm{~cm}^{-1}$ using FTIR IRAffinity-1 spectrometer (Shimadzu, Japan) and MIRacle ATR (PikeTechnologies) equipped with a diamond/ZnSe crystal plate. High resolution and accurate mass measurements were carried out using a BrukermicroTOF-Q ${ }^{\mathrm{TM}}$ ESITOF (Electro Spray Ionization / Time of Flight) and Thermo Scientific ${ }^{\mathrm{TM}}$ LTQ Orbitrap mass spectrometers. Melting points (mp) were determined using Electrothermal IA 9100 capillary melting point apparatus. Microwave reactions were performed in a Monowave 300-Anton Paar microwave reactor in sealed reaction vessels. Analytical thin layer chromatography (TLC) was carried out with silica gel plates (silica gel $60, F_{254}$, supported on aluminium) visualized with UV lamp (254 nm). Column chromatography was performed on silica gel 60 (230-400 mesh). 3Diazooxindoles $\mathbf{S 1},{ }^{1}$ cyclopropanes $\mathbf{1 a}^{2}$ and $\mathbf{2}^{3}$ as well as dimethyl 2-methylenemalonate ${ }^{4}$ were synthesized by published procedures. Spectral data for compounds $\mathbf{1 a},{ }^{2} \mathbf{S 1 a},{ }^{5} \mathbf{S 1 f}^{6}$ and $\mathbf{2} \mathbf{c}^{7}$ are consistent with those reported previously.

DFT calculations were carried out with ORCA 3.0 suite of programs, ${ }^{8}$ using B3LYP 9,10 functional. The standard $\mathrm{SVP}^{11}$ basis set has been used in all cases together with continuous solvation model $\operatorname{COSMO}^{12}(\varepsilon=38)$. Frequency analysis was carried out to check whether optimized structures were local minima or transition states. No imaginary frequencies were found for local minima, and only one imaginary frequency was found for each transition state. Computed structures were visualized using CYLview 1.0b. ${ }^{13}$ 


\section{Two-step synthesis of cyclopropanes 2}

Cyclopropanes $\mathbf{2}$ were synthesized via two-step procedure starting from isatin derivatives which were initially transformed into 3-diazooxindoles ${ }^{1} \mathbf{S 1}$ followed by cycloaddition $^{3}$ of $\mathbf{S 1}$ to

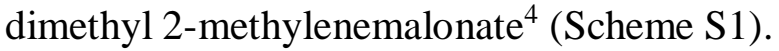

\section{Scheme S1. Synthesis of starting cyclopropanes 2}

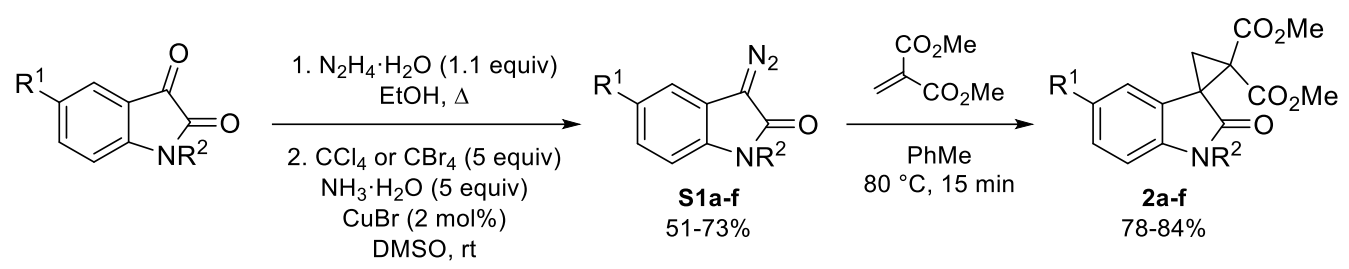

\section{A. General procedure for the synthesis of 3-diazooxindoles $\mathrm{S1}^{1}$}

The suspension of substituted isatin ( 1 equiv) and hydrazine hydrate (1.1 equiv) in ethanol (0.2 M) was heated under reflux (oil bath) for a specified time $\left(t_{1}\right)$ and then the solvent was evaporated under reduced pressure. The residue was dissolved in DMSO (0.25 M) and aqueous $\mathrm{NH}_{3}(25 \%)$ (5 equiv) and $\mathrm{CuBr}\left(2 \%\right.$ mol) were added subsequently. After 5 min stirring $\mathrm{CCl}_{4}$ or $\mathrm{CBr}_{4}$ (5 equiv) was added. The reaction mixture was stirred for a specified time $\left(t_{2}\right)$ and then diluted with water, extracted with ethyl acetate, washed with water and dried with sodium sulfate. Solvent was evaporated under reduced pressure. The residue was purified by column chromatography.

\section{3-Diazo-1-(4-methoxybenzyl)-1,3-dihydro-2H-indol-2-one ${ }^{5}$ (S1a)}

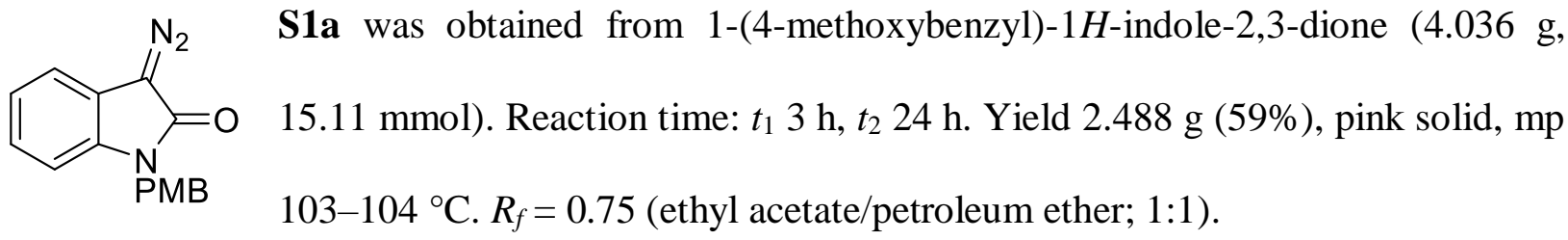

${ }^{1} \mathrm{H}$ NMR $\left(\mathrm{CDCl}_{3}, 600 \mathrm{MHz}\right): \delta=3.77\left(\mathrm{~s}, 3 \mathrm{H}, \mathrm{OCH}_{3}\right), 4.97\left(\mathrm{~s}, 2 \mathrm{H}, \mathrm{NCH}_{2}\right), 6.83-6.88(\mathrm{~m}, 3 \mathrm{H}$, Ar), $7.06\left(\mathrm{ddd},{ }^{3} J=7.6,{ }^{3} J=7.5,{ }^{4} J=1.1 \mathrm{~Hz}, 1 \mathrm{H}, \mathrm{Ar}\right), 7.11\left(\mathrm{ddd},{ }^{3} J=7.7,{ }^{3} J=7.6,{ }^{4} J=1.3 \mathrm{~Hz}\right.$, $1 \mathrm{H}, \mathrm{Ar}), 7.20\left(\mathrm{ddd},{ }^{3} J=7.5,{ }^{4} J=1.3,{ }^{5} J=0.6 \mathrm{~Hz}, 1 \mathrm{H}, \mathrm{Ar}\right), 7.26\left(\mathrm{~d},{ }^{3} J=8.9 \mathrm{~Hz}, 2 \mathrm{H}, \mathrm{Ar}\right)$. 
${ }^{13} \mathrm{C} \mathrm{NMR}\left(\mathrm{CDCl}_{3}, 150 \mathrm{MHz}\right): \delta=43.8\left(\mathrm{NCH}_{2}\right), 55.2\left(\mathrm{OCH}_{3}\right), 60.8\left(\mathrm{C}^{2} \mathrm{~N}_{2}\right), 109.5(\mathrm{CH}, \mathrm{Ar})$, $114.1(2 \times \mathrm{CH}, \mathrm{Ar}), 116.8(\mathrm{C}, \mathrm{Ar}), 118.2(\mathrm{CH}, \mathrm{Ar}), 122.1(\mathrm{CH}, \mathrm{Ar}), 125.4(\mathrm{CH}, \mathrm{Ar}), 128.1(\mathrm{C}$, Ar), $128.7(2 \times \mathrm{CH}, \mathrm{Ar}), 133.6(\mathrm{C}, \mathrm{Ar}), 159.1(\mathrm{C}, \mathrm{Ar}), 166.8(\mathrm{C}=\mathrm{O})$.

\section{3-Diazo-1-(4-methoxybenzyl)-5-methyl-1,3-dihydro-2H-indol-2-one (S1b)}<smiles>Cc1ccc2c(c1)C(=N)C(=O)N2c1ccccc1</smiles>

S1b was obtained from 1-(4-methoxybenzyl)-5-methyl-1H-indole-2,3dione $(5.045 \mathrm{~g}, 17.95 \mathrm{mmol})$. Reaction time: $t_{1} 3 \mathrm{~h}, t_{2} 24 \mathrm{~h}$. Yield $3.419 \mathrm{~g}$ (65\%), beige solid, mp $133-134{ }^{\circ} \mathrm{C} . R_{f}=0.71$ (ethyl acetate/petroleum

ether; $1: 1)$.

${ }^{1} \mathrm{H} \mathrm{NMR}\left(\mathrm{CDCl}_{3}, 600 \mathrm{MHz}\right): \delta=2.34\left(\mathrm{~s}, 3 \mathrm{H}, \mathrm{CH}_{3}\right), 3.76\left(\mathrm{~s}, 3 \mathrm{H}, \mathrm{OCH}_{3}\right), 4.93\left(\mathrm{~s}, 2 \mathrm{H}, \mathrm{NCH}_{2}\right)$, $6.74\left(\mathrm{~d},{ }^{3} \mathrm{~J}=8.0 \mathrm{~Hz}, 1 \mathrm{H}, \mathrm{Ar}\right), 6.84\left(\mathrm{~d},{ }^{3} \mathrm{~J}=8.7 \mathrm{~Hz}, 2 \mathrm{H}, \mathrm{Ar}\right), 6.90$ (br. d, $\left.{ }^{3} J=8.0 \mathrm{~Hz}, 1 \mathrm{H}, \mathrm{Ar}\right), 7.01$ (br.s, $1 \mathrm{H}, \mathrm{Ar}), 7.25\left(\mathrm{~d},{ }^{3} \mathrm{~J}=8.7 \mathrm{~Hz}, 2 \mathrm{H}, \mathrm{Ar}\right)$.

${ }^{13} \mathrm{C} \mathrm{NMR}\left(\mathrm{CDCl}_{3}, 150 \mathrm{MHz}\right): \delta=21.0\left(\mathrm{CH}_{3}\right), 43.7\left(\mathrm{NCH}_{2}\right), 55.1\left(\mathrm{OCH}_{3}\right), 60.5\left(\mathrm{C}=\mathrm{N}_{2}\right), 109.2$ $(\mathrm{CH}, \mathrm{Ar}), 114.0(2 \times \mathrm{CH}, \mathrm{Ar}), 116.7(\mathrm{C}, \mathrm{Ar}), 118.8(\mathrm{CH}, \mathrm{Ar}), 125.9(\mathrm{CH}, \mathrm{Ar}), 128.1(\mathrm{C}, \mathrm{Ar})$, $128.6(2 \times \mathrm{CH}, \mathrm{Ar}), 131.4(\mathrm{C}, \mathrm{Ar}), 131.6(\mathrm{C}, \mathrm{Ar}), 159.0(\mathrm{C}, \mathrm{Ar}), 166.8(\mathrm{C}=\mathrm{O})$.

HRMS (ESI-TOF) $m / z:[\mathrm{M}+\mathrm{H}]^{+}$calcd for $\mathrm{C}_{17} \mathrm{H}_{16} \mathrm{~N}_{3} \mathrm{O}_{2}{ }^{+} 294.1237$, found 294.1233.

\section{3-Diazo-1-methyl-1,3-dihydro-2H-indol-2-one ${ }^{6}$ (S1c)}

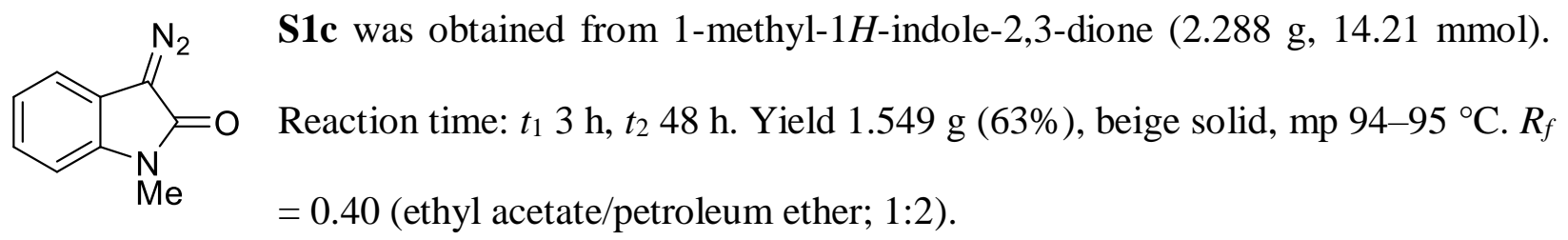

${ }^{1} \mathrm{H} \mathrm{NMR}\left(\mathrm{CDCl}_{3}, 600 \mathrm{MHz}\right): \delta=3.25\left(\mathrm{~s}, 3 \mathrm{H}, \mathrm{NCH}_{3}\right), 6.85\left(\mathrm{~d},{ }^{3} J=7.9 \mathrm{~Hz}, 1 \mathrm{H}, \mathrm{Ar}\right), 7.04\left(\mathrm{dd},{ }^{3} J\right.$ $\left.=7.6,{ }^{3} \mathrm{~J}=7.5 \mathrm{~Hz}, 1 \mathrm{H}, \mathrm{Ar}\right), 7.11-7.17(\mathrm{~m}, 2 \mathrm{H}, \mathrm{Ar})$.

${ }^{13} \mathrm{C} \mathrm{NMR}\left(\mathrm{CDCl}_{3}, 150 \mathrm{MHz}\right): \delta=26.5\left(\mathrm{NCH}_{3}\right), 60.5\left(\mathrm{C}=\mathrm{N}_{2}\right), 108.3(\mathrm{CH}, \mathrm{Ar}), 116.4(\mathrm{C}, \mathrm{Ar})$, $118.0(\mathrm{CH}, \mathrm{Ar}), 121.8(\mathrm{CH}, \mathrm{Ar}), 125.2(\mathrm{CH}, \mathrm{Ar}), 134.2(\mathrm{C}, \mathrm{Ar}), 166.5(\mathrm{C}=\mathrm{O})$. 


\section{3-Diazo-5-fluoro-1-(4-methoxybenzyl)-1,3-dihydro-2H-indol-2-one (S1d)}<smiles>[R15]N1C(=O)C(=N)c2cc(F)ccc21</smiles>

S1d was obtained from 5-fluoro-1-(4-methoxybenzyl)-1H-indole-2,3-dione (4.518 g, $15.85 \mathrm{mmol})$. Reaction time: $t_{1} 3 \mathrm{~h}, t_{2} 24 \mathrm{~h}$. Yield $2.731 \mathrm{~g}(58 \%)$, beige solid, mp $132-133{ }^{\circ} \mathrm{C} . R_{f}=0.78$ (ethyl acetate/petroleum ether; $1: 1$ ).

${ }^{1} \mathrm{H} \mathrm{NMR}\left(\mathrm{CDCl}_{3}, 600 \mathrm{MHz}\right): \delta=3.76\left(\mathrm{~s}, 3 \mathrm{H}, \mathrm{OCH}_{3}\right), 4.93\left(\mathrm{~s}, 2 \mathrm{H}, \mathrm{NCH}_{2}\right), 6.73\left(\mathrm{dd},{ }^{3} J=8.6\right.$, $\left.{ }^{4} J_{\mathrm{HF}}=4.2 \mathrm{~Hz}, 1 \mathrm{H}, \mathrm{Ar}\right), 6.78\left(\mathrm{ddd},{ }^{3} J=9.3,{ }^{3} J=8.6,{ }^{4} J=2.4 \mathrm{~Hz}, 1 \mathrm{H}, \mathrm{Ar}\right), 6.84\left(\mathrm{~d},{ }^{3} J=8.8 \mathrm{~Hz}\right.$, $2 \mathrm{H}, \mathrm{Ar}), 6.92\left(\mathrm{dd},{ }^{3} \mathrm{~J}=8.0,{ }^{4} \mathrm{~J}=2.4 \mathrm{~Hz}, 1 \mathrm{H}, \mathrm{Ar}\right), 7.22\left(\mathrm{~d},{ }^{3} \mathrm{~J}=8.8 \mathrm{~Hz}, 2 \mathrm{H}, \mathrm{Ar}\right)$.

${ }^{13} \mathrm{C} \mathrm{NMR}\left(\mathrm{CDCl}_{3}, 150 \mathrm{MHz}\right): \delta=43.8\left(\mathrm{NCH}_{2}\right), 55.1\left(\mathrm{OCH}_{3}\right), 61.3\left(\mathrm{C}=\mathrm{N}_{2}\right), 105.7\left({ }^{2} J_{\mathrm{CF}}=27 \mathrm{~Hz}\right.$, $\mathrm{CH}, \mathrm{Ar}), 109.9\left({ }^{3} J_{\mathrm{CF}}=9 \mathrm{~Hz}, \mathrm{CH}, \mathrm{Ar}\right), 111.9\left({ }^{2} J_{\mathrm{CF}}=24 \mathrm{~Hz}, \mathrm{CH}, \mathrm{Ar}\right), 114.1(2 \times \mathrm{CH}, \mathrm{Ar}), 117.9$ $\left({ }^{3} J_{\mathrm{CF}}=10 \mathrm{~Hz}, \mathrm{C}, \mathrm{Ar}\right), 127.75(\mathrm{C}, \mathrm{Ar}), 128.6(2 \times \mathrm{CH}, \mathrm{Ar}), 129.7(\mathrm{C}, \mathrm{Ar}), 158.7\left({ }^{1} J_{\mathrm{CF}}=240 \mathrm{~Hz}, \mathrm{C}\right.$, Ar), $159.1(\mathrm{C}, \mathrm{Ar}), 166.3(\mathrm{C}=\mathrm{O})$.

HRMS (ESI-TOF) $m / z:[\mathrm{M}+\mathrm{H}]^{+}$calcd for $\mathrm{C}_{16} \mathrm{H}_{15} \mathrm{FN}_{3} \mathrm{O}_{2}{ }^{+} 298.0986$, found 298.0988.

\section{5-Chloro-3-diazo-1-(4-methoxybenzyl)-1,3-dihydro-2H-indol-2-one (S1e)}<smiles>N#[R16]C1=Nc2ccc(Cl)cc2C1=N</smiles>

$1: 1)$.

${ }^{1} \mathrm{H} \mathrm{NMR}\left(\mathrm{CDCl}_{3}, 600 \mathrm{MHz}\right): \delta=3.74\left(\mathrm{~s}, 3 \mathrm{H}, \mathrm{OCH}_{3}\right), 4.90\left(\mathrm{~s}, 2 \mathrm{H}, \mathrm{NCH}_{2}\right), 6.72\left(\mathrm{dd},{ }^{3} J=8.4,{ }^{5} J\right.$ $=0.3 \mathrm{~Hz}, 1 \mathrm{H}, \mathrm{Ar}), 6.82\left(\mathrm{~d},{ }^{3} \mathrm{~J}=8.8 \mathrm{~Hz}, 2 \mathrm{H}, \mathrm{Ar}\right), 7.01\left(\mathrm{dd},{ }^{3} \mathrm{~J}=8.4,{ }^{4} \mathrm{~J}=2.0 \mathrm{~Hz}, 1 \mathrm{H}, \mathrm{Ar}\right), 7.12$ $\left(\mathrm{dd},{ }^{4} J=2.0,{ }^{5} \mathrm{~J}=0.3 \mathrm{~Hz}, 1 \mathrm{H}, \mathrm{Ar}\right), 7.21\left(\mathrm{~d},{ }^{3} \mathrm{~J}=8.8 \mathrm{~Hz}, 2 \mathrm{H}, \mathrm{Ar}\right)$.

${ }^{13} \mathrm{C}$ NMR $\left(\mathrm{CDCl}_{3}, 150 \mathrm{MHz}\right): \delta=43.7\left(\mathrm{NCH}_{2}\right), 55.0\left(\mathrm{OCH}_{3}\right), 60.8\left(\mathrm{C}=\mathrm{N}_{2}\right), 110.1(\mathrm{CH}, \mathrm{Ar})$, $114.0(2 \times \mathrm{CH}, \mathrm{Ar}), 118.0(\mathrm{CH}, \mathrm{Ar}), 118.1$ (C, Ar), $125.1(\mathrm{CH}, \mathrm{Ar}), 127.2(\mathrm{C}, \mathrm{Ar}), 127.5(\mathrm{C}, \mathrm{Ar})$, 128.5 (2×CH, Ar), $131.9(\mathrm{C}, \mathrm{Ar}), 159.0(\mathrm{C}, \mathrm{Ar}), 166.0(\mathrm{C}=\mathrm{O})$.

HRMS (ESI-TOF) $m / z:[\mathrm{M}+\mathrm{H}]^{+}$calcd for $\mathrm{C}_{16} \mathrm{H}_{15} \mathrm{ClN}_{3} \mathrm{O}_{2}{ }^{+} 314.0691$, found 314.0689. 


\section{5-Bromo-3-diazo-1-(4-methoxybenzyl)-1,3-dihydro-2H-indol-2-one (S1f)}<smiles>[R15]N1C(=O)C(=N)c2cc(Br)ccc21</smiles>

S1f was obtained from 5-bromo-1-(4-methoxybenzyl)-1H-indole-2,3-dione (1.292 g, $3.75 \mathrm{mmol})$. Reaction time: $t_{1} 11 \mathrm{~h}, t_{2} 24 \mathrm{~h}$. Yield $682 \mathrm{mg}(51 \%)$, orange solid, mp $132-133{ }^{\circ} \mathrm{C} . R_{f}=0.73$ (ethyl acetate/petroleum ether;

$1: 1)$.

${ }^{1} \mathrm{H} \mathrm{NMR}\left(\mathrm{CDCl}_{3}, 600 \mathrm{MHz}\right): \delta=3.71\left(\mathrm{~s}, 3 \mathrm{H}, \mathrm{OCH}_{3}\right), 4.87\left(\mathrm{~s}, 2 \mathrm{H}, \mathrm{NCH}_{2}\right), 6.65\left(\mathrm{dd},{ }^{3} J=8.4 \mathrm{~Hz}\right.$, $1 \mathrm{H}, \mathrm{Ar}), 6.80\left(\mathrm{~d},{ }^{3} J=8.9 \mathrm{~Hz}, 2 \mathrm{H}, \mathrm{Ar}\right), 7.12\left(\mathrm{dd},{ }^{3} J=8.4,{ }^{4} J=1.9 \mathrm{~Hz}, 1 \mathrm{H}, \mathrm{Ar}\right), 7.19\left(\mathrm{~d},{ }^{3} J=8.9\right.$ $\mathrm{Hz}, 2 \mathrm{H}, \mathrm{Ar}), 7.22\left(\mathrm{~d},{ }^{4} \mathrm{~J}=1.9 \mathrm{~Hz}, 1 \mathrm{H}, \mathrm{Ar}\right)$.

${ }^{13} \mathrm{C} \mathrm{NMR}\left(\mathrm{CDCl}_{3}, 150 \mathrm{MHz}\right): \delta=43.5\left(\mathrm{NCH}_{2}\right), 54.9\left(\mathrm{OCH}_{3}\right), 60.5\left(\mathrm{C}=\mathrm{N}_{2}\right), 110.4(\mathrm{CH}, \mathrm{Ar})$, $113.9(2 \times \mathrm{CH}, \mathrm{Ar}), 114.2(\mathrm{C}, \mathrm{Ar}), 118.4(\mathrm{C}, \mathrm{Ar}), 120.6(\mathrm{CH}, \mathrm{Ar}), 127.4(\mathrm{C}, \mathrm{Ar}), 127.7(\mathrm{CH}, \mathrm{Ar})$, 128.5 (2×CH, Ar), $132.2(\mathrm{C}, \mathrm{Ar}), 158.9(\mathrm{C}, \mathrm{Ar}), 165.7(\mathrm{C}=\mathrm{O})$.

HRMS (ESI-TOF) $m / z:[\mathrm{M}+\mathrm{H}]^{+}$calcd for $\mathrm{C}_{16} \mathrm{H}_{15} \mathrm{BrN}_{3} \mathrm{O}_{2}{ }^{+}$358.0186, found 358.0184.

\section{B. General procedure for the synthesis of cyclopropanes $2^{3}$}

The mixture of diazo compound $\mathbf{S 1}$ (1.0 equiv) and dimethyl 2-methylenemalonate ${ }^{4}$ (1.2 equiv) in toluene (4 M) was heated at $80{ }^{\circ} \mathrm{C}$ (oil bath) under vigorous stirring. In $15 \mathrm{~min}$, the reaction mixture was cooled to the room temperature. Solvent was removed under reduced pressure. Cyclopropane 2 was purified by column chromatography on silica gel.

\section{Dimethyl 1'-(4-methoxybenzyl)-2'-oxo-1',2'-dihydro-2H-spiro[cyclopropane-1,3'-indole]-}

\section{2,2-dicarboxylate (2a)}

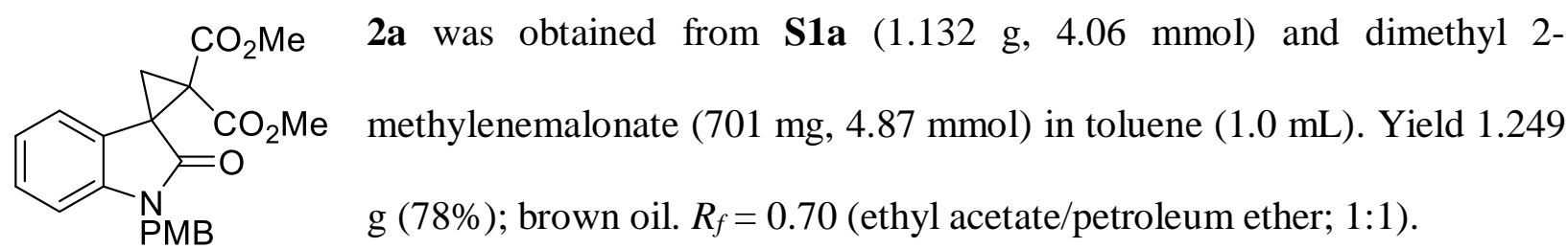

${ }^{1} \mathrm{H} \mathrm{NMR}\left(\mathrm{CDCl}_{3}, 600 \mathrm{MHz}\right): \delta=2.51\left(\mathrm{~d},{ }^{2} J=5.2 \mathrm{~Hz}, 1 \mathrm{H}, \mathrm{CH}_{2}\right), 2.52\left(\mathrm{~d},{ }^{2} J=5.2 \mathrm{~Hz}, 1 \mathrm{H}, \mathrm{CH}_{2}\right)$, $3.72\left(\mathrm{~s}, 3 \mathrm{H}, \mathrm{OCH}_{3}\right), 3.73\left(\mathrm{~s}, 3 \mathrm{H}, \mathrm{OCH}_{3}\right), 3.79\left(\mathrm{~s}, 3 \mathrm{H}, \mathrm{OCH}_{3}\right), 4.70\left(\mathrm{~d},{ }^{2} \mathrm{~J}=15.5 \mathrm{~Hz}, 1 \mathrm{H}, \mathrm{NCH}_{2}\right)$, $5.03\left(\mathrm{~d},{ }^{2} J=15.5 \mathrm{~Hz}, 1 \mathrm{H}, \mathrm{NCH}_{2}\right), 6.82\left(\mathrm{~d},{ }^{3} J=7.8 \mathrm{~Hz}, 1 \mathrm{H}, \mathrm{Ar}\right), 6.84\left(\mathrm{~d},{ }^{3} J=8.7 \mathrm{~Hz}, 2 \mathrm{H}, \mathrm{Ar}\right)$, 
$6.97\left(\mathrm{dd},{ }^{3} J=7.7,{ }^{3} J=7.7 \mathrm{~Hz}, 1 \mathrm{H}, \mathrm{Ar}\right), 7.19\left(\mathrm{dd},{ }^{3} J=7.8,{ }^{3} J=7.7 \mathrm{~Hz}, 1 \mathrm{H}\right.$, Ar $), 7.26\left(\mathrm{~d},{ }^{3} J=8.7\right.$ $\mathrm{Hz}, 2 \mathrm{H}, \mathrm{Ar}), 7.39\left(\mathrm{~d},{ }^{3} \mathrm{~J}=7.7 \mathrm{~Hz}, 1 \mathrm{H}, \mathrm{Ar}\right)$.

${ }^{13} \mathrm{C} \mathrm{NMR}\left(\mathrm{CDCl}_{3}, 150 \mathrm{MHz}\right): \delta=24.3\left(\mathrm{CH}_{2}\right), 37.9(\mathrm{C}), 43.3\left(\mathrm{NCH}_{2}\right), 45.3(\mathrm{C}), 52.7\left(\mathrm{OCH}_{3}\right)$, $53.0\left(\mathrm{OCH}_{3}\right), 54.9\left(\mathrm{OCH}_{3}\right), 108.9(\mathrm{CH}, \mathrm{Ar}), 113.9(2 \times \mathrm{CH}, \mathrm{Ar}), 122.0(\mathrm{CH}, \mathrm{Ar}), 123.2(\mathrm{CH}, \mathrm{Ar})$, 124.1 (C, Ar), 127.4 (C, Ar), $128.2(\mathrm{CH}, \mathrm{Ar}), 128.4(2 \times \mathrm{CH}, \mathrm{Ar}), 143.6(\mathrm{C}, \mathrm{Ar}), 158.9(\mathrm{C}, \mathrm{Ar})$, $165.7\left(\mathrm{CO}_{2} \mathrm{Me}\right), 165.8\left(\mathrm{CO}_{2} \mathrm{Me}\right), 172.5(\mathrm{C}=\mathrm{O})$.

HRMS (ESI-TOF) $m / z:[\mathrm{M}+\mathrm{H}]^{+}$calcd for $\mathrm{C}_{22} \mathrm{H}_{22} \mathrm{NO}_{6}{ }^{+}$396.1442, found 396.1446.

\section{Dimethyl 1'-(4-methoxybenzyl)-5' -methyl-2'-oxo-1',2' -dihydro-2H-spiro[cyclopropane-1,3'-} indole]-2,2-dicarboxylate (2b)

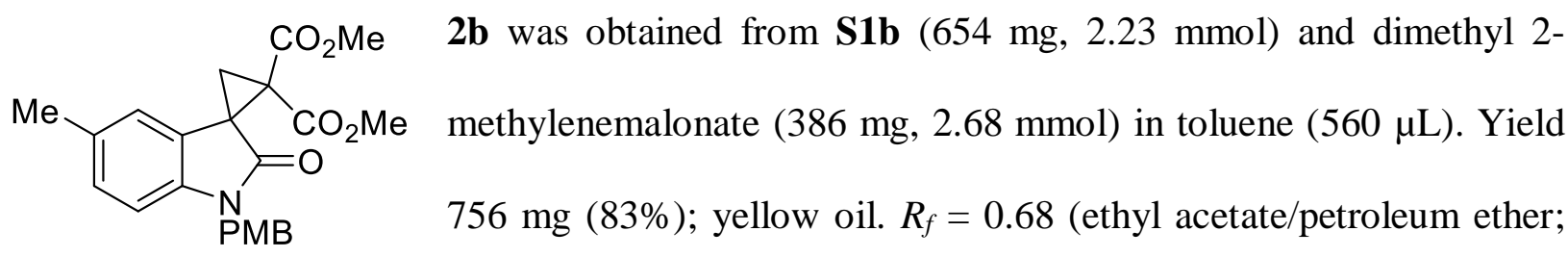
$1: 1)$.

${ }^{1} \mathrm{H} \mathrm{NMR}\left(\mathrm{CDCl}_{3}, 600 \mathrm{MHz}\right): \delta=2.27\left(\mathrm{~d},{ }^{4} J=0.7 \mathrm{~Hz}, 3 \mathrm{H}, \mathrm{CH}_{3}\right), 2.48\left(\mathrm{~d},{ }^{2} J=5.0 \mathrm{~Hz}, 1 \mathrm{H}, \mathrm{CH}_{2}\right)$, $2.49\left(\mathrm{~d},{ }^{2} J=5.0 \mathrm{~Hz}, 1 \mathrm{H}, \mathrm{CH}_{2}\right), 3.70\left(\mathrm{~s}, 3 \mathrm{H}, \mathrm{OCH}_{3}\right), 3.72\left(\mathrm{~s}, 3 \mathrm{H}, \mathrm{OCH}_{3}\right), 3.78\left(\mathrm{~s}, 3 \mathrm{H}, \mathrm{OCH}_{3}\right)$, $4.66\left(\mathrm{~d},{ }^{2} J=15.5 \mathrm{~Hz}, 1 \mathrm{H}, \mathrm{NCH}_{2}\right), 5.01\left(\mathrm{~d},{ }^{2} J=15.5 \mathrm{~Hz}, 1 \mathrm{H}, \mathrm{NCH}_{2}\right), 6.70\left(\mathrm{~d},{ }^{3} J=8.0 \mathrm{~Hz}, 1 \mathrm{H}\right.$, Ar), $6.82\left(\mathrm{~d},{ }^{3} \mathrm{~J}=8.9 \mathrm{~Hz}, 2 \mathrm{H}, \mathrm{Ar}\right), 6.98\left(\mathrm{ddd},{ }^{3} \mathrm{~J}=8.0,{ }^{4} \mathrm{~J}=1.7,{ }^{4} \mathrm{~J}=0.7 \mathrm{~Hz}, 1 \mathrm{H}, \mathrm{Ar}\right), 7.22\left(\mathrm{~d},{ }^{4} \mathrm{~J}=\right.$ $1.7 \mathrm{~Hz}, 1 \mathrm{H}, \mathrm{Ar}), 7.24\left(\mathrm{~d},{ }^{3} \mathrm{~J}=8.9 \mathrm{~Hz}, 2 \mathrm{H}, \mathrm{Ar}\right)$.

${ }^{13} \mathrm{C} \mathrm{NMR}\left(\mathrm{CDCl}_{3}, 150 \mathrm{MHz}\right): \delta=20.8\left(\mathrm{CH}_{3}\right), 24.1\left(\mathrm{CH}_{2}\right), 37.8(\mathrm{C}), 43.2\left(\mathrm{NCH}_{2}\right), 45.2(\mathrm{C}), 52.6$ $\left(\mathrm{OCH}_{3}\right), 52.8\left(\mathrm{OCH}_{3}\right), 54.8\left(\mathrm{OCH}_{3}\right), 108.6(\mathrm{CH}, \mathrm{Ar}), 113.8(2 \times \mathrm{CH}, \mathrm{Ar}), 123.9(\mathrm{CH}, \mathrm{Ar}), 124.0$ (C, Ar), 127.5 (C, Ar), 128.27 (2×CH, Ar), 128.34 (CH, Ar), 131.4 (C, Ar), 141.1 (C, Ar), 158.7 $(\mathrm{C}, \mathrm{Ar}), 165.6\left(\mathrm{CO}_{2} \mathrm{Me}\right), 165.8\left(\mathrm{CO}_{2} \mathrm{Me}\right), 172.3(\mathrm{C}=\mathrm{O})$. IR (film) 2954, 2917, 2850, 1740, 1715, 1513, 1490, 1247, $1121 \mathrm{~cm}^{-1}$. HRMS (ESI-TOF) $m / z:[\mathrm{M}+\mathrm{H}]^{+}$calcd for $\mathrm{C}_{23} \mathrm{H}_{24} \mathrm{NO}_{6}{ }^{+} 410.1598$, found 410.1600. 


\section{Dimethyl 1'-methyl-2' -oxo-1',2'-dihydro-2H-spiro[cyclopropane-1,3'-indole]-2,2-}

dicarboxylate $^{7}(2 c)$

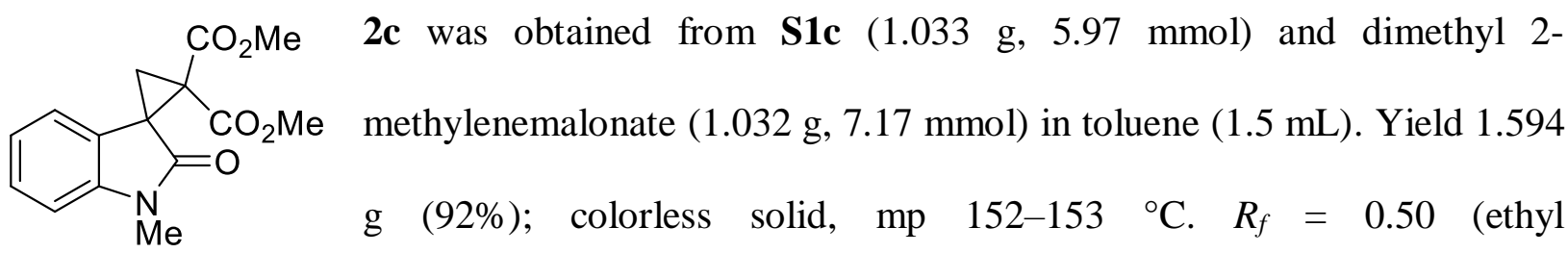
acetate/petroleum ether; $1: 1)$.

${ }^{1} \mathrm{H} \mathrm{NMR}\left(\mathrm{CDCl}_{3}, 600 \mathrm{MHz}\right): \delta=2.42\left(\mathrm{~d},{ }^{2} J=5.2 \mathrm{~Hz}, 1 \mathrm{H}, \mathrm{CH}_{2}\right), 2.46\left(\mathrm{~d},{ }^{2} J=5.2 \mathrm{~Hz}, 1 \mathrm{H}, \mathrm{CH}_{2}\right)$, $3.23\left(\mathrm{~s}, 3 \mathrm{H}, \mathrm{NCH}_{3}\right), 3.72\left(\mathrm{~s}, 3 \mathrm{H}, \mathrm{OCH}_{3}\right), 3.77\left(\mathrm{~s}, 3 \mathrm{H}, \mathrm{OCH}_{3}\right), 6.87\left(\mathrm{~d},{ }^{3} \mathrm{~J}=7.8 \mathrm{~Hz}, 1 \mathrm{H}, \mathrm{Ar}\right), 6.99$ (ddd, ${ }^{3} J=7.7,{ }^{3} J=7.6,{ }^{4} J=0.9 \mathrm{~Hz}, 1 \mathrm{H}$, Ar), $7.29\left(\mathrm{ddd},{ }^{3} J=7.8,{ }^{3} J=7.7,{ }^{4} J=1.2 \mathrm{~Hz}, 1 \mathrm{H}\right.$, Ar), $7.32\left(\mathrm{ddd},{ }^{3} J=7.6,{ }^{4} \mathrm{~J}=1.2,{ }^{5} \mathrm{~J}=0.6 \mathrm{~Hz}, 1 \mathrm{H}, \mathrm{Ar}\right)$.

${ }^{13} \mathrm{C} \mathrm{NMR}\left(\mathrm{CDCl}_{3}, 150 \mathrm{MHz}\right): \delta=24.2\left(\mathrm{CH}_{2}\right), 26.6\left(\mathrm{NCH}_{3}\right), 37.8(\mathrm{C}), 45.2(\mathrm{C}), 52.9\left(\mathrm{OCH}_{3}\right)$, $53.1\left(\mathrm{OCH}_{3}\right), 108.2(\mathrm{CH}, \mathrm{Ar}), 122.2(\mathrm{CH}, \mathrm{Ar}), 122.9(\mathrm{CH}, \mathrm{Ar}), 124.2(\mathrm{C}, \mathrm{Ar}), 128.4(\mathrm{CH}, \mathrm{Ar})$, $144.6(\mathrm{C}, \mathrm{Ar}), 165.6\left(\mathrm{CO}_{2} \mathrm{Me}\right), 165.8\left(\mathrm{CO}_{2} \mathrm{Me}\right), 172.4(\mathrm{C}=\mathrm{O})$.

IR (film) 2950, 2910, 1718, 1710, 1612, 1436, 1264, $1251 \mathrm{~cm}^{-1}$.

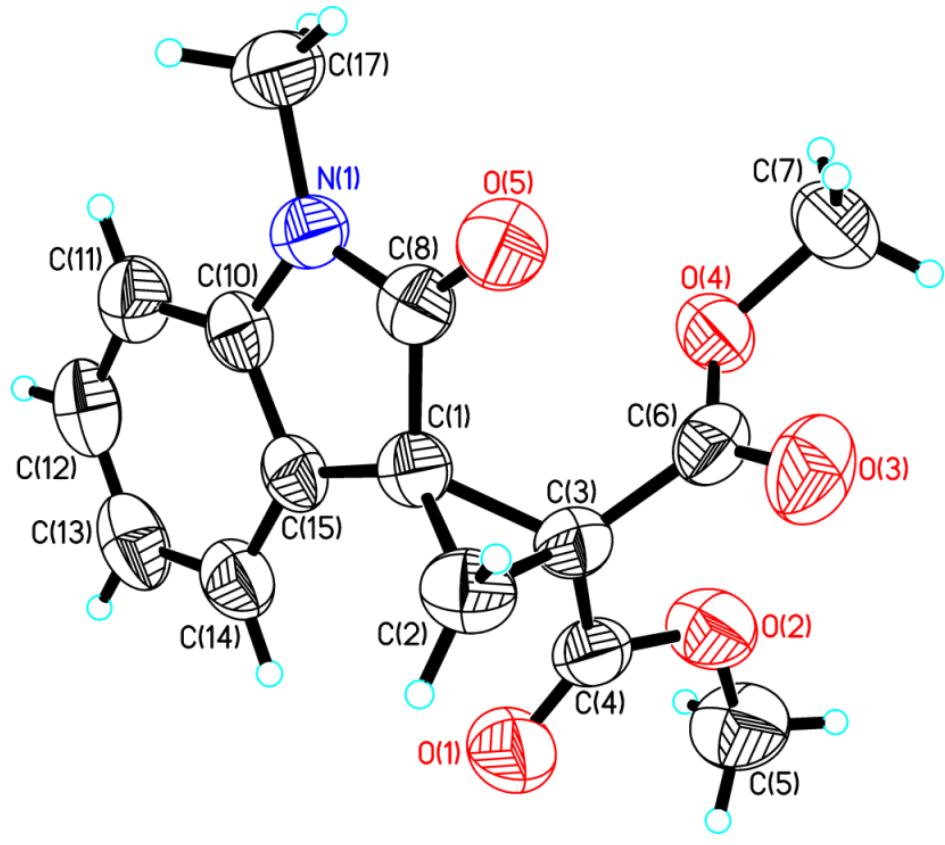

Figure S1. Molecular structure from single crystal X-ray study of 2c (thermal ellipsoids are drawn at the 50\% probability level); CCDC 1954544. 


\section{Dimethyl 5'-fluoro-1'-(4-methoxybenzyl)-2' -oxo-1', $2^{\prime}$-dihydro-2H-spiro[cyclopropane-1,3'-}

indole]-2,2-dicarboxylate (2d)

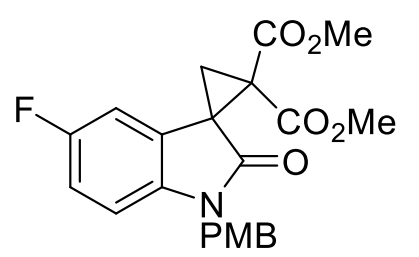

2d was obtained from S1d (1.067 g, $3.59 \mathrm{mmol})$ and dimethyl 2methylenemalonate $(621 \mathrm{mg}, 4.31 \mathrm{mmol})$ in toluene $(900 \mu \mathrm{L})$. Yield 1.394 g (94\%); orange oil. $R_{f}=0.71$ (ethyl acetate/petroleum ether; $1: 1)$

${ }^{1} \mathrm{H} \mathrm{NMR}\left(\mathrm{CDCl}_{3}, 600 \mathrm{MHz}\right): \delta=2.44\left(\mathrm{~d},{ }^{2} J=5.1 \mathrm{~Hz}, 1 \mathrm{H}, \mathrm{CH}_{2}\right), 2.47\left(\mathrm{~d},{ }^{2} J=5.1 \mathrm{~Hz}, 1 \mathrm{H}, \mathrm{CH}_{2}\right)$, $3.68\left(\mathrm{~s}, 3 \mathrm{H}, \mathrm{OCH}_{3}\right), 3.70\left(\mathrm{~s}, 3 \mathrm{H}, \mathrm{OCH}_{3}\right), 3.74\left(\mathrm{~s}, 3 \mathrm{H}, \mathrm{OCH}_{3}\right), 4.62\left(\mathrm{~d},{ }^{2} J=15.6 \mathrm{~Hz}, 1 \mathrm{H}, \mathrm{NCH}_{2}\right)$, $4.99\left(\mathrm{~d},{ }^{2} J=15.6 \mathrm{~Hz}, 1 \mathrm{H}, \mathrm{NCH}_{2}\right), 6.69\left(\mathrm{dd},{ }^{3} J=8.6,{ }^{4} J=4.3 \mathrm{~Hz}, 1 \mathrm{H}, \mathrm{Ar}\right), 6.79\left(\mathrm{~d},{ }^{3} J=8.7 \mathrm{~Hz}\right.$, $2 \mathrm{H}, \mathrm{Ar}), 6.84\left(\mathrm{ddd},{ }^{3} J=8.8,{ }^{3} J=8.6,{ }^{4} \mathrm{~J}=2.6 \mathrm{~Hz}, 1 \mathrm{H}, \mathrm{Ar}\right), 7.18\left(\mathrm{dd},{ }^{3} J=9.0,{ }^{4} J=2.6 \mathrm{~Hz}, 1 \mathrm{H}\right.$, Ar), $7.20\left(\mathrm{~d},{ }^{3} \mathrm{~J}=8.7 \mathrm{~Hz}, 2 \mathrm{H}, \mathrm{Ar}\right)$.

${ }^{13} \mathrm{C} \mathrm{NMR}\left(\mathrm{CDCl}_{3}, 150 \mathrm{MHz}\right): \delta=24.6\left(\mathrm{CH}_{2}\right), 37.9(\mathrm{C}), 43.3\left(\mathrm{NCH}_{2}\right), 45.3(\mathrm{C}), 52.6\left(\mathrm{OCH}_{3}\right)$, $53.0\left(\mathrm{OCH}_{3}\right), 54.8\left(\mathrm{OCH}_{3}\right), 109.2\left({ }^{3} J_{\mathrm{CF}}=8 \mathrm{~Hz}, \mathrm{CH}, \mathrm{Ar}\right), 111.6\left({ }^{2} J_{\mathrm{CF}}=27 \mathrm{~Hz}, \mathrm{CH}, \mathrm{Ar}\right), 113.8$ $(2 \times \mathrm{CH}, \mathrm{Ar}), 114.3\left({ }^{2} J_{\mathrm{CF}}=24 \mathrm{~Hz}, \mathrm{CH}, \mathrm{Ar}\right), 125.6\left({ }^{3} J_{\mathrm{CF}}=9 \mathrm{~Hz}, \mathrm{C}, \mathrm{Ar}\right), 127.1(\mathrm{C}, \mathrm{Ar}), 128.3$ $(2 \times \mathrm{CH}, \mathrm{Ar}), 139.5(\mathrm{C}, \mathrm{Ar}), 158.4\left({ }^{1} J_{\mathrm{CF}}=239 \mathrm{~Hz}, \mathrm{C}, \mathrm{Ar}\right), 158.8(\mathrm{C}, \mathrm{Ar}), 165.3\left(\mathrm{CO}_{2} \mathrm{Me}\right), 165.7$ $\left(\mathrm{CO}_{2} \mathrm{Me}\right), 172.1(\mathrm{C}=\mathrm{O})$.

HRMS (ESI-TOF) $m / z:[\mathrm{M}+\mathrm{H}]^{+}$calcd for $\mathrm{C}_{22} \mathrm{H}_{21} \mathrm{FNO}_{6}{ }^{+} 414.1347$, found 414.1345 .

\section{Dimethyl 5'-chloro-1'-(4-methoxybenzyl)-2'-oxo-1',2'-dihydro-2H-spiro[cyclopropane-1,3'-} indole]-2,2-dicarboxylate (2e)

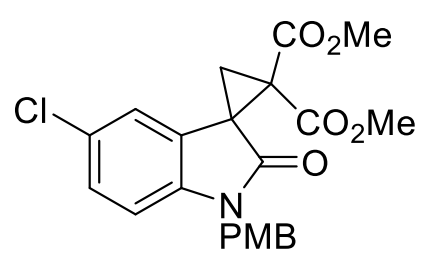

2e was obtained from S1e (666 $\mathrm{mg}, 2.13 \mathrm{mmol})$ and dimethyl 2methylenemalonate $(368 \mathrm{mg}, 2.56 \mathrm{mmol})$ in toluene $(535 \mu \mathrm{L})$. Yield $824 \mathrm{mg}(90 \%)$; orange oil. $R_{f}=0.68$ (ethyl acetate/petroleum ether; $1: 1)$.

${ }^{1} \mathrm{H} \mathrm{NMR}\left(\mathrm{CDCl}_{3}, 600 \mathrm{MHz}\right): \delta=2.49\left(\mathrm{~d},{ }^{2} J=5.2 \mathrm{~Hz}, 1 \mathrm{H}, \mathrm{CH}_{2}\right), 2.51\left(\mathrm{~d},{ }^{2} J=5.2 \mathrm{~Hz}, 1 \mathrm{H}, \mathrm{CH}_{2}\right)$, $3.74\left(\mathrm{~s}, 3 \mathrm{H}, \mathrm{OCH}_{3}\right), 3.76\left(\mathrm{~s}, 3 \mathrm{H}, \mathrm{OCH}_{3}\right), 3.78\left(\mathrm{~s}, 3 \mathrm{H}, \mathrm{OCH}_{3}\right), 4.67\left(\mathrm{~d},{ }^{2} J=15.6 \mathrm{~Hz}, 1 \mathrm{H}, \mathrm{NCH}_{2}\right)$, $5.02\left(\mathrm{~d},{ }^{2} J=15.6 \mathrm{~Hz}, 1 \mathrm{H}, \mathrm{NCH}_{2}\right), 6.71\left(\mathrm{~d},{ }^{3} J=8.4 \mathrm{~Hz}, 1 \mathrm{H}, \mathrm{Ar}\right), 6.83\left(\mathrm{~d},{ }^{3} J=8.7 \mathrm{~Hz}, 2 \mathrm{H}, \mathrm{Ar}\right)$, 
$7.14\left(\mathrm{dd},{ }^{3} J=8.4,{ }^{4} J=2.1 \mathrm{~Hz}, 1 \mathrm{H}, \mathrm{Ar}\right), 7.21\left(\mathrm{~d},{ }^{3} J=8.7 \mathrm{~Hz}, 2 \mathrm{H}, \mathrm{Ar}\right), 7.42\left(\mathrm{~d},{ }^{4} J=2.1 \mathrm{~Hz}, 1 \mathrm{H}\right.$, $\operatorname{Ar})$.

${ }^{13} \mathrm{C} \mathrm{NMR}\left(\mathrm{CDCl}_{3}, 150 \mathrm{MHz}\right): \delta=24.7\left(\mathrm{CH}_{2}\right), 37.8(\mathrm{C}), 43.5\left(\mathrm{NCH}_{2}\right), 45.6(\mathrm{C}), 52.9\left(\mathrm{OCH}_{3}\right)$, $53.2\left(\mathrm{OCH}_{3}\right), 55.0\left(\mathrm{OCH}_{3}\right), 109.8(\mathrm{CH}, \mathrm{Ar}), 114.1(2 \times \mathrm{CH}, \mathrm{Ar}), 123.9(\mathrm{CH}, \mathrm{Ar}), 125.9(\mathrm{C}, \mathrm{Ar})$, $127.1(\mathrm{C}, \mathrm{Ar}), 127.6(\mathrm{C}, \mathrm{Ar}), 128.1(\mathrm{CH}, \mathrm{Ar}), 128.4(2 \times \mathrm{CH}, \mathrm{Ar}), 142.2(\mathrm{C}, \mathrm{Ar}), 159.1(\mathrm{C}, \mathrm{Ar})$, $165.4\left(\mathrm{CO}_{2} \mathrm{Me}\right), 165.8\left(\mathrm{CO}_{2} \mathrm{Me}\right), 172.2(\mathrm{C}=\mathrm{O})$.

HRMS (ESI-TOF) $m / z:[\mathrm{M}+\mathrm{H}]^{+}$calcd for $\mathrm{C}_{22} \mathrm{H}_{21} \mathrm{ClNO}_{6}{ }^{+} 430.1052$, found 430.1059 .

\section{Dimethyl 5'-bromo-1'-(4-methoxybenzyl)-2'-oxo-1',2'-dihydro-2H-spiro[cyclopropane-1,3'-} indole]-2,2-dicarboxylate (2f)

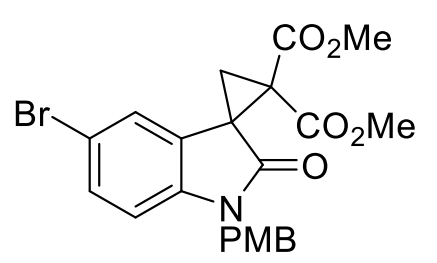

2f was obtained from S1f (454 $\mathrm{mg}, 1.27 \mathrm{mmol}$ ) and dimethyl 2methylenemalonate $(219 \mathrm{mg}, 1.52 \mathrm{mmol})$ in toluene $(320 \mu \mathrm{L})$. Yield $513 \mathrm{mg}(85 \%)$; yellow oil. $R_{f}=0.67$ (ethyl acetate/petroleum ether; $1: 1)$

${ }^{1} \mathrm{H} \mathrm{NMR}\left(\mathrm{CDCl}_{3}, 600 \mathrm{MHz}\right): \delta=2.47\left(\mathrm{~d},{ }^{2} J=5.2 \mathrm{~Hz}, 1 \mathrm{H}, \mathrm{CH}_{2}\right), 2.50\left(\mathrm{~d},{ }^{2} J=5.2 \mathrm{~Hz}, 1 \mathrm{H}, \mathrm{CH}_{2}\right)$, $3.71\left(\mathrm{~s}, 3 \mathrm{H}, \mathrm{OCH}_{3}\right), 3.74\left(\mathrm{~s}, 3 \mathrm{H}, \mathrm{OCH}_{3}\right), 3.76\left(\mathrm{~s}, 3 \mathrm{H}, \mathrm{OCH}_{3}\right), 4.65\left(\mathrm{~d},{ }^{2} \mathrm{~J}=15.5 \mathrm{~Hz}, 1 \mathrm{H}, \mathrm{NCH}_{2}\right)$, $5.00\left(\mathrm{~d},{ }^{2} J=15.5 \mathrm{~Hz}, 1 \mathrm{H}, \mathrm{NCH}_{2}\right), 6.67\left(\mathrm{~d},{ }^{3} J=8.4 \mathrm{~Hz}, 1 \mathrm{H}, \mathrm{Ar}\right), 6.81\left(\mathrm{~d},{ }^{3} J=8.6 \mathrm{~Hz}, 2 \mathrm{H}, \mathrm{Ar}\right)$, $7.20\left(\mathrm{~d},{ }^{3} J=8.6 \mathrm{~Hz}, 2 \mathrm{H}, \mathrm{Ar}\right), 7.28\left(\mathrm{dd},{ }^{3} J=8.4,{ }^{4} J=2.0 \mathrm{~Hz}, 1 \mathrm{H}, \mathrm{Ar}\right), 7.54\left(\mathrm{~d},{ }^{4} J=2.0 \mathrm{~Hz}, 1 \mathrm{H}\right.$, $\operatorname{Ar})$.

${ }^{13} \mathrm{C} \mathrm{NMR}\left(\mathrm{CDCl}_{3}, 150 \mathrm{MHz}\right): \delta=24.7\left(\mathrm{CH}_{2}\right), 37.6(\mathrm{C}), 43.4\left(\mathrm{NCH}_{2}\right), 45.5(\mathrm{C}), 52.8\left(\mathrm{OCH}_{3}\right)$, $53.2\left(\mathrm{OCH}_{3}\right), 54.9\left(\mathrm{OCH}_{3}\right), 110.3(\mathrm{CH}, \mathrm{Ar}), 113.9(2 \times \mathrm{CH}, \mathrm{Ar}), 114.8(\mathrm{C}, \mathrm{Ar}), 126.1(\mathrm{C}, \mathrm{Ar})$, $126.6(\mathrm{CH}, \mathrm{Ar}), 126.9$ (C, Ar), $128.3(2 \times \mathrm{CH}, \mathrm{Ar}), 130.9(\mathrm{CH}, \mathrm{Ar}), 142.6(\mathrm{C}, \mathrm{Ar}), 158.9(\mathrm{C}, \mathrm{Ar})$, $165.3\left(\mathrm{CO}_{2} \mathrm{Me}\right), 165.7\left(\mathrm{CO}_{2} \mathrm{Me}\right), 171.9(\mathrm{C}=\mathrm{O})$.

HRMS (ESI-TOF) $m / z:[\mathrm{M}+\mathrm{H}]^{+}$calcd for $\mathrm{C}_{22} \mathrm{H}_{21} \mathrm{BrNO}_{6}{ }^{+}$474.0547, found 474.0544. 


\section{3-(2-Azidoethyl)-1-methyl-1,3-dihydro-2H-indol-2-one (3b)}

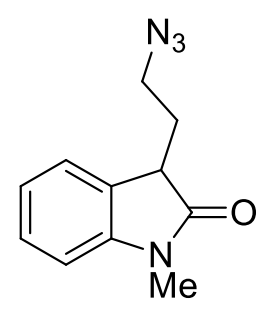

A mixture of cyclopropane $\mathbf{1 b}(323 \mathrm{mg}, 1.87 \mathrm{mmol}), \mathrm{NaN}_{3}(243 \mathrm{mg}, 3.73$ $\mathrm{mmol})$, and $\mathrm{Et}_{3} \mathrm{~N} \cdot \mathrm{HCl}(514 \mathrm{mg}, 3.73 \mathrm{mmol})$ in $\mathrm{DMSO}(0.5 \mathrm{M})$ was stirred at 140

${ }^{\circ} \mathrm{C}$ under microwave irradiation for $7 \mathrm{~h}$. Under these conditions, $78 \%$ conversion of $\mathbf{1 b}$ was achieved. Then, the reaction mixture was diluted with water, extracted with ethyl acetate, washed with water and dried with sodium sulfate. Solvent was evaporated under reduced pressure. Azide $\mathbf{3 b}$ was purified by column chromatography on silica gel. Yield $110 \mathrm{mg}$ (35\%); yellowish oil. $R_{f}=0.34$ (ethyl acetate/petroleum ether; 1:3).

${ }^{1} \mathrm{H}$ NMR $\left(\mathrm{CDCl}_{3}, 600 \mathrm{MHz}\right): \delta=2.14-2.25\left(\mathrm{~m}, 2 \mathrm{H}, \mathrm{CH}_{2}\right), 3.22\left(\mathrm{~s}, 3 \mathrm{H}, \mathrm{NCH}_{3}\right), 3.45-3.56(\mathrm{~m}$, $\left.3 \mathrm{H}, \mathrm{CH}_{2}, \mathrm{CH}\right), 6.85\left(\mathrm{~d},{ }^{3} \mathrm{~J}=7.7 \mathrm{~Hz}, 1 \mathrm{H}, \mathrm{Ar}\right), 7.08\left(\mathrm{ddd},{ }^{3} J=7.6,{ }^{3} J=7.5,{ }^{4} J=0.9 \mathrm{~Hz}, 1 \mathrm{H}, \mathrm{Ar}\right)$, 7.23-7.25 (m, 1H, Ar), 7.29-7.32 (m, 1H, Ar).

${ }^{13} \mathrm{C} \mathrm{NMR}\left(\mathrm{CDCl}_{3}, 150 \mathrm{MHz}\right): \delta=26.2\left(\mathrm{NCH}_{3}\right), 29.8\left(\mathrm{CH}_{2}\right), 42.7(\mathrm{CH}), 48.2\left(\mathrm{CH}_{2}\right), 108.2(\mathrm{CH}$, Ar), 122.5 (CH, Ar), $123.7(\mathrm{CH}, \mathrm{Ar}), 127.8$ (C, Ar), $128.2(\mathrm{CH}, \mathrm{Ar}), 144.3(\mathrm{C}, \mathrm{Ar}), 177.0(\mathrm{C}=\mathrm{O})$. IR (film) 2092, 1718, 1700, 1610, 1465, 1374, 1341, $1261 \mathrm{~cm}^{-1}$. HRMS (ESI-TOF) $m / z:[\mathrm{M}+\mathrm{H}]^{+}$calcd for $\mathrm{C}_{11} \mathrm{H}_{13} \mathrm{~N}_{4} \mathrm{O}^{+} 217.1084$, found 217.1082.

\section{Nucleophilic ring opening of cyclopropanes 2 with the azide ion ${ }^{14}$}

A mixture of cyclopropane 2 (1.0 equiv), $\mathrm{NaN}_{3}$ (2.0 equiv), and $\mathrm{Et}_{3} \mathrm{~N} \cdot \mathrm{HCl}$ (2.0 equiv) in DMSO $(0.5 \mathrm{M})$ was stirred at $80{ }^{\circ} \mathrm{C}$ under microwave irradiation for $8 \mathrm{~h}$. Then, the reaction mixture was diluted with water, extracted with ethyl acetate, washed with water and dried with sodium sulfate. Solvent was evaporated under reduced pressure. Azide 4 was purified by column chromatography on silica gel. 


\section{Dimethyl \{[3-azido-1-(4-methoxybenzyl)-2-oxo-2,3-dihydro-1H-indol-3-yl $]$ methyl $\}$ malonate}

(4a)<smiles>COC(C)CC1(N)C(=O)Nc2ccccc21</smiles>
4a was obtained from $\mathbf{2 a}(269 \mathrm{mg}, 0.68 \mathrm{mmol}), \mathrm{NaN}_{3}(89 \mathrm{mg}, 1.36 \mathrm{mmol})$, and $\mathrm{Et}_{3} \mathrm{~N} \cdot \mathrm{HCl}(187 \mathrm{mg}, 1.36 \mathrm{mmol})$ in DMSO $(1.35 \mathrm{~mL})$. Yield $103 \mathrm{mg}$ (35\%); yellow oil. $R_{f}=0.42$ (ethyl acetate/petroleum ether; 1:2).

${ }^{1} \mathrm{H}$ NMR $\left(\mathrm{CDCl}_{3}, 600 \mathrm{MHz}\right): \delta=2.68-2.75\left(\mathrm{~m}, 2 \mathrm{H}, \mathrm{CH}_{2}\right), 3.54(\mathrm{~s}, 3 \mathrm{H}$, $\left.\mathrm{OCH}_{3}\right), 3.71\left(\mathrm{dd},{ }^{3} \mathrm{~J}=7.1,{ }^{3} \mathrm{~J}=6.1 \mathrm{~Hz}, 1 \mathrm{H}, \mathrm{CH}\right), 3.74\left(\mathrm{~s}, 3 \mathrm{H}, \mathrm{OCH}_{3}\right), 3.76\left(\mathrm{~s}, 3 \mathrm{H}, \mathrm{OCH}_{3}\right), 4.83$ $\left(\mathrm{d},{ }^{2} J=15.6 \mathrm{~Hz}, 1 \mathrm{H}, \mathrm{NCH}_{2}\right), 4.86\left(\mathrm{~d},{ }^{2} J=15.6 \mathrm{~Hz}, 1 \mathrm{H}, \mathrm{NCH}_{2}\right), 6.79\left(\mathrm{~d},{ }^{3} J=7.8 \mathrm{~Hz}, 1 \mathrm{H}, \mathrm{Ar}\right)$, $6.86\left(\mathrm{~d},{ }^{3} J=8.8 \mathrm{~Hz}, 2 \mathrm{H}, \mathrm{Ar}\right), 7.08\left(\mathrm{ddd},{ }^{3} J=7.7,{ }^{3} J=7.5,{ }^{4} J=0.9 \mathrm{~Hz}, 1 \mathrm{H}\right.$, Ar $), 7.23-7.28(\mathrm{~m}$, $3 \mathrm{H}, \mathrm{Ar}), 7.34\left(\mathrm{ddd},{ }^{3} J=7.5,{ }^{4} \mathrm{~J}=1.2,{ }^{5} \mathrm{~J}=0.5 \mathrm{~Hz}, 1 \mathrm{H}, \mathrm{Ar}\right)$.

${ }^{13} \mathrm{C} \mathrm{NMR}\left(\mathrm{CDCl}_{3}, 150 \mathrm{MHz}\right): \delta=34.3\left(\mathrm{CH}_{2}\right), 43.5\left(\mathrm{NCH}_{2}\right), 46.7(\mathrm{CH}), 52.5\left(\mathrm{OCH}_{3}\right), 52.8$ $\left(\mathrm{OCH}_{3}\right), 55.1\left(\mathrm{OCH}_{3}\right), 65.3(\mathrm{C}), 110.0(\mathrm{CH}, \mathrm{Ar}), 114.2(2 \times \mathrm{CH}, \mathrm{Ar}), 123.1(\mathrm{CH}, \mathrm{Ar}), 124.4(\mathrm{CH}$, Ar), $125.8(\mathrm{C}, \mathrm{Ar}), 126.8(\mathrm{C}, \mathrm{Ar}), 128.5(2 \times \mathrm{CH}, \mathrm{Ar}), 130.4(\mathrm{CH}, \mathrm{Ar}), 142.4(\mathrm{C}, \mathrm{Ar}), 159.2(\mathrm{C}$, $\mathrm{Ar}), 168.7\left(\mathrm{CO}_{2} \mathrm{Me}\right), 168.8\left(\mathrm{CO}_{2} \mathrm{Me}\right), 173.2(\mathrm{C}=\mathrm{O})$.

HRMS (ESI-TOF) $m / z:[\mathrm{M}+\mathrm{H}]^{+}$calcd for $\mathrm{C}_{22} \mathrm{H}_{23} \mathrm{~N}_{4} \mathrm{O}_{6}{ }^{+} 439.1612$, found 439.1608 .

\section{Dimethyl \{[3-azido-1-(4-methoxybenzyl)-5-methyl-2-oxo-2,3-dihydro-1H-indol-3- yl]methyl\}malonate (4b)}

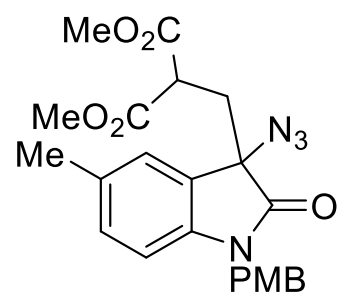

4b was obtained from $\mathbf{2 b}(181 \mathrm{mg}, 0.44 \mathrm{mmol}), \mathrm{NaN}_{3}(58 \mathrm{mg}, 0.89$ $\mathrm{mmol})$, and $\mathrm{Et}_{3} \mathrm{~N} \cdot \mathrm{HCl}(122 \mathrm{mg}, 0.89 \mathrm{mmol})$ in DMSO $(0.9 \mathrm{~mL})$. Yield 80 mg (40\%); yellow oil. $R_{f}=0.40$ (ethyl acetate/petroleum ether; $1: 2$ ).

${ }^{1} \mathrm{H}$ NMR $\left(\mathrm{CDCl}_{3}, 600 \mathrm{MHz}\right): \delta=2.31\left(\mathrm{~s}, 3 \mathrm{H}, \mathrm{CH}_{3}\right), 2.65-2.73(\mathrm{~m}, 2 \mathrm{H}$, $\left.\mathrm{CH}_{2}\right), 3.56$ (s, $\left.3 \mathrm{H}, \mathrm{OCH}_{3}\right), 3.71-3.75\left(\mathrm{~m}, 4 \mathrm{H}, \mathrm{CH}, \mathrm{OCH}_{3}\right), 3.76\left(\mathrm{~s}, 3 \mathrm{H}, \mathrm{OCH}_{3}\right), 4.79\left(\mathrm{~d},{ }^{2} \mathrm{~J}=15.5\right.$ $\left.\mathrm{Hz}, 1 \mathrm{H}, \mathrm{NCH}_{2}\right), 4.85\left(\mathrm{~d},{ }^{2} J=15.6 \mathrm{~Hz}, 1 \mathrm{H}, \mathrm{NCH}_{2}\right), 6.66\left(\mathrm{~d},{ }^{3} J=8.0 \mathrm{~Hz}, 1 \mathrm{H}, \mathrm{Ar}\right), 6.85\left(\mathrm{~d},{ }^{3} J=8.8\right.$ $\mathrm{Hz}, 2 \mathrm{H}, \mathrm{Ar}), 7.05\left(\mathrm{~d},{ }^{3} J=8.0 \mathrm{~Hz}, 1 \mathrm{H}, \mathrm{Ar}\right), 7.15(\mathrm{~s}, 1 \mathrm{H}, \mathrm{Ar}), 7.23\left(\mathrm{~d},{ }^{3} J=8.8 \mathrm{~Hz}, 2 \mathrm{H}, \mathrm{Ar}\right)$. 
${ }^{13} \mathrm{C} \mathrm{NMR}\left(\mathrm{CDCl}_{3}, 150 \mathrm{MHz}\right): \delta=20.9\left(\mathrm{CH}_{3}\right), 34.4\left(\mathrm{CH}_{2}\right), 43.5\left(\mathrm{NCH}_{2}\right), 46.7(\mathrm{CH}), 52.6$ $\left(\mathrm{OCH}_{3}\right), 52.8\left(\mathrm{OCH}_{3}\right), 55.1\left(\mathrm{OCH}_{3}\right), 65.5(\mathrm{C}), 109.8(\mathrm{CH}, \mathrm{Ar}), 114.1(2 \times \mathrm{CH}, \mathrm{Ar}), 125.0(\mathrm{CH}$, Ar), 125.9 (C, Ar), 126.9 (C, Ar), 128.5 (2×CH, Ar), 130.7 (CH, Ar), 132.9 (C, Ar), 139.9 (C, Ar), $159.1(\mathrm{C}, \mathrm{Ar}), 168.8\left(\mathrm{CO}_{2} \mathrm{Me}\right), 168.9\left(\mathrm{CO}_{2} \mathrm{Me}\right), 173.2(\mathrm{C}=\mathrm{O})$.

IR (film) 2101, 1734, 1718, 1700, 1610, 1507, $1437 \mathrm{~cm}^{-1}$.

HRMS (ESI-TOF) $m / z:[\mathrm{M}+\mathrm{H}]^{+}$calcd for $\mathrm{C}_{23} \mathrm{H}_{25} \mathrm{~N}_{4} \mathrm{O}_{6}{ }^{+} 453.1769$, found 453.1765 .

\section{Dimethyl $\{[3$-azido-1-methyl-2-oxo-2,3-dihydro-1H-indol-3-yl]methyl $\}$ malonate (4c)}

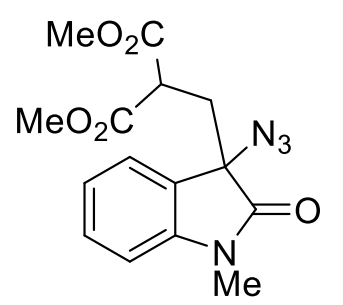

4c was obtained from $2 \mathbf{c}(183 \mathrm{mg}, 0.63 \mathrm{mmol}), \mathrm{NaN}_{3}(82 \mathrm{mg}, 1.27 \mathrm{mmol})$, and $\mathrm{Et}_{3} \mathrm{~N} \cdot \mathrm{HCl}(174 \mathrm{mg}, 1.27 \mathrm{mmol})$ in DMSO $(1.25 \mathrm{~mL})$. Yield $59 \mathrm{mg}$ (28\%); yellow oil. $R_{f}=0.30$ (ethyl acetate/petroleum ether; $1: 2$ ).

${ }^{1} \mathrm{H}$ NMR $\left(\mathrm{CDCl}_{3}, 600 \mathrm{MHz}\right): \delta=2.62-2.70\left(\mathrm{~m}, 2 \mathrm{H}, \mathrm{CH}_{2}\right), 3.20(\mathrm{~s}, 3 \mathrm{H}$, $\left.\mathrm{NCH}_{3}\right), 3.54\left(\mathrm{~s}, 3 \mathrm{H}, \mathrm{OCH}_{3}\right), 3.57\left(\mathrm{dd},{ }^{3} J=6.7,{ }^{3} \mathrm{~J}=6.7 \mathrm{~Hz}, 1 \mathrm{H}, \mathrm{CH}\right), 3.71\left(\mathrm{~s}, 3 \mathrm{H}, \mathrm{OCH}_{3}\right), 6.88$ $\left(\mathrm{d},{ }^{3} J=7.8 \mathrm{~Hz}, 1 \mathrm{H}, \mathrm{Ar}\right), 7.12\left(\mathrm{ddd},{ }^{3} J=7.6,{ }^{3} J=7.5,{ }^{4} J=0.9 \mathrm{~Hz}, 1 \mathrm{H}, \mathrm{Ar}\right), 7.31\left(\mathrm{ddd},{ }^{3} J=7.5,{ }^{4} J\right.$ $\left.=1.2,{ }^{5} \mathrm{~J}=0.5 \mathrm{~Hz}, 1 \mathrm{H}, \mathrm{Ar}\right), 7.37\left(\mathrm{ddd},{ }^{3} J=7.8,{ }^{3} \mathrm{~J}=7.6,{ }^{4} \mathrm{~J}=1.2 \mathrm{~Hz}, 1 \mathrm{H}, \mathrm{Ar}\right)$.

${ }^{13} \mathrm{C} \mathrm{NMR}\left(\mathrm{CDCl}_{3}, 150 \mathrm{MHz}\right): \delta=26.3\left(\mathrm{NCH}_{3}\right), 34.2\left(\mathrm{CH}_{2}\right), 46.8(\mathrm{CH}), 52.7\left(\mathrm{OCH}_{3}\right), 52.8$ $\left(\mathrm{OCH}_{3}\right), 65.2(\mathrm{C}), 108.9(\mathrm{CH}, \mathrm{Ar}), 123.2(\mathrm{CH}, \mathrm{Ar}), 124.3(\mathrm{CH}, \mathrm{Ar}), 125.6(\mathrm{C}, \mathrm{Ar}), 130.6(\mathrm{CH}$, $\mathrm{Ar}), 143.3(\mathrm{C}, \mathrm{Ar}), 168.67\left(\mathrm{CO}_{2} \mathrm{Me}\right), 168.71\left(\mathrm{CO}_{2} \mathrm{Me}\right), 173.1(\mathrm{C}=\mathrm{O})$.

HRMS (ESI-TOF) $m / z:[\mathrm{M}+\mathrm{H}]^{+}$calcd for $\mathrm{C}_{15} \mathrm{H}_{17} \mathrm{~N}_{4} \mathrm{O}_{5}{ }^{+} 333.1193$, found 333.1190.

\section{Methyl 3-(3-azido-1-methyl-2-oxo-2,3-dihydro-1H-indol-3-yl)propanoate (4c')}

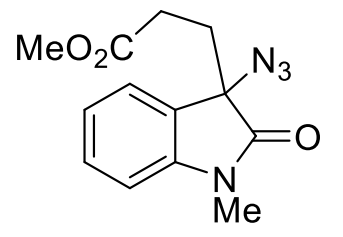

$\mathbf{4} \mathbf{c}^{\prime}$ was obtained as side-product in the synthesis of azide $\mathbf{4 c}$. Yield $13 \mathrm{mg}$

(7\%); yellowish oil. $R_{f}=0.35$ (ethyl acetate/petroleum ether; $1: 2$ ).

${ }^{1} \mathrm{H}$ NMR $\left(\mathrm{CDCl}_{3}, 600 \mathrm{MHz}\right): \delta=2.20-2.26\left(\mathrm{~m}, 1 \mathrm{H}, \mathrm{CH}_{2}\right), 2.30-2.41(\mathrm{~m}$, $\left.3 \mathrm{H}, \mathrm{CH}_{2}\right), 3.24\left(\mathrm{~s}, 3 \mathrm{H}, \mathrm{NCH}_{3}\right), 3.60\left(\mathrm{~s}, 3 \mathrm{H}, \mathrm{OCH}_{3}\right), 6.89\left(\mathrm{~d},{ }^{3} \mathrm{~J}=7.8 \mathrm{~Hz}, 1 \mathrm{H}, \mathrm{Ar}\right), 7.14$ (ddd, ${ }^{3} \mathrm{~J}=$ $7.6,{ }^{3} J=7.5,{ }^{4} J=0.9 \mathrm{~Hz}, 1 \mathrm{H}$, Ar), $7.32\left(\mathrm{ddd},{ }^{3} J=7.5,{ }^{4} J=1.2,{ }^{5} J=0.5 \mathrm{~Hz}, 1 \mathrm{H}\right.$, Ar), 7.39 (ddd, $\left.{ }^{3} J=7.8,{ }^{3} J=7.6,{ }^{4} J=1.2 \mathrm{~Hz}, 1 \mathrm{H}, \mathrm{Ar}\right)$. 
${ }^{13} \mathrm{C} \mathrm{NMR}\left(\mathrm{CDCl}_{3}, 150 \mathrm{MHz}\right): \delta=26.4\left(\mathrm{NCH}_{3}\right), 28.3\left(\mathrm{CH}_{2}\right), 30.8\left(\mathrm{CH}_{2}\right), 51.8\left(\mathrm{OCH}_{3}\right), 66.0(\mathrm{C})$, $108.9(\mathrm{CH}, \mathrm{Ar}), 123.4(\mathrm{CH}, \mathrm{Ar}), 124.1(\mathrm{CH}, \mathrm{Ar}), 126.2$ (C, Ar), $130.5(\mathrm{CH}, \mathrm{Ar}), 143.3(\mathrm{C}, \mathrm{Ar})$, $172.4(\mathrm{C}=\mathrm{O}), 173.7(\mathrm{C}=\mathrm{O})$.

HRMS (ESI-TOF) $m / z:[\mathrm{M}+\mathrm{H}]^{+}$calcd for $\mathrm{C}_{13} \mathrm{H}_{15} \mathrm{~N}_{4} \mathrm{O}_{3}{ }^{+} 275.1139$, found 275.1134 .

\section{Nucleophilic ring opening of cyclopropane 1a with the aniline}

A mixture of cyclopropane $1 \mathbf{a}(89 \mathrm{mg}, 0.25 \mathrm{mmol})$, aniline $(46 \mu \mathrm{L}, 0.50 \mathrm{mmol})$, and Lewis acid $(0.025 \mathrm{mmol})$ in toluene $(2 \mathrm{~mL})$ was heated under reflux (oil bath) for specified time. Then, the reaction mixture was concentrated under reduced pressure. Amines $\mathbf{5}$ and $\mathbf{6}$ were purified by column chromatography on silica gel.

\section{1-(4-Methoxybenzyl)-3-[2-(phenylamino)-2-phenylethyl]-1,3-dihydro-2H-indol-2-one (5)}

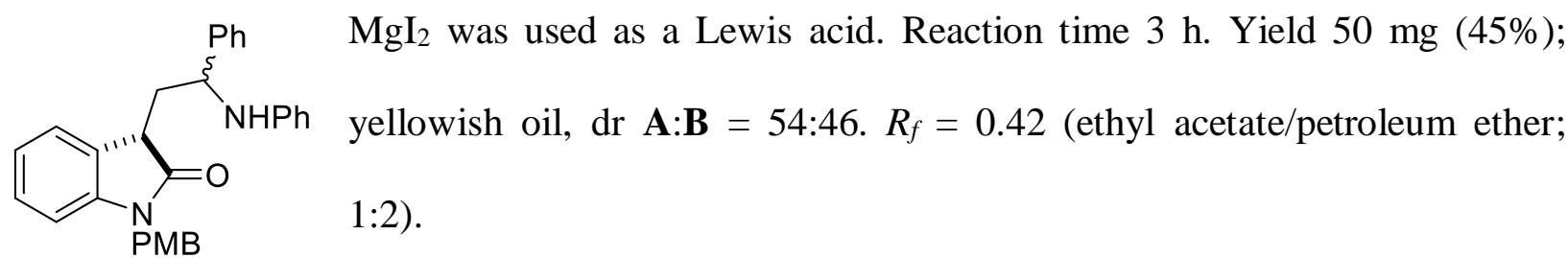

A: ${ }^{1} \mathrm{H}$ NMR $\left(\mathrm{CDCl}_{3}, 600 \mathrm{MHz}\right): \delta=2.56\left(\mathrm{ddd},{ }^{2} J=14.7,{ }^{3} J=6.6,{ }^{3} \mathrm{~J}=3.7 \mathrm{~Hz}, 1 \mathrm{H}, \mathrm{CH}_{2}\right), 2.63$ $\left(\mathrm{ddd},{ }^{2} J=14.7,{ }^{3} J=8.9,{ }^{3} J=3.7 \mathrm{~Hz}, 1 \mathrm{H}, \mathrm{CH}_{2}\right), 3.69-3.71(\mathrm{~m}, 1 \mathrm{H}, \mathrm{CH}), 3.73\left(\mathrm{~s}, 3 \mathrm{H}, \mathrm{OCH}_{3}\right)$, $4.57\left(\mathrm{dd},{ }^{3} J=8.9,{ }^{3} J=3.7 \mathrm{~Hz}, 1 \mathrm{H}, \mathrm{NCH}\right), 4.76\left(\mathrm{~d},{ }^{2} J=15.5 \mathrm{~Hz}, 1 \mathrm{H}, \mathrm{NCH}_{2}\right), 4.82\left(\mathrm{~d},{ }^{2} J=15.5\right.$ $\mathrm{Hz}, 1 \mathrm{H}, \mathrm{NCH}_{2}$ ), 4.89 (br. s, 1H, NH), 6.39-6.42 (m, 2H, Ar), 6.61 (t, $\left.{ }^{3} \mathrm{~J}=7.3 \mathrm{~Hz}, 1 \mathrm{H}, \mathrm{Ar}\right), 6.68$ $\left(\mathrm{d},{ }^{3} J=8.7 \mathrm{~Hz}, 2 \mathrm{H}, \mathrm{Ar}\right), 7.02-7.05(\mathrm{~m}, 2 \mathrm{H}, \mathrm{Ar}), 7.07-7.08(\mathrm{~m}, 1 \mathrm{H}, \mathrm{Ar}), 7.14-7.22(\mathrm{~m}, 5 \mathrm{H}, \mathrm{Ar})$, 7.22-7.26 (m, 1H, Ar), $7.29\left(\mathrm{~d},{ }^{3} J=7.4 \mathrm{~Hz}, 1 \mathrm{H}, \mathrm{Ar}\right), 7.31-7.36(\mathrm{~m}, 3 \mathrm{H}, \mathrm{Ar})$.

${ }^{13} \mathrm{C} \mathrm{NMR}\left(\mathrm{CDCl}_{3}, 150 \mathrm{MHz}\right): \delta=38.4\left(\mathrm{CH}_{2}\right), 43.3\left(\mathrm{NCH}_{2}\right), 43.5(\mathrm{CH}), 55.0(\mathrm{NCH}), 55.17$ $\left(\mathrm{OCH}_{3}\right), 109.4(\mathrm{CH}, \mathrm{Ar}), 113.5(2 \times \mathrm{CH}, \mathrm{Ar}), 114.1(2 \times \mathrm{CH}, \mathrm{Ar}), 117.1(\mathrm{CH}, \mathrm{Ar}), 122.5(\mathrm{CH}, \mathrm{Ar})$, $123.3(\mathrm{CH}, \mathrm{Ar}), 126.2(2 \times \mathrm{CH}, \mathrm{Ar}), 127.1(\mathrm{CH}, \mathrm{Ar}), 127.6(\mathrm{C}, \mathrm{Ar}), 127.98(\mathrm{CH}, \mathrm{Ar}), 128.2(\mathrm{C}$, Ar), $128.47(2 \times \mathrm{CH}, \mathrm{Ar}), 128.69(2 \times \mathrm{CH}, \mathrm{Ar}), 129.0(2 \times \mathrm{CH}, \mathrm{Ar}), 143.4(\mathrm{C}, \mathrm{Ar}), 143.6(\mathrm{C}, \mathrm{Ar})$, $146.9(\mathrm{C}, \mathrm{Ar}), 158.9(\mathrm{C}, \mathrm{Ar}), 178.5(\mathrm{C}=\mathrm{O})$. 
B: ${ }^{1} \mathrm{H}$ NMR $\left(\mathrm{CDCl}_{3}, 600 \mathrm{MHz}\right): \delta=2.32-2.37\left(\mathrm{~m}, 1 \mathrm{H}, \mathrm{CH}_{2}\right), 2.43\left(\mathrm{ddd},{ }^{2} J=14.5,{ }^{3} J=9.8,{ }^{3} J=\right.$ $\left.6.2 \mathrm{~Hz}, 1 \mathrm{H}, \mathrm{CH}_{2}\right), 3.71-3.75(\mathrm{~m}, 1 \mathrm{H}, \mathrm{CH}), 3.76\left(\mathrm{~s}, 3 \mathrm{H}, \mathrm{OCH}_{3}\right), 4.77\left(\mathrm{~d},{ }^{2} \mathrm{~J}=15.5 \mathrm{~Hz}, 1 \mathrm{H}\right.$, $\left.\mathrm{NCH}_{2}\right), 4.81-4.83(\mathrm{~m}, 1 \mathrm{H}, \mathrm{NCH}), 4.84\left(\mathrm{~d},{ }^{2} J=15.5 \mathrm{~Hz}, 1 \mathrm{H}, \mathrm{NCH}_{2}\right), 4.89$ (br. s, 1H, NH), 6.49$6.51(\mathrm{~m}, 2 \mathrm{H}, \mathrm{Ar}), 6.63-6.66(\mathrm{~m}, 2 \mathrm{H}, \mathrm{Ar}), 6.74\left(\mathrm{~d},{ }^{3} \mathrm{~J}=7.8 \mathrm{~Hz}, 1 \mathrm{H}, \mathrm{Ar}\right), 6.78\left(\mathrm{~d},{ }^{3} \mathrm{~J}=8.7 \mathrm{~Hz}, 2 \mathrm{H}\right.$, Ar), 7.04-7.07 (m, 1H, Ar), 7.08-7.11 (m, 2H, Ar), 7.14-7.22 (m, 2H, Ar), 7.22-7.26 (m, 1H, Ar), 7.31-7.36 (m, 3H, Ar), 7.42-7.45 (m, 2H, Ar).

${ }^{13} \mathrm{C} \mathrm{NMR}\left(\mathrm{CDCl}_{3}, 150 \mathrm{MHz}\right): \delta=39.7\left(\mathrm{CH}_{2}\right), 43.2\left(\mathrm{NCH}_{2}\right), 43.7(\mathrm{CH}), 55.20\left(\mathrm{OCH}_{3}\right), 56.7$ $(\mathrm{NCH}), 109.3(\mathrm{CH}, \mathrm{Ar}), 113.3(2 \times \mathrm{CH}, \mathrm{Ar}), 114.2(2 \times \mathrm{CH}, \mathrm{Ar}), 117.2(\mathrm{CH}, \mathrm{Ar}), 122.6(\mathrm{CH}, \mathrm{Ar})$, 123.6 (CH, Ar), $126.4(2 \times \mathrm{CH}, \mathrm{Ar}), 127.2(\mathrm{CH}, \mathrm{Ar}), 127.7(\mathrm{C}, \mathrm{Ar}), 128.01(\mathrm{CH}, \mathrm{Ar}), 128.46$ (2×CH, Ar), $128.67(2 \times \mathrm{CH}, \mathrm{Ar}), 129.1(2 \times \mathrm{CH}, \mathrm{Ar}), 129.2(\mathrm{C}, \mathrm{Ar}), 143.2(\mathrm{C}, \mathrm{Ar}), 143.5(\mathrm{C}, \mathrm{Ar})$, $147.1(\mathrm{C}, \mathrm{Ar}), 159.0(\mathrm{C}, \mathrm{Ar}), 177.9(\mathrm{C}=\mathrm{O})$.

HRMS (ESI-TOF) $m / z:[\mathrm{M}+\mathrm{H}]^{+}$calcd for $\mathrm{C}_{30} \mathrm{H}_{29} \mathrm{~N}_{2} \mathrm{O}_{2}{ }^{+} 449.2224$, found 449.2208.

\section{3-Hydroxy-1-(4-methoxybenzyl)-3-[2-(phenylamino)-2-phenylethyl]-1,3-dihydro-2H-indol-}

2-one (6)

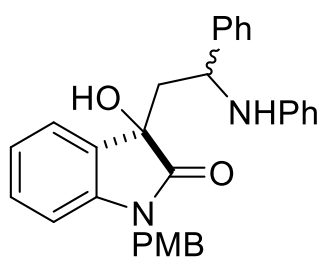

$\mathrm{Sc}(\mathrm{OTf})_{3}$ was used as a Lewis acid. Reaction time $12 \mathrm{~h}$. Yield $34 \mathrm{mg}$ (29\%); yellowish oil, dr A:B $=80: 20 . R_{f}=0.20$ (ethyl acetate/petroleum ether; $1: 2)$.

A: ${ }^{1} \mathrm{H} \mathrm{NMR}\left(\mathrm{CDCl}_{3}, 600 \mathrm{MHz}\right): \delta=2.25\left(\mathrm{dd},{ }^{2} J=14.7,{ }^{3} J=3.9 \mathrm{~Hz}, 1 \mathrm{H}, \mathrm{CH}_{2}\right), 2.58\left(\mathrm{dd},{ }^{2} J=\right.$ $\left.14.7,{ }^{3} \mathrm{~J}=10.8 \mathrm{~Hz}, 1 \mathrm{H}, \mathrm{CH}_{2}\right), 3.75\left(\mathrm{~s}, 3 \mathrm{H}, \mathrm{OCH}_{3}\right), 4.63-4.67\left(\mathrm{~m}, 2 \mathrm{H}, \mathrm{NCH}+\mathrm{NCH}_{2}\right), 4.74$ (br. s, $1 \mathrm{H}, \mathrm{NH}), 4.79\left(\mathrm{~d},{ }^{2} \mathrm{~J}=15.6 \mathrm{~Hz}, 1 \mathrm{H}, \mathrm{NCH}_{2}\right), 6.39-6.42(\mathrm{~m}, 2 \mathrm{H}, \mathrm{Ar}), 6.68\left(\mathrm{t},{ }^{3} \mathrm{~J}=7.4 \mathrm{~Hz}, 1 \mathrm{H}, \mathrm{Ar}\right)$, 6.72-6.75 (m, 3H, Ar), 7.05-7.08 (m, 2H, Ar), 7.10-7.14 (m, 3H, Ar), 7.25-7.30 (m, 6H, Ar), 7.47 (ddd, ${ }^{3} J=7.4,{ }^{4} J=1.2,{ }^{5} J=0.5 \mathrm{~Hz}, 1 \mathrm{H}$, Ar).

${ }^{13} \mathrm{C} \mathrm{NMR}\left(\mathrm{CDCl}_{3}, 150 \mathrm{MHz}\right): \delta=43.4\left(\mathrm{NCH}_{2}\right), 45.6\left(\mathrm{CH}_{2}\right), 55.2(\mathrm{NCH}), 55.5\left(\mathrm{OCH}_{3}\right), 76.1(\mathrm{C})$, $110.0(\mathrm{CH}), 114.1(2 \times \mathrm{CH}, \mathrm{Ar}), 114.2(2 \times \mathrm{CH}, \mathrm{Ar}), 118.0(\mathrm{CH}, \mathrm{Ar}), 123.3(\mathrm{CH}, \mathrm{Ar}), 124.0(\mathrm{CH}$, Ar), $126.2(2 \times \mathrm{CH}, \mathrm{Ar}), 127.2(\mathrm{C}, \mathrm{Ar}), 127.3(\mathrm{CH}, \mathrm{Ar}), 128.5(2 \times \mathrm{CH}, \mathrm{Ar}), 128.7(2 \times \mathrm{CH}, \mathrm{Ar})$, 
$129.1(2 \times \mathrm{CH}, \mathrm{Ar}), 129.95$ (CH, Ar), 130.04 (C, Ar), 142.6 (C, Ar), 143.1 (C, Ar), 146.4 (C, Ar), 159.1 (C, Ar), $177.4(\mathrm{C}=\mathrm{O})$.

HRMS (ESI-TOF) $m / z:[\mathrm{M}+\mathrm{H}]^{+}$calcd for $\mathrm{C}_{30} \mathrm{H}_{29} \mathrm{~N}_{2} \mathrm{O}_{3}{ }^{+} 465.2173$, found 465.2124.

\section{General procedure for the nucleophilic ring opening of cyclopropanes 2 with anilines}

A mixture of cyclopropane 2 (1.0 equiv), aromatic amine (2.0 equiv), and $\mathrm{Ni}\left(\mathrm{ClO}_{4}\right)_{2} \cdot 6 \mathrm{H}_{2} \mathrm{O}(0.1$ equiv) in $\mathrm{CH}_{2} \mathrm{Cl}_{2}\left(2 \mathrm{M}\right.$ ) was stirred at $40{ }^{\circ} \mathrm{C}$ (oil bath) for specified time. Then, the reaction mixture was concentrated under reduced pressure. Amine 7 was purified by column chromatography on silica gel.

\section{Dimethyl \{[1-(4-methoxybenzyl)-2-oxo-3-(phenylamino)-2,3-dihydro-1H-indol-3-}

yl]methyl\}malonate (7a)

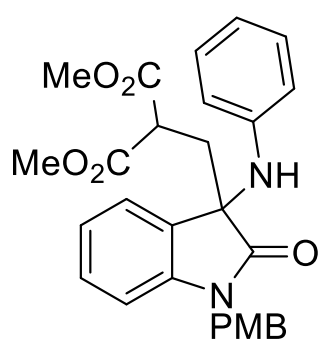

7a was obtained from $\mathbf{2 a}(186 \mathrm{mg}, 0.47 \mathrm{mmol})$, aniline $(86 \mu \mathrm{L}, 0.94 \mathrm{mmol})$, and $\mathrm{Ni}\left(\mathrm{ClO}_{4}\right)_{2} \cdot 6 \mathrm{H}_{2} \mathrm{O}(17 \mathrm{mg}, 0.05 \mathrm{mmol})$ in $\mathrm{CH}_{2} \mathrm{Cl}_{2}(235 \mu \mathrm{L})$; reaction time 4.5 h. Yield $179 \mathrm{mg}(78 \%)$; yellow oil. $R_{f}=0.32$ (ethyl acetate/petroleum

${ }^{1} \mathrm{H} \mathrm{NMR}\left(\mathrm{CDCl}_{3}, 600 \mathrm{MHz}\right): \delta=2.59\left(\mathrm{dd},{ }^{2} J=14.5,{ }^{3} J=5.4 \mathrm{~Hz}, 1 \mathrm{H}, \mathrm{CH}_{2}\right), 2.71\left(\mathrm{dd},{ }^{2} J=14.5\right.$, $\left.{ }^{3} J=7.4 \mathrm{~Hz}, 1 \mathrm{H}, \mathrm{CH}_{2}\right), 3.67\left(\mathrm{~s}, 3 \mathrm{H}, \mathrm{OCH}_{3}\right), 3.74\left(\mathrm{~s}, 3 \mathrm{H}, \mathrm{OCH}_{3}\right), 3.78\left(\mathrm{~s}, 3 \mathrm{H}, \mathrm{OCH}_{3}\right), 3.84\left(\mathrm{dd},{ }^{3} \boldsymbol{J}\right.$ $\left.=7.4,{ }^{3} J=5.4 \mathrm{~Hz}, 1 \mathrm{H}, \mathrm{CH}\right), 4.67\left(\mathrm{~d},{ }^{2} J=15.3 \mathrm{~Hz}, 1 \mathrm{H}, \mathrm{NCH}_{2}\right), 4.72($ br.s, $1 \mathrm{H}, \mathrm{NH}), 5.09\left(\mathrm{~d},{ }^{2} J=\right.$ $\left.15.3 \mathrm{~Hz}, 1 \mathrm{H}, \mathrm{NCH}_{2}\right), 6.18-6.22(\mathrm{~m}, 2 \mathrm{H}, \mathrm{Ar}), 6.69\left(\mathrm{t},{ }^{3} \mathrm{~J}=7.4 \mathrm{~Hz}, 1 \mathrm{H}, \mathrm{Ar}\right), 6.83\left(\mathrm{~d},{ }^{3} J=8.7 \mathrm{~Hz}\right.$, 2H, Ar), $6.85\left(\mathrm{~d},{ }^{3} J=7.9 \mathrm{~Hz}, 1 \mathrm{H}, \mathrm{Ar}\right), 6.92\left(\mathrm{dd},{ }^{3} J=8.6,{ }^{3} J=7.4 \mathrm{~Hz}, 2 \mathrm{H}\right.$, Ar), $7.03\left(\mathrm{ddd},{ }^{3} J=\right.$ $\left.7.6,{ }^{3} J=7.5,{ }^{4} J=0.8 \mathrm{~Hz}, 1 \mathrm{H}, \mathrm{Ar}\right), 7.19\left(\mathrm{~d},{ }^{3} J=8.7 \mathrm{~Hz}, 2 \mathrm{H}, \mathrm{Ar}\right), 7.23\left(\mathrm{ddd},{ }^{3} J=7.9,{ }^{3} J=7.6,{ }^{4} J\right.$ $=1.2 \mathrm{~Hz}, 1 \mathrm{H}, \mathrm{Ar}), 7.34\left(\mathrm{ddd},{ }^{3} J=7.5,{ }^{4} \mathrm{~J}=1.2,{ }^{5} \mathrm{~J}=0.6 \mathrm{~Hz}, 1 \mathrm{H}, \mathrm{Ar}\right)$.

${ }^{13} \mathrm{C} \mathrm{NMR}\left(\mathrm{CDCl}_{3}, 150 \mathrm{MHz}\right): \delta=38.0\left(\mathrm{CH}_{2}\right), 43.5\left(\mathrm{NCH}_{2}\right), 46.6(\mathrm{CH}), 52.7\left(\mathrm{OCH}_{3}\right), 52.9$ $\left(\mathrm{OCH}_{3}\right), 55.1\left(\mathrm{OCH}_{3}\right), 63.4(\mathrm{C}), 109.9(\mathrm{CH}, \mathrm{Ar}), 114.0(2 \times \mathrm{CH}, \mathrm{Ar}), 115.8(2 \times \mathrm{CH}, \mathrm{Ar}), 119.5$ $(\mathrm{CH}, \mathrm{Ar}), 122.9(\mathrm{CH}, \mathrm{Ar}), 124.1(\mathrm{CH}, \mathrm{Ar}), 127.3$ (C, Ar), 128.7 (C, Ar), $128.8(2 \times \mathrm{CH}, \mathrm{Ar})$, 
$129.0(2 \times \mathrm{CH}, \mathrm{Ar}), 129.2(\mathrm{CH}, \mathrm{Ar}), 141.8(\mathrm{C}, \mathrm{Ar}), 144.8$ (C, Ar), $159.0(\mathrm{C}, \mathrm{Ar}), 169.47\left(\mathrm{CO}_{2} \mathrm{Me}\right)$, $169.52\left(\mathrm{CO}_{2} \mathrm{Me}\right), 176.8(\mathrm{C}=\mathrm{O})$.

HRMS (ESI-TOF) $m / z:[\mathrm{M}+\mathrm{H}]^{+}$calcd for $\mathrm{C}_{28} \mathrm{H}_{29} \mathrm{~N}_{2} \mathrm{O}_{6}{ }^{+} 489.2020$, found 489.2020.

\section{Dimethyl \{[1-(4-methoxybenzyl)-5-methyl-2-oxo-3-(phenylamino)-2,3-dihydro-1H-indol-3-} yl]methyl\}malonate (7b)

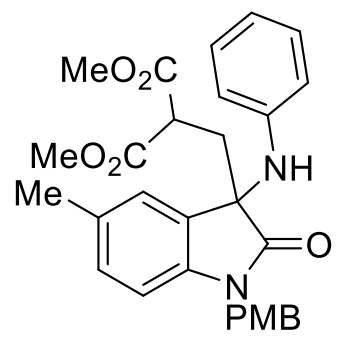

7b was obtained from $\mathbf{2 b}(187 \mathrm{mg}, 0.46 \mathrm{mmol})$, aniline $(89 \mu \mathrm{L}, 0.91$ mmol), and $\mathrm{Ni}\left(\mathrm{ClO}_{4}\right)_{2} \cdot 6 \mathrm{H}_{2} \mathrm{O}(17 \mathrm{mg}, 0.05 \mathrm{mmol})$ in $\mathrm{CH}_{2} \mathrm{Cl}_{2}(230 \mu \mathrm{L})$; reaction time $4.5 \mathrm{~h}$. Yield $167 \mathrm{mg}$ (73\%); colorless oil. $R_{f}=0.20$ (ethyl acetate/petroleum ether; $1: 2)$.

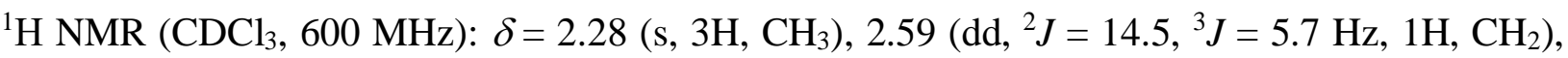
$2.68\left(\mathrm{dd},{ }^{2} J=14.5,{ }^{3} \mathrm{~J}=7.1 \mathrm{~Hz}, 1 \mathrm{H}, \mathrm{CH}_{2}\right), 3.68\left(\mathrm{~s}, 3 \mathrm{H}, \mathrm{OCH}_{3}\right), 3.75\left(\mathrm{~s}, 3 \mathrm{H}, \mathrm{OCH}_{3}\right), 3.78(\mathrm{~s}, 3 \mathrm{H}$, $\left.\mathrm{OCH}_{3}\right), 3.86\left(\mathrm{dd},{ }^{3} J=7.1,{ }^{3} J=5.7 \mathrm{~Hz}, 1 \mathrm{H}, \mathrm{CH}\right), 4.65\left(\mathrm{~d},{ }^{2} J=15.3 \mathrm{~Hz}, 1 \mathrm{H}, \mathrm{NCH}_{2}\right), 4.73$ (br.s, $1 \mathrm{H}, \mathrm{NH}), 5.08\left(\mathrm{~d},{ }^{2} J=15.3 \mathrm{~Hz}, 1 \mathrm{H}, \mathrm{NCH}_{2}\right), 6.20-6.22(\mathrm{~m}, 2 \mathrm{H}, \mathrm{Ar}), 6.69$ (t, $\left.{ }^{3} J=7.4 \mathrm{~Hz}, 1 \mathrm{H}, \mathrm{Ar}\right)$, $6.75\left(\mathrm{~d},{ }^{3} \mathrm{~J}=7.9 \mathrm{~Hz}, 1 \mathrm{H}, \mathrm{Ar}\right), 6.83\left(\mathrm{~d},{ }^{3} J=8.7 \mathrm{~Hz}, 2 \mathrm{H}, \mathrm{Ar}\right), 6.93\left(\mathrm{dd},{ }^{3} J=8.5,{ }^{3} J=7.4 \mathrm{~Hz}, 2 \mathrm{H}\right.$, Ar), $7.03\left(\mathrm{dd},{ }^{3} J=7.9,{ }^{4} J=1.6 \mathrm{~Hz}, 1 \mathrm{H}, \mathrm{Ar}\right), 7.15$ (br.s, $\left.1 \mathrm{H}, \mathrm{Ar}\right), 7.20\left(\mathrm{~d},{ }^{3} J=8.7 \mathrm{~Hz}, 2 \mathrm{H}, \mathrm{Ar}\right)$.

${ }^{13} \mathrm{C}$ NMR $\left(\mathrm{CDCl}_{3}, 150 \mathrm{MHz}\right): \delta=20.9\left(\mathrm{CH}_{3}\right), 38.1\left(\mathrm{CH}_{2}\right), 43.5\left(\mathrm{NCH}_{2}\right), 46.6(\mathrm{CH}), 52.7$ $\left(\mathrm{OCH}_{3}\right), 52.8\left(\mathrm{OCH}_{3}\right), 55.1\left(\mathrm{OCH}_{3}\right), 63.4(\mathrm{C}), 109.6(\mathrm{CH}, \mathrm{Ar}), 114.0(2 \times \mathrm{CH}, \mathrm{Ar}), 115.6(2 \times \mathrm{CH}$, Ar), $119.3(\mathrm{CH}, \mathrm{Ar}), 124.8(\mathrm{CH}, \mathrm{Ar}), 127.5$ (C, Ar), 128.81 (2×CH, Ar), 128.84 (C, Ar), 129.0 (2×CH, Ar), 129.5 (CH, Ar), 132.4 (C, Ar), 139.4 (C, Ar), 144.9 (C, Ar), 159.0 (C, Ar), 169.4 $\left(\mathrm{CO}_{2} \mathrm{Me}\right), 169.6\left(\mathrm{CO}_{2} \mathrm{Me}\right), 176.7(\mathrm{C}=\mathrm{O})$.

HRMS (ESI-TOF) $m / z:[\mathrm{M}+\mathrm{H}]^{+}$calcd for $\mathrm{C}_{29} \mathrm{H}_{31} \mathrm{~N}_{2} \mathrm{O}_{6}{ }^{+}$503.2177, found 503.2175.

\section{Dimethyl $\{[1$-methyl-2-oxo-3-(phenylamino)-2,3-dihydro-1H-indol-3-yl]methyl $\}$ malonate}

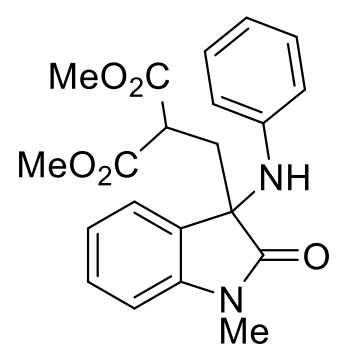

7c was obtained from $2 \mathbf{c}(123 \mathrm{mg}, 0.43 \mathrm{mmol})$, aniline $(77 \mu \mathrm{L}, 0.85 \mathrm{mmol})$, and $\mathrm{Ni}\left(\mathrm{ClO}_{4}\right)_{2} \cdot 6 \mathrm{H}_{2} \mathrm{O}(16 \mathrm{mg}, 0.04 \mathrm{mmol})$ in $\mathrm{CH}_{2} \mathrm{Cl}_{2}(215 \mu \mathrm{L})$; reaction 
time 4.5 h. Yield $128 \mathrm{mg}$ (79\%); yellowish oil. $R_{f}=0.17$ (ethyl acetate/petroleum ether; 1:2).

2-mmol-scale procedure: a mixture of cyclopropane $2 \mathrm{c}(578 \mathrm{mg}, 2.00 \mathrm{mmol})$, aniline $(365 \mu \mathrm{L}$, $4.00 \mathrm{mmol})$, and $\mathrm{Ni}\left(\mathrm{ClO}_{4}\right)_{2} \cdot 6 \mathrm{H}_{2} \mathrm{O}(73 \mathrm{mg}, 0.20 \mathrm{mmol})$ in $\mathrm{CH}_{2} \mathrm{Cl}_{2}(1.0 \mathrm{~mL})$ was stirred at $40{ }^{\circ} \mathrm{C}$ for $4.5 \mathrm{~h}$. Then, the reaction mixture was concentrated under reduced pressure. Amine 7c was purified by column chromatography on silica gel. Yield $641 \mathrm{mg}(84 \%)$.

${ }^{1} \mathrm{H} \mathrm{NMR}\left(\mathrm{CDCl}_{3}, 600 \mathrm{MHz}\right): \delta=2.55\left(\mathrm{dd},{ }^{2} J=14.5,{ }^{3} J=5.5 \mathrm{~Hz}, 1 \mathrm{H}, \mathrm{CH}_{2}\right), 2.66\left(\mathrm{dd},{ }^{2} J=14.5\right.$, $\left.{ }^{3} J=7.4 \mathrm{~Hz}, 1 \mathrm{H}, \mathrm{CH}_{2}\right), 3.25\left(\mathrm{~s}, 3 \mathrm{H}, \mathrm{NCH}_{3}\right), 3.64\left(\mathrm{~s}, 3 \mathrm{H}, \mathrm{OCH}_{3}\right), 3.71\left(\mathrm{~s}, 3 \mathrm{H}, \mathrm{OCH}_{3}\right), 3.73\left(\mathrm{dd},{ }^{3} \boldsymbol{J}\right.$ $\left.=7.4,{ }^{3} \mathrm{~J}=5.5 \mathrm{~Hz}, 1 \mathrm{H}, \mathrm{CH}\right), 4.69$ (br.s, $\left.1 \mathrm{H}, \mathrm{NH}\right), 6.16-6.19(\mathrm{~m}, 2 \mathrm{H}, \mathrm{Ar}), 6.64\left(\mathrm{t},{ }^{3} \mathrm{~J}=7.4 \mathrm{~Hz}, 1 \mathrm{H}\right.$, Ar), 6.91-6.96 (m, 3H, Ar), $7.06\left(\mathrm{dd},{ }^{3} J=7.6,{ }^{3} J=7.5 \mathrm{~Hz}, 1 \mathrm{H}\right.$, Ar), $7.31\left(\mathrm{~d},{ }^{3} J=7.5 \mathrm{~Hz}, 1 \mathrm{H}\right.$, Ar), $7.35\left(\mathrm{dd},{ }^{3} J=7.8,{ }^{3} \mathrm{~J}=7.6 \mathrm{~Hz}, 1 \mathrm{H}, \mathrm{Ar}\right)$.

${ }^{13} \mathrm{C} \mathrm{NMR}\left(\mathrm{CDCl}_{3}, 150 \mathrm{MHz}\right): \delta=26.3\left(\mathrm{NCH}_{3}\right), 38.0\left(\mathrm{CH}_{2}\right), 46.6(\mathrm{CH}), 52.7\left(\mathrm{OCH}_{3}\right), 52.8$ $\left(\mathrm{OCH}_{3}\right), 63.2(\mathrm{C}), 108.7(\mathrm{CH}, \mathrm{Ar}), 115.1(2 \times \mathrm{CH}, \mathrm{Ar}), 119.2(\mathrm{CH}, \mathrm{Ar}), 122.9(\mathrm{CH}, \mathrm{Ar}), 124.1$ (CH, Ar), 128.6 (C, Ar), 128.8 (2×CH, Ar), $129.4(\mathrm{CH}, \mathrm{Ar}), 142.7$ (C, Ar), 144.8 (C, Ar), 169.38 $\left(\mathrm{CO}_{2} \mathrm{Me}\right), 169.39\left(\mathrm{CO}_{2} \mathrm{Me}\right), 176.8(\mathrm{C}=\mathrm{O})$.

IR (film) 1734, 17718, 1700, 1616, 1560, 1507, $1490 \mathrm{~cm}^{-1}$.

HRMS (ESI-TOF) $m / z:[\mathrm{M}+\mathrm{H}]^{+}$calcd for $\mathrm{C}_{21} \mathrm{H}_{23} \mathrm{~N}_{2} \mathrm{O}_{5}{ }^{+} 383.1601$, found 383.1602.

\section{Dimethyl \{[5-fluoro-1-(4-methoxybenzyl)-2-oxo-3-(phenylamino)-2,3-dihydro-1H-indol-3-}

\section{yl]methyl\}malonate (7d)}

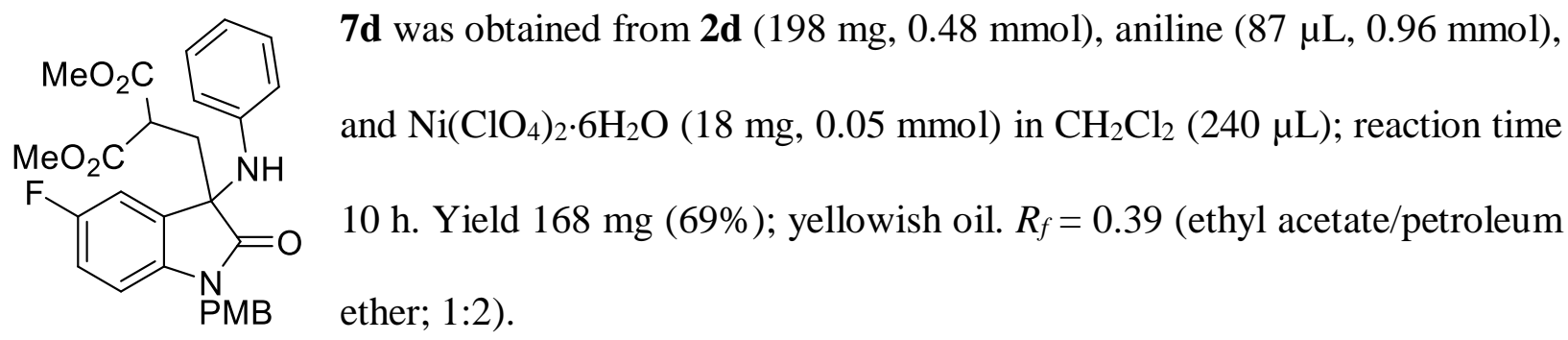

${ }^{1} \mathrm{H} \mathrm{NMR}\left(\mathrm{CDCl}_{3}, 600 \mathrm{MHz}\right): \delta=2.60\left(\mathrm{dd},{ }^{2} J=14.6,{ }^{3} J=5.8 \mathrm{~Hz}, 1 \mathrm{H}, \mathrm{CH}_{2}\right), 2.67\left(\mathrm{dd},{ }^{2} J=14.6\right.$, $\left.{ }^{3} J=7.1 \mathrm{~Hz}, 1 \mathrm{H}, \mathrm{CH}_{2}\right), 3.70\left(\mathrm{~s}, 3 \mathrm{H}, \mathrm{OCH}_{3}\right), 3.76\left(\mathrm{~s}, 3 \mathrm{H}, \mathrm{OCH}_{3}\right), 3.79\left(\mathrm{~s}, 3 \mathrm{H}, \mathrm{OCH}_{3}\right), 3.85\left(\mathrm{dd},{ }^{3} \boldsymbol{J}\right.$ $\left.=7.1,{ }^{3} J=5.8 \mathrm{~Hz}, 1 \mathrm{H}, \mathrm{CH}\right), 4.65\left(\mathrm{~d},{ }^{2} J=15.3 \mathrm{~Hz}, 1 \mathrm{H}, \mathrm{NCH}_{2}\right), 4.74$ (br.s, $\left.1 \mathrm{H}, \mathrm{NH}\right), 5.08\left(\mathrm{~d},{ }^{2} J=\right.$ $\left.15.3 \mathrm{~Hz}, 1 \mathrm{H}, \mathrm{NCH}_{2}\right), 6.19-6.21(\mathrm{~m}, 2 \mathrm{H}, \mathrm{Ar}), 6.72\left(\mathrm{t},{ }^{3} J=7.4 \mathrm{~Hz}, 1 \mathrm{H}, \mathrm{Ar}\right), 6.77\left(\mathrm{dd},{ }^{3} J=8.6,{ }^{4} J=\right.$ 
$4.0 \mathrm{~Hz}, 1 \mathrm{H}, \mathrm{Ar}), 6.83\left(\mathrm{~d},{ }^{3} J=8.8 \mathrm{~Hz}, 2 \mathrm{H}, \mathrm{Ar}\right), 6.91-6.96(\mathrm{~m}, 3 \mathrm{H}, \mathrm{Ar}), 7.09$ (dd, ${ }^{3} J=7.6,{ }^{4} J=2.5$ $\mathrm{Hz}, 1 \mathrm{H}, \mathrm{Ar}), 7.17\left(\mathrm{~d},{ }^{3} \mathrm{~J}=8.8 \mathrm{~Hz}, 2 \mathrm{H}, \mathrm{Ar}\right)$.

${ }^{13} \mathrm{C} \mathrm{NMR}\left(\mathrm{CDCl}_{3}, 150 \mathrm{MHz}\right): \delta=38.0\left(\mathrm{CH}_{2}\right), 43.7\left(\mathrm{NCH}_{2}\right), 46.5(\mathrm{CH}), 52.8\left(\mathrm{OCH}_{3}\right), 53.0$ $\left(\mathrm{OCH}_{3}\right), 55.2\left(\mathrm{OCH}_{3}\right), 63.7(\mathrm{C}), 110.6\left({ }^{3} J_{\mathrm{CF}}=8 \mathrm{~Hz}, \mathrm{CH}, \mathrm{Ar}\right), 112.2\left({ }^{2} J_{\mathrm{CF}}=25 \mathrm{~Hz}, \mathrm{CH}, \mathrm{Ar}\right)$, $114.1(2 \times \mathrm{CH}, \mathrm{Ar}), 115.6\left({ }^{2} J_{\mathrm{CF}}=24 \mathrm{~Hz}, \mathrm{CH}, \mathrm{Ar}\right), 115.8(2 \times \mathrm{CH}, \mathrm{Ar}), 119.8(\mathrm{CH}, \mathrm{Ar}), 127.1(\mathrm{C}$, Ar), $128.9(2 \times \mathrm{CH}, \mathrm{Ar}), 129.0(2 \times \mathrm{CH}, \mathrm{Ar}), 130.8\left({ }^{3} J_{\mathrm{CF}}=7 \mathrm{~Hz}, \mathrm{C}, \mathrm{Ar}\right), 137.7\left({ }^{4} J_{\mathrm{CF}}=1 \mathrm{~Hz}, \mathrm{C}, \mathrm{Ar}\right)$, $144.5(\mathrm{C}, \mathrm{Ar}), 159.2(\mathrm{C}, \mathrm{Ar}), 159.3\left({ }^{1} J_{\mathrm{CF}}=243 \mathrm{~Hz}, \mathrm{C}, \mathrm{Ar}\right), 169.3\left(C_{2} \mathrm{Me}\right), 169.5\left(\mathrm{CO}_{2} \mathrm{Me}\right)$, $176.6(\mathrm{C}=\mathrm{O})$.

HRMS (ESI-TOF) $m / z:[\mathrm{M}+\mathrm{H}]^{+}$calcd for $\mathrm{C}_{28} \mathrm{H}_{28} \mathrm{FN}_{2} \mathrm{O}_{6}{ }^{+}$507.1926, found 507.1928.

\section{Dimethyl \{[5-chloro-1-(4-methoxybenzyl)-2-oxo-3-(phenylamino)-2,3-dihydro-1H-indol-3-}

\section{yl]methyl\}malonate (7e)}

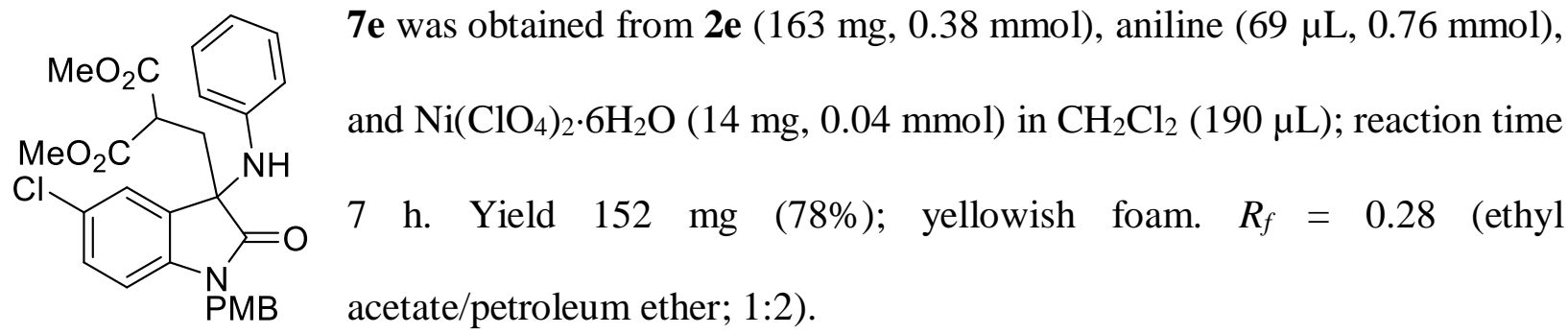

${ }^{1} \mathrm{H} \mathrm{NMR}\left(\mathrm{CDCl}_{3}, 600 \mathrm{MHz}\right): \delta=2.62\left(\mathrm{dd},{ }^{2} J=14.6,{ }^{3} J=5.9 \mathrm{~Hz}, 1 \mathrm{H}, \mathrm{CH}_{2}\right), 2.67\left(\mathrm{dd},{ }^{2} J=14.6\right.$, $\left.{ }^{3} J=7.0 \mathrm{~Hz}, 1 \mathrm{H}, \mathrm{CH}_{2}\right), 3.70\left(\mathrm{~s}, 3 \mathrm{H}, \mathrm{OCH}_{3}\right), 3.77\left(\mathrm{~s}, 3 \mathrm{H}, \mathrm{OCH}_{3}\right), 3.79\left(\mathrm{~s}, 3 \mathrm{H}, \mathrm{OCH}_{3}\right), 3.84\left(\mathrm{dd},{ }^{3} J\right.$ $\left.=7.0,{ }^{3} J=5.9 \mathrm{~Hz}, 1 \mathrm{H}, \mathrm{CH}\right), 4.63\left(\mathrm{~d},{ }^{2} J=15.3 \mathrm{~Hz}, 1 \mathrm{H}, \mathrm{NCH}_{2}\right), 4.76$ (br.s, $\left.1 \mathrm{H}, \mathrm{NH}\right), 5.10\left(\mathrm{~d},{ }^{2} J=\right.$ $\left.15.3 \mathrm{~Hz}, 1 \mathrm{H}, \mathrm{NCH}_{2}\right), 6.18-6.21(\mathrm{~m}, 2 \mathrm{H}, \mathrm{Ar}), 6.72\left(\mathrm{t},{ }^{3} \mathrm{~J}=7.4 \mathrm{~Hz}, 1 \mathrm{H}, \mathrm{Ar}\right), 6.78\left(\mathrm{dd},{ }^{3} \mathrm{~J}=8.4 \mathrm{~Hz}\right.$, $1 \mathrm{H}, \mathrm{Ar}), 6.84\left(\mathrm{~d},{ }^{3} J=8.8 \mathrm{~Hz}, 2 \mathrm{H}, \mathrm{Ar}\right), 6.94\left(\mathrm{dd},{ }^{3} J=8.6,{ }^{3} J=7.4 \mathrm{~Hz}, 2 \mathrm{H}, \mathrm{Ar}\right), 7.17\left(\mathrm{~d},{ }^{3} J=8.8\right.$ $\mathrm{Hz}, 2 \mathrm{H}, \mathrm{Ar}), 7.21\left(\mathrm{dd},{ }^{3} J=8.4,{ }^{4} \mathrm{~J}=2.2 \mathrm{~Hz}, 1 \mathrm{H}, \mathrm{Ar}\right), 7.31\left(\mathrm{~d},{ }^{4} \mathrm{~J}=2.2 \mathrm{~Hz}, 1 \mathrm{H}, \mathrm{Ar}\right)$.

${ }^{13} \mathrm{C} \mathrm{NMR}\left(\mathrm{CDCl}_{3}, 150 \mathrm{MHz}\right): \delta=38.0\left(\mathrm{CH}_{2}\right), 43.6\left(\mathrm{NCH}_{2}\right), 46.4(\mathrm{CH}), 52.8\left(\mathrm{OCH}_{3}\right), 53.0$ $\left(\mathrm{OCH}_{3}\right), 55.1\left(\mathrm{OCH}_{3}\right), 63.4(\mathrm{C}), 110.9(\mathrm{CH}, \mathrm{Ar}), 114.1(2 \times \mathrm{CH}, \mathrm{Ar}), 115.6(2 \times \mathrm{CH}, \mathrm{Ar}), 119.7$ $(\mathrm{CH}, \mathrm{Ar}), 124.4(\mathrm{CH}, \mathrm{Ar}), 126.9(\mathrm{C}, \mathrm{Ar}), 128.4(\mathrm{C}, \mathrm{Ar}), 128.9(2 \times \mathrm{CH}, \mathrm{Ar}), 129.1(2 \times \mathrm{CH}, \mathrm{Ar})$, $129.3(\mathrm{CH}, \mathrm{Ar}), 130.7(\mathrm{C}, \mathrm{Ar}), 140.2$ (C, Ar), $144.4(\mathrm{C}, \mathrm{Ar}), 159.1(\mathrm{C}, \mathrm{Ar}), 169.2\left(\mathrm{CO}_{2} \mathrm{Me}\right)$, $169.5\left(\mathrm{CO}_{2} \mathrm{Me}\right), 176.4(\mathrm{C}=\mathrm{O})$. 
HRMS (ESI-TOF) $m / z:[\mathrm{M}+\mathrm{H}]^{+}$calcd for $\mathrm{C}_{28} \mathrm{H}_{28} \mathrm{ClN}_{2} \mathrm{O}_{6}{ }^{+} 523.1630$, found 523.1640.

\section{Dimethyl \{[5-bromo-1-(4-methoxybenzyl)-2-oxo-3-(phenylamino)-2,3-dihydro-1H-indol-3-} yl]methyl\}malonate (7f)

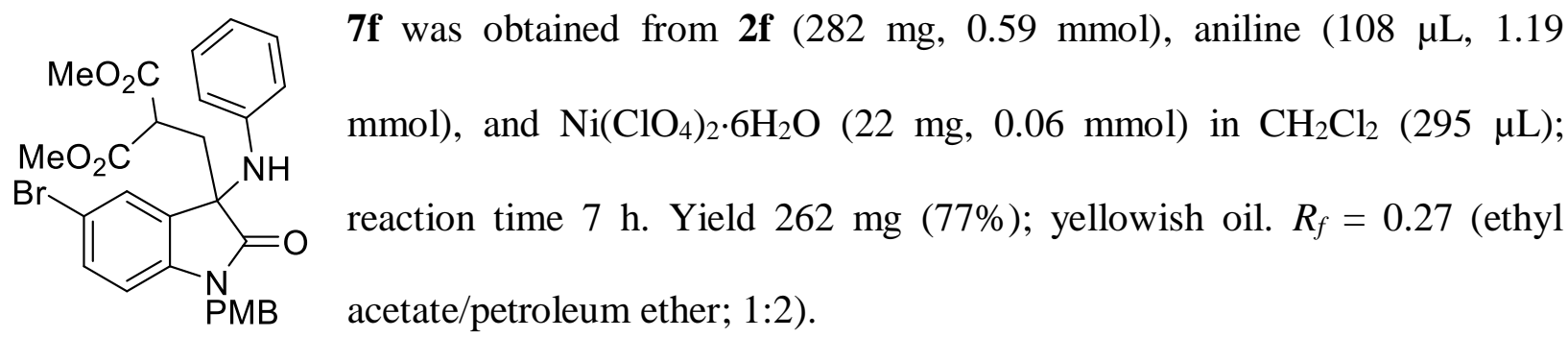

${ }^{1} \mathrm{H} \mathrm{NMR}\left(\mathrm{CDCl}_{3}, 600 \mathrm{MHz}\right): \delta=2.62\left(\mathrm{dd},{ }^{2} J=14.6,{ }^{3} J=5.9 \mathrm{~Hz}, 1 \mathrm{H}, \mathrm{CH}_{2}\right), 2.66\left(\mathrm{dd},{ }^{2} J=14.6\right.$, $\left.{ }^{3} J=6.9 \mathrm{~Hz}, 1 \mathrm{H}, \mathrm{CH}_{2}\right), 3.70\left(\mathrm{~s}, 3 \mathrm{H}, \mathrm{OCH}_{3}\right), 3.77\left(\mathrm{~s}, 3 \mathrm{H}, \mathrm{OCH}_{3}\right), 3.79\left(\mathrm{~s}, 3 \mathrm{H}, \mathrm{OCH}_{3}\right), 3.84\left(\mathrm{dd},{ }^{3} \boldsymbol{J}\right.$ $\left.=6.9,{ }^{3} J=5.9 \mathrm{~Hz}, 1 \mathrm{H}, \mathrm{CH}\right), 4.63\left(\mathrm{~d},{ }^{2} J=15.3 \mathrm{~Hz}, 1 \mathrm{H}, \mathrm{NCH}_{2}\right), 4.77$ (br.s, $\left.1 \mathrm{H}, \mathrm{NH}\right), 5.10\left(\mathrm{~d},{ }^{2} J=\right.$ $\left.15.3 \mathrm{~Hz}, 1 \mathrm{H}, \mathrm{NCH}_{2}\right), 6.18-6.20(\mathrm{~m}, 2 \mathrm{H}, \mathrm{Ar}), 6.72\left(\mathrm{t},{ }^{3} \mathrm{~J}=7.4 \mathrm{~Hz}, 1 \mathrm{H}, \mathrm{Ar}\right), 6.73\left(\mathrm{~d},{ }^{3} \mathrm{~J}=8.3 \mathrm{~Hz}\right.$, $1 \mathrm{H}, \mathrm{Ar}), 6.84\left(\mathrm{~d},{ }^{3} J=8.9 \mathrm{~Hz}, 2 \mathrm{H}, \mathrm{Ar}\right), 6.95\left(\mathrm{dd},{ }^{3} J=8.6,{ }^{3} J=7.4 \mathrm{~Hz}, 2 \mathrm{H}, \mathrm{Ar}\right), 7.17\left(\mathrm{~d},{ }^{3} J=8.9\right.$ $\mathrm{Hz}, 2 \mathrm{H}, \mathrm{Ar}), 7.35\left(\mathrm{dd},{ }^{3} \mathrm{~J}=8.3,{ }^{4} \mathrm{~J}=2.0 \mathrm{~Hz}, 1 \mathrm{H}, \mathrm{Ar}\right), 7.45\left(\mathrm{~d},{ }^{4} \mathrm{~J}=2.0 \mathrm{~Hz}, 1 \mathrm{H}, \mathrm{Ar}\right)$.

${ }^{13} \mathrm{C}$ NMR $\left(\mathrm{CDCl}_{3}, 150 \mathrm{MHz}\right): \delta=38.0\left(\mathrm{CH}_{2}\right), 43.6\left(\mathrm{NCH}_{2}\right), 46.4(\mathrm{CH}), 52.9\left(\mathrm{OCH}_{3}\right), 53.0$ $\left(\mathrm{OCH}_{3}\right), 55.1\left(\mathrm{OCH}_{3}\right), 63.3(\mathrm{C}), 111.4(\mathrm{CH}, \mathrm{Ar}), 114.0(2 \times \mathrm{CH}, \mathrm{Ar}), 115.6(2 \times \mathrm{CH}, \mathrm{Ar}), 115.8(\mathrm{C}$, Ar), $119.7(\mathrm{CH}, \mathrm{Ar}), 126.8(\mathrm{C}, \mathrm{Ar}), 127.1(\mathrm{CH}, \mathrm{Ar}), 128.9(2 \times \mathrm{CH}, \mathrm{Ar}), 129.0(2 \times \mathrm{CH}, \mathrm{Ar}), 131.1$ (C, Ar), $132.1(\mathrm{CH}, \mathrm{Ar}), 140.7$ (C, Ar), 144.4 (C, Ar), 159.1 (C, Ar), $169.2\left(\mathrm{CO}_{2} \mathrm{Me}\right), 169.5$ $\left(\mathrm{CO}_{2} \mathrm{Me}\right), 176.2(\mathrm{C}=\mathrm{O})$.

HRMS (ESI-TOF) $m / z:[\mathrm{M}+\mathrm{H}]^{+}$calcd for $\mathrm{C}_{28} \mathrm{H}_{28} \mathrm{BrN}_{2} \mathrm{O}_{6}{ }^{+} 567.1125$, found 567.1130.

Dimethyl (\{3-[(4-fluorophenyl)amino]-1-(4-methoxybenzyl)-2-oxo-2,3-dihydro-1H-indol-3yl\}methyl)malonate (7g)

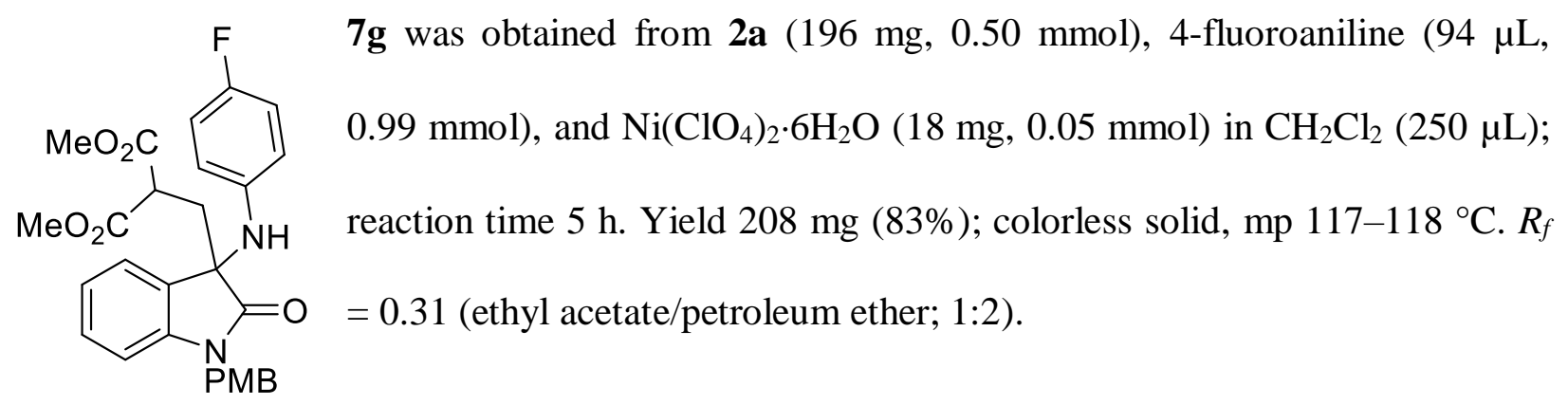


${ }^{1} \mathrm{H} \mathrm{NMR}\left(\mathrm{CDCl}_{3}, 600 \mathrm{MHz}\right): \delta=2.58\left(\mathrm{dd},{ }^{2} J=14.5,{ }^{3} J=5.4 \mathrm{~Hz}, 1 \mathrm{H}, \mathrm{CH}_{2}\right), 2.73\left(\mathrm{dd},{ }^{2} J=14.5\right.$, $\left.{ }^{3} J=7.7 \mathrm{~Hz}, 1 \mathrm{H}, \mathrm{CH}_{2}\right), 3.62\left(\mathrm{~s}, 3 \mathrm{H}, \mathrm{OCH}_{3}\right), 3.71\left(\mathrm{~s}, 3 \mathrm{H}, \mathrm{OCH}_{3}\right), 3.74-3.77\left(\mathrm{~m}, 4 \mathrm{H}, \mathrm{CH}, \mathrm{OCH}_{3}\right)$, 4.53 (br.s, $1 \mathrm{H}, \mathrm{NH}), 4.58\left(\mathrm{~d},{ }^{2} J=15.4 \mathrm{~Hz}, 1 \mathrm{H}, \mathrm{NCH}_{2}\right), 5.05\left(\mathrm{~d},{ }^{2} J=15.4 \mathrm{~Hz}, 1 \mathrm{H}, \mathrm{NCH}_{2}\right), 6.22$ $\left(\mathrm{dd},{ }^{3} J=8.9,{ }^{4} J=4.5 \mathrm{~Hz}, 2 \mathrm{H}, \mathrm{Ar}\right), 6.60\left(\mathrm{dd},{ }^{3} J=8.9,{ }^{3} J=8.6 \mathrm{~Hz}, 2 \mathrm{H}, \mathrm{Ar}\right), 6.77-6.80(\mathrm{~m}, 3 \mathrm{H}$, Ar), 7.02-7.06 (m, 3H, Ar), $7.21\left(\mathrm{ddd},{ }^{3} J=7.8,{ }^{3} J=7.7,{ }^{4} J=1.2 \mathrm{~Hz}, 1 \mathrm{H}\right.$, Ar) 7.37 (ddd, ${ }^{3} J=$ $\left.7.4,{ }^{4} \mathrm{~J}=1.2,{ }^{5} \mathrm{~J}=0.6 \mathrm{~Hz}, 1 \mathrm{H}, \mathrm{Ar}\right)$.

${ }^{13} \mathrm{C} \mathrm{NMR}\left(\mathrm{CDCl}_{3}, 150 \mathrm{MHz}\right): \delta=37.6\left(\mathrm{CH}_{2}\right), 43.3\left(\mathrm{NCH}_{2}\right), 46.7(\mathrm{CH}), 52.6\left(\mathrm{OCH}_{3}\right), 52.8$ $\left(\mathrm{OCH}_{3}\right), 55.0\left(\mathrm{OCH}_{3}\right), 64.4(\mathrm{C}), 109.9(\mathrm{CH}, \mathrm{Ar}), 113.9(2 \times \mathrm{CH}, \mathrm{Ar}), 115.3\left({ }^{2} J_{\mathrm{CF}}=22 \mathrm{~Hz}, 2 \times \mathrm{CH}\right.$, Ar), $118.9\left({ }^{3} J_{\mathrm{CF}}=7 \mathrm{~Hz}, 2 \times \mathrm{CH}, \mathrm{Ar}\right), 122.8(\mathrm{CH}, \mathrm{Ar}), 124.4(\mathrm{CH}, \mathrm{Ar}), 127.2(\mathrm{C}, \mathrm{Ar}), 128.3(\mathrm{C}$, Ar), $128.8(2 \times \mathrm{CH}, \mathrm{Ar}), 129.4(\mathrm{CH}, \mathrm{Ar}), 140.8\left({ }^{4} J_{\mathrm{CF}}=2 \mathrm{~Hz}, \mathrm{C}, \mathrm{Ar}\right), 142.1(\mathrm{C}, \mathrm{Ar}), 157.4\left({ }^{1} J_{\mathrm{CF}}=\right.$ $238 \mathrm{~Hz}, \mathrm{C}, \mathrm{Ar}), 159.0(\mathrm{C}, \mathrm{Ar}), 169.32\left(\mathrm{CO}_{2} \mathrm{Me}\right), 169.34\left(\mathrm{CO}_{2} \mathrm{Me}\right), 176.7(\mathrm{C}=\mathrm{O})$.

HRMS (ESI-TOF) $m / z:[\mathrm{M}+\mathrm{H}]^{+}$calcd for $\mathrm{C}_{28} \mathrm{H}_{28} \mathrm{FN}_{2} \mathrm{O}_{6}{ }^{+}$507.1926, found 507.1925.

\section{Dimethyl (\{3-[(4-chlorophenyl)amino]-1-(4-methoxybenzyl)-2-oxo-2,3-dihydro-1H-indol-3-}

\section{yl\}methyl)malonate (7h)}

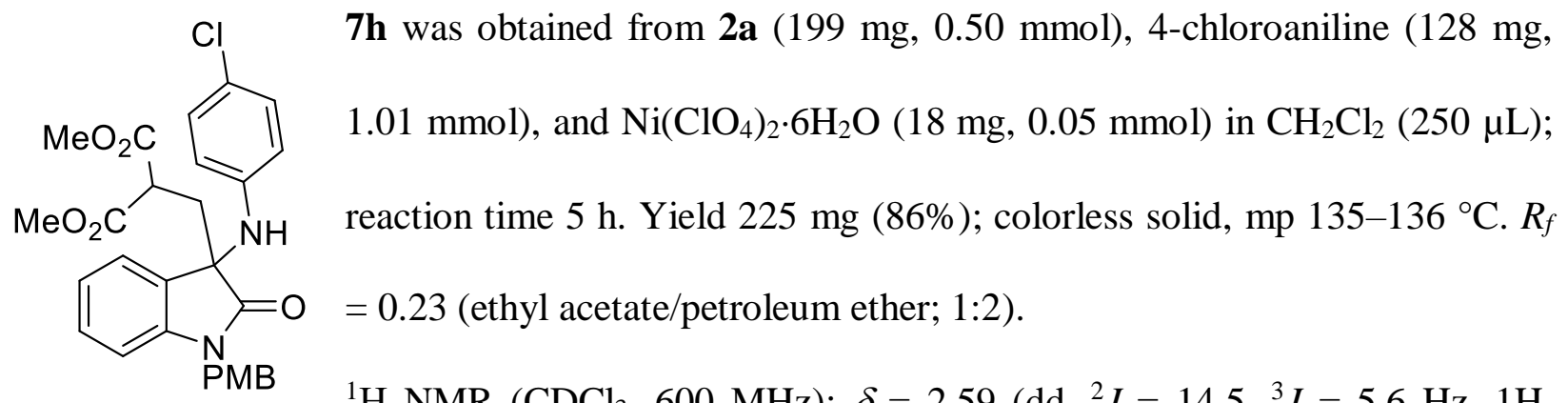

${ }^{1} \mathrm{H} \mathrm{NMR}\left(\mathrm{CDCl}_{3}, 600 \mathrm{MHz}\right): \delta=2.59\left(\mathrm{dd},{ }^{2} J=14.5,{ }^{3} J=5.6 \mathrm{~Hz}, 1 \mathrm{H}\right.$,

$\left.\mathrm{CH}_{2}\right), 2.70\left(\mathrm{dd},{ }^{2} J=14.5,{ }^{3} \mathrm{~J}=7.4 \mathrm{~Hz}, 1 \mathrm{H}, \mathrm{CH}_{2}\right), 3.64\left(\mathrm{~s}, 3 \mathrm{H}, \mathrm{OCH}_{3}\right), 3.73\left(\mathrm{~s}, 3 \mathrm{H}, \mathrm{OCH}_{3}\right), 3.78$ $\left(\mathrm{s}, 3 \mathrm{H}, \mathrm{OCH}_{3}\right), 3.79\left(\mathrm{dd},{ }^{3} J=7.4,{ }^{3} J=5.6 \mathrm{~Hz}, 1 \mathrm{H}, \mathrm{CH}\right), 4.62\left(\mathrm{~d},{ }^{2} J=15.3 \mathrm{~Hz}, 1 \mathrm{H}, \mathrm{NCH}_{2}\right), 4.81$ (br.s, 1H, NH), 5.09 (d, $\left.{ }^{2} J=15.3 \mathrm{~Hz}, 1 \mathrm{H}, \mathrm{NCH}_{2}\right), 6.12$ (d, $\left.{ }^{3} J=9.0 \mathrm{~Hz}, 2 \mathrm{H}, \mathrm{Ar}\right), 6.81-6.86$ (m, $5 \mathrm{H}, \mathrm{Ar}), 7.03\left(\mathrm{ddd},{ }^{3} J=7.6,{ }^{3} J=7.5,{ }^{4} \mathrm{~J}=0.8 \mathrm{~Hz}, 1 \mathrm{H}, \mathrm{Ar}\right), 7.12\left(\mathrm{~d},{ }^{3} J=8.8 \mathrm{~Hz}, 2 \mathrm{H}, \mathrm{Ar}\right), 7.23$ $\left(\mathrm{ddd},{ }^{3} J=7.8,{ }^{3} J=7.6,{ }^{4} J=1.3 \mathrm{~Hz}, 1 \mathrm{H}, \mathrm{Ar}\right), 7.32\left(\mathrm{ddd},{ }^{3} J=7.5,{ }^{4} \mathrm{~J}=1.3,{ }^{5} \mathrm{~J}=0.5 \mathrm{~Hz}, 1 \mathrm{H}, \mathrm{Ar}\right)$.

${ }^{13} \mathrm{C} \mathrm{NMR}\left(\mathrm{CDCl}_{3}, 150 \mathrm{MHz}\right): \delta=37.7\left(\mathrm{CH}_{2}\right), 43.4\left(\mathrm{NCH}_{2}\right), 46.5(\mathrm{CH}), 52.7\left(\mathrm{OCH}_{3}\right), 52.9$ $\left(\mathrm{OCH}_{3}\right), 55.1\left(\mathrm{OCH}_{3}\right), 63.6(\mathrm{C}), 109.9(\mathrm{CH}, \mathrm{Ar}), 113.9(2 \times \mathrm{CH}, \mathrm{Ar}), 117.5(2 \times \mathrm{CH}, \mathrm{Ar}), 123.0$ 
(CH, Ar), $124.2(\mathrm{CH}, \mathrm{Ar}), 124.5$ (C, Ar), 127.1 (C, Ar), 128.2 (C, Ar), $128.6(2 \times \mathrm{CH}, \mathrm{Ar}), 129.0$ (2×CH, Ar), $129.4(\mathrm{CH}, \mathrm{Ar}), 141.8(\mathrm{C}, \mathrm{Ar}), 143.4(\mathrm{C}, \mathrm{Ar}), 159.1(\mathrm{C}, \mathrm{Ar}), 169.3\left(\mathrm{CO}_{2} \mathrm{Me}\right), 169.4$ $\left(\mathrm{CO}_{2} \mathrm{Me}\right), 176.5(\mathrm{C}=\mathrm{O})$.

HRMS (ESI-TOF) $m / z:[\mathrm{M}+\mathrm{H}]^{+}$calcd for $\mathrm{C}_{28} \mathrm{H}_{28} \mathrm{ClN}_{2} \mathrm{O}_{6}{ }^{+}$523.1630, found 523.1635.

\section{Dimethyl (\{3-[(3-chlorophenyl)amino]-1-(4-methoxybenzyl)-2-oxo-2,3-dihydro-1H-indol-3-} yl\}methyl)malonate (7i)

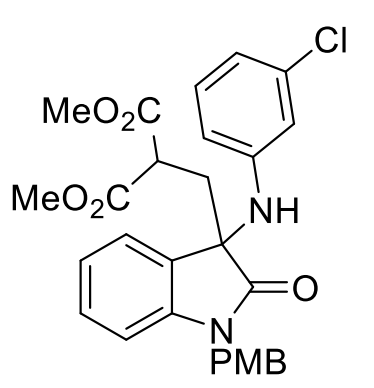

$7 \mathbf{i}$ was obtained from $\mathbf{2 a}(201 \mathrm{mg}, 0.51 \mathrm{mmol}), 3$-chloroaniline $(108 \mu \mathrm{L}$, $1.02 \mathrm{mmol})$, and $\mathrm{Ni}\left(\mathrm{ClO}_{4}\right)_{2} \cdot 6 \mathrm{H}_{2} \mathrm{O}(19 \mathrm{mg}, 0.05 \mathrm{mmol})$ in $\mathrm{CH}_{2} \mathrm{Cl}_{2}(255$ $\mu \mathrm{L}$ ); reaction time $5 \mathrm{~h}$. Yield $182 \mathrm{mg}(68 \%)$; white foam. $R_{f}=0.22$ (ethyl acetate/petroleum ether; $1: 2$ ).

${ }^{1} \mathrm{H} \mathrm{NMR}\left(\mathrm{CDCl}_{3}, 600 \mathrm{MHz}\right): \delta=2.60\left(\mathrm{dd},{ }^{2} J=14.6,{ }^{3} \mathrm{~J}=5.7 \mathrm{~Hz}, 1 \mathrm{H}, \mathrm{CH}_{2}\right), 2.66\left(\mathrm{dd},{ }^{2} J=14.6\right.$, $\left.{ }^{3} J=7.2 \mathrm{~Hz}, 1 \mathrm{H}, \mathrm{CH}_{2}\right), 3.66\left(\mathrm{~s}, 3 \mathrm{H}, \mathrm{OCH}_{3}\right), 3.74\left(\mathrm{~s}, 3 \mathrm{H}, \mathrm{OCH}_{3}\right), 3.77\left(\mathrm{~s}, 3 \mathrm{H}, \mathrm{OCH}_{3}\right), 3.84\left(\mathrm{dd},{ }^{3} \boldsymbol{J}\right.$ $\left.=7.2,{ }^{3} J=5.7 \mathrm{~Hz}, 1 \mathrm{H}, \mathrm{CH}\right), 4.69\left(\mathrm{~d},{ }^{2} J=15.3 \mathrm{~Hz}, 1 \mathrm{H}, \mathrm{NCH}_{2}\right), 4.99$ (br.s, $\left.1 \mathrm{H}, \mathrm{NH}\right), 5.10\left(\mathrm{~d},{ }^{2} J=\right.$ $\left.15.3 \mathrm{~Hz}, 1 \mathrm{H}, \mathrm{NCH}_{2}\right), 5.97\left(\mathrm{ddd},{ }^{3} J=8.2,{ }^{4} J=2.4,{ }^{4} J=0.9 \mathrm{~Hz}, 1 \mathrm{H}, \mathrm{Ar}\right), 6.23\left(\mathrm{dd},{ }^{4} J=2.4,{ }^{4} J=\right.$ $2.0 \mathrm{~Hz}, 1 \mathrm{H}, \mathrm{Ar}), 6.62\left(\mathrm{ddd},{ }^{3} J=8.0,{ }^{4} J=2.0,{ }^{4} J=0.9 \mathrm{~Hz}, 1 \mathrm{H}, \mathrm{Ar}\right), 6.78\left(\mathrm{dd},{ }^{3} J=8.2,{ }^{3} J=8.0\right.$ $\mathrm{Hz}, 1 \mathrm{H}, \mathrm{Ar}), 6.85\left(\mathrm{~d},{ }^{3} J=8.9 \mathrm{~Hz}, 2 \mathrm{H}, \mathrm{Ar}\right), 6.87\left(\mathrm{~d},{ }^{3} J=7.9 \mathrm{~Hz}, 1 \mathrm{H}, \mathrm{Ar}\right), 7.03\left(\mathrm{ddd},{ }^{3} J=7.6,{ }^{3} J=\right.$ $\left.7.5,{ }^{4} J=0.9 \mathrm{~Hz}, 1 \mathrm{H}, \mathrm{Ar}\right), 7.22-7.26(\mathrm{~m}, 3 \mathrm{H}, \mathrm{Ar}), 7.29\left(\mathrm{ddd},{ }^{3} J=7.5,{ }^{4} J=1.2,{ }^{5} J=0.5 \mathrm{~Hz}, 1 \mathrm{H}\right.$, $\operatorname{Ar})$.

${ }^{13} \mathrm{C} \mathrm{NMR}\left(\mathrm{CDCl}_{3}, 150 \mathrm{MHz}\right): \delta=38.0\left(\mathrm{CH}_{2}\right), 43.6\left(\mathrm{NCH}_{2}\right), 46.5(\mathrm{CH}), 52.8\left(\mathrm{OCH}_{3}\right), 53.0$ $\left(\mathrm{OCH}_{3}\right), 55.1\left(\mathrm{OCH}_{3}\right), 63.1(\mathrm{C}), 110.1(\mathrm{CH}, \mathrm{Ar}), 113.1(\mathrm{CH}, \mathrm{Ar}), 114.1(2 \times \mathrm{CH}, \mathrm{Ar}), 115.2(\mathrm{CH}$, Ar), $119.1(\mathrm{CH}, \mathrm{Ar}), 123.1(\mathrm{CH}, \mathrm{Ar}), 124.0(\mathrm{CH}, \mathrm{Ar}), 127.2(\mathrm{C}, \mathrm{Ar}), 128.2(\mathrm{C}, \mathrm{Ar}), 128.9$ (2×CH, Ar), $129.5(\mathrm{CH}, \mathrm{Ar}), 129.8(\mathrm{CH}, \mathrm{Ar}), 134.4(\mathrm{C}, \mathrm{Ar}), 141.6(\mathrm{C}, \mathrm{Ar}), 146.1$ (C, Ar), 159.1 $(\mathrm{C}, \mathrm{Ar}), 169.4\left(\mathrm{CO}_{2} \mathrm{Me}\right), 169.6\left(\mathrm{CO}_{2} \mathrm{Me}\right), 176.4(\mathrm{C}=\mathrm{O})$. HRMS (ESI-TOF) $m / z:[\mathrm{M}+\mathrm{H}]^{+}$calcd for $\mathrm{C}_{28} \mathrm{H}_{28} \mathrm{ClN}_{2} \mathrm{O}_{6}{ }^{+}$523.1630, found 523.1619. 


\section{Dimethyl (\{3-[(4-bromophenyl)amino]-1-(4-methoxybenzyl)-2-oxo-2,3-dihydro-1H-indol-3-}

yl\}methyl)malonate (7j)

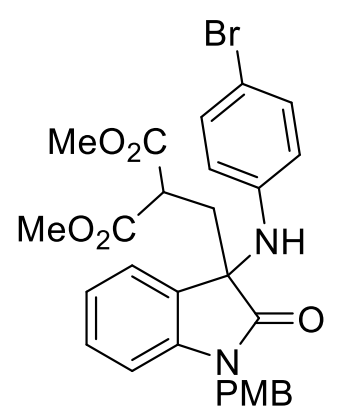

7j was obtained from $\mathbf{2 a}(113 \mathrm{mg}, 0.29 \mathrm{mmol})$, 4-bromoaniline (98 mg, $0.57 \mathrm{mmol})$, and $\mathrm{Ni}\left(\mathrm{ClO}_{4}\right)_{2} \cdot 6 \mathrm{H}_{2} \mathrm{O}(10 \mathrm{mg}, 0.03 \mathrm{mmol})$ in $\mathrm{CH}_{2} \mathrm{Cl}_{2}(145 \mu \mathrm{L})$; reaction time $4.5 \mathrm{~h}$. Yield $134 \mathrm{mg}(83 \%)$; colorless solid, mp $131-132{ }^{\circ} \mathrm{C}$. $R_{f}=0.27$ (ethyl acetate/petroleum ether; $1: 2$ ).

${ }^{1} \mathrm{H}$ NMR $\left(\mathrm{CDCl}_{3}, 600 \mathrm{MHz}\right): \delta=2.58\left(\mathrm{dd},{ }^{2} J=14.5,{ }^{3} J=5.5 \mathrm{~Hz}, 1 \mathrm{H}\right.$,

$\left.\mathrm{CH}_{2}\right), 2.68\left(\mathrm{dd},{ }^{2} J=14.5,{ }^{3} \mathrm{~J}=7.4 \mathrm{~Hz}, 1 \mathrm{H}, \mathrm{CH}_{2}\right), 3.65\left(\mathrm{~s}, 3 \mathrm{H}, \mathrm{OCH}_{3}\right), 3.74\left(\mathrm{~s}, 3 \mathrm{H}, \mathrm{OCH}_{3}\right), 3.77-$ $3.81\left(\mathrm{~m}, 4 \mathrm{H}, \mathrm{CH}, \mathrm{OCH}_{3}\right), 4.61\left(\mathrm{~d},{ }^{2} J=15.3 \mathrm{~Hz}, 1 \mathrm{H}, \mathrm{NCH}_{2}\right), 4.79$ (br.s, $\left.1 \mathrm{H}, \mathrm{NH}\right), 5.09\left(\mathrm{~d},{ }^{2} J=\right.$ $\left.15.3 \mathrm{~Hz}, 1 \mathrm{H}, \mathrm{NCH}_{2}\right), 6.06\left(\mathrm{~d},{ }^{3} J=9.0 \mathrm{~Hz}, 2 \mathrm{H}, \mathrm{Ar}\right), 6.83\left(\mathrm{~d},{ }^{3} J=8.8 \mathrm{~Hz}, 2 \mathrm{H}, \mathrm{Ar}\right), 6.85\left(\mathrm{~d},{ }^{3} J=\right.$ $7.8 \mathrm{~Hz}, 1 \mathrm{H}, \mathrm{Ar}), 6.97\left(\mathrm{~d},{ }^{3} \mathrm{~J}=9.0 \mathrm{~Hz}, 2 \mathrm{H}, \mathrm{Ar}\right), 7.04\left(\mathrm{ddd},{ }^{3} J=7.6,{ }^{3} J=7.5,{ }^{4} J=0.8 \mathrm{~Hz}, 1 \mathrm{H}, \mathrm{Ar}\right)$, $7.12\left(\mathrm{~d},{ }^{3} \mathrm{~J}=8.8 \mathrm{~Hz}, 2 \mathrm{H}, \mathrm{Ar}\right), 7.24\left(\mathrm{ddd},{ }^{3} \mathrm{~J}=7.8,{ }^{3} \mathrm{~J}=7.6,{ }^{4} \mathrm{~J}=1.2 \mathrm{~Hz}, 1 \mathrm{H}\right.$, Ar), 7.31 (ddd, ${ }^{3} J=$ $\left.7.5,{ }^{4} \mathrm{~J}=1.2,{ }^{5} \mathrm{~J}=0.5 \mathrm{~Hz}, 1 \mathrm{H}, \mathrm{Ar}\right)$.

${ }^{13} \mathrm{C} \mathrm{NMR}\left(\mathrm{CDCl}_{3}, 150 \mathrm{MHz}\right): \delta=37.7\left(\mathrm{CH}_{2}\right), 43.5\left(\mathrm{NCH}_{2}\right), 46.6(\mathrm{CH}), 52.8\left(\mathrm{OCH}_{3}\right), 53.0$ $\left(\mathrm{OCH}_{3}\right), 55.2\left(\mathrm{OCH}_{3}\right), 63.6(\mathrm{C}), 110.0(\mathrm{CH}, \mathrm{Ar}), 111.8(\mathrm{C}, \mathrm{Ar}), 114.0(2 \times \mathrm{CH}, \mathrm{Ar}), 117.9(2 \times \mathrm{CH}$, Ar), $123.0(\mathrm{CH}, \mathrm{Ar}), 124.2(\mathrm{CH}, \mathrm{Ar}), 127.2(\mathrm{C}, \mathrm{Ar}), 128.3(\mathrm{C}, \mathrm{Ar}), 129.0(2 \times \mathrm{CH}, \mathrm{Ar}), 129.5$ (CH, Ar), $131.6(2 \times \mathrm{CH}, \mathrm{Ar}), 141.8(\mathrm{C}, \mathrm{Ar}), 143.9(\mathrm{C}, \mathrm{Ar}), 159.1(\mathrm{C}, \mathrm{Ar}), 169.4\left(\mathrm{CO}_{2} \mathrm{Me}\right), 169.5$ $\left(\mathrm{CO}_{2} \mathrm{Me}\right), 176.5(\mathrm{C}=\mathrm{O})$.

HRMS (ESI-TOF) $m / z:[\mathrm{M}+\mathrm{H}]^{+}$calcd for $\mathrm{C}_{28} \mathrm{H}_{28} \mathrm{BrN}_{2} \mathrm{O}_{6}{ }^{+}$567.1125, found 567.1128.

\section{Dimethyl (\{3-1-(4-methoxybenzyl)-[(4-methylphenyl)amino]-2-oxo-2,3-dihydro-1H-indol-3-} yl\}methyl)malonate (7k)

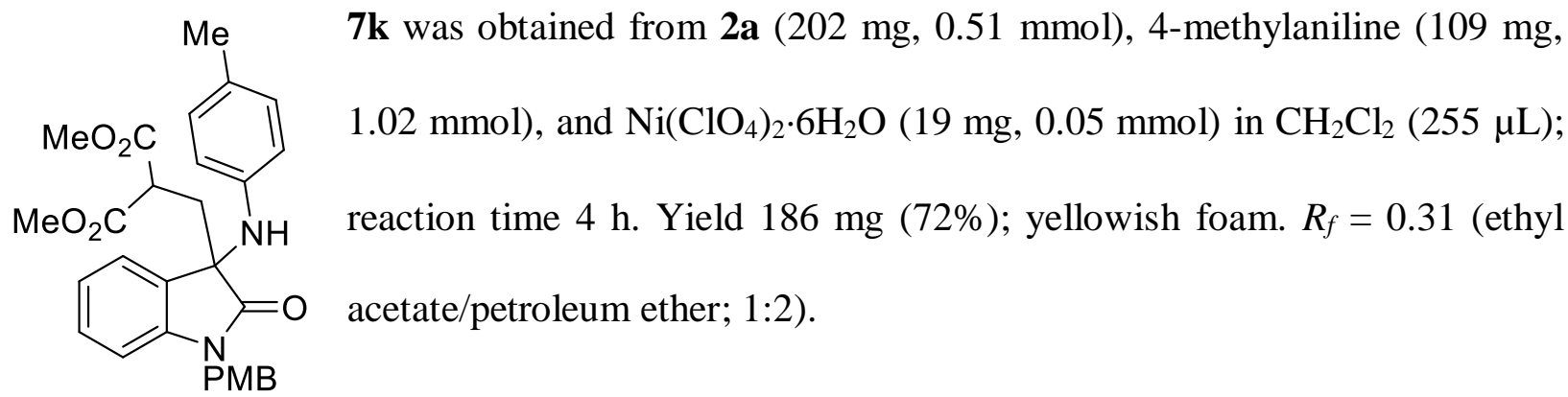


${ }^{1} \mathrm{H} \mathrm{NMR}\left(\mathrm{CDCl}_{3}, 600 \mathrm{MHz}\right): \delta=2.16\left(\mathrm{~s}, 3 \mathrm{H}, \mathrm{CH}_{3}\right), 2.57\left(\mathrm{dd},{ }^{2} J=14.5,{ }^{3} \mathrm{~J}=5.3 \mathrm{~Hz}, 1 \mathrm{H}, \mathrm{CH}_{2}\right)$, $2.74\left(\mathrm{dd},{ }^{2} J=14.5,{ }^{3} \mathrm{~J}=7.8 \mathrm{~Hz}, 1 \mathrm{H}, \mathrm{CH}_{2}\right), 3.65\left(\mathrm{~s}, 3 \mathrm{H}, \mathrm{OCH}_{3}\right), 3.74\left(\mathrm{~s}, 3 \mathrm{H}, \mathrm{OCH}_{3}\right), 3.78(\mathrm{~s}, 3 \mathrm{H}$, $\left.\mathrm{OCH}_{3}\right), 3.80\left(\mathrm{dd},{ }^{3} J=7.8,{ }^{3} J=5.3 \mathrm{~Hz}, 1 \mathrm{H}, \mathrm{CH}\right), 4.49$ (br.s, $\left.1 \mathrm{H}, \mathrm{NH}\right), 4.63\left(\mathrm{~d},{ }^{2} J=15.4 \mathrm{~Hz}, 1 \mathrm{H}\right.$, $\left.\mathrm{NCH}_{2}\right), 5.08\left(\mathrm{~d},{ }^{2} J=15.4 \mathrm{~Hz}, 1 \mathrm{H}, \mathrm{NCH}_{2}\right), 6.18\left(\mathrm{~d},{ }^{3} J=8.3 \mathrm{~Hz}, 2 \mathrm{H}, \mathrm{Ar}\right), 6.74\left(\mathrm{~d},{ }^{3} J=8.3 \mathrm{~Hz}, 2 \mathrm{H}\right.$, Ar), 6.79-6.82 (m, 3H, Ar), $7.04\left(\mathrm{dd},{ }^{3} J=7.6,{ }^{3} J=7.2 \mathrm{~Hz}, 1 \mathrm{H}\right.$, Ar), $7.10\left(\mathrm{~d},{ }^{3} J=8.5 \mathrm{~Hz}, 2 \mathrm{H}\right.$, Ar), $7.22\left(\mathrm{ddd},{ }^{3} J=7.8,{ }^{3} J=7.6,{ }^{4} J=0.9 \mathrm{~Hz}, 1 \mathrm{H}, \mathrm{Ar}\right), 7.37\left(\mathrm{~d},{ }^{3} J=7.2 \mathrm{~Hz}, 1 \mathrm{H}, \mathrm{Ar}\right)$.

${ }^{13} \mathrm{C}$ NMR $\left(\mathrm{CDCl}_{3}, 150 \mathrm{MHz}\right): \delta=20.3\left(\mathrm{CH}_{3}\right), 37.8\left(\mathrm{CH}_{2}\right), 43.3\left(\mathrm{NCH}_{2}\right), 46.7(\mathrm{CH}), 52.7$ $\left(\mathrm{OCH}_{3}\right), 52.8\left(\mathrm{OCH}_{3}\right), 55.1\left(\mathrm{OCH}_{3}\right), 64.0(\mathrm{C}), 109.8(\mathrm{CH}, \mathrm{Ar}), 113.9(2 \times \mathrm{CH}, \mathrm{Ar}), 117.0(2 \times \mathrm{CH}$, Ar), $122.7(\mathrm{CH}, \mathrm{Ar}), 124.3(\mathrm{CH}, \mathrm{Ar}), 127.3$ (C, Ar), 128.7 (C, Ar), 128.8 (2×CH, Ar), 129.19 (CH, Ar), 129.21 (C, Ar), 129.3 (2×CH, Ar), 142.0 (C, Ar), 142.3 (C, Ar), 158.9 (C, Ar), 169.40 $\left(\mathrm{CO}_{2} \mathrm{Me}\right), 169.44\left(\mathrm{CO}_{2} \mathrm{Me}\right), 177.0(\mathrm{C}=\mathrm{O})$.

HRMS (ESI-TOF) $m / z:[\mathrm{M}+\mathrm{H}]^{+}$calcd for $\mathrm{C}_{29} \mathrm{H}_{31} \mathrm{~N}_{2} \mathrm{O}_{6}{ }^{+}$503.2177, found 503.2169.

\section{Dimethyl (\{3-1-(4-methoxybenzyl)-[(4-methoxyphenyl)amino]-2-oxo-2,3-dihydro-1H-indol-}

\section{3-yl\}methyl)malonate (7l)}

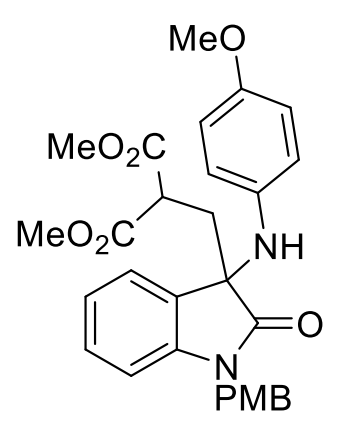

$7 \mathbf{l}$ was obtained from $\mathbf{2 a}(202 \mathrm{mg}, 0.51 \mathrm{mmol})$, 4-methoxyaniline (126 mg, $1.02 \mathrm{mmol})$, and $\mathrm{Ni}\left(\mathrm{ClO}_{4}\right)_{2} \cdot 6 \mathrm{H}_{2} \mathrm{O}(19 \mathrm{mg}, 0.05 \mathrm{mmol})$ in $\mathrm{CH}_{2} \mathrm{Cl}_{2}(255 \mu \mathrm{L})$; reaction time $3 \mathrm{~h}$. Yield $237 \mathrm{mg}(89 \%)$; brown solid, mp $114-115^{\circ} \mathrm{C} . R_{f}=$ 0.15 (ethyl acetate/petroleum ether; 1:2).

${ }^{1} \mathrm{H}$ NMR $\left(\mathrm{CDCl}_{3}, 600 \mathrm{MHz}\right): \delta=2.54\left(\mathrm{dd},{ }^{2} J=14.4,{ }^{3} J=5.3 \mathrm{~Hz}, 1 \mathrm{H}\right.$, $\left.\mathrm{CH}_{2}\right), 2.77\left(\mathrm{dd},{ }^{2} J=14.4,{ }^{3} \mathrm{~J}=8.1 \mathrm{~Hz}, 1 \mathrm{H}, \mathrm{CH}_{2}\right), 3.61\left(\mathrm{~s}, 3 \mathrm{H}, \mathrm{OCH}_{3}\right), 3.65\left(\mathrm{~s}, 3 \mathrm{H}, \mathrm{OCH}_{3}\right), 3.70$ $\left(\mathrm{dd},{ }^{3} J=8.1,{ }^{3} J=5.3 \mathrm{~Hz}, 1 \mathrm{H}, \mathrm{CH}\right), 3.71\left(\mathrm{~s}, 3 \mathrm{H}, \mathrm{OCH}_{3}\right), 3.75\left(\mathrm{~s}, 3 \mathrm{H}, \mathrm{OCH}_{3}\right), 4.20$ (br.s, $\left.1 \mathrm{H}, \mathrm{NH}\right)$, $4.51\left(\mathrm{~d},{ }^{2} J=15.5 \mathrm{~Hz}, 1 \mathrm{H}, \mathrm{NCH}_{2}\right), 5.06\left(\mathrm{~d},{ }^{2} J=15.5 \mathrm{~Hz}, 1 \mathrm{H}, \mathrm{NCH}_{2}\right), 6.32\left(\mathrm{~d},{ }^{3} J=9.1 \mathrm{~Hz}, 2 \mathrm{H}\right.$, Ar), $6.50\left(\mathrm{~d},{ }^{3} J=9.1 \mathrm{~Hz}, 2 \mathrm{H}, \mathrm{Ar}\right), 6.69\left(\mathrm{~d},{ }^{3} J=7.8 \mathrm{~Hz}, 1 \mathrm{H}, \mathrm{Ar}\right), 6.73\left(\mathrm{~d},{ }^{3} J=8.8 \mathrm{~Hz}, 2 \mathrm{H}, \mathrm{Ar}\right)$, $6.89\left(\mathrm{~d},{ }^{3} \mathrm{~J}=8.8 \mathrm{~Hz}, 2 \mathrm{H}, \mathrm{Ar}\right), 7.05\left(\mathrm{ddd},{ }^{3} \mathrm{~J}=7.6,{ }^{3} \mathrm{~J}=7.5,{ }^{4} \mathrm{~J}=0.9 \mathrm{~Hz}, 1 \mathrm{H}\right.$, Ar), 7.19 (ddd, ${ }^{3} J=$ $\left.7.8,{ }^{3} J=7.6,{ }^{4} J=1.2 \mathrm{~Hz}, 1 \mathrm{H}, \mathrm{Ar}\right), 7.43\left(\mathrm{ddd},{ }^{3} J=7.5,{ }^{4} \mathrm{~J}=1.2,{ }^{5} \mathrm{~J}=0.6 \mathrm{~Hz}, 1 \mathrm{H}, \mathrm{Ar}\right)$. 
${ }^{13} \mathrm{C} \mathrm{NMR}\left(\mathrm{CDCl}_{3}, 150 \mathrm{MHz}\right): \delta=37.3\left(\mathrm{CH}_{2}\right), 43.1\left(\mathrm{NCH}_{2}\right), 46.9(\mathrm{CH}), 52.6\left(\mathrm{OCH}_{3}\right), 52.8$ $\left(\mathrm{OCH}_{3}\right), 55.0\left(\mathrm{OCH}_{3}\right), 55.1\left(\mathrm{OCH}_{3}\right), 65.2(\mathrm{C}), 109.8(\mathrm{CH}, \mathrm{Ar}), 113.8(2 \times \mathrm{CH}, \mathrm{Ar}), 114.0(2 \times \mathrm{CH}$, Ar), $120.8(2 \times \mathrm{CH}, \mathrm{Ar}), 122.7(\mathrm{CH}, \mathrm{Ar}), 124.7(\mathrm{CH}, \mathrm{Ar}), 127.1$ (C, Ar), 128.5 (2×CH, C, Ar), $129.3(\mathrm{CH}, \mathrm{Ar}), 137.9$ (C, Ar), 142.3 (C, Ar), 154.5 (C, Ar), 158.8 (C, Ar), $169.3\left(\mathrm{CO}_{2} \mathrm{Me}\right)$, $169.4\left(\mathrm{CO}_{2} \mathrm{Me}\right), 177.0(\mathrm{C}=\mathrm{O})$.

HRMS (ESI-TOF) $m / z:[\mathrm{M}+\mathrm{H}]^{+}$calcd for $\mathrm{C}_{29} \mathrm{H}_{31} \mathrm{~N}_{2} \mathrm{O}_{7}{ }^{+}$519.2126, found 519.2127.

\section{Dimethyl (\{3-1-(4-methoxybenzyl)-[(2-methoxyphenyl)amino]-2-oxo-2,3-dihydro-1H-indol-}

\section{3-yl\}methyl)malonate (7m)}<smiles>COCC(C)CC1(Nc2ccccc2C(=O)OC)C(=O)Nc2ccccc21</smiles>

7m was obtained from 2a (160 mg, $0.41 \mathrm{mmol})$, 2-methoxyaniline (92 $\mu \mathrm{L}, 0.81 \mathrm{mmol})$, and $\mathrm{Ni}\left(\mathrm{ClO}_{4}\right)_{2} \cdot 6 \mathrm{H}_{2} \mathrm{O}(15 \mathrm{mg}, 0.04 \mathrm{mmol})$ in $\mathrm{CH}_{2} \mathrm{Cl}_{2}$ $(205 \mu \mathrm{L})$; reaction time $9 \mathrm{~h}$. Yield $168 \mathrm{mg}(80 \%)$; yellow oil. $R_{f}=0.23$ (ethyl acetate/petroleum ether; 1:2).

${ }^{1} \mathrm{H} \mathrm{NMR}\left(\mathrm{CDCl}_{3}, 600 \mathrm{MHz}\right): \delta=2.63\left(\mathrm{dd},{ }^{2} J=14.5,{ }^{3} J=5.2 \mathrm{~Hz}, 1 \mathrm{H}, \mathrm{CH}_{2}\right), 2.75\left(\mathrm{dd},{ }^{2} J=14.5\right.$, $\left.{ }^{3} J=7.7 \mathrm{~Hz}, 1 \mathrm{H}, \mathrm{CH}_{2}\right), 3.67\left(\mathrm{~s}, 3 \mathrm{H}, \mathrm{OCH}_{3}\right), 3.75\left(\mathrm{~s}, 3 \mathrm{H}, \mathrm{OCH}_{3}\right), 3.79\left(\mathrm{~s}, 3 \mathrm{H}, \mathrm{OCH}_{3}\right), 3.83\left(\mathrm{dd},{ }^{3} J\right.$ $\left.=7.7,{ }^{3} \mathrm{~J}=5.2 \mathrm{~Hz}, 1 \mathrm{H}, \mathrm{CH}\right), 3.88\left(\mathrm{~s}, 3 \mathrm{H}, \mathrm{OCH}_{3}\right), 4.70\left(\mathrm{~d},{ }^{2} J=15.3 \mathrm{~Hz}, 1 \mathrm{H}, \mathrm{NCH}_{2}\right), 5.11\left(\mathrm{~d},{ }^{2} J=\right.$ $15.3 \mathrm{~Hz}, 1 \mathrm{H}, \mathrm{NCH}_{2}$ ), 5.23 (br.s, $\left.1 \mathrm{H}, \mathrm{NH}\right), 5.55$ (dd, $\left.{ }^{3} J=7.9,{ }^{4} J=1.5 \mathrm{~Hz}, 1 \mathrm{H}, \mathrm{Ar}\right), 6.34$ (ddd, ${ }^{3} J=$ $\left.7.9,{ }^{3} J=7.6,{ }^{4} J=1.3 \mathrm{~Hz}, 1 \mathrm{H}, \mathrm{Ar}\right), 6.61\left(\mathrm{ddd},{ }^{3} J=8.1,{ }^{3} J=7.6,{ }^{4} J=1.5 \mathrm{~Hz}, 1 \mathrm{H}, \mathrm{Ar}\right), 6.73\left(\mathrm{dd},{ }^{3} J\right.$ $\left.=8.1,{ }^{4} J=1.3 \mathrm{~Hz}, 1 \mathrm{H}, \mathrm{Ar}\right), 6.86\left(\mathrm{~d},{ }^{3} J=8.8 \mathrm{~Hz}, 2 \mathrm{H}, \mathrm{Ar}\right), 6.88\left(\mathrm{~d},{ }^{3} J=7.8 \mathrm{~Hz}, 1 \mathrm{H}, \mathrm{Ar}\right), 7.01$ (ddd, ${ }^{3} J=7.6,{ }^{3} J=7.5,{ }^{4} J=0.9 \mathrm{~Hz}, 1 \mathrm{H}$, Ar), $7.23\left(\mathrm{ddd},{ }^{3} J=7.8,{ }^{3} J=7.6,{ }^{4} J=1.2 \mathrm{~Hz}, 1 \mathrm{H}\right.$, Ar), 7.28-7.31 (m, 3H, Ar).

${ }^{13} \mathrm{C} \mathrm{NMR}\left(\mathrm{CDCl}_{3}, 150 \mathrm{MHz}\right): \delta=38.2\left(\mathrm{CH}_{2}\right), 43.6\left(\mathrm{NCH}_{2}\right), 46.6(\mathrm{CH}), 52.7\left(\mathrm{OCH}_{3}\right), 52.8$ $\left(\mathrm{OCH}_{3}\right), 55.1\left(\mathrm{OCH}_{3}\right), 55.4\left(\mathrm{OCH}_{3}\right), 62.8(\mathrm{C}), 109.5(\mathrm{CH}, \mathrm{Ar}), 109.8(\mathrm{CH}, \mathrm{Ar}), 111.9(\mathrm{CH}, \mathrm{Ar})$, $114.0(2 \times \mathrm{CH}, \mathrm{Ar}), 118.3(\mathrm{CH}, \mathrm{Ar}), 120.6(\mathrm{CH}, \mathrm{Ar}), 122.9(\mathrm{CH}, \mathrm{Ar}), 124.1(\mathrm{CH}, \mathrm{Ar}), 127.5(\mathrm{C}$, Ar), $129.0(\mathrm{C}, \mathrm{Ar}), 129.15(\mathrm{CH}, \mathrm{Ar}), 129.21(2 \times \mathrm{CH}, \mathrm{Ar}), 134.7(\mathrm{C}, \mathrm{Ar}), 141.6(\mathrm{C}, \mathrm{Ar}), 147.4(\mathrm{C}$, $\mathrm{Ar}), 159.1(\mathrm{C}, \mathrm{Ar}), 169.2\left(\mathrm{CO}_{2} \mathrm{Me}\right), 169.3\left(\mathrm{CO}_{2} \mathrm{Me}\right), 177.0(\mathrm{C}=\mathrm{O})$.

HRMS (ESI-TOF) $m / z:[\mathrm{M}+\mathrm{H}]^{+}$calcd for $\mathrm{C}_{29} \mathrm{H}_{31} \mathrm{~N}_{2} \mathrm{O}_{7}{ }^{+}$519.2126, found 519.2126. 


\section{Dimethyl (\{3-1-(4-methoxybenzyl)-[(4-nitrophenyl)amino]-2-oxo-2,3-dihydro-1H-indol-3-}

yl\}methyl)malonate (7n)<smiles>CC(=O)CCC1(Nc2ccc([N+](=O)[O-])cc2)C(=O)Nc2ccccc21</smiles>

7n was obtained from $\mathbf{2 a}(270 \mathrm{mg}, 0.68 \mathrm{mmol})$, 4-nitroaniline (189 mg, $1.37 \mathrm{mmol})$, and $\mathrm{Ni}\left(\mathrm{ClO}_{4}\right)_{2} \cdot 6 \mathrm{H}_{2} \mathrm{O} \quad(25 \mathrm{mg}, \quad 0.07 \mathrm{mmol})$ in $1,2-$ dichloroethane $(340 \mu \mathrm{L})$ at $83{ }^{\circ} \mathrm{C}$; reaction time $9.5 \mathrm{~h}$. Yield $246 \mathrm{mg}$ (70\%); yellow oil. $R_{f}=0.23$ (ethyl acetate/petroleum ether; $1: 2$ ).

${ }^{1} \mathrm{H} \mathrm{NMR}\left(\mathrm{CDCl}_{3}, 600 \mathrm{MHz}\right): \delta=2.60\left(\mathrm{dd},{ }^{2} J=14.7,{ }^{3} J=6.4 \mathrm{~Hz}, 1 \mathrm{H}\right.$,

$\left.\mathrm{CH}_{2}\right), 2.72\left(\mathrm{dd},{ }^{2} J=14.7,{ }^{3} \mathrm{~J}=6.0 \mathrm{~Hz}, 1 \mathrm{H}, \mathrm{CH}_{2}\right), 3.63\left(\mathrm{~s}, 3 \mathrm{H}, \mathrm{OCH}_{3}\right), 3.74\left(\mathrm{~s}, 3 \mathrm{H}, \mathrm{OCH}_{3}\right), 3.78$ $\left(\mathrm{s}, 3 \mathrm{H}, \mathrm{OCH}_{3}\right), 3.87\left(\mathrm{dd},{ }^{3} J=6.4,{ }^{3} J=6.0 \mathrm{~Hz}, 1 \mathrm{H}, \mathrm{CH}\right), 4.73\left(\mathrm{~d},{ }^{2} J=15.1 \mathrm{~Hz}, 1 \mathrm{H}, \mathrm{NCH}_{2}\right), 5.10$ $\left(\mathrm{d},{ }^{2} J=15.1 \mathrm{~Hz}, 1 \mathrm{H}, \mathrm{NCH}_{2}\right), 6.01\left(\mathrm{~d},{ }^{3} J=9.2 \mathrm{~Hz}, 2 \mathrm{H}, \mathrm{Ar}\right), 6.20$ (br.s, $\left.1 \mathrm{H}, \mathrm{NH}\right), 6.87$ (d, ${ }^{3} J=8.7$ $\mathrm{Hz}, 2 \mathrm{H}, \mathrm{Ar}), 7.00-7.03(\mathrm{~m}, 2 \mathrm{H}, \mathrm{Ar}), 7.20\left(\mathrm{dd},{ }^{3} J=7.5,{ }^{4} J=1.1 \mathrm{~Hz}, 1 \mathrm{H}, \mathrm{Ar}\right), 7.28$ (ddd, ${ }^{3} J=7.8$, $\left.{ }^{3} J=7.6,{ }^{4} J=1.1 \mathrm{~Hz}, 1 \mathrm{H}, \mathrm{Ar}\right), 7.33\left(\mathrm{~d},{ }^{3} J=8.7 \mathrm{~Hz}, 2 \mathrm{H}, \mathrm{Ar}\right), 7.71\left(\mathrm{~d},{ }^{3} J=9.2 \mathrm{~Hz}, 2 \mathrm{H}, \mathrm{Ar}\right)$.

${ }^{13} \mathrm{C} \mathrm{NMR}\left(\mathrm{CDCl}_{3}, 150 \mathrm{MHz}\right): \delta=37.7\left(\mathrm{CH}_{2}\right), 43.7\left(\mathrm{NCH}_{2}\right), 46.1(\mathrm{CH}), 52.8\left(\mathrm{OCH}_{3}\right), 53.1$ $\left(\mathrm{OCH}_{3}\right), 55.1\left(\mathrm{OCH}_{3}\right), 62.4(\mathrm{C}), 110.2(\mathrm{CH}, \mathrm{Ar}), 112.8(2 \times \mathrm{CH}, \mathrm{Ar}), 114.0(2 \times \mathrm{CH}, \mathrm{Ar}), 123.3$ $(\mathrm{CH}, \mathrm{Ar}), 123.6(\mathrm{CH}, \mathrm{Ar}), 125.5(2 \times \mathrm{CH}, \mathrm{Ar}), 127.0$ (C, Ar), $127.6(\mathrm{C}, \mathrm{Ar}), 129.4(2 \times \mathrm{CH}, \mathrm{Ar})$, $129.8(\mathrm{CH}, \mathrm{Ar}), 138.8$ (C, Ar), $141.3(\mathrm{C}, \mathrm{Ar}), 150.4(\mathrm{C}, \mathrm{Ar}), 159.3(\mathrm{C}, \mathrm{Ar}), 169.1\left(\mathrm{CO}_{2} \mathrm{Me}\right)$, $169.8\left(\mathrm{CO}_{2} \mathrm{Me}\right), 175.4(\mathrm{C}=\mathrm{O})$.

HRMS (ESI-TOF) $m / z:[\mathrm{M}+\mathrm{H}]^{+}$calcd for $\mathrm{C}_{28} \mathrm{H}_{28} \mathrm{~N}_{3} \mathrm{O}_{8}{ }^{+}$534.1871, found 534.1857.

\section{General procedure for the $\gamma$-lactamization of 7}

A mixture of amine 7 (1.0 equiv) and $\mathrm{TsOH}(0.2$ equiv) in toluene $(0.15 \mathrm{M})$ was heated under reflux (oil bath) for specified time. Then, the reaction mixture was washed with sodium hydrocarbonate (saturated solution in water), dried with sodium sulfate, and concentrated under reduced pressure. Lactame $\mathbf{8}$ was purified by column chromatography on silica gel. 
Methyl 1-(4-methoxybenzyl)-2,5' -dioxo-1'-phenyl-1,2-dihydrospiro[indole-3,2' -pyrrolidine]4'-carboxylate (8a)

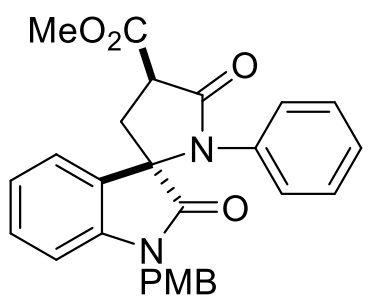

8a was obtained from 7a $(173 \mathrm{mg}, 0.35 \mathrm{mmol})$ and $\mathrm{TsOH}(12 \mathrm{mg}, 0.07$ $\mathrm{mmol})$ in toluene $(2.4 \mathrm{~mL})$; reaction time $1 \mathrm{~h}$. Yield $130 \mathrm{mg}(80 \%)$; colorless oil; dr A:B $=80: 20 . R_{f}=0.40$ (ethyl acetate/petroleum ether; $1: 1)$.

A: ${ }^{1} \mathrm{H}$ NMR $\left(\mathrm{CDCl}_{3}, 600 \mathrm{MHz}\right): \delta=2.77\left(\mathrm{dd},{ }^{2} J=13.5,{ }^{3} \mathrm{~J}=9.5 \mathrm{~Hz}, 1 \mathrm{H}, \mathrm{CH}_{2}\right), 2.87\left(\mathrm{dd},{ }^{2} J=\right.$ $\left.13.5,{ }^{3} J=9.2 \mathrm{~Hz}, 1 \mathrm{H}, \mathrm{CH}_{2}\right), 3.75\left(\mathrm{~s}, 3 \mathrm{H}, \mathrm{OCH}_{3}\right), 3.88\left(\mathrm{~s}, 3 \mathrm{H}, \mathrm{OCH}_{3}\right), 4.25\left(\mathrm{dd},{ }^{3} J=9.5,{ }^{3} J=9.2\right.$ $\mathrm{Hz}, 1 \mathrm{H}, \mathrm{CH}), 4.51\left(\mathrm{~d},{ }^{2} J=15.5 \mathrm{~Hz}, 1 \mathrm{H}, \mathrm{NCH}_{2}\right), 5.00\left(\mathrm{~d},{ }^{2} J=15.5 \mathrm{~Hz}, 1 \mathrm{H}, \mathrm{NCH}_{2}\right), 6.61\left(\mathrm{~d},{ }^{3} J=\right.$ $7.7 \mathrm{~Hz}, 1 \mathrm{H}, \mathrm{Ar}), 6.69-6.73(\mathrm{~m}, 2 \mathrm{H}, \mathrm{Ar}), 6.80\left(\mathrm{~d},{ }^{3} \mathrm{~J}=8.8 \mathrm{~Hz}, 2 \mathrm{H}, \mathrm{Ar}\right), 6.95-6.98$ (m, 2H, Ar), $7.09\left(\mathrm{ddd},{ }^{3} J=7.6,{ }^{3} J=7.5,{ }^{4} J=0.9 \mathrm{~Hz}, 1 \mathrm{H}, \mathrm{Ar}\right), 7.14-7.23(\mathrm{~m}, 4 \mathrm{H}, \mathrm{Ar}), 7.56\left(\mathrm{ddd},{ }^{3} J=7.5,{ }^{4} J\right.$ $\left.=1.2,{ }^{5} \mathrm{~J}=0.5 \mathrm{~Hz}, 1 \mathrm{H}, \mathrm{Ar}\right)$.

${ }^{13} \mathrm{C}$ NMR $\left(\mathrm{CDCl}_{3}, 150 \mathrm{MHz}\right): \delta=33.7\left(\mathrm{CH}_{2}\right), 43.2\left(\mathrm{NCH}_{2}\right), 46.97(\mathrm{CH}), 52.9\left(\mathrm{OCH}_{3}\right), 55.15$ $\left(\mathrm{OCH}_{3}\right), 68.68(\mathrm{C}), 109.8(\mathrm{CH}, \mathrm{Ar}), 114.04(2 \times \mathrm{CH}, \mathrm{Ar}), 123.5(\mathrm{CH}, \mathrm{Ar}), 124.9(\mathrm{CH}, \mathrm{Ar}), 126.54$ (C, Ar), $127.3(\mathrm{C}, \mathrm{Ar}), 127.5(2 \times \mathrm{CH}, \mathrm{Ar}), 127.89(\mathrm{CH}, \mathrm{Ar}), 128.2(2 \times \mathrm{CH}, \mathrm{Ar}), 129.0(2 \times \mathrm{CH}$, Ar), $130.22(\mathrm{CH}, \mathrm{Ar}), 135.55(\mathrm{C}, \mathrm{Ar}), 142.4(\mathrm{C}, \mathrm{Ar}), 159.0(\mathrm{C}, \mathrm{Ar}), 170.2(\mathrm{C}=\mathrm{O}), 170.5(\mathrm{C}=\mathrm{O})$, $175.8(\mathrm{C}=\mathrm{O})$.

B: ${ }^{1} \mathrm{H}$ NMR $\left(\mathrm{CDCl}_{3}, 600 \mathrm{MHz}\right): \delta=2.56\left(\mathrm{dd},{ }^{2} J=13.1,{ }^{3} \mathrm{~J}=9.3 \mathrm{~Hz}, 1 \mathrm{H}, \mathrm{CH}_{2}\right), 3.21\left(\mathrm{dd},{ }^{2} J=\right.$ $\left.13.1,{ }^{3} J=9.1 \mathrm{~Hz}, 1 \mathrm{H}, \mathrm{CH}_{2}\right), 3.75\left(\mathrm{~s}, 3 \mathrm{H}, \mathrm{OCH}_{3}\right), 3.90\left(\mathrm{~s}, 3 \mathrm{H}, \mathrm{OCH}_{3}\right), 4.06\left(\mathrm{dd},{ }^{3} J=9.3,{ }^{3} J=9.1\right.$ $\mathrm{Hz}, 1 \mathrm{H}, \mathrm{CH}), 4.43\left(\mathrm{~d},{ }^{2} J=15.5 \mathrm{~Hz}, 1 \mathrm{H}, \mathrm{NCH}_{2}\right), 5.04\left(\mathrm{~d},{ }^{2} J=15.5 \mathrm{~Hz}, 1 \mathrm{H}, \mathrm{NCH}_{2}\right), 6.64\left(\mathrm{~d},{ }^{3} J=\right.$ $7.8 \mathrm{~Hz}, 1 \mathrm{H}, \mathrm{Ar}), 6.66\left(\mathrm{~d},{ }^{3} J=8.9 \mathrm{~Hz}, 2 \mathrm{H}, \mathrm{Ar}\right), 6.69-6.73(\mathrm{~m}, 2 \mathrm{H}, \mathrm{Ar}), 6.95-6.98(\mathrm{~m}, 2 \mathrm{H}, \mathrm{Ar})$, $7.10\left(\mathrm{ddd},{ }^{3} J=7.6,{ }^{3} J=7.5,{ }^{4} J=0.9 \mathrm{~Hz}, 1 \mathrm{H}, \mathrm{Ar}\right), 7.14-7.23(\mathrm{~m}, 4 \mathrm{H}, \mathrm{Ar}), 7.40\left(\mathrm{ddd},{ }^{3} J=7.5,{ }^{4} J\right.$ $\left.=1.2,{ }^{5} \mathrm{~J}=0.5 \mathrm{~Hz}, 1 \mathrm{H}, \mathrm{Ar}\right)$.

${ }^{13} \mathrm{C} \mathrm{NMR}\left(\mathrm{CDCl}_{3}, 150 \mathrm{MHz}\right): \delta=34.4\left(\mathrm{CH}_{2}\right), 43.3\left(\mathrm{NCH}_{2}\right), 47.04(\mathrm{CH}), 53.0\left(\mathrm{OCH}_{3}\right), 55.15$ $\left(\mathrm{OCH}_{3}\right), 68.68(\mathrm{C}), 110.2(\mathrm{CH}, \mathrm{Ar}), 113.99(2 \times \mathrm{CH}, \mathrm{Ar}), 123.35(\mathrm{CH}, \mathrm{Ar}), 123.40(\mathrm{CH}, \mathrm{Ar})$, 126.54 (C, Ar), $127.6(2 \times \mathrm{CH}, \mathrm{Ar}), 127.89(\mathrm{CH}, \mathrm{Ar}), 128.1(2 \times \mathrm{CH}, \mathrm{Ar}), 128.7(\mathrm{C}, \mathrm{Ar}), 129.1$ 
(2×CH, Ar), 130.22 (CH, Ar), 135.55 (C, Ar), 142.0 (C, Ar), 158.9 (C, Ar), $168.9(\mathrm{C}=\mathrm{O}), 169.9$ $(\mathrm{C}=\mathrm{O}), 174.1(\mathrm{C}=\mathrm{O})$.

HRMS (ESI-TOF) $m / z:[\mathrm{M}+\mathrm{H}]^{+}$calcd for $\mathrm{C}_{27} \mathrm{H}_{25} \mathrm{~N}_{2} \mathrm{O}_{5}{ }^{+} 456.1758$, found 457.1756.

\section{Methyl 1-(4-methoxybenzyl)-5-methyl-2,5'-dioxo-1'-phenyl-1,2-dihydrospiro[indole-3,2' -} pyrrolidine]-4' -carboxylate $(8 b)$<smiles>COC(=O)c1ccc(C)cc1C12C[C@H](C(=O)Nc3ccccc3)C(=O)N1c1ccccc12</smiles>

$\mathbf{8 b}$ was obtained from $\mathbf{7 b}(152 \mathrm{mg}, 0.30 \mathrm{mmol})$ and $\mathrm{TsOH}(10 \mathrm{mg}$, $0.06 \mathrm{mmol})$ in toluene $(2.0 \mathrm{~mL})$; reaction time $1 \mathrm{~h}$. Yield $116 \mathrm{mg}$ (82\%); colorless solid, mp $162-163{ }^{\circ} \mathrm{C} ; \mathrm{dr} \mathbf{A}: \mathbf{B}=80: 20 . R_{f}=0.33$ (ethyl acetate/petroleum ether; $1: 1$ ).

A: ${ }^{1} \mathrm{H}$ NMR $\left(\mathrm{CDCl}_{3}, 600 \mathrm{MHz}\right): \delta=2.31\left(\mathrm{~s}, 3 \mathrm{H}, \mathrm{CH}_{3}\right), 2.74\left(\mathrm{dd},{ }^{2} J=13.4,{ }^{3} J=9.5 \mathrm{~Hz}, 1 \mathrm{H}\right.$, $\left.\mathrm{CH}_{2}\right), 2.85\left(\mathrm{dd},{ }^{2} J=13.4,{ }^{3} J=9.5 \mathrm{~Hz}, 1 \mathrm{H}, \mathrm{CH}_{2}\right), 3.74\left(\mathrm{~s}, 3 \mathrm{H}, \mathrm{OCH}_{3}\right), 3.87\left(\mathrm{~s}, 3 \mathrm{H}, \mathrm{OCH}_{3}\right), 4.24$ $\left(\mathrm{dd},{ }^{3} J=9.5,{ }^{3} J=9.5 \mathrm{~Hz}, 1 \mathrm{H}, \mathrm{CH}\right), 4.49\left(\mathrm{~d},{ }^{2} J=15.5 \mathrm{~Hz}, 1 \mathrm{H}, \mathrm{NCH}_{2}\right), 4.98\left(\mathrm{~d},{ }^{2} J=15.5 \mathrm{~Hz}, 1 \mathrm{H}\right.$, $\left.\mathrm{NCH}_{2}\right), 6.50\left(\mathrm{~d},{ }^{3} \mathrm{~J}=8.0 \mathrm{~Hz}, 1 \mathrm{H}, \mathrm{Ar}\right), 6.65-6.73(\mathrm{~m}, 2 \mathrm{H}, \mathrm{Ar}), 6.80\left(\mathrm{~d},{ }^{3} \mathrm{~J}=8.4 \mathrm{~Hz}, 2 \mathrm{H}, \mathrm{Ar}\right), 6.95-$ 7.03 (m, 3H, Ar), 7.14-7.24 (m, 3H, Ar), 7.35 (s, 1H, Ar).

${ }^{13} \mathrm{C} \mathrm{NMR}\left(\mathrm{CDCl}_{3}, 150 \mathrm{MHz}\right): \delta=20.94\left(\mathrm{CH}_{3}\right), 33.8\left(\mathrm{CH}_{2}\right), 43.1\left(\mathrm{NCH}_{2}\right), 46.9(\mathrm{CH}), 52.8$ $\left(\mathrm{OCH}_{3}\right), 55.07\left(\mathrm{OCH}_{3}\right), 68.66(\mathrm{C}), 109.5(\mathrm{CH}, \mathrm{Ar}), 114.0(2 \times \mathrm{CH}, \mathrm{Ar}), 125.3(\mathrm{CH}, \mathrm{Ar}), 126.62$ (C, Ar), $127.3(2 \times \mathrm{CH}, \mathrm{Ar}), 127.5(\mathrm{C}, \mathrm{Ar}), 127.70(\mathrm{CH}, \mathrm{Ar}), 128.2(2 \times \mathrm{CH}, \mathrm{Ar}), 128.9(2 \times \mathrm{CH}$, Ar), $130.49(\mathrm{CH}, \mathrm{Ar}), 133.2$ (C, Ar), 135.62 (C, Ar), $140.0(\mathrm{C}, \mathrm{Ar}), 158.9(\mathrm{C}, \mathrm{Ar}), 170.2(\mathrm{C}=\mathrm{O})$, $170.5(\mathrm{C}=\mathrm{O}), 175.6(\mathrm{C}=\mathrm{O})$.

B: ${ }^{1} \mathrm{H}$ NMR $\left(\mathrm{CDCl}_{3}, 600 \mathrm{MHz}\right): \delta=2.32\left(\mathrm{~s}, 3 \mathrm{H}, \mathrm{CH}_{3}\right), 2.54\left(\mathrm{dd},{ }^{2} J=13.0,{ }^{3} J=9.1 \mathrm{~Hz}, 1 \mathrm{H}\right.$, $\left.\mathrm{CH}_{2}\right), 3.19\left(\mathrm{dd},{ }^{2} J=13.0,{ }^{3} J=9.4 \mathrm{~Hz}, 1 \mathrm{H}, \mathrm{CH}_{2}\right), 3.73\left(\mathrm{~s}, 3 \mathrm{H}, \mathrm{OCH}_{3}\right), 3.88\left(\mathrm{~s}, 3 \mathrm{H}, \mathrm{OCH}_{3}\right), 4.07$ $\left(\mathrm{dd},{ }^{3} J=9.4,{ }^{3} J=9.1 \mathrm{~Hz}, 1 \mathrm{H}, \mathrm{CH}\right), 4.40\left(\mathrm{~d},{ }^{2} J=15.6 \mathrm{~Hz}, 1 \mathrm{H}, \mathrm{NCH}_{2}\right), 5.02\left(\mathrm{~d},{ }^{2} J=15.6 \mathrm{~Hz}, 1 \mathrm{H}\right.$, $\left.\mathrm{NCH}_{2}\right), 6.54\left(\mathrm{~d},{ }^{3} \mathrm{~J}=8.0 \mathrm{~Hz}, 1 \mathrm{H}, \mathrm{Ar}\right), 6.65-6.73$ (m, 4H, Ar), 6.95-7.03 (m, 3H, Ar), 7.14-7.24 (m, 4H, Ar). 
${ }^{13} \mathrm{C} \mathrm{NMR}\left(\mathrm{CDCl}_{3}, 150 \mathrm{MHz}\right): \delta=20.94\left(\mathrm{CH}_{3}\right), 34.5\left(\mathrm{CH}_{2}\right), 43.2\left(\mathrm{NCH}_{2}\right), 47.0(\mathrm{CH}), 52.9$ $\left(\mathrm{OCH}_{3}\right), 55.07\left(\mathrm{OCH}_{3}\right), 68.68(\mathrm{C}), 109.9(\mathrm{CH}, \mathrm{Ar}), 123.9(\mathrm{CH}, \mathrm{Ar}), 113.9(2 \times \mathrm{CH}, \mathrm{Ar}), 126.62$ (C, Ar), $127.0(2 \times \mathrm{CH}, \mathrm{Ar}), 127.70(\mathrm{CH}, \mathrm{Ar}), 128.1(2 \times \mathrm{CH}, \mathrm{Ar}), 128.8(\mathrm{C}, \mathrm{Ar}), 129.0(2 \times \mathrm{CH}$, Ar), 130.46 (CH, Ar), 133.1 (C, Ar), 135.62 (C, Ar), 139.4 (C, Ar), 158.8 (C, Ar), $168.9(\mathrm{C}=\mathrm{O})$, $169.8(\mathrm{C}=\mathrm{O}), 173.9(\mathrm{C}=\mathrm{O})$.

HRMS (ESI-TOF) $m / z:[\mathrm{M}+\mathrm{H}]^{+}$calcd for $\mathrm{C}_{28} \mathrm{H}_{27} \mathrm{~N}_{2} \mathrm{O}_{5}{ }^{+} 471.1914$, found 471.1918.

\section{Methyl 1-methyl-2,5'-dioxo-1'-phenyl-1,2-dihydrospiro[indole-3,2' -pyrrolidine]-4' -}

carboxylate (8c)

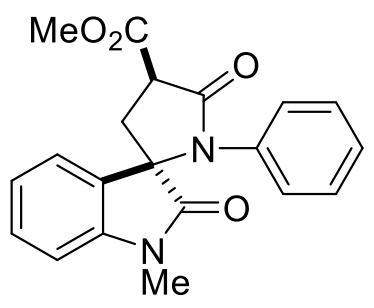

8c was obtained from 7c (115 mg, $0.30 \mathrm{mmol})$ and $\mathrm{TsOH}(10 \mathrm{mg}, 0.06$ mmol) in toluene $(2.0 \mathrm{~mL})$; reaction time $1 \mathrm{~h}$. Yield $79 \mathrm{mg}(75 \%)$; colorless solid, mp $194-195{ }^{\circ} \mathrm{C} ; \mathrm{dr} \mathbf{A}: \mathbf{B}=79: 21 . R_{f}=0.27$ (ethyl acetate/petroleum ether; $1: 1)$.

A: ${ }^{1} \mathrm{H} \mathrm{NMR}\left(\mathrm{CDCl}_{3}, 600 \mathrm{MHz}\right): \delta=2.69\left(\mathrm{dd},{ }^{2} J=13.4,{ }^{3} J=9.4 \mathrm{~Hz}, 1 \mathrm{H}, \mathrm{CH}_{2}\right), 2.81\left(\mathrm{dd},{ }^{2} J=\right.$ $\left.13.4,{ }^{3} J=9.5 \mathrm{~Hz}, 1 \mathrm{H}, \mathrm{CH}_{2}\right), 3.11-3.14\left(\mathrm{~m}, 3 \mathrm{H}, \mathrm{NCH}_{3}\right), 3.85\left(\mathrm{~s}, 3 \mathrm{H}, \mathrm{OCH}_{3}\right), 4.22\left(\mathrm{dd},{ }^{3} J=9.5,{ }^{3} J\right.$ $=9.4 \mathrm{~Hz}, 1 \mathrm{H}, \mathrm{CH}), 6.73\left(\mathrm{~d},{ }^{3} \mathrm{~J}=7.8 \mathrm{~Hz}, 1 \mathrm{H}, \mathrm{Ar}\right), 6.94-6.98(\mathrm{~m}, 2 \mathrm{H}, \mathrm{Ar}), 7.08-7.18(\mathrm{~m}, 4 \mathrm{H}, \mathrm{Ar})$, $7.27\left(\mathrm{ddd},{ }^{3} \mathrm{~J}=7.8,{ }^{3} \mathrm{~J}=7.7,{ }^{4} \mathrm{~J}=1.2 \mathrm{~Hz}, 1 \mathrm{H}, \mathrm{Ar}\right), 7.48\left(\mathrm{dd},{ }^{3} \mathrm{~J}=7.5,{ }^{4} \mathrm{~J}=1.2 \mathrm{~Hz}, 1 \mathrm{H}, \mathrm{Ar}\right)$.

${ }^{13} \mathrm{C} \mathrm{NMR}\left(\mathrm{CDCl}_{3}, 150 \mathrm{MHz}\right): \delta=26.4\left(\mathrm{NCH}_{3}\right), 34.0\left(\mathrm{CH}_{2}\right), 46.9(\mathrm{CH}), 52.85\left(\mathrm{OCH}_{3}\right), 68.5(\mathrm{C})$, $108.7(\mathrm{CH}, \mathrm{Ar}), 123.5(\mathrm{CH}, \mathrm{Ar}), 124.6(\mathrm{CH}, \mathrm{Ar}), 127.0(2 \times \mathrm{CH}, \mathrm{Ar}), 127.8(\mathrm{CH}, \mathrm{Ar}), 128.90$ (2×CH, C, Ar), $130.2(\mathrm{CH}, \mathrm{Ar}), 135.5(\mathrm{C}, \mathrm{Ar}), 143.2(\mathrm{C}, \mathrm{Ar}), 170.2(\mathrm{C}=\mathrm{O}), 170.4(\mathrm{C}=\mathrm{O}), 175.9$ $(\mathrm{C}=\mathrm{O})$.

B: ${ }^{1} \mathrm{H}$ NMR $\left(\mathrm{CDCl}_{3}, 600 \mathrm{MHz}\right): \delta=2.50\left(\mathrm{dd},{ }^{2} \mathrm{~J}=13.1,{ }^{3} \mathrm{~J}=9.3 \mathrm{~Hz}, 1 \mathrm{H}, \mathrm{CH}_{2}\right), 3.11-3.14(\mathrm{~m}$, $\left.1 \mathrm{H}, \mathrm{CH}_{2}\right), 3.09\left(\mathrm{~s}, 3 \mathrm{H}, \mathrm{NCH}_{3}\right), 3.86\left(\mathrm{~s}, 3 \mathrm{H}, \mathrm{OCH}_{3}\right), 4.01\left(\mathrm{dd},{ }^{3} J=9.3,{ }^{3} \mathrm{~J}=8.7 \mathrm{~Hz}, 1 \mathrm{H}, \mathrm{CH}\right), 6.79$ $\left(\mathrm{d},{ }^{3} J=7.7 \mathrm{~Hz}, 1 \mathrm{H}, \mathrm{Ar}\right), 6.94-6.98(\mathrm{~m}, 2 \mathrm{H}, \mathrm{Ar}), 7.08-7.18(\mathrm{~m}, 4 \mathrm{H}, \mathrm{Ar}), 7.33\left(\mathrm{ddd},{ }^{3} J=7.7,{ }^{3} J=\right.$ $\left.7.7,{ }^{4} J=1.2 \mathrm{~Hz}, 1 \mathrm{H}, \mathrm{Ar}\right), 7.36\left(\mathrm{dd},{ }^{3} J=7.5,{ }^{4} J=1.2,{ }^{5} J=0.5 \mathrm{~Hz}, 1 \mathrm{H}, \mathrm{Ar}\right)$. 
${ }^{13} \mathrm{C} \mathrm{NMR}\left(\mathrm{CDCl}_{3}, 150 \mathrm{MHz}\right): \delta=26.3\left(\mathrm{NCH}_{3}\right), 34.5\left(\mathrm{CH}_{2}\right), 47.0(\mathrm{CH}), 52.91\left(\mathrm{OCH}_{3}\right), 68.7(\mathrm{C})$, $109.0(\mathrm{CH}, \mathrm{Ar}), 123.2(\mathrm{CH}, \mathrm{Ar}), 123.4(\mathrm{CH}, \mathrm{Ar}), 126.6(2 \times \mathrm{CH}, \mathrm{Ar}), 127.2(2 \times \mathrm{CH}, \mathrm{Ar}), 127.7$ $(\mathrm{CH}, \mathrm{Ar}), 128.5(\mathrm{C}, \mathrm{Ar}), 130.3(\mathrm{CH}, \mathrm{Ar}), 135.6(\mathrm{C}, \mathrm{Ar}), 142.8(\mathrm{C}, \mathrm{Ar}), 168.9(\mathrm{C}=\mathrm{O}), 169.8$ $(\mathrm{C}=\mathrm{O}), 174.2(\mathrm{C}=\mathrm{O})$.

IR (film) 1718, 1710, 1613, 1472, 1344, $1261 \mathrm{~cm}^{-1}$.

HRMS (ESI-TOF) $m / z:[\mathrm{M}+\mathrm{H}]^{+}$calcd for $\mathrm{C}_{20} \mathrm{H}_{19} \mathrm{~N}_{2} \mathrm{O}_{4}{ }^{+} 351.1339$, found 351.1342.

\section{Methyl 5-fluoro-1-(4-methoxybenzyl)-2,5' -dioxo-1'-phenyl-1,2-dihydrospiro[indole-3,2' -}

pyrrolidine]-4' -carboxylate (8d)

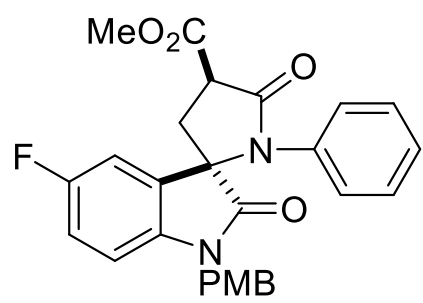

8d was obtained from $7 \mathbf{d}(121 \mathrm{mg}, 0.24 \mathrm{mmol})$ and $\mathrm{TsOH}(8 \mathrm{mg}$, $0.05 \mathrm{mmol})$ in toluene $(1.6 \mathrm{~mL})$; reaction time $1 \mathrm{~h}$. Yield $86 \mathrm{mg}$ (76\%); colorless oil; $\mathrm{dr} \mathbf{A}: \mathbf{B}=78: 22 . \quad R_{f}=0.30$ (ethyl acetate/petroleum ether; $1: 1)$.

A: ${ }^{1} \mathrm{H} \mathrm{NMR}\left(\mathrm{CDCl}_{3}, 600 \mathrm{MHz}\right): \delta=2.79\left(\mathrm{dd},{ }^{2} J=13.5,{ }^{3} \mathrm{~J}=9.5 \mathrm{~Hz}, 1 \mathrm{H}, \mathrm{CH}_{2}\right), 2.83\left(\mathrm{dd},{ }^{2} J=\right.$ $\left.13.5,{ }^{3} \mathrm{~J}=8.7 \mathrm{~Hz}, 1 \mathrm{H}, \mathrm{CH}_{2}\right), 3.75\left(\mathrm{~s}, 3 \mathrm{H}, \mathrm{OCH}_{3}\right), 3.87\left(\mathrm{~s}, 3 \mathrm{H}, \mathrm{OCH}_{3}\right), 4.21\left(\mathrm{dd},{ }^{3} J=9.5,{ }^{3} J=8.7\right.$ $\mathrm{Hz}, 1 \mathrm{H}, \mathrm{CH}), 4.48\left(\mathrm{~d},{ }^{2} J=15.6 \mathrm{~Hz}, 1 \mathrm{H}, \mathrm{NCH}_{2}\right), 5.00\left(\mathrm{~d},{ }^{2} J=15.6 \mathrm{~Hz}, 1 \mathrm{H}, \mathrm{NCH}_{2}\right), 6.53\left(\mathrm{dd},{ }^{3} J=\right.$ $\left.8.7,{ }^{4} J=4.0 \mathrm{~Hz}, 1 \mathrm{H}, \mathrm{Ar}\right), 6.65-6.72(\mathrm{~m}, 2 \mathrm{H}, \mathrm{Ar}), 6.77\left(\mathrm{~d},{ }^{3} J=8.8 \mathrm{~Hz}, 2 \mathrm{H}, \mathrm{Ar}\right), 6.87$ (ddd, ${ }^{3} J=$ $\left.9.0,{ }^{3} J=8.7,{ }^{4} J=2.6 \mathrm{~Hz}, 1 \mathrm{H}, \mathrm{Ar}\right), 6.96-7.00(\mathrm{~m}, 2 \mathrm{H}, \mathrm{Ar}), 7.15-7.26(\mathrm{~m}, 3 \mathrm{H}, \mathrm{Ar}), 7.35$ (dd, ${ }^{3} J=$ $\left.7.6,{ }^{4} \mathrm{~J}=2.6 \mathrm{~Hz}, 1 \mathrm{H}, \mathrm{Ar}\right)$.

${ }^{13} \mathrm{C} \mathrm{NMR}\left(\mathrm{CDCl}_{3}, 150 \mathrm{MHz}\right): \delta=33.6\left(\mathrm{CH}_{2}\right), 43.3\left(\mathrm{NCH}_{2}\right), 46.9(\mathrm{CH}), 52.99\left(\mathrm{OCH}_{3}\right), 55.13$ $\left(\mathrm{OCH}_{3}\right), 68.80(\mathrm{C}), 110.7\left({ }^{3} J_{\mathrm{CF}}=8 \mathrm{~Hz}, \mathrm{CH}, \mathrm{Ar}\right), 112.9\left({ }^{2} J_{\mathrm{CF}}=25 \mathrm{~Hz}, \mathrm{CH}, \mathrm{Ar}\right), 114.1(2 \times \mathrm{CH}$, Ar), $116.72\left({ }^{2} J_{\mathrm{CF}}=23 \mathrm{~Hz}, \mathrm{CH}, \mathrm{Ar}\right), 126.14(\mathrm{C}, \mathrm{Ar}), 127.5(2 \times \mathrm{CH}, \mathrm{Ar}), 128.07(\mathrm{CH}, \mathrm{Ar}), 128.14$ (2×CH, Ar), $129.15(2 \times \mathrm{CH}, \mathrm{Ar}), 129.3\left({ }^{3} J_{\mathrm{CF}}=8 \mathrm{~Hz}, \mathrm{C}, \mathrm{Ar}\right), 135.3(\mathrm{C}, \mathrm{Ar}), 138.3(\mathrm{C}, \mathrm{Ar}), 159.0$ $(\mathrm{C}, \mathrm{Ar}), 159.5\left({ }^{1} J_{\mathrm{CF}}=243 \mathrm{~Hz}, \mathrm{C}, \mathrm{Ar}\right), 170.1(\mathrm{C}=\mathrm{O}), 170.3(\mathrm{C}=\mathrm{O}), 175.5(\mathrm{C}=\mathrm{O})$.

B: ${ }^{1} \mathrm{H} \mathrm{NMR}\left(\mathrm{CDCl}_{3}, 600 \mathrm{MHz}\right): \delta=2.55\left(\mathrm{dd},{ }^{2} J=13.2,{ }^{3} J=9.4 \mathrm{~Hz}, 1 \mathrm{H}, \mathrm{CH}_{2}\right), 3.21\left(\mathrm{dd},{ }^{2} J=\right.$ $\left.13.2,{ }^{3} \mathrm{~J}=8.7 \mathrm{~Hz}, 1 \mathrm{H}, \mathrm{CH}_{2}\right), 3.74\left(\mathrm{~s}, 3 \mathrm{H}, \mathrm{OCH}_{3}\right), 3.89\left(\mathrm{~s}, 3 \mathrm{H}, \mathrm{OCH}_{3}\right), 4.02\left(\mathrm{dd},{ }^{3} J=8.7,{ }^{3} J=9.4\right.$ 
$\mathrm{Hz}, 1 \mathrm{H}, \mathrm{CH}), 4.40\left(\mathrm{~d},{ }^{2} J=15.6 \mathrm{~Hz}, 1 \mathrm{H}, \mathrm{NCH}_{2}\right), 5.04\left(\mathrm{~d},{ }^{2} J=15.6 \mathrm{~Hz}, 1 \mathrm{H}, \mathrm{NCH}_{2}\right), 6.56\left(\mathrm{dd},{ }^{3} J=\right.$ $\left.8.7,{ }^{4} \mathrm{~J}=4.1 \mathrm{~Hz}, 1 \mathrm{H}, \mathrm{Ar}\right), 6.65-6.72(\mathrm{~m}, 4 \mathrm{H}, \mathrm{Ar}), 6.91\left(\mathrm{ddd},{ }^{3} \mathrm{~J}=8.9,{ }^{3} \mathrm{~J}=8.7,{ }^{4} \mathrm{~J}=2.5 \mathrm{~Hz}, 1 \mathrm{H}\right.$, Ar), 6.96-7.00 (m, 2H, Ar), 7.15-7.26 (m, 4H, Ar).

${ }^{13} \mathrm{C} \mathrm{NMR}\left(\mathrm{CDCl}_{3}, 150 \mathrm{MHz}\right): \delta=34.1\left(\mathrm{CH}_{2}\right), 43.4\left(\mathrm{NCH}_{2}\right), 46.8(\mathrm{CH}), 53.02\left(\mathrm{OCH}_{3}\right), 55.13$ $\left(\mathrm{OCH}_{3}\right), 68.80(\mathrm{C}), 111.0\left({ }^{3} J_{\mathrm{CF}}=8 \mathrm{~Hz}, \mathrm{CH}, \mathrm{Ar}\right), 111.5\left({ }^{2} J_{\mathrm{CF}}=25 \mathrm{~Hz}, \mathrm{CH}, \mathrm{Ar}\right), 114.0(2 \times \mathrm{CH}$, Ar), $116.69\left({ }^{2} J_{\mathrm{CF}}=23 \mathrm{~Hz}, \mathrm{CH}, \mathrm{Ar}\right), 126.14(\mathrm{C}, \mathrm{Ar}), 127.3(2 \times \mathrm{CH}, \mathrm{Ar}), 128.07(\mathrm{CH}, \mathrm{Ar}), 128.10$ $(2 \times \mathrm{CH}, \mathrm{Ar}), 129.19(2 \times \mathrm{CH}, \mathrm{Ar}), 130.2\left({ }^{3} J_{\mathrm{CF}}=7 \mathrm{~Hz}, \mathrm{C}, \mathrm{Ar}\right), 135.4(\mathrm{C}, \mathrm{Ar}), 137.9(\mathrm{C}, \mathrm{Ar}), 158.9$ (C, Ar), $159.3\left({ }^{1} J_{\mathrm{CF}}=244 \mathrm{~Hz}, \mathrm{C}, \mathrm{Ar}\right), 168.7(\mathrm{C}=\mathrm{O}), 169.7(\mathrm{C}=\mathrm{O}), 174.0(\mathrm{C}=\mathrm{O})$.

HRMS (ESI-TOF) $m / z:[\mathrm{M}+\mathrm{H}]^{+}$calcd for $\mathrm{C}_{27} \mathrm{H}_{24} \mathrm{FN}_{2} \mathrm{O}_{5}{ }^{+} 475.1664$, found 475.1671.

\section{Methyl 5-chloro-1-(4-methoxybenzyl)-2,5'-dioxo-1'-phenyl-1,2-dihydrospiro[indole-3,2' -}

\section{pyrrolidine]-4' -carboxylate (8e)}

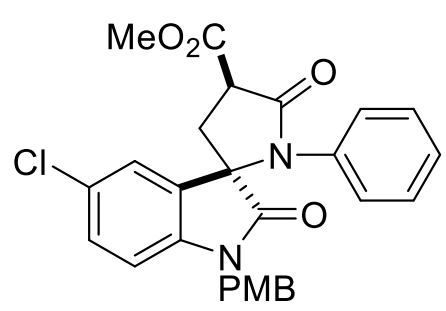

8e was obtained from $7 \mathbf{e}(154 \mathrm{mg}, 0.29 \mathrm{mmol})$ and $\mathrm{TsOH}(10 \mathrm{mg}$,

$0.06 \mathrm{mmol})$ in toluene $(2.0 \mathrm{~mL})$; reaction time $1 \mathrm{~h}$. Yield $127 \mathrm{mg}$ (87\%); colorless oil; dr $\mathbf{A}: \mathbf{B}=78: 22 . \quad R_{f}=0.38$ (ethyl acetate/petroleum ether; $1: 1)$.

A: ${ }^{1} \mathrm{H}$ NMR $\left(\mathrm{CDCl}_{3}, 600 \mathrm{MHz}\right): \delta=2.77\left(\mathrm{dd},{ }^{2} J=13.5,{ }^{3} \mathrm{~J}=9.5 \mathrm{~Hz}, 1 \mathrm{H}, \mathrm{CH}_{2}\right), 2.83\left(\mathrm{dd},{ }^{2} J=\right.$ $\left.13.5,{ }^{3} J=8.9 \mathrm{~Hz}, 1 \mathrm{H}, \mathrm{CH}_{2}\right), 3.74\left(\mathrm{~s}, 3 \mathrm{H}, \mathrm{OCH}_{3}\right), 3.87\left(\mathrm{~s}, 3 \mathrm{H}, \mathrm{OCH}_{3}\right), 4.20\left(\mathrm{dd},{ }^{3} J=9.5,{ }^{3} J=8.9\right.$ $\mathrm{Hz}, 1 \mathrm{H}, \mathrm{CH}), 4.48\left(\mathrm{~d},{ }^{2} J=15.6 \mathrm{~Hz}, 1 \mathrm{H}, \mathrm{NCH}_{2}\right), 4.99\left(\mathrm{~d},{ }^{2} J=15.6 \mathrm{~Hz}, 1 \mathrm{H}, \mathrm{NCH}_{2}\right), 6.53\left(\mathrm{~d},{ }^{3} J=\right.$ $8.4 \mathrm{~Hz}, 1 \mathrm{H}, \mathrm{Ar}), 6.65-6.71(\mathrm{~m}, 2 \mathrm{H}, \mathrm{Ar}), 6.77\left(\mathrm{~d},{ }^{3} \mathrm{~J}=8.6 \mathrm{~Hz}, 2 \mathrm{H}, \mathrm{Ar}\right), 6.95-6.98$ (m, 2H, Ar), $7.14\left(\mathrm{dd},{ }^{3} J=8.4,{ }^{4} J=2.2 \mathrm{~Hz}, 1 \mathrm{H}, \mathrm{Ar}\right), 7.16-7.25(\mathrm{~m}, 3 \mathrm{H}, \mathrm{Ar}), 7.56\left(\mathrm{~d},{ }^{4} J=2.2 \mathrm{~Hz}, 1 \mathrm{H}, \mathrm{Ar}\right)$.

${ }^{13} \mathrm{C} \mathrm{NMR}\left(\mathrm{CDCl}_{3}, 150 \mathrm{MHz}\right): \delta=33.6\left(\mathrm{CH}_{2}\right), 43.3\left(\mathrm{NCH}_{2}\right), 46.82(\mathrm{CH}), 52.92\left(\mathrm{OCH}_{3}\right), 55.12$ $\left(\mathrm{OCH}_{3}\right), 68.56(\mathrm{C}), 110.9(\mathrm{CH}, \mathrm{Ar}), 114.10(2 \times \mathrm{CH}, \mathrm{Ar}), 125.2(\mathrm{CH}, \mathrm{Ar}), 126.05(\mathrm{C}, \mathrm{Ar}), 127.4$ $(2 \times \mathrm{CH}, \mathrm{Ar}), 128.04(\mathrm{CH}, \mathrm{Ar}), 128.2(2 \times \mathrm{CH}, \mathrm{Ar}), 128.9(\mathrm{C}, \mathrm{Ar}), 129.1(2 \times \mathrm{CH}, \mathrm{Ar}), 129.4(\mathrm{C}$, Ar), $130.10(\mathrm{CH}, \mathrm{Ar}), 135.3(\mathrm{C}, \mathrm{Ar}), 140.9$ (C, Ar), 159.1 (C, Ar), $170.0(\mathrm{C}=\mathrm{O}), 170.3(\mathrm{C}=\mathrm{O})$, $175.3(\mathrm{C}=\mathrm{O})$. 
B: ${ }^{1} \mathrm{H} \mathrm{NMR}\left(\mathrm{CDCl}_{3}, 600 \mathrm{MHz}\right): \delta=2.54\left(\mathrm{dd},{ }^{2} J=13.1,{ }^{3} J=9.3 \mathrm{~Hz}, 1 \mathrm{H}, \mathrm{CH}_{2}\right), 3.20\left(\mathrm{dd},{ }^{2} J=\right.$ $\left.13.1,{ }^{3} J=9.0 \mathrm{~Hz}, 1 \mathrm{H}, \mathrm{CH}_{2}\right), 3.74\left(\mathrm{~s}, 3 \mathrm{H}, \mathrm{OCH}_{3}\right), 3.88\left(\mathrm{~s}, 3 \mathrm{H}, \mathrm{OCH}_{3}\right), 4.03\left(\mathrm{dd},{ }^{3} J=9.3,{ }^{3} J=9.0\right.$ $\mathrm{Hz}, 1 \mathrm{H}, \mathrm{CH}), 4.40\left(\mathrm{~d},{ }^{2} J=15.6 \mathrm{~Hz}, 1 \mathrm{H}, \mathrm{NCH}_{2}\right), 5.02\left(\mathrm{~d},{ }^{2} J=15.6 \mathrm{~Hz}, 1 \mathrm{H}, \mathrm{NCH}_{2}\right), 6.56\left(\mathrm{~d},{ }^{3} J=\right.$ $8.4 \mathrm{~Hz}, 1 \mathrm{H}, \mathrm{Ar}), 6.65-6.71(\mathrm{~m}, 4 \mathrm{H}, \mathrm{Ar}), 6.95-6.98$ (m, 2H, Ar), 7.16-7.25 (m, 4H, Ar), 7.40 (d, $\left.{ }^{4} J=2.2 \mathrm{~Hz}, 1 \mathrm{H}, \mathrm{Ar}\right)$.

${ }^{13} \mathrm{C} \mathrm{NMR}\left(\mathrm{CDCl}_{3}, 150 \mathrm{MHz}\right): \delta=34.2\left(\mathrm{CH}_{2}\right), 43.4\left(\mathrm{NCH}_{2}\right), 46.77(\mathrm{CH}), 52.92\left(\mathrm{OCH}_{3}\right), 55.12$ $\left(\mathrm{OCH}_{3}\right), 68.56(\mathrm{C}), 111.2(\mathrm{CH}, \mathrm{Ar}), 114.06(2 \times \mathrm{CH}, \mathrm{Ar}), 123.8(\mathrm{CH}, \mathrm{Ar}), 126.05(\mathrm{C}, \mathrm{Ar}), 127.2$ $(2 \times \mathrm{CH}, \mathrm{Ar}), 128.04(\mathrm{CH}, \mathrm{Ar}), 128.1(2 \times \mathrm{CH}, \mathrm{Ar}), 128.8(\mathrm{C}, \mathrm{Ar}), 129.2(2 \times \mathrm{CH}, \mathrm{Ar}), 130.16(\mathrm{CH}$, Ar), 130.4 (C, Ar), 135.4 (C, Ar), 140.5 (C, Ar), 159.0 (C, Ar), 168.6 (C=O), 169.6 (C=O), 173.7 $(\mathrm{C}=\mathrm{O})$.

HRMS (ESI-TOF) $m / z:[\mathrm{M}+\mathrm{H}]^{+}$calcd for $\mathrm{C}_{27} \mathrm{H}_{24} \mathrm{ClN}_{2} \mathrm{O}_{5}{ }^{+} 491.1368$, found 491.1380.

\section{Methyl 5-bromo-1-(4-methoxybenzyl)-2,5' -dioxo-1'-phenyl-1,2-dihydrospiro[indole-3,2' -}

\section{pyrrolidine]-4' -carboxylate (8f)}

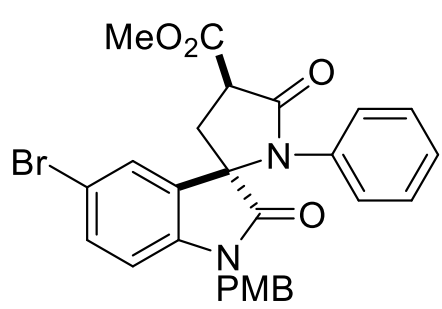

8f was obtained from $7 \mathbf{f}(187 \mathrm{mg}, 0.33 \mathrm{mmol})$ and $\mathrm{TsOH}(11 \mathrm{mg}$, $0.07 \mathrm{mmol})$ in toluene $(2.2 \mathrm{~mL})$; reaction time $1 \mathrm{~h}$. Yield $151 \mathrm{mg}$ $(86 \%) ;$ colorless oil; dr $\mathbf{A}: \mathbf{B}=79: 21 . \quad R_{f}=0.40$ (ethyl acetate/petroleum ether; $1: 1)$

A: ${ }^{1} \mathrm{H} \mathrm{NMR}\left(\mathrm{CDCl}_{3}, 600 \mathrm{MHz}\right): \delta=2.77\left(\mathrm{dd},{ }^{2} J=13.5,{ }^{3} J=9.6 \mathrm{~Hz}, 1 \mathrm{H}, \mathrm{CH}_{2}\right), 2.82\left(\mathrm{dd},{ }^{2} J=\right.$ $\left.13.5,{ }^{3} J=8.9 \mathrm{~Hz}, 1 \mathrm{H}, \mathrm{CH}_{2}\right), 3.73\left(\mathrm{~s}, 3 \mathrm{H}, \mathrm{OCH}_{3}\right), 3.88\left(\mathrm{~s}, 3 \mathrm{H}, \mathrm{OCH}_{3}\right), 4.20\left(\mathrm{dd},{ }^{3} J=9.6,{ }^{3} J=8.9\right.$ $\mathrm{Hz}, 1 \mathrm{H}, \mathrm{CH}), 4.47\left(\mathrm{~d},{ }^{2} J=15.5 \mathrm{~Hz}, 1 \mathrm{H}, \mathrm{NCH}_{2}\right), 4.98\left(\mathrm{~d},{ }^{2} J=15.5 \mathrm{~Hz}, 1 \mathrm{H}, \mathrm{NCH}_{2}\right), 6.48\left(\mathrm{~d},{ }^{3} J=\right.$ $8.4 \mathrm{~Hz}, 1 \mathrm{H}, \mathrm{Ar}), 6.65-6.71(\mathrm{~m}, 2 \mathrm{H}, \mathrm{Ar}), 6.77\left(\mathrm{~d},{ }^{3} \mathrm{~J}=8.8 \mathrm{~Hz}, 2 \mathrm{H}, \mathrm{Ar}\right), 6.95-6.98$ (m, 2H, Ar), 7.16-7.25 (m, 3H, Ar), $7.28\left(\mathrm{dd},{ }^{3} J=8.4,{ }^{4} J=2.0 \mathrm{~Hz}, 1 \mathrm{H}, \mathrm{Ar}\right), 7.69\left(\mathrm{~d},{ }^{4} J=2.0 \mathrm{~Hz}, 1 \mathrm{H}, \mathrm{Ar}\right)$.

${ }^{13} \mathrm{C} \mathrm{NMR}\left(\mathrm{CDCl}_{3}, 150 \mathrm{MHz}\right): \delta=33.6\left(\mathrm{CH}_{2}\right), 43.3\left(\mathrm{NCH}_{2}\right), 46.80(\mathrm{CH}), 52.91\left(\mathrm{OCH}_{3}\right), 55.11$ $\left(\mathrm{OCH}_{3}\right), 68.47(\mathrm{C}), 111.4(\mathrm{CH}, \mathrm{Ar}), 114.09(2 \times \mathrm{CH}, \mathrm{Ar}), 116.1(\mathrm{C}, \mathrm{Ar}), 126.00(\mathrm{C}, \mathrm{Ar}), 127.4$ $(2 \times \mathrm{CH}, \mathrm{Ar}), 127.9(\mathrm{CH}, \mathrm{Ar}), 128.03(\mathrm{CH}, \mathrm{Ar}), 128.14(2 \times \mathrm{CH}, \mathrm{Ar}), 129.1(2 \times \mathrm{CH}, \mathrm{Ar}), 129.7(\mathrm{C}$, 
Ar), $133.1(\mathrm{CH}, \mathrm{Ar}), 135.31(\mathrm{C}, \mathrm{Ar}), 141.4(\mathrm{C}, \mathrm{Ar}), 159.04(\mathrm{C}, \mathrm{Ar}), 169.9(\mathrm{C}=\mathrm{O}), 170.3(\mathrm{C}=\mathrm{O})$, $175.2(\mathrm{C}=\mathrm{O})$.

B: ${ }^{1} \mathrm{H}$ NMR $\left(\mathrm{CDCl}_{3}, 600 \mathrm{MHz}\right): \delta=2.54\left(\mathrm{dd},{ }^{2} J=13.1,{ }^{3} \mathrm{~J}=9.3 \mathrm{~Hz}, 1 \mathrm{H}, \mathrm{CH}_{2}\right), 3.20\left(\mathrm{dd},{ }^{2} J=\right.$ $\left.13.1,{ }^{3} J=9.1 \mathrm{~Hz}, 1 \mathrm{H}, \mathrm{CH}_{2}\right), 3.74\left(\mathrm{~s}, 3 \mathrm{H}, \mathrm{OCH}_{3}\right), 3.87\left(\mathrm{~s}, 3 \mathrm{H}, \mathrm{OCH}_{3}\right), 4.03\left(\mathrm{dd},{ }^{3} J=9.3,{ }^{3} J=9.1\right.$ $\mathrm{Hz}, 1 \mathrm{H}, \mathrm{CH}), 4.39\left(\mathrm{~d},{ }^{2} J=15.6 \mathrm{~Hz}, 1 \mathrm{H}, \mathrm{NCH}_{2}\right), 5.01\left(\mathrm{~d},{ }^{2} J=15.6 \mathrm{~Hz}, 1 \mathrm{H}, \mathrm{NCH}_{2}\right), 6.51\left(\mathrm{~d},{ }^{3} J=\right.$ 8.4 Hz, 1H, Ar), 6.65-6.71 (m, 4H, Ar), 6.95-6.98 (m, 2H, Ar), 7.16-7.25 (m, 3H, Ar), 7.32 (dd, $\left.{ }^{3} J=8.4,{ }^{4} J=2.0 \mathrm{~Hz}, 1 \mathrm{H}, \mathrm{Ar}\right), 7.53\left(\mathrm{~d},{ }^{4} J=2.0 \mathrm{~Hz}, 1 \mathrm{H}, \mathrm{Ar}\right)$.

${ }^{13} \mathrm{C} \mathrm{NMR}\left(\mathrm{CDCl}_{3}, 150 \mathrm{MHz}\right): \delta=34.2\left(\mathrm{CH}_{2}\right), 43.4\left(\mathrm{NCH}_{2}\right), 46.75(\mathrm{CH}), 52.91\left(\mathrm{OCH}_{3}\right), 55.11$ $\left(\mathrm{OCH}_{3}\right), 68.47(\mathrm{C}), 111.7(\mathrm{CH}, \mathrm{Ar}), 114.05(2 \times \mathrm{CH}, \mathrm{Ar}), 115.9(\mathrm{C}, \mathrm{Ar}), 126.00(\mathrm{C}, \mathrm{Ar}), 126.5$ $(\mathrm{CH}, \mathrm{Ar}), 127.2(2 \times \mathrm{CH}, \mathrm{Ar}), 128.03(\mathrm{CH}, \mathrm{Ar}), 128.09(2 \times \mathrm{CH}, \mathrm{Ar}), 129.2(2 \times \mathrm{CH}, \mathrm{Ar}), 130.8(\mathrm{C}$, Ar), 133.0 (CH, Ar), 135.33 (C, Ar), 141.0 (C, Ar), 158.98 (C, Ar), $168.6(\mathrm{C}=\mathrm{O}), 169.6(\mathrm{C}=\mathrm{O})$, $173.6(\mathrm{C}=\mathrm{O})$.

HRMS (ESI-TOF) $m / z:[\mathrm{M}+\mathrm{H}]^{+}$calcd for $\mathrm{C}_{27} \mathrm{H}_{24} \mathrm{BrN}_{2} \mathrm{O}_{5}{ }^{+}$535.0863, found 535.0877.

\section{Methyl 1'-(4-fluorophenyl)-1-(4-methoxybenzyl)-2,5'-dioxo-1,2-dihydrospiro[indole-3,2'-} pyrrolidine]-4' -carboxylate $(8 g)$

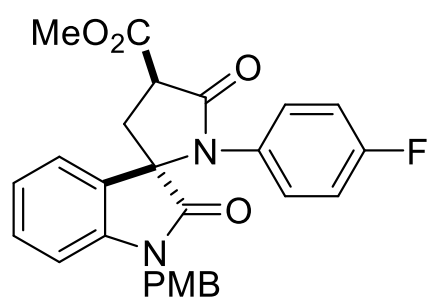

$\mathbf{8 g}$ was obtained from $\mathbf{7 g}(190 \mathrm{mg}, 0.38 \mathrm{mmol})$ and $\mathrm{TsOH}(13 \mathrm{mg}$, $0.08 \mathrm{mmol})$ in toluene $(2.5 \mathrm{~mL})$; reaction time $1 \mathrm{~h}$. Yield $163 \mathrm{mg}$ (92\%); colorless solid, mp $189-190{ }^{\circ} \mathrm{C} ; \mathrm{dr} \mathbf{A}: \mathbf{B}=77: 23 . R_{f}=0.29$ (ethyl acetate/petroleum ether; 1:1).

A: ${ }^{1} \mathrm{H}$ NMR $\left(\mathrm{CDCl}_{3}, 600 \mathrm{MHz}\right): \delta=2.77\left(\mathrm{dd},{ }^{2} J=13.5,{ }^{3} J=9.6 \mathrm{~Hz}, 1 \mathrm{H}, \mathrm{CH}_{2}\right), 2.85\left(\mathrm{dd},{ }^{2} J=\right.$ $\left.13.5,{ }^{3} \mathrm{~J}=9.0 \mathrm{~Hz}, 1 \mathrm{H}, \mathrm{CH}_{2}\right), 3.73\left(\mathrm{~s}, 3 \mathrm{H}, \mathrm{OCH}_{3}\right), 3.85\left(\mathrm{~s}, 3 \mathrm{H}, \mathrm{OCH}_{3}\right), 4.21\left(\mathrm{dd},{ }^{3} J=9.6,{ }^{3} J=9.0\right.$ $\mathrm{Hz}, 1 \mathrm{H}, \mathrm{CH}), 4.48\left(\mathrm{~d},{ }^{2} J=15.5 \mathrm{~Hz}, 1 \mathrm{H}, \mathrm{NCH}_{2}\right), 4.97\left(\mathrm{~d},{ }^{2} J=15.5 \mathrm{~Hz}, 1 \mathrm{H}, \mathrm{NCH}_{2}\right), 6.64\left(\mathrm{~d},{ }^{3} J=\right.$ $7.8 \mathrm{~Hz}, 1 \mathrm{H}, \mathrm{Ar}), 6.69-6.72$ (m, 2H, Ar), 6.78-6.85 (m, 4H, Ar), 6.89-6.94 (m, 2H, Ar), 7.08$7.12(\mathrm{~m}, 1 \mathrm{H}, \mathrm{Ar}), 7.18-7.24(\mathrm{~m}, 1 \mathrm{H}, \mathrm{Ar}), 7.57\left(\mathrm{~d},{ }^{3} J=7.4 \mathrm{~Hz}, 1 \mathrm{H}, \mathrm{Ar}\right)$. 
${ }^{13} \mathrm{C} \mathrm{NMR}\left(\mathrm{CDCl}_{3}, 150 \mathrm{MHz}\right): \delta=33.3\left(\mathrm{CH}_{2}\right), 43.1\left(\mathrm{NCH}_{2}\right), 46.79(\mathrm{CH}), 52.8\left(\mathrm{OCH}_{3}\right), 55.01$ $\left(\mathrm{OCH}_{3}\right), 68.64(\mathrm{C}), 109.8(\mathrm{CH}, \mathrm{Ar}), 113.9(2 \times \mathrm{CH}, \mathrm{Ar}), 115.86\left({ }^{2} J_{\mathrm{CF}}=22 \mathrm{~Hz}, 2 \times \mathrm{CH}, \mathrm{Ar}\right), 123.5$ $(\mathrm{CH}, \mathrm{Ar}), 124.8(\mathrm{CH}, \mathrm{Ar}), 126.42(\mathrm{C}, \mathrm{Ar}), 127.3(\mathrm{C}, \mathrm{Ar}), 128.11(2 \times \mathrm{CH}, \mathrm{Ar}), 129.6\left({ }^{3} J_{\mathrm{CF}}=8 \mathrm{~Hz}\right.$, $2 \times \mathrm{CH}, \mathrm{Ar}), 130.33(\mathrm{CH}, \mathrm{Ar}), 131.35\left({ }^{4} J_{\mathrm{CF}}=2 \mathrm{~Hz}, \mathrm{C}, \mathrm{Ar}\right), 142.4(\mathrm{C}, \mathrm{Ar}), 158.95(\mathrm{C}, \mathrm{Ar}), 161.7$ $\left({ }^{1} J_{\mathrm{CF}}=248 \mathrm{~Hz}, \mathrm{C}, \mathrm{Ar}\right), 170.0(\mathrm{C}=\mathrm{O}), 170.6(\mathrm{C}=\mathrm{O}), 175.5(\mathrm{C}=\mathrm{O})$.

B: ${ }^{1} \mathrm{H}$ NMR $\left(\mathrm{CDCl}_{3}, 600 \mathrm{MHz}\right): \delta=2.56\left(\mathrm{dd},{ }^{2} J=13.1,{ }^{3} J=9.3 \mathrm{~Hz}, 1 \mathrm{H}, \mathrm{CH}_{2}\right), 3.19\left(\mathrm{dd},{ }^{2} J=\right.$ $\left.13.1,{ }^{3} J=9.0 \mathrm{~Hz}, 1 \mathrm{H}, \mathrm{CH}_{2}\right), 3.72\left(\mathrm{~s}, 3 \mathrm{H}, \mathrm{OCH}_{3}\right), 3.87\left(\mathrm{~s}, 3 \mathrm{H}, \mathrm{OCH}_{3}\right), 4.05\left(\mathrm{dd},{ }^{3} J=9.3,{ }^{3} J=9.0\right.$ $\mathrm{Hz}, 1 \mathrm{H}, \mathrm{CH}), 4.41\left(\mathrm{~d},{ }^{2} \mathrm{~J}=15.5 \mathrm{~Hz}, 1 \mathrm{H}, \mathrm{NCH}_{2}\right), 5.01\left(\mathrm{~d},{ }^{2} \mathrm{~J}=15.5 \mathrm{~Hz}, 1 \mathrm{H}, \mathrm{NCH}_{2}\right), 6.66-6.68(\mathrm{~m}$, 3H, Ar), 6.69-6.72 (m, 2H, Ar), 6.78-6.85 (m, 2H, Ar), 6.89-6.94 (m, 2H, Ar), 7.08-7.12 (m, 1H, Ar), 7.18-7.24 (m, 1H, Ar), $7.40\left(\mathrm{~d},{ }^{3} J=7.4 \mathrm{~Hz}, 1 \mathrm{H}, \mathrm{Ar}\right)$.

${ }^{13} \mathrm{C} \mathrm{NMR}\left(\mathrm{CDCl}_{3}, 150 \mathrm{MHz}\right): \delta=33.9\left(\mathrm{CH}_{2}\right), 43.2\left(\mathrm{NCH}_{2}\right), 46.79(\mathrm{CH}), 52.9\left(\mathrm{OCH}_{3}\right), 54.99$ $\left(\mathrm{OCH}_{3}\right), 68.59(\mathrm{C}), 110.1(\mathrm{CH}, \mathrm{Ar}), 113.8(2 \times \mathrm{CH}, \mathrm{Ar}), 115.89\left({ }^{2} J_{\mathrm{CF}}=22 \mathrm{~Hz}, 2 \times \mathrm{CH}, \mathrm{Ar}\right), 123.3$ $(\mathrm{CH}, \mathrm{Ar}), 123.4(\mathrm{CH}, \mathrm{Ar}), 126.42(\mathrm{C}, \mathrm{Ar}), 128.06(2 \times \mathrm{CH}, \mathrm{Ar}), 128.3(\mathrm{C}, \mathrm{Ar}), 129.5\left({ }^{3} J_{\mathrm{CF}}=8 \mathrm{~Hz}\right.$, $2 \times \mathrm{CH}, \mathrm{Ar}), 130.33(\mathrm{CH}, \mathrm{Ar}), 131.28\left({ }^{4} J_{\mathrm{CF}}=2 \mathrm{~Hz}, \mathrm{C}, \mathrm{Ar}\right), 142.0(\mathrm{C}, \mathrm{Ar}), 158.90(\mathrm{C}, \mathrm{Ar}), 161.8$ $\left({ }^{1} J_{\mathrm{CF}}=248 \mathrm{~Hz}, \mathrm{C}, \mathrm{Ar}\right), 168.7(\mathrm{C}=\mathrm{O}), 169.9(\mathrm{C}=\mathrm{O}), 173.9(\mathrm{C}=\mathrm{O})$.

HRMS (ESI-TOF) $m / z:[\mathrm{M}+\mathrm{H}]^{+}$calcd for $\mathrm{C}_{27} \mathrm{H}_{24} \mathrm{FN}_{2} \mathrm{O}_{5}{ }^{+} 475.1664$, found 475.1670.

\section{Methyl 1'-(4-chlorophenyl)-1-(4-methoxybenzyl)-2,5'-dioxo-1,2-dihydrospiro[indole-3,2'-} pyrrolidine]-4' -carboxylate $(8 \mathrm{~h})$

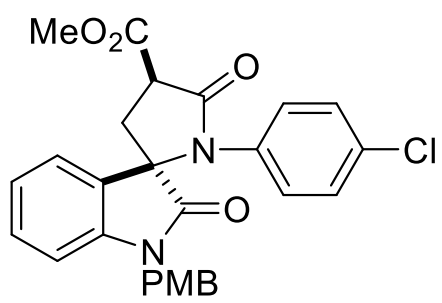

8h was obtained from $7 \mathbf{h}(174 \mathrm{mg}, 0.33 \mathrm{mmol})$ and $\mathrm{TsOH}(11 \mathrm{mg}$, $0.07 \mathrm{mmol})$ in toluene $(2.2 \mathrm{~mL})$; reaction time $1 \mathrm{~h}$. Yield $144 \mathrm{mg}$ (88\%); colorless solid, mp $139-140{ }^{\circ} \mathrm{C} ; \mathrm{dr} \mathbf{A}: \mathbf{B}=78: 22 . R_{f}=0.30$ (ethyl acetate/petroleum ether; 1:1).

A: ${ }^{1} \mathrm{H}$ NMR $\left(\mathrm{CDCl}_{3}, 600 \mathrm{MHz}\right): \delta=2.77\left(\mathrm{dd},{ }^{2} J=13.6,{ }^{3} J=9.6 \mathrm{~Hz}, 1 \mathrm{H}, \mathrm{CH}_{2}\right), 2.86\left(\mathrm{dd},{ }^{2} J=\right.$ $\left.13.6,{ }^{3} J=9.0 \mathrm{~Hz}, 1 \mathrm{H}, \mathrm{CH}_{2}\right), 3.75\left(\mathrm{~s}, 3 \mathrm{H}, \mathrm{OCH}_{3}\right), 3.86\left(\mathrm{~s}, 3 \mathrm{H}, \mathrm{OCH}_{3}\right), 4.21\left(\mathrm{dd},{ }^{3} J=9.6,{ }^{3} J=9.0\right.$ $\mathrm{Hz}, 1 \mathrm{H}, \mathrm{CH}), 4.46\left(\mathrm{~d},{ }^{2} J=15.5 \mathrm{~Hz}, 1 \mathrm{H}, \mathrm{NCH}_{2}\right), 5.01\left(\mathrm{~d},{ }^{2} \mathrm{~J}=15.5 \mathrm{~Hz}, 1 \mathrm{H}, \mathrm{NCH}_{2}\right), 6.64\left(\mathrm{~d},{ }^{3} J=\right.$ 
$7.8 \mathrm{~Hz}, 1 \mathrm{H}, \mathrm{Ar}), 6.67-6.76(\mathrm{~m}, 4 \mathrm{H}, \mathrm{Ar}), 6.85-6.89$ (m, 2H, Ar), 7.08-7.14 (m, 3H, Ar), 7.21 $\left(\mathrm{ddd},{ }^{3} J=7.8,{ }^{3} \mathrm{~J}=7.6,{ }^{4} \mathrm{~J}=1.2 \mathrm{~Hz}, 1 \mathrm{H}, \mathrm{Ar}\right), 7.56\left(\mathrm{ddd},{ }^{3} \mathrm{~J}=7.5,{ }^{4} \mathrm{~J}=1.2,{ }^{5} \mathrm{~J}=0.4 \mathrm{~Hz}, 1 \mathrm{H}, \mathrm{Ar}\right)$.

${ }^{13} \mathrm{C} \mathrm{NMR}\left(\mathrm{CDCl}_{3}, 150 \mathrm{MHz}\right): \delta=33.3\left(\mathrm{CH}_{2}\right), 43.1\left(\mathrm{NCH}_{2}\right), 46.83(\mathrm{CH}), 52.88\left(\mathrm{OCH}_{3}\right), 55.05$ $\left(\mathrm{OCH}_{3}\right), 68.54(\mathrm{C}), 109.9(\mathrm{CH}, \mathrm{Ar}), 113.93(2 \times \mathrm{CH}, \mathrm{Ar}), 123.6(\mathrm{CH}, \mathrm{Ar}), 124.7(\mathrm{CH}, \mathrm{Ar}), 126.27$ (C, Ar), $128.1(2 \times \mathrm{CH}, \mathrm{C}, \mathrm{Ar}), 128.9(2 \times \mathrm{CH}, \mathrm{Ar}), 129.15(2 \times \mathrm{CH}, \mathrm{Ar}), 130.41(\mathrm{CH}, \mathrm{Ar}), 133.6(\mathrm{C}$, Ar), 134.04 (C, Ar), 142.4 (C, Ar), 159.0 (C, Ar), 169.9 (C=O), 170.5 (C=O), $175.4(\mathrm{C}=\mathrm{O})$.

B: ${ }^{1} \mathrm{H} \mathrm{NMR}\left(\mathrm{CDCl}_{3}, 600 \mathrm{MHz}\right): \delta=2.57\left(\mathrm{dd},{ }^{2} J=13.1,{ }^{3} J=9.3 \mathrm{~Hz}, 1 \mathrm{H}, \mathrm{CH}_{2}\right), 3.19\left(\mathrm{dd},{ }^{2} J=\right.$ $\left.13.1,{ }^{3} J=9.1 \mathrm{~Hz}, 1 \mathrm{H}, \mathrm{CH}_{2}\right), 3.74\left(\mathrm{~s}, 3 \mathrm{H}, \mathrm{OCH}_{3}\right), 3.88\left(\mathrm{~s}, 3 \mathrm{H}, \mathrm{OCH}_{3}\right), 4.06\left(\mathrm{dd},{ }^{3} J=9.3,{ }^{3} J=9.1\right.$ $\mathrm{Hz}, 1 \mathrm{H}, \mathrm{CH}), 4.39\left(\mathrm{~d},{ }^{2} \mathrm{~J}=15.5 \mathrm{~Hz}, 1 \mathrm{H}, \mathrm{NCH}_{2}\right), 5.05\left(\mathrm{~d},{ }^{2} \mathrm{~J}=15.5 \mathrm{~Hz}, 1 \mathrm{H}, \mathrm{NCH}_{2}\right), 6.67-6.76(\mathrm{~m}$, 5H, Ar), 6.85-6.89 (m, 2H, Ar), 7.08-7.14 (m, 3H, Ar), $7.23\left(\mathrm{ddd},{ }^{3} J=7.8,{ }^{3} J=7.6,{ }^{4} J=1.2 \mathrm{~Hz}\right.$, $1 \mathrm{H}, \mathrm{Ar}), 7.40\left(\mathrm{ddd},{ }^{3} J=7.5,{ }^{4} \mathrm{~J}=1.2,{ }^{5} \mathrm{~J}=0.4 \mathrm{~Hz}, 1 \mathrm{H}, \mathrm{Ar}\right)$.

${ }^{13} \mathrm{C} \mathrm{NMR}\left(\mathrm{CDCl}_{3}, 150 \mathrm{MHz}\right): \delta=34.0\left(\mathrm{CH}_{2}\right), 43.2\left(\mathrm{NCH}_{2}\right), 46.83(\mathrm{CH}), 52.92\left(\mathrm{OCH}_{3}\right), 55.05$ $\left(\mathrm{OCH}_{3}\right), 68.49(\mathrm{C}), 110.2(\mathrm{CH}, \mathrm{Ar}), 113.87(2 \times \mathrm{CH}, \mathrm{Ar}), 123.3(\mathrm{CH}, \mathrm{Ar}), 123.5(\mathrm{CH}, \mathrm{Ar}), 126.31$ (C, Ar), $127.2(2 \times \mathrm{CH}, \mathrm{Ar}), 128.3(\mathrm{C}, \mathrm{Ar}), 128.7(2 \times \mathrm{CH}, \mathrm{Ar}), 129.22(2 \times \mathrm{CH}, \mathrm{Ar}), 130.39(\mathrm{CH}$, Ar), $133.7(\mathrm{C}, \mathrm{Ar}), 133.99(\mathrm{C}, \mathrm{Ar}), 142.0(\mathrm{C}, \mathrm{Ar}), 158.9(\mathrm{C}, \mathrm{Ar}), 168.7(\mathrm{C}=\mathrm{O}), 169.8(\mathrm{C}=\mathrm{O})$, $173.8(\mathrm{C}=\mathrm{O})$.

HRMS (ESI-TOF) $m / z:[\mathrm{M}+\mathrm{H}]^{+}$calcd for $\mathrm{C}_{27} \mathrm{H}_{24} \mathrm{ClN}_{2} \mathrm{O}_{5}{ }^{+} 491.1368$, found 491.1376 .

\section{Methyl 1'-(3-chlorophenyl)-1-(4-methoxybenzyl)-2,5'-dioxo-1,2-dihydrospiro[indole-3,2'-}

\section{pyrrolidine]-4' -carboxylate (8i)}

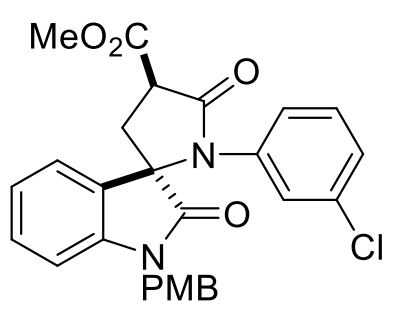

8i was obtained from $7 \mathbf{i}(185 \mathrm{mg}, 0.35 \mathrm{mmol})$ and TsOH (12 mg, 0.07 mmol) in toluene $(2.4 \mathrm{~mL})$; reaction time $1 \mathrm{~h}$. Yield $162 \mathrm{mg}(93 \%)$; colorless solid, mp $150-151{ }^{\circ} \mathrm{C} ; \mathrm{dr} \mathbf{A}: \mathbf{B}=78: 22 . R_{f}=0.35$ (ethyl acetate/petroleum ether; $1: 1)$.

A: ${ }^{1} \mathrm{H} \mathrm{NMR}\left(\mathrm{CDCl}_{3}, 600 \mathrm{MHz}\right): \delta=2.76\left(\mathrm{dd},{ }^{2} J=13.4,{ }^{3} J=9.4 \mathrm{~Hz}, 1 \mathrm{H}, \mathrm{CH}_{2}\right), 2.85\left(\mathrm{dd},{ }^{2} J=\right.$ $\left.13.4,{ }^{3} J=9.4 \mathrm{~Hz}, 1 \mathrm{H}, \mathrm{CH}_{2}\right), 3.74\left(\mathrm{~s}, 3 \mathrm{H}, \mathrm{OCH}_{3}\right), 3.86\left(\mathrm{~s}, 3 \mathrm{H}, \mathrm{OCH}_{3}\right), 4.24\left(\mathrm{dd},{ }^{3} J=9.4,{ }^{3} J=9.4\right.$ 
$\mathrm{Hz}, 1 \mathrm{H}, \mathrm{CH}), 4.53\left(\mathrm{~d},{ }^{2} J=15.3 \mathrm{~Hz}, 1 \mathrm{H}, \mathrm{NCH}_{2}\right), 5.02\left(\mathrm{~d},{ }^{2} J=15.3 \mathrm{~Hz}, 1 \mathrm{H}, \mathrm{NCH}_{2}\right), 6.68\left(\mathrm{~d},{ }^{3} J=\right.$ $7.8 \mathrm{~Hz}, 1 \mathrm{H}, \mathrm{Ar}), 6.75\left(\mathrm{~d},{ }^{3} \mathrm{~J}=8.8 \mathrm{~Hz}, 2 \mathrm{H}, \mathrm{Ar}\right), 6.78-6.81(\mathrm{~m}, 1 \mathrm{H}, \mathrm{Ar}), 6.82\left(\mathrm{~d},{ }^{3} J=8.8 \mathrm{~Hz}, 2 \mathrm{H}\right.$, Ar), 7.01-7.11 (m, 3H, Ar), $7.13\left(\mathrm{ddd},{ }^{3} J=8.1,{ }^{4} J=2.1,{ }^{4} J=1.0 \mathrm{~Hz}, 1 \mathrm{H}\right.$, Ar $), 7.20\left(\mathrm{ddd},{ }^{3} J=\right.$ $\left.7.8,{ }^{3} J=7.6,{ }^{4} J=1.2 \mathrm{~Hz}, 1 \mathrm{H}, \mathrm{Ar}\right), 7.49\left(\mathrm{ddd},{ }^{3} J=7.5,{ }^{4} \mathrm{~J}=1.2,{ }^{5} \mathrm{~J}=0.4 \mathrm{~Hz}, 1 \mathrm{H}, \mathrm{Ar}\right)$.

${ }^{13} \mathrm{C} \mathrm{NMR}\left(\mathrm{CDCl}_{3}, 150 \mathrm{MHz}\right): \delta=34.0\left(\mathrm{CH}_{2}\right), 43.3\left(\mathrm{NCH}_{2}\right), 46.76(\mathrm{CH}), 52.87\left(\mathrm{OCH}_{3}\right), 55.06$ $\left(\mathrm{OCH}_{3}\right), 68.4(\mathrm{C}), 109.9(\mathrm{CH}, \mathrm{Ar}), 114.1(2 \times \mathrm{CH}, \mathrm{Ar}), 123.6(\mathrm{CH}, \mathrm{Ar}), 124.6(\mathrm{CH}, \mathrm{Ar}), 125.3$ $(\mathrm{CH}, \mathrm{Ar}), 126.50$ (C, Ar), 126.9 (C, Ar), $127.4(\mathrm{CH}, \mathrm{Ar}), 128.02(\mathrm{CH}, \mathrm{Ar}), 128.3$ (2×CH, Ar), 129.85 (CH, Ar), 130.39 (CH, Ar), 134.3 (C, Ar), 136.68 (C, Ar), 142.4 (C, Ar), 159.0 (C, Ar), $169.9(\mathrm{C}=\mathrm{O}), 170.4(\mathrm{C}=\mathrm{O}), 175.5(\mathrm{C}=\mathrm{O})$.

B: ${ }^{1} \mathrm{H}$ NMR $\left(\mathrm{CDCl}_{3}, 600 \mathrm{MHz}\right): \delta=2.55\left(\mathrm{dd},{ }^{2} J=13.0,{ }^{3} \mathrm{~J}=9.3 \mathrm{~Hz}, 1 \mathrm{H}, \mathrm{CH}_{2}\right), 3.18\left(\mathrm{dd},{ }^{2} J=\right.$ $\left.13.0,{ }^{3} J=9.0 \mathrm{~Hz}, 1 \mathrm{H}, \mathrm{CH}_{2}\right), 3.74\left(\mathrm{~s}, 3 \mathrm{H}, \mathrm{OCH}_{3}\right), 3.87\left(\mathrm{~s}, 3 \mathrm{H}, \mathrm{OCH}_{3}\right), 4.04\left(\mathrm{dd},{ }^{3} J=9.3,{ }^{3} J=9.0\right.$ $\mathrm{Hz}, 1 \mathrm{H}, \mathrm{CH}), 4.46\left(\mathrm{~d},{ }^{2} J=15.4 \mathrm{~Hz}, 1 \mathrm{H}, \mathrm{NCH}_{2}\right), 5.04\left(\mathrm{~d},{ }^{2} J=15.4 \mathrm{~Hz}, 1 \mathrm{H}, \mathrm{NCH}_{2}\right), 6.70-6.73(\mathrm{~m}$, $3 \mathrm{H}, \mathrm{Ar}), 6.78-6.81(\mathrm{~m}, 1 \mathrm{H}, \mathrm{Ar}), 6.84\left(\mathrm{~d},{ }^{3} \mathrm{~J}=8.7 \mathrm{~Hz}, 2 \mathrm{H}, \mathrm{Ar}\right), 7.01-7.11(\mathrm{~m}, 3 \mathrm{H}, \mathrm{Ar}), 7.16$ (ddd, ${ }^{3} J=8.1,{ }^{4} J=2.0,{ }^{4} J=0.9 \mathrm{~Hz}, 1 \mathrm{H}$, Ar $), 7.23\left(\mathrm{ddd},{ }^{3} J=7.8,{ }^{3} J=7.6,{ }^{4} J=1.2 \mathrm{~Hz}, 1 \mathrm{H}\right.$, Ar $), 7.37$ (ddd, $\left.{ }^{3} J=7.5,{ }^{4} J=1.2,{ }^{5} \mathrm{~J}=0.4 \mathrm{~Hz}, 1 \mathrm{H}, \mathrm{Ar}\right)$.

${ }^{13} \mathrm{C} \mathrm{NMR}\left(\mathrm{CDCl}_{3}, 150 \mathrm{MHz}\right): \delta=34.5\left(\mathrm{CH}_{2}\right), 43.4\left(\mathrm{NCH}_{2}\right), 46.83(\mathrm{CH}), 52.94\left(\mathrm{OCH}_{3}\right), 55.06$ $\left(\mathrm{OCH}_{3}\right), 68.5(\mathrm{C}), 110.3(\mathrm{CH}, \mathrm{Ar}), 114.0(2 \times \mathrm{CH}, \mathrm{Ar}), 123.2(\mathrm{CH}, \mathrm{Ar}), 123.5(\mathrm{CH}, \mathrm{Ar}), 125.0$ $(\mathrm{CH}, \mathrm{Ar}), 126.50(\mathrm{C}, \mathrm{Ar}), 127.1(\mathrm{CH}, \mathrm{Ar}), 127.96(\mathrm{CH}, \mathrm{Ar}), 128.1(\mathrm{C}, \mathrm{Ar}), 128.2(2 \times \mathrm{CH}, \mathrm{Ar})$, 129.90 (CH, Ar), 130.39 (CH, Ar), 134.4 (C, Ar), 136.73 (C, Ar), 141.8 (C, Ar), 158.9 (C, Ar), $168.6(\mathrm{C}=\mathrm{O}), 169.7(\mathrm{C}=\mathrm{O}), 173.9(\mathrm{C}=\mathrm{O})$.

HRMS (ESI-TOF) $m / z:[\mathrm{M}+\mathrm{H}]^{+}$calcd for $\mathrm{C}_{27} \mathrm{H}_{24} \mathrm{ClN}_{2} \mathrm{O}_{5}{ }^{+} 491.1368$, found 491.1376.

\section{Methyl 1'-(4-bromophenyl)-1-(4-methoxybenzyl)-2,5'-dioxo-1,2-dihydrospiro[indole-3,2' -}

\section{pyrrolidine]-4' -carboxylate $(8 \mathbf{j})$}

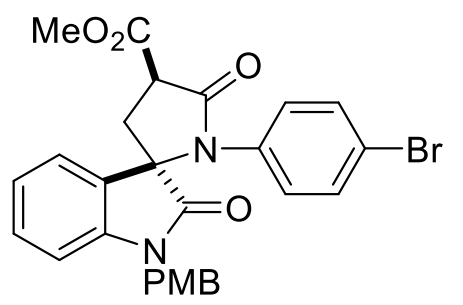

8j was obtained from $7 \mathbf{j}$ (105 mg, $0.19 \mathrm{mmol})$ and $\mathrm{TsOH}(6 \mathrm{mg}$,

$0.04 \mathrm{mmol})$ in toluene $(1.2 \mathrm{~mL})$; reaction time $1 \mathrm{~h}$. Yield $79 \mathrm{mg}$ 
(80\%); colorless solid, mp $168-169^{\circ} \mathrm{C}, \mathrm{dr} \mathbf{A}: \mathbf{B}=79: 21 . R_{f}=0.35$ (ethyl acetate/petroleum ether; 1:1).

A: ${ }^{1} \mathrm{H}$ NMR $\left(\mathrm{CDCl}_{3}, 600 \mathrm{MHz}\right): \delta=2.77\left(\mathrm{dd},{ }^{2} J=13.5,{ }^{3} J=9.6 \mathrm{~Hz}, 1 \mathrm{H}, \mathrm{CH}_{2}\right), 2.86\left(\mathrm{dd},{ }^{2} J=\right.$ $\left.13.5,{ }^{3} \mathrm{~J}=9.0 \mathrm{~Hz}, 1 \mathrm{H}, \mathrm{CH}_{2}\right), 3.77\left(\mathrm{~s}, 3 \mathrm{H}, \mathrm{OCH}_{3}\right), 3.87\left(\mathrm{~s}, 3 \mathrm{H}, \mathrm{OCH}_{3}\right), 4.21\left(\mathrm{dd},{ }^{3} J=9.6,{ }^{3} J=9.0\right.$ $\mathrm{Hz}, 1 \mathrm{H}, \mathrm{CH}), 4.45\left(\mathrm{~d},{ }^{2} J=15.5 \mathrm{~Hz}, 1 \mathrm{H}, \mathrm{NCH}_{2}\right), 5.03\left(\mathrm{~d},{ }^{2} J=15.5 \mathrm{~Hz}, 1 \mathrm{H}, \mathrm{NCH}_{2}\right), 6.64\left(\mathrm{~d},{ }^{3} J=\right.$ $7.8 \mathrm{~Hz}, 1 \mathrm{H}, \mathrm{Ar}), 6.70-6.77$ (m, 4H, Ar), 6.79-6.83 (m, 2H, Ar), 7.09-7.13 (m, 1H, Ar), 7.207.25 (m, 1H, Ar), 7.26-7.31 (m, 2H, Ar), 7.57 (d, $\left.{ }^{3} \mathrm{~J}=7.4 \mathrm{~Hz}, 1 \mathrm{H}, \mathrm{Ar}\right)$.

${ }^{13} \mathrm{C} \mathrm{NMR}\left(\mathrm{CDCl}_{3}, 150 \mathrm{MHz}\right): \delta=33.4\left(\mathrm{CH}_{2}\right), 43.2\left(\mathrm{NCH}_{2}\right), 46.89(\mathrm{CH}), 52.95\left(\mathrm{OCH}_{3}\right), 55.18$ $\left(\mathrm{OCH}_{3}\right), 68.6(\mathrm{C}), 110.0(\mathrm{CH}, \mathrm{Ar}), 114.1(2 \times \mathrm{CH}, \mathrm{Ar}), 121.85(\mathrm{C}, \mathrm{Ar}), 123.7(\mathrm{CH}, \mathrm{Ar}), 124.8$ $(\mathrm{CH}, \mathrm{Ar}), 126.27$ (C, Ar), $127.3(\mathrm{C}, \mathrm{Ar}), 128.08(2 \times \mathrm{CH}, \mathrm{Ar}), 129.2(2 \times \mathrm{CH}, \mathrm{Ar}), 130.48(\mathrm{CH}$, Ar), $132.2(2 \times \mathrm{CH}, \mathrm{Ar}), 134.62(\mathrm{C}, \mathrm{Ar}), 142.5(\mathrm{C}, \mathrm{Ar}), 159.04(\mathrm{C}, \mathrm{Ar}), 170.0(\mathrm{C}=\mathrm{O}), 170.5$ $(\mathrm{C}=\mathrm{O}), 175.4(\mathrm{C}=\mathrm{O})$.

B: ${ }^{1} \mathrm{H}$ NMR $\left(\mathrm{CDCl}_{3}, 600 \mathrm{MHz}\right): \delta=2.57\left(\mathrm{dd},{ }^{2} J=13.1,{ }^{3} J=9.3 \mathrm{~Hz}, 1 \mathrm{H}, \mathrm{CH}_{2}\right), 3.20\left(\mathrm{dd},{ }^{2} J=\right.$ $\left.13.1,{ }^{3} J=9.1 \mathrm{~Hz}, 1 \mathrm{H}, \mathrm{CH}_{2}\right), 3.76\left(\mathrm{~s}, 3 \mathrm{H}, \mathrm{OCH}_{3}\right), 3.89\left(\mathrm{~s}, 3 \mathrm{H}, \mathrm{OCH}_{3}\right), 4.05\left(\mathrm{dd},{ }^{3} J=9.3,{ }^{3} J=9.1\right.$ $\mathrm{Hz}, 1 \mathrm{H}, \mathrm{CH}), 4.39\left(\mathrm{~d},{ }^{2} \mathrm{~J}=15.5 \mathrm{~Hz}, 1 \mathrm{H}, \mathrm{NCH}_{2}\right), 5.07\left(\mathrm{~d},{ }^{2} J=15.5 \mathrm{~Hz}, 1 \mathrm{H}, \mathrm{NCH}_{2}\right), 6.66-6.69(\mathrm{~m}$, 3H, Ar), 6.70-6.77 (m, 2H, Ar), 6.79-6.83 (m, 2H, Ar), 7.09-7.13 (m, 1H, Ar), 7.20-7.25 (m, 1H, Ar), 7.26-7.31 (m, 2H, Ar), $7.39\left(\mathrm{~d},{ }^{3} J=7.4 \mathrm{~Hz}, 1 \mathrm{H}, \mathrm{Ar}\right)$.

${ }^{13} \mathrm{C} \mathrm{NMR}\left(\mathrm{CDCl}_{3}, 150 \mathrm{MHz}\right): \delta=34.0\left(\mathrm{CH}_{2}\right), 43.3\left(\mathrm{NCH}_{2}\right), 46.89(\mathrm{CH}), 53.01\left(\mathrm{OCH}_{3}\right), 55.18$ $\left(\mathrm{OCH}_{3}\right), 68.5(\mathrm{C}), 110.3(\mathrm{CH}, \mathrm{Ar}), 114.0(2 \times \mathrm{CH}, \mathrm{Ar}), 121.89(\mathrm{C}, \mathrm{Ar}), 123.3(\mathrm{CH}, \mathrm{Ar}), 123.5$ (CH, Ar), 126.31 (C, Ar), $128.08(2 \times \mathrm{CH}, \mathrm{Ar}), 128.4(\mathrm{C}, \mathrm{Ar}), 129.1(2 \times \mathrm{CH}, \mathrm{Ar}), 130.48(\mathrm{CH}$, Ar), $132.3(2 \times \mathrm{CH}, \mathrm{Ar}), 134.58(\mathrm{C}, \mathrm{Ar}), 142.0(\mathrm{C}, \mathrm{Ar}), 150.01(\mathrm{C}, \mathrm{Ar}), 168.7(\mathrm{C}=\mathrm{O}), 169.8$ $(\mathrm{C}=\mathrm{O}), 173.8(\mathrm{C}=\mathrm{O})$.

HRMS (ESI-TOF) $m / z:[\mathrm{M}+\mathrm{H}]^{+}$calcd for $\mathrm{C}_{27} \mathrm{H}_{24} \mathrm{BrN}_{2} \mathrm{O}_{5}{ }^{+} 535.0863$, found 535.0875. 


\section{Methyl 1-(4-methoxybenzyl)-1' -(4-methylphenyl)-2,5' -dioxo-1,2-dihydrospiro[indole-3,2'-}

\section{pyrrolidine]-4' -carboxylate (8k)}

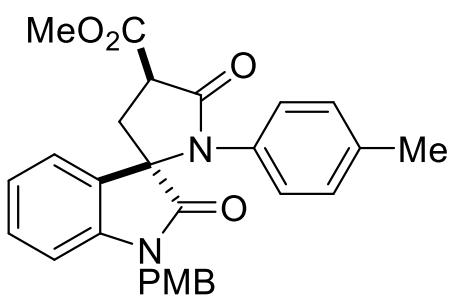

8k was obtained from 7k (135 mg, $0.27 \mathrm{mmol})$ and $\mathrm{TsOH}(9 \mathrm{mg}$, $0.05 \mathrm{mmol})$ in toluene $(1.8 \mathrm{~mL})$; reaction time $1 \mathrm{~h}$. Yield $118 \mathrm{mg}$ (94\%); grey solid, mp $172-173{ }^{\circ} \mathrm{C} ; \mathrm{dr} \mathbf{A}: \mathbf{B}=80: 20 . R_{f}=0.36$ (ethyl acetate/petroleum ether; $1: 1)$.

A: ${ }^{1} \mathrm{H}$ NMR $\left(\mathrm{CDCl}_{3}, 600 \mathrm{MHz}\right): \delta=2.26\left(\mathrm{~s}, 3 \mathrm{H}, \mathrm{CH}_{3}\right), 2.76\left(\mathrm{dd},{ }^{2} J=13.5,{ }^{3} J=9.5 \mathrm{~Hz}, 1 \mathrm{H}\right.$, $\left.\mathrm{CH}_{2}\right), 2.86\left(\mathrm{dd},{ }^{2} J=13.5,{ }^{3} J=9.0 \mathrm{~Hz}, 1 \mathrm{H}, \mathrm{CH}_{2}\right), 3.75\left(\mathrm{~s}, 3 \mathrm{H}, \mathrm{OCH}_{3}\right), 3.87\left(\mathrm{~s}, 3 \mathrm{H}, \mathrm{OCH}_{3}\right), 4.22$ $\left(\mathrm{dd},{ }^{3} J=9.5,{ }^{3} J=9.0 \mathrm{~Hz}, 1 \mathrm{H}, \mathrm{CH}\right), 4.49\left(\mathrm{~d},{ }^{2} J=15.5 \mathrm{~Hz}, 1 \mathrm{H}, \mathrm{NCH}_{2}\right), 5.01\left(\mathrm{~d},{ }^{2} J=15.5 \mathrm{~Hz}, 1 \mathrm{H}\right.$, $\left.\mathrm{NCH}_{2}\right), 6.60\left(\mathrm{~d},{ }^{3} J=7.8 \mathrm{~Hz}, 1 \mathrm{H}, \mathrm{Ar}\right), 6.64-6.71(\mathrm{~m}, 2 \mathrm{H}, \mathrm{Ar}), 6.79\left(\mathrm{~d},{ }^{3} J=8.5 \mathrm{~Hz}, 2 \mathrm{H}, \mathrm{Ar}\right), 6.82-$ $6.87(\mathrm{~m}, 2 \mathrm{H}, \mathrm{Ar}), 6.96\left(\mathrm{~d},{ }^{3} \mathrm{~J}=8.2 \mathrm{~Hz}, 2 \mathrm{H}, \mathrm{Ar}\right), 7.08-7.12(\mathrm{~m}, 1 \mathrm{H}, \mathrm{Ar}), 7.18\left(\mathrm{ddd},{ }^{3} J=7.8,{ }^{3} J=\right.$ $\left.7.7,{ }^{4} J=1.2 \mathrm{~Hz}, 1 \mathrm{H}, \mathrm{Ar}\right), 7.58\left(\mathrm{~d},{ }^{3} J=7.4 \mathrm{~Hz}, 1 \mathrm{H}, \mathrm{Ar}\right)$.

${ }^{13} \mathrm{C}$ NMR $\left(\mathrm{CDCl}_{3}, 150 \mathrm{MHz}\right): \delta=20.98\left(\mathrm{CH}_{3}\right), 33.5\left(\mathrm{CH}_{2}\right), 43.1\left(\mathrm{NCH}_{2}\right), 46.95(\mathrm{CH}), 52.85$ $\left(\mathrm{OCH}_{3}\right), 55.1\left(\mathrm{OCH}_{3}\right), 68.71(\mathrm{C}), 109.7(\mathrm{CH}, \mathrm{Ar}), 113.9(2 \times \mathrm{CH}, \mathrm{Ar}), 123.5(\mathrm{CH}, \mathrm{Ar}), 124.9(\mathrm{CH}$, Ar), $126.52(\mathrm{C}, \mathrm{Ar}), 127.5(2 \times \mathrm{CH}, \mathrm{Ar}), 127.7(\mathrm{C}, \mathrm{Ar}), 128.14(2 \times \mathrm{CH}, \mathrm{Ar}), 129.6(2 \times \mathrm{CH}, \mathrm{Ar})$, $130.14(\mathrm{CH}, \mathrm{Ar}), 132.84$ (C, Ar), 137.77 (C, Ar), 142.5 (C, Ar), 158.9 (C, Ar), 170.2 (C=O), $170.6(\mathrm{C}=\mathrm{O}), 175.8(\mathrm{C}=\mathrm{O})$.

B: ${ }^{1} \mathrm{H}$ NMR $\left(\mathrm{CDCl}_{3}, 600 \mathrm{MHz}\right): \delta=2.29\left(\mathrm{~s}, 3 \mathrm{H}, \mathrm{CH}_{3}\right), 2.56\left(\mathrm{dd},{ }^{2} J=13.0,{ }^{3} J=9.3 \mathrm{~Hz}, 1 \mathrm{H}\right.$, $\left.\mathrm{CH}_{2}\right), 3.20\left(\mathrm{dd},{ }^{2} J=13.0,{ }^{3} J=9.1 \mathrm{~Hz}, 1 \mathrm{H}, \mathrm{CH}_{2}\right), 3.74\left(\mathrm{~s}, 3 \mathrm{H}, \mathrm{OCH}_{3}\right), 3.89\left(\mathrm{~s}, 3 \mathrm{H}, \mathrm{OCH}_{3}\right), 4.05$ $\left(\mathrm{dd},{ }^{3} J=9.3,{ }^{3} J=9.1 \mathrm{~Hz}, 1 \mathrm{H}, \mathrm{CH}\right), 4.42\left(\mathrm{~d},{ }^{2} J=15.5 \mathrm{~Hz}, 1 \mathrm{H}, \mathrm{NCH}_{2}\right), 5.04\left(\mathrm{~d},{ }^{2} J=15.5 \mathrm{~Hz}, 1 \mathrm{H}\right.$, $\left.\mathrm{NCH}_{2}\right), 6.62\left(\mathrm{~d},{ }^{3} J=7.8 \mathrm{~Hz}, 1 \mathrm{H}, \mathrm{Ar}\right), 6.64-6.71(\mathrm{~m}, 4 \mathrm{H}, \mathrm{Ar}), 6.82-6.87$ (m, 2H, Ar), 6.99 (d, ${ }^{3} J$ $=8.2 \mathrm{~Hz}, 2 \mathrm{H}, \mathrm{Ar}), 7.08-7.12(\mathrm{~m}, 1 \mathrm{H}, \mathrm{Ar}), 7.21\left(\mathrm{ddd},{ }^{3} \mathrm{~J}=7.8,{ }^{3} \mathrm{~J}=7.7,{ }^{4} \mathrm{~J}=1.2 \mathrm{~Hz}, 1 \mathrm{H}, \mathrm{Ar}\right), 7.41$ $\left(\mathrm{d},{ }^{3} \mathrm{~J}=7.4 \mathrm{~Hz}, 1 \mathrm{H}, \mathrm{Ar}\right)$.

${ }^{13} \mathrm{C} \mathrm{NMR}\left(\mathrm{CDCl}_{3}, 150 \mathrm{MHz}\right): \delta=21.01\left(\mathrm{CH}_{3}\right), 34.1\left(\mathrm{CH}_{2}\right), 43.2\left(\mathrm{NCH}_{2}\right), 46.99(\mathrm{CH}), 52.91$ $\left(\mathrm{OCH}_{3}\right), 55.0\left(\mathrm{OCH}_{3}\right), 68.71(\mathrm{C}), 110.1(\mathrm{CH}, \mathrm{Ar}), 113.8(2 \times \mathrm{CH}, \mathrm{Ar}), 123.3(\mathrm{CH}, \mathrm{Ar}), 123.4(\mathrm{CH}$, Ar), $126.52(\mathrm{C}, \mathrm{Ar}), 127.3(2 \times \mathrm{CH}, \mathrm{Ar}), 128.08$ (2×CH, Ar), $128.8(\mathrm{C}, \mathrm{Ar}), 129.7(2 \times \mathrm{CH}, \mathrm{Ar})$, 
$130.14(\mathrm{CH}, \mathrm{Ar}), 132.79$ (C, Ar), 137.83 (C, Ar), 142.1 (C, Ar), 158.8 (C, Ar), 169.0 (C=O), $169.9(\mathrm{C}=\mathrm{O}), 174.2(\mathrm{C}=\mathrm{O})$.

IR (film) 1718, 1714, 1613, 1513, 1349, 1248, $1174 \mathrm{~cm}^{-1}$.

HRMS (ESI-TOF) $m / z:[\mathrm{M}+\mathrm{H}]^{+}$calcd for $\mathrm{C}_{28} \mathrm{H}_{27} \mathrm{~N}_{2} \mathrm{O}_{5}{ }^{+}$471.1914, found 457.1918.

\section{Methyl 1-(4-methoxybenzyl)-1'-(4-methoxyphenyl)-2,5'-dioxo-1,2-dihydrospiro[indole-3,2'-}

\section{pyrrolidine]-4' -carboxylate $(81)$}

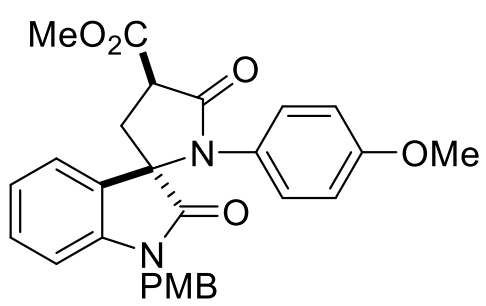

$8 \mathbf{l}$ was obtained from 71 (172 mg, $0.33 \mathrm{mmol})$ and $\mathrm{TsOH}(11 \mathrm{mg}$, $0.07 \mathrm{mmol})$ in toluene $(2.2 \mathrm{~mL})$; reaction time $1 \mathrm{~h}$. Yield $136 \mathrm{mg}$ (84\%); brown solid, mp 190-191 ${ }^{\circ} \mathrm{C} ; \mathrm{dr} \mathbf{A}: \mathbf{B}=77: 23 . R_{f}=0.22$ (ethyl acetate/petroleum ether; 1:1).

A: ${ }^{1} \mathrm{H}$ NMR $\left(\mathrm{CDCl}_{3}, 600 \mathrm{MHz}\right): \delta=2.76\left(\mathrm{dd},{ }^{2} J=13.6,{ }^{3} \mathrm{~J}=9.7 \mathrm{~Hz}, 1 \mathrm{H}, \mathrm{CH}_{2}\right), 2.85\left(\mathrm{dd},{ }^{2} J=\right.$ $\left.13.6,{ }^{3} J=8.8 \mathrm{~Hz}, 1 \mathrm{H}, \mathrm{CH}_{2}\right), 3.69\left(\mathrm{~s}, 3 \mathrm{H}, \mathrm{OCH}_{3}\right), 3.72\left(\mathrm{~s}, 3 \mathrm{H}, \mathrm{OCH}_{3}\right), 3.85\left(\mathrm{~s}, 3 \mathrm{H}, \mathrm{OCH}_{3}\right), 4.20$ $\left(\mathrm{dd},{ }^{3} J=9.7,{ }^{3} \mathrm{~J}=8.8 \mathrm{~Hz}, 1 \mathrm{H}, \mathrm{CH}\right), 4.45\left(\mathrm{~d},{ }^{2} J=15.6 \mathrm{~Hz}, 1 \mathrm{H}, \mathrm{NCH}_{2}\right), 5.00\left(\mathrm{~d},{ }^{2} J=15.6 \mathrm{~Hz}, 1 \mathrm{H}\right.$, $\left.\mathrm{NCH}_{2}\right), 6.58\left(\mathrm{~d},{ }^{3} \mathrm{~J}=7.6 \mathrm{~Hz}, 1 \mathrm{H}, \mathrm{Ar}\right), 6.63-6.69(\mathrm{~m}, 4 \mathrm{H}, \mathrm{Ar}), 6.72\left(\mathrm{~d},{ }^{3} \mathrm{~J}=8.9 \mathrm{~Hz}, 2 \mathrm{H}, \mathrm{Ar}\right), 6.87$ $\left(\mathrm{d},{ }^{3} J=9.1 \mathrm{~Hz}, 2 \mathrm{H}, \mathrm{Ar}\right), 7.08-7.12(\mathrm{~m}, 1 \mathrm{H}, \mathrm{Ar}), 7.16-7.22(\mathrm{~m}, 1 \mathrm{H}, \mathrm{Ar}), 7.60\left(\mathrm{ddd},{ }^{3} J=7.4,{ }^{4} J=\right.$ $\left.1.3,{ }^{5} \mathrm{~J}=0.5 \mathrm{~Hz}, 1 \mathrm{H}, \mathrm{Ar}\right)$.

${ }^{13} \mathrm{C} \mathrm{NMR}\left(\mathrm{CDCl}_{3}, 150 \mathrm{MHz}\right): \delta=33.1\left(\mathrm{CH}_{2}\right), 42.97\left(\mathrm{NCH}_{2}\right), 46.87(\mathrm{CH}), 52.8\left(\mathrm{OCH}_{3}\right), 55.0$ $\left(\mathrm{OCH}_{3}\right), 55.05\left(\mathrm{OCH}_{3}\right), 68.8(\mathrm{C}), 109.7(\mathrm{CH}, \mathrm{Ar}), 113.81(2 \times \mathrm{CH}, \mathrm{Ar}), 114.15(2 \times \mathrm{CH}, \mathrm{Ar})$, $123.40(\mathrm{CH}, \mathrm{Ar}), 124.8(\mathrm{CH}, \mathrm{Ar}), 126.37$ (C, Ar), 127.7 (C, Ar), $128.0(2 \times \mathrm{CH}, \mathrm{C}, \mathrm{Ar}), 129.1$ (2×CH, Ar), $130.14(\mathrm{CH}, \mathrm{Ar}), 142.4(\mathrm{C}, \mathrm{Ar}), 158.8(\mathrm{C}, \mathrm{Ar}), 159.0(\mathrm{C}, \mathrm{Ar}), 170.2(\mathrm{C}=\mathrm{O}), 170.7$ $(\mathrm{C}=\mathrm{O}), 175.7(\mathrm{C}=\mathrm{O})$.

B: ${ }^{1} \mathrm{H}$ NMR $\left(\mathrm{CDCl}_{3}, 600 \mathrm{MHz}\right): \delta=2.56\left(\mathrm{dd},{ }^{2} J=13.1,{ }^{3} \mathrm{~J}=9.3 \mathrm{~Hz}, 1 \mathrm{H}, \mathrm{CH}_{2}\right), 3.19\left(\mathrm{dd},{ }^{2} J=\right.$ $\left.13.1,{ }^{3} J=9.1 \mathrm{~Hz}, 1 \mathrm{H}, \mathrm{CH}_{2}\right), 3.70\left(\mathrm{~s}, 3 \mathrm{H}, \mathrm{OCH}_{3}\right), 3.72\left(\mathrm{~s}, 3 \mathrm{H}, \mathrm{OCH}_{3}\right), 3.87\left(\mathrm{~s}, 3 \mathrm{H}, \mathrm{OCH}_{3}\right), 4.05$ $\left(\mathrm{dd},{ }^{3} J=9.3,{ }^{3} J=9.1 \mathrm{~Hz}, 1 \mathrm{H}, \mathrm{CH}\right), 4.37\left(\mathrm{~d},{ }^{2} J=15.5 \mathrm{~Hz}, 1 \mathrm{H}, \mathrm{NCH}_{2}\right), 5.04\left(\mathrm{~d},{ }^{2} J=15.5 \mathrm{~Hz}, 1 \mathrm{H}\right.$, $\left.\mathrm{NCH}_{2}\right), 6.61\left(\mathrm{~d},{ }^{3} J=7.7 \mathrm{~Hz}, 1 \mathrm{H}, \mathrm{Ar}\right), 6.63-6.69(\mathrm{~m}, 6 \mathrm{H}, \mathrm{Ar}), 6.84\left(\mathrm{~d},{ }^{3} J=9.1 \mathrm{~Hz}, 2 \mathrm{H}, \mathrm{Ar}\right), 7.08-$ $7.12(\mathrm{~m}, 1 \mathrm{H}, \mathrm{Ar}), 7.16-7.22(\mathrm{~m}, 1 \mathrm{H}, \mathrm{Ar}), 7.41\left(\mathrm{ddd},{ }^{3} J=7.4,{ }^{4} J=1.3,{ }^{5} J=0.5 \mathrm{~Hz}, 1 \mathrm{H}, \mathrm{Ar}\right)$. 
${ }^{13} \mathrm{C} \mathrm{NMR}\left(\mathrm{CDCl}_{3}, 150 \mathrm{MHz}\right): \delta=33.6\left(\mathrm{CH}_{2}\right), 43.04\left(\mathrm{NCH}_{2}\right), 46.87(\mathrm{CH}), 52.9\left(\mathrm{OCH}_{3}\right), 54.9$ $\left(\mathrm{OCH}_{3}\right), 55.07\left(\mathrm{OCH}_{3}\right), 68.7(\mathrm{C}), 110.0(\mathrm{CH}, \mathrm{Ar}), 113.76(2 \times \mathrm{CH}, \mathrm{Ar}), 114.21(2 \times \mathrm{CH}, \mathrm{Ar}), 123.3$ $(\mathrm{CH}, \mathrm{Ar}), 123.35(\mathrm{CH}, \mathrm{Ar}), 126.37(\mathrm{C}, \mathrm{Ar}), 127.8$ (C, Ar), 127.9 (2×CH, Ar), $128.7(\mathrm{C}, \mathrm{Ar})$, $129.0(2 \times \mathrm{CH}, \mathrm{Ar}), 130.14(\mathrm{CH}, \mathrm{Ar}), 142.1$ (C, Ar), 158.7 (C, Ar), $159.1(\mathrm{C}, \mathrm{Ar}), 168.9(\mathrm{C}=\mathrm{O})$, $170.0(\mathrm{C}=\mathrm{O}), 174.1(\mathrm{C}=\mathrm{O})$.

IR (film) 1718, 1700, 1560, 1507, 1250, $1168 \mathrm{~cm}^{-1}$.

HRMS (ESI-TOF) $m / z:[\mathrm{M}+\mathrm{H}]^{+}$calcd for $\mathrm{C}_{28} \mathrm{H}_{27} \mathrm{~N}_{2} \mathrm{O}_{6}{ }^{+}$487.1864, found 487.1872.

\section{Methyl 1-(4-methoxybenzyl)-1'-(2-methoxyphenyl)-2,5'-dioxo-1,2-dihydrospiro[indole-3,2'-}

\section{pyrrolidine]-4' -carboxylate $(8 \mathrm{~m})$}

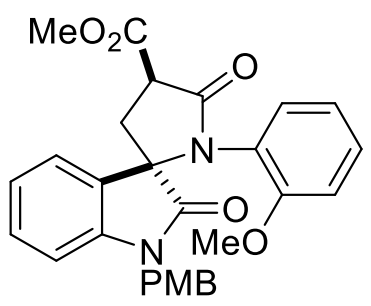

$\mathbf{8 m}$ was obtained from $\mathbf{7 m}(132 \mathrm{mg}, 0.25 \mathrm{mmol})$ and $\mathrm{TsOH}(9 \mathrm{mg}, 0.05$ mmol) in toluene $(1.7 \mathrm{~mL})$; reaction time $7 \mathrm{~h}$. Yield $76 \mathrm{mg}(61 \%)$; yellowish oil; dr $\mathbf{A}: \mathbf{B}=76: 24 . R_{f}=0.20$ (ethyl acetate/petroleum ether; $1: 1)$.

A: ${ }^{1} \mathrm{H} \mathrm{NMR}\left(\mathrm{CDCl}_{3}, 600 \mathrm{MHz}\right): \delta=2.73\left(\mathrm{dd},{ }^{2} J=13.3,{ }^{3} J=9.1 \mathrm{~Hz}, 1 \mathrm{H}, \mathrm{CH}_{2}\right), 2.91\left(\mathrm{dd},{ }^{2} J=\right.$ $\left.13.3,{ }^{3} J=9.7 \mathrm{~Hz}, 1 \mathrm{H}, \mathrm{CH}_{2}\right), 3.66\left(\mathrm{~s}, 3 \mathrm{H}, \mathrm{OCH}_{3}\right), 3.76\left(\mathrm{~s}, 3 \mathrm{H}, \mathrm{OCH}_{3}\right), 3.87\left(\mathrm{~s}, 3 \mathrm{H}, \mathrm{OCH}_{3}\right), 4.28$ $\left(\mathrm{dd},{ }^{3} J=9.7,{ }^{3} J=9.1 \mathrm{~Hz}, 1 \mathrm{H}, \mathrm{CH}\right), 4.65\left(\mathrm{~d},{ }^{2} J=15.5 \mathrm{~Hz}, 1 \mathrm{H}, \mathrm{NCH}_{2}\right), 4.93\left(\mathrm{~d},{ }^{2} J=15.5 \mathrm{~Hz}, 1 \mathrm{H}\right.$, $\left.\mathrm{NCH}_{2}\right), 6.57\left(\mathrm{~d},{ }^{3} \mathrm{~J}=7.9 \mathrm{~Hz}, 1 \mathrm{H}, \mathrm{Ar}\right), 6.70-6.78(\mathrm{~m}, 4 \mathrm{H}, \mathrm{Ar}), 6.95-7.02$ (m, 4H, Ar), 7.10-7.13 (m, 1H, Ar), 7.14-7.17 (m, 1H, Ar), $7.54\left(\mathrm{dd},{ }^{3} J=7.4 \mathrm{~Hz}, 1 \mathrm{H}, \mathrm{Ar}\right)$.

${ }^{13} \mathrm{C} \mathrm{NMR}\left(\mathrm{CDCl}_{3}, 150 \mathrm{MHz}\right): \delta=34.48\left(\mathrm{CH}_{2}\right), 43.2\left(\mathrm{NCH}_{2}\right), 46.90(\mathrm{CH}), 52.8\left(\mathrm{OCH}_{3}\right), 55.18$ $\left(\mathrm{OCH}_{3}\right), 55.4\left(\mathrm{OCH}_{3}\right), 68.6(\mathrm{C}), 109.2(\mathrm{CH}, \mathrm{Ar}), 112.02(\mathrm{CH}, \mathrm{Ar}), 114.1(2 \times \mathrm{CH}, \mathrm{Ar}), 120.5(\mathrm{CH}$, Ar), $122.7(\mathrm{CH}, \mathrm{Ar}), 124.4(\mathrm{C}, \mathrm{Ar}), 125.4(\mathrm{CH}, \mathrm{Ar}), 127.0(\mathrm{C}, \mathrm{Ar}), 128.29(\mathrm{C}), 128.34(2 \times \mathrm{CH}$, Ar), $129.0(\mathrm{CH}, \mathrm{Ar}), 129.78(\mathrm{CH}, \mathrm{Ar}), 130.0(\mathrm{CH}, \mathrm{Ar}), 142.6(\mathrm{C}, \mathrm{Ar}), 155.6(\mathrm{C}, \mathrm{Ar}), 159.0(\mathrm{C}$, Ar), $169.9(\mathrm{C}=\mathrm{O}), 170.2(\mathrm{C}=\mathrm{O}), 176.4(\mathrm{C}=\mathrm{O})$.

B: ${ }^{1} \mathrm{H}$ NMR $\left(\mathrm{CDCl}_{3}, 600 \mathrm{MHz}\right): \delta=2.56\left(\mathrm{dd},{ }^{2} J=13.0,{ }^{3} J=9.4 \mathrm{~Hz}, 1 \mathrm{H}, \mathrm{CH}_{2}\right), 3.24\left(\mathrm{dd},{ }^{2} J=\right.$ $\left.13.0,{ }^{3} \mathrm{~J}=8.8 \mathrm{~Hz}, 1 \mathrm{H}, \mathrm{CH}_{2}\right), 3.60\left(\mathrm{~s}, 3 \mathrm{H}, \mathrm{OCH}_{3}\right), 3.75\left(\mathrm{~s}, 3 \mathrm{H}, \mathrm{OCH}_{3}\right), 3.88\left(\mathrm{~s}, 3 \mathrm{H}, \mathrm{OCH}_{3}\right), 4.06$ 
$\left(\mathrm{dd},{ }^{3} J=9.4,{ }^{3} J=8.8 \mathrm{~Hz}, 1 \mathrm{H}, \mathrm{CH}\right), 4.51\left(\mathrm{~d},{ }^{2} J=15.5 \mathrm{~Hz}, 1 \mathrm{H}, \mathrm{NCH}_{2}\right), 5.02\left(\mathrm{~d},{ }^{2} J=15.5 \mathrm{~Hz}, 1 \mathrm{H}\right.$, $\left.\mathrm{NCH}_{2}\right), 6.55\left(\mathrm{~d},{ }^{3} \mathrm{~J}=7.9 \mathrm{~Hz}, 1 \mathrm{H}, \mathrm{Ar}\right), 6.70-6.78(\mathrm{~m}, 4 \mathrm{H}, \mathrm{Ar}), 6.85\left(\mathrm{~d},{ }^{3} \mathrm{~J}=8.6 \mathrm{~Hz}, 2 \mathrm{H}, \mathrm{Ar}\right), 6.95-$ 7.02 (m, 2H, Ar), 7.11-7.14 (m, 1H, Ar), 7.19-7.22 (m, 1H, Ar), 7.43-7.44 (m, 1H, Ar).

${ }^{13} \mathrm{C} \mathrm{NMR}\left(\mathrm{CDCl}_{3}, 150 \mathrm{MHz}\right): \delta=34.49\left(\mathrm{CH}_{2}\right), 43.3\left(\mathrm{NCH}_{2}\right), 46.88(\mathrm{CH}), 52.9\left(\mathrm{OCH}_{3}\right), 55.15$ $\left(\mathrm{OCH}_{3}\right), 55.3\left(\mathrm{OCH}_{3}\right), 69.1(\mathrm{C}), 109.4(\mathrm{CH}, \mathrm{Ar}), 111.97(\mathrm{CH}, \mathrm{Ar}), 114.0(2 \times \mathrm{CH}, \mathrm{Ar}), 122.45$ $(\mathrm{CH}, \mathrm{Ar}), 122.50(\mathrm{CH}, \mathrm{Ar}), 124.2(\mathrm{C}, \mathrm{Ar}), 124.7$ (CH, Ar), 126.96 (C, Ar), 128.0 (C, Ar), 128.2 (2×CH, Ar), $129.79(\mathrm{CH}, \mathrm{Ar}), 129.82(\mathrm{CH}, \mathrm{Ar}), 129.9(\mathrm{CH}, \mathrm{Ar}), 142.1(\mathrm{C}, \mathrm{Ar}), 155.3(\mathrm{C}, \mathrm{Ar})$, $158.9(\mathrm{C}, \mathrm{Ar}), 169.0(\mathrm{C}=\mathrm{O}), 169.5(\mathrm{C}=\mathrm{O}), 174.8(\mathrm{C}=\mathrm{O})$.

HRMS (ESI-TOF) $m / z:[\mathrm{M}+\mathrm{H}]^{+}$calcd for $\mathrm{C}_{28} \mathrm{H}_{27} \mathrm{~N}_{2} \mathrm{O}_{6}{ }^{+} 487.1864$, found 487.1878.

\section{Methyl 1-(4-methoxybenzyl)-1' -(4-nitrophenyl)-2,5' -dioxo-1,2-dihydrospiro[indole-3,2'-}

\section{pyrrolidine]-4' -carboxylate (8n)}

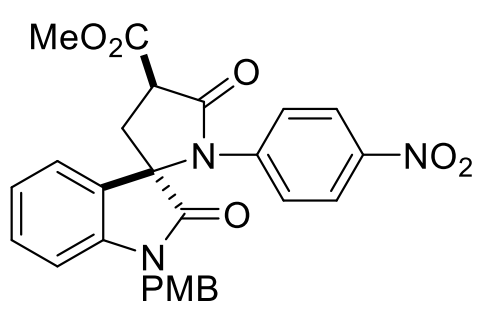
8n was obtained from 7n $(268 \mathrm{mg}, 0.50 \mathrm{mmol})$ and $\mathrm{TsOH}(17 \mathrm{mg}$, $0.10 \mathrm{mmol})$ in toluene $(3.4 \mathrm{~mL})$; reaction time $1 \mathrm{~h}$. Yield $197 \mathrm{mg}$ (78\%); yellowish foam, $\operatorname{dr} \mathbf{A}: \mathbf{B}=78: 22 . R_{f}=0.33$ (ethyl acetate/petroleum ether; $1: 1$ ).

A: ${ }^{1} \mathrm{H}$ NMR $\left(\mathrm{CDCl}_{3}, 600 \mathrm{MHz}\right): \delta=2.76\left(\mathrm{dd},{ }^{2} J=13.4,{ }^{3} \mathrm{~J}=9.2 \mathrm{~Hz}, 1 \mathrm{H}, \mathrm{CH}_{2}\right), 2.85\left(\mathrm{dd},{ }^{2} J=\right.$ $\left.13.4,{ }^{3} J=10.0 \mathrm{~Hz}, 1 \mathrm{H}, \mathrm{CH}_{2}\right), 3.73\left(\mathrm{~s}, 3 \mathrm{H}, \mathrm{OCH}_{3}\right), 3.85\left(\mathrm{~s}, 3 \mathrm{H}, \mathrm{OCH}_{3}\right), 4.28\left(\mathrm{dd},{ }^{3} J=10.0,{ }^{3} J=\right.$ $9.2 \mathrm{~Hz}, 1 \mathrm{H}, \mathrm{CH}), 4.61\left(\mathrm{~d},{ }^{2} J=15.2 \mathrm{~Hz}, 1 \mathrm{H}, \mathrm{NCH}_{2}\right), 4.97\left(\mathrm{~d},{ }^{2} J=15.2 \mathrm{~Hz}, 1 \mathrm{H}, \mathrm{NCH}_{2}\right), 6.74\left(\mathrm{~d},{ }^{3} J\right.$ $=8.8 \mathrm{~Hz}, 2 \mathrm{H}, \mathrm{Ar}), 6.84\left(\mathrm{~d},{ }^{3} J=7.8 \mathrm{~Hz}, 1 \mathrm{H}, \mathrm{Ar}\right), 7.02-7.10(\mathrm{~m}, 5 \mathrm{H}, \mathrm{Ar}), 7.25\left(\mathrm{ddd},{ }^{3} J=7.8,{ }^{3} J=\right.$ $\left.7.7,{ }^{4} J=1.2 \mathrm{~Hz}, 1 \mathrm{H}, \mathrm{Ar}\right), 7.39\left(\mathrm{ddd},{ }^{3} J=7.5,{ }^{4} J=1.2,{ }^{5} J=0.5 \mathrm{~Hz}, 1 \mathrm{H}, \mathrm{Ar}\right), 7.86-7.91(\mathrm{~m}, 2 \mathrm{H}$, Ar).

${ }^{13} \mathrm{C} \mathrm{NMR}\left(\mathrm{CDCl}_{3}, 150 \mathrm{MHz}\right): \delta=34.3\left(\mathrm{CH}_{2}\right), 43.3\left(\mathrm{NCH}_{2}\right), 46.7(\mathrm{CH}), 52.9\left(\mathrm{OCH}_{3}\right), 55.0$ $\left(\mathrm{OCH}_{3}\right), 67.9(\mathrm{C}), 110.0(\mathrm{CH}, \mathrm{Ar}), 113.9(2 \times \mathrm{CH}, \mathrm{Ar}), 123.8(\mathrm{CH}, \mathrm{Ar}), 124.1(2 \times \mathrm{CH}, \mathrm{Ar}), 124.2$ (C, Ar), $124.4(\mathrm{CH}, \mathrm{Ar}), 125.9(2 \times \mathrm{CH}, \mathrm{Ar}), 126.3$ (C, Ar), 126.47 (C, Ar), $128.78(2 \times \mathrm{CH}, \mathrm{Ar})$, $130.61(\mathrm{CH}, \mathrm{Ar}), 141.38$ (C, Ar), 142.2 (C, Ar), 145.7 (C, Ar), 159.19 (C, Ar), $169.4(\mathrm{C}=\mathrm{O})$, $170.3(\mathrm{C}=\mathrm{O}), 175.0(\mathrm{C}=\mathrm{O})$. 
B: ${ }^{1} \mathrm{H}$ NMR $\left(\mathrm{CDCl}_{3}, 600 \mathrm{MHz}\right): \delta=2.55\left(\mathrm{dd},{ }^{2} J=12.9,{ }^{3} \mathrm{~J}=9.1 \mathrm{~Hz}, 1 \mathrm{H}, \mathrm{CH}_{2}\right), 3.17\left(\mathrm{dd},{ }^{2} J=\right.$ $\left.12.9,{ }^{3} J=9.4 \mathrm{~Hz}, 1 \mathrm{H}, \mathrm{CH}_{2}\right), 3.72\left(\mathrm{~s}, 3 \mathrm{H}, \mathrm{OCH}_{3}\right), 3.86\left(\mathrm{~s}, 3 \mathrm{H}, \mathrm{OCH}_{3}\right), 4.09\left(\mathrm{dd},{ }^{3} J=9.4,{ }^{3} J=9.1\right.$ $\mathrm{Hz}, 1 \mathrm{H}, \mathrm{CH}), 4.53\left(\mathrm{~d},{ }^{2} J=15.2 \mathrm{~Hz}, 1 \mathrm{H}, \mathrm{NCH}_{2}\right), 5.00\left(\mathrm{~d},{ }^{2} J=15.2 \mathrm{~Hz}, 1 \mathrm{H}, \mathrm{NCH}_{2}\right), 6.70\left(\mathrm{~d},{ }^{3} J=\right.$ $8.7 \mathrm{~Hz}, 2 \mathrm{H}, \mathrm{Ar}), 6.89$ (d, $\left.{ }^{3} \mathrm{~J}=7.8 \mathrm{~Hz}, 1 \mathrm{H}, \mathrm{Ar}\right), 6.97$ (d, $\left.{ }^{3} \mathrm{~J}=8.7 \mathrm{~Hz}, 2 \mathrm{H}, \mathrm{Ar}\right), 7.02-7.10$ (m, 3H, Ar), $7.29\left(\mathrm{ddd},{ }^{3} J=7.8,{ }^{3} J=7.7,{ }^{4} J=1.1 \mathrm{~Hz}, 1 \mathrm{H}, \mathrm{Ar}\right), 7.35\left(\mathrm{ddd},{ }^{3} J=7.5,{ }^{4} J=1.1,{ }^{5} J=0.5 \mathrm{~Hz}\right.$, 1H, Ar), 7.86-7.91 (m, 2H, Ar).

${ }^{13} \mathrm{C} \mathrm{NMR}\left(\mathrm{CDCl}_{3}, 150 \mathrm{MHz}\right): \delta=34.8\left(\mathrm{CH}_{2}\right), 43.4\left(\mathrm{NCH}_{2}\right), 46.9(\mathrm{CH}), 53.0\left(\mathrm{OCH}_{3}\right), 54.98$ $\left(\mathrm{OCH}_{3}\right), 68.1(\mathrm{C}), 110.4(\mathrm{CH}, \mathrm{Ar}), 113.8(2 \times \mathrm{CH}, \mathrm{Ar}), 123.1(\mathrm{CH}, \mathrm{Ar}), 123.7(\mathrm{CH}, \mathrm{Ar}), 124.2$ (2×CH, Ar), $125.2(2 \times \mathrm{CH}, \mathrm{Ar}), 126.51(\mathrm{C}, \mathrm{Ar}), 127.9(\mathrm{C}, \mathrm{Ar}), 128.84(2 \times \mathrm{CH}, \mathrm{Ar}), 130.57(\mathrm{CH}$, Ar), 141.44 (C, Ar), 141.7 (C, Ar), 145.5 (C, Ar), 159.15 (C, Ar), $168.1(\mathrm{C}=\mathrm{O}), 169.7(\mathrm{C}=\mathrm{O})$, $173.4(\mathrm{C}=\mathrm{O})$.

HRMS (ESI-TOF) $m / z:[\mathrm{M}+\mathrm{H}]^{+}$calcd for $\mathrm{C}_{27} \mathrm{H}_{24} \mathrm{~N}_{3} \mathrm{O}_{7}{ }^{+}$502.1609, found 502.1617.

\section{General procedure for the PMB- $N$-deprotection of lactames $8^{15}$}

A mixture of lactame 8 (1.0 equiv), TFA (50 equiv) and TfOH (2.5 equiv) in $\mathrm{CH}_{2} \mathrm{Cl}_{2}(0.08 \mathrm{M})$ was stirred at room temperature for specified time. The reaction mixture was then poured into an ice - water mixture and extracted with EtOAc $(3 \times 20 \mathrm{~mL})$. The combined organic layers were washed with $\mathrm{NaHCO}_{3}$ solution, dried over $\mathrm{Na}_{2} \mathrm{SO}_{4}$, and concentrated under reduced pressure. Lactames 9 were purified by column chromatography on silica gel.

\section{Methyl 2,5'-dioxo-1'-phenyl-1,2-dihydrospiro[indole-3,2'-pyrrolidine]-4' -carboxylate (9a)}

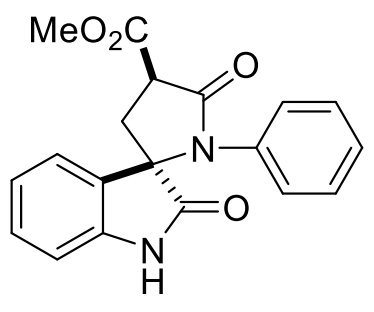

9a was obtained from 8a (102 mg, $0.22 \mathrm{mmol})$, TFA $(855 \mu \mathrm{L}, 11.18$ mmol) and TfOH $(50 \mu \mathrm{L}, 0.56 \mathrm{mmol})$ in $\mathrm{CH}_{2} \mathrm{Cl}_{2}(2.8 \mathrm{~mL})$; reaction time 68 h. Yield $35 \mathrm{mg}$ (47\%); yellowish oil; dr A:B = 80:20. $R_{f}=0.20$ (ethyl acetate/petroleum ether; $1: 1)$.

A: ${ }^{1} \mathrm{H} \mathrm{NMR}\left(\mathrm{CDCl}_{3}, 600 \mathrm{MHz}\right): \delta=2.74\left(\mathrm{dd},{ }^{2} J=13.5,{ }^{3} \mathrm{~J}=9.2 \mathrm{~Hz}, 1 \mathrm{H}, \mathrm{CH}_{2}\right), 2.83\left(\mathrm{dd},{ }^{2} J=\right.$ $\left.13.5,{ }^{3} J=9.7 \mathrm{~Hz}, 1 \mathrm{H}, \mathrm{CH}_{2}\right), 3.85\left(\mathrm{~s}, 3 \mathrm{H}, \mathrm{OCH}_{3}\right), 4.23\left(\mathrm{dd},{ }^{3} J=9.7,{ }^{3} J=9.2 \mathrm{~Hz}, 1 \mathrm{H}, \mathrm{CH}\right), 6.77$ 
$\left(\mathrm{d},{ }^{3} J=7.8 \mathrm{~Hz}, 1 \mathrm{H}, \mathrm{Ar}\right), 7.02-7.04(\mathrm{~m}, 2 \mathrm{H}, \mathrm{Ar}), 7.07\left(\mathrm{ddd},{ }^{3} J=7.6,{ }^{3} J=7.5,{ }^{4} \mathrm{~J}=0.9 \mathrm{~Hz}, 1 \mathrm{H}\right.$, Ar), 7.10-7.18 (m, 3H, Ar), $7.21\left(\mathrm{ddd},{ }^{3} J=7.8,{ }^{3} J=7.6,{ }^{4} J=1.2 \mathrm{~Hz}, 1 \mathrm{H}, \mathrm{Ar}\right), 7.44\left(\mathrm{~d},{ }^{3} J=7.5\right.$ Hz, 1H, Ar), 8.59 (br.s, 1H, NH).

${ }^{13} \mathrm{C} \mathrm{NMR}\left(\mathrm{CDCl}_{3}, 150 \mathrm{MHz}\right): \delta=34.3\left(\mathrm{CH}_{2}\right), 46.9(\mathrm{CH}), 52.98\left(\mathrm{OCH}_{3}\right), 68.9(\mathrm{C}), 110.7(\mathrm{CH}$, Ar), $123.53(\mathrm{CH}, \mathrm{Ar}), 125.0(\mathrm{CH}, \mathrm{Ar}), 126.9(2 \times \mathrm{CH}, \mathrm{Ar}), 127.5(\mathrm{C}, \mathrm{Ar}), 127.85(\mathrm{CH}, \mathrm{Ar})$, $129.03(2 \times \mathrm{CH}, \mathrm{Ar}), 130.28(\mathrm{CH}, \mathrm{Ar}), 135.57(\mathrm{C}, \mathrm{Ar}), 140.52$ (C, Ar), $170.1(\mathrm{C}=\mathrm{O}), 170.7$ $(\mathrm{C}=\mathrm{O}), 178.1(\mathrm{C}=\mathrm{O})$.

B: ${ }^{1} \mathrm{H}$ NMR $\left(\mathrm{CDCl}_{3}, 600 \mathrm{MHz}\right): \delta=2.54\left(\mathrm{dd},{ }^{2} J=13.1,{ }^{3} J=9.3 \mathrm{~Hz}, 1 \mathrm{H}, \mathrm{CH}_{2}\right), 3.15\left(\mathrm{dd},{ }^{2} J=\right.$ $\left.13.1,{ }^{3} J=8.8 \mathrm{~Hz}, 1 \mathrm{H}, \mathrm{CH}_{2}\right), 3.85\left(\mathrm{~s}, 3 \mathrm{H}, \mathrm{OCH}_{3}\right), 4.04\left(\mathrm{dd},{ }^{3} \mathrm{~J}=9.3,{ }^{3} \mathrm{~J}=8.8 \mathrm{~Hz}, 1 \mathrm{H}, \mathrm{CH}\right), 6.84$ (d, $\left.{ }^{3} J=7.7 \mathrm{~Hz}, 1 \mathrm{H}, \mathrm{Ar}\right), 6.99-7.01(\mathrm{~m}, 2 \mathrm{H}, \mathrm{Ar}), 7.10-7.18$ (m, 4H, Ar), 7.26-7.29 (m, 1H, Ar), $7.36\left(\mathrm{~d},{ }^{3} \mathrm{~J}=7.5 \mathrm{~Hz}, 1 \mathrm{H}, \mathrm{Ar}\right), 8.65$ (br.s, $\left.1 \mathrm{H}, \mathrm{NH}\right)$.

${ }^{13} \mathrm{C} \mathrm{NMR}\left(\mathrm{CDCl}_{3}, 150 \mathrm{MHz}\right): \delta=34.7\left(\mathrm{CH}_{2}\right), 47.0(\mathrm{CH}), 53.03\left(\mathrm{OCH}_{3}\right), 69.1(\mathrm{C}), 111.2(\mathrm{CH}$, Ar), $123.49(\mathrm{CH}, \mathrm{Ar}), 123.6(\mathrm{CH}, \mathrm{Ar}), 126.6(2 \times \mathrm{CH}, \mathrm{Ar}), 127.85(\mathrm{CH}, \mathrm{Ar}), 128.9(\mathrm{C}, \mathrm{Ar})$, $129.03(2 \times \mathrm{CH}, \mathrm{Ar}), 130.34(\mathrm{CH}, \mathrm{Ar}), 135.6$ (C, Ar), 140.1 (C, Ar), $168.9(\mathrm{C}=\mathrm{O}), 170.2(\mathrm{C}=\mathrm{O})$, $176.4(\mathrm{C}=\mathrm{O})$.

HRMS (ESI-TOF) $m / z:[\mathrm{M}+\mathrm{H}]^{+}$calcd for $\mathrm{C}_{19} \mathrm{H}_{17} \mathrm{~N}_{2} \mathrm{O}_{4}{ }^{+} 337.1183$, found 337.1189.

Methyl 5-methyl-2,5'-dioxo-1'-phenyl-1,2-dihydrospiro[indole-3,2' -pyrrolidine]-4' carboxylate (9b)

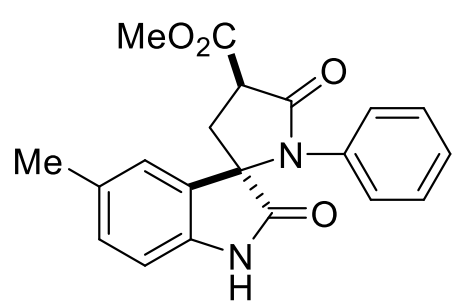

9b was obtained from $\mathbf{8 b}$ (94 mg, $0.20 \mathrm{mmol})$, TFA (765 $\mu \mathrm{L}, 10.00$ $\mathrm{mmol})$ and $\mathrm{TfOH}(44 \mu \mathrm{L}, 0.50 \mathrm{mmol})$ in $\mathrm{CH}_{2} \mathrm{Cl}_{2}(2.5 \mathrm{~mL})$; reaction time $118 \mathrm{~h}$. Yield $35 \mathrm{mg}$ (50\%); orange solid, mp 104-105 ${ }^{\circ} \mathrm{C}$; dr $\mathbf{A}: \mathbf{B}=75: 25 . R_{f}=0.22$ (ethyl acetate/petroleum ether; $\left.1: 1\right)$.

A: ${ }^{1} \mathrm{H} \mathrm{NMR}\left(\mathrm{CDCl}_{3}, 600 \mathrm{MHz}\right): \delta=2.31\left(\mathrm{~s}, 3 \mathrm{H}, \mathrm{CH}_{3}\right), 2.72\left(\mathrm{dd},{ }^{2} J=13.4,{ }^{3} J=9.3 \mathrm{~Hz}, 1 \mathrm{H}\right.$, $\left.\mathrm{CH}_{2}\right), 2.81\left(\mathrm{dd},{ }^{2} J=13.4,{ }^{3} \mathrm{~J}=9.9 \mathrm{~Hz}, 1 \mathrm{H}, \mathrm{CH}_{2}\right), 3.86\left(\mathrm{~s}, 3 \mathrm{H}, \mathrm{OCH}_{3}\right), 4.22\left(\mathrm{dd},{ }^{3} J=9.9,{ }^{3} J=9.3\right.$ $\mathrm{Hz}, 1 \mathrm{H}, \mathrm{CH}), 6.67\left(\mathrm{~d},{ }^{3} \mathrm{~J}=7.9 \mathrm{~Hz}, 1 \mathrm{H}, \mathrm{Ar}\right), 6.99-7.02$ (m, 1H, Ar), 7.02-7.05 (m, 2H, Ar), 7.127.19 (m, 3H, Ar), 7.23-7.24 (m, 1H, Ar), 8.43 (br.s, 1H, NH). 
${ }^{13} \mathrm{C} \mathrm{NMR}\left(\mathrm{CDCl}_{3}, 150 \mathrm{MHz}\right): \delta=21.09\left(\mathrm{CH}_{3}\right), 34.4\left(\mathrm{CH}_{2}\right), 46.9(\mathrm{CH}), 52.95\left(\mathrm{OCH}_{3}\right), 68.9(\mathrm{C})$, $110.4(\mathrm{CH}, \mathrm{Ar}), 125.5(\mathrm{CH}, \mathrm{Ar}), 126.7(2 \times \mathrm{CH}, \mathrm{Ar}), 127.6(\mathrm{C}, \mathrm{Ar}), 127.70(\mathrm{CH}, \mathrm{Ar}), 129.00$ (2×CH, Ar), $130.67(\mathrm{CH}, \mathrm{Ar}), 133.3(\mathrm{C}, \mathrm{Ar}), 135.7$ (C, Ar), $138.0(\mathrm{C}, \mathrm{Ar}), 170.2(\mathrm{C}=\mathrm{O}), 170.6$ $(\mathrm{C}=\mathrm{O}), 178.1(\mathrm{C}=\mathrm{O})$.

B: ${ }^{1} \mathrm{H}$ NMR $\left(\mathrm{CDCl}_{3}, 600 \mathrm{MHz}\right): \delta=2.35\left(\mathrm{~s}, 3 \mathrm{H}, \mathrm{CH}_{3}\right), 2.51\left(\mathrm{dd},{ }^{2} J=13.0,{ }^{3} J=9.1 \mathrm{~Hz}, 1 \mathrm{H}\right.$, $\left.\mathrm{CH}_{2}\right), 3.13\left(\mathrm{dd},{ }^{2} J=13.0,{ }^{3} J=9.2 \mathrm{~Hz}, 1 \mathrm{H}, \mathrm{CH}_{2}\right), 3.85\left(\mathrm{~s}, 3 \mathrm{H}, \mathrm{OCH}_{3}\right), 4.05\left(\mathrm{dd},{ }^{3} J=9.2,{ }^{3} J=9.1\right.$ $\mathrm{Hz}, 1 \mathrm{H}, \mathrm{CH}), 6.74\left(\mathrm{~d},{ }^{3} \mathrm{~J}=7.9 \mathrm{~Hz}, 1 \mathrm{H}, \mathrm{Ar}\right), 6.99-7.02$ (m, 2H, Ar), 7.07-7.09 (m, 1H, Ar), 7.127.19 (m, 4H, Ar), 8.49 (br.s, 1H, NH).

${ }^{13} \mathrm{C} \mathrm{NMR}\left(\mathrm{CDCl}_{3}, 150 \mathrm{MHz}\right): \delta=21.10\left(\mathrm{CH}_{3}\right), 34.9\left(\mathrm{CH}_{2}\right), 47.0(\mathrm{CH}), 53.00\left(\mathrm{OCH}_{3}\right), 69.1(\mathrm{C})$, $110.9(\mathrm{CH}, \mathrm{Ar}), 124.2$ (CH, Ar), $126.3(2 \times \mathrm{CH}, \mathrm{Ar}), 127.67$ (CH, Ar), $129.02(2 \times \mathrm{CH}, \mathrm{Ar}), 129.1$ (C, Ar), 130.67 (CH, Ar), 133.2 (C, Ar), 135.8 (C, Ar), 137.5 (C, Ar), 169.0 (C=O), 170.0 $(\mathrm{C}=\mathrm{O}), 176.2(\mathrm{C}=\mathrm{O})$.

HRMS (ESI-TOF) $m / z:[\mathrm{M}+\mathrm{H}]^{+}$calcd for $\mathrm{C}_{20} \mathrm{H}_{19} \mathrm{~N}_{2} \mathrm{O}_{4}{ }^{+} 351.1339$, found 351.1342.

\section{Methyl 5-fluoro-2,5' -dioxo-1'-phenyl-1,2-dihydrospiro[indole-3,2' -pyrrolidine]-4'-} carboxylate (9c)

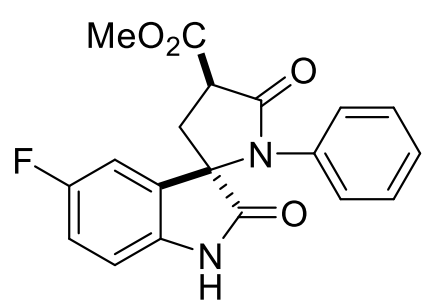

9c was obtained from $8 d$ (78 mg, $0.16 \mathrm{mmol})$, TFA $(630 \mu \mathrm{L}, 8.23$ mmol) and $\mathrm{TfOH}(36 \mu \mathrm{L}, 0.41 \mathrm{mmol})$ in $\mathrm{CH}_{2} \mathrm{Cl}_{2}(2.1 \mathrm{~mL})$; reaction time 118 h. Yield $35 \mathrm{mg}(60 \%)$; yellowish oil; dr $\mathbf{A}: \mathbf{B}=82: 18 . R_{f}=$ 0.13 (ethyl acetate/petroleum ether; 1:1).

A: ${ }^{1} \mathrm{H} \mathrm{NMR}\left(\mathrm{CDCl}_{3}, 600 \mathrm{MHz}\right): \delta=2.76\left(\mathrm{dd},{ }^{2} J=13.5,{ }^{3} \mathrm{~J}=9.4 \mathrm{~Hz}, 1 \mathrm{H}, \mathrm{CH}_{2}\right), 2.79\left(\mathrm{dd},{ }^{2} J=\right.$ $\left.13.5,{ }^{3} J=9.4 \mathrm{~Hz}, 1 \mathrm{H}, \mathrm{CH}_{2}\right), 3.85\left(\mathrm{~s}, 3 \mathrm{H}, \mathrm{OCH}_{3}\right), 4.20\left(\mathrm{dd},{ }^{3} J=9.4,{ }^{3} J=9.4 \mathrm{~Hz}, 1 \mathrm{H}, \mathrm{CH}\right), 6.70$ $\left(\mathrm{dd},{ }^{3} J=8.6,{ }^{4} J=4.1 \mathrm{~Hz}, 1 \mathrm{H}, \mathrm{Ar}\right), 6.91\left(\mathrm{ddd},{ }^{3} J=9.0,{ }^{3} J=8.6,{ }^{4} \mathrm{~J}=2.6 \mathrm{~Hz}, 1 \mathrm{H}, \mathrm{Ar}\right), 7.00-7.04$ (m, 2H, Ar), 7.14-7.22 (m, 4H, Ar), 8.66 (br.s, 1H, NH).

${ }^{13} \mathrm{C} \mathrm{NMR}\left(\mathrm{CDCl}_{3}, 150 \mathrm{MHz}\right): \delta=34.2\left(\mathrm{CH}_{2}\right), 46.81(\mathrm{CH}), 53.06\left(\mathrm{OCH}_{3}\right), 69.2(\mathrm{C}), 111.63\left({ }^{3} J_{\mathrm{CF}}\right.$ $=8 \mathrm{~Hz}, \mathrm{CH}, \mathrm{Ar}), 112.8\left({ }^{2} J_{\mathrm{CF}}=25 \mathrm{~Hz}, \mathrm{CH}, \mathrm{Ar}\right), 116.97\left({ }^{2} J_{\mathrm{CF}}=24 \mathrm{~Hz}, \mathrm{CH}, \mathrm{Ar}\right), 126.8(2 \times \mathrm{CH}$, 
Ar), $128.06(\mathrm{CH}, \mathrm{Ar}), 128.20(2 \times \mathrm{CH}, \mathrm{C}, \mathrm{Ar}), 135.4(\mathrm{C}, \mathrm{Ar}), 136.4(\mathrm{C}, \mathrm{Ar}), 159.4\left({ }^{1} J_{\mathrm{CF}}=243 \mathrm{~Hz}\right.$ C, Ar), $170.0(\mathrm{C}=\mathrm{O}), 170.6(\mathrm{C}=\mathrm{O}), 178.0(\mathrm{C}=\mathrm{O})$.

B: ${ }^{1} \mathrm{H}$ NMR $\left(\mathrm{CDCl}_{3}, 600 \mathrm{MHz}\right): \delta=2.53\left(\mathrm{dd},{ }^{2} J=13.2,{ }^{3} \mathrm{~J}=9.4 \mathrm{~Hz}, 1 \mathrm{H}, \mathrm{CH}_{2}\right), 3.15\left(\mathrm{dd},{ }^{2} J=\right.$ $\left.13.2,{ }^{3} \mathrm{~J}=8.5 \mathrm{~Hz}, 1 \mathrm{H}, \mathrm{CH}_{2}\right), 3.84\left(\mathrm{~s}, 3 \mathrm{H}, \mathrm{OCH}_{3}\right), 4.01\left(\mathrm{dd},{ }^{3} \mathrm{~J}=9.4,{ }^{3} \mathrm{~J}=8.5 \mathrm{~Hz}, 1 \mathrm{H}, \mathrm{CH}\right), 6.76$ $\left(\mathrm{dd},{ }^{3} J=8.5,{ }^{4} J=4.0 \mathrm{~Hz}, 1 \mathrm{H}, \mathrm{Ar}\right), 6.97\left(\mathrm{ddd},{ }^{3} J=8.9,{ }^{3} J=8.5,{ }^{4} \mathrm{~J}=2.6 \mathrm{~Hz}, 1 \mathrm{H}\right.$, Ar $), 7.00-7.04$ (m, 2H, Ar), $7.11\left(\mathrm{dd},{ }^{3} J=7.4,{ }^{4} J=2.6 \mathrm{~Hz}, 1 \mathrm{H}, \mathrm{Ar}\right), 7.14-7.22(\mathrm{~m}, 3 \mathrm{H}, \mathrm{Ar}), 8.72$ (br.s, $\left.1 \mathrm{H}, \mathrm{NH}\right)$. ${ }^{13} \mathrm{C} \mathrm{NMR}\left(\mathrm{CDCl}_{3}, 150 \mathrm{MHz}\right): \delta=34.5\left(\mathrm{CH}_{2}\right), 46.76(\mathrm{CH}), 53.06\left(\mathrm{OCH}_{3}\right), 69.4(\mathrm{C}), 111.59\left({ }^{2} J_{\mathrm{CF}}\right.$ $=25 \mathrm{~Hz}, \mathrm{CH}, \mathrm{Ar}), 112.0\left({ }^{3} J_{\mathrm{CF}}=8 \mathrm{~Hz}, \mathrm{CH}, \mathrm{Ar}\right), 116.97\left({ }^{2} J_{\mathrm{CF}}=24 \mathrm{~Hz}, \mathrm{CH}, \mathrm{Ar}\right), 126.6(2 \times \mathrm{CH}$, Ar), $128.06(\mathrm{CH}, \mathrm{Ar}), 128.20(2 \times \mathrm{CH}, \mathrm{C}, \mathrm{Ar}), 135.5(\mathrm{C}, \mathrm{Ar}), 136.1(\mathrm{C}, \mathrm{Ar}), 159.3\left({ }^{1} J_{\mathrm{CF}}=244 \mathrm{~Hz}\right.$ C, Ar), $168.7(\mathrm{C}=\mathrm{O}), 170.1(\mathrm{C}=\mathrm{O}), 176.4(\mathrm{C}=\mathrm{O})$.

HRMS (ESI-TOF) $m / z:[\mathrm{M}+\mathrm{H}]^{+}$calcd for $\mathrm{C}_{19} \mathrm{H}_{16} \mathrm{FN}_{2} \mathrm{O}_{4}{ }^{+} 355.1089$, found 355.1089.

\section{Methyl 5-chloro-2,5'-dioxo-1'-phenyl-1,2-dihydrospiro[indole-3,2' -pyrrolidine]-4' -} carboxylate (9d)<smiles>COC(=O)C1C[C@]2(C(=O)Nc3ccc(Cl)cc32)N(c2ccccc2)C1=O</smiles>

9d was obtained from 8e (123 mg, $0.25 \mathrm{mmol})$, TFA (960 $\mu \mathrm{L}, 12.54$ $\mathrm{mmol})$ and $\mathrm{TfOH}(55 \mu \mathrm{L}, 0.63 \mathrm{mmol})$ in $\mathrm{CH}_{2} \mathrm{Cl}_{2}(3.1 \mathrm{~mL})$; reaction time 118 h. Yield $43 \mathrm{mg}$ (46\%); yellowish solid, mp 191-192 ${ }^{\circ} \mathrm{C}$; dr $\mathbf{A}: \mathbf{B}=78: 22 . R_{f}=0.16$ (ethyl acetate/petroleum ether; $\left.1: 1\right)$.

A: ${ }^{1} \mathrm{H}$ NMR $\left(\mathrm{CDCl}_{3}, 600 \mathrm{MHz}\right): \delta=2.74\left(\mathrm{dd},{ }^{2} J=13.5,{ }^{3} \mathrm{~J}=9.4 \mathrm{~Hz}, 1 \mathrm{H}, \mathrm{CH}_{2}\right), 2.79\left(\mathrm{dd},{ }^{2} J=\right.$ $\left.13.5,{ }^{3} J=9.4 \mathrm{~Hz}, 1 \mathrm{H}, \mathrm{CH}_{2}\right), 3.85\left(\mathrm{~s}, 3 \mathrm{H}, \mathrm{OCH}_{3}\right), 4.19\left(\mathrm{dd},{ }^{3} J=9.4,{ }^{3} \mathrm{~J}=9.4 \mathrm{~Hz}, 1 \mathrm{H}, \mathrm{CH}\right), 6.68$ $\left(\mathrm{d},{ }^{3} J=8.3 \mathrm{~Hz}, 1 \mathrm{H}, \mathrm{Ar}\right), 6.99-7.03(\mathrm{~m}, 2 \mathrm{H}, \mathrm{Ar}), 7.15-7.21(\mathrm{~m}, 4 \mathrm{H}, \mathrm{Ar}), 7.44\left(\mathrm{~d},{ }^{4} \mathrm{~J}=2.0 \mathrm{~Hz}, 1 \mathrm{H}\right.$, Ar), 8.62 (br.s, 1H, NH).

${ }^{13} \mathrm{C} \mathrm{NMR}\left(\mathrm{CDCl}_{3}, 150 \mathrm{MHz}\right): \delta=34.2\left(\mathrm{CH}_{2}\right), 46.80(\mathrm{CH}), 53.06\left(\mathrm{OCH}_{3}\right), 68.9(\mathrm{C}), 111.9(\mathrm{CH}$, Ar), $125.3(\mathrm{CH}, \mathrm{Ar}), 126.9(2 \times \mathrm{CH}, \mathrm{Ar}), 128.10(\mathrm{CH}, \mathrm{Ar}), 128.9(\mathrm{C}, \mathrm{Ar}), 129.23(2 \times \mathrm{CH}, \mathrm{Ar})$, 129.3 (C, Ar), 130.38 (CH, Ar), 135.36 (C, Ar), 139.1 (C, Ar), 169.9 (C=O), $170.6(\mathrm{C}=\mathrm{O}), 177.6$ $(\mathrm{C}=\mathrm{O})$. 
B: ${ }^{1} \mathrm{H} \mathrm{NMR}\left(\mathrm{CDCl}_{3}, 600 \mathrm{MHz}\right): \delta=2.52\left(\mathrm{dd},{ }^{2} J=13.2,{ }^{3} J=9.4 \mathrm{~Hz}, 1 \mathrm{H}, \mathrm{CH}_{2}\right), 3.14\left(\mathrm{dd},{ }^{2} J=\right.$ $\left.13.2,{ }^{3} J=8.7 \mathrm{~Hz}, 1 \mathrm{H}, \mathrm{CH}_{2}\right), 3.83\left(\mathrm{~s}, 3 \mathrm{H}, \mathrm{OCH}_{3}\right), 4.02\left(\mathrm{dd},{ }^{3} J=9.4,{ }^{3} \mathrm{~J}=8.7 \mathrm{~Hz}, 1 \mathrm{H}, \mathrm{CH}\right), 6.75$ $\left(\mathrm{d},{ }^{3} J=8.3 \mathrm{~Hz}, 1 \mathrm{H}, \mathrm{Ar}\right), 6.99-7.03(\mathrm{~m}, 2 \mathrm{H}, \mathrm{Ar}), 7.15-7.21(\mathrm{~m}, 3 \mathrm{H}, \mathrm{Ar}), 7.23\left(\mathrm{dd},{ }^{3} J=8.3,{ }^{4} J=\right.$ $2.1 \mathrm{~Hz}, 1 \mathrm{H}, \mathrm{Ar}), 7.36\left(\mathrm{~d},{ }^{4} \mathrm{~J}=2.1 \mathrm{~Hz}, 1 \mathrm{H}, \mathrm{Ar}\right), 8.68$ (br.s, $\left.1 \mathrm{H}, \mathrm{NH}\right)$.

${ }^{13} \mathrm{C} \mathrm{NMR}\left(\mathrm{CDCl}_{3}, 150 \mathrm{MHz}\right): \delta=34.5\left(\mathrm{CH}_{2}\right), 46.77(\mathrm{CH}), 53.06\left(\mathrm{OCH}_{3}\right), 69.2(\mathrm{C}), 112.3(\mathrm{CH}$, Ar), $124.1(\mathrm{CH}, \mathrm{Ar}), 126.6(2 \times \mathrm{CH}, \mathrm{Ar}), 128.08(\mathrm{CH}, \mathrm{Ar}), 128.8(\mathrm{C}, \mathrm{Ar}), 129.23(2 \times \mathrm{CH}, \mathrm{Ar})$, 130.38 (CH, Ar), 130.5 (C, Ar), 135.44 (C, Ar), 138.7 (C, Ar), 168.7 (C=O), $170.1(\mathrm{C}=\mathrm{O}), 176.0$ $(\mathrm{C}=\mathrm{O})$.

IR (film) 3266, 1739, 1700, 1696, 1479, 1363, 1293, 1268, $1170 \mathrm{~cm}^{-1}$.

HRMS (ESI-TOF) $m / z:[\mathrm{M}+\mathrm{H}]^{+}$calcd for $\mathrm{C}_{19} \mathrm{H}_{16} \mathrm{ClN}_{2} \mathrm{O}_{4}{ }^{+} 371.0793$, found 371.0796.

\section{Methyl 5-bromo-2,5'-dioxo-1'-phenyl-1,2-dihydrospiro[indole-3,2' -pyrrolidine]-4' -}

carboxylate (9e)

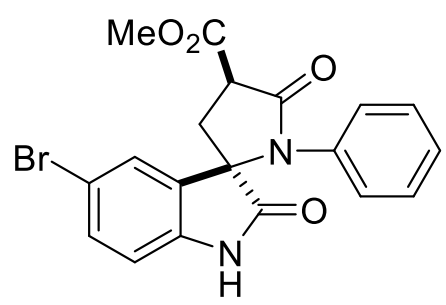

9e was obtained from $8 \mathbf{8}$ (146 mg, $0.27 \mathrm{mmol})$, TFA (1.0 mL, 13.64 mmol) and $\mathrm{TfOH}(60 \mu \mathrm{L}, 0.68 \mathrm{mmol})$ in $\mathrm{CH}_{2} \mathrm{Cl}_{2}(3.4 \mathrm{~mL})$; reaction time 118 h. Yield $54 \mathrm{mg}(48 \%)$; colorless solid, mp 197-198 ${ }^{\circ} \mathrm{C}$; $\mathrm{dr}$ $\mathbf{A}: \mathbf{B}=77: 23 . R_{f}=0.20$ (ethyl acetate/petroleum ether; $\left.1: 1\right)$.

A: ${ }^{1} \mathrm{H}$ NMR $\left(\mathrm{CDCl}_{3}, 600 \mathrm{MHz}\right): \delta=2.74\left(\mathrm{dd},{ }^{2} J=13.5,{ }^{3} \mathrm{~J}=9.4 \mathrm{~Hz}, 1 \mathrm{H}, \mathrm{CH}_{2}\right), 2.79\left(\mathrm{dd},{ }^{2} J=\right.$ $\left.13.5,{ }^{3} J=9.4 \mathrm{~Hz}, 1 \mathrm{H}, \mathrm{CH}_{2}\right), 3.86\left(\mathrm{~s}, 3 \mathrm{H}, \mathrm{OCH}_{3}\right), 4.19\left(\mathrm{dd},{ }^{3} J=9.4,{ }^{3} J=9.4 \mathrm{~Hz}, 1 \mathrm{H}, \mathrm{CH}\right), 6.64$ $\left(\mathrm{d},{ }^{3} J=8.3 \mathrm{~Hz}, 1 \mathrm{H}, \mathrm{Ar}\right), 7.01-7.03(\mathrm{~m}, 2 \mathrm{H}, \mathrm{Ar}), 7.16-7.22(\mathrm{~m}, 3 \mathrm{H}, \mathrm{Ar}), 7.33\left(\mathrm{dd},{ }^{3} J=8.3,{ }^{4} J=\right.$ $2.0 \mathrm{~Hz}, 1 \mathrm{H}, \mathrm{Ar}), 7.58\left(\mathrm{~d},{ }^{4} \mathrm{~J}=2.0 \mathrm{~Hz}, 1 \mathrm{H}, \mathrm{Ar}\right), 8.50$ (br.s, $\left.1 \mathrm{H}, \mathrm{NH}\right)$.

${ }^{13} \mathrm{C} \mathrm{NMR}\left(\mathrm{CDCl}_{3}, 150 \mathrm{MHz}\right): \delta=34.2\left(\mathrm{CH}_{2}\right), 46.79(\mathrm{CH}), 53.08\left(\mathrm{OCH}_{3}\right), 68.9(\mathrm{C}), 112.3(\mathrm{CH}$, $\mathrm{Ar}), 116.1(\mathrm{C}, \mathrm{Ar}), 126.9(2 \times \mathrm{CH}, \mathrm{Ar}), 128.14(\mathrm{CH}, \mathrm{Ar}), 128.18(\mathrm{CH}, \mathrm{Ar}), 129.26(2 \times \mathrm{CH}, \mathrm{Ar})$, 129.67 (C, Ar), 133.28 (CH, Ar), 135.36 (C, Ar), 139.6 (C, Ar), $169.9(\mathrm{C}=\mathrm{O}), 170.6(\mathrm{C}=\mathrm{O})$, $177.4(\mathrm{C}=\mathrm{O})$. 
B: ${ }^{1} \mathrm{H} \mathrm{NMR}\left(\mathrm{CDCl}_{3}, 600 \mathrm{MHz}\right): \delta=2.53\left(\mathrm{dd},{ }^{2} J=13.1,{ }^{3} J=9.3 \mathrm{~Hz}, 1 \mathrm{H}, \mathrm{CH}_{2}\right), 3.14\left(\mathrm{dd},{ }^{2} J=\right.$ $\left.13.1,{ }^{3} J=8.8 \mathrm{~Hz}, 1 \mathrm{H}, \mathrm{CH}_{2}\right), 3.84\left(\mathrm{~s}, 3 \mathrm{H}, \mathrm{OCH}_{3}\right), 4.02\left(\mathrm{dd},{ }^{3} J=9.3,{ }^{3} J=8.8 \mathrm{~Hz}, 1 \mathrm{H}, \mathrm{CH}\right), 6.71$ $\left(\mathrm{d},{ }^{3} J=8.3 \mathrm{~Hz}, 1 \mathrm{H}, \mathrm{Ar}\right), 6.98-7.00(\mathrm{~m}, 2 \mathrm{H}, \mathrm{Ar}), 7.16-7.22(\mathrm{~m}, 3 \mathrm{H}, \mathrm{Ar}), 7.39\left(\mathrm{dd},{ }^{3} J=8.3,{ }^{4} J=\right.$ $2.0 \mathrm{~Hz}, 1 \mathrm{H}, \mathrm{Ar}), 7.50\left(\mathrm{~d},{ }^{4} \mathrm{~J}=2.0 \mathrm{~Hz}, 1 \mathrm{H}, \mathrm{Ar}\right), 8.56$ (br.s, $\left.1 \mathrm{H}, \mathrm{NH}\right)$.

${ }^{13} \mathrm{C} \mathrm{NMR}\left(\mathrm{CDCl}_{3}, 150 \mathrm{MHz}\right): \delta=34.5\left(\mathrm{CH}_{2}\right), 46.79(\mathrm{CH}), 53.10\left(\mathrm{OCH}_{3}\right), 69.1(\mathrm{C}), 112.7(\mathrm{CH}$, Ar), $116.0(\mathrm{C}, \mathrm{Ar}), 126.6(2 \times \mathrm{CH}, \mathrm{Ar}), 128.12(\mathrm{CH}, \mathrm{Ar}), 128.18(\mathrm{CH}, \mathrm{Ar}), 129.26(2 \times \mathrm{CH}, \mathrm{Ar})$, 129.67 (C, Ar), 133.28 (CH, Ar), 135.42 (C, Ar), 139.1 (C, Ar), $168.7(\mathrm{C}=\mathrm{O}), 170.1(\mathrm{C}=\mathrm{O})$, $175.8(\mathrm{C}=\mathrm{O})$.

HRMS (ESI-TOF) $m / z:[\mathrm{M}+\mathrm{H}]^{+}$calcd for $\mathrm{C}_{19} \mathrm{H}_{16} \mathrm{BrN}_{2} \mathrm{O}_{4}{ }^{+}$415.0288, found 415.0300.

\section{Methyl 1'-(4-fluorophenyl)-2,5'-dioxo-1,2-dihydrospiro[indole-3,2' -pyrrolidine]-4' -}

carboxylate (9f)

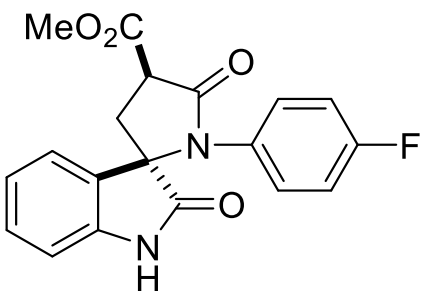

9f was obtained from $8 \mathrm{~g}$ (137 mg, $0.29 \mathrm{mmol})$, TFA (1.1 mL, 14.45 mmol) and $\mathrm{TfOH}(64 \mu \mathrm{L}, 0.72 \mathrm{mmol})$ in $\mathrm{CH}_{2} \mathrm{Cl}_{2}(3.6 \mathrm{~mL})$; reaction time 140 h. Yield $61 \mathrm{mg}(60 \%)$; yellowish oil; dr $\mathbf{A}: \mathbf{B}=80: 20 . R_{f}=$ 0.21 (ethyl acetate/petroleum ether; 1:1).

A: ${ }^{1} \mathrm{H} \mathrm{NMR}\left(\mathrm{CDCl}_{3}, 600 \mathrm{MHz}\right): \delta=2.75\left(\mathrm{dd},{ }^{2} J=13.5,{ }^{3} J=9.3 \mathrm{~Hz}, 1 \mathrm{H}, \mathrm{CH}_{2}\right), 2.82\left(\mathrm{dd},{ }^{2} J=\right.$ $\left.13.5,{ }^{3} J=9.5 \mathrm{~Hz}, 1 \mathrm{H}, \mathrm{CH}_{2}\right), 3.84\left(\mathrm{~s}, 3 \mathrm{H}, \mathrm{OCH}_{3}\right), 4.20\left(\mathrm{dd},{ }^{3} J=9.5,{ }^{3} \mathrm{~J}=9.3 \mathrm{~Hz}, 1 \mathrm{H}, \mathrm{CH}\right), 6.77$ $\left(\mathrm{d},{ }^{3} J=7.8 \mathrm{~Hz}, 1 \mathrm{H}, \mathrm{Ar}\right), 6.81-6.85(\mathrm{~m}, 2 \mathrm{H}, \mathrm{Ar}), 7.00\left(\mathrm{dd},{ }^{3} J=9.1,{ }^{4} J=4.9 \mathrm{~Hz}, 2 \mathrm{H}, \mathrm{Ar}\right), 7.08-$ $7.13(\mathrm{~m}, 1 \mathrm{H}, \mathrm{Ar}), 7.23\left(\mathrm{ddd},{ }^{3} J=7.8,{ }^{3} J=7.6,{ }^{4} J=1.1 \mathrm{~Hz}, 1 \mathrm{H}, \mathrm{Ar}\right), 7.46\left(\mathrm{~d},{ }^{3} J=7.4 \mathrm{~Hz}, 1 \mathrm{H}\right.$, Ar), 8.70 (br.s, 1H, NH).

${ }^{13} \mathrm{C} \mathrm{NMR}\left(\mathrm{CDCl}_{3}, 150 \mathrm{MHz}\right): \delta=34.0\left(\mathrm{CH}_{2}\right), 46.79(\mathrm{CH}), 52.99\left(\mathrm{OCH}_{3}\right), 69.1(\mathrm{C}), 110.8(\mathrm{CH}$, Ar), $116.05\left({ }^{2} J_{\mathrm{CF}}=23 \mathrm{~Hz}, 2 \times \mathrm{CH}, \mathrm{Ar}\right), 123.61(\mathrm{CH}, \mathrm{Ar}), 125.0(\mathrm{CH}, \mathrm{Ar}), 127.3(\mathrm{C}, \mathrm{Ar}), 129.1$ $\left({ }^{3} J_{\mathrm{CF}}=8 \mathrm{~Hz}, 2 \times \mathrm{CH}, \mathrm{Ar}\right), 130.45(\mathrm{CH}, \mathrm{Ar}), 131.39\left({ }^{4} J_{\mathrm{CF}}=2 \mathrm{~Hz}, \mathrm{C}, \mathrm{Ar}\right), 140.6(\mathrm{C}, \mathrm{Ar}), 161.76$ $\left({ }^{1} J_{\mathrm{CF}}=248 \mathrm{~Hz}, \mathrm{C}, \mathrm{Ar}\right), 170.0(\mathrm{C}=\mathrm{O}), 171.0(\mathrm{C}=\mathrm{O}), 177.9(\mathrm{C}=\mathrm{O})$. 
B: ${ }^{1} \mathrm{H} \mathrm{NMR}\left(\mathrm{CDCl}_{3}, 600 \mathrm{MHz}\right): \delta=2.54\left(\mathrm{dd},{ }^{2} J=13.2,{ }^{3} J=9.4 \mathrm{~Hz}, 1 \mathrm{H}, \mathrm{CH}_{2}\right), 3.14\left(\mathrm{dd},{ }^{2} J=\right.$ $\left.13.2,{ }^{3} \mathrm{~J}=8.7 \mathrm{~Hz}, 1 \mathrm{H}, \mathrm{CH}_{2}\right), 3.84\left(\mathrm{~s}, 3 \mathrm{H}, \mathrm{OCH}_{3}\right), 4.04\left(\mathrm{dd},{ }^{3} \mathrm{~J}=9.4,{ }^{3} \mathrm{~J}=8.7 \mathrm{~Hz}, 1 \mathrm{H}, \mathrm{CH}\right), 6.81-$ $6.85(\mathrm{~m}, 3 \mathrm{H}, \mathrm{Ar}), 6.96\left(\mathrm{dd},{ }^{3} J=9.1,{ }^{4} \mathrm{~J}=4.9 \mathrm{~Hz}, 2 \mathrm{H}, \mathrm{Ar}\right), 7.08-7.13(\mathrm{~m}, 1 \mathrm{H}, \mathrm{Ar}), 7.28\left(\mathrm{ddd},{ }^{3} J=\right.$ $\left.7.7,{ }^{3} J=7.7,{ }^{4} J=1.1 \mathrm{~Hz}, 1 \mathrm{H}, \mathrm{Ar}\right), 7.36\left(\mathrm{~d},{ }^{3} \mathrm{~J}=7.4 \mathrm{~Hz}, 1 \mathrm{H}, \mathrm{Ar}\right), 8.77$ (br.s, $\left.1 \mathrm{H}, \mathrm{NH}\right)$.

${ }^{13} \mathrm{C} \mathrm{NMR}\left(\mathrm{CDCl}_{3}, 150 \mathrm{MHz}\right): \delta=34.4\left(\mathrm{CH}_{2}\right), 46.79(\mathrm{CH}), 53.03\left(\mathrm{OCH}_{3}\right), 69.2(\mathrm{C}), 111.2(\mathrm{CH}$, Ar), $116.05\left({ }^{2} J_{\mathrm{CF}}=23 \mathrm{~Hz}, 2 \times \mathrm{CH}, \mathrm{Ar}\right), 123.55(\mathrm{CH}, \mathrm{Ar}), 123.61(\mathrm{CH}, \mathrm{Ar}), 128.5(\mathrm{C}, \mathrm{Ar}), 129.0$ $\left({ }^{3} J_{\mathrm{CF}}=8 \mathrm{~Hz}, 2 \times \mathrm{CH}, \mathrm{Ar}\right), 130.49(\mathrm{CH}, \mathrm{Ar}), 131.39\left({ }^{4} J_{\mathrm{CF}}=2 \mathrm{~Hz}, \mathrm{C}, \mathrm{Ar}\right), 140.2(\mathrm{C}, \mathrm{Ar}), 161.76$ $\left({ }^{1} J_{\mathrm{CF}}=248 \mathrm{~Hz}, \mathrm{C}, \mathrm{Ar}\right), 168.8(\mathrm{C}=\mathrm{O}), 170.4(\mathrm{C}=\mathrm{O}), 176.3(\mathrm{C}=\mathrm{O})$.

HRMS (ESI-TOF) $m / z:[\mathrm{M}+\mathrm{H}]^{+}$calcd for $\mathrm{C}_{19} \mathrm{H}_{16} \mathrm{FN}_{2} \mathrm{O}_{4}{ }^{+} 355.1089$, found 355.1094.

\section{Methyl 1'-(4-chlorophenyl)-2,5' -dioxo-1,2-dihydrospiro[indole-3,2' -pyrrolidine]-4' -}

carboxylate (9g)

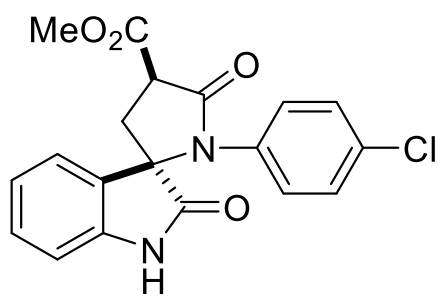

9g was obtained from $\mathbf{8 h}(135 \mathrm{mg}, 0.28 \mathrm{mmol})$, TFA (1.1 mL, 13.76 mmol) and $\mathrm{TfOH}(61 \mu \mathrm{L}, 0.69 \mathrm{mmol})$ in $\mathrm{CH}_{2} \mathrm{Cl}_{2}(3.4 \mathrm{~mL})$; reaction time 140 h. Yield $56 \mathrm{mg}$ (55\%); yellowish oil; dr A:B = 80:20. $R_{f}=$ 0.25 (ethyl acetate/petroleum ether; $1: 1$ ).

A: ${ }^{1} \mathrm{H}$ NMR $\left(\mathrm{CDCl}_{3}, 600 \mathrm{MHz}\right): \delta=2.75\left(\mathrm{dd},{ }^{2} J=13.5,{ }^{3} \mathrm{~J}=9.3 \mathrm{~Hz}, 1 \mathrm{H}, \mathrm{CH}_{2}\right), 2.82\left(\mathrm{dd},{ }^{2} J=\right.$ $\left.13.5,{ }^{3} J=9.7 \mathrm{~Hz}, 1 \mathrm{H}, \mathrm{CH}_{2}\right), 3.85\left(\mathrm{~s}, 3 \mathrm{H}, \mathrm{OCH}_{3}\right), 4.21\left(\mathrm{dd},{ }^{3} J=9.7,{ }^{3} J=9.3 \mathrm{~Hz}, 1 \mathrm{H}, \mathrm{CH}\right), 6.79$ $\left(\mathrm{d},{ }^{3} J=7.8 \mathrm{~Hz}, 1 \mathrm{H}, \mathrm{Ar}\right), 6.97\left(\mathrm{~d},{ }^{3} J=8.8 \mathrm{~Hz}, 2 \mathrm{H}, \mathrm{Ar}\right), 7.09\left(\mathrm{ddd},{ }^{3} J=7.6,{ }^{3} J=7.5,{ }^{4} J=0.9 \mathrm{~Hz}\right.$, $1 \mathrm{H}, \mathrm{Ar}), 7.11-7.15(\mathrm{~m}, 2 \mathrm{H}, \mathrm{Ar}), 7.24\left(\mathrm{ddd},{ }^{3} J=7.8,{ }^{3} J=7.6,{ }^{4} J=1.2 \mathrm{~Hz}, 1 \mathrm{H}, \mathrm{Ar}\right), 7.43\left(\mathrm{~d},{ }^{3} J=\right.$ $7.5 \mathrm{~Hz}, 1 \mathrm{H}, \mathrm{Ar}), 8.62$ (br.s, 1H, NH).

${ }^{13} \mathrm{C} \mathrm{NMR}\left(\mathrm{CDCl}_{3}, 150 \mathrm{MHz}\right): \delta=34.2\left(\mathrm{CH}_{2}\right), 46.8(\mathrm{CH}), 53.0\left(\mathrm{OCH}_{3}\right), 68.8(\mathrm{C}), 110.9(\mathrm{CH}$, Ar), $123.7(\mathrm{CH}, \mathrm{Ar}), 124.8(\mathrm{CH}, \mathrm{Ar}), 127.2(\mathrm{C}, \mathrm{Ar}), 128.2(2 \times \mathrm{CH}, \mathrm{Ar}), 129.28(2 \times \mathrm{CH}, \mathrm{Ar})$, $130.51(\mathrm{CH}, \mathrm{Ar}), 133.65$ (C, Ar), 134.1 (C, Ar), 140.5 (C, Ar), 169.9 (C=O), 170.7 (C=O), 177.8 $(\mathrm{C}=\mathrm{O})$. 
B: ${ }^{1} \mathrm{H} \mathrm{NMR}\left(\mathrm{CDCl}_{3}, 600 \mathrm{MHz}\right): \delta=2.54\left(\mathrm{dd},{ }^{2} J=13.2,{ }^{3} J=9.3 \mathrm{~Hz}, 1 \mathrm{H}, \mathrm{CH}_{2}\right), 3.14\left(\mathrm{dd},{ }^{2} J=\right.$ $\left.13.2,{ }^{3} J=8.8 \mathrm{~Hz}, 1 \mathrm{H}, \mathrm{CH}_{2}\right), 3.85\left(\mathrm{~s}, 3 \mathrm{H}, \mathrm{OCH}_{3}\right), 4.04\left(\mathrm{dd},{ }^{3} J=9.3,{ }^{3} \mathrm{~J}=8.8 \mathrm{~Hz}, 1 \mathrm{H}, \mathrm{CH}\right), 6.85$ $\left(\mathrm{d},{ }^{3} J=7.8 \mathrm{~Hz}, 1 \mathrm{H}, \mathrm{Ar}\right), 6.94\left(\mathrm{~d},{ }^{3} J=8.8 \mathrm{~Hz}, 2 \mathrm{H}, \mathrm{Ar}\right), 7.11-7.15(\mathrm{~m}, 3 \mathrm{H}, \mathrm{Ar}), 7.29\left(\mathrm{ddd},{ }^{3} J=\right.$ $\left.7.8,{ }^{3} J=7.6,{ }^{4} J=1.1 \mathrm{~Hz}, 1 \mathrm{H}, \mathrm{Ar}\right), 7.34\left(\mathrm{~d},{ }^{3} J=7.4 \mathrm{~Hz}, 1 \mathrm{H}, \mathrm{Ar}\right), 8.70$ (br.s, $\left.1 \mathrm{H}, \mathrm{NH}\right)$.

${ }^{13} \mathrm{C} \mathrm{NMR}\left(\mathrm{CDCl}_{3}, 150 \mathrm{MHz}\right): \delta=34.6\left(\mathrm{CH}_{2}\right), 46.9(\mathrm{CH}), 53.1\left(\mathrm{OCH}_{3}\right), 69.1(\mathrm{C}), 111.3(\mathrm{CH}$, Ar), $123.57(\mathrm{CH}, \mathrm{Ar}), 123.63(\mathrm{CH}, \mathrm{Ar}), 127.9$ (2×CH, Ar), $128.5(\mathrm{C}, \mathrm{Ar}), 129.28(2 \times \mathrm{CH}, \mathrm{Ar})$, 130.54 (CH, Ar), 133.65 (C, Ar), 134.2 (C, Ar), 140.1 (C, Ar), $168.7(\mathrm{C}=\mathrm{O}), 170.2(\mathrm{C}=\mathrm{O}), 176.2$ $(\mathrm{C}=\mathrm{O})$.

HRMS (ESI-TOF) $m / z:[\mathrm{M}+\mathrm{H}]^{+}$calcd for $\mathrm{C}_{19} \mathrm{H}_{16} \mathrm{ClN}_{2} \mathrm{O}_{4}{ }^{+} 371.0793$, found 371.0795.

\section{Methyl 1'-(3-chlorophenyl)-2,5' -dioxo-1,2-dihydrospiro[indole-3,2' -pyrrolidine]-4' -}

carboxylate (9h)

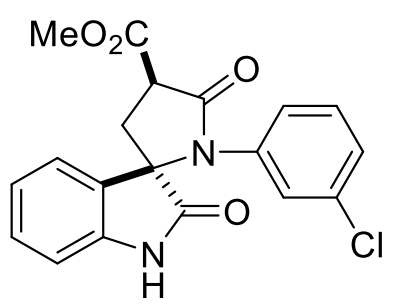

9h was obtained from $8 \mathbf{i}(123 \mathrm{mg}, 0.25 \mathrm{mmol})$, TFA $(960 \mu \mathrm{L}, 12.54$ mmol) and TfOH $(55 \mu \mathrm{L}, 0.63 \mathrm{mmol})$ in $\mathrm{CH}_{2} \mathrm{Cl}_{2}(3.1 \mathrm{~mL})$; reaction time 140 h. Yield $42 \mathrm{mg}$ (45\%); yellowish oil; $\mathrm{dr} \mathbf{A}: \mathbf{B}=79: 21 . R_{f}=$ 0.23 (ethyl acetate/petroleum ether; $1: 1$ ).

A: ${ }^{1} \mathrm{H}$ NMR $\left(\mathrm{CDCl}_{3}, 600 \mathrm{MHz}\right): \delta=2.75\left(\mathrm{dd},{ }^{2} J=13.5,{ }^{3} \mathrm{~J}=9.2 \mathrm{~Hz}, 1 \mathrm{H}, \mathrm{CH}_{2}\right), 2.83\left(\mathrm{dd},{ }^{2} J=\right.$ $\left.13.5,{ }^{3} J=9.9 \mathrm{~Hz}, 1 \mathrm{H}, \mathrm{CH}_{2}\right), 3.86\left(\mathrm{~s}, 3 \mathrm{H}, \mathrm{OCH}_{3}\right), 4.23\left(\mathrm{dd},{ }^{3} J=9.9,{ }^{3} \mathrm{~J}=9.2 \mathrm{~Hz}, 1 \mathrm{H}, \mathrm{CH}\right), 6.82$ $\left(\mathrm{d},{ }^{3} J=7.8 \mathrm{~Hz}, 1 \mathrm{H}, \mathrm{Ar}\right), 6.87-6.90(\mathrm{~m}, 1 \mathrm{H}, \mathrm{Ar}), 7.06-7.14(\mathrm{~m}, 4 \mathrm{H}, \mathrm{Ar}), 7.24\left(\mathrm{ddd},{ }^{3} J=7.8,{ }^{3} J=\right.$ 7.6, $\left.{ }^{4} J=1.1 \mathrm{~Hz}, 1 \mathrm{H}, \mathrm{Ar}\right), 7.41\left(\mathrm{~d},{ }^{3} J=7.5 \mathrm{~Hz}, 1 \mathrm{H}, \mathrm{Ar}\right), 8.64$ (br.s, $\left.1 \mathrm{H}, \mathrm{NH}\right)$.

${ }^{13} \mathrm{C} \mathrm{NMR}\left(\mathrm{CDCl}_{3}, 150 \mathrm{MHz}\right): \delta=34.4\left(\mathrm{CH}_{2}\right), 46.8(\mathrm{CH}), 53.0\left(\mathrm{OCH}_{3}\right), 68.8(\mathrm{C}), 110.9(\mathrm{CH}$, Ar), $123.74(\mathrm{CH}, \mathrm{Ar}), 124.7(\mathrm{CH}, \mathrm{Ar}), 124.9(\mathrm{CH}, \mathrm{Ar}), 127.0(\mathrm{C}, \mathrm{Ar}), 127.1(\mathrm{CH}, \mathrm{Ar}), 128.1$ (CH, Ar), 129.98 (CH, Ar), 130.55 (CH, Ar), 134.49 (C, Ar), 136.8 (C, Ar), 140.5 (C, Ar), 169.9 $(\mathrm{C}=\mathrm{O}), 170.6(\mathrm{C}=\mathrm{O}), 177.8(\mathrm{C}=\mathrm{O})$.

B: ${ }^{1} \mathrm{H} \mathrm{NMR}\left(\mathrm{CDCl}_{3}, 600 \mathrm{MHz}\right): \delta=2.55\left(\mathrm{dd},{ }^{2} J=13.1,{ }^{3} J=9.3 \mathrm{~Hz}, 1 \mathrm{H}, \mathrm{CH}_{2}\right), 3.15\left(\mathrm{dd},{ }^{2} J=\right.$ $\left.13.1,{ }^{3} J=8.8 \mathrm{~Hz}, 1 \mathrm{H}, \mathrm{CH}_{2}\right), 3.85\left(\mathrm{~s}, 3 \mathrm{H}, \mathrm{OCH}_{3}\right), 4.04\left(\mathrm{dd},{ }^{3} J=9.3,{ }^{3} \mathrm{~J}=8.8 \mathrm{~Hz}, 1 \mathrm{H}, \mathrm{CH}\right), 6.84$ 
(ddd, $\left.{ }^{3} J=8.0,{ }^{4} J=1.8,{ }^{4} J=1.0 \mathrm{~Hz}, 1 \mathrm{H}, \mathrm{Ar}\right), 6.87-6.90(\mathrm{~m}, 1 \mathrm{H}, \mathrm{Ar}), 7.06-7.14(\mathrm{~m}, 4 \mathrm{H}, \mathrm{Ar}), 7.30$ (ddd, $\left.{ }^{3} J=7.8,{ }^{3} J=7.6,{ }^{4} J=1.1 \mathrm{~Hz}, 1 \mathrm{H}, \mathrm{Ar}\right), 7.34\left(\mathrm{~d},{ }^{3} J=7.5 \mathrm{~Hz}, 1 \mathrm{H}, \mathrm{Ar}\right), 8.72$ (br.s, $\left.1 \mathrm{H}, \mathrm{NH}\right)$.

${ }^{13} \mathrm{C} \mathrm{NMR}\left(\mathrm{CDCl}_{3}, 150 \mathrm{MHz}\right): \delta=34.8\left(\mathrm{CH}_{2}\right), 46.9(\mathrm{CH}), 53.1\left(\mathrm{OCH}_{3}\right), 69.0(\mathrm{C}), 111.3(\mathrm{CH}$, Ar), $123.5(\mathrm{CH}, \mathrm{Ar}), 123.69(\mathrm{CH}, \mathrm{Ar}), 124.3(\mathrm{CH}, \mathrm{Ar}), 126.8(\mathrm{CH}, \mathrm{Ar}), 128.0(\mathrm{CH}, \mathrm{Ar}), 128.4$ (C, Ar), 129.98 (CH, Ar), 130.60 (CH, Ar), 134.51 (C, Ar), 136.9 (C, Ar), 140.0 (C, Ar), 168.7 $(\mathrm{C}=\mathrm{O}), 170.1(\mathrm{C}=\mathrm{O}), 176.1(\mathrm{C}=\mathrm{O})$.

HRMS (ESI-TOF) $m / z:[\mathrm{M}+\mathrm{H}]^{+}$calcd for $\mathrm{C}_{19} \mathrm{H}_{16} \mathrm{ClN}_{2} \mathrm{O}_{4}{ }^{+}$371.0793, found 371.0799.

\section{Methyl 1'-(4-bromophenyl)-2,5'-dioxo-1,2-dihydrospiro[indole-3,2' -pyrrolidine]-4' -}

carboxylate $(9 \mathbf{i})$

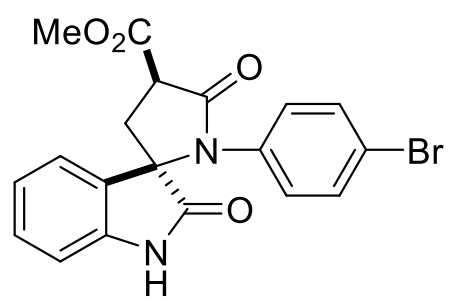

9i was obtained from $8 \mathbf{j}$ (70 mg, $0.13 \mathrm{mmol})$, TFA (500 $\mu \mathrm{L}, 6.54$ mmol) and $\mathrm{TfOH}(29 \mu \mathrm{L}, 0.33 \mathrm{mmol})$ in $\mathrm{CH}_{2} \mathrm{Cl}_{2}(1.6 \mathrm{~mL})$; reaction time 140 h. Yield $29 \mathrm{mg}(54 \%)$; as yellowish oil; dr A:B = 79:21. $R_{f}$ $=0.24$ (ethyl acetate/petroleum ether; $1: 1)$.

A: ${ }^{1} \mathrm{H} \mathrm{NMR}\left(\mathrm{CDCl}_{3}, 600 \mathrm{MHz}\right): \delta=2.75\left(\mathrm{dd},{ }^{2} J=13.5,{ }^{3} \mathrm{~J}=9.4 \mathrm{~Hz}, 1 \mathrm{H}, \mathrm{CH}_{2}\right), 2.82\left(\mathrm{dd},{ }^{2} J=\right.$ $\left.13.5,{ }^{3} J=9.7 \mathrm{~Hz}, 1 \mathrm{H}, \mathrm{CH}_{2}\right), 3.86\left(\mathrm{~s}, 3 \mathrm{H}, \mathrm{OCH}_{3}\right), 4.20\left(\mathrm{dd},{ }^{3} J=9.7,{ }^{3} \mathrm{~J}=9.4 \mathrm{~Hz}, 1 \mathrm{H}, \mathrm{CH}\right), 6.80$ (ddd, ${ }^{3} J=7.8,{ }^{4} J=0.9,{ }^{5} J=0.6 \mathrm{~Hz}, 1 \mathrm{H}$, Ar) $, 6.91\left(\mathrm{~d},{ }^{3} J=8.9 \mathrm{~Hz}, 2 \mathrm{H}, \mathrm{Ar}\right), 7.10\left(\mathrm{ddd},{ }^{3} J=7.6,{ }^{3} J\right.$ $\left.=7.5,{ }^{4} J=0.9 \mathrm{~Hz}, 1 \mathrm{H}, \mathrm{Ar}\right), 7.25\left(\mathrm{ddd},{ }^{3} J=7.8,{ }^{3} \mathrm{~J}=7.6,{ }^{4} \mathrm{~J}=1.2 \mathrm{~Hz}, 1 \mathrm{H}, \mathrm{Ar}\right), 7.29-7.32(\mathrm{~m}, 2 \mathrm{H}$, Ar), $7.43\left(\mathrm{ddd},{ }^{3} J=7.5,{ }^{4} \mathrm{~J}=1.2,{ }^{5} \mathrm{~J}=0.6 \mathrm{~Hz}, 1 \mathrm{H}, \mathrm{Ar}\right.$ ), 8.30 (br.s, $1 \mathrm{H}, \mathrm{NH}$ ).

${ }^{13} \mathrm{C} \mathrm{NMR}\left(\mathrm{CDCl}_{3}, 150 \mathrm{MHz}\right): \delta=34.3\left(\mathrm{CH}_{2}\right), 46.8(\mathrm{CH}), 53.0\left(\mathrm{OCH}_{3}\right), 68.8(\mathrm{C}), 110.8(\mathrm{CH}$, Ar), $121.74(\mathrm{C}, \mathrm{Ar}), 123.8(\mathrm{CH}, \mathrm{Ar}), 125.0(\mathrm{CH}, \mathrm{Ar}), 127.3(\mathrm{C}, \mathrm{Ar}), 128.5(2 \times \mathrm{CH}, \mathrm{Ar}), 130.55$ $(\mathrm{CH}, \mathrm{Ar}), 132.28(2 \times \mathrm{CH}, \mathrm{Ar}), 134.7(\mathrm{C}, \mathrm{Ar}), 140.4(\mathrm{C}, \mathrm{Ar}), 169.9(\mathrm{C}=\mathrm{O}), 170.6(\mathrm{C}=\mathrm{O}), 177.7$ $(\mathrm{C}=\mathrm{O})$.

B: ${ }^{1} \mathrm{H}$ NMR $\left(\mathrm{CDCl}_{3}, 600 \mathrm{MHz}\right): \delta=2.54\left(\mathrm{dd},{ }^{2} J=13.1,{ }^{3} \mathrm{~J}=9.4 \mathrm{~Hz}, 1 \mathrm{H}, \mathrm{CH}_{2}\right), 3.15\left(\mathrm{dd},{ }^{2} J=\right.$ $\left.13.1,{ }^{3} \mathrm{~J}=8.8 \mathrm{~Hz}, 1 \mathrm{H}, \mathrm{CH}_{2}\right), 3.86\left(\mathrm{~s}, 3 \mathrm{H}, \mathrm{OCH}_{3}\right), 4.02\left(\mathrm{dd},{ }^{3} \mathrm{~J}=9.4,{ }^{3} \mathrm{~J}=8.8 \mathrm{~Hz}, 1 \mathrm{H}, \mathrm{CH}\right), 6.85$ (ddd, $\left.{ }^{3} J=7.8,{ }^{4} J=0.9,{ }^{5} J=0.7 \mathrm{~Hz}, 1 \mathrm{H}, \mathrm{Ar}\right), 6.89\left(\mathrm{~d},{ }^{3} J=9.0 \mathrm{~Hz}, 2 \mathrm{H}, \mathrm{Ar}\right), 7.13\left(\mathrm{ddd},{ }^{3} J=7.6,{ }^{3} J\right.$ 
$\left.=7.5,{ }^{4} J=0.9 \mathrm{~Hz}, 1 \mathrm{H}, \mathrm{Ar}\right), 7.29-7.32(\mathrm{~m}, 3 \mathrm{H}, \mathrm{Ar}), 7.34\left(\mathrm{ddd},{ }^{3} J=7.5,{ }^{4} J=1.2,{ }^{5} \mathrm{~J}=0.7 \mathrm{~Hz}, 1 \mathrm{H}\right.$, Ar), 8.39 (br.s, 1H, NH).

${ }^{13} \mathrm{C} \mathrm{NMR}\left(\mathrm{CDCl}_{3}, 150 \mathrm{MHz}\right): \delta=34.7\left(\mathrm{CH}_{2}\right), 46.9(\mathrm{CH}), 53.1\left(\mathrm{OCH}_{3}\right), 69.0(\mathrm{C}), 111.2(\mathrm{CH}$, Ar), $121.72(\mathrm{C}, \mathrm{Ar}), 123.6(\mathrm{CH}, \mathrm{Ar}), 123.7(\mathrm{CH}, \mathrm{Ar}), 128.2(2 \times \mathrm{CH}, \mathrm{Ar}), 128.6(\mathrm{C}, \mathrm{Ar}), 130.58$ (CH, Ar), 132.28 (2×CH, Ar), $134.8(\mathrm{C}, \mathrm{Ar}), 140.0(\mathrm{C}, \mathrm{Ar}), 168.7(\mathrm{C}=\mathrm{O}), 170.0(\mathrm{C}=\mathrm{O}), 176.0$ $(\mathrm{C}=\mathrm{O})$.

HRMS (ESI-TOF) $m / z:[\mathrm{M}+\mathrm{H}]^{+}$calcd for $\mathrm{C}_{19} \mathrm{H}_{16} \mathrm{BrN}_{2} \mathrm{O}_{4}{ }^{+} 415.0288$, found 415.0288.

\section{Methyl 1'-(4-methylphenyl)-2,5'-dioxo-1,2-dihydrospiro[indole-3,2' -pyrrolidine]-4' -}

carboxylate $(9 \mathbf{j})$

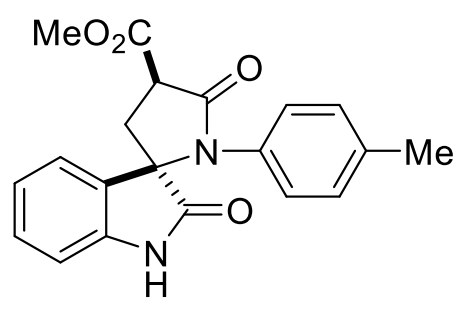

9j was obtained from $\mathbf{8 k}(84 \mathrm{mg}, 0.18 \mathrm{mmol})$, TFA $(685 \mu \mathrm{L}, 8.94$ mmol) and $\mathrm{TfOH}(39 \mu \mathrm{L}, 0.45 \mathrm{mmol})$ in $\mathrm{CH}_{2} \mathrm{Cl}_{2}(2.2 \mathrm{~mL})$; reaction time 100 h. Yield $39 \mathrm{mg}(62 \%)$; brown solid, mp 247-248 ${ }^{\circ} \mathrm{C}$; dr $\mathbf{A}: \mathbf{B}=80: 20 . R_{f}=0.23$ (ethyl acetate/petroleum ether; 1:1).

A: ${ }^{1} \mathrm{H}$ NMR $\left(\mathrm{CDCl}_{3}, 600 \mathrm{MHz}\right): \delta=2.19\left(\mathrm{~s}, 3 \mathrm{H}, \mathrm{CH}_{3}\right), 2.73\left(\mathrm{dd},{ }^{2} J=13.5,{ }^{3} J=9.3 \mathrm{~Hz}, 1 \mathrm{H}\right.$, $\left.\mathrm{CH}_{2}\right), 2.81\left(\mathrm{dd},{ }^{2} J=13.5,{ }^{3} J=9.6 \mathrm{~Hz}, 1 \mathrm{H}, \mathrm{CH}_{2}\right), 3.84\left(\mathrm{~s}, 3 \mathrm{H}, \mathrm{OCH}_{3}\right), 4.21\left(\mathrm{dd},{ }^{3} J=9.6,{ }^{3} J=9.3\right.$ $\mathrm{Hz}, 1 \mathrm{H}, \mathrm{CH}), 6.75\left(\mathrm{~d},{ }^{3} \mathrm{~J}=7.8 \mathrm{~Hz}, 1 \mathrm{H}, \mathrm{Ar}\right), 6.91\left(\mathrm{~d},{ }^{3} \mathrm{~J}=8.4 \mathrm{~Hz}, 2 \mathrm{H}, \mathrm{Ar}\right), 6.93-6.96$ (m, 2H, Ar), $7.08\left(\mathrm{dd},{ }^{3} J=7.6,{ }^{3} J=7.5 \mathrm{~Hz}, 1 \mathrm{H}\right.$, Ar $), 7.20\left(\mathrm{ddd},{ }^{3} J=7.8,{ }^{3} J=7.6,{ }^{4} \mathrm{~J}=1.0 \mathrm{~Hz}, 1 \mathrm{H}\right.$, Ar $), 7.45$ $\left(\mathrm{d},{ }^{3} J=7.5 \mathrm{~Hz}, 1 \mathrm{H}, \mathrm{Ar}\right), 8.59$ (br.s, $\left.1 \mathrm{H}, \mathrm{NH}\right)$.

${ }^{13} \mathrm{C} \mathrm{NMR}\left(\mathrm{CDCl}_{3}, 150 \mathrm{MHz}\right): \delta=20.96\left(\mathrm{CH}_{3}\right), 34.1\left(\mathrm{CH}_{2}\right), 46.88(\mathrm{CH}), 52.9\left(\mathrm{OCH}_{3}\right), 69.0(\mathrm{C})$, $110.7(\mathrm{CH}, \mathrm{Ar}), 123.5(\mathrm{CH}, \mathrm{Ar}), 125.0(\mathrm{CH}, \mathrm{Ar}), 126.9(2 \times \mathrm{CH}, \mathrm{Ar}), 127.7(\mathrm{C}, \mathrm{Ar}), 129.69$ (2×CH, Ar), 130.2 (CH, Ar), 132.90 (C, Ar), 137.79 (C, Ar), 140.6 (C, Ar), 170.19 (C=O), 170.7 $(\mathrm{C}=\mathrm{O}), 178.1(\mathrm{C}=\mathrm{O})$.

B: ${ }^{1} \mathrm{H}$ NMR $\left(\mathrm{CDCl}_{3}, 600 \mathrm{MHz}\right): \delta=2.19\left(\mathrm{~s}, 3 \mathrm{H}, \mathrm{CH}_{3}\right), 2.52\left(\mathrm{dd},{ }^{2} J=13.1,{ }^{3} J=9.3 \mathrm{~Hz}, 1 \mathrm{H}\right.$, $\left.\mathrm{CH}_{2}\right), 3.14\left(\mathrm{dd},{ }^{2} J=13.1,{ }^{3} \mathrm{~J}=8.8 \mathrm{~Hz}, 1 \mathrm{H}, \mathrm{CH}_{2}\right), 3.83\left(\mathrm{~s}, 3 \mathrm{H}, \mathrm{OCH}_{3}\right), 4.03\left(\mathrm{dd},{ }^{3} J=9.3,{ }^{3} J=8.8\right.$ $\mathrm{Hz}, 1 \mathrm{H}, \mathrm{CH}), 6.82\left(\mathrm{~d},{ }^{3} \mathrm{~J}=7.8 \mathrm{~Hz}, 1 \mathrm{H}, \mathrm{Ar}\right), 6.87\left(\mathrm{~d},{ }^{3} \mathrm{~J}=8.4 \mathrm{~Hz}, 2 \mathrm{H}, \mathrm{Ar}\right), 6.93-6.96$ (m, 2H, Ar), 
$7.11\left(\mathrm{dd},{ }^{3} J=7.6,{ }^{3} J=7.5 \mathrm{~Hz}, 1 \mathrm{H}\right.$, Ar $), 7.26\left(\mathrm{ddd},{ }^{3} J=7.8,{ }^{3} J=7.6,{ }^{4} J=1.0 \mathrm{~Hz}, 1 \mathrm{H}\right.$, Ar $), 7.36$ $\left(\mathrm{d},{ }^{3} J=7.5 \mathrm{~Hz}, 1 \mathrm{H}, \mathrm{Ar}\right), 8.65$ (br.s, $\left.1 \mathrm{H}, \mathrm{NH}\right)$.

${ }^{13} \mathrm{C} \mathrm{NMR}\left(\mathrm{CDCl}_{3}, 150 \mathrm{MHz}\right): \delta=20.96\left(\mathrm{CH}_{3}\right), 34.5\left(\mathrm{CH}_{2}\right), 46.94(\mathrm{CH}), 53.0\left(\mathrm{OCH}_{3}\right), 69.2(\mathrm{C})$, $111.1(\mathrm{CH}, \mathrm{Ar}), 123.4(\mathrm{CH}, \mathrm{Ar}), 123.7(\mathrm{CH}, \mathrm{Ar}), 126.6(2 \times \mathrm{CH}, \mathrm{Ar}), 129.0(\mathrm{C}, \mathrm{Ar}), 129.69$ (2×CH, Ar), 130.3 (CH, Ar), 132.93 (C, Ar), 137.79 (C, Ar), 140.2 (C, Ar), 169.0 (C=O), 170.19 $(\mathrm{C}=\mathrm{O}), 176.4(\mathrm{C}=\mathrm{O})$.

HRMS (ESI-TOF) $m / z:[\mathrm{M}+\mathrm{H}]^{+}$calcd for $\mathrm{C}_{20} \mathrm{H}_{19} \mathrm{~N}_{2} \mathrm{O}_{4}{ }^{+} 351.1339$, found 351.1345.

\section{Methyl 1'-(4-methoxyphenyl)-2,5' -dioxo-1,2-dihydrospiro[indole-3,2' -pyrrolidine]-4' -} carboxylate (9k)

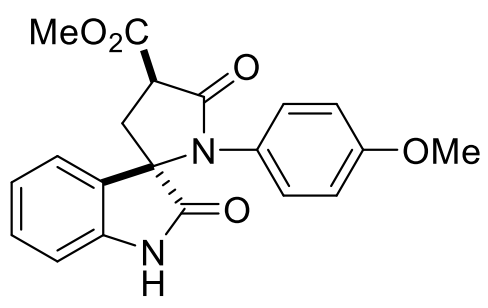

9k was obtained from $8 \mathbf{l}(121 \mathrm{mg}, 0.25 \mathrm{mmol})$, TFA $(950 \mu \mathrm{L}$, $12.45 \mathrm{mmol})$ and $\mathrm{TfOH}(55 \mu \mathrm{L}, 0.62 \mathrm{mmol})$ in $\mathrm{CH}_{2} \mathrm{Cl}_{2}(3.1 \mathrm{~mL})$; reaction time $100 \mathrm{~h}$. Yield $51 \mathrm{mg}(56 \%)$; yellowish oil; dr A:B = 82:18. $R_{f}=0.16($ ethyl acetate/petroleum ether; $1: 1)$.

A: ${ }^{1} \mathrm{H}$ NMR $\left(\mathrm{CDCl}_{3}, 600 \mathrm{MHz}\right): \delta=2.73\left(\mathrm{dd},{ }^{2} J=13.5,{ }^{3} \mathrm{~J}=9.4 \mathrm{~Hz}, 1 \mathrm{H}, \mathrm{CH}_{2}\right), 2.80\left(\mathrm{dd},{ }^{2} J=\right.$ $\left.13.5,{ }^{3} J=9.4 \mathrm{~Hz}, 1 \mathrm{H}, \mathrm{CH}_{2}\right), 3.62\left(\mathrm{~s}, 3 \mathrm{H}, \mathrm{OCH}_{3}\right), 3.83\left(\mathrm{~s}, 3 \mathrm{H}, \mathrm{OCH}_{3}\right), 4.19\left(\mathrm{dd},{ }^{3} J=9.4,{ }^{3} J=9.4\right.$ $\mathrm{Hz}, 1 \mathrm{H}, \mathrm{CH}), 6.60-6.63(\mathrm{~m}, 2 \mathrm{H}, \mathrm{Ar}), 6.73\left(\mathrm{~d},{ }^{3} \mathrm{~J}=7.8 \mathrm{~Hz}, 1 \mathrm{H}, \mathrm{Ar}\right), 6.92\left(\mathrm{~d},{ }^{3} \mathrm{~J}=9.0 \mathrm{~Hz}, 2 \mathrm{H}, \mathrm{Ar}\right)$, $7.08\left(\mathrm{ddd},{ }^{3} J=7.6,{ }^{3} J=7.5,{ }^{4} \mathrm{~J}=0.8 \mathrm{~Hz}, 1 \mathrm{H}, \mathrm{Ar}\right), 7.19\left(\mathrm{ddd},{ }^{3} J=7.8,{ }^{3} J=7.6,{ }^{4} J=1.2 \mathrm{~Hz}, 1 \mathrm{H}\right.$, Ar), $7.47\left(\mathrm{~d},{ }^{3} J=7.5 \mathrm{~Hz}, 1 \mathrm{H}, \mathrm{Ar}\right), 8.69$ (br.s, $\left.1 \mathrm{H}, \mathrm{NH}\right)$.

${ }^{13} \mathrm{C} \mathrm{NMR}\left(\mathrm{CDCl}_{3}, 150 \mathrm{MHz}\right): \delta=33.8\left(\mathrm{CH}_{2}\right), 46.84(\mathrm{CH}), 52.91\left(\mathrm{OCH}_{3}\right), 55.13\left(\mathrm{OCH}_{3}\right), 69.2$ (C), $110.8(\mathrm{CH}, \mathrm{Ar}), 114.26(2 \times \mathrm{CH}, \mathrm{Ar}), 123.4(\mathrm{CH}, \mathrm{Ar}), 125.0(\mathrm{CH}, \mathrm{Ar}), 127.7$ (C, Ar), 128.1 (C, Ar), $128.6(2 \times \mathrm{CH}, \mathrm{Ar}), 130.2(\mathrm{CH}, \mathrm{Ar}), 140.7(\mathrm{C}, \mathrm{Ar}), 158.92(\mathrm{C}, \mathrm{Ar}), 170.2(\mathrm{C}=\mathrm{O}), 171.0$ $(\mathrm{C}=\mathrm{O}), 178.1(\mathrm{C}=\mathrm{O})$.

B: ${ }^{1} \mathrm{H}$ NMR $\left(\mathrm{CDCl}_{3}, 600 \mathrm{MHz}\right): \delta=2.52\left(\mathrm{dd},{ }^{2} J=13.1,{ }^{3} J=9.3 \mathrm{~Hz}, 1 \mathrm{H}, \mathrm{CH}_{2}\right), 3.12\left(\mathrm{dd},{ }^{2} J=\right.$ $\left.13.1,{ }^{3} J=8.8 \mathrm{~Hz}, 1 \mathrm{H}, \mathrm{CH}_{2}\right), 3.61\left(\mathrm{~s}, 3 \mathrm{H}, \mathrm{OCH}_{3}\right), 3.82\left(\mathrm{~s}, 3 \mathrm{H}, \mathrm{OCH}_{3}\right), 4.02\left(\mathrm{dd},{ }^{3} J=9.3,{ }^{3} J=8.8\right.$ $\mathrm{Hz}, 1 \mathrm{H}, \mathrm{CH}), 6.60-6.63(\mathrm{~m}, 2 \mathrm{H}, \mathrm{Ar}), 6.80\left(\mathrm{~d},{ }^{3} \mathrm{~J}=7.8 \mathrm{~Hz}, 1 \mathrm{H}, \mathrm{Ar}\right), 6.89\left(\mathrm{~d},{ }^{3} \mathrm{~J}=9.0 \mathrm{~Hz}, 2 \mathrm{H}, \mathrm{Ar}\right)$, 
$7.11\left(\mathrm{ddd},{ }^{3} \mathrm{~J}=7.6,{ }^{3} \mathrm{~J}=7.5,{ }^{4} \mathrm{~J}=0.8 \mathrm{~Hz}, 1 \mathrm{H}, \mathrm{Ar}\right), 7.25\left(\mathrm{ddd},{ }^{3} J=7.8,{ }^{3} \mathrm{~J}=7.6,{ }^{4} \mathrm{~J}=1.2 \mathrm{~Hz}, 1 \mathrm{H}\right.$, Ar), $7.36\left(\mathrm{~d},{ }^{3} J=7.5 \mathrm{~Hz}, 1 \mathrm{H}, \mathrm{Ar}\right), 8.76$ (br.s, $\left.1 \mathrm{H}, \mathrm{NH}\right)$.

${ }^{13} \mathrm{C} \mathrm{NMR}\left(\mathrm{CDCl}_{3}, 150 \mathrm{MHz}\right): \delta=34.2\left(\mathrm{CH}_{2}\right), 46.84(\mathrm{CH}), 52.94\left(\mathrm{OCH}_{3}\right), 55.13\left(\mathrm{OCH}_{3}\right), 69.3$ (C), $111.1(\mathrm{CH}, \mathrm{Ar}), 114.26(2 \times \mathrm{CH}, \mathrm{Ar}), 123.3(\mathrm{CH}, \mathrm{Ar}), 123.7(\mathrm{CH}, \mathrm{Ar}), 128.0(\mathrm{C}, \mathrm{Ar}), 128.4$ (2×CH, Ar), 128.9 (C, Ar), 130.3 (CH, Ar), 140.3 (C, Ar), 158.92 (C, Ar), $169.0(\mathrm{C}=\mathrm{O}), 170.5$ $(\mathrm{C}=\mathrm{O}), 176.5(\mathrm{C}=\mathrm{O})$.

HRMS (ESI-TOF) $m / z:[\mathrm{M}+\mathrm{H}]^{+}$calcd for $\mathrm{C}_{20} \mathrm{H}_{19} \mathrm{~N}_{2} \mathrm{O}_{5}{ }^{+}$367.1288, found 367.1294.

\section{Methyl 1'-(4-nitrophenyl)-2,5'-dioxo-1,2-dihydrospiro[indole-3,2' -pyrrolidine]-4' -}

carboxylate (91)

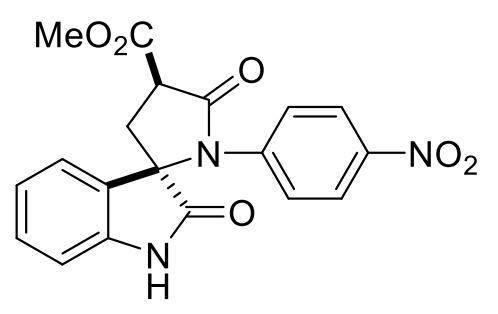

91 was obtained from 8 n (206 mg, $0.41 \mathrm{mmol})$, TFA (1.6 mL, $20.56 \mathrm{mmol})$ and $\mathrm{TfOH}(91 \mu \mathrm{L}, 1.03 \mathrm{mmol})$ in $\mathrm{CH}_{2} \mathrm{Cl}_{2}(5.1 \mathrm{~mL})$; reaction time $100 \mathrm{~h}$. Yield $86 \mathrm{mg}(55 \%)$; yellowish oil; dr $\mathbf{A}: \mathbf{B}=$ 79:21. $R_{f}=0.25$ (ethyl acetate/petroleum ether; $\left.1: 1\right)$.

A: ${ }^{1} \mathrm{H}$ NMR $\left(\mathrm{CDCl}_{3}, 600 \mathrm{MHz}\right): \delta=2.78\left(\mathrm{dd},{ }^{2} J=13.3,{ }^{3} \mathrm{~J}=9.0 \mathrm{~Hz}, 1 \mathrm{H}, \mathrm{CH}_{2}\right), 2.82\left(\mathrm{dd},{ }^{2} J=\right.$ $\left.13.3,{ }^{3} \mathrm{~J}=10.5 \mathrm{~Hz}, 1 \mathrm{H}, \mathrm{CH}_{2}\right), 3.87\left(\mathrm{~s}, 3 \mathrm{H}, \mathrm{OCH}_{3}\right), 4.28\left(\mathrm{dd},{ }^{3} \mathrm{~J}=10.5,{ }^{3} \mathrm{~J}=9.0 \mathrm{~Hz}, 1 \mathrm{H}, \mathrm{CH}\right), 6.89$ $\left(\mathrm{d},{ }^{3} J=7.8 \mathrm{~Hz}, 1 \mathrm{H}, \mathrm{Ar}\right), 7.06\left(\mathrm{ddd},{ }^{3} J=7.6,{ }^{3} J=7.6,{ }^{4} J=1.0 \mathrm{~Hz}, 1 \mathrm{H}, \mathrm{Ar}\right), 7.21-7.28(\mathrm{~m}, 3 \mathrm{H}$, Ar), 7.30-7.34 (m, 1H, Ar), 8.01-8.04 (m, 2H, Ar), 8.80 (br.s, 1H, NH).

${ }^{13} \mathrm{C} \mathrm{NMR}\left(\mathrm{CDCl}_{3}, 150 \mathrm{MHz}\right): \delta=34.9\left(\mathrm{CH}_{2}\right), 46.8(\mathrm{CH}), 53.15\left(\mathrm{OCH}_{3}\right), 68.3(\mathrm{C}), 111.2(\mathrm{CH}$, Ar), $124.1(\mathrm{CH}, \mathrm{Ar}), 124.4(2 \times \mathrm{CH}, \mathrm{Ar}), 124.6(\mathrm{CH}, \mathrm{Ar}), 125.5(2 \times \mathrm{CH}, \mathrm{Ar}), 126.4(\mathrm{C}, \mathrm{Ar})$, $130.82(\mathrm{CH}, \mathrm{Ar}), 140.3$ (C, Ar), $141.6(\mathrm{C}, \mathrm{Ar}), 145.8$ (C, Ar), $169.5(\mathrm{C}=\mathrm{O}), 170.6(\mathrm{C}=\mathrm{O}), 177.4$ $(\mathrm{C}=\mathrm{O})$.

B: ${ }^{1} \mathrm{H}$ NMR $\left(\mathrm{CDCl}_{3}, 600 \mathrm{MHz}\right): \delta=2.57\left(\mathrm{dd},{ }^{2} J=13.0,{ }^{3} J=9.2 \mathrm{~Hz}, 1 \mathrm{H}, \mathrm{CH}_{2}\right), 3.15\left(\mathrm{dd},{ }^{2} J=\right.$ $\left.13.0,{ }^{3} \mathrm{~J}=8.9 \mathrm{~Hz}, 1 \mathrm{H}, \mathrm{CH}_{2}\right), 3.87\left(\mathrm{~s}, 3 \mathrm{H}, \mathrm{OCH}_{3}\right), 4.09\left(\mathrm{dd},{ }^{3} \mathrm{~J}=9.2,{ }^{3} \mathrm{~J}=8.9 \mathrm{~Hz}, 1 \mathrm{H}, \mathrm{CH}\right), 6.95$ $\left(\mathrm{d},{ }^{3} J=7.8 \mathrm{~Hz}, 1 \mathrm{H}, \mathrm{Ar}\right), 7.12\left(\mathrm{ddd},{ }^{3} J=7.6,{ }^{3} J=7.5,{ }^{4} \mathrm{~J}=1.0 \mathrm{~Hz}, 1 \mathrm{H}, \mathrm{Ar}\right), 7.21-7.28(\mathrm{~m}, 2 \mathrm{H}$, Ar), 7.30-7.34 (m, 2H, Ar), 8.01-8.04 (m, 2H, Ar), 8.90 (br.s, 1H, NH). 
${ }^{13} \mathrm{C} \mathrm{NMR}\left(\mathrm{CDCl}_{3}, 150 \mathrm{MHz}\right): \delta=35.3\left(\mathrm{CH}_{2}\right), 46.9(\mathrm{CH}), 53.21\left(\mathrm{OCH}_{3}\right), 68.8(\mathrm{C}), 111.6(\mathrm{CH}$, Ar), $123.3(\mathrm{CH}, \mathrm{Ar}), 124.0(\mathrm{CH}, \mathrm{Ar}), 124.5(2 \times \mathrm{CH}, \mathrm{Ar}), 124.9(2 \times \mathrm{CH}, \mathrm{Ar}), 128.1(\mathrm{C}, \mathrm{Ar})$, $130.84(\mathrm{CH}, \mathrm{Ar}), 139.6$ (C, Ar), 141.9 (C, Ar), 145.7 (C, Ar), $168.3(\mathrm{C}=\mathrm{O}), 170.0(\mathrm{C}=\mathrm{O}), 175.8$ $(\mathrm{C}=\mathrm{O})$.

HRMS (ESI-TOF) $m / z:[\mathrm{M}+\mathrm{H}]^{+}$calcd for $\mathrm{C}_{19} \mathrm{H}_{16} \mathrm{~N}_{3} \mathrm{O}_{6}{ }^{+} 382.1034$, found 382.1040.

\section{General procedure for the synthesis of cyclopropanes 10}

A mixture of cyclopropane $2 \mathbf{a}$ (1.0 equiv), aliphatic amine (10.0 equiv), and $\mathrm{Ni}\left(\mathrm{ClO}_{4}\right)_{2} \cdot 6 \mathrm{H}_{2} \mathrm{O}$ (0.1 equiv) in $\mathrm{CH}_{2} \mathrm{Cl}_{2}(2 \mathrm{M})$ was stirred at $40{ }^{\circ} \mathrm{C}$ (oil bath) for specified time. Then, the reaction mixture was concentrated under reduced pressure. Cyclopropanes $\mathbf{1 0}$ were purified by column chromatography on silica gel.

\section{Methyl (1RS,2RS)-2-(benzylcarbamoyl)-1' -(4-methoxybenzyl)-2' -oxo-1',2'-}

\section{dihydrospiro[cyclopropane-1,3'-indole]-2-carboxylate (10a)}

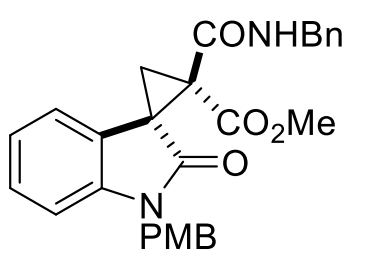

10a was obtained from $2 \mathbf{a}(216 \mathrm{mg}, 0.55 \mathrm{mmol})$, benzylamine $(595 \mu \mathrm{L}$, $5.47 \mathrm{mmol})$, and $\mathrm{Ni}\left(\mathrm{ClO}_{4}\right)_{2} \cdot 6 \mathrm{H}_{2} \mathrm{O}(20 \mathrm{mg}, 0.05 \mathrm{mmol})$ in $\mathrm{CH}_{2} \mathrm{Cl}_{2}(275$ $\mu \mathrm{L})$; reaction time $12 \mathrm{~h}$. Yield $170 \mathrm{mg}(66 \%)$; colorless solid, mp 169-

$170{ }^{\circ} \mathrm{C} . R_{f}=0.38$ (ethyl acetate/petroleum ether; $1: 2$ ).

${ }^{1} \mathrm{H} \mathrm{NMR}\left(\mathrm{CDCl}_{3}, 600 \mathrm{MHz}\right): \delta=2.62\left(\mathrm{~d},{ }^{2} J=5.3 \mathrm{~Hz}, 1 \mathrm{H}, \mathrm{CH}_{2}\right), 2.77\left(\mathrm{~d},{ }^{2} J=5.3 \mathrm{~Hz}, 1 \mathrm{H}, \mathrm{CH}_{2}\right)$, $3.76\left(\mathrm{~s}, 3 \mathrm{H}, \mathrm{OCH}_{3}\right), 3.88\left(\mathrm{~s}, 3 \mathrm{H}, \mathrm{OCH}_{3}\right), 4.33\left(\mathrm{dd},{ }^{2} J=15.0,{ }^{3} J=5.3 \mathrm{~Hz}, 1 \mathrm{H}, \mathrm{NCH}_{2} \mathrm{Ph}\right), 4.50$ $\left(\mathrm{dd},{ }^{2} J=15.0,{ }^{3} J=6.4 \mathrm{~Hz}, 1 \mathrm{H}, \mathrm{NCH}_{2} \mathrm{Ph}\right), 4.86-4.92\left(\mathrm{~m}, 2 \mathrm{H}, \mathrm{NCH}_{2} \mathrm{PMP}\right), 6.81-6.85$ (m, 3H, Ar), $6.94\left(\mathrm{ddd},{ }^{3} J=7.7,{ }^{3} \mathrm{~J}=7.6,{ }^{4} \mathrm{~J}=1.0 \mathrm{~Hz}, 1 \mathrm{H}, \mathrm{Ar}\right), 6.96-6.99(\mathrm{~m}, 2 \mathrm{H}, \mathrm{Ar}), 7.15-7.25$ (m, 7H, Ar), $7.53\left(\mathrm{dd},{ }^{3} J=6.4,{ }^{3} \mathrm{~J}=5.3 \mathrm{~Hz}, 1 \mathrm{H}, \mathrm{NH}\right)$.

${ }^{13} \mathrm{C} \mathrm{NMR}\left(\mathrm{CDCl}_{3}, 150 \mathrm{MHz}\right): \delta=21.7\left(\mathrm{CH}_{2}\right), 37.9(\mathrm{C}), 43.6\left(\mathrm{NCH}_{2}\right), 43.9\left(\mathrm{NCH}_{2}\right), 46.4(\mathrm{C})$, $53.1\left(\mathrm{OCH}_{3}\right), 55.1\left(\mathrm{OCH}_{3}\right), 109.1(\mathrm{CH}, \mathrm{Ar}), 114.1(2 \times \mathrm{CH}, \mathrm{Ar}), 122.3(\mathrm{CH}, \mathrm{Ar}), 122.5(\mathrm{CH}, \mathrm{Ar})$, $124.5(\mathrm{C}, \mathrm{Ar}), 127.2(\mathrm{CH}, \mathrm{Ar}), 127.3(2 \times \mathrm{CH}, \mathrm{Ar}), 127.6(\mathrm{C}, \mathrm{Ar}), 128.2(\mathrm{CH}, \mathrm{Ar}), 128.4(2 \times \mathrm{CH}$, 
Ar), $128.6(2 \times C H, A r), 137.4(\mathrm{C}, \mathrm{Ar}), 143.3$ (C, Ar), 159.0 (C, Ar), 161.8 (CONHBn), 169.1 $\left(\mathrm{CO}_{2} \mathrm{Me}\right), 172.8(\mathrm{CONPMB})$.

HRMS (ESI-TOF) $m / z:[\mathrm{M}+\mathrm{H}]^{+}$calcd for $\mathrm{C}_{28} \mathrm{H}_{27} \mathrm{~N}_{2} \mathrm{O}_{5}{ }^{+}$471.1914, found 471.1927.

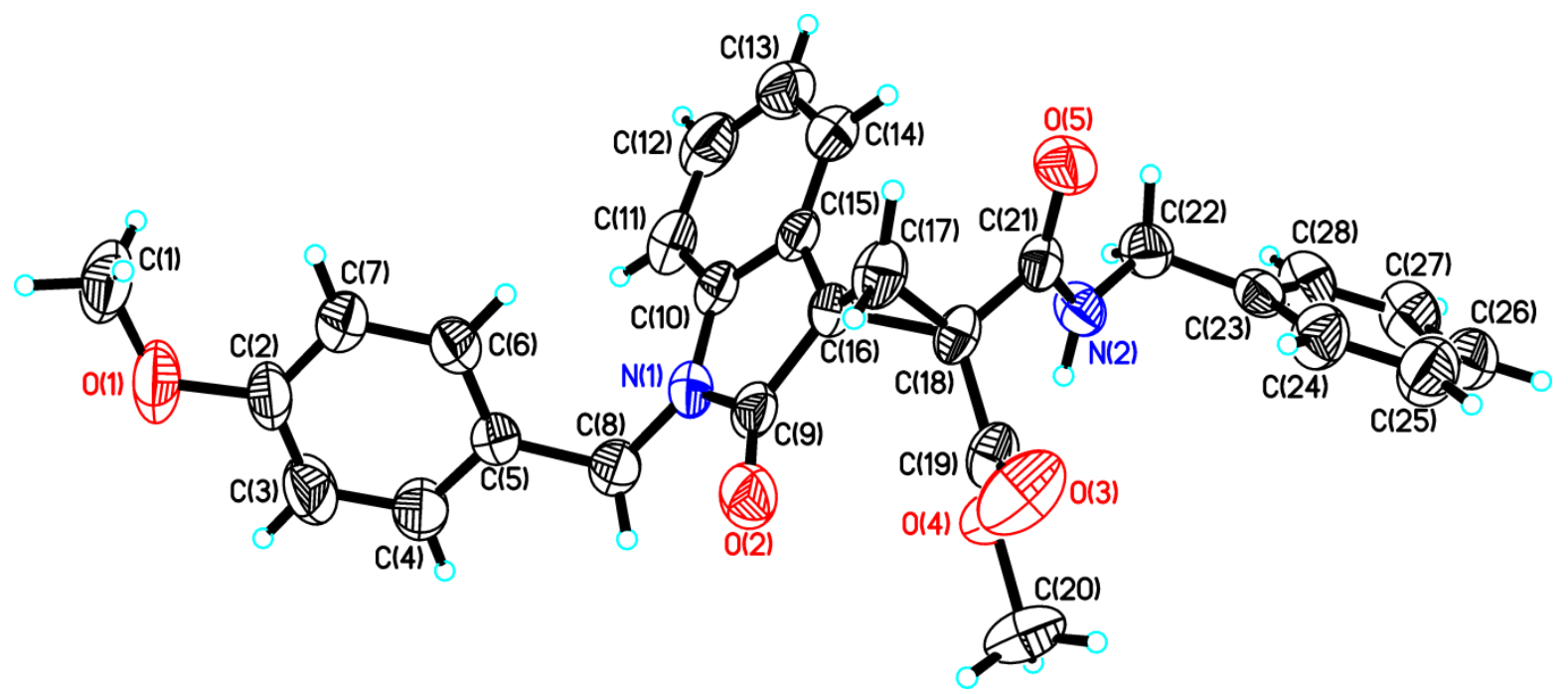

Figure S2. Molecular structure from single crystal X-ray study of 10a (thermal ellipsoids are drawn at the 50\% probability level); CCDC 1948999.

Methyl (1RS,2RS)-2-(butylcarbamoyl)-1'-(4-methoxybenzyl)-2' -oxospiro[cyclopropane-1,3' indoline]-2-carboxylate (10b)

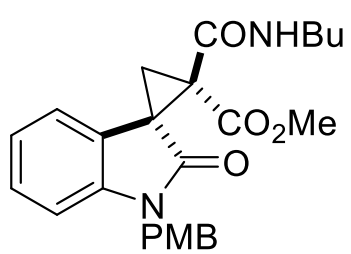

10b was obtained from $2 \mathbf{a}(111 \mathrm{mg}, 0.28 \mathrm{mmol}), n$-butylamine $(275 \mu \mathrm{L}$, $2.81 \mathrm{mmol})$, and $\mathrm{Ni}\left(\mathrm{ClO}_{4}\right)_{2} \cdot 6 \mathrm{H}_{2} \mathrm{O}(10 \mathrm{mg}, 0.03 \mathrm{mmol})$ in $\mathrm{CH}_{2} \mathrm{Cl}_{2}(140$ $\mu \mathrm{L})$; reaction time 4 h. Yield $86 \mathrm{mg}$ (70\%); colorless solid, mp 153-154 ${ }^{\circ} \mathrm{C} . R_{f}=0.73$ (ethyl acetate/petroleum ether; $1: 1$ ).

${ }^{1} \mathrm{H}$ NMR $\left(\mathrm{CDCl}_{3}, 600 \mathrm{MHz}\right): \delta=0.78\left(\mathrm{t},{ }^{3} \mathrm{~J}=7.4 \mathrm{~Hz}, 3 \mathrm{H}, \mathrm{CH}_{3}\right), 1.06-1.16\left(\mathrm{~m}, 2 \mathrm{H}, \mathrm{CH}_{2}\right), 1.28-$ $1.36\left(\mathrm{~m}, 2 \mathrm{H}, \mathrm{CH}_{2}\right), 2.54\left(\mathrm{~d},{ }^{2} J=5.2 \mathrm{~Hz}, 1 \mathrm{H}, \mathrm{CH}_{2}\right), 2.70\left(\mathrm{~d},{ }^{2} J=5.2 \mathrm{~Hz}, 1 \mathrm{H}, \mathrm{CH}_{2}\right), 3.12-3.18(\mathrm{~m}$, $\left.1 \mathrm{H}, \mathrm{NCH}_{2} \mathrm{Pr}\right), 3.19-3.25\left(\mathrm{~m}, 1 \mathrm{H}, \mathrm{NCH}_{2} \mathrm{Pr}\right), 3.76\left(\mathrm{~s}, 3 \mathrm{H}, \mathrm{OCH}_{3}\right), 3.87\left(\mathrm{~s}, 3 \mathrm{H}, \mathrm{OCH}_{3}\right), 4.86\left(\mathrm{~d},{ }^{2} \boldsymbol{J}\right.$ $\left.=15.4 \mathrm{~Hz}, 1 \mathrm{H}, \mathrm{NCH}_{2} \mathrm{PMP}\right), 4.89\left(\mathrm{~d},{ }^{2} J=15.4 \mathrm{~Hz}, 1 \mathrm{H}, \mathrm{NCH}_{2} \mathrm{PMP}\right), 6.79\left(\mathrm{~d},{ }^{3} J=7.8 \mathrm{~Hz}, 1 \mathrm{H}\right.$, Ar), $6.85\left(\mathrm{~d},{ }^{3} J=8.8 \mathrm{~Hz}, 2 \mathrm{H}, \mathrm{Ar}\right), 6.94\left(\mathrm{ddd},{ }^{3} J=7.7,{ }^{3} \mathrm{~J}=7.6,{ }^{4} \mathrm{~J}=1.0 \mathrm{~Hz}, 1 \mathrm{H}, \mathrm{Ar}\right), 7.14-7.18$ 
(m, 2H, Ar, NH), $7.20\left(\mathrm{ddd},{ }^{3} J=7.7,{ }^{4} J=1.2,{ }^{5} J=0.4 \mathrm{~Hz}, 1 \mathrm{H}, \mathrm{Ar}\right), 7.23\left(\mathrm{~d},{ }^{3} J=8.8 \mathrm{~Hz}, 2 \mathrm{H}\right.$, Ar).

${ }^{13} \mathrm{C} \mathrm{NMR}\left(\mathrm{CDCl}_{3}, 150 \mathrm{MHz}\right): \delta=13.5\left(\mathrm{CH}_{3}\right), 19.7\left(\mathrm{CH}_{2}\right), 21.8\left(\mathrm{CH}_{2}\right.$, cyclopropane $), 31.2\left(\mathrm{CH}_{2}\right)$, $37.6(\mathrm{C}), 39.8\left(\mathrm{CH}_{2}\right), 43.6\left(\mathrm{NCH}_{2}\right), 46.3(\mathrm{C}), 53.1\left(\mathrm{OCH}_{3}\right), 55.2\left(\mathrm{OCH}_{3}\right), 109.0(\mathrm{CH}, \mathrm{Ar}), 114.1$ $(2 \times \mathrm{CH}, \mathrm{Ar}), 122.1(\mathrm{CH}, \mathrm{Ar}), 122.3(\mathrm{CH}, \mathrm{Ar}), 124.6(\mathrm{C}, \mathrm{Ar}), 127.7(\mathrm{C}, \mathrm{Ar}), 128.1(\mathrm{CH}, \mathrm{Ar})$, $128.6(2 \times \mathrm{CH}, \mathrm{Ar}), 143.2(\mathrm{C}, \mathrm{Ar}), 159.1(\mathrm{C}, \mathrm{Ar}), 161.6(\mathrm{CONHBu}), 169.3\left(\mathrm{CO}_{2} \mathrm{Me}\right), 173.0$ (CONPMB).

HRMS (ESI-TOF) $m / z:[\mathrm{M}+\mathrm{H}]^{+}$calcd for $\mathrm{C}_{25} \mathrm{H}_{29} \mathrm{~N}_{2} \mathrm{O}_{5}{ }^{+}$437.2071, found 437.2074.

\section{General procedure for the synthesis of lactames 11}

A mixture of cyclopropane $\mathbf{1 0}$ (1.0 equiv), aromatic amine (2.0 equiv), and $\mathrm{Ni}\left(\mathrm{ClO}_{4}\right)_{2} \cdot 6 \mathrm{H}_{2} \mathrm{O}(0.1$ equiv) in $o$-xylene $(2 \mathrm{M})$ was stirred at $140{ }^{\circ} \mathrm{C}$ in microwave reactor for specified time. Then, the reaction mixture was concentrated under reduced pressure. Lactames $\mathbf{1 0}$ were purified by column chromatography on silica gel.

\section{$N$-benzyl-1-(4-methoxybenzyl)-2,5'-dioxo-1'-phenyl-1,2-dihydrospiro[indole-3,2' -}

\section{pyrrolidine]-4' -carboxamide (11a)}

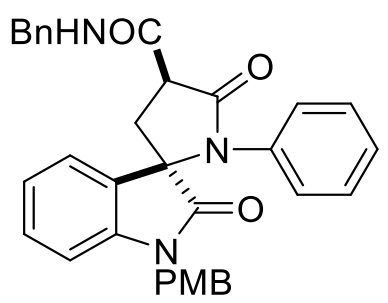

11a was obtained from $10 a(103 \mathrm{mg}, 0.22 \mathrm{mmol})$, aniline $(40 \mu \mathrm{L}, 0.44$ $\mathrm{mmol})$, and $\mathrm{Ni}\left(\mathrm{ClO}_{4}\right)_{2} \cdot 6 \mathrm{H}_{2} \mathrm{O}(8 \mathrm{mg}, 0.02 \mathrm{mmol})$ in $o$-xylene $(115 \mu \mathrm{L})$ at $140{ }^{\circ} \mathrm{C}$; reaction time $4 \mathrm{~h}$. Yield $40 \mathrm{mg}(34 \%)$; yellow oil, dr $\mathbf{A}: \mathbf{B}=$ 78:22. $R_{f}=0.23$ (ethyl acetate/petroleum ether; $1: 1$ ).

A: ${ }^{1} \mathrm{H}$ NMR $\left(\mathrm{CDCl}_{3}, 600 \mathrm{MHz}\right): \delta=2.81\left(\mathrm{dd},{ }^{2} J=13.7,{ }^{3} \mathrm{~J}=9.6 \mathrm{~Hz}, 1 \mathrm{H}, \mathrm{CH}_{2}\right), 2.98\left(\mathrm{dd},{ }^{2} J=\right.$ $\left.13.7,{ }^{3} \mathrm{~J}=9.7 \mathrm{~Hz}, 1 \mathrm{H}, \mathrm{CH}_{2}\right), 3.76\left(\mathrm{~s}, 3 \mathrm{H}, \mathrm{OCH}_{3}\right), 4.15\left(\mathrm{dd},{ }^{3} \mathrm{~J}=9.7,{ }^{3} \mathrm{~J}=9.6 \mathrm{~Hz}, 1 \mathrm{H}, \mathrm{CH}\right), 4.49$ $4.55\left(\mathrm{~m}, 2 \mathrm{H}, \mathrm{NCH}_{2}\right), 4.62\left(\mathrm{dd},{ }^{2} J=14.9,{ }^{3} \mathrm{~J}=6.0 \mathrm{~Hz}, 1 \mathrm{H}, \mathrm{NCH}_{2} \mathrm{Ph}\right), 5.00\left(\mathrm{~d},{ }^{2} J=15.5 \mathrm{~Hz}, 1 \mathrm{H}\right.$, $\left.\mathrm{NCH}_{2} \mathrm{PMP}\right), 6.60\left(\mathrm{~d},{ }^{3} \mathrm{~J}=7.7 \mathrm{~Hz}, 1 \mathrm{H}, \mathrm{Ar}\right), 6.70-6.73(\mathrm{~m}, 2 \mathrm{H}, \mathrm{Ar}), 6.80\left(\mathrm{~d},{ }^{3} \mathrm{~J}=8.8 \mathrm{~Hz}, 2 \mathrm{H}, \mathrm{Ar}\right)$, 6.95-6.98 (m, 2H, Ar), $7.07\left(\mathrm{ddd},{ }^{3} J=7.7,{ }^{3} J=7.6,{ }^{4} J=1.0 \mathrm{~Hz}, 1 \mathrm{H}, \mathrm{Ar}\right), 7.16-7.25$ (m, 4H, Ar), 
7.28-7.31 (m, 1H, Ar), 7.33-7.40 (m, 4H, Ar), 7.41-7.43 (m, 1H, Ar), 7.87 (dd, ${ }^{3} J=6.0,{ }^{3} J=5.4$ $\mathrm{Hz}, 1 \mathrm{H}, \mathrm{NH})$.

${ }^{13} \mathrm{C} \mathrm{NMR}\left(\mathrm{CDCl}_{3}, 150 \mathrm{MHz}\right): \delta=32.6\left(\mathrm{CH}_{2}\right), 43.2\left(\mathrm{NCH}_{2}\right), 43.74\left(\mathrm{NCH}_{2}\right), 46.0(\mathrm{CH}), 55.17$ $\left(\mathrm{OCH}_{3}\right), 68.6(\mathrm{C}), 109.9(\mathrm{CH}, \mathrm{Ar}), 114.1(2 \times \mathrm{CH}, \mathrm{Ar}), 123.52(\mathrm{CH}, \mathrm{Ar}), 124.8(\mathrm{CH}, \mathrm{Ar}), 126.54$ (C, Ar), $127.25(\mathrm{C}, \mathrm{Ar}), 127.36(\mathrm{CH}, \mathrm{Ar}), 127.50(2 \times \mathrm{CH}, \mathrm{Ar}), 127.65(2 \times \mathrm{CH}, \mathrm{Ar}), 128.11(\mathrm{CH}$, Ar), $128.15(2 \times \mathrm{CH}, \mathrm{Ar}), 128.64(2 \times \mathrm{CH}, \mathrm{Ar}), 129.15(2 \times \mathrm{CH}, \mathrm{Ar}), 130.2(\mathrm{CH}, \mathrm{Ar}), 135.5(\mathrm{C}, \mathrm{Ar})$, $137.9(\mathrm{C}, \mathrm{Ar}), 142.5(\mathrm{C}, \mathrm{Ar}), 159.0(\mathrm{C}, \mathrm{Ar}), 167.0(\mathrm{C}=\mathrm{O}), 173.5(\mathrm{C}=\mathrm{O}), 175.7(\mathrm{C}=\mathrm{O})$.

B: ${ }^{1} \mathrm{H}$ NMR $\left(\mathrm{CDCl}_{3}, 600 \mathrm{MHz}\right): \delta=2.67\left(\mathrm{dd},{ }^{2} J=13.5,{ }^{3} J=10.0 \mathrm{~Hz}, 1 \mathrm{H}, \mathrm{CH}_{2}\right), 3.25\left(\mathrm{dd},{ }^{2} J=\right.$ $\left.13.5,{ }^{3} \mathrm{~J}=8.4 \mathrm{~Hz}, 1 \mathrm{H}, \mathrm{CH}_{2}\right), 3.76\left(\mathrm{~s}, 3 \mathrm{H}, \mathrm{OCH}_{3}\right), 3.93\left(\mathrm{dd},{ }^{3} \mathrm{~J}=10.0,{ }^{3} J=8.4 \mathrm{~Hz}, 1 \mathrm{H}, \mathrm{CH}\right), 4.46$ $\left(\mathrm{d},{ }^{2} J=15.5 \mathrm{~Hz}, 1 \mathrm{H}, \mathrm{NCH}_{2} \mathrm{PMP}\right), 4.49-4.55\left(\mathrm{~m}, 1 \mathrm{H}, \mathrm{NCH}_{2} \mathrm{Ph}\right), 4.67\left(\mathrm{dd},{ }^{2} J=14.8,{ }^{3} J=6.0 \mathrm{~Hz}\right.$, $\left.1 \mathrm{H}, \mathrm{NCH}_{2} \mathrm{Ph}\right), 5.05\left(\mathrm{~d},{ }^{2} \mathrm{~J}=15.5 \mathrm{~Hz}, 1 \mathrm{H}, \mathrm{NCH}_{2} \mathrm{PMP}\right), 6.63\left(\mathrm{~d},{ }^{3} \mathrm{~J}=7.7 \mathrm{~Hz}, 1 \mathrm{H}, \mathrm{Ar}\right), 6.67\left(\mathrm{~d},{ }^{3} \mathrm{~J}=\right.$ $8.8 \mathrm{~Hz}, 2 \mathrm{H}, \mathrm{Ar}), 6.70-6.73$ (m, 2H, Ar), 6.95-6.98 (m, 2H, Ar), $7.10\left(\mathrm{ddd},{ }^{3} J=7.7,{ }^{3} J=7.6,{ }^{4} \mathrm{~J}=\right.$ $0.9 \mathrm{~Hz}, 1 \mathrm{H}, \mathrm{Ar}), 7.16-7.25(\mathrm{~m}, 4 \mathrm{H}, \mathrm{Ar}), 7.28-7.31$ (m, 1H, Ar), 7.33-7.40 (m, 4H, Ar), 7.41$7.43(\mathrm{~m}, 1 \mathrm{H}, \mathrm{Ar}), 8.11\left(\mathrm{dd},{ }^{3} J=6.0,{ }^{3} J=5.4 \mathrm{~Hz}, 1 \mathrm{H}, \mathrm{NH}\right)$.

${ }^{13} \mathrm{C}$ NMR $\left(\mathrm{CDCl}_{3}, 150 \mathrm{MHz}\right): \delta=33.1\left(\mathrm{CH}_{2}\right), 43.3\left(\mathrm{NCH}_{2}\right), 43.68\left(\mathrm{NCH}_{2}\right), 45.8(\mathrm{CH}), 55.17$ $\left(\mathrm{OCH}_{3}\right), 68.8(\mathrm{C}), 110.2(\mathrm{CH}, \mathrm{Ar}), 114.0(2 \times \mathrm{CH}, \mathrm{Ar}), 123.4(\mathrm{CH}, \mathrm{Ar}), 123.49(\mathrm{CH}, \mathrm{Ar}), 126.53$ $(\mathrm{C}, \mathrm{Ar}), 127.30(\mathrm{C}, \mathrm{Ar}), 127.36(\mathrm{CH}, \mathrm{Ar}), 127.46(2 \times \mathrm{CH}, \mathrm{Ar}), 127.70(2 \times \mathrm{CH}, \mathrm{Ar}), 128.11$ $(2 \times \mathrm{CH}, \mathrm{Ar}), 127.50(2 \times \mathrm{CH}, \mathrm{Ar}), 128.22(\mathrm{CH}, \mathrm{Ar}), 128.61(2 \times \mathrm{CH}, \mathrm{Ar}), 129.21(2 \times \mathrm{CH}, \mathrm{Ar})$, $130.3(\mathrm{CH}, \mathrm{Ar}), 135.3$ (C, Ar), 138.0 (C, Ar), 142.2 (C, Ar), 158.9 (C, Ar), 166.3 (C=O), 173.3 $(\mathrm{C}=\mathrm{O}), 174.4(\mathrm{C}=\mathrm{O})$.

IR (film) 3356, 1715, 1700, 1696, 1675, 1612, 1510, 1360, $1247 \mathrm{~cm}^{-1}$. HRMS (ESI-TOF) $m / z:[\mathrm{M}+\mathrm{H}]^{+}$calcd for $\mathrm{C}_{33} \mathrm{H}_{30} \mathrm{~N}_{3} \mathrm{O}_{4}{ }^{+}$532.2231, found 532.2247. 


\section{$N$-Benzyl-1-(4-methoxybenzyl)-1'-(4-methoxyphenyl)-2,5'-dioxo-1,2-dihydrospiro[indole-}

\section{3,2'-pyrrolidine]-4' -carboxamide (11b)}

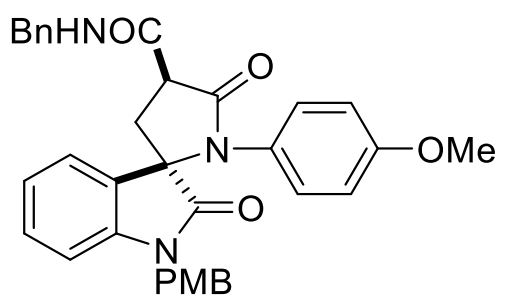

11b was obtained from 10a (175 $\mathrm{mg}, 0.37 \mathrm{mmol})$, 4methoxyaniline (92 $\mathrm{mg}, 0.74 \mathrm{mmol})$, and $\mathrm{Ni}\left(\mathrm{ClO}_{4}\right)_{2} \cdot 6 \mathrm{H}_{2} \mathrm{O}(14$ $\mathrm{mg}, 0.04 \mathrm{mmol})$ in $o$-xylene $(185 \mu \mathrm{L})$ at $140{ }^{\circ} \mathrm{C}$; reaction time 2 h. Yield $102 \mathrm{mg}$ (49\%); brown oil, dr $\mathbf{A}: \mathbf{B}=77: 23 . R_{f}=0.19$ (ethyl acetate/petroleum ether; 1:1).

A: ${ }^{1} \mathrm{H}$ NMR $\left(\mathrm{CDCl}_{3}, 600 \mathrm{MHz}\right): \delta=2.79\left(\mathrm{dd},{ }^{2} J=13.8,{ }^{3} J=9.7 \mathrm{~Hz}, 1 \mathrm{H}, \mathrm{CH}_{2}\right), 2.98\left(\mathrm{dd},{ }^{2} J=\right.$ $\left.13.8,{ }^{3} J=9.3 \mathrm{~Hz}, 1 \mathrm{H}, \mathrm{CH}_{2}\right), 3.71\left(\mathrm{~s}, 3 \mathrm{H}, \mathrm{OCH}_{3}\right), 3.75\left(\mathrm{~s}, 3 \mathrm{H}, \mathrm{OCH}_{3}\right), 4.11\left(\mathrm{dd},{ }^{3} J=9.7,{ }^{3} J=9.3\right.$ $\mathrm{Hz}, 1 \mathrm{H}, \mathrm{CH}), 4.47-4.54\left(\mathrm{~m}, 2 \mathrm{H}, \mathrm{NCH}_{2}\right), 4.60\left(\mathrm{dd},{ }^{2} J=14.9,{ }^{3} \mathrm{~J}=5.9 \mathrm{~Hz}, 1 \mathrm{H}, \mathrm{NCH}_{2} \mathrm{Ph}\right), 5.00(\mathrm{~d}$, $\left.{ }^{2} J=15.7 \mathrm{~Hz}, 1 \mathrm{H}, \mathrm{NCH}_{2} \mathrm{PMP}\right), 6.59\left(\mathrm{~d},{ }^{3} J=7.8 \mathrm{~Hz}, 1 \mathrm{H}, \mathrm{Ar}\right), 6.68-6.72(\mathrm{~m}, 4 \mathrm{H}, \mathrm{Ar}), 6.75\left(\mathrm{~d},{ }^{3} J=\right.$ $8.8 \mathrm{~Hz}, 2 \mathrm{H}, \mathrm{Ar}), 6.86-6.89(\mathrm{~m}, 2 \mathrm{H}, \mathrm{Ar}), 7.07-7.12(\mathrm{~m}, 1 \mathrm{H}, \mathrm{Ar}), 7.18\left(\mathrm{ddd},{ }^{3} J=7.8,{ }^{3} J=7.7,{ }^{4} \mathrm{~J}=\right.$ $1.2 \mathrm{~Hz}, 1 \mathrm{H}, \mathrm{Ar}), 7.25-7.29(\mathrm{~m}, 1 \mathrm{H}, \mathrm{Ar}), 7.31-7.39(\mathrm{~m}, 4 \mathrm{H}, \mathrm{Ar}), 7.45\left(\mathrm{ddd},{ }^{3} J=7.5,{ }^{4} J=1.1,{ }^{5} J=\right.$ $0.4 \mathrm{~Hz}, 1 \mathrm{H}, \mathrm{Ar}), 7.89\left(\mathrm{dd},{ }^{3} J=5.9,{ }^{3} J=5.5 \mathrm{~Hz}, 1 \mathrm{H}, \mathrm{NH}\right)$.

${ }^{13} \mathrm{C} \mathrm{NMR}\left(\mathrm{CDCl}_{3}, 150 \mathrm{MHz}\right): \delta=31.9\left(\mathrm{CH}_{2}\right), 43.0\left(\mathrm{NCH}_{2}\right), 43.7\left(\mathrm{NCH}_{2}\right), 46.0(\mathrm{CH}), 55.02$ $\left(\mathrm{OCH}_{3}\right), 55.14\left(\mathrm{OCH}_{3}\right), 68.8(\mathrm{C}), 109.8(\mathrm{CH}, \mathrm{Ar}), 113.9(2 \times \mathrm{CH}, \mathrm{Ar}), 114.30(2 \times \mathrm{CH}, \mathrm{Ar}), 123.4$ $(\mathrm{CH}, \mathrm{Ar}), 124.8(\mathrm{CH}, \mathrm{Ar}), 126.5(\mathrm{C}, \mathrm{Ar}), 127.3(\mathrm{CH}, \mathrm{Ar}), 127.5(\mathrm{C}, \mathrm{Ar}), 127.55(2 \times \mathrm{CH}, \mathrm{Ar})$, $127.9(\mathrm{C}, \mathrm{Ar}), 128.01(2 \times \mathrm{CH}, \mathrm{Ar}), 128.54(2 \times \mathrm{CH}, \mathrm{Ar}), 129.05(2 \times \mathrm{CH}, \mathrm{Ar}), 130.1(\mathrm{CH}, \mathrm{Ar})$, $137.9(\mathrm{C}, \mathrm{Ar}), 142.5(\mathrm{C}, \mathrm{Ar}), 158.9(\mathrm{C}, \mathrm{Ar}), 159.1(\mathrm{C}, \mathrm{Ar}), 167.1(\mathrm{C}=\mathrm{O}), 173.6(\mathrm{C}=\mathrm{O}), 175.7$ $(\mathrm{C}=\mathrm{O})$.

B: ${ }^{1} \mathrm{H}$ NMR $\left(\mathrm{CDCl}_{3}, 600 \mathrm{MHz}\right): \delta=2.66\left(\mathrm{dd},{ }^{2} J=13.6,{ }^{3} J=10.1 \mathrm{~Hz}, 1 \mathrm{H}, \mathrm{CH}_{2}\right), 3.23\left(\mathrm{dd},{ }^{2} J=\right.$ $\left.13.6,{ }^{3} J=8.5 \mathrm{~Hz}, 1 \mathrm{H}, \mathrm{CH}_{2}\right), 3.73\left(\mathrm{~s}, 3 \mathrm{H}, \mathrm{OCH}_{3}\right), 3.74\left(\mathrm{~s}, 3 \mathrm{H}, \mathrm{OCH}_{3}\right), 3.92\left(\mathrm{dd},{ }^{3} J=10.1,{ }^{3} J=\right.$ $8.5 \mathrm{~Hz}, 1 \mathrm{H}, \mathrm{CH}), 4.42\left(\mathrm{~d},{ }^{2} J=15.6 \mathrm{~Hz}, 1 \mathrm{H}, \mathrm{NCH}_{2} \mathrm{PMP}\right), 4.47-4.54\left(\mathrm{~m}, 1 \mathrm{H}, \mathrm{NCH}_{2} \mathrm{Ph}\right), 4.65(\mathrm{dd}$, $\left.{ }^{2} J=14.9,{ }^{3} J=6.0 \mathrm{~Hz}, 1 \mathrm{H}, \mathrm{NCH}_{2} \mathrm{Ph}\right), 5.05\left(\mathrm{~d},{ }^{2} J=15.6 \mathrm{~Hz}, 1 \mathrm{H}, \mathrm{NCH}_{2} \mathrm{PMP}\right), 6.61\left(\mathrm{~d},{ }^{3} J=7.7\right.$ $\mathrm{Hz}, 1 \mathrm{H}, \mathrm{Ar}), 6.65-6.67(\mathrm{~m}, 4 \mathrm{H}, \mathrm{Ar}), 6.68-6.72$ (m, 2H, Ar), 6.86-6.89 (m, 2H, Ar), 7.07-7.12 (m, 1H, Ar), 7.21 (ddd, ${ }^{3} J=7.8,{ }^{3} J=7.7,{ }^{4} J=1.2 \mathrm{~Hz}, 1 \mathrm{H}, \mathrm{Ar}$ ), 7.25-7.29 (m, 1H, Ar), 7.31-7.39 
(m, 4H, Ar), $7.42\left(\mathrm{ddd},{ }^{3} J=7.5,{ }^{4} J=1.1,{ }^{5} J=0.4 \mathrm{~Hz}, 1 \mathrm{H}, \mathrm{Ar}\right), 8.14\left(\mathrm{dd},{ }^{3} J=6.0,{ }^{3} J=5.3 \mathrm{~Hz}\right.$, 1H, NH).

${ }^{13} \mathrm{C} \mathrm{NMR}\left(\mathrm{CDCl}_{3}, 150 \mathrm{MHz}\right): \delta=32.5\left(\mathrm{CH}_{2}\right), 43.1\left(\mathrm{NCH}_{2}\right), 43.6\left(\mathrm{NCH}_{2}\right), 45.7(\mathrm{CH}), 54.99$ $\left(\mathrm{OCH}_{3}\right), 55.14\left(\mathrm{OCH}_{3}\right), 68.9(\mathrm{C}), 110.1(\mathrm{CH}, \mathrm{Ar}), 113.8(2 \times \mathrm{CH}, \mathrm{Ar}), 114.34(2 \times \mathrm{CH}, \mathrm{Ar}), 123.3$ $(\mathrm{CH}, \mathrm{Ar}), 123.5(\mathrm{CH}, \mathrm{Ar}), 126.4(\mathrm{C}, \mathrm{Ar}), 127.2(\mathrm{CH}, \mathrm{Ar}), 127.4(\mathrm{C}, \mathrm{Ar}), 127.60(2 \times \mathrm{CH}, \mathrm{Ar})$, 127.9 (C, Ar), 127.97 (2×CH, C, Ar), 128.52 (2×CH, C, Ar), $129.10(2 \times \mathrm{CH}, \mathrm{C}, \mathrm{Ar}), 130.2(\mathrm{CH}$, Ar), 138.0 (C, Ar), 142.3 (C, Ar), 158.8 (C, Ar), 159.3 (C, Ar), $166.5(\mathrm{C}=\mathrm{O}), 173.4(\mathrm{C}=\mathrm{O}), 174.4$ $(\mathrm{C}=\mathrm{O})$.

HRMS (ESI-TOF) $m / z:[\mathrm{M}+\mathrm{H}]^{+}$calcd for $\mathrm{C}_{34} \mathrm{H}_{32} \mathrm{~N}_{3} \mathrm{O}_{5}{ }^{+}$562.2336, found 562.2355.

\section{$N$-Butyl-1-(4-methoxybenzyl)-1'-(4-methoxyphenyl)-2,5'-dioxo-1,2-dihydrospiro[indole-}

\section{3,2'-pyrrolidine]-4'-carboxamide (11c)}

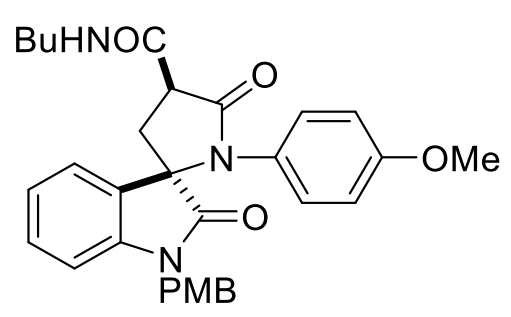

11c was obtained from $\mathbf{1 0 b}(73 \mathrm{mg}, 0.17 \mathrm{mmol})$, 4methoxyaniline $(41 \mathrm{mg}, 0.33 \mathrm{mmol})$, and $\mathrm{Ni}\left(\mathrm{ClO}_{4}\right)_{2} \cdot 6 \mathrm{H}_{2} \mathrm{O}(6 \mathrm{mg}$, $0.02 \mathrm{mmol})$ in $o$-xylene $(85 \mu \mathrm{L})$ at $140{ }^{\circ} \mathrm{C}$; reaction time $2 \mathrm{~h} /$ Yield $31 \mathrm{mg}$ (35\%); brown oil, dr $\mathbf{A}: \mathbf{B}=73: 27 . R_{f}=0.21$ (ethyl acetate/petroleum ether; $1: 1)$.

A: ${ }^{1} \mathrm{H}$ NMR $\left(\mathrm{CDCl}_{3}, 600 \mathrm{MHz}\right): \delta=0.92-0.96\left(\mathrm{~m}, 3 \mathrm{H}, \mathrm{CH}_{3}\right), 1.37-1.43\left(\mathrm{~m}, 2 \mathrm{H}, \mathrm{CH}_{2}\right), 1.54-$ $1.59\left(\mathrm{~m}, 2 \mathrm{H}, \mathrm{CH}_{2}\right), 2.77\left(\mathrm{dd},{ }^{2} J=13.7,{ }^{3} \mathrm{~J}=9.8 \mathrm{~Hz}, 1 \mathrm{H}, \mathrm{CH}_{2}\right), 2.93\left(\mathrm{dd},{ }^{2} J=13.7,{ }^{3} J=9.3 \mathrm{~Hz}\right.$, $\left.1 \mathrm{H}, \mathrm{CH}_{2}\right), 3.30-3.42\left(\mathrm{~m}, 2 \mathrm{H}, \mathrm{NCH}_{2} \mathrm{Pr}\right), 3.71\left(\mathrm{~s}, 3 \mathrm{H}, \mathrm{OCH}_{3}\right), 3.74\left(\mathrm{~s}, 3 \mathrm{H}, \mathrm{OCH}_{3}\right), 4.02\left(\mathrm{dd},{ }^{3} J=\right.$ $\left.9.8,{ }^{3} J=9.3 \mathrm{~Hz}, 1 \mathrm{H}, \mathrm{CH}\right), 4.48\left(\mathrm{~d},{ }^{2} J=15.6 \mathrm{~Hz}, 1 \mathrm{H}, \mathrm{NCH}_{2}\right), 4.98\left(\mathrm{~d},{ }^{2} J=15.6 \mathrm{~Hz}, 1 \mathrm{H}, \mathrm{NCH}_{2}\right)$, $6.57\left(\mathrm{~d},{ }^{3} J=7.8 \mathrm{~Hz}, 1 \mathrm{H}, \mathrm{Ar}\right), 6.67-6.69(\mathrm{~m}, 4 \mathrm{H}, \mathrm{Ar}), 6.74\left(\mathrm{~d},{ }^{3} J=8.7 \mathrm{~Hz}, 2 \mathrm{H}, \mathrm{Ar}\right), 6.84-6.88$ (m, 2H, Ar), 7.06-7.10 (m, 1H, Ar), 7.15-7.21 (m, 1H, Ar), $7.45\left(\mathrm{~d},{ }^{3} J=7.5 \mathrm{~Hz}, 1 \mathrm{H}, \mathrm{Ar}\right), 7.49$ (t, $\left.{ }^{3} J=5.3 \mathrm{~Hz}, 1 \mathrm{H}, \mathrm{NH}\right)$.

${ }^{13} \mathrm{C}$ NMR $\left(\mathrm{CDCl}_{3}, 150 \mathrm{MHz}\right): \delta=13.64\left(\mathrm{CH}_{3}\right), 19.99\left(\mathrm{CH}_{2}\right), 31.35\left(\mathrm{CH}_{2}\right), 32.0\left(\mathrm{CH}_{2}\right), 39.4$ $\left(\mathrm{NCH}_{2}\right), 43.1\left(\mathrm{NCH}_{2}\right), 45.8(\mathrm{CH}), 55.04\left(\mathrm{OCH}_{3}\right), 55.16\left(\mathrm{OCH}_{3}\right), 68.8(\mathrm{C}), 109.8(\mathrm{CH}, \mathrm{Ar})$, 
$113.94(2 \times \mathrm{CH}, \mathrm{Ar}), 114.3(2 \times \mathrm{CH}, \mathrm{Ar}), 123.4(\mathrm{CH}, \mathrm{Ar}), 124.8(\mathrm{CH}, \mathrm{Ar}), 126.53(\mathrm{C}, \mathrm{Ar}), 127.6$ (C, Ar), $128.04(2 \times \mathrm{CH}, \mathrm{C}, \mathrm{Ar}), 129.06(2 \times \mathrm{CH}, \mathrm{Ar}), 130.1(\mathrm{CH}, \mathrm{Ar}), 142.6(\mathrm{C}, \mathrm{Ar}), 158.92(\mathrm{C}$, Ar), $159.2(\mathrm{C}, \mathrm{Ar}), 167.0(\mathrm{C}=\mathrm{O}), 173.9(\mathrm{C}=\mathrm{O}), 175.8(\mathrm{C}=\mathrm{O})$.

B: ${ }^{1} \mathrm{H}$ NMR $\left(\mathrm{CDCl}_{3}, 600 \mathrm{MHz}\right): \delta=0.92-0.96\left(\mathrm{~m}, 3 \mathrm{H}, \mathrm{CH}_{3}\right), 1.37-1.43\left(\mathrm{~m}, 2 \mathrm{H}, \mathrm{CH}_{2}\right), 1.54-$ $1.59\left(\mathrm{~m}, 2 \mathrm{H}, \mathrm{CH}_{2}\right), 2.63\left(\mathrm{dd},{ }^{2} J=13.6,{ }^{3} J=10.1 \mathrm{~Hz}, 1 \mathrm{H}, \mathrm{CH}_{2}\right), 3.16\left(\mathrm{dd},{ }^{2} J=13.6,{ }^{3} J=8.6 \mathrm{~Hz}\right.$, $\left.1 \mathrm{H}, \mathrm{CH}_{2}\right), 3.30-3.42\left(\mathrm{~m}, 2 \mathrm{H}, \mathrm{NCH}_{2} \mathrm{Pr}\right), 3.73\left(\mathrm{~s}, 6 \mathrm{H}, \mathrm{OCH}_{3}\right), 3.85\left(\mathrm{dd},{ }^{3} J=10.1,{ }^{3} J=8.6 \mathrm{~Hz}, 1 \mathrm{H}\right.$, $\mathrm{CH}), 4.41\left(\mathrm{~d},{ }^{2} J=15.6 \mathrm{~Hz}, 1 \mathrm{H}, \mathrm{NCH}_{2}\right), 5.02\left(\mathrm{~d},{ }^{2} J=15.6 \mathrm{~Hz}, 1 \mathrm{H}, \mathrm{NCH}_{2}\right), 6.60\left(\mathrm{~d},{ }^{3} J=7.8 \mathrm{~Hz}\right.$, 1H, Ar), 6.63-6,65 (m, 4H, Ar), 6.67-6.69 (m, 2H, Ar), 6.84-6.88 (m, 2H, Ar), 7.06-7.10 (m, $1 \mathrm{H}, \mathrm{Ar}), 7.15-7.21(\mathrm{~m}, 1 \mathrm{H}, \mathrm{Ar}), 7.40\left(\mathrm{dd},{ }^{3} \mathrm{~J}=7.5,{ }^{4} \mathrm{~J}=0.9 \mathrm{~Hz}, 1 \mathrm{H}, \mathrm{Ar}\right), 7.77\left(\mathrm{t},{ }^{3} \mathrm{~J}=5.3 \mathrm{~Hz}, 1 \mathrm{H}\right.$, $\mathrm{NH})$.

${ }^{13} \mathrm{C}$ NMR $\left(\mathrm{CDCl}_{3}, 150 \mathrm{MHz}\right): \delta=13.64\left(\mathrm{CH}_{3}\right), 20.02\left(\mathrm{CH}_{2}\right), 31.35\left(\mathrm{CH}_{2}\right), 32.7\left(\mathrm{CH}_{2}\right), 39.3$ $\left(\mathrm{NCH}_{2}\right), 43.2\left(\mathrm{NCH}_{2}\right), 45.6(\mathrm{CH}), 55.02\left(\mathrm{OCH}_{3}\right), 55.18\left(\mathrm{OCH}_{3}\right), 68.9(\mathrm{C}), 110.1(\mathrm{CH}, \mathrm{Ar})$, $113.88(2 \times \mathrm{CH}, \mathrm{Ar}), 114.4(2 \times \mathrm{CH}, \mathrm{Ar}), 123.3(\mathrm{CH}, \mathrm{Ar}), 123.5(\mathrm{CH}, \mathrm{Ar}), 126.49(\mathrm{C}, \mathrm{Ar}), 127.7$ (C, Ar), 127.99 (2×CH, Ar), 128.21 (C, Ar), $129.11(2 \times \mathrm{CH}, \mathrm{Ar}), 130.2(\mathrm{CH}, \mathrm{Ar}), 142.3(\mathrm{C}, \mathrm{Ar})$, $158.86(\mathrm{C}, \mathrm{Ar}), 159.3(\mathrm{C}, \mathrm{Ar}), 166.4(\mathrm{C}=\mathrm{O}), 173.7(\mathrm{C}=\mathrm{O}), 174.5(\mathrm{C}=\mathrm{O})$.

HRMS (ESI-TOF) $m / z:[\mathrm{M}+\mathrm{H}]^{+}$calcd for $\mathrm{C}_{31} \mathrm{H}_{34} \mathrm{~N}_{3} \mathrm{O}_{5}{ }^{+}$528.2493, found 528.2508. 


\section{References}

1. V. M. Muzalevskiy, E. S. Balenkova, A. V. Shastin, A. M. Magerramov, N. G. Shikhaliev and V. G. Nenajdenko, Russ. Chem. Bull., Int. Ed., 2011, 60, 2343-2346.

2. S. V. Zaytsev, K. L. Ivanov, D. A. Skvortsov, S. I. Bezzubov, M. Ya. Melnikov and E. M. Budynina, J. Org. Chem., 2018, 83, 8695-8709.

3. G. Karthik, T. Rajasekaran, B. Sridhar and B. V. Subba Reddy, Tetrahedron Lett., 2014, 55, 7064-7067.

4. F. De Nanteuil and J. Waser, Angew. Chem. Int. Ed., 2013, 52, 9009-9013.

5. B. M. Trost, J. Xie and J. D. Sieber, J. Am. Chem. Soc., 2011, 133, 20611-20622.

6. S. M. Nicolle and C. J. Moody, Chem. Eur. J., 2014, 20, 4420-4425.

7. E. E. Wilson, K. X. Rodrigues and B. L. Ashfeld, Tetrahedron, 2015, 71, 5765-5775.

8. F. Neese, WIREs Comput. Mol. Sci., 2012, 2, 73-78.

9. A. D. Becke, J. Chem. Phys., 1993, 98, 5648-5652.

10. P. J. Stephens, F. J. Devlin, C. F. Chabalowski and M. J. Frisch, J. Phys. Chem., 1994, 98, $11623-11627$.

11. A. Schäfer, H. Horn and R. Ahlrichs, J. Chem. Phys., 1992, 97, 2571-2577.

12. S. Sinnecker, A. Rajendran, A. Klamt, M. Diedenhofen and F. Neese, J. Phys. Chem. A, 2006, 110, 2235-2245.

13. C. Y. Legault, Université de Sherbrooke, 2009, http://www.cylview.org.

14. A. A. Akaev, E. V. Villemson, N. S. Vorobyeva, A. G. Majouga, E. M. Budynina and M. Ya. Melnikov, J. Org. Chem., 2017, 82, 5689-5701.

15. G. Li, G. Zhou, D. Zhang-Negrerie, Y. Du, J. Huang and K. Zhao, Adv. Synth. Catal., 2016, 358, 3534-3540. 


\section{Cartesian coordinates and energies of stationary points}

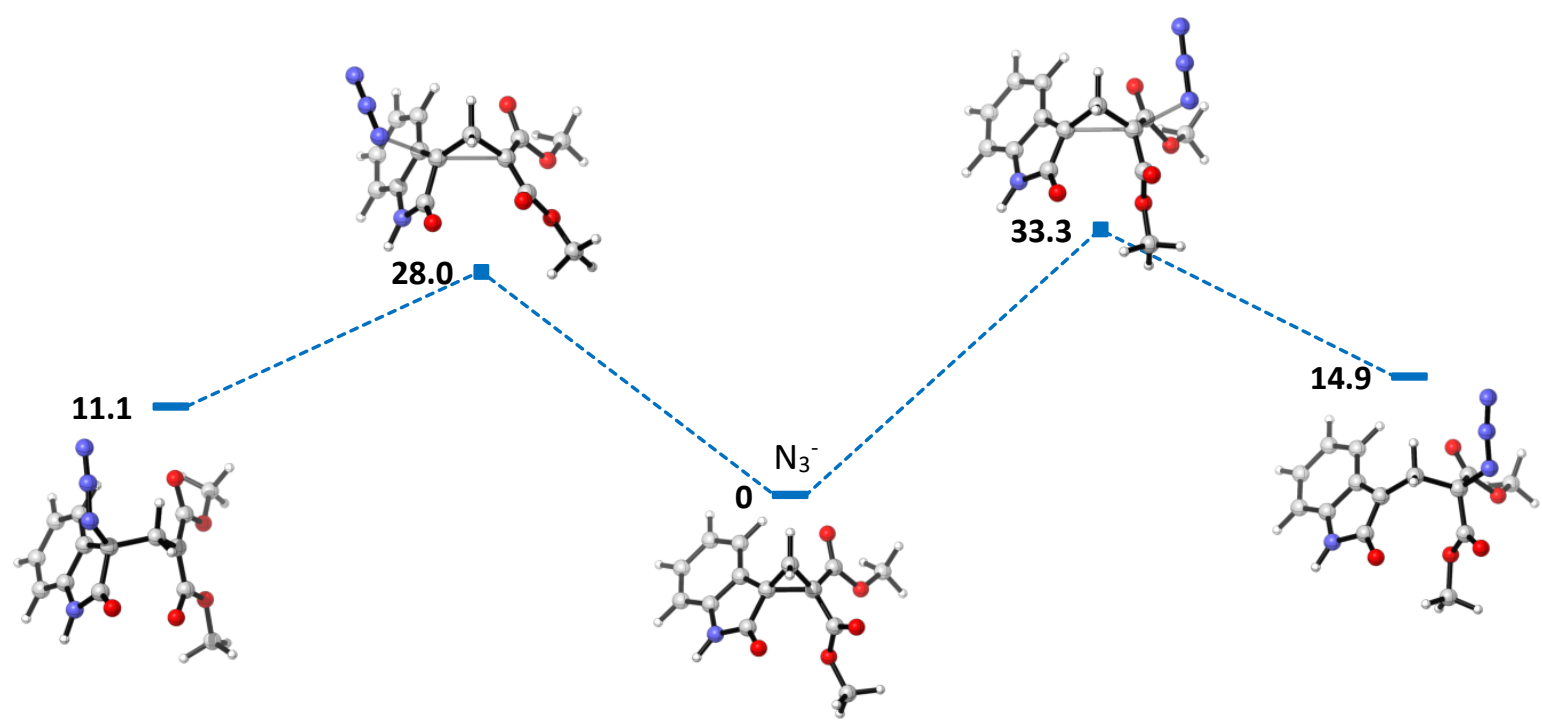

Figure S3. Energy diagram for $\mathbf{2} \mathbf{a}^{\prime}$ ring openings along $\mathrm{C} 1-\mathrm{C} 2$ bond with the azide ion $\left(\Delta \mathrm{G}_{298}\right.$, $\mathrm{kcal} / \mathrm{mol})$. 
$\mathbf{N}^{-}$

$\mathrm{SPE}=-164.1132 ; \mathrm{H}_{298}=-164.1000 ; \mathrm{G}_{298}=-164.1240$

$1 b^{\prime}$

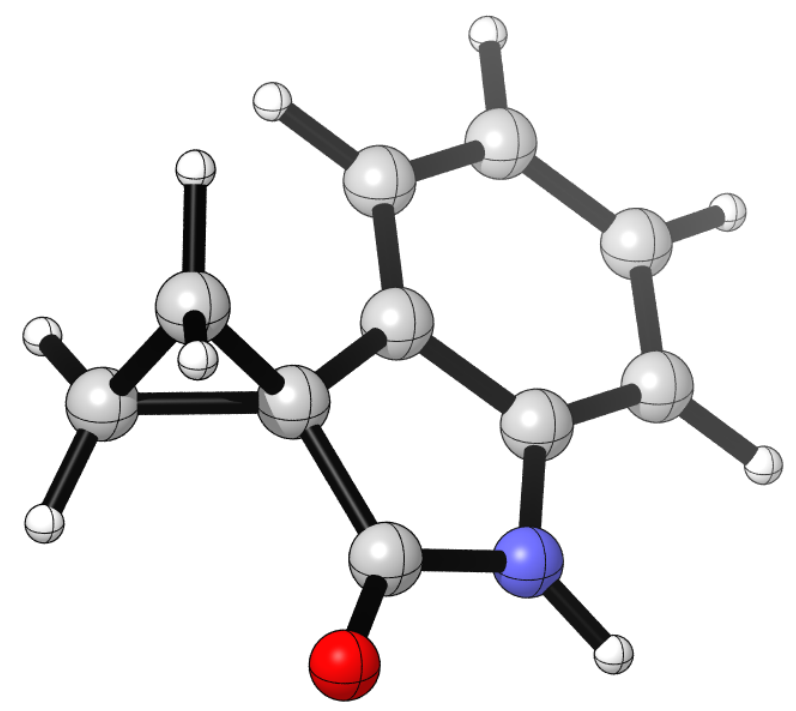

$\mathrm{SPE}=-515.7915 ; \mathrm{H}_{298}=-515.6138 ; \mathrm{G}_{298}=-515.6573$

$\begin{array}{llll}6 & -2.578969000 & 5.904333000 & 2.509787000 \\ 1 & -3.130677000 & 6.562432000 & 3.187903000 \\ 6 & -1.117189000 & 5.648689000 & 2.862219000 \\ 6 & -0.425165000 & 6.160369000 & 4.074473000 \\ 6 & -0.555805000 & 4.266114000 & 2.632859000 \\ 8 & -0.843530000 & 3.481357000 & 1.740963000 \\ 6 & -1.511882000 & 6.484407000 & 1.651073000 \\ 1 & -1.303075000 & 7.556258000 & 1.716444000 \\ 1 & -1.325181000 & 6.024356000 & 0.675982000 \\ 6 & 0.459212000 & 5.146058000 & 4.504964000\end{array}$




$\begin{array}{llll}6 & 1.265439000 & 5.302102000 & 5.630732000 \\ 1 & 1.943986000 & 4.508922000 & 5.953988000 \\ 6 & 1.174050000 & 6.513275000 & 6.336578000 \\ 6 & -0.503056000 & 7.356761000 & 4.782214000 \\ 1 & -1.180143000 & 8.154036000 & 4.462418000 \\ 6 & 0.304070000 & 7.529313000 & 5.920665000 \\ 1 & 1.793215000 & 6.659909000 & 7.225696000 \\ 1 & 0.250311000 & 8.463730000 & 6.485231000 \\ 7 & 0.358714000 & 4.051365000 & 3.638053000 \\ 1 & 0.886824000 & 3.188460000 & 3.726747000 \\ 1 & -3.134209000 & 5.039889000 & 2.133103000\end{array}$


TS_1 $b^{\prime}$

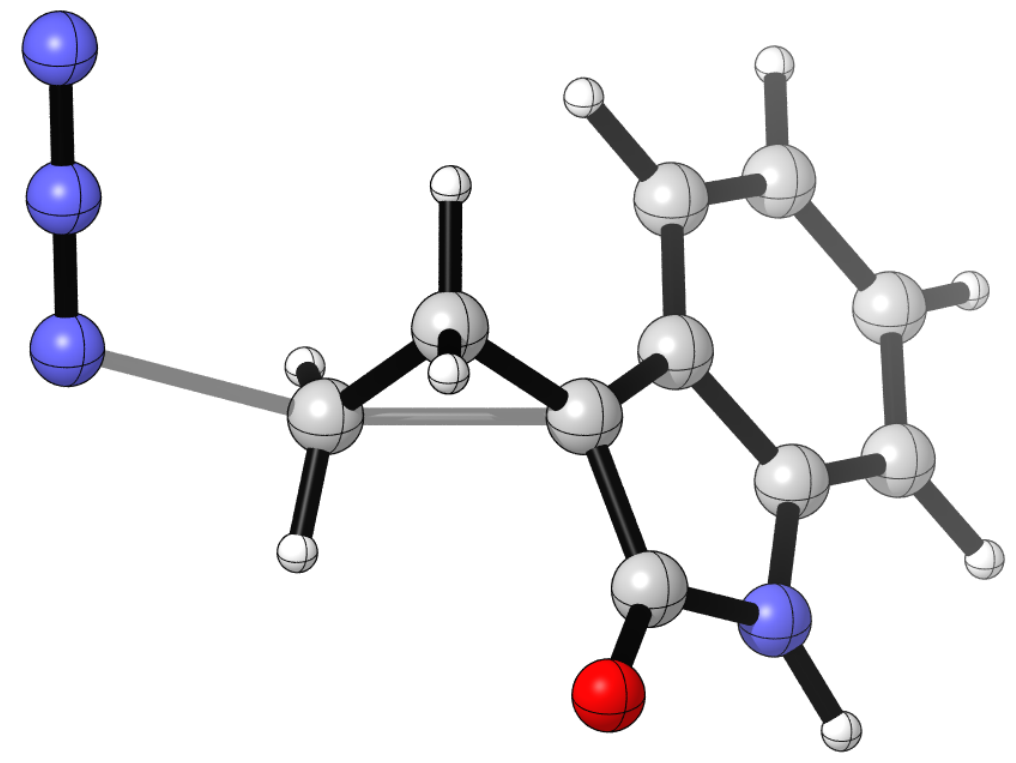

$\mathrm{SPE}=-679.8710 ; \mathrm{H}_{298}=-679.6799 ; \mathrm{G}_{298}=-679.7317$
$6 \quad-2.492140000$
$6.116540000 \quad 2.458900000$
$1 \quad-3.054743000$
$6.841036000 \quad 3.062731000$
$6 \quad-1.279819000$
5.548685000
3.131882000
$6 \quad-0.522250000$
6.069493000
4.248187000
$6 \quad-0.704095000$
4.265952000
2.763653000
$-1.032113000$
3.469251000
1.871038000
$6 \quad-1.539714000$
6.692440000
1.503384000
$1-0.958444000$
7.566963000
1.792264000
$1-1.135641000$
6.048171000
0.724515000
6
0.497045000
5.109268000
4.540938000
6
1.409555000
5.295379000
5.577707000
$1 \quad 2.180492000$
4.548095000
5.786896000
$6 \quad 1.310920000$
6.468275000
6.348361000 


$\begin{array}{llll}6 & -0.602394000 & 7.229852000 & 5.033582000 \\ 1 & -1.370993000 & 7.983228000 & 4.835244000 \\ 6 & 0.316925000 & 7.421745000 & 6.077853000 \\ 1 & 2.016811000 & 6.633575000 & 7.167458000 \\ 1 & 0.256321000 & 8.327670000 & 6.688340000 \\ 7 & 0.364299000 & 4.057917000 & 3.645917000 \\ 1 & 0.949711000 & 3.230320000 & 3.616237000 \\ 7 & -4.296847000 & 9.038459000 & 1.117944000 \\ 7 & -3.444815000 & 8.443050000 & 0.584428000 \\ 7 & -2.576743000 & 7.834357000 & 0.037872000 \\ 1 & -3.153256000 & 5.348264000 & 2.035225000\end{array}$




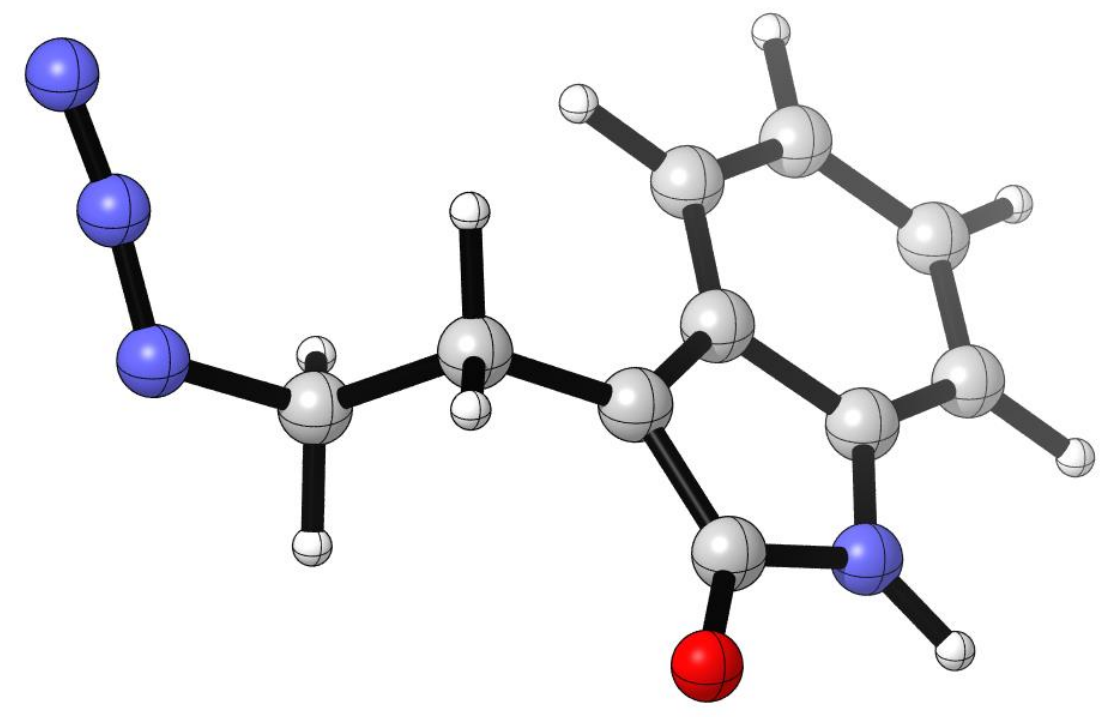

$\mathrm{SPE}=-679.9090 ; \mathrm{H}_{298}=-679.7164 ; \mathrm{G}_{298}=-679.7659$
$\begin{array}{llll}6 & -2.278751000 & 6.083005000 & 2.290536000\end{array}$
$\begin{array}{llll}1 & -2.982619000 & 6.647096000 & 2.933792000\end{array}$
$\begin{array}{llll}6 & -1.187073000 & 5.457547000 & 3.101473000\end{array}$
$\begin{array}{llll}6 & -0.465634000 & 6.012874000 & 4.203052000\end{array}$
$\begin{array}{llll}6 & -0.666230000 & 4.153567000 & 2.839867000\end{array}$
$\begin{array}{llll}8 & -0.976893000 & 3.288204000 & 1.985617000\end{array}$
$\begin{array}{llll}6 & -1.754410000 & 7.036441000 & 1.200091000\end{array}$
$\begin{array}{llll}1 & -1.148241000 & 7.844014000 & 1.648184000\end{array}$
$\begin{array}{llll}1 & -1.111883000 & 6.485173000 & 0.500166000\end{array}$
$\begin{array}{llll}6 & 0.498076000 & 5.027003000 & 4.630800000\end{array}$
$\begin{array}{llll}6 & 1.359915000 & 5.246133000 & 5.702845000\end{array}$
$\begin{array}{llll}1 & 2.079841000 & 4.478416000 & 6.004830000\end{array}$
$\begin{array}{llll}6 & 1.283432000 & 6.474416000 & 6.391014000\end{array}$ 


$\begin{array}{llll}6 & -0.513804000 & 7.234165000 & 4.911539000 \\ 1 & -1.225265000 & 8.013679000 & 4.619186000 \\ 6 & 0.354741000 & 7.451527000 & 5.992927000 \\ 1 & 1.950288000 & 6.662482000 & 7.237790000 \\ 1 & 0.307985000 & 8.401738000 & 6.535438000 \\ 7 & 0.355293000 & 3.936250000 & 3.798408000 \\ 1 & 0.884281000 & 3.073450000 & 3.852362000 \\ 7 & -4.342668000 & 9.221149000 & 1.229137000 \\ 7 & -3.583376000 & 8.445659000 & 0.872920000 \\ 7 & -2.828156000 & 7.631307000 & 0.365762000 \\ 1 & -2.863060000 & 5.287866000 & 1.795311000\end{array}$




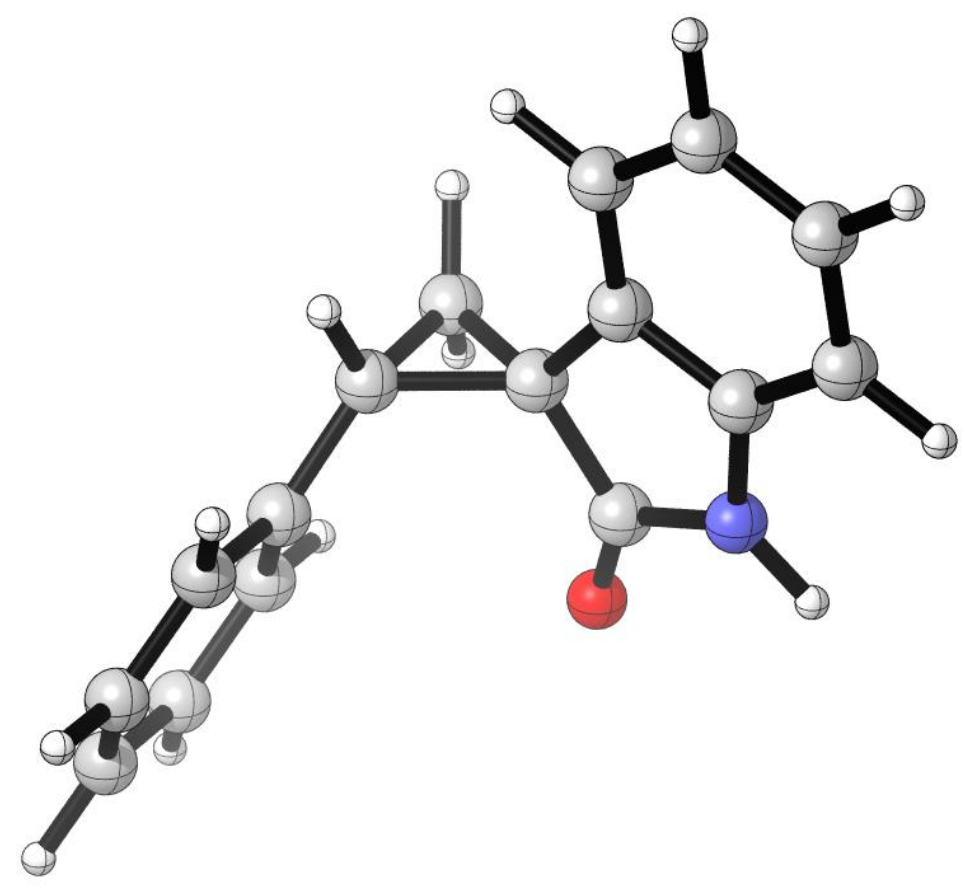

$$
\begin{array}{lrrr}
\multicolumn{3}{c}{\mathrm{SPE}=-746.5334 ; \mathrm{H}_{298}=-746.2711 ; \mathrm{G}_{298}=-746.3244} \\
6 & -2.587554000 & 6.029086000 & 2.815678000 \\
1 & -2.977929000 & 6.474202000 & 3.737075000 \\
6 & -3.518210000 & 5.016344000 & 2.227927000 \\
6 & -4.348019000 & 4.289051000 & 3.102473000 \\
1 & -4.284919000 & 4.473910000 & 4.179452000 \\
6 & -5.253043000 & 3.340876000 & 2.616967000 \\
1 & -5.888122000 & 2.788015000 & 3.314955000 \\
6 & -5.348949000 & 3.104707000 & 1.240355000 \\
1 & -6.057171000 & 2.365625000 & 0.854970000 \\
6 & -4.535149000 & 3.826684000 & 0.360262000 \\
1 & -4.606614000 & 3.653636000 & -0.717562000 \\
6 & -3.628331000 & 4.773115000 & 0.848262000
\end{array}
$$




\begin{tabular}{llll}
1 & -3.004308000 & 5.325120000 & 0.143737000 \\
6 & -1.048564000 & 5.818298000 & 2.939371000 \\
6 & -0.329465000 & 6.037182000 & 4.221844000 \\
6 & -0.377830000 & 4.621584000 & 2.305852000 \\
8 & -0.590076000 & 4.126980000 & 1.209581000 \\
6 & -1.645066000 & 6.894724000 & 2.055784000 \\
1 & -1.513287000 & 7.931059000 & 2.380158000 \\
1 & -1.531457000 & 6.725761000 & 0.980766000 \\
6 & 0.628949000 & 5.006890000 & 4.344587000 \\
6 & 1.467676000 & 4.910418000 & 5.453127000 \\
\hline 1 & 2.204333000 & 4.107411000 & 5.536300000 \\
6 & 1.334118000 & 5.881228000 & 6.459157000 \\
\hline 6 & -0.449824000 & 6.993374000 & 5.226789000 \\
\hline 1 & -1.184893000 & 7.800109000 & 5.149109000 \\
\hline 6 & 0.390721000 & 6.910860000 & 6.350687000 \\
\hline 1 & 1.979564000 & 5.828682000 & 7.340488000 \\
\hline 1 & 0.305982000 & 7.656665000 & 7.145729000 \\
\hline 6 & 0.563305000 & 4.186700000 & 3.214130000 \\
\hline 6 & 1.171799000 & 3.394056000 & 3.033351000
\end{tabular}




\section{1a'_TS1}

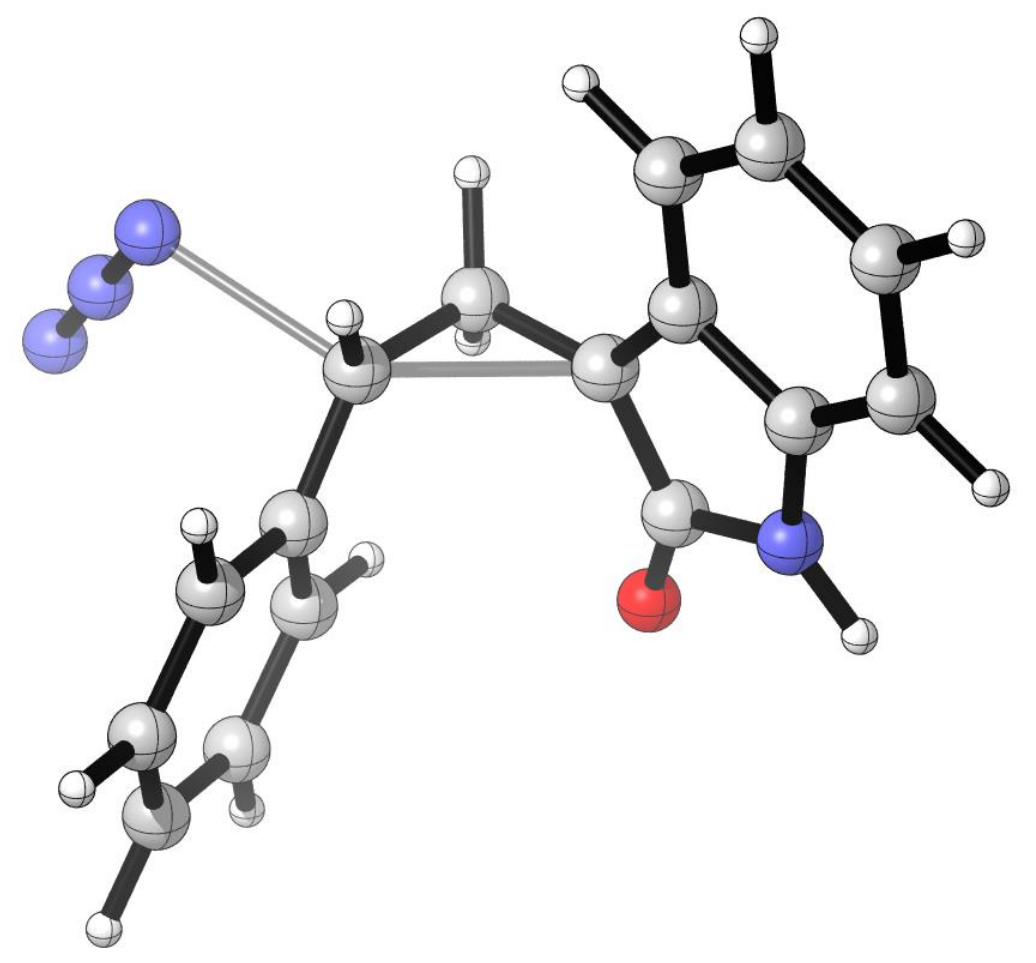

$\mathrm{SPE}=-910.6199 ; \mathrm{H}_{298}=-910.3442 ; \mathrm{G}_{298}=-910.4044$
$6 \quad-2.761892000$
6.354042000
2.756245000
$1 \quad-2.755592000$
6.488884000
3.838192000
$6 \quad-3.481660000$
5.185268000
2.274425000
$6 \quad-4.190804000$
4.392901000
3.210203000
$1 \quad-4.149620000$
4.660032000
4.270253000
$6 \quad-4.934397000$
3.290195000
2.800331000
$1 \quad-5.470747000$
2.688129000
3.538956000
$6 \quad-4.992092000$
2.950060000
1.438990000
$1 \quad-5.571601000$
2.081236000
1.114336000
$6 \quad-4.304524000$
3.726117000
0.501134000
$1 \quad-4.348084000$
3.465209000
$-0.560186000$
$6 \quad-3.556562000$
4.834802000
0.908137000
$1 \quad-3.012514000$
5.416164000
0.165901000 


\begin{tabular}{llll}
6 & -0.625151000 & 6.177446000 & 2.496893000 \\
6 & 0.138776000 & 6.235202000 & 3.716954000 \\
6 & -0.186377000 & 5.011137000 & 1.755176000 \\
8 & -0.540036000 & 4.584243000 & 0.644180000 \\
6 & -1.709466000 & 7.097934000 & 2.030390000 \\
1 & -1.602487000 & 8.130433000 & 2.391684000 \\
1 & -1.822455000 & 7.096896000 & 0.937362000 \\
6 & 1.022600000 & 5.107124000 & 3.713519000 \\
\hline 6 & 1.919642000 & 4.869978000 & 4.753193000 \\
\hline 1 & 2.585918000 & 4.002635000 & 4.731821000 \\
\hline 6 & 1.944957000 & 5.774080000 & 5.830662000 \\
\hline 6 & 0.187443000 & 7.125305000 & 4.804300000 \\
\hline 1 & -0.472780000 & 7.997833000 & 4.838498000 \\
\hline 6 & 1.089578000 & 6.887662000 & 5.852478000 \\
\hline 1 & 2.639680000 & 5.604297000 & 6.658569000 \\
\hline 1 & 1.125857000 & 7.579655000 & 6.699564000 \\
\hline 7 & 0.797932000 & 4.396749000 & 2.546864000 \\
\hline 7 & 1.294345000 & 3.561454000 & 2.257480000 \\
\hline 6 & -4.460679000 & 7.847493000 & 2.736843000 \\
\hline 6.544148000 & 7.634471000 & 0.651049000
\end{tabular}




\section{1a'_TS2}

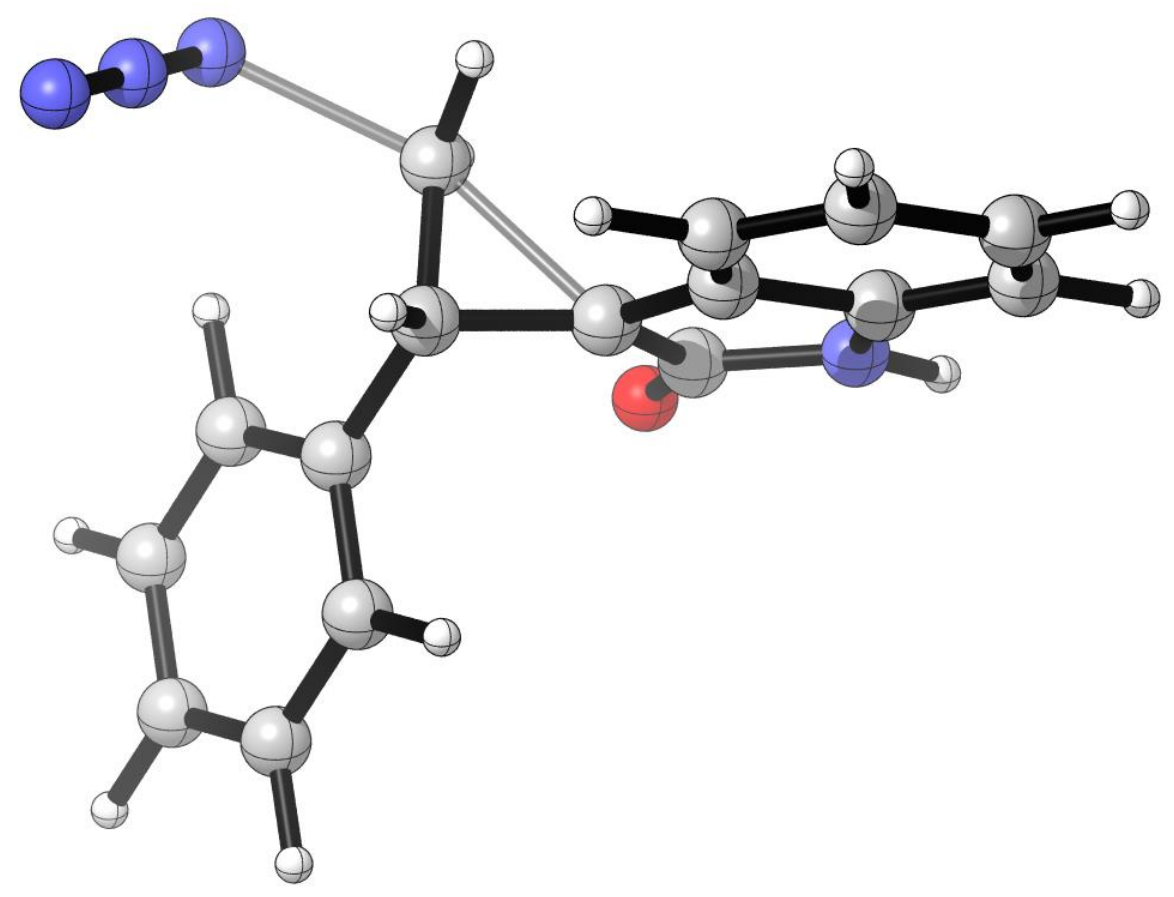

$\mathrm{SPE}=-910.6107 ; \mathrm{H}_{298}=-910.3341 ; \mathrm{G}_{298}=-910.3970$
$\begin{array}{llll}6 & -2.563525000 & 6.088561000 & 2.411480000\end{array}$
$1 \quad-3.007111000 \quad 6.810683000 \quad 3.115158000$
$\begin{array}{llll}6 & -3.625818000 & 5.110039000 & 1.950052000\end{array}$
$\begin{array}{llll}6 & -4.257796000 & 4.300849000 & 2.909754000\end{array}$
$\begin{array}{llll}1 & -3.949593000 & 4.372280000 & 3.957526000\end{array}$
$\begin{array}{llll}6 & -5.265538000 & 3.405101000 & 2.543473000\end{array}$
$\begin{array}{llll}1 & -5.747083000 & 2.786076000 & 3.306085000\end{array}$
$\begin{array}{llll}6 & -5.659576000 & 3.301204000 & 1.202537000\end{array}$
$\begin{array}{llll}1 & -6.449525000 & 2.602320000 & 0.912145000\end{array}$
$\begin{array}{llll}6 & -5.037404000 & 4.102989000 & 0.240758000\end{array}$
$\begin{array}{llll}1 & -5.341298000 & 4.034328000 & -0.808085000\end{array}$
$\begin{array}{llll}6 & -4.028711000 & 5.001641000 & 0.611467000\end{array}$
$\begin{array}{llll}1 & -3.566094000 & 5.643447000 & -0.141157000\end{array}$ 


\begin{tabular}{llll}
6 & -1.298060000 & 5.517106000 & 2.999145000 \\
6 & -0.581975000 & 5.985426000 & 4.165099000 \\
6 & -0.603623000 & 4.342938000 & 2.491431000 \\
8 & -0.834049000 & 3.627170000 & 1.507009000 \\
6 & -1.664219000 & 6.746837000 & 1.449352000 \\
1 & -1.093641000 & 7.608573000 & 1.787032000 \\
1 & -1.260409000 & 6.153410000 & 0.630825000 \\
6 & 0.521567000 & 5.096969000 & 4.361440000 \\
6 & 1.419996000 & 5.257728000 & 5.414841000 \\
1 & 2.257550000 & 4.566916000 & 5.547983000 \\
\hline 6 & 1.220735000 & 6.332003000 & 6.301196000 \\
\hline 6 & -0.762261000 & 7.046552000 & 5.065680000 \\
\hline 1 & -1.598285000 & 7.742933000 & 4.946875000 \\
\hline 6 & 0.142470000 & 7.213421000 & 6.127198000 \\
\hline 1 & 1.914577000 & 6.476841000 & 7.134411000 \\
\hline 7 & 0.003226000 & 8.042545000 & 6.827519000 \\
\hline 7 & 0.478340000 & 4.137972000 & 3.362179000 \\
\hline 7 & -2.695392000 & 7.984548000 & 0.103761000
\end{tabular}




\section{1a'_TS3}

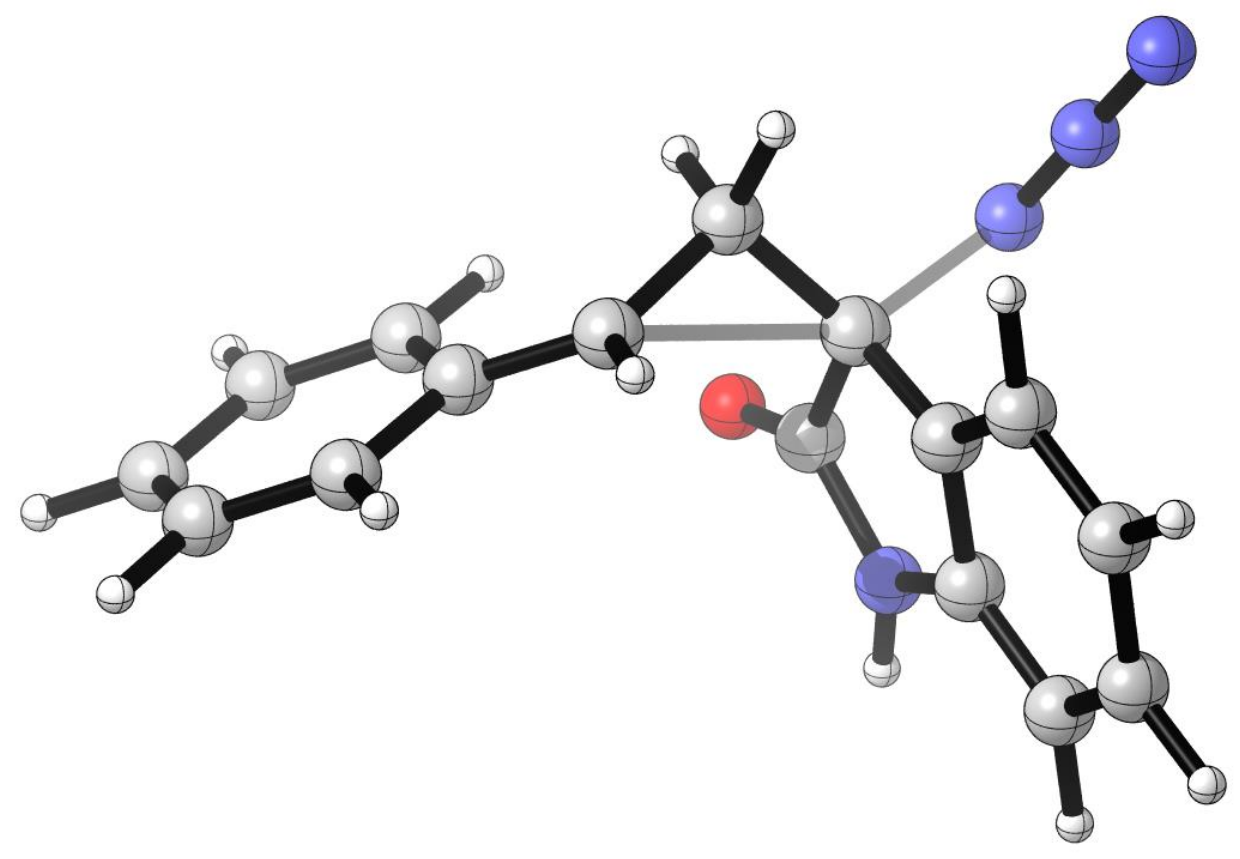

$\mathrm{SPE}=-910.5892 ; \mathrm{H}_{298}=-910.3141 ; \mathrm{G}_{298}=-910.3757$

\begin{tabular}{llll}
6 & -2.725734000 & 6.019040000 & 2.761062000 \\
1 & -2.846091000 & 6.270862000 & 3.818899000 \\
6 & -3.580273000 & 5.022740000 & 2.232588000 \\
6 & -4.548618000 & 4.375058000 & 3.079467000 \\
1 & -4.587677000 & 4.662140000 & 4.136403000 \\
6 & -5.431164000 & 3.414853000 & 2.601413000 \\
1 & -6.149147000 & 2.958442000 & 3.292404000 \\
6 & -5.417548000 & 3.021485000 & 1.248637000 \\
1 & -6.111879000 & 2.263855000 & 0.874632000 \\
6 & -4.479118000 & 3.628487000 & 0.397399000 \\
\hline 1 & -4.439016000 & 3.334892000 & -0.657953000 \\
6 & -3.585578000 & 4.592167000 & 0.860476000 \\
\hline 1 & -2.855425000 & 5.012089000 & 0.167070000
\end{tabular}




\begin{tabular}{llll}
6 & -0.430126000 & 6.219118000 & 2.456961000 \\
6 & 0.009599000 & 6.263881000 & 3.878541000 \\
6 & -0.199610000 & 4.765729000 & 2.049483000 \\
8 & -0.454935000 & 4.242504000 & 0.976486000 \\
6 & -1.716734000 & 6.835363000 & 2.002952000 \\
1 & -1.747302000 & 7.916194000 & 2.237494000 \\
1 & -1.806484000 & 6.736366000 & 0.909954000 \\
6 & 0.603764000 & 5.016768000 & 4.191279000 \\
6 & 1.181280000 & 4.771318000 & 5.434642000 \\
\hline 1 & 1.633152000 & 3.804007000 & 5.666983000 \\
\hline 6 & 1.157713000 & 5.807957000 & 6.384036000 \\
\hline 6 & -0.004974000 & 7.282044000 & 4.831302000 \\
\hline 1 & -0.461060000 & 8.249763000 & 4.607801000 \\
\hline 6 & 0.570331000 & 7.046206000 & 6.091946000 \\
\hline 1 & 1.604664000 & 5.639292000 & 7.367675000 \\
\hline 1 & 0.560437000 & 7.835330000 & 6.848626000 \\
\hline 7 & 0.472455000 & 4.164266000 & 3.097772000 \\
\hline 7 & 0.795726000 & 3.202990000 & 3.064283000 \\
\hline 7 & 1.584424000 & 9.084786000 & 2.328176000 \\
\hline 6 & 1.250941000 & 8.047613000 & 1.937950000 \\
\hline 6 & & & \\
\hline
\end{tabular}




\section{1a'_TS4}

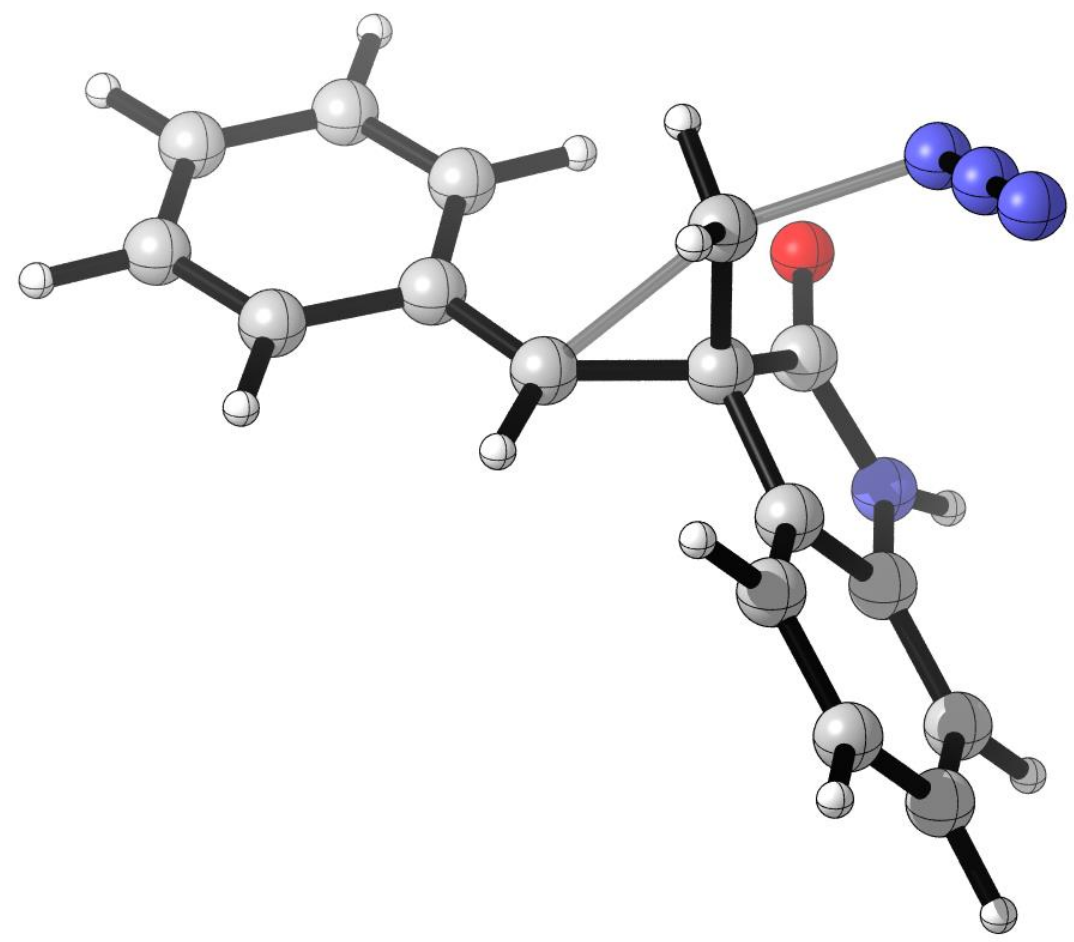

$\mathrm{SPE}=-910.5763 ; \mathrm{H}_{298}=-910.3017 ; \mathrm{G}_{298}=-910.3618$

\begin{tabular}{llll}
6 & -2.522217000 & 5.623729000 & 2.681817000 \\
1 & -2.841601000 & 6.511399000 & 3.234151000 \\
6 & -3.577453000 & 4.833854000 & 2.119068000 \\
6 & -3.433497000 & 3.645187000 & 1.334655000 \\
1 & -2.434504000 & 3.256737000 & 1.139673000 \\
6 & -4.540872000 & 2.967888000 & 0.821578000 \\
1 & -4.373091000 & 2.061726000 & 0.228821000 \\
6 & -5.851032000 & 3.413619000 & 1.050050000 \\
1 & -6.710636000 & 2.872328000 & 0.646405000 \\
6 & -6.022876000 & 4.578742000 & 1.817304000 \\
1 & -7.031452000 & 4.957014000 & 2.016735000 \\
6 & -4.928698000 & 5.265238000 & 2.334023000 \\
\hline 1 & -5.098958000 & 6.170771000 & 2.925919000
\end{tabular}




\begin{tabular}{llll}
6 & -1.010861000 & 5.449736000 & 2.698758000 \\
6 & -0.364012000 & 6.179068000 & 3.860530000 \\
6 & -0.400531000 & 4.032168000 & 2.829857000 \\
8 & -0.478864000 & 3.096559000 & 2.050580000 \\
6 & -0.923516000 & 6.075387000 & 1.359919000 \\
1 & -1.073247000 & 7.155112000 & 1.315245000 \\
1 & -1.311598000 & 5.488256000 & 0.528188000 \\
6 & 0.368376000 & 5.253429000 & 4.625672000 \\
\hline 6 & 1.037592000 & 5.619625000 & 5.791614000 \\
1 & 1.598284000 & 4.886559000 & 6.376500000 \\
\hline 6 & 0.969579000 & 6.966466000 & 6.185238000 \\
\hline 6 & -0.417200000 & 7.510040000 & 4.257796000 \\
\hline 1 & -0.971928000 & 8.249057000 & 3.672764000 \\
\hline 6 & 0.256553000 & 7.903542000 & 5.428678000 \\
\hline 1 & 1.484331000 & 7.283425000 & 7.096400000 \\
\hline 1 & 0.221535000 & 8.948225000 & 5.748785000 \\
\hline 7 & 0.296526000 & 3.999033000 & 4.012776000 \\
\hline 1 & 0.798236000 & 3.171921000 & 4.322929000 \\
\hline 7 & 1.859839000 & 8.231447000 & 0.818776000 \\
\hline 6 & 1.405860000 & 7.160114000 & 0.773765000 \\
\hline 6 & 0.930588000 & 6.060735000 & 0.729599000
\end{tabular}




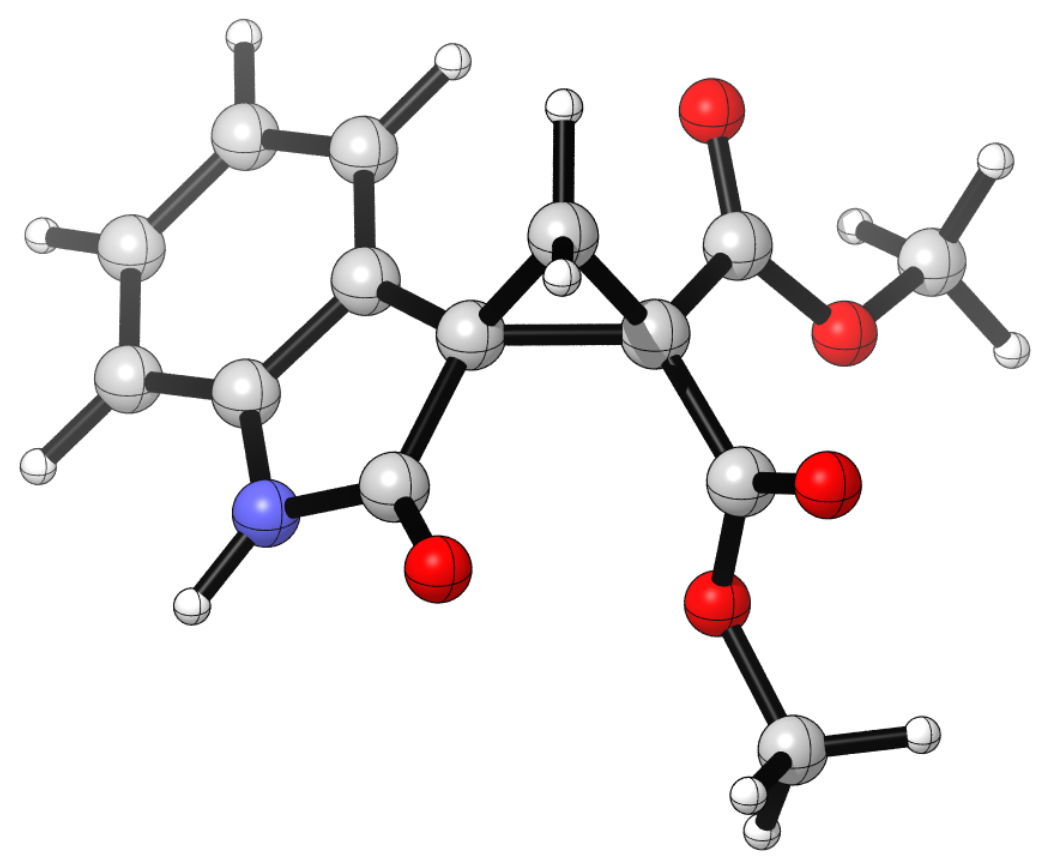

SPE $=-970.9817 ; H_{298}=-970.7107 ; \mathrm{G}_{298}=-970.7739$
$6 \quad-0.524159000$
2.798024000
4.462069000
$6 \quad 0.063961000$
3.339904000
5.725931000
$\begin{array}{ll}6 & -0.508597000\end{array}$
4.616878000
5.925497000
$6 \quad-0.191598000$
5.409771000
7.025470000
$1-0.645462000$
6.394056000
7.159582000
$6 \quad 0.722151000$
4.895555000
7.957922000
$1 \quad 0.989240000$
5.496734000
8.831261000
$6 \quad 1.283902000$
3.624891000
7.788936000
$1 \quad 1.984492000$
3.237957000
8.533449000
6
0.954936000
2.836051000
6.673592000
$1 \quad 1.395577000$
1.847664000
6.548222000
$6 \quad 0.214935000$
2.047299000
3.316937000
$\begin{array}{ll}6 & -0.085745000\end{array}$
$2.447664000 \quad 1.880314000$ 


\begin{tabular}{llll}
8 & -0.599654000 & 1.705273000 & 1.075516000 \\
8 & 0.323883000 & 3.684609000 & 1.611595000 \\
6 & 0.083330000 & 4.171273000 & 0.283041000 \\
1 & 0.566849000 & 3.521862000 & -0.460892000 \\
1 & 0.512909000 & 5.179023000 & 0.246804000 \\
1 & -0.998049000 & 4.209371000 & 0.085614000 \\
6 & 1.634130000 & 1.590020000 & 3.558064000 \\
8 & 1.986438000 & 0.927137000 & 4.508629000 \\
8 & 2.450177000 & 1.977636000 & 2.579346000 \\
6 & 3.825546000 & 1.567463000 & 2.670145000 \\
\hline 1 & 4.288152000 & 1.983382000 & 3.576683000 \\
\hline 1 & 4.316362000 & 1.964561000 & 1.774152000 \\
\hline 1 & 3.896451000 & 0.470360000 & 2.694312000 \\
\hline 6 & -0.851467000 & 1.369509000 & 4.116781000 \\
\hline 1 & -1.810248000 & 1.200001000 & 3.619108000 \\
\hline 1 & -0.519991000 & 0.599077000 & 4.816741000 \\
\hline 6 & -1.519019000 & 3.860879000 & 4.005440000 \\
\hline 1 & -1.412987000 & 4.894820000 & 4.893459000 \\
\hline 6 & -1.981299000 & 5.734498000 & 4.830471000 \\
\hline 1 & & & \\
\hline & & & \\
\hline
\end{tabular}




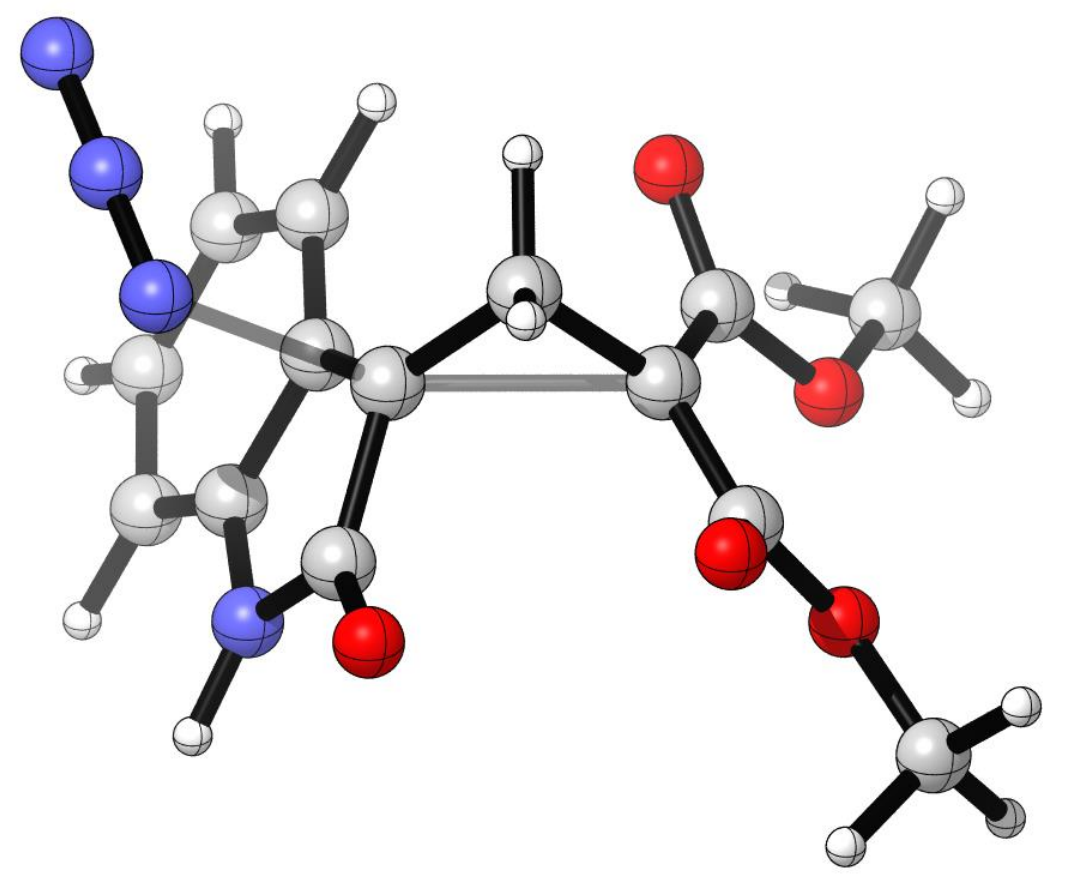

$\mathrm{SPE}=-1135.0679 ; \mathrm{H}_{298}=-1134.7838 ; \mathrm{G}_{298}=-1134.8532$

$\begin{array}{llll}6 & -0.897160000 & 2.642544000 & 4.863281000 \\ 6 & -0.049004000 & 3.160573000 & 5.917977000 \\ 6 & -0.362753000 & 4.540224000 & 6.080924000 \\ 6 & 0.246375000 & 5.318631000 & 7.057285000 \\ 1 & 0.000294000 & 6.376629000 & 7.173554000 \\ 6 & 1.187563000 & 4.694054000 & 7.896978000 \\ 1 & 1.681795000 & 5.287434000 & 8.671521000 \\ 6 & 1.495013000 & 3.334457000 & 7.766777000 \\ 1 & 2.223331000 & 2.876162000 & 8.440724000 \\ 6 & 0.873187000 & 2.553362000 & 6.780624000 \\ 1 & 1.117452000 & 1.499225000 & 6.666021000 \\ 6 & 0.242843000 & 2.005462000 & 3.105590000 \\ 6 & -0.270374000 & 2.472743000 & 1.821465000\end{array}$




\begin{tabular}{|c|c|c|c|}
\hline & 1.389262000 & 2.209530000 & 5000 \\
\hline & 0.582859000 & 3.234615000 & 1.091669000 \\
\hline & 119153000 & 3.63 & 1000 \\
\hline & -0.109644000 & 1000 & 4000 \\
\hline & .933141000 & 9000 & -0.64 \\
\hline & -0.788891000 & 2000 & -0.1 \\
\hline & 1.639999000 & 1.8510 & 5000 \\
\hline & 2.020473000 & 1.183126000 & 7000 \\
\hline & 2.541168000 & 2.476903000 & 2.6 \\
\hline & 3.914224000 & 2.273688000 & 2.999843000 \\
\hline & 4.145847000 & 2.64 & 4.008758000 \\
\hline & 8000 & 24000 & 2.251681000 \\
\hline & 000 & 1.20 & 3000 \\
\hline & -0.766192000 & 1.40 & 4.06 \\
\hline & -1.705252000 & $1.15 \mathrm{c}$ & 18000 \\
\hline & -0.379773000 & 0.556266000 & 4.631467000 \\
\hline & -1.734057000 & 3.822315000 & 4.387033000 \\
\hline & -1.331877000 & 4.901235000 & 5.152762000 \\
\hline & -1.770518000 & 5.813968000 & 5.08879100 \\
\hline & -2.599796000 & 3.840901000 & 3.53387900 \\
\hline & -1.764570000 & 1.106539000 & 8.12078300 \\
\hline & -2.696066000 & 2.072240000 & 6.18006700 \\
\hline & -2.226792000 & 1.583442000 & 7.16000300 \\
\hline
\end{tabular}




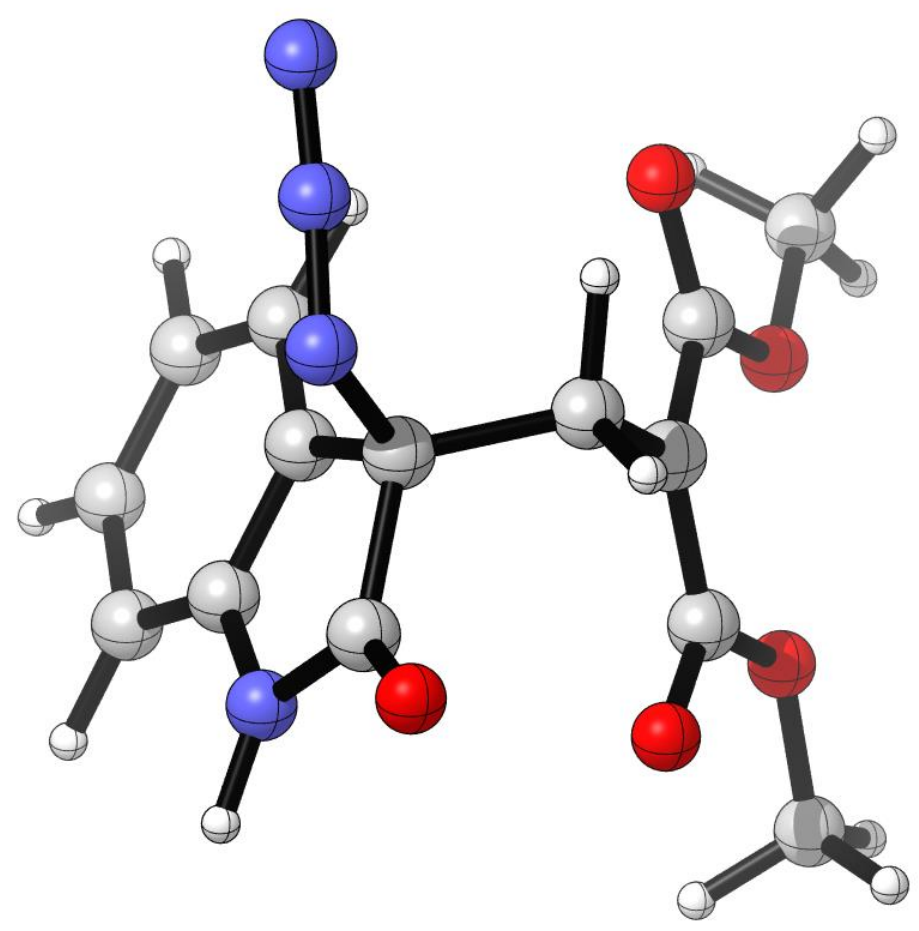

$\mathrm{SPE}=-1135.0975 ; \mathrm{H}_{298}=-1134.8112 ; \mathrm{G}_{298}=-1134.8801$
$6 \quad-1.366414000 \quad 2.373496000 \quad 5.263152000$
$\begin{array}{llll}6 & -0.337129000 & 3.284669000 & 5.905405000\end{array}$
$\begin{array}{llll}6 & -0.790652000 & 4.613391000 & 5.803267000\end{array}$
$\begin{array}{llll}6 & -0.054946000 & 5.682235000 & 6.310827000\end{array}$
$\begin{array}{llll}1 & -0.416967000 & 6.709825000 & 6.226196000\end{array}$
$\begin{array}{llll}6 & 1.170327000 & 5.392186000 & 6.933565000\end{array}$
$\begin{array}{llll}1 & 1.767235000 & 6.212376000 & 7.341901000\end{array}$
$\begin{array}{llll}6 & 1.641034000 & 4.077331000 & 7.028526000\end{array}$
$1 \quad 2.605694000 \quad 3.877563000 \quad 7.501615000$
$\begin{array}{llll}6 & 0.888106000 & 3.011803000 & 6.503458000\end{array}$
$\begin{array}{llll}1 & 1.274517000 & 1.990764000 & 6.529261000\end{array}$
$\begin{array}{llll}6 & 0.284276000 & 1.948524000 & 3.266910000\end{array}$
$\begin{array}{llll}6 & -0.049813000 & 2.952543000 & 2.308199000\end{array}$ 


\begin{tabular}{|c|c|c|c|}
\hline & 1.186218000 & 3.430 & 1000 \\
\hline & 0.957690000 & 3.400096000 & 1.487865000 \\
\hline & 386000 & 4000 & 000 \\
\hline & -0.170518000 & 9000 & +200 \\
\hline & .516434000 & 4000 & 344000 \\
\hline & 0.209249000 & 5.313508000 & .070728000 \\
\hline & 1.567620000 & 1.391 & 7000 \\
\hline & 000 & 3000 & 1000 \\
\hline & 2.616175000 & 22000 & 3000 \\
\hline & 3.88 & 1.25 & 3.08 \\
\hline & 000 & 1.48 & 5000 \\
\hline & 3000 & 1000 & 2.376405000 \\
\hline & 000 & 0.1 & 000 \\
\hline & 065000 & 1.443 & 4.116 \\
\hline & 2000 & 1.205158000 & 3.513029000 \\
\hline & -0.515444000 & 0.515385000 & 4.598714000 \\
\hline & -2.452451000 & 3.388717000 & 4.786358000 \\
\hline & -2.024250000 & 4.639639000 & 5.143810000 \\
\hline & -2.555881000 & 5.480205000 & 4.94069900 \\
\hline & -3.516206000 & 3.109525000 & 4.271242000 \\
\hline & -1.007106000 & -0.043231000 & 7.60965100 \\
\hline & -2.113901000 & 1.560668000 & 6.27953100 \\
\hline & -1.484965000 & 0.742654000 & 6.93522500 \\
\hline
\end{tabular}




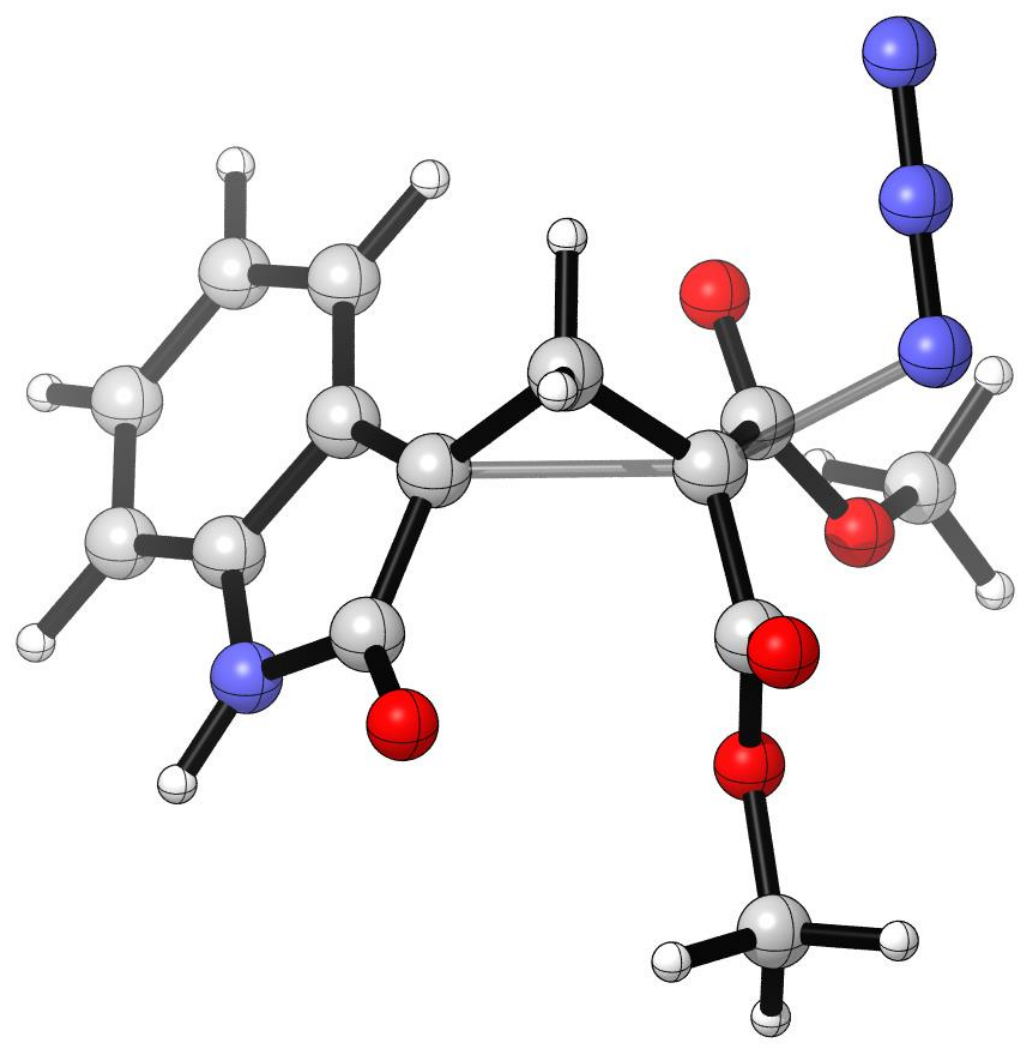

$\mathrm{SPE}=-1135.0600 ; \mathrm{H}_{298}=-1134.7760 ; \mathrm{G}_{298}=-1134.8448$
$\begin{array}{llll}6 & -0.500981000 & 2.382402000 & 4.194464000\end{array}$
$\begin{array}{llll}6 & 0.128140000 & 2.782850000 & 5.429404000\end{array}$
$\begin{array}{llll}6 & -0.257864000 & 4.139132000 & 5.678190000\end{array}$
$\begin{array}{llll}6 & 0.153794000 & 4.830992000 & 6.814631000\end{array}$
$\begin{array}{llll}1 & -0.156805000 & 5.865286000 & 6.985283000\end{array}$
$\begin{array}{llll}6 & 0.969025000 & 4.156949000 & 7.741292000\end{array}$
$\begin{array}{llll}1 & 1.303090000 & 4.679244000 & 8.642294000\end{array}$
$\begin{array}{llll}6 & 1.349253000 & 2.823209000 & 7.524837000\end{array}$
$\begin{array}{llll}1 & 1.978385000 & 2.315651000 & 8.262288000\end{array}$
$\begin{array}{llll}6 & 0.936065000 & 2.130532000 & 6.377423000\end{array}$
$\begin{array}{llll}1 & 1.250177000 & 1.099783000 & 6.210103000\end{array}$
$\begin{array}{llll}6 & 0.529980000 & 1.430693000 & 2.508003000\end{array}$
$\begin{array}{llll}6 & 0.002042000 & 2.174973000 & 1.295550000\end{array}$ 


\begin{tabular}{|c|c|c|c|}
\hline & -0.879291000 & 1.736206000 & 0.59 \\
\hline & 0.582455000 & 3.358289000 & 1.098534000 \\
\hline & 0.049653000 & 56000 & 7000 \\
\hline & 0.118236000 & 3.623067000 & 9000 \\
\hline & 655660000 & 5000 & 3000 \\
\hline & -1.0020 & 4.397850000 & 7000 \\
\hline & 1.949202000 & 1.490368000 & 9000 \\
\hline & 2.336089000 & 7000 & 53000 \\
\hline & 2.770994000 & 57000 & 76000 \\
\hline & 4.165052000 & 2000 & 37000 \\
\hline & 4.350338000 & 2.536378000 & 3.416545000 \\
\hline & 4.670738000 & 50000 & 1.647327000 \\
\hline & 000 & 000 & 1000 \\
\hline & 206000 & 56000 & 3.515899000 \\
\hline & -1.450080000 & 2000 & 6000 \\
\hline & -0.155338000 & 0.232557000 & 4.171943000 \\
\hline & -1.294654000 & 3.515579000 & 3.722899000 \\
\hline & -1.079109000 & 4.548623000 & 4.640506000 \\
\hline & -1.536936000 & 5.450500000 & 4.568908000 \\
\hline & -2.041862000 & 3.615668000 & 2.739717000 \\
\hline & 0.992746000 & -0.298090000 & 1.39700100 \\
\hline & 1.087174000 & -1.218726000 & 2.15777800 \\
\hline & 1.178127000 & -2.125081000 & 2.88098700 \\
\hline
\end{tabular}




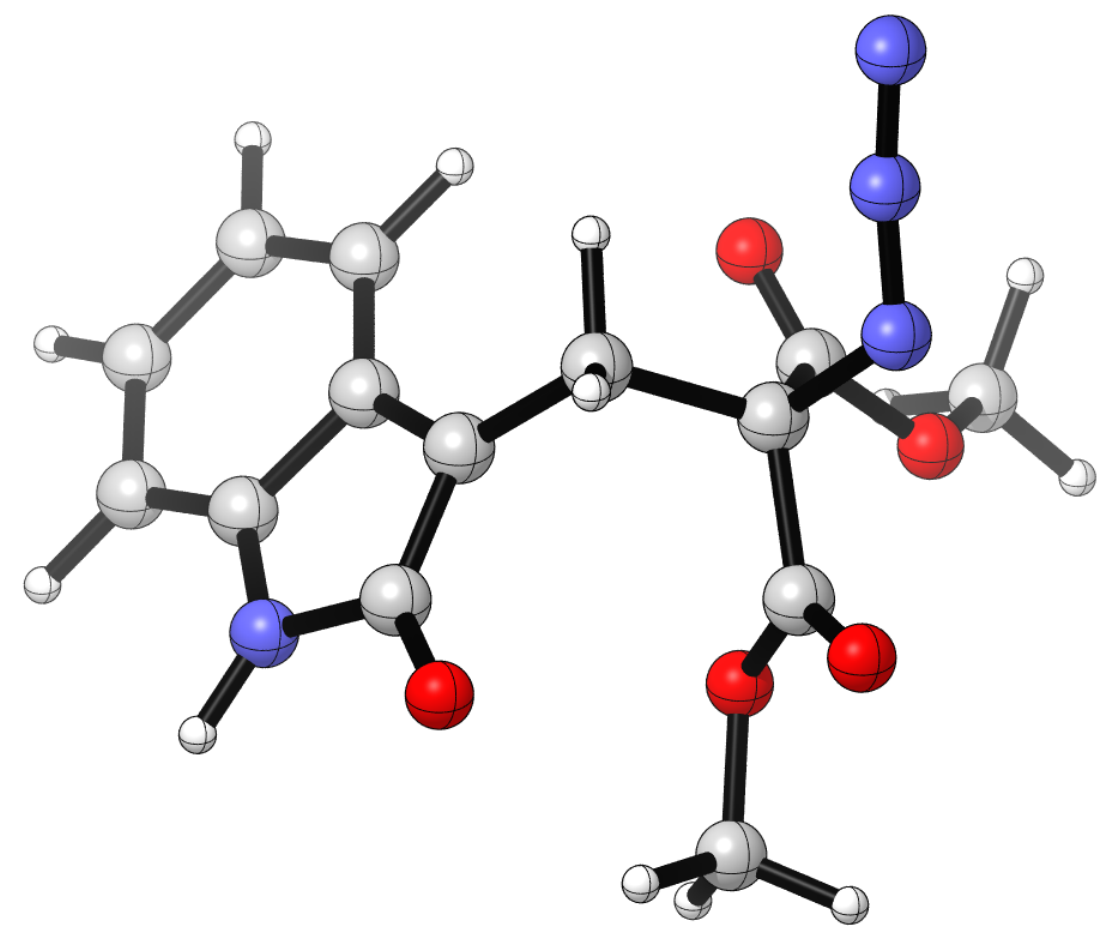

$\mathrm{SPE}=-1135.0920 ; \mathrm{H}_{298}=-1134.8060 ; \mathrm{G}_{298}=-1134.8742$
$\begin{array}{llll}6 & -0.497918000 & 2.415677000 & 4.189307000\end{array}$
$\begin{array}{llll}6 & 0.078114000 & 2.881812000 & 5.414294000\end{array}$
$\begin{array}{llll}6 & -0.507792000 & 4.169337000 & 5.698716000\end{array}$
$\begin{array}{llll}6 & -0.177186000 & 4.905579000 & 6.834684000\end{array}$
$\begin{array}{llll}1 & -0.638459000 & 5.880579000 & 7.020859000\end{array}$
$\begin{array}{llll}6 & 0.759866000 & 4.366940000 & 7.737574000\end{array}$
$\begin{array}{llll}1 & 1.032389000 & 4.929354000 & 8.635597000\end{array}$
$\begin{array}{llll}6 & 1.342210000 & 3.112811000 & 7.485403000\end{array}$
$\begin{array}{llll}1 & 2.070930000 & 2.707137000 & 8.195663000\end{array}$
$\begin{array}{llll}6 & 1.014410000 & 2.371394000 & 6.340229000\end{array}$
$\begin{array}{llll}1 & 1.496842000 & 1.409569000 & 6.151972000\end{array}$
$\begin{array}{llll}6 & 0.692481000 & 1.072814000 & 2.267603000\end{array}$
$\begin{array}{llll}6 & 0.256258000 & 2.067938000 & 1.183200000\end{array}$ 


\begin{tabular}{|c|c|c|c|}
\hline & 0.412522000 & 1.765094000 & 5000 \\
\hline & 0.668930000 & 3.297957000 & 1.462464000 \\
\hline & 0.142672000 & 000 & 9000 \\
\hline & 0.317819000 & 4.159392000 & 2000 \\
\hline & 673751000 & 0000 & 9000 \\
\hline & 60000 & 4.4533 & 1000 \\
\hline & 2.154358000 & 1.259432000 & 6000 \\
\hline & 2.5509 & 0.962 & 7000 \\
\hline & 2.945784000 & 1.656669000 & 1.729894000 \\
\hline & 4.350 & 3000 & 31000 \\
\hline & 000 & 000 & 77000 \\
\hline & 4.823084000 & 73000 & 1.083468000 \\
\hline & 5000 & 000 & 5000 \\
\hline & -0.310020000 & 1.119 & 3.476 \\
\hline & $-1.2707^{\prime}$ & 0.7870 & 3.045852000 \\
\hline & 0.031102000 & 0.338527000 & 4.175747000 \\
\hline & -1.423344000 & 3.401385000 & 3.732211000 \\
\hline & -1.391474000 & 4.450402000 & 4.676197000 \\
\hline & -1.952100000 & 5.290090000 & 4.588174000 \\
\hline & -2.164405000 & 3.422704000 & 2.717744000 \\
\hline & 0.654177000 & -0.260818000 & 1.61011900 \\
\hline & 0.742127000 & -1.262631000 & 2.31201700 \\
\hline & 0.821000000 & -2.273450000 & 2.83111900 \\
\hline
\end{tabular}




\section{2a' $a^{\prime}$ TS3}

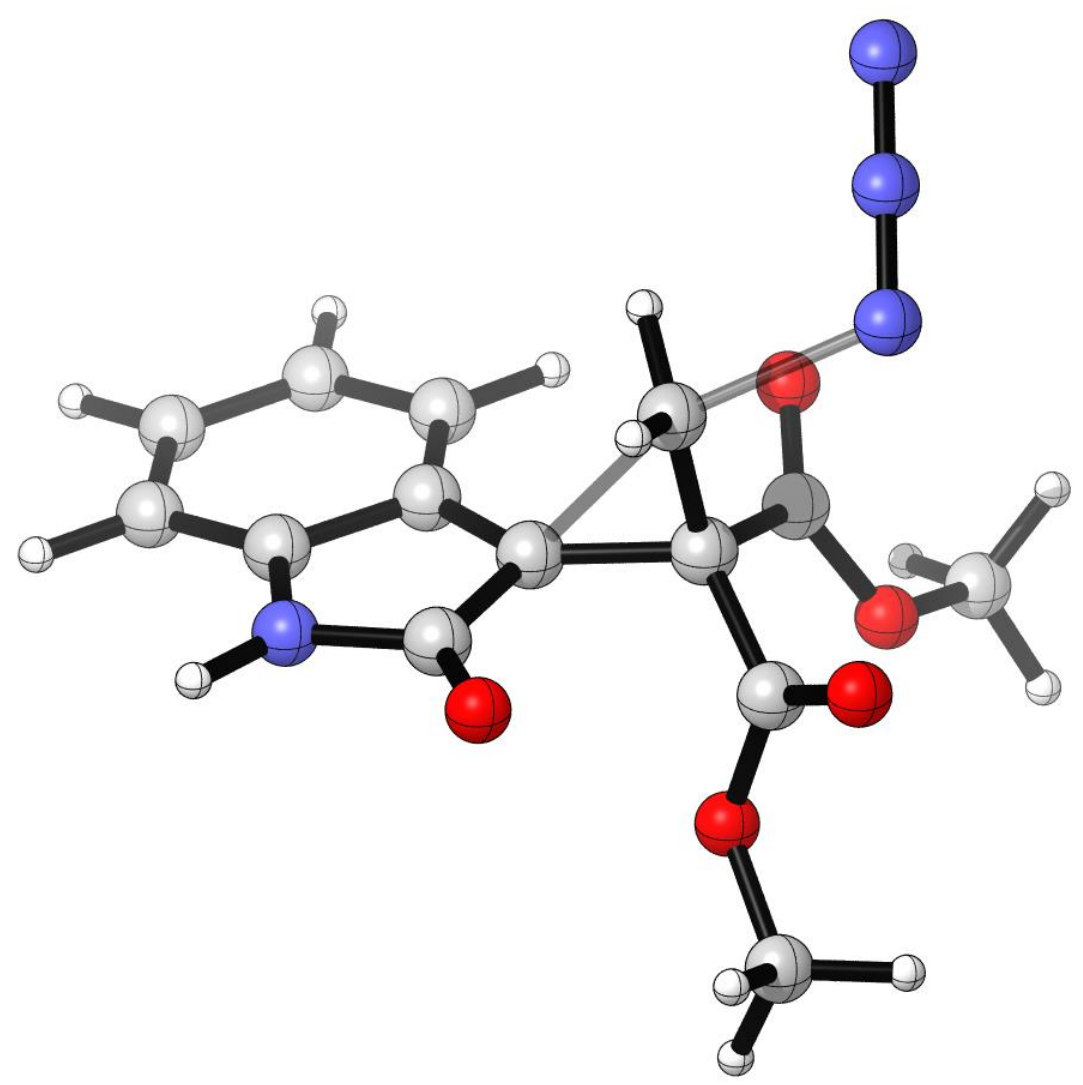

$\mathrm{SPE}=-1135.0555 ; \mathrm{H}_{298}=-1134.7722 ; \mathrm{G}_{298}=-1134.8393$

$\begin{array}{llll}6 & -0.082567000 & 2.874950000 & 4.052021000 \\ 6 & 0.360001000 & 3.217596000 & 5.397530000 \\ 6 & -0.606132000 & 4.140661000 & 5.910833000 \\ 6 & -0.513927000 & 4.689146000 & 7.188463000 \\ 1 & -1.270368000 & 5.392405000 & 7.548188000 \\ 6 & 0.571024000 & 4.313803000 & 7.996615000 \\ 1 & 0.663611000 & 4.730759000 & 9.003101000 \\ 6 & 1.529592000 & 3.409051000 & 7.518772000 \\ 1 & 2.368172000 & 3.121409000 & 8.159831000 \\ 6 & 1.433751000 & 2.858959000 & 6.230964000 \\ 1 & 2.185970000 & 2.148794000 & 5.888554000 \\ 6 & 0.512431000 & 2.031775000 & 2.944164000 \\ 6 & 0.197509000 & 2.557206000 & 1.534596000\end{array}$




\begin{tabular}{|c|c|c|c|}
\hline & 0.281413000 & 1.892770000 & 0.64 \\
\hline & 0.569698000 & 3.830438000 & 1.398279000 \\
\hline & 0.293050000 & 0000 & 4000 \\
\hline & 0.7759 & 46000 & 9000 \\
\hline & 695630000 & 2000 & 4000 \\
\hline & -0.793011000 & 22000 & -0.0 \\
\hline & 1.986182000 & 8000 & 1000 \\
\hline & 1000 & 1000 & 81000 \\
\hline & 2.741483000 & 2.133015000 & 2.11 \\
\hline & 4.1396 & 5000 & 3000 \\
\hline & 000 & 00 & 8000 \\
\hline & 4.575605000 & 8000 & 7000 \\
\hline & 000 & 000 & 3000 \\
\hline & 0000 & 8000 & 3.469186000 \\
\hline & -1.440592000 & 4000 & 3.182078000 \\
\hline & -0.090990000 & 0.486258000 & 4.379314000 \\
\hline & -1.316018000 & 3.612683000 & 3.792722000 \\
\hline & -1.573469000 & 4.353691000 & 4.940493000 \\
\hline & -2.397596000 & 4.935315000 & 5.046509000 \\
\hline & -2.038366000 & 3.606848000 & 2.78552700 \\
\hline & -0.078586000 & -0.777868000 & 2.37139900 \\
\hline & 0.121078000 & -1.653590000 & 3.15869000 \\
\hline & 0.314385000 & -2.506018000 & $3.9327370 \mathrm{C}$ \\
\hline
\end{tabular}




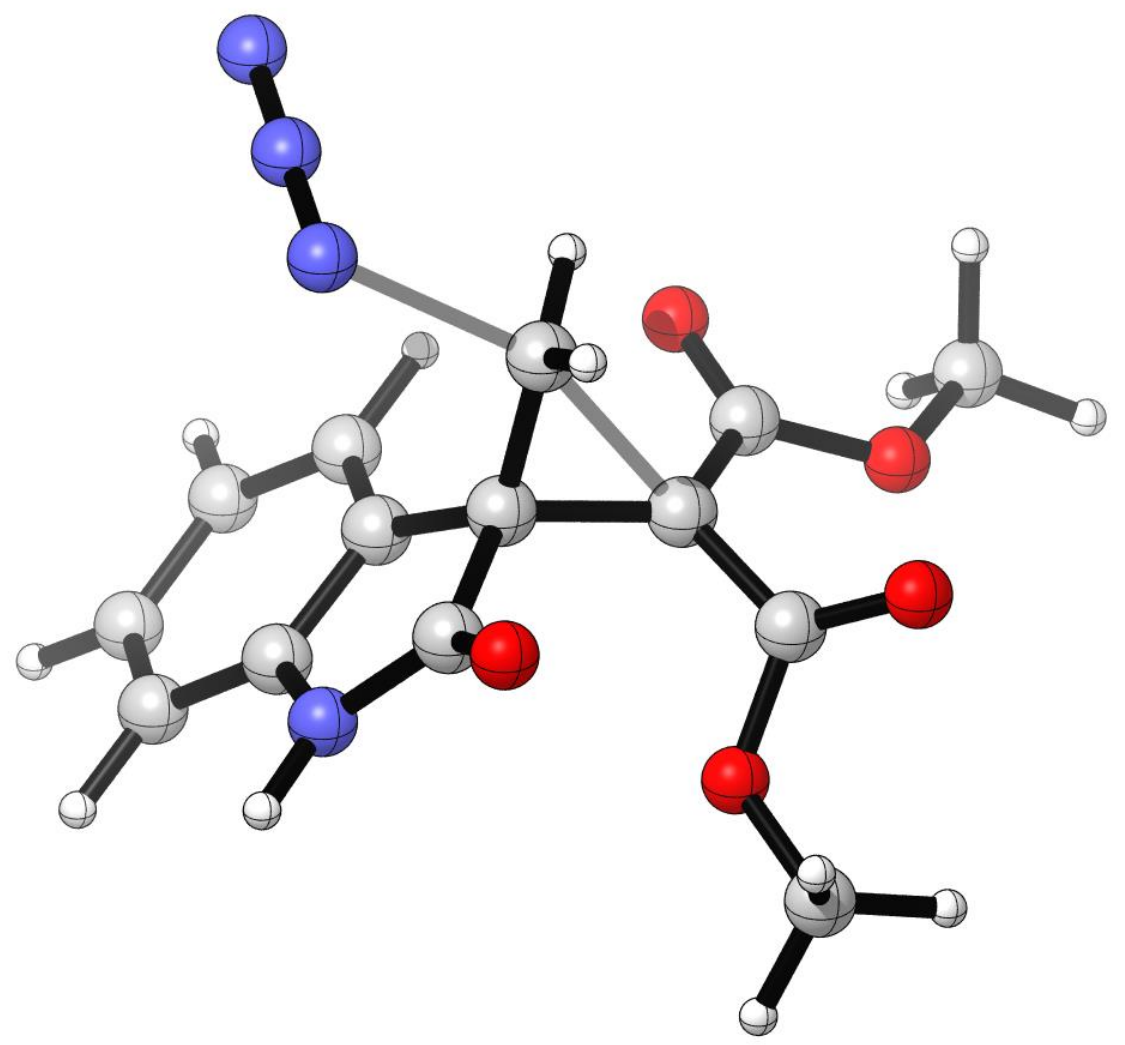

$\mathrm{SPE}=-1135.0493 ; \mathrm{H}_{298}=-1134.7653 ; \mathrm{G}_{298}=-1134.8356$
$6 \quad-0.352175000$
2.714976000
4.187964000
$\begin{array}{ll}6 & 0.104228000\end{array}$
3.329607000
5.496400000
$6-0.577949000$
4.553946000
5.655019000
$6 \quad-0.371439000$
5.389343000
6.750708000
$1-0.910594000$
6.334503000
6.847874000
$\begin{array}{ll}6 & 0.543594000\end{array}$
4.967414000
7.727894000
$1 \quad 0.725196000$
5.601672000
8.600210000
$6 \quad 1.213862000$
3.745772000
7.600793000
$1 \quad 1.913377000$
3.427756000
8.378722000
6
0.996436000
2.918486000
6.484250000
$1 \quad 1.518173000$
1.968735000
6.380198000
$6 \quad 0.619333000$
2.300320000
3.076463000
6
0.448331000
2.831354000
1.701079000 


\begin{tabular}{|c|c|c|c|}
\hline 8 & 0.429023000 & 2.184082000 & 0.671651000 \\
\hline 8 & 0.337913000 & 4.180726000 & 1.691754000 \\
\hline 6 & 0.003694000 & 4.791404000 & 0.444233000 \\
\hline 1 & 0.763460000 & 4.568642000 & -0.320342000 \\
\hline 1 & -0.039207000 & 5.872251000 & 0.631496000 \\
\hline 1 & -0.975215000 & 4.432728000 & 0.090067000 \\
\hline 6 & 1.817463000 & 1.549282000 & 3.380625000 \\
\hline 8 & 2.093400000 & 0.994436000 & 4.442921000 \\
\hline 8 & 2.685628000 & 1.497966000 & 2.331105000 \\
\hline 6 & 3.874108000 & 0.734993000 & 2.525103000 \\
\hline 1 & 4.488191000 & 1.151719000 & 3.339331000 \\
\hline 1 & 4.427377000 & 0.785393000 & 1.577699000 \\
\hline 1 & 3.641228000 & -0.314216000 & 2.766932000 \\
\hline 6 & -0.818225000 & 1.325237000 & 4.030711000 \\
\hline 1 & -1.539046000 & 1.140747000 & 3.237576000 \\
\hline 1 & -0.209917000 & 0.514768000 & 4.425303000 \\
\hline 6 & -1.459699000 & 3.694397000 & 3.716029000 \\
\hline 7 & -1.464319000 & 4.744091000 & 4.588029000 \\
\hline 1 & -2.129635000 & 5.508552000 & 4.518279000 \\
\hline 0 & -2.221795000 & 3.531521000 & 2.779595000 \\
\hline 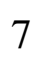 & -2.297713000 & 1.031781000 & 5.477015000 \\
\hline I & -1.826755000 & 0.383751000 & 6.363384000 \\
\hline 7 & -1.366232000 & -0.253964000 & 7.224490000 \\
\hline
\end{tabular}


3-Diazo-1-(4-methoxybenzyl)-5-methyl-1,3-dihydro-2H-indol-2-one (S1b)

${ }^{1} \mathrm{H}$ NMR $\left(\mathrm{CDCl}_{3}, 600 \mathrm{MHz}\right)$

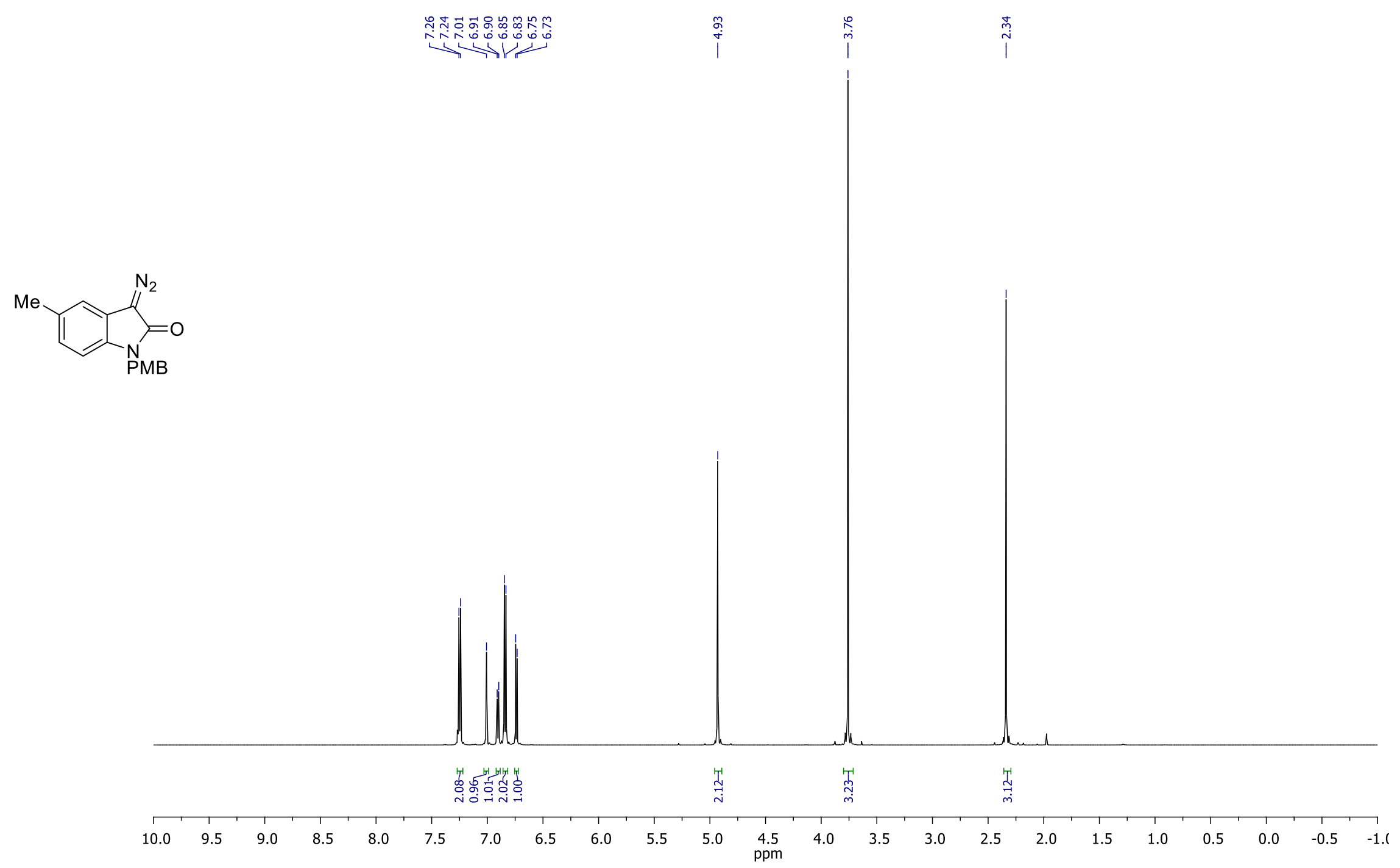


3-Diazo-1-(4-methoxybenzyl)-5-methyl-1,3-dihydro-2H-indol-2-one (S1b)

${ }^{13} \mathrm{C} \mathrm{NMR}\left(\mathrm{CDCl}_{3}, 150 \mathrm{MHz}\right)$

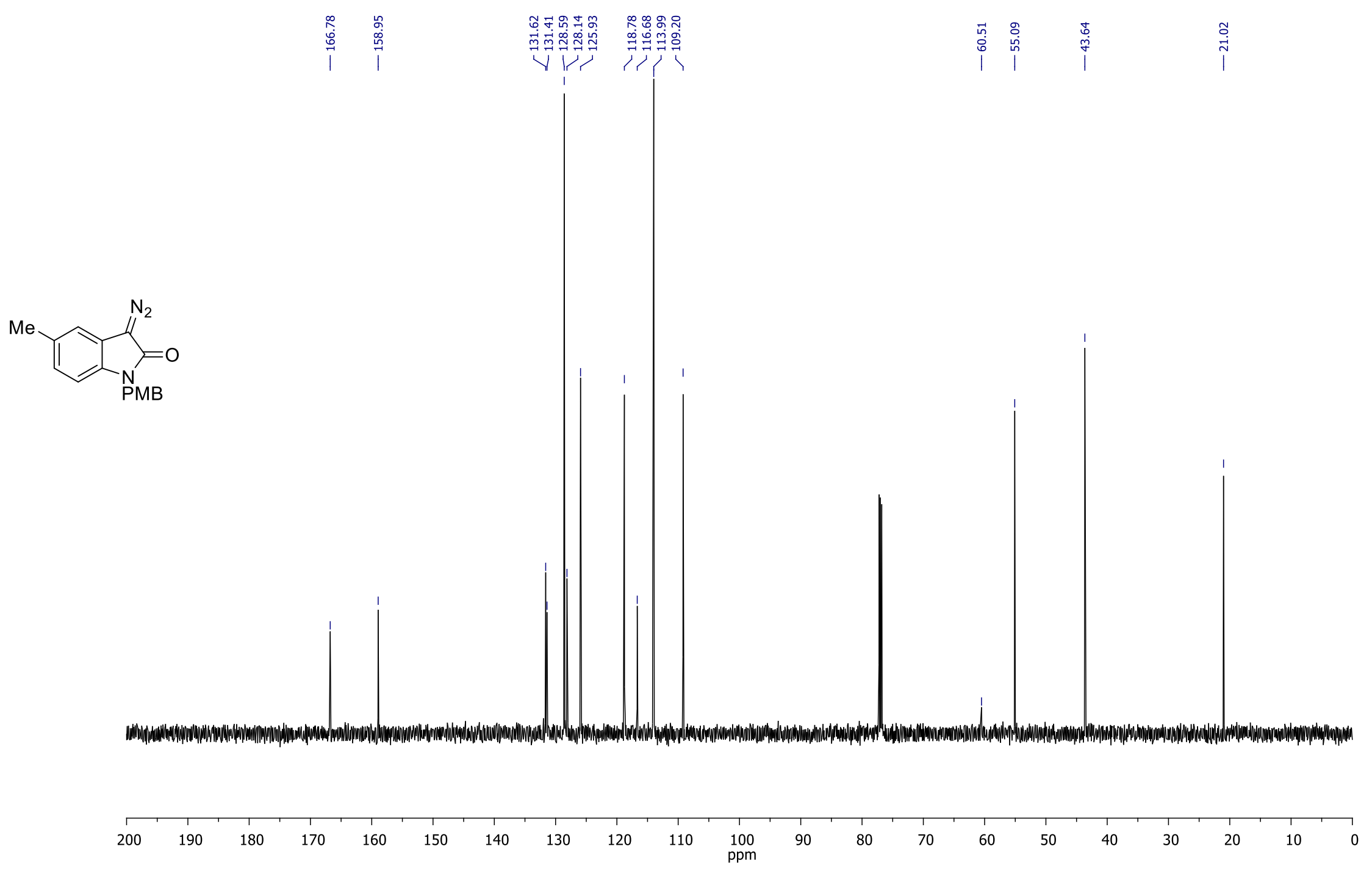


${ }^{1} \mathrm{H}$ NMR $\left(\mathrm{CDCl}_{3}, 600 \mathrm{MHz}\right)$
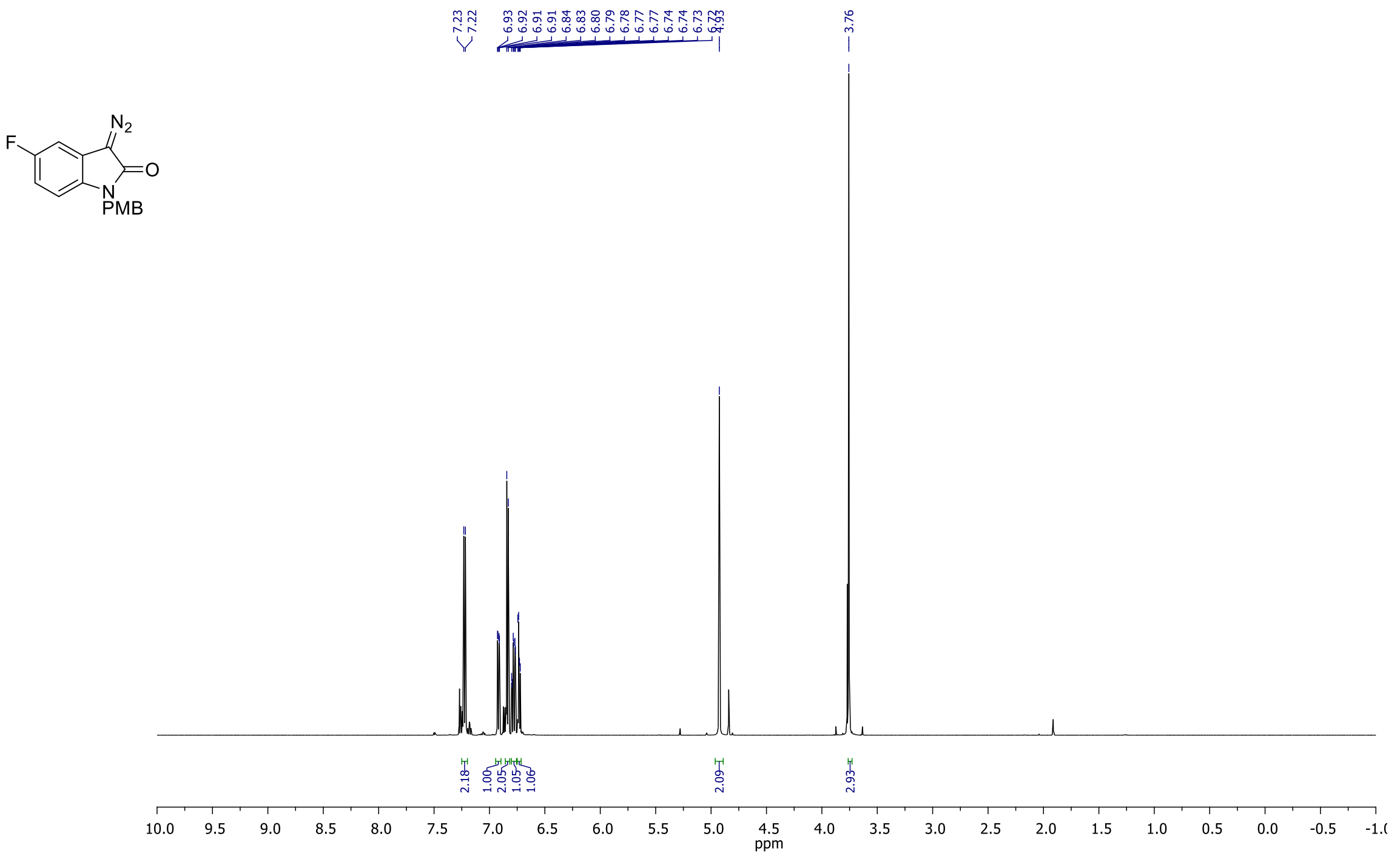
3-Diazo-5-fluoro-1-(4-methoxybenzyl)-1,3-dihydro-2H-indol-2-one (S1d)

${ }^{13} \mathrm{C} \mathrm{NMR}\left(\mathrm{CDCl}_{3}, 150 \mathrm{MHz}\right)$

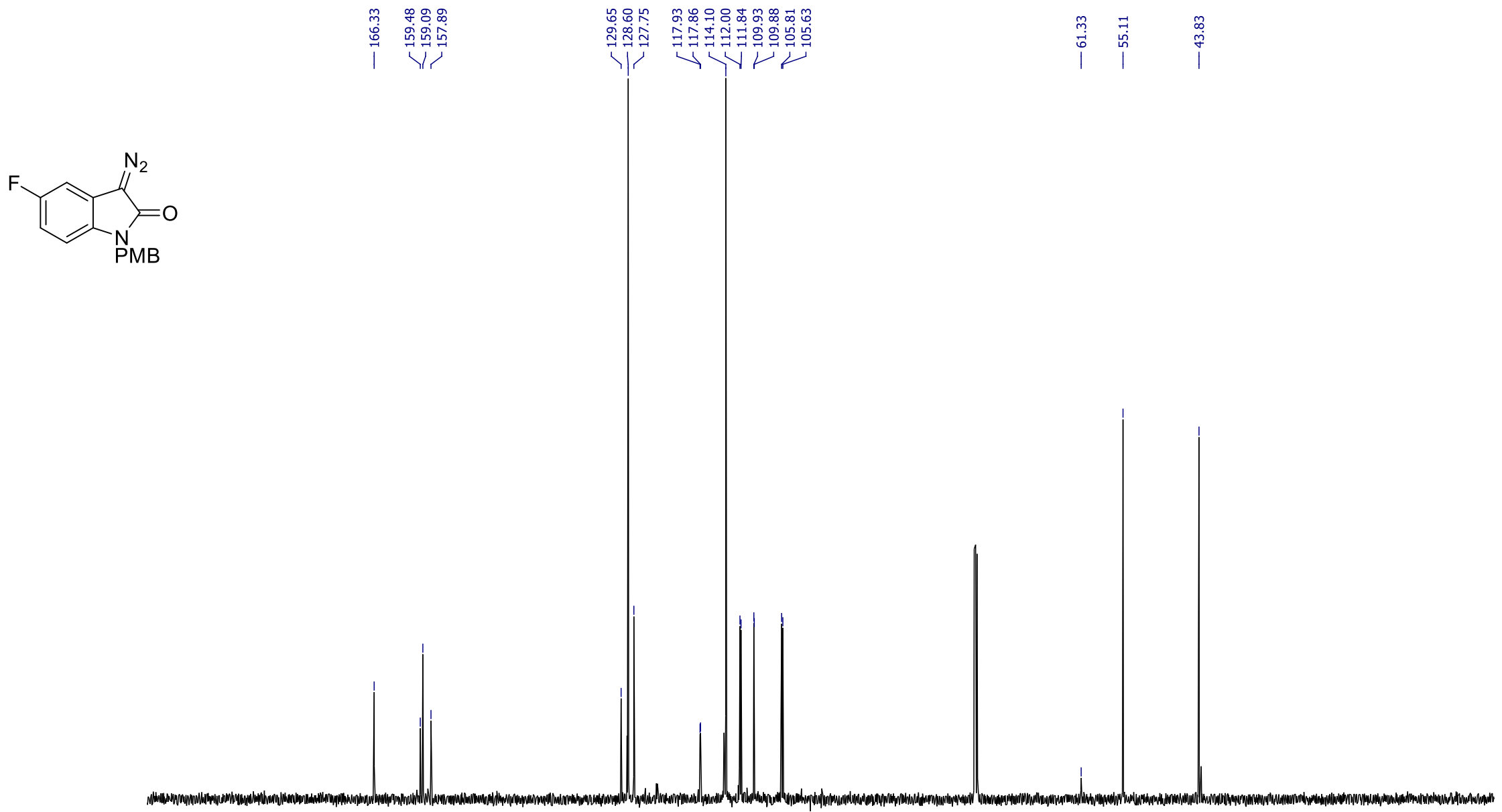


5-Chloro-3-diazo-1-(4-methoxybenzyl)-1,3-dihydro-2H-indol-2-one (S1e)

${ }^{1} \mathrm{H} \mathrm{NMR}\left(\mathrm{CDCl}_{3}, 600 \mathrm{MHz}\right)$

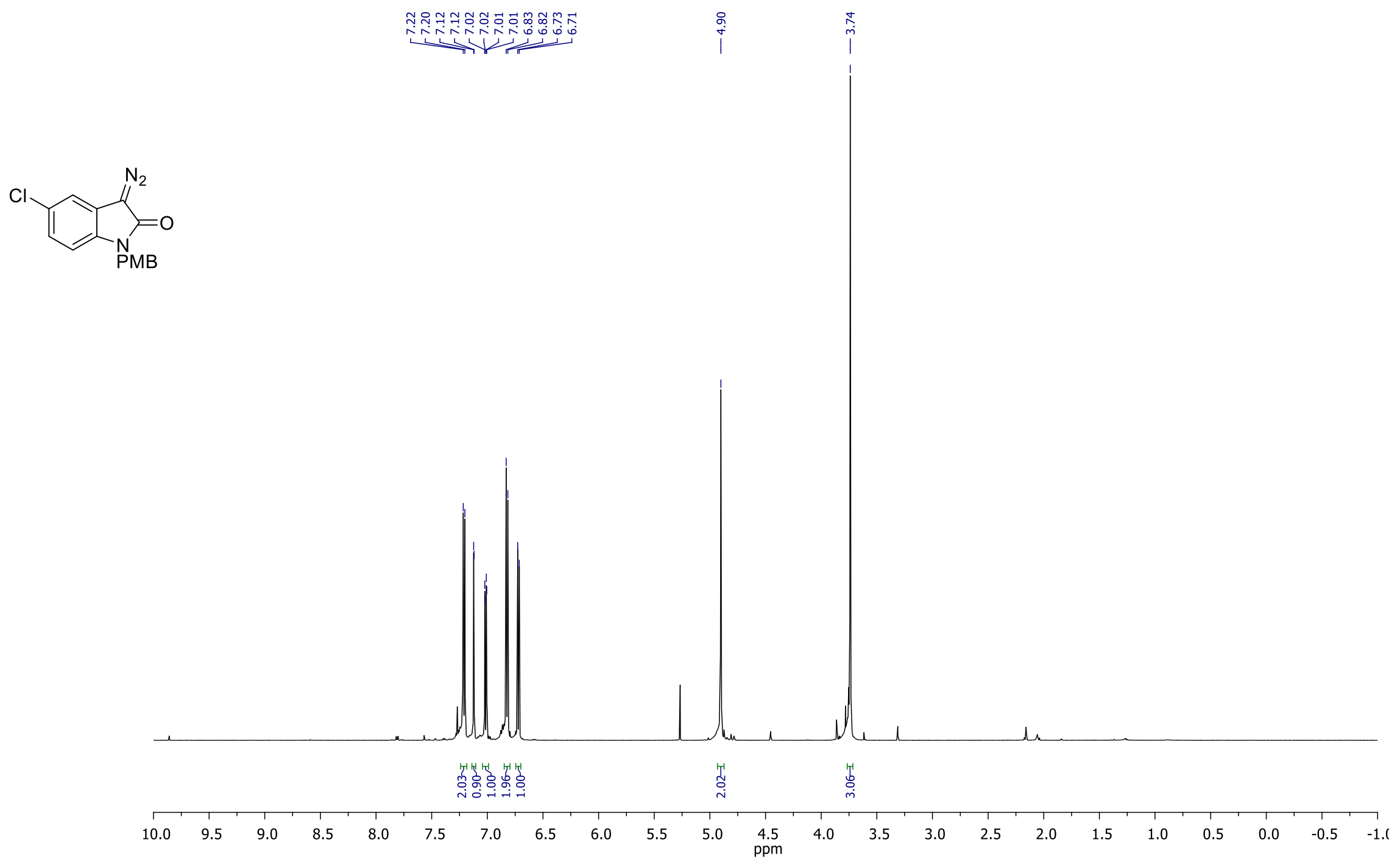


5-Chloro-3-diazo-1-(4-methoxybenzyl)-1,3-dihydro-2H-indol-2-one (S1e)

${ }^{13} \mathrm{C} \mathrm{NMR}\left(\mathrm{CDCl}_{3}, 150 \mathrm{MHz}\right)$
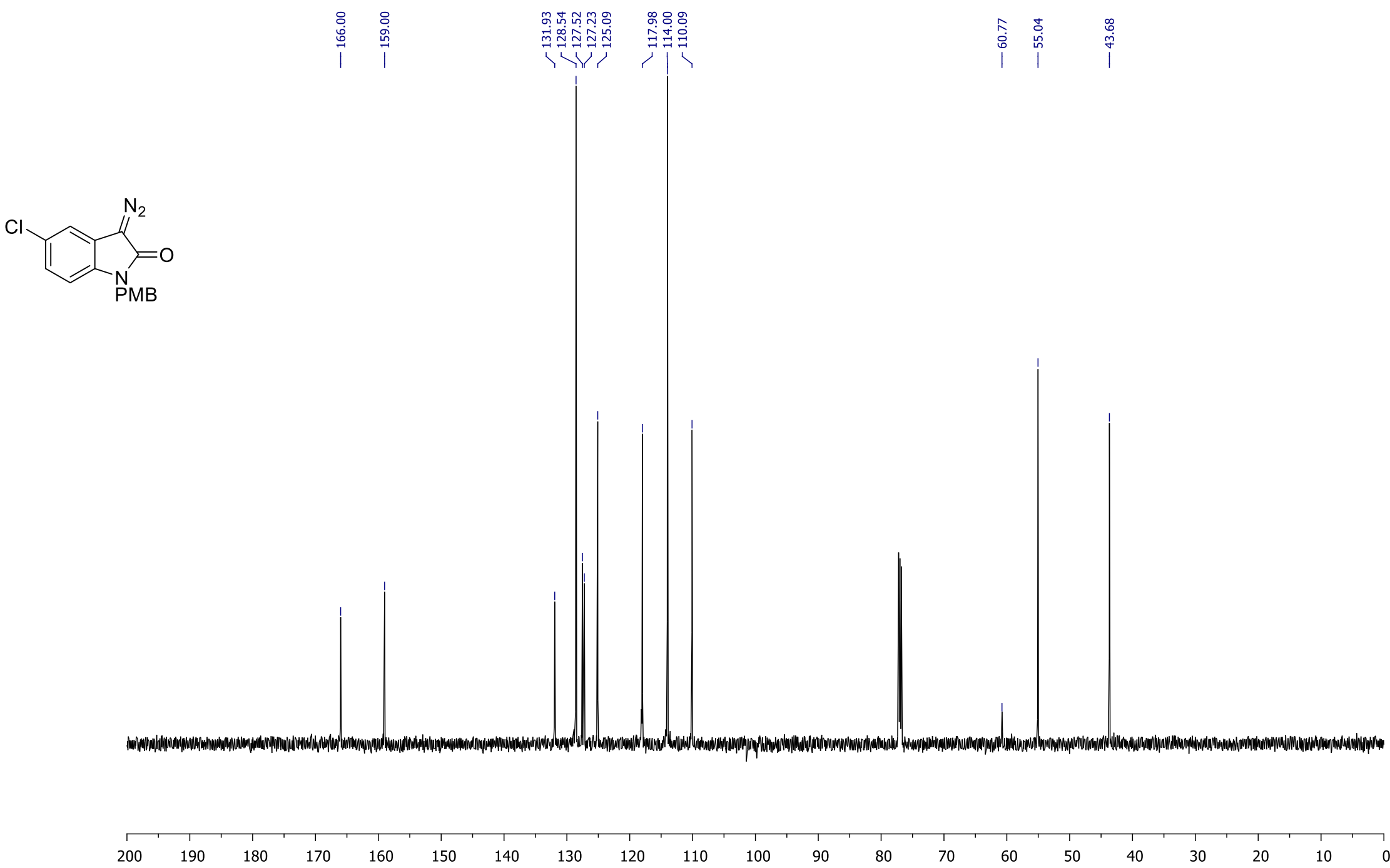

160

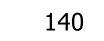

130

110

100
$\mathrm{ppm}$

90

$80 \quad 70$

60

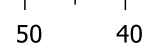

30

$20 \quad 10$ 
${ }^{1} \mathrm{H}$ NMR $\left(\mathrm{CDCl}_{3}, 600 \mathrm{MHz}\right)$

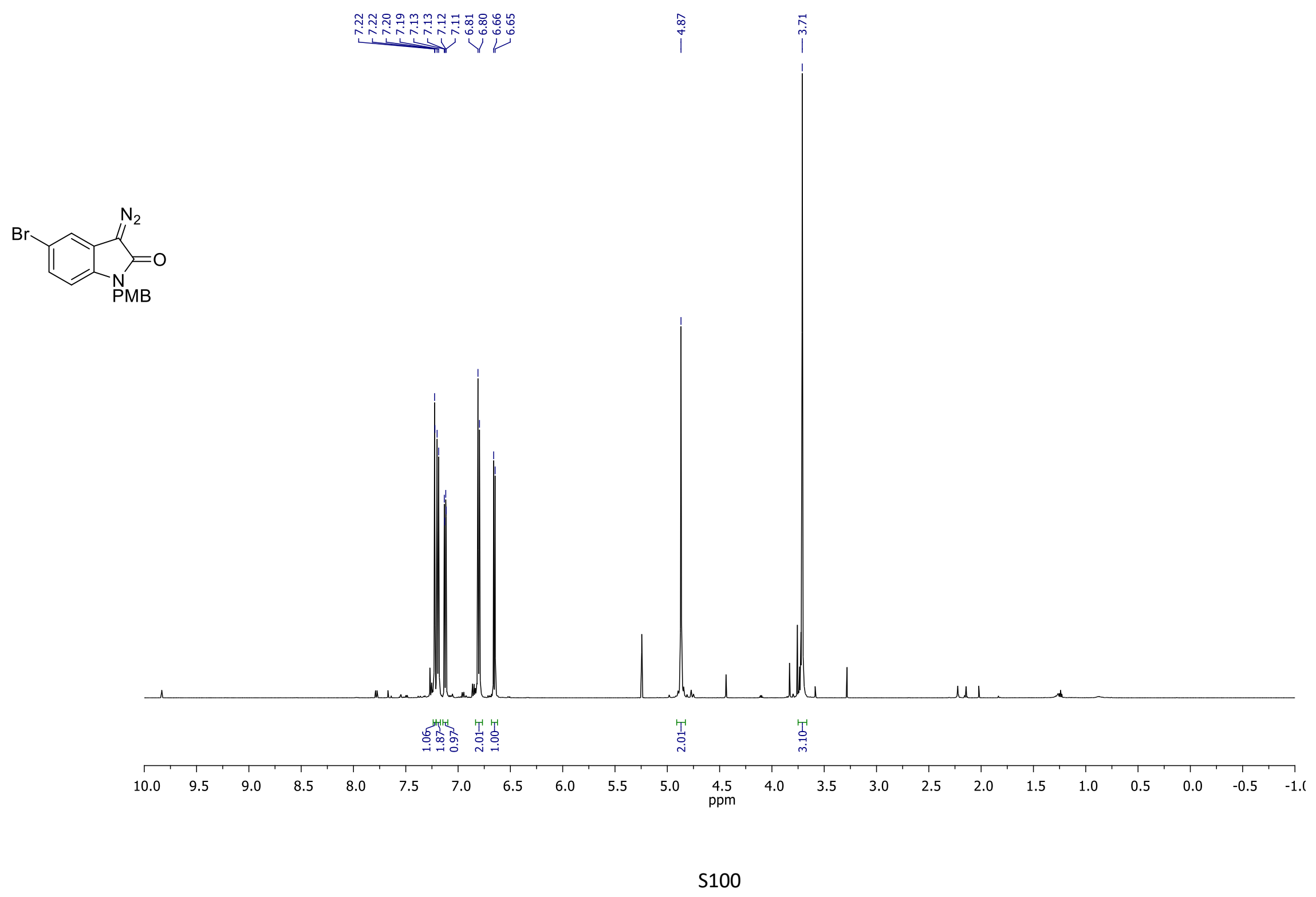


5-Bromo-3-diazo-1-(4-methoxybenzyl)-1,3-dihydro-2H-indol-2-one (S1f)

${ }^{13} \mathrm{C} \mathrm{NMR}\left(\mathrm{CDCl}_{3}, 150 \mathrm{MHz}\right)$

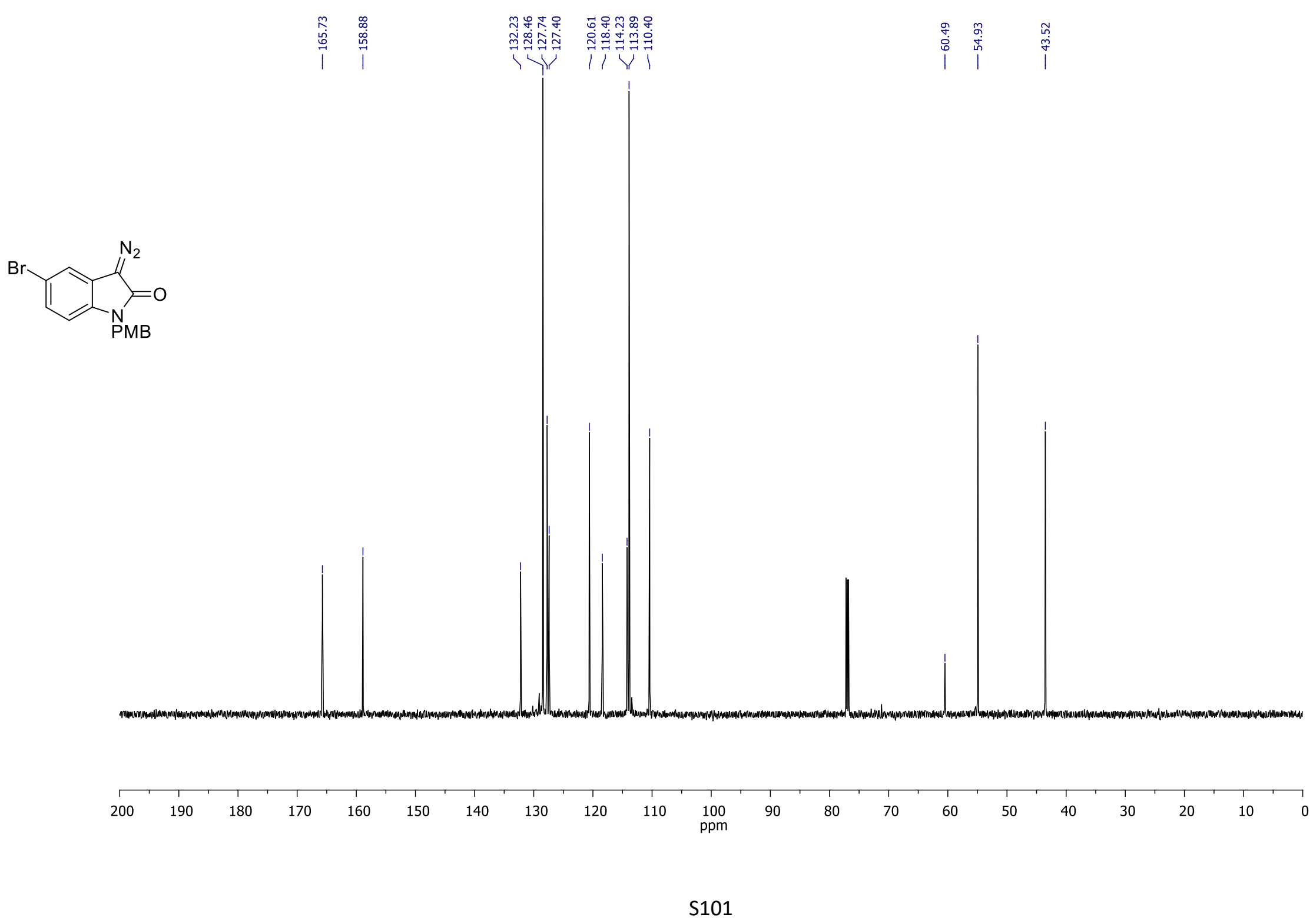


Dimethyl 1'-(4-methoxybenzyl)-2'-oxo-1',2'-dihydro-2H-spiro[cyclopropane-1,3'-indole]-2,2-dicarboxylate (2a)

${ }^{1} \mathrm{H} \mathrm{NMR}\left(\mathrm{CDCl}_{3}, 600 \mathrm{MHz}\right)$

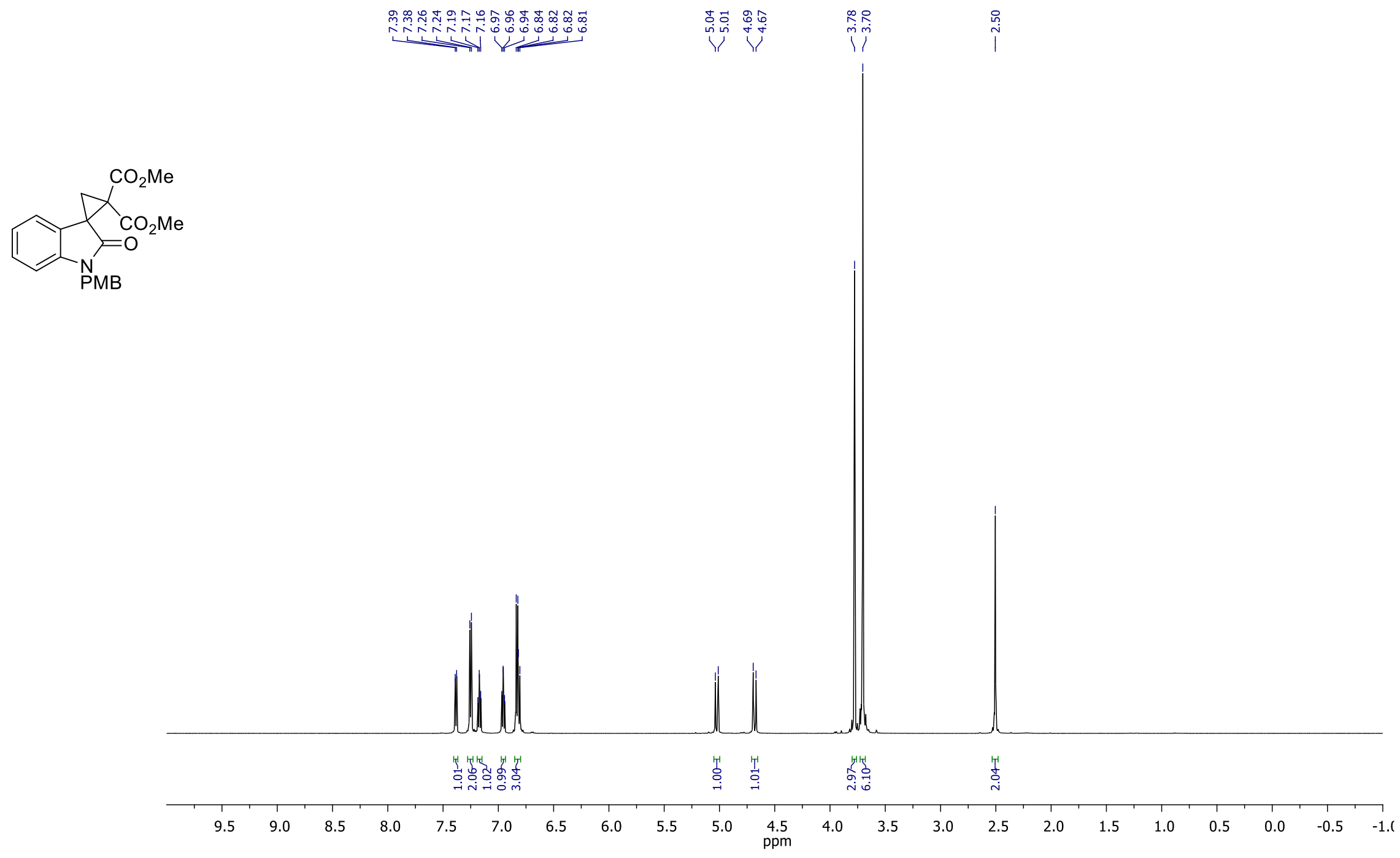


Dimethyl 1'-(4-methoxybenzyl)-2'-oxo-1',2'-dihydro-2H-spiro[cyclopropane-1,3'-indole]-2,2-dicarboxylate (2a)

${ }^{13} \mathrm{C} \mathrm{NMR}\left(\mathrm{CDCl}_{3}, 150 \mathrm{MHz}\right)$

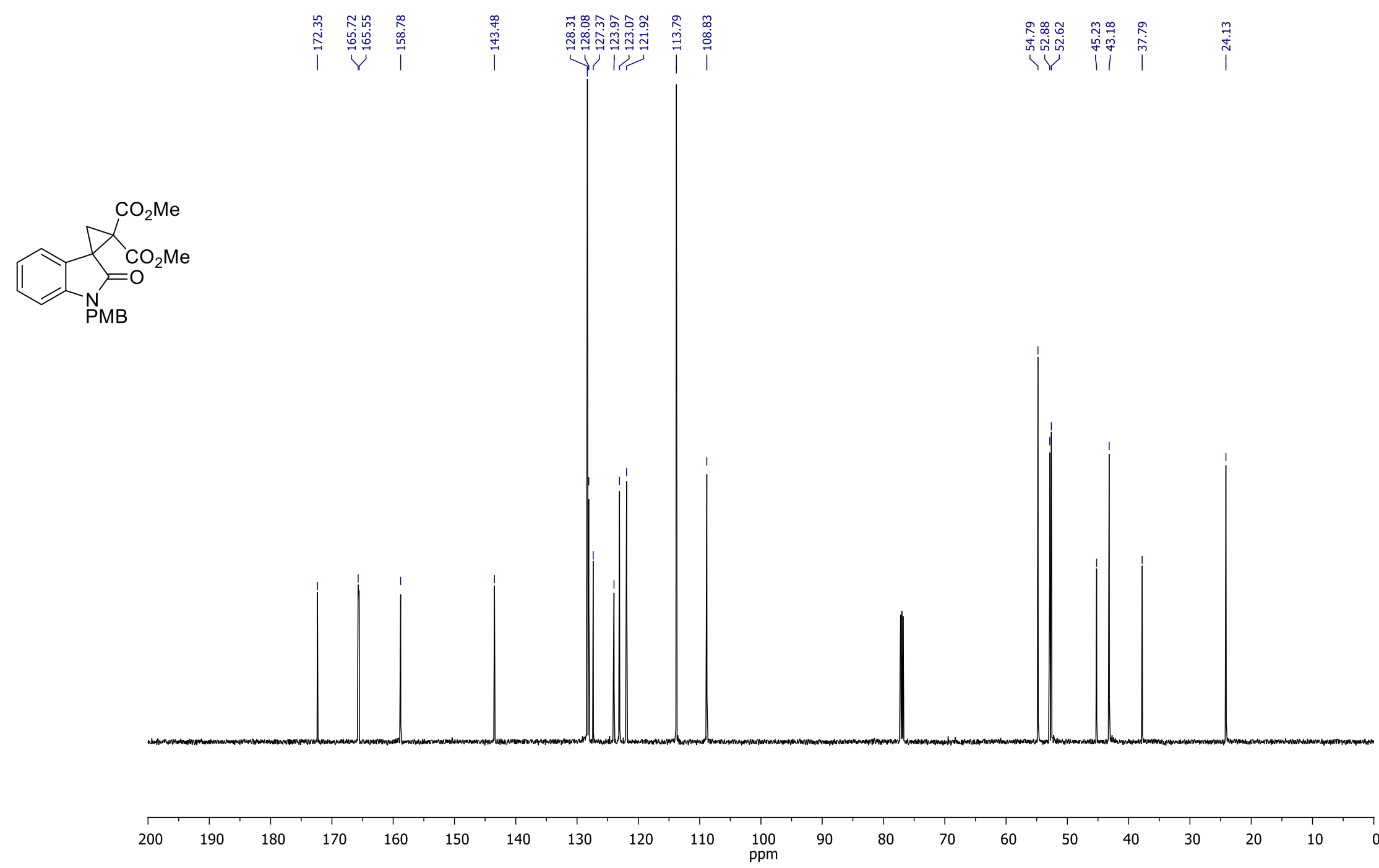


Dimethyl 1'-(4-methoxybenzyl)-5'-methyl-2'-oxo-1',2'-dihydro-2H-spiro[cyclopropane-1,3'-indole]-2,2-dicarboxylate (2b)

${ }^{1} \mathrm{H} \mathrm{NMR}\left(\mathrm{CDCl}_{3}, 600 \mathrm{MHz}\right)$

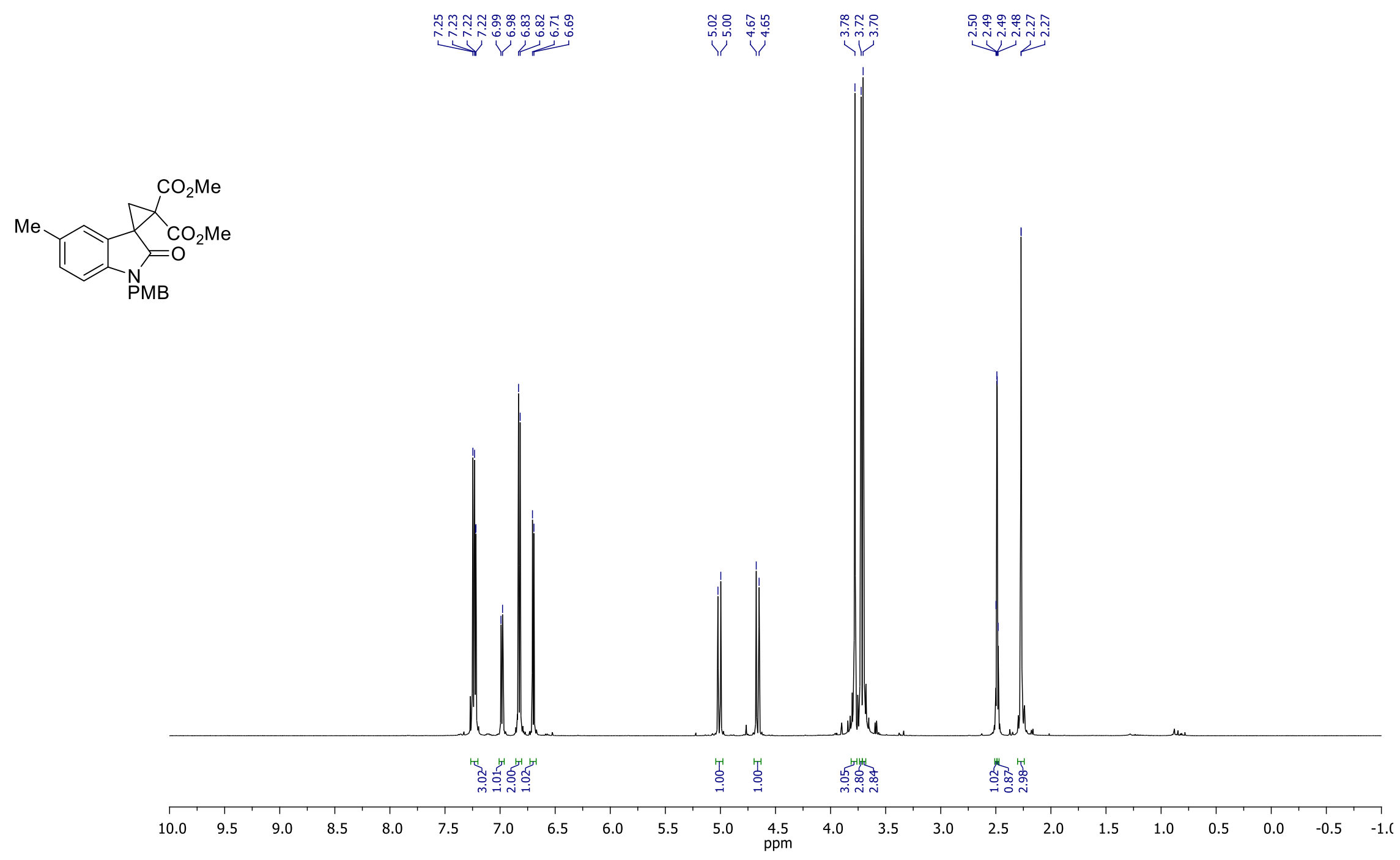


Dimethyl 1'-(4-methoxybenzyl)-5'-methyl-2'-oxo-1',2'-dihydro-2H-spiro[cyclopropane-1,3'-indole]-2,2-dicarboxylate (2b)

${ }^{13} \mathrm{C} \mathrm{NMR}\left(\mathrm{CDCl}_{3}, 150 \mathrm{MHz}\right)$

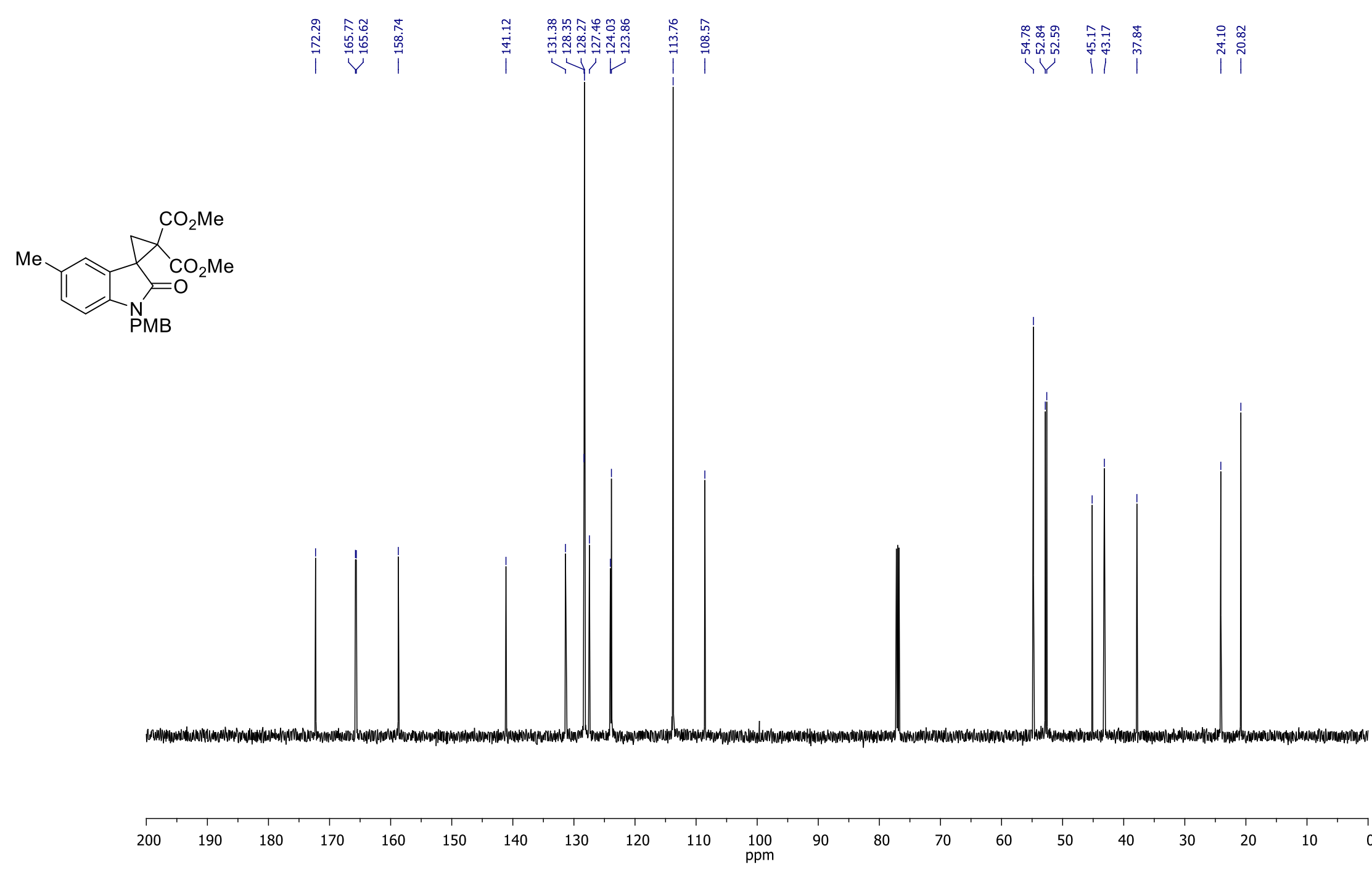


Dimethyl 5'-fluoro-1'-(4-methoxybenzyl)-2'-oxo-1',2'-dihydro-2H-spiro[cyclopropane-1,3'-indole]-2,2-dicarboxylate (2d)

${ }^{1} \mathrm{H} \mathrm{NMR}\left(\mathrm{CDCl}_{3}, 600 \mathrm{MHz}\right)$

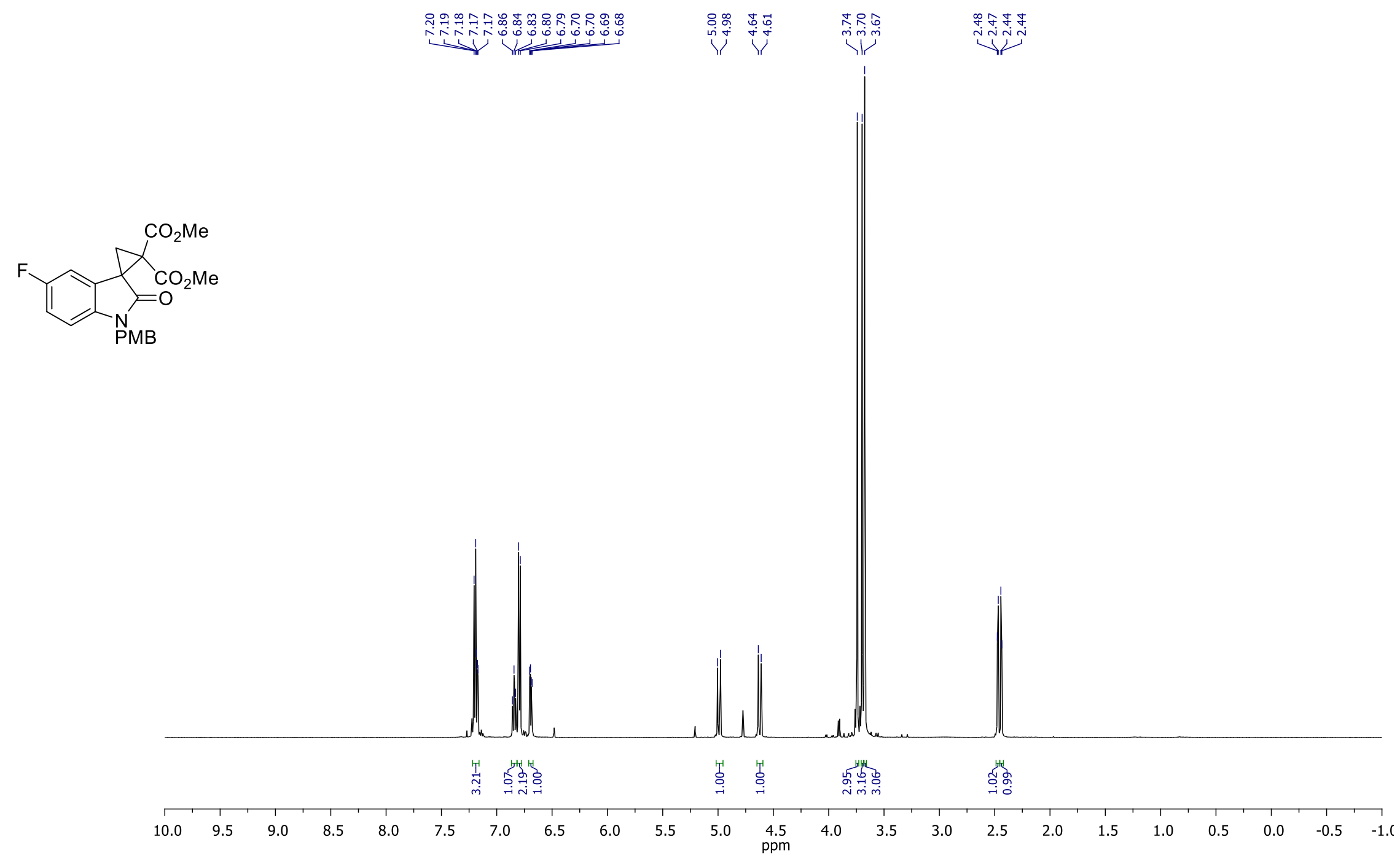


Dimethyl 5' -fluoro-1' -(4-methoxybenzyl)-2' -oxo-1',2'-dihydro-2H-spiro[cyclopropane-1,3'-indole]-2,2-dicarboxylate (2d)

${ }^{13} \mathrm{C} \mathrm{NMR}\left(\mathrm{CDCl}_{3}, 150 \mathrm{MHz}\right)$

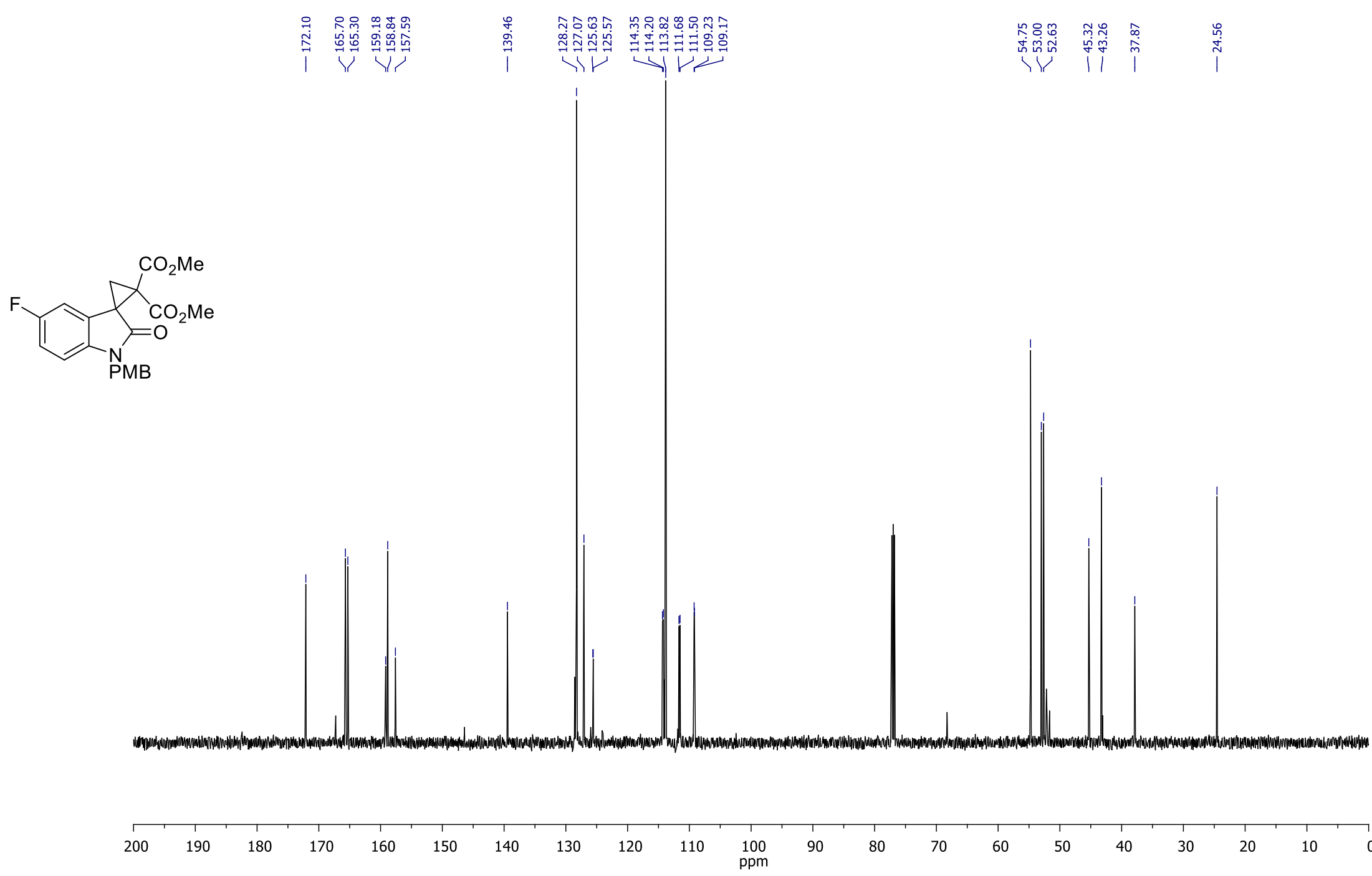


Dimethyl 5' -chloro-1'-(4-methoxybenzyl)-2'-oxo-1',2'-dihydro-2H-spiro[cyclopropane-1,3'-indole]-2,2-dicarboxylate (2e)

${ }^{1} \mathrm{H} \mathrm{NMR}\left(\mathrm{CDCl}_{3}, 600 \mathrm{MHz}\right)$

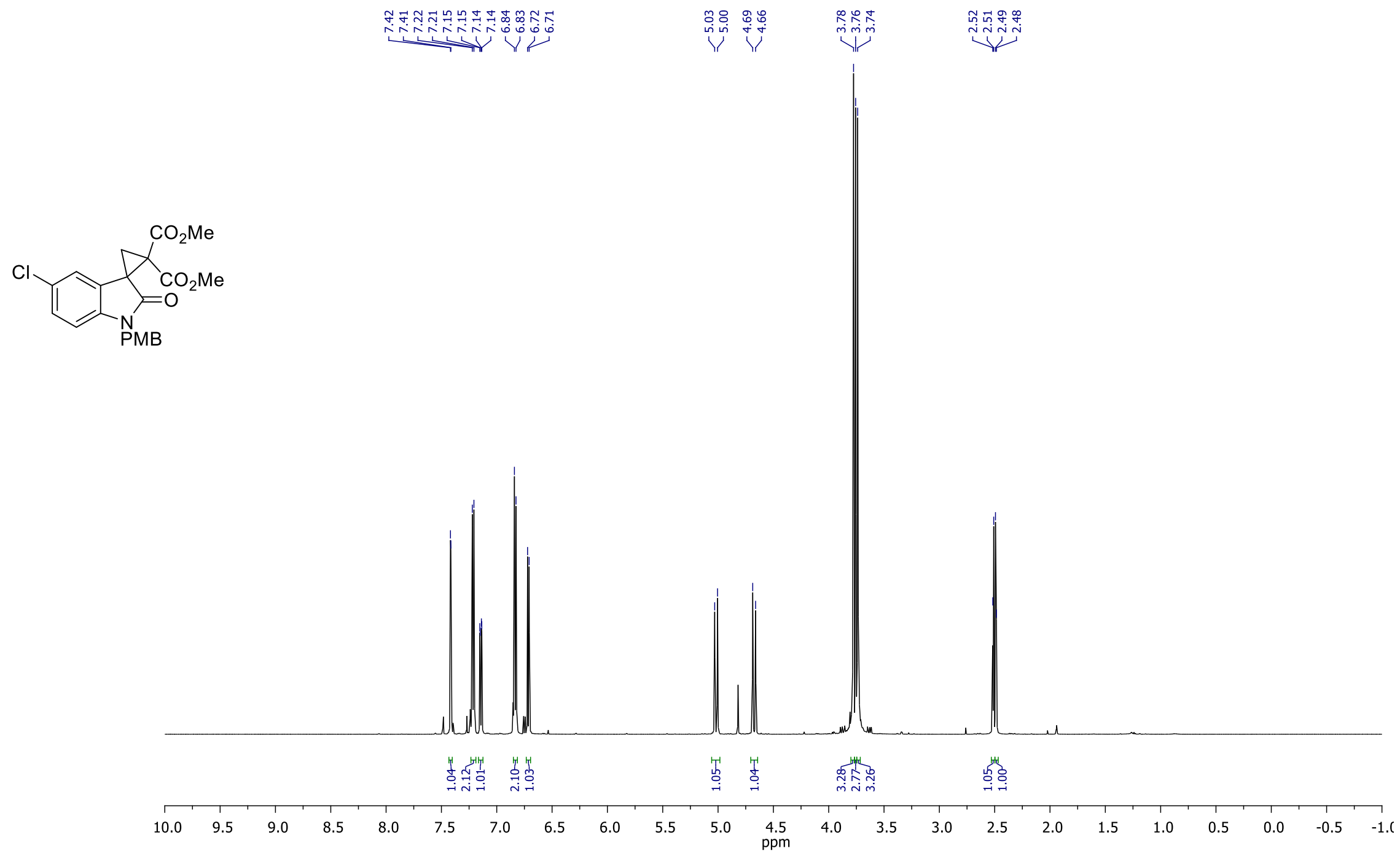


Dimethyl 5'-chloro-1'-(4-methoxybenzyl)-2'-oxo-1',2'-dihydro-2H-spiro[cyclopropane-1,3'-indole]-2,2-dicarboxylate (2e)

${ }^{13} \mathrm{C} \mathrm{NMR}\left(\mathrm{CDCl}_{3}, 150 \mathrm{MHz}\right)$

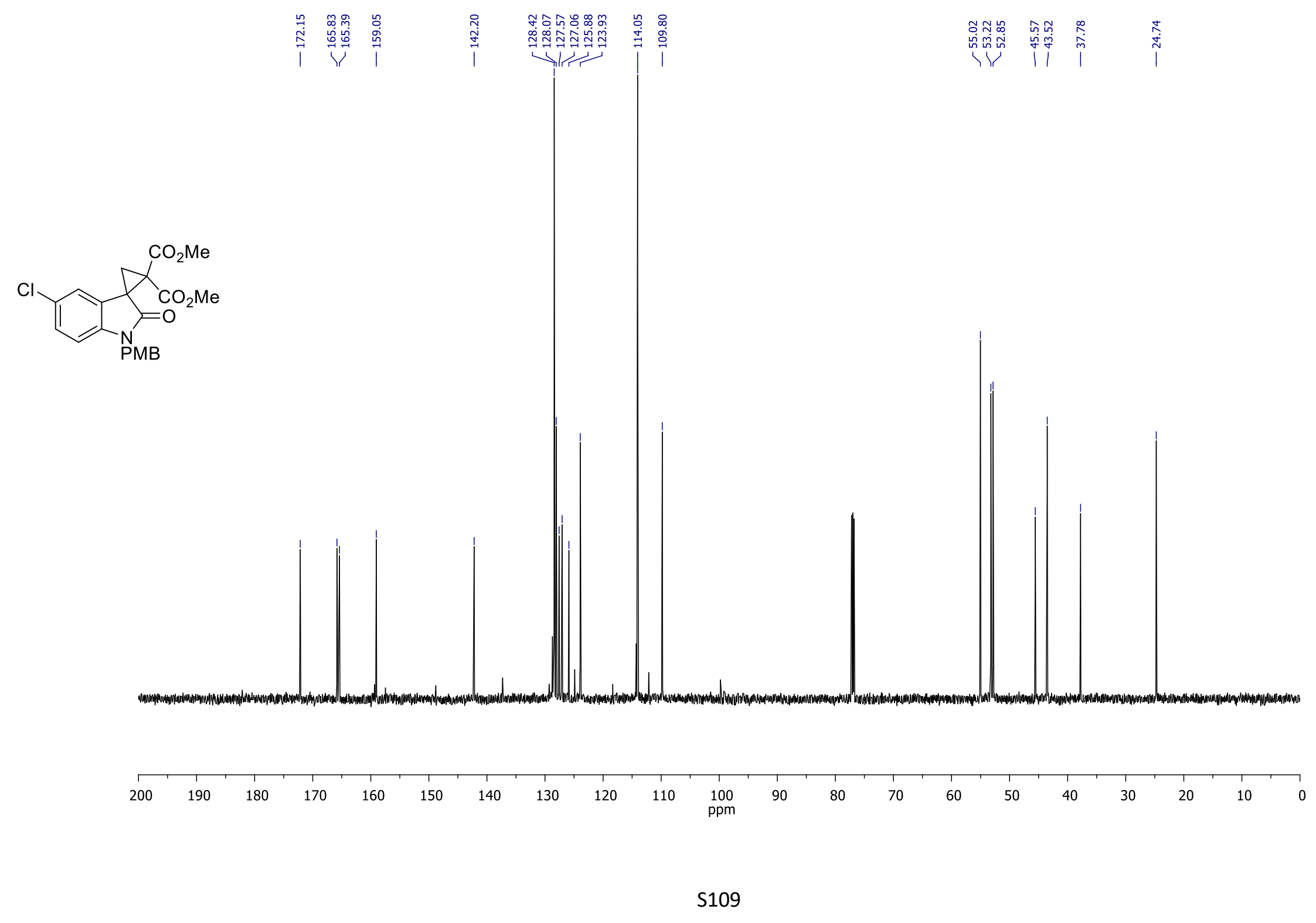


Dimethyl 5' -bromo-1' -(4-methoxybenzyl)-2' -oxo-1',2'-dihydro-2H-spiro[cyclopropane-1,3'-indole]-2,2-dicarboxylate (2f)

${ }^{1} \mathrm{H} \mathrm{NMR}\left(\mathrm{CDCl}_{3}, 600 \mathrm{MHz}\right)$

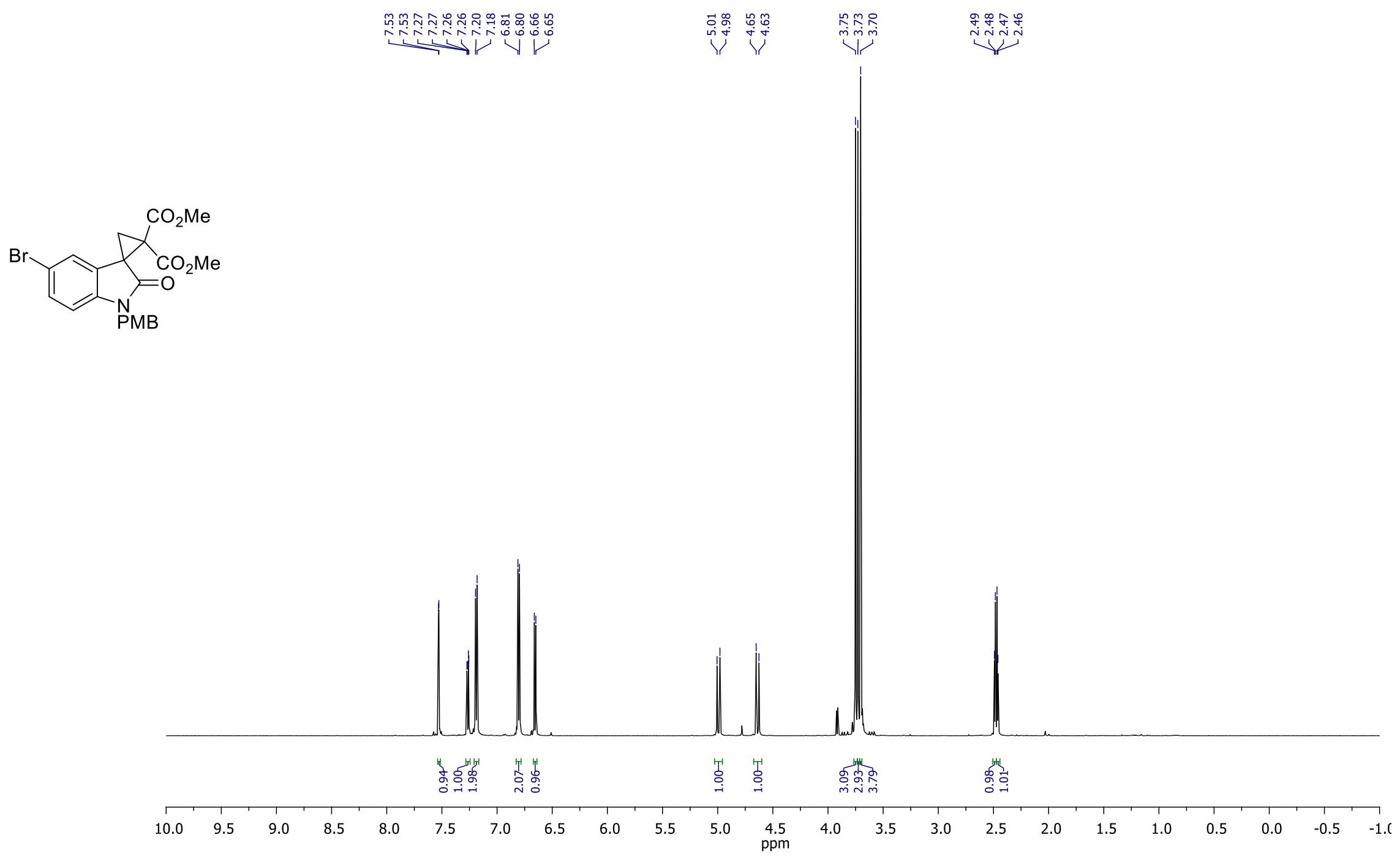


Dimethyl 5'-bromo-1'-(4-methoxybenzyl)-2' -oxo-1',2'-dihydro-2H-spiro[cyclopropane-1,3'-indole]-2,2-dicarboxylate (2f)

${ }^{13} \mathrm{C} \mathrm{NMR}\left(\mathrm{CDCl}_{3}, 150 \mathrm{MHz}\right)$

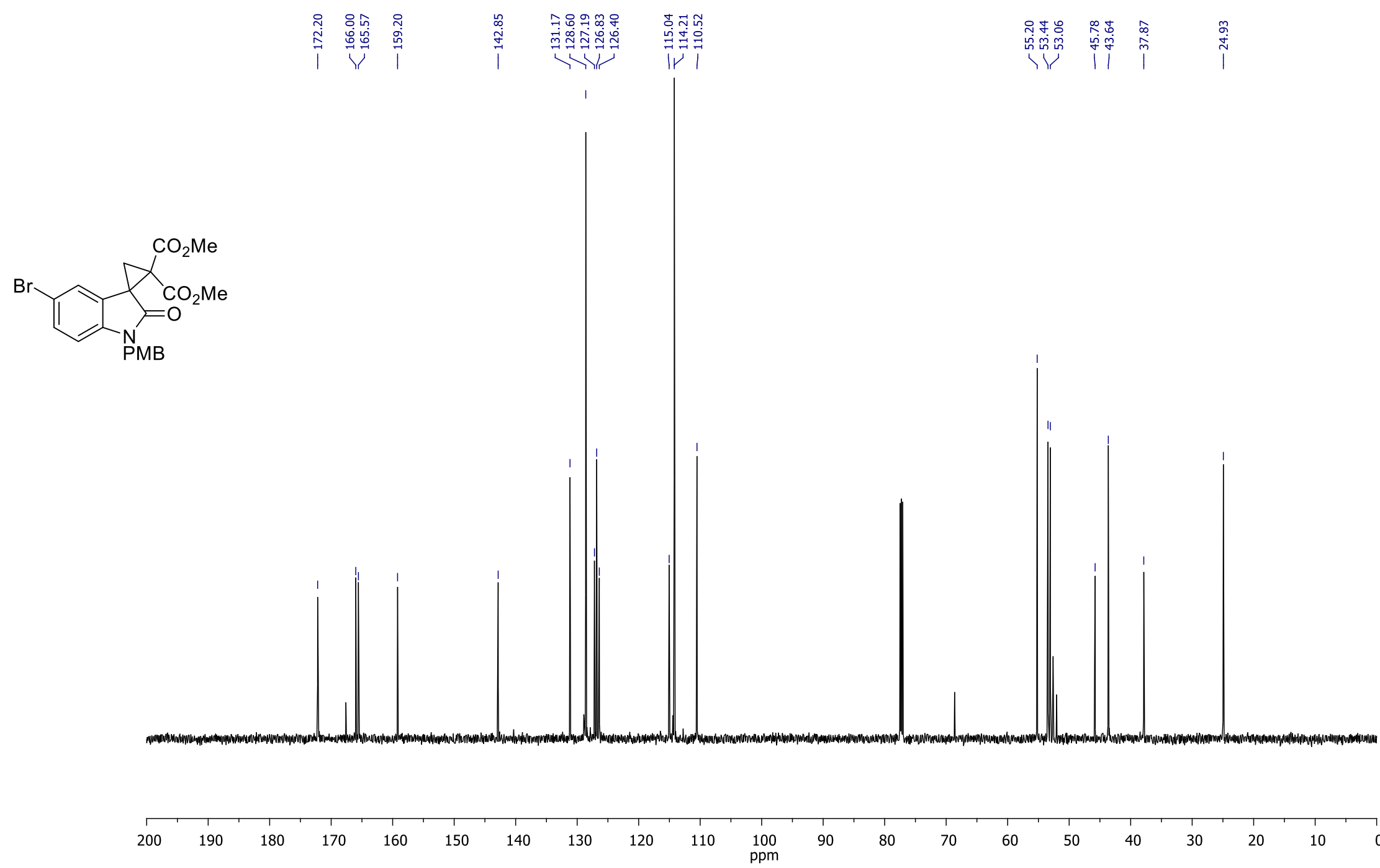


${ }^{1} \mathrm{H} \mathrm{NMR}\left(\mathrm{CDCl}_{3}, 600 \mathrm{MHz}\right)$

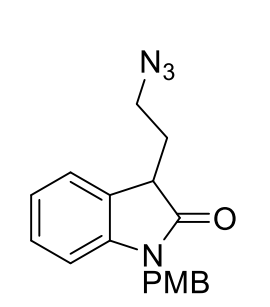

+ starting $1 \mathrm{~b}(20 \mathrm{~mol} \%)$

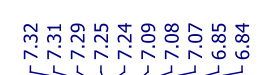

(1)

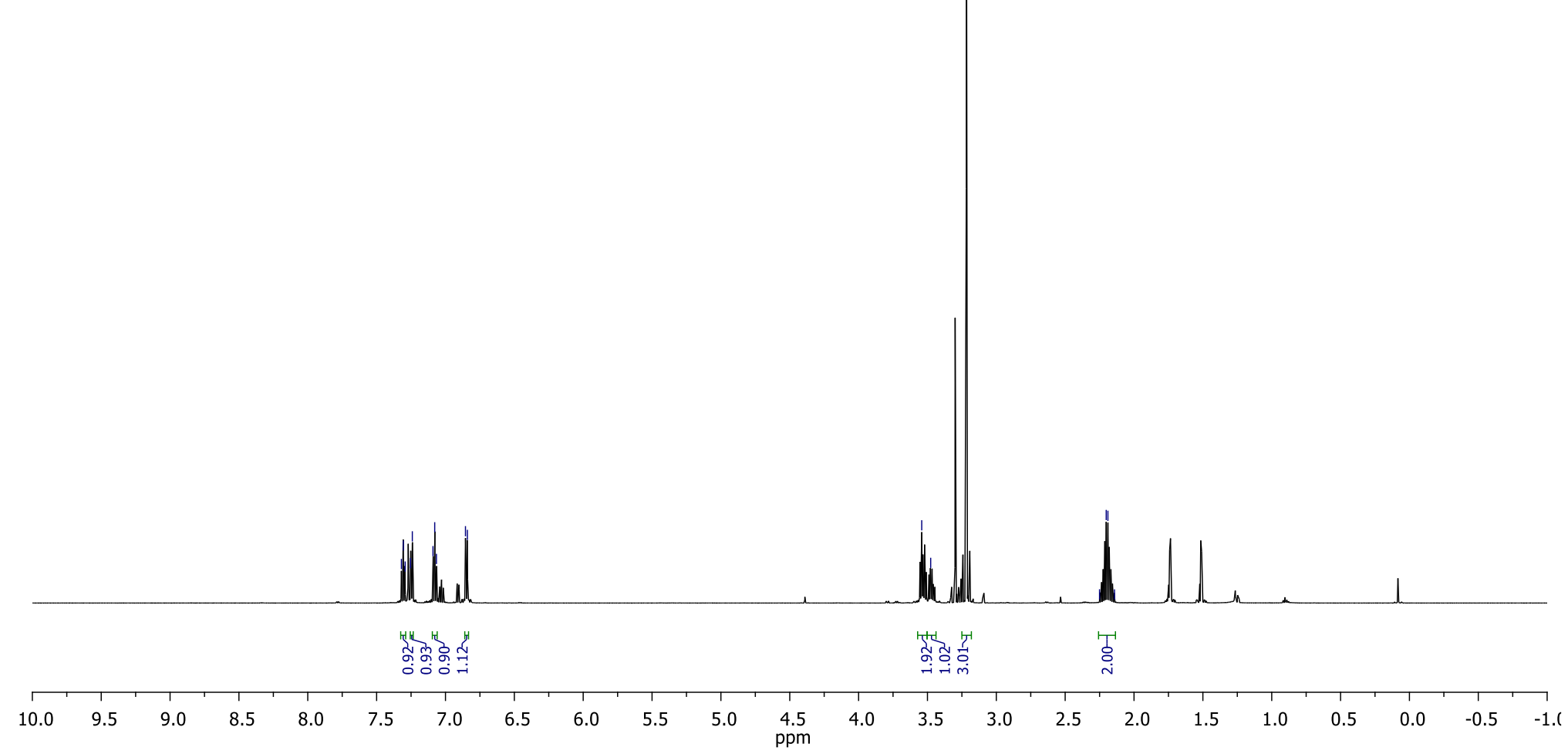


3-(2-Azidoethyl)-1-methyl-1,3-dihydro-2H-indol-2-one (3b)

${ }^{13} \mathrm{C} \mathrm{NMR}\left(\mathrm{CDCl}_{3}, 150 \mathrm{MHz}\right)$<smiles>[Y5]N1C(=O)C(CCN)c2ccccc21</smiles>

+ starting 1 b (20 mol\%)

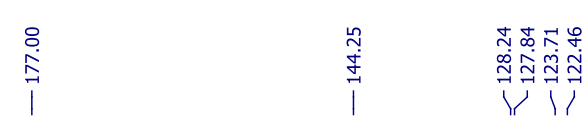

|
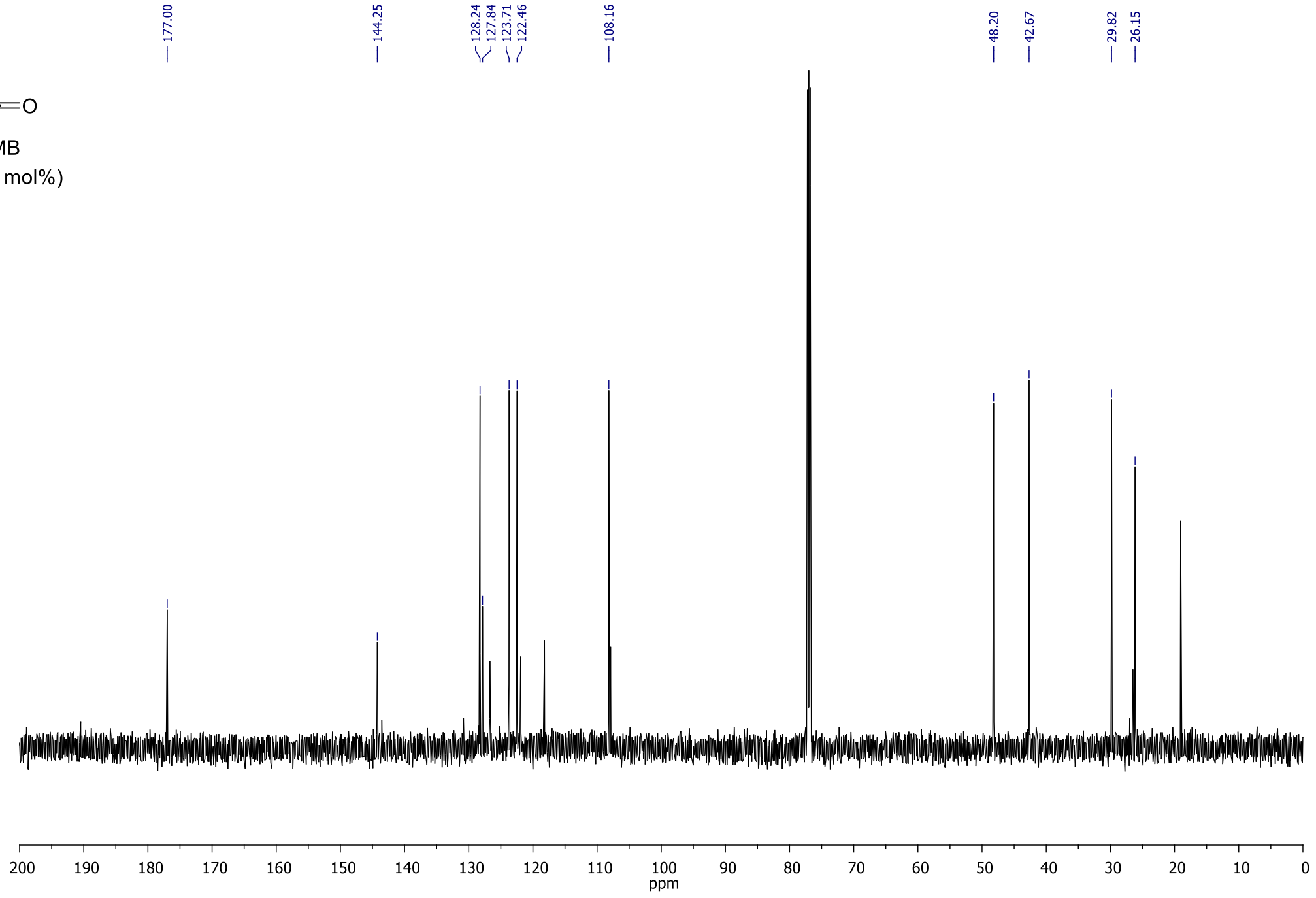

S113 
Dimethyl $\{[3$-azido-1-(4-methoxybenzyl)-2-oxo-2,3-dihydro-1H-indol-3-yl]methyl $\}$ malonate (4a)

${ }^{1} \mathrm{H}$ NMR $\left(\mathrm{CDCl}_{3}, 600 \mathrm{MHz}\right)$

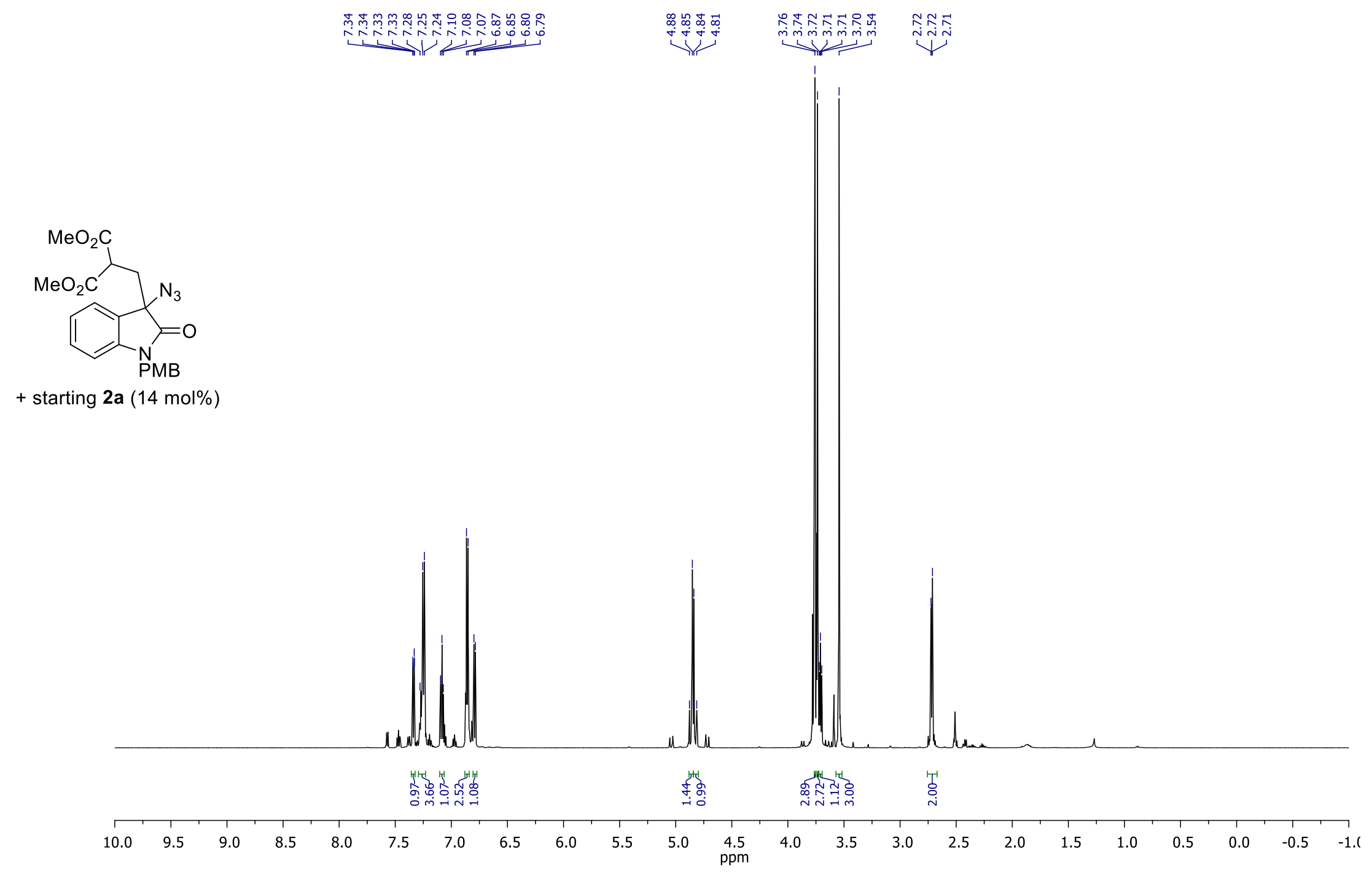


Dimethyl $\{[3$-azido-1-(4-methoxybenzyl)-2-oxo-2,3-dihydro-1H-indol-3-yl]methyl $\}$ malonate (4a)

${ }^{13} \mathrm{C} \mathrm{NMR}\left(\mathrm{CDCl}_{3}, 150 \mathrm{MHz}\right)$

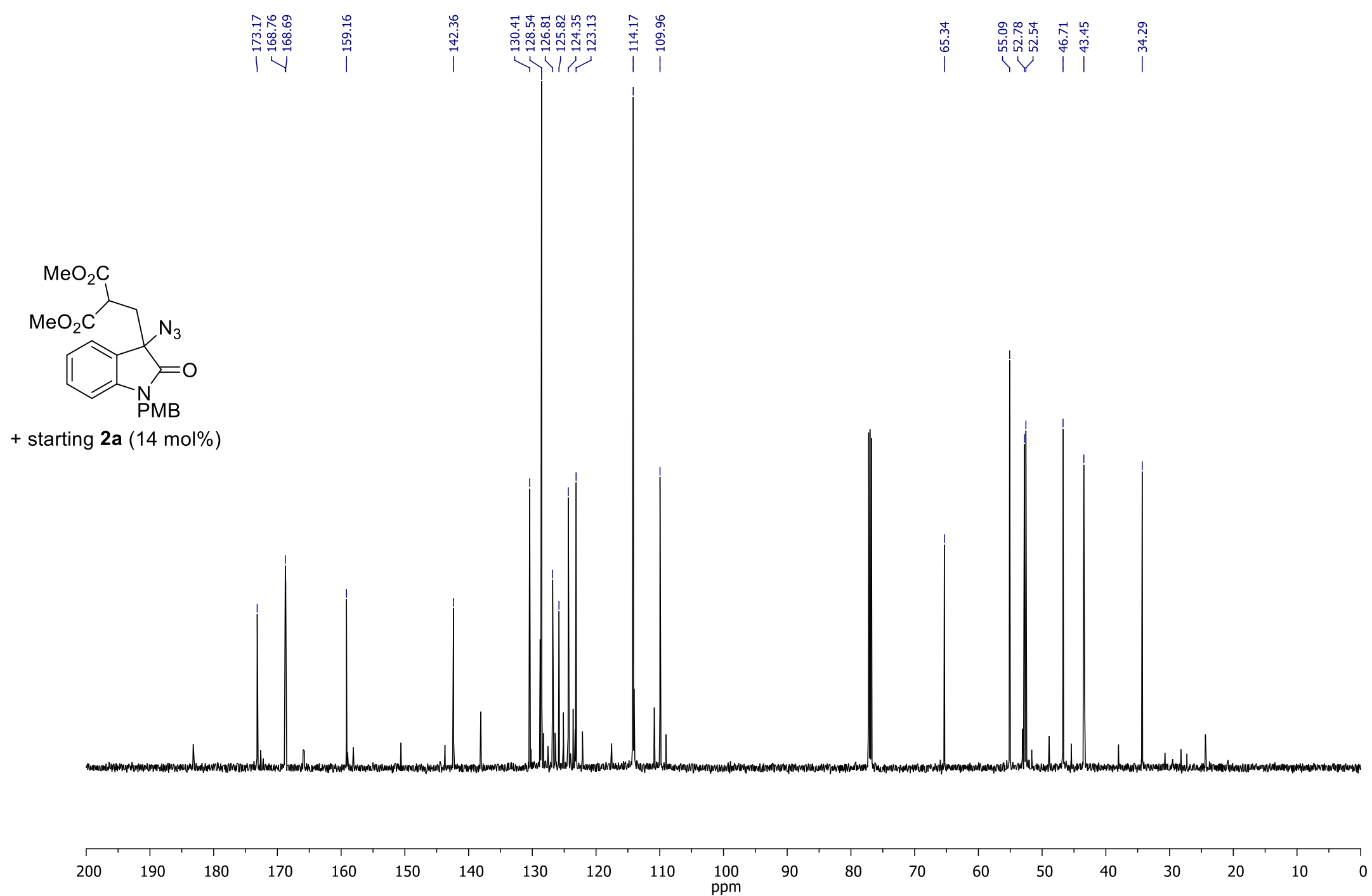


Dimethyl \{[3-azido-1-(4-methoxybenzyl)-5-methyl-2-oxo-2,3-dihydro-1H-indol-3-yl]methyl\}malonate (4b)

${ }^{1} \mathrm{H}$ NMR $\left(\mathrm{CDCl}_{3}, 600 \mathrm{MHz}\right)$

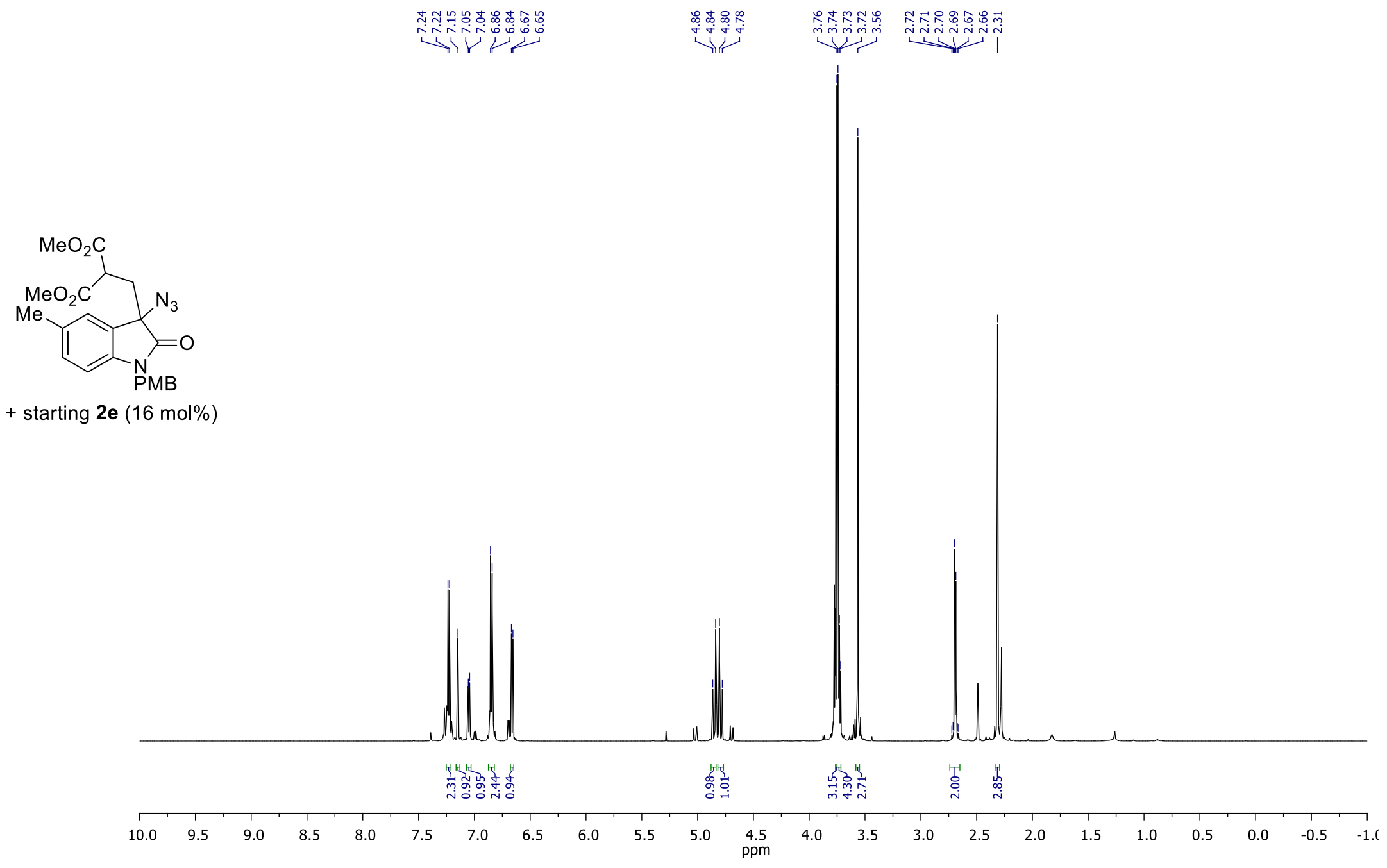


Dimethyl $\{[3$-azido-1-(4-methoxybenzyl)-5-methyl-2-oxo-2,3-dihydro-1H-indol-3-yl]methyl $\}$ malonate (4b)

${ }^{13} \mathrm{C} \mathrm{NMR}\left(\mathrm{CDCl}_{3}, 150 \mathrm{MHz}\right)$
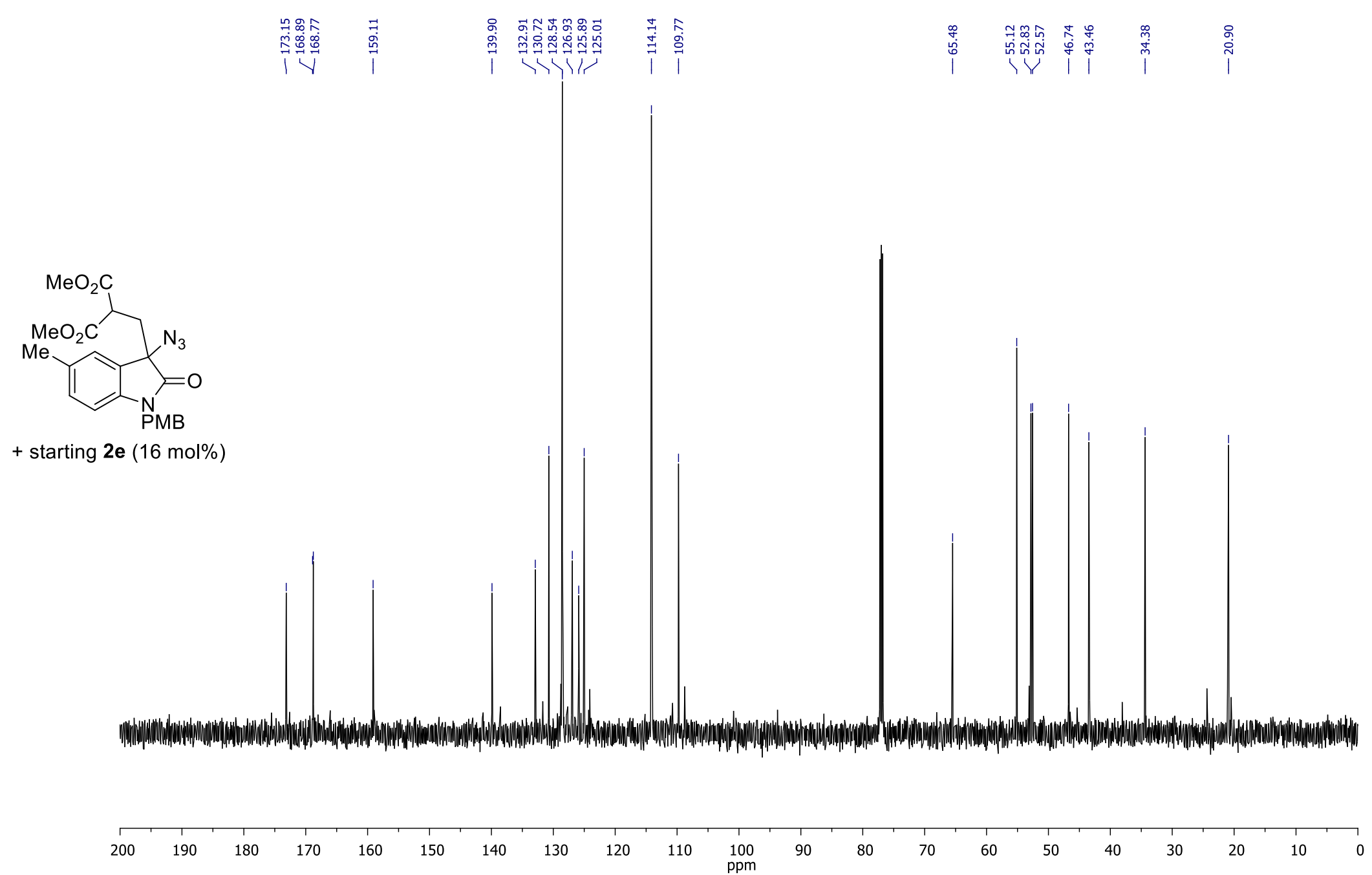
Dimethyl $\{[3-a z i d o-1-m e t h y l-2-o x o-2,3-d i h y d r o-1 H$-indol-3-yl $]$ methyl $\}$ malonate $(4 c)$

${ }^{1} \mathrm{H}$ NMR $\left(\mathrm{CDCl}_{3}, 600 \mathrm{MHz}\right)$

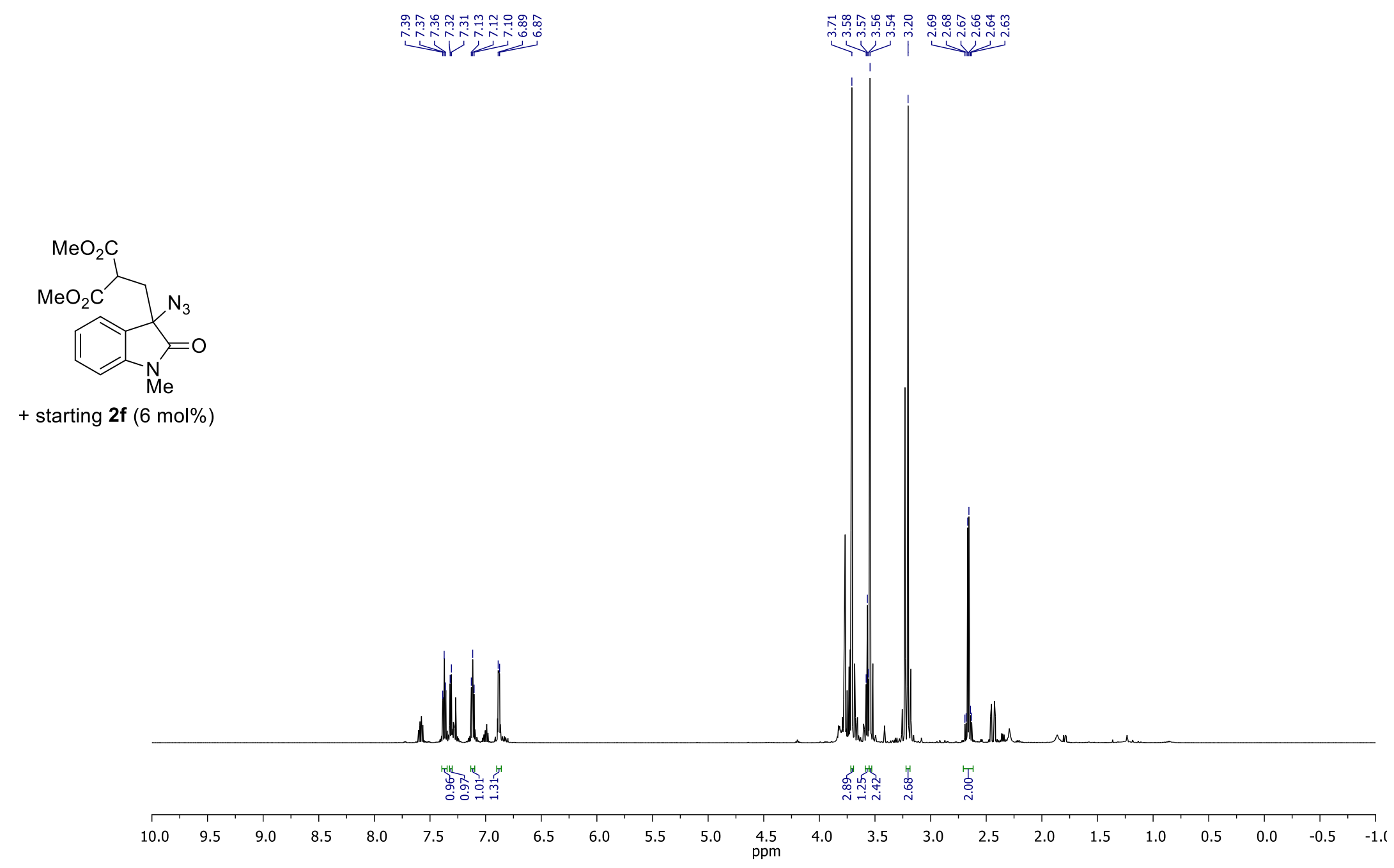


Dimethyl $\{[3$-azido-1-methyl-2-oxo-2,3-dihydro-1H-indol-3-yl]methyl $\}$ malonate $(4 c)$

${ }^{13} \mathrm{C} \mathrm{NMR}\left(\mathrm{CDCl}_{3}, 150 \mathrm{MHz}\right)$
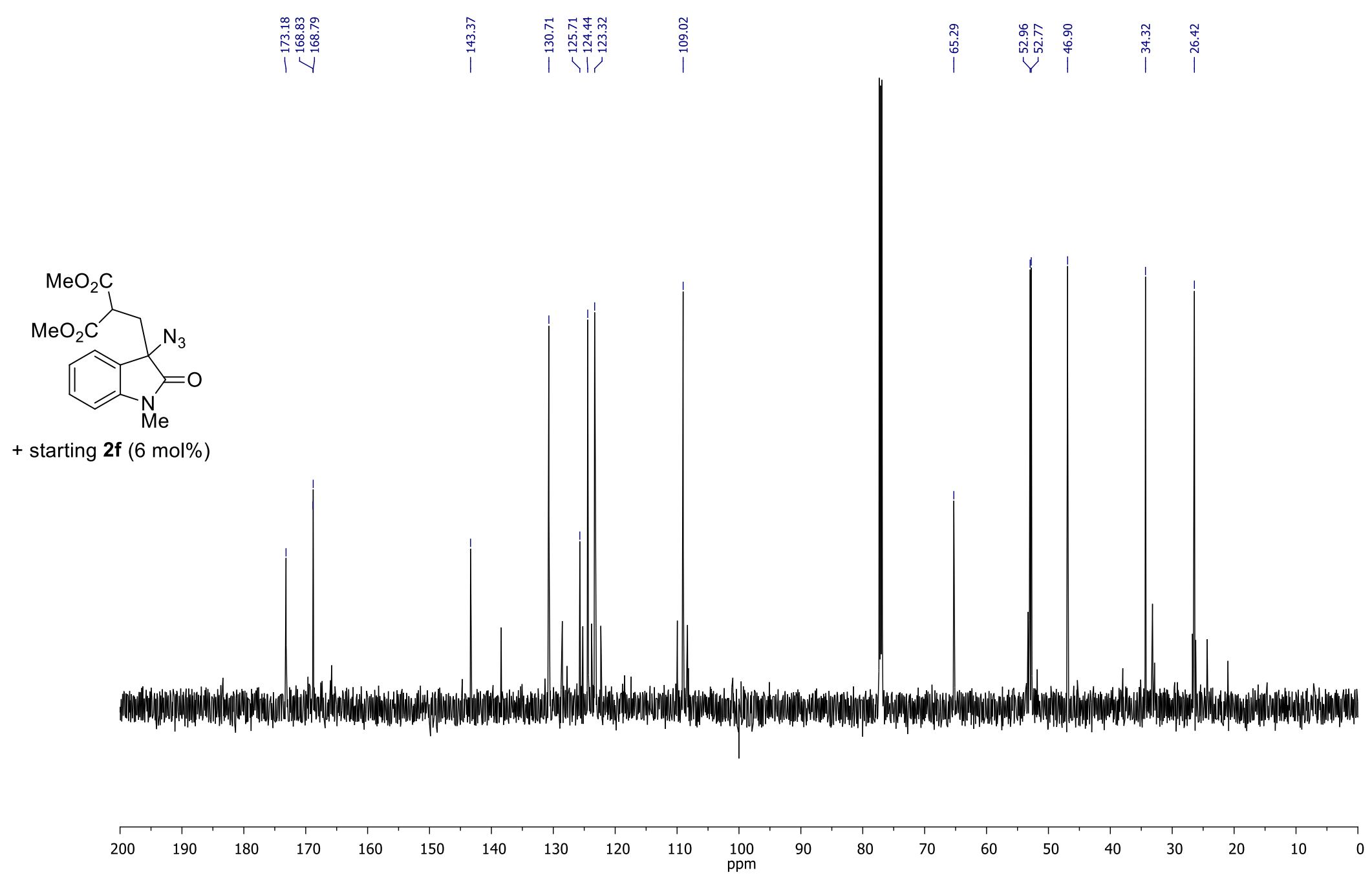
Methyl 3-(3-azido-1-methyl-2-oxo-2,3-dihydro-1H-indol-3-yl)propanoate $\left(4 \mathrm{c}^{\prime}\right)$

${ }^{1} \mathrm{H} \mathrm{NMR}\left(\mathrm{CDCl}_{3}, 600 \mathrm{MHz}\right)$

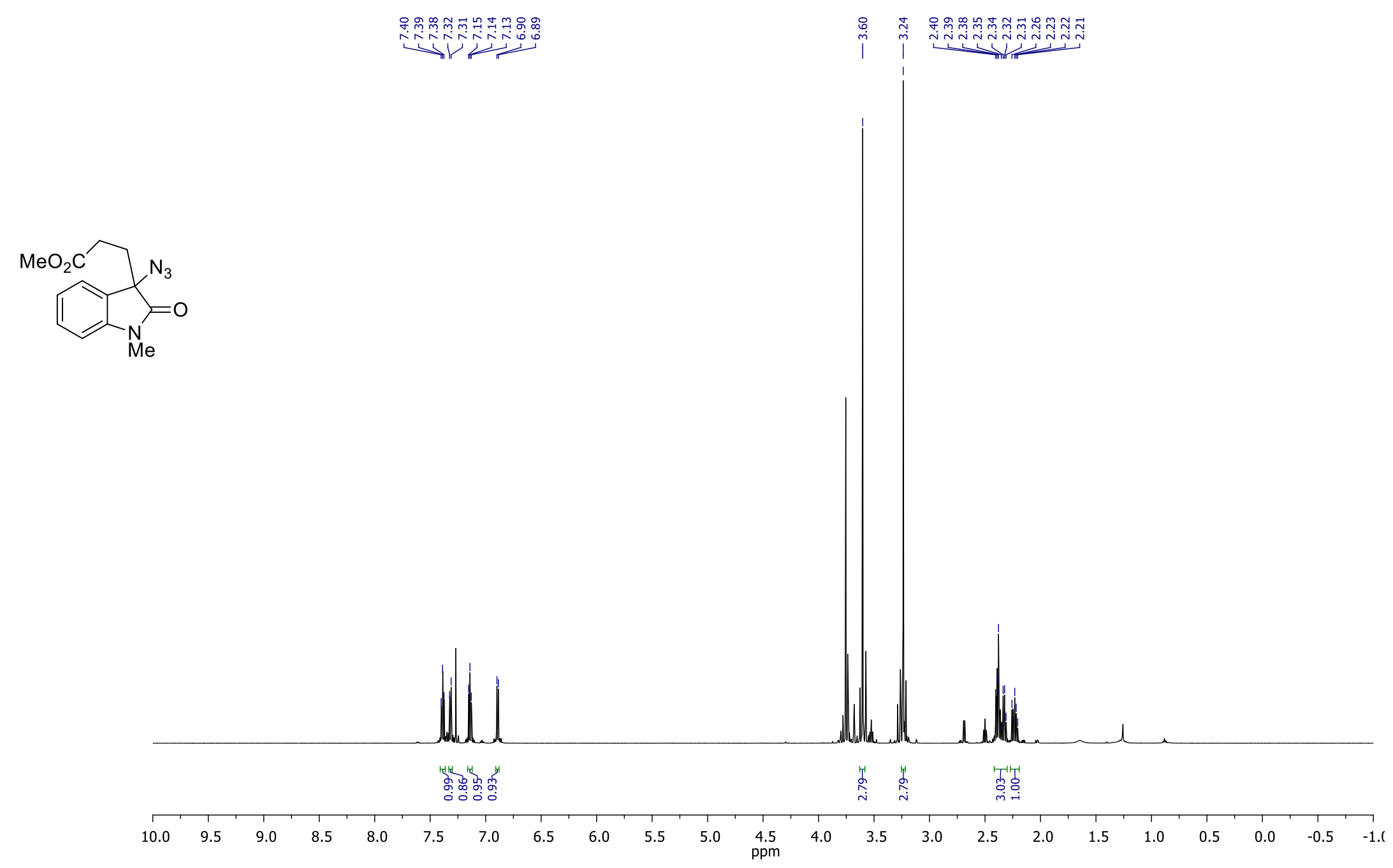


Methyl 3-(3-azido-1-methyl-2-oxo-2,3-dihydro-1H-indol-3-yl)propanoate (4c')

${ }^{13} \mathrm{C} \mathrm{NMR}\left(\mathrm{CDCl}_{3}, 150 \mathrm{MHz}\right)$

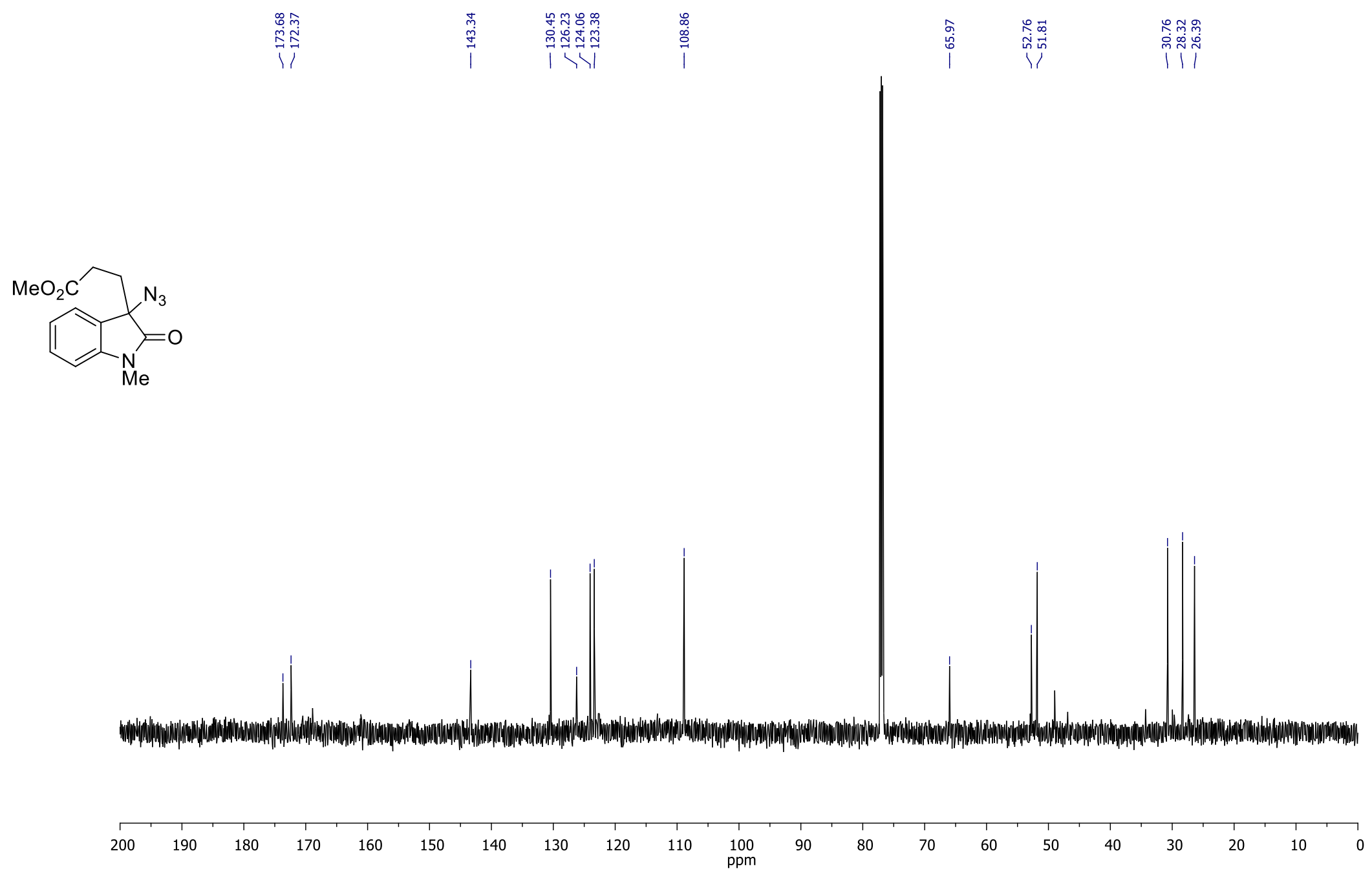


${ }^{1} \mathrm{H}$ NMR $\left(\mathrm{CDCl}_{3}, 600 \mathrm{MHz}\right)$

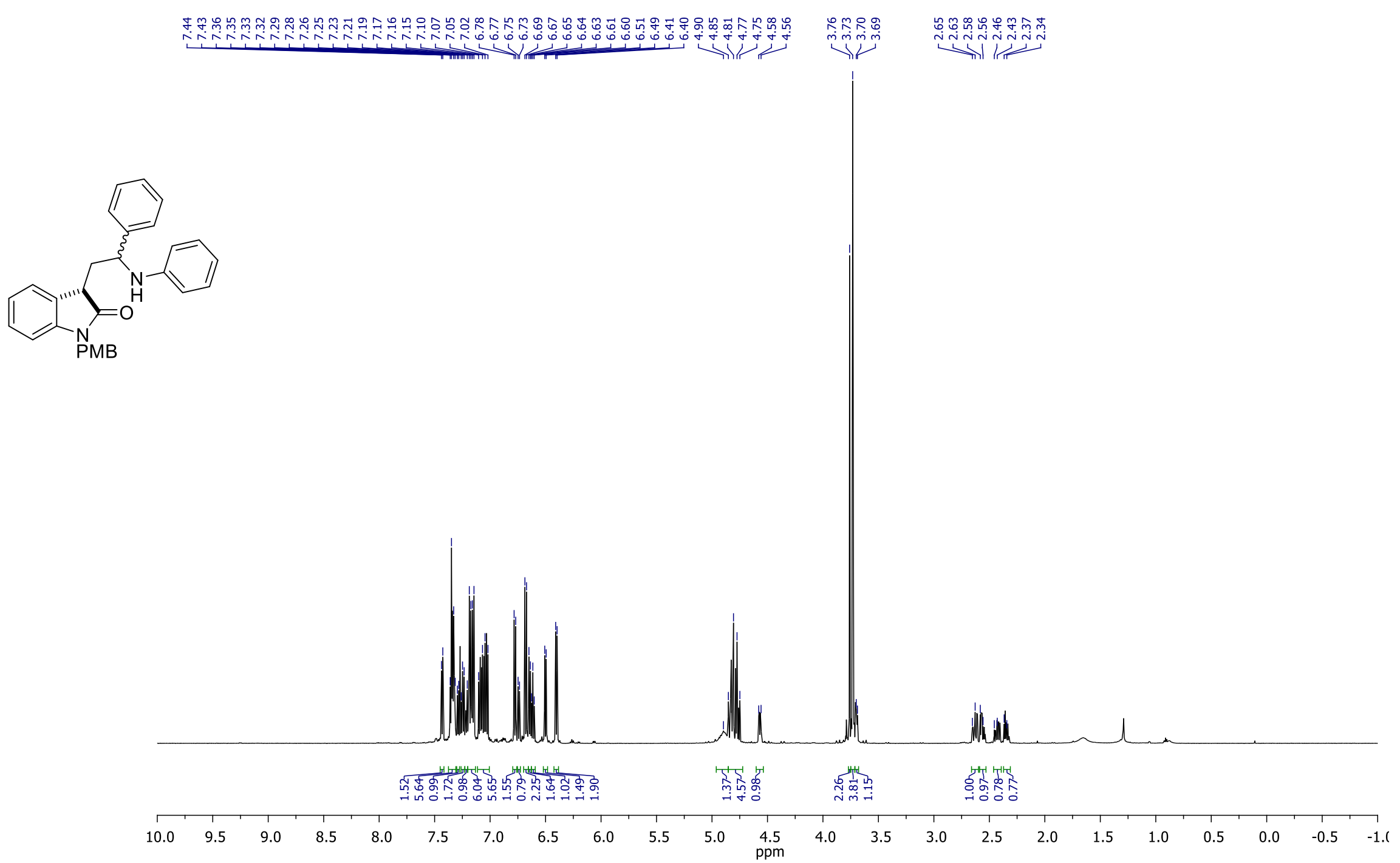


1-(4-Methoxybenzyl)-3-[2-(phenylamino)-2-phenylethyl]-1,3-dihydro-2H-indol-2-one (5)

${ }^{13} \mathrm{C} \mathrm{NMR}\left(\mathrm{CDCl}_{3}, 150 \mathrm{MHz}\right)$
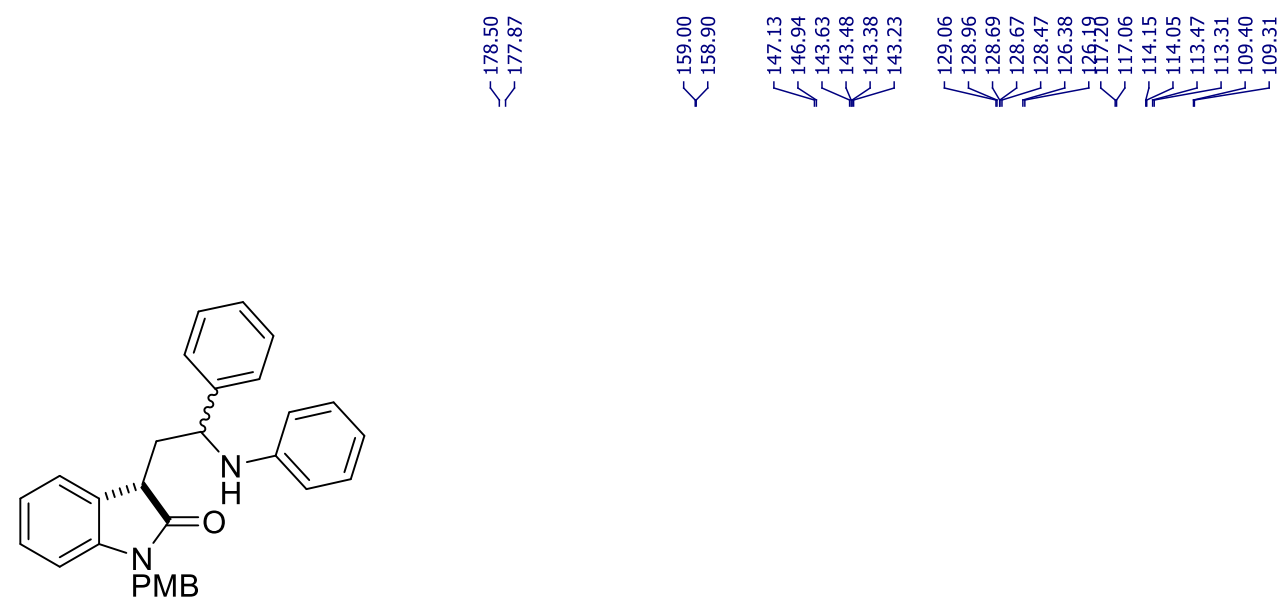

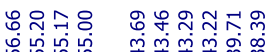

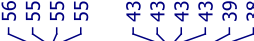

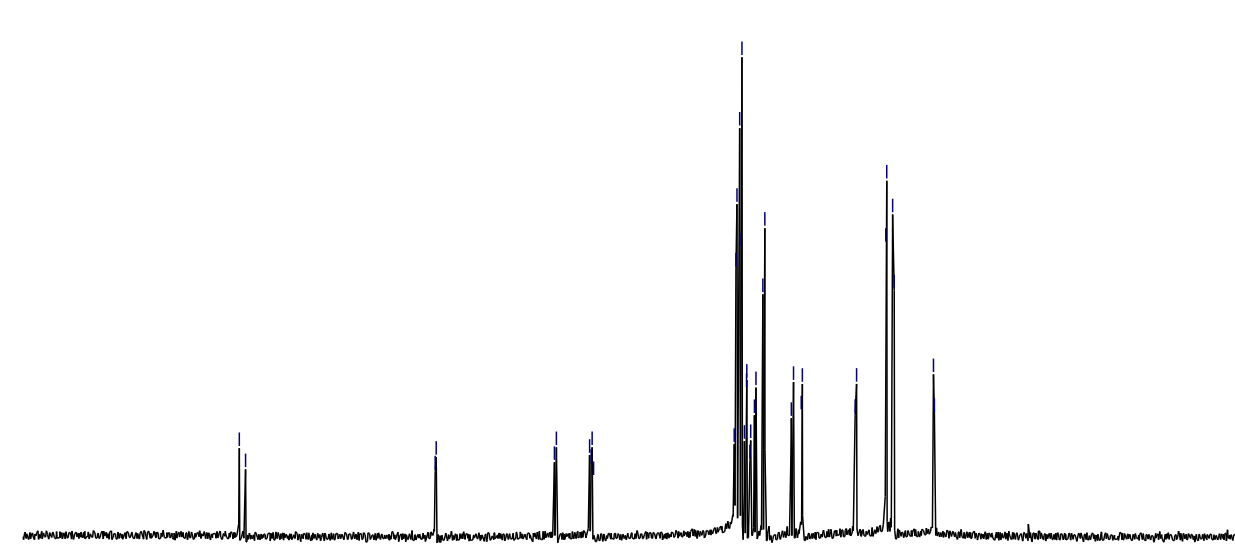


3-Hydroxy-1-(4-methoxybenzyl)-3-[2-(phenylamino)-2-phenylethyl]-1,3-dihydro-2H-indol-2-one (6)

${ }^{1} \mathrm{H} \mathrm{NMR}\left(\mathrm{CDCl}_{3}, 600 \mathrm{MHz}\right)$

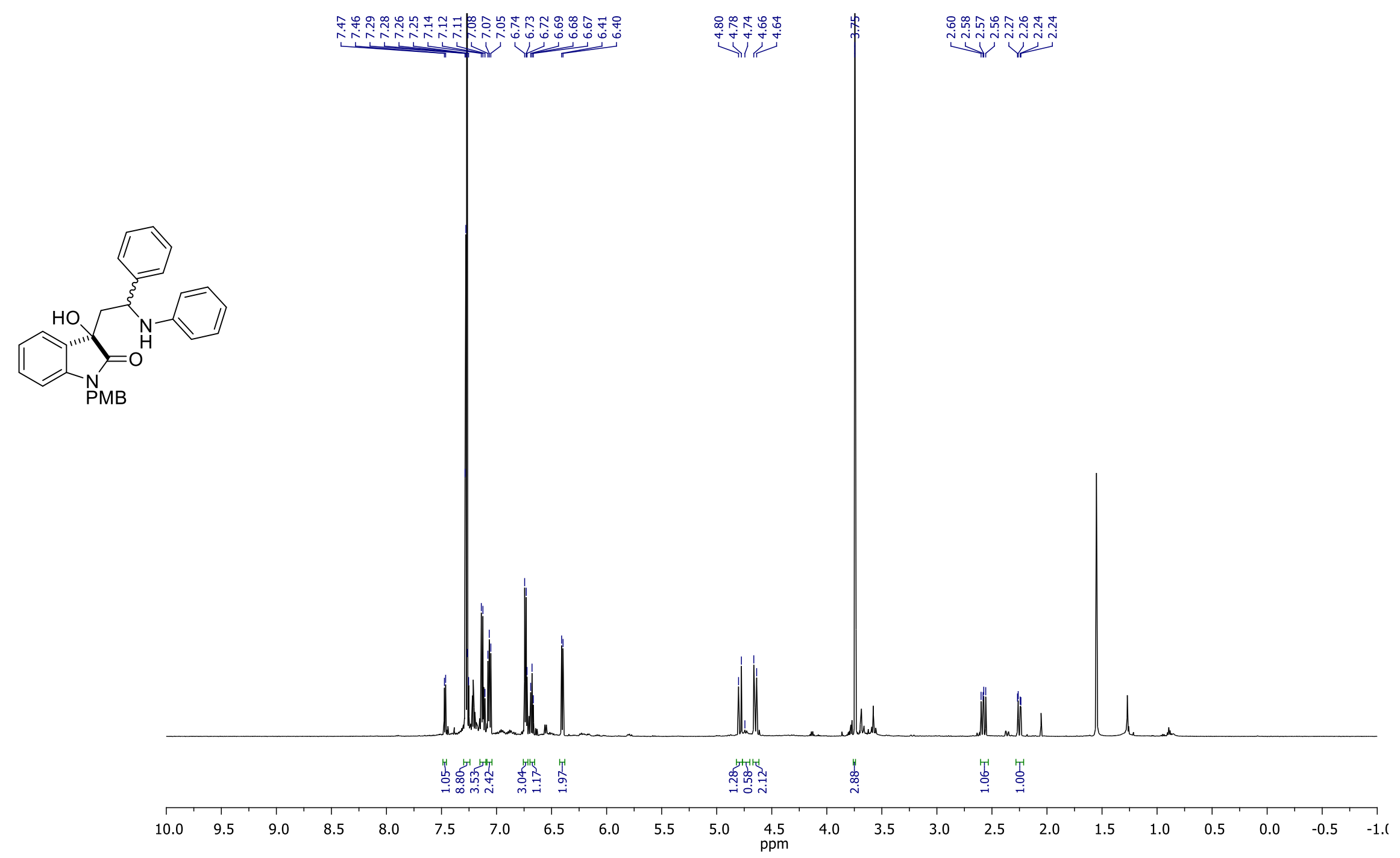


${ }^{13} \mathrm{C} \mathrm{NMR}\left(\mathrm{CDCl}_{3}, 150 \mathrm{MHz}\right)$

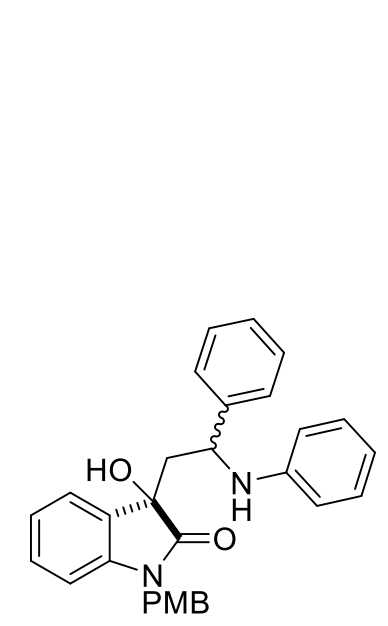

| l|
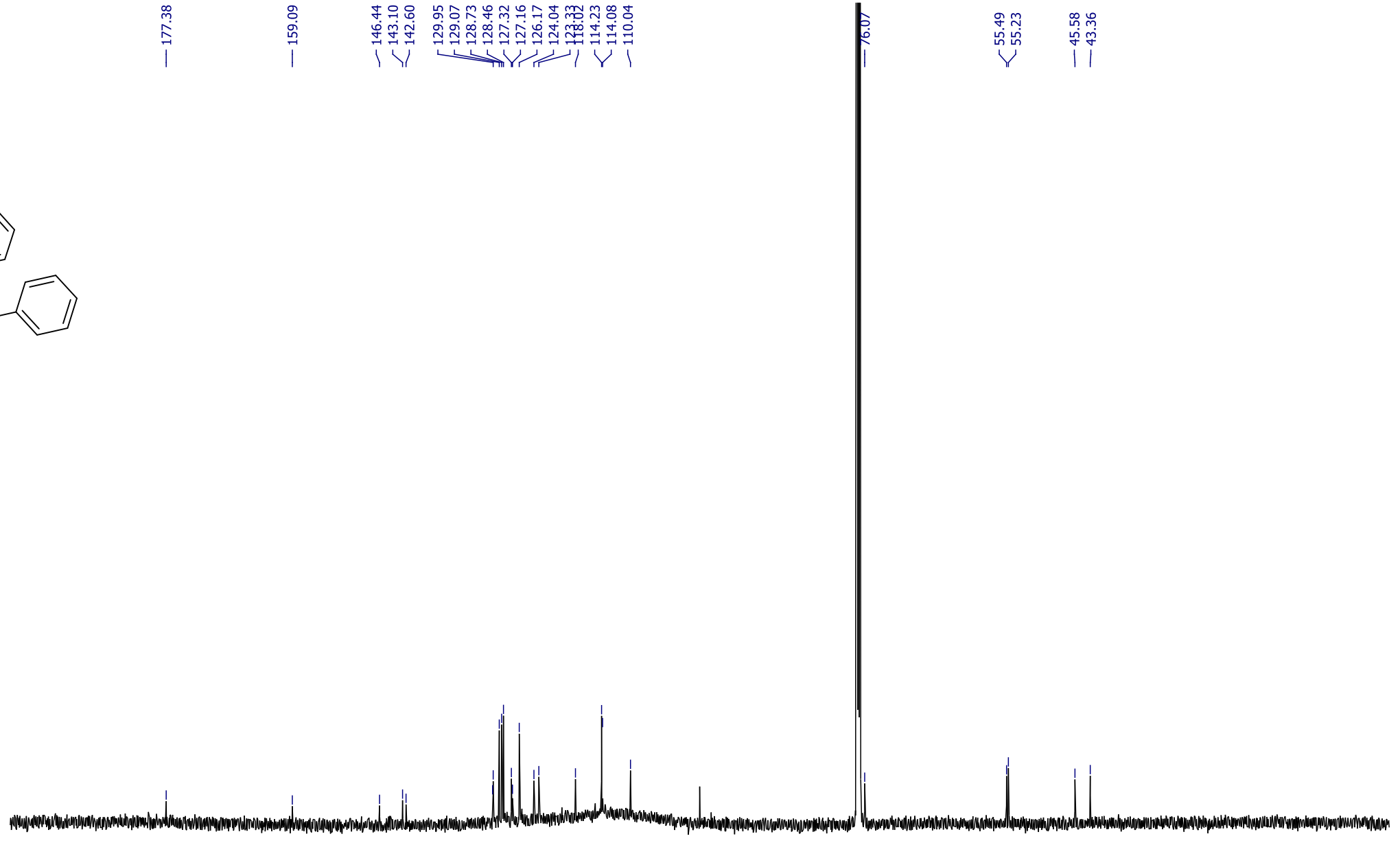

200

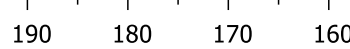

$160 \quad 150 \quad 140 \quad 130 \quad 120$

$110 \quad 100$

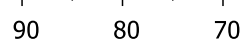

60

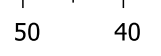

30

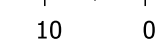


Dimethyl \{[1-(4-methoxybenzyl)-2-oxo-3-(phenylamino)-2,3-dihydro-1H-indol-3-yl]methyl $\}$ malonate (7a)

${ }^{1} \mathrm{H}$ NMR $\left(\mathrm{CDCl}_{3}, 600 \mathrm{MHz}\right)$

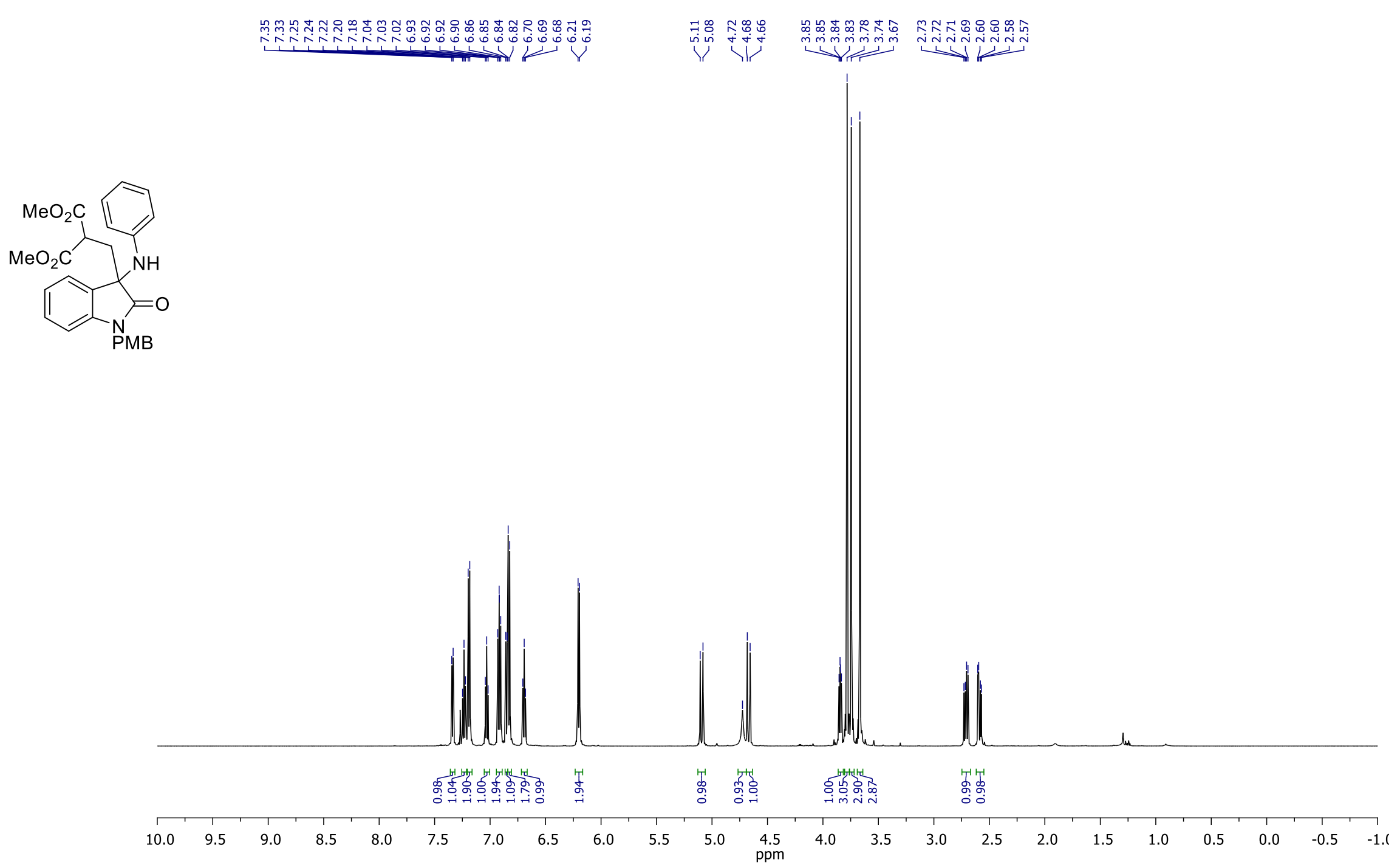


Dimethyl \{[1-(4-methoxybenzyl)-2-oxo-3-(phenylamino)-2,3-dihydro-1H-indol-3-yl]methyl $\}$ malonate (7a)

${ }^{13} \mathrm{C} \mathrm{NMR}\left(\mathrm{CDCl}_{3}, 150 \mathrm{MHz}\right)$

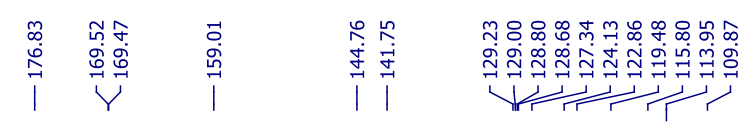

|

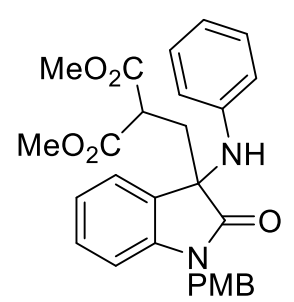

200 
Dimethyl \{[1-(4-methoxybenzyl)-5-methyl-2-oxo-3-(phenylamino)-2,3-dihydro-1H-indol-3-yl]methyl $\}$ malonate (7b)

${ }^{1} \mathrm{H} \mathrm{NMR}\left(\mathrm{CDCl}_{3}, 600 \mathrm{MHz}\right)$

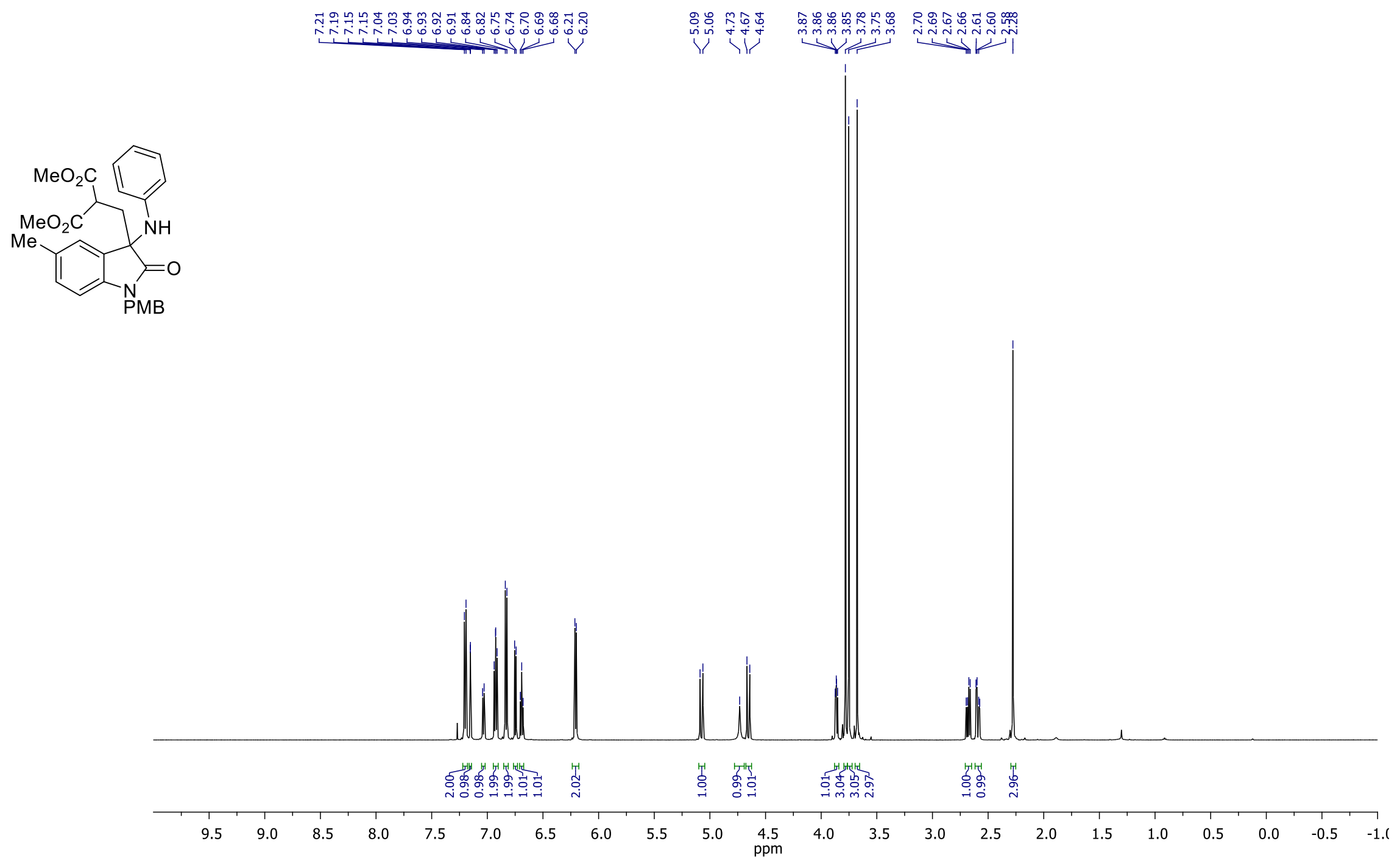


Dimethyl \{[1-(4-methoxybenzyl)-5-methyl-2-oxo-3-(phenylamino)-2,3-dihydro-1H-indol-3-yl]methyl $\}$ malonate (7b)

${ }^{13} \mathrm{C} \mathrm{NMR}\left(\mathrm{CDCl}_{3}, 150 \mathrm{MHz}\right)$

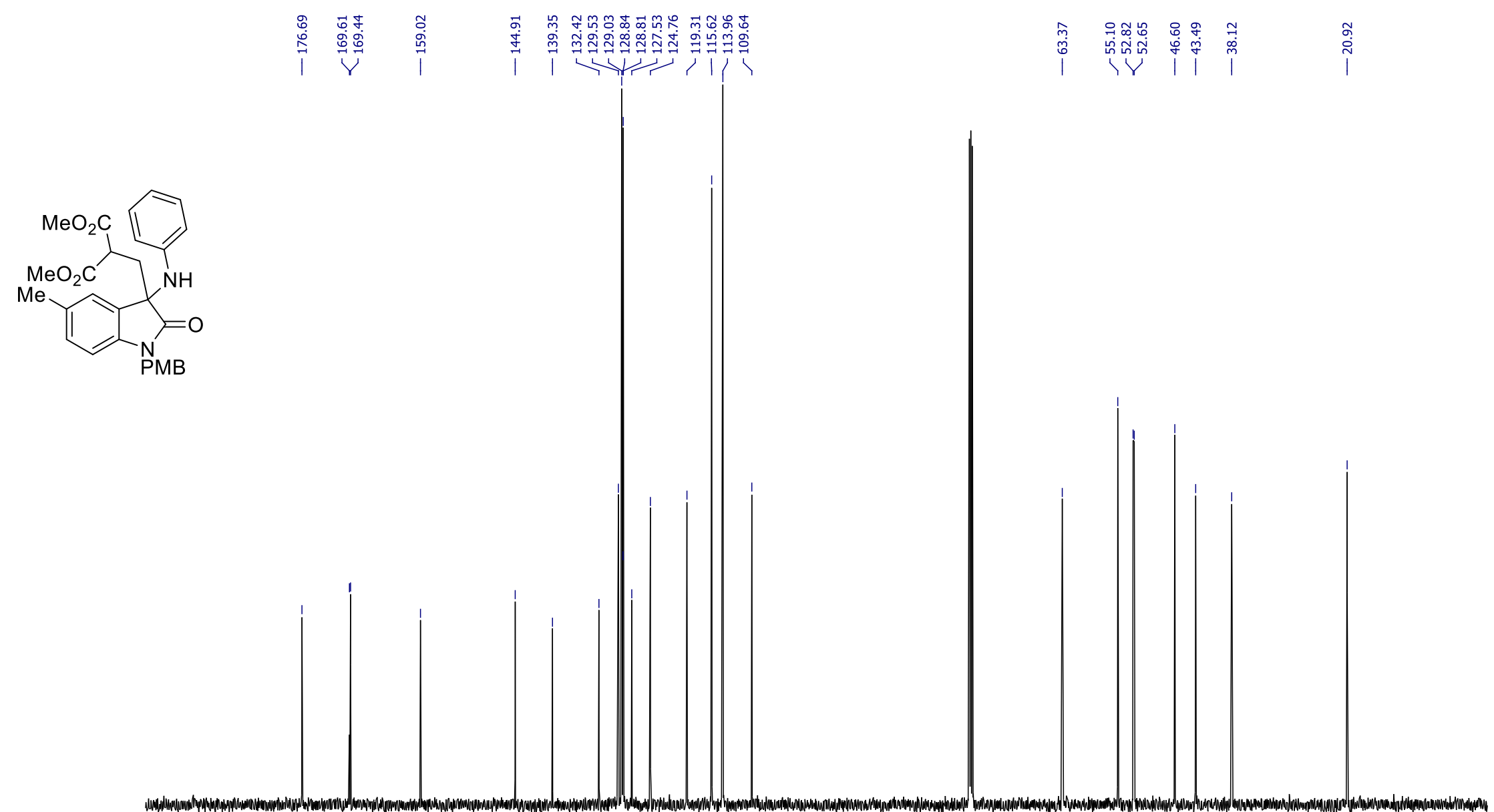

$\begin{array}{llllllllll}190 & 180 & 170 & 160 & 150 & 140 & 130 & 120 & 110 & 100 \\ \mathrm{ppm}\end{array}$

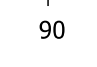

$80 \quad 70$

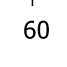

$50 \quad 40$

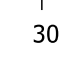

20 
Dimethyl \{[1-methyl-2-oxo-3-(phenylamino)-2,3-dihydro-1H-indol-3-yl]methyl $\}$ malonate (7c)

${ }^{1} \mathrm{H} \mathrm{NMR}\left(\mathrm{CDCl}_{3}, 600 \mathrm{MHz}\right)$
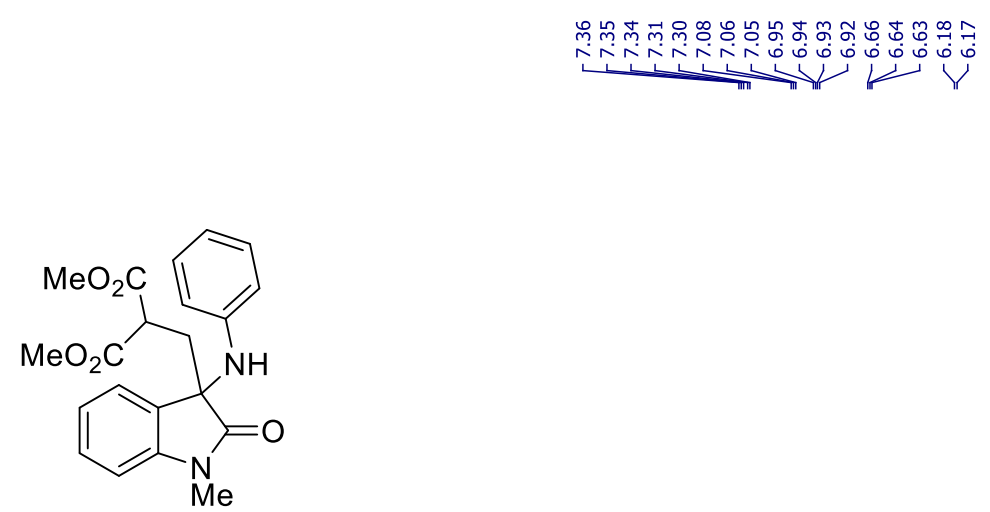

草

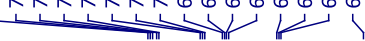

1 U

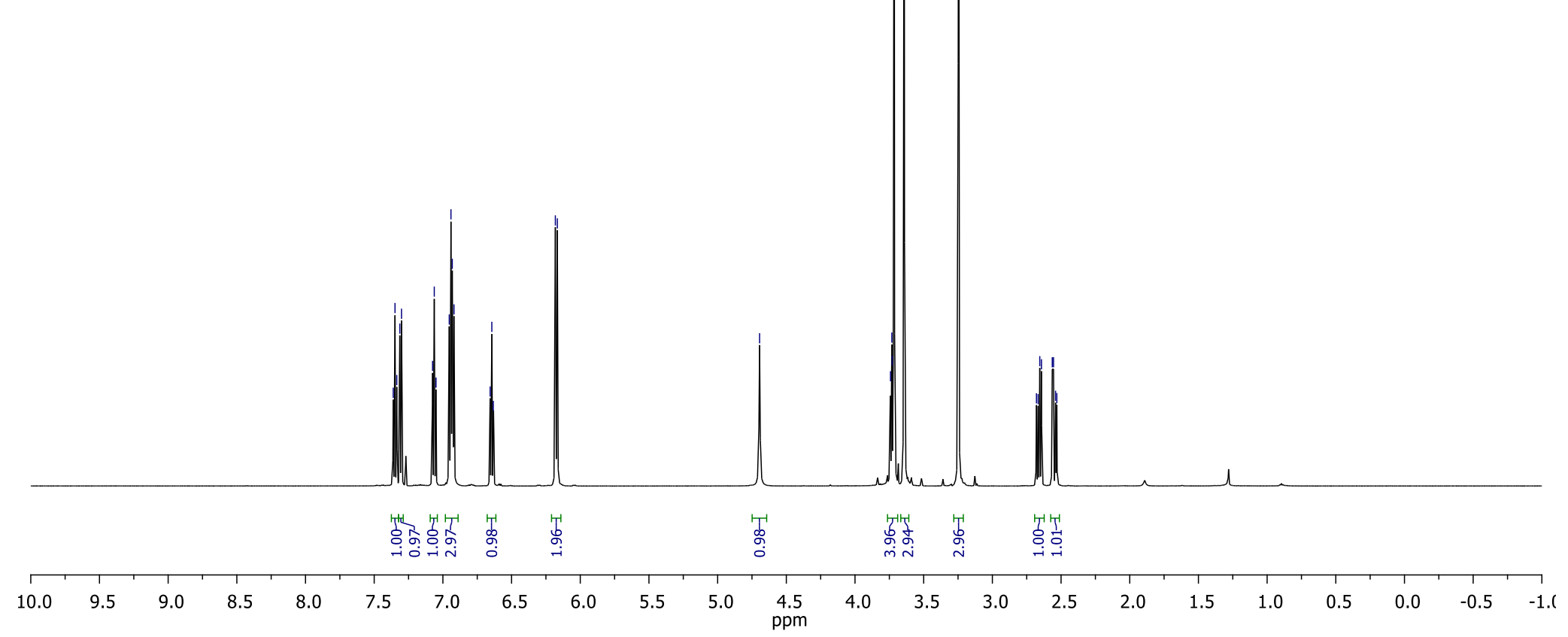


Dimethyl \{[1-methyl-2-oxo-3-(phenylamino)-2,3-dihydro-1H-indol-3-yl]methyl $\}$ malonate (7c)

${ }^{13} \mathrm{C} \mathrm{NMR}\left(\mathrm{CDCl}_{3}, 150 \mathrm{MHz}\right)$

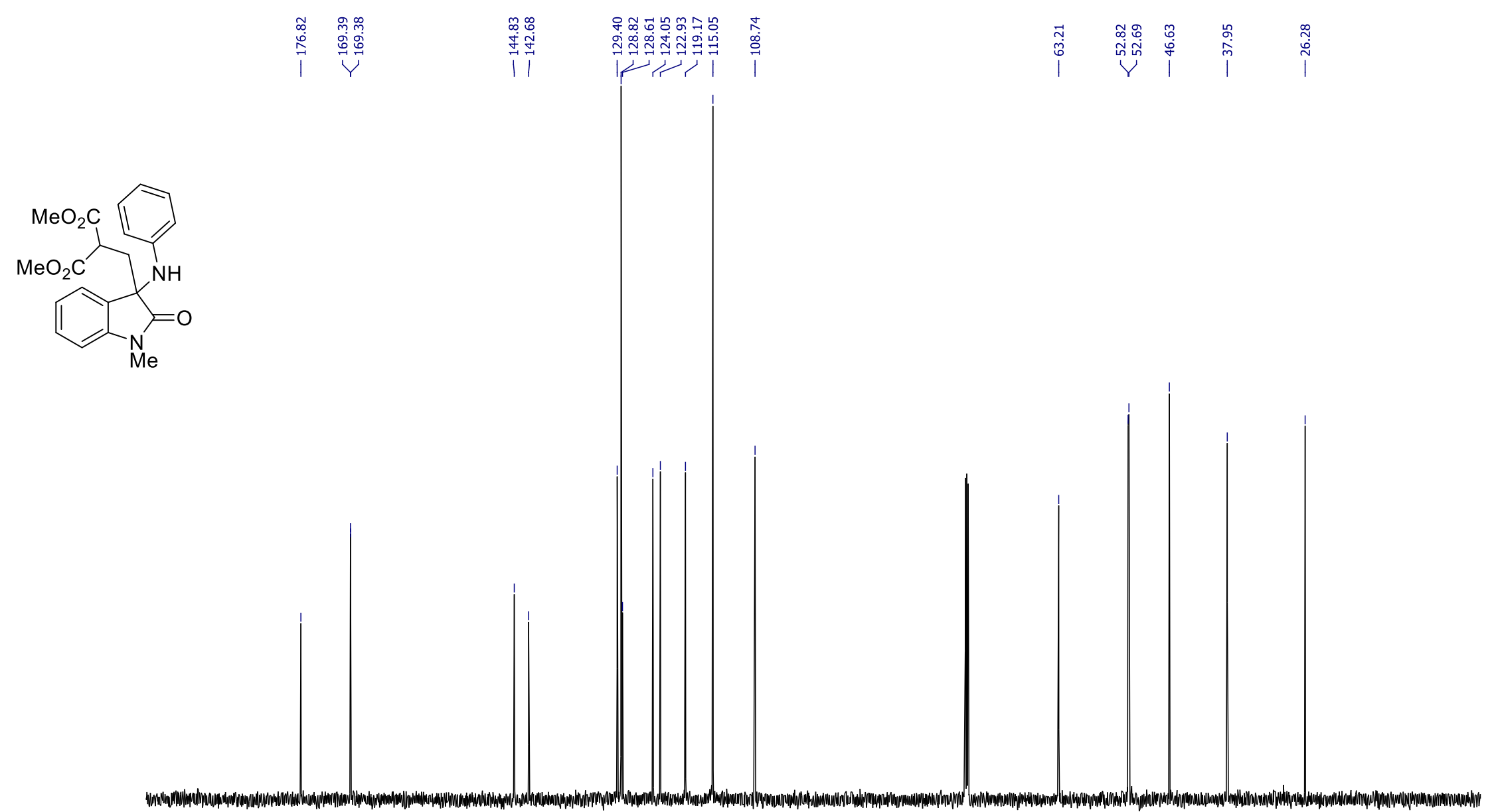

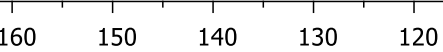

110

ppm

$90 \quad 80 \quad 70$

$60 \quad 50$

40

30

20

$10 \quad 0$ 
Dimethyl \{[5-fluoro-1-(4-methoxybenzyl)-2-oxo-3-(phenylamino)-2,3-dihydro-1H-indol-3-yl]methyl\}malonate (7d)

${ }^{1} \mathrm{H} \mathrm{NMR}\left(\mathrm{CDCl}_{3}, 600 \mathrm{MHz}\right)$

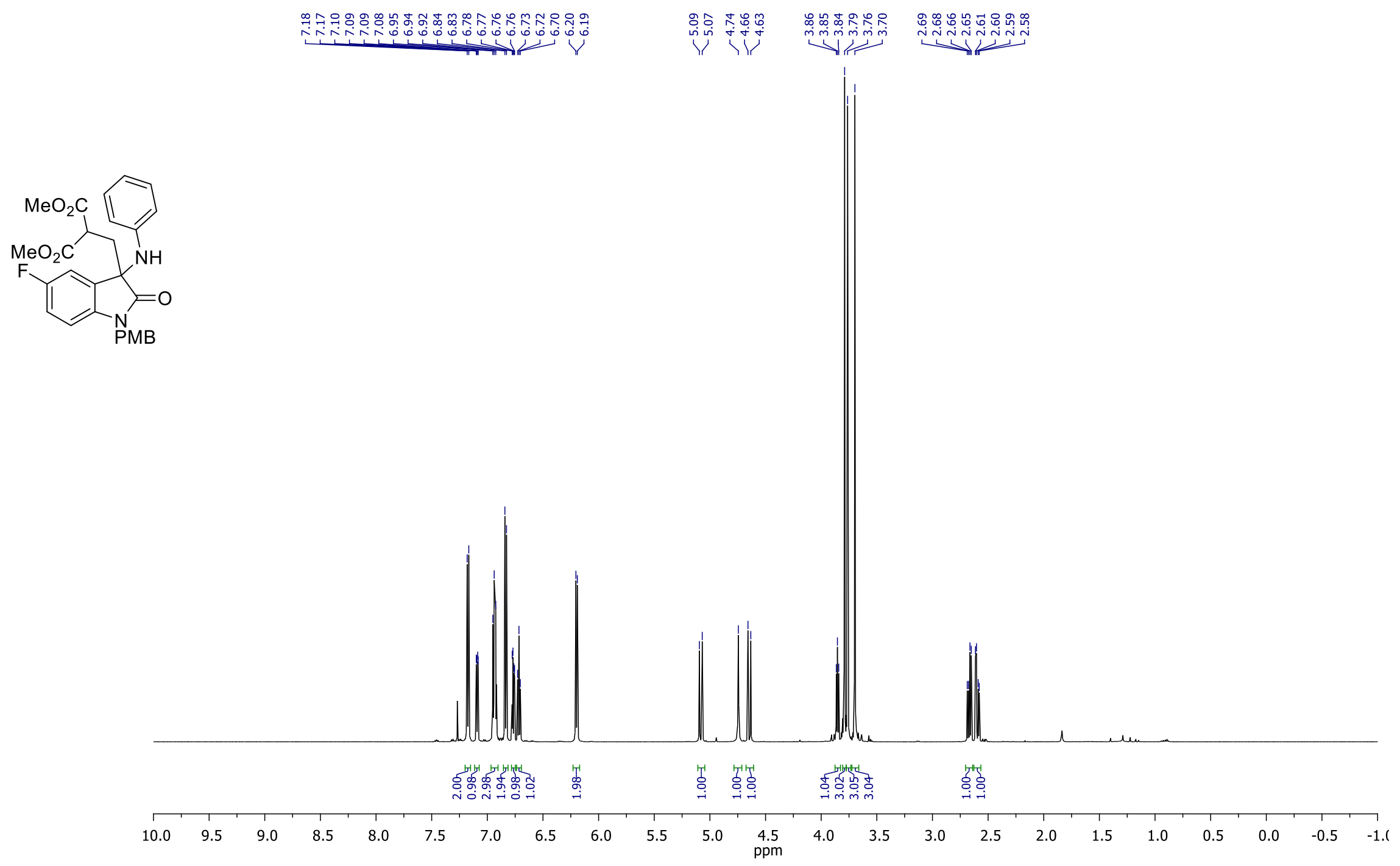


Dimethyl \{[5-fluoro-1-(4-methoxybenzyl)-2-oxo-3-(phenylamino)-2,3-dihydro-1H-indol-3-yl]methyl\}malonate (7d)

${ }^{13} \mathrm{C} \mathrm{NMR}\left(\mathrm{CDCl}_{3}, 150 \mathrm{MHz}\right)$
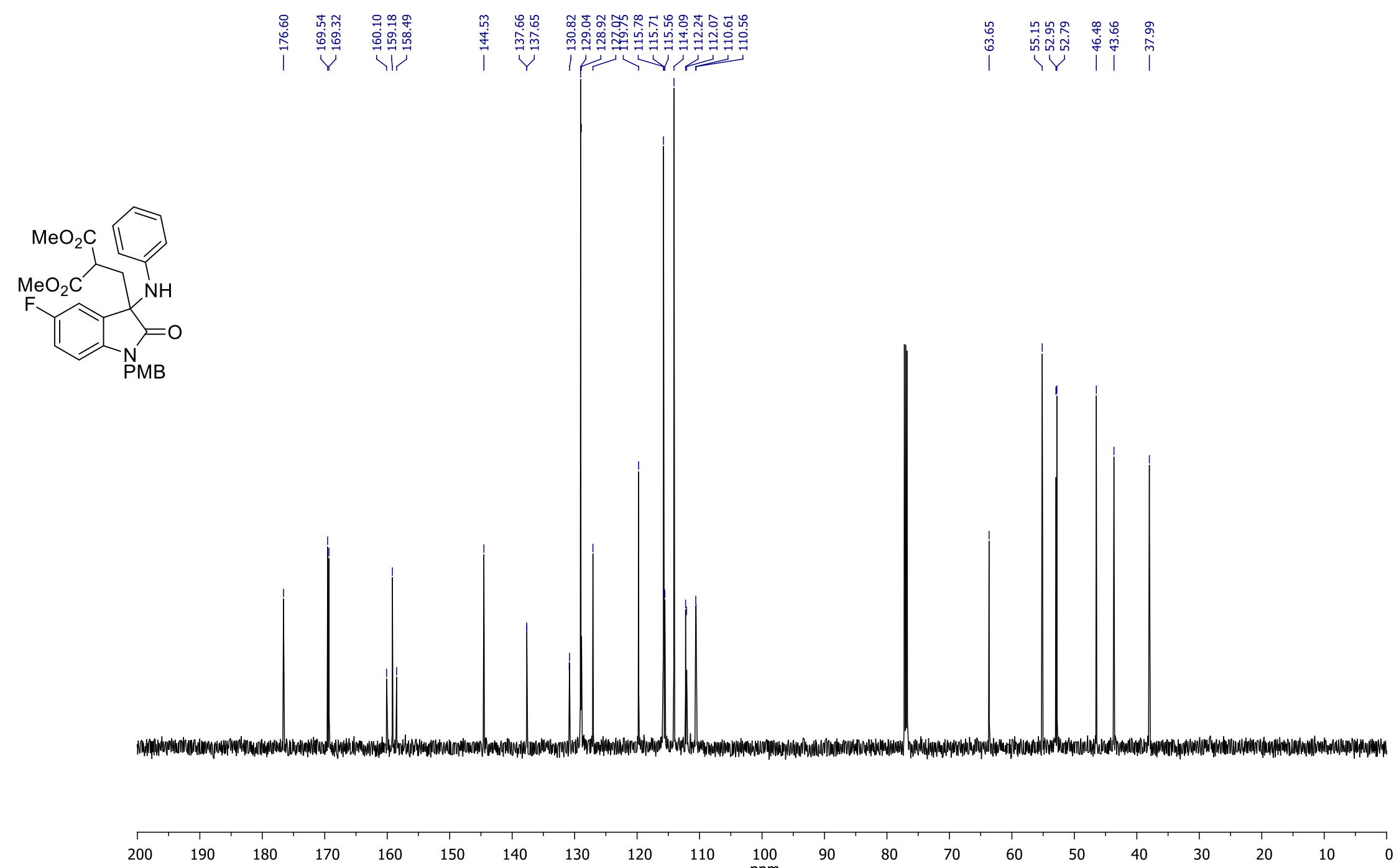

100

$90 \quad 80 \quad 70$

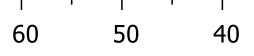

30

20

$10 \quad 0$ 
Dimethyl \{[5-chloro-1-(4-methoxybenzyl)-2-oxo-3-(phenylamino)-2,3-dihydro-1H-indol-3-yl]methyl $\}$ malonate (7e)

${ }^{1} \mathrm{H} \mathrm{NMR}\left(\mathrm{CDCl}_{3}, 600 \mathrm{MHz}\right)$

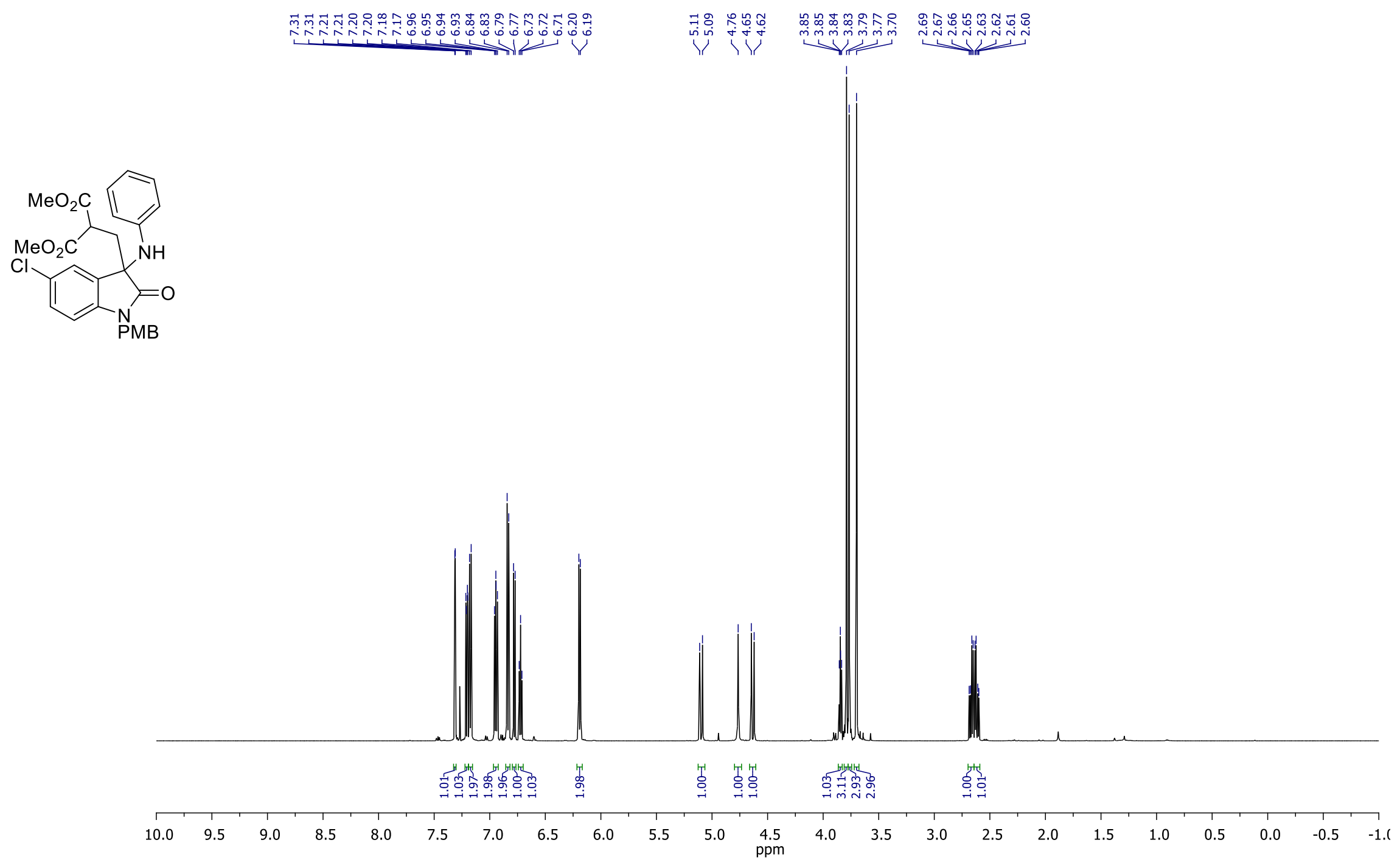


Dimethyl \{[5-chloro-1-(4-methoxybenzyl)-2-oxo-3-(phenylamino)-2,3-dihydro-1H-indol-3-yl]methyl $\}$ malonate (7e)

${ }^{13} \mathrm{C} \mathrm{NMR}\left(\mathrm{CDCl}_{3}, 150 \mathrm{MHz}\right)$
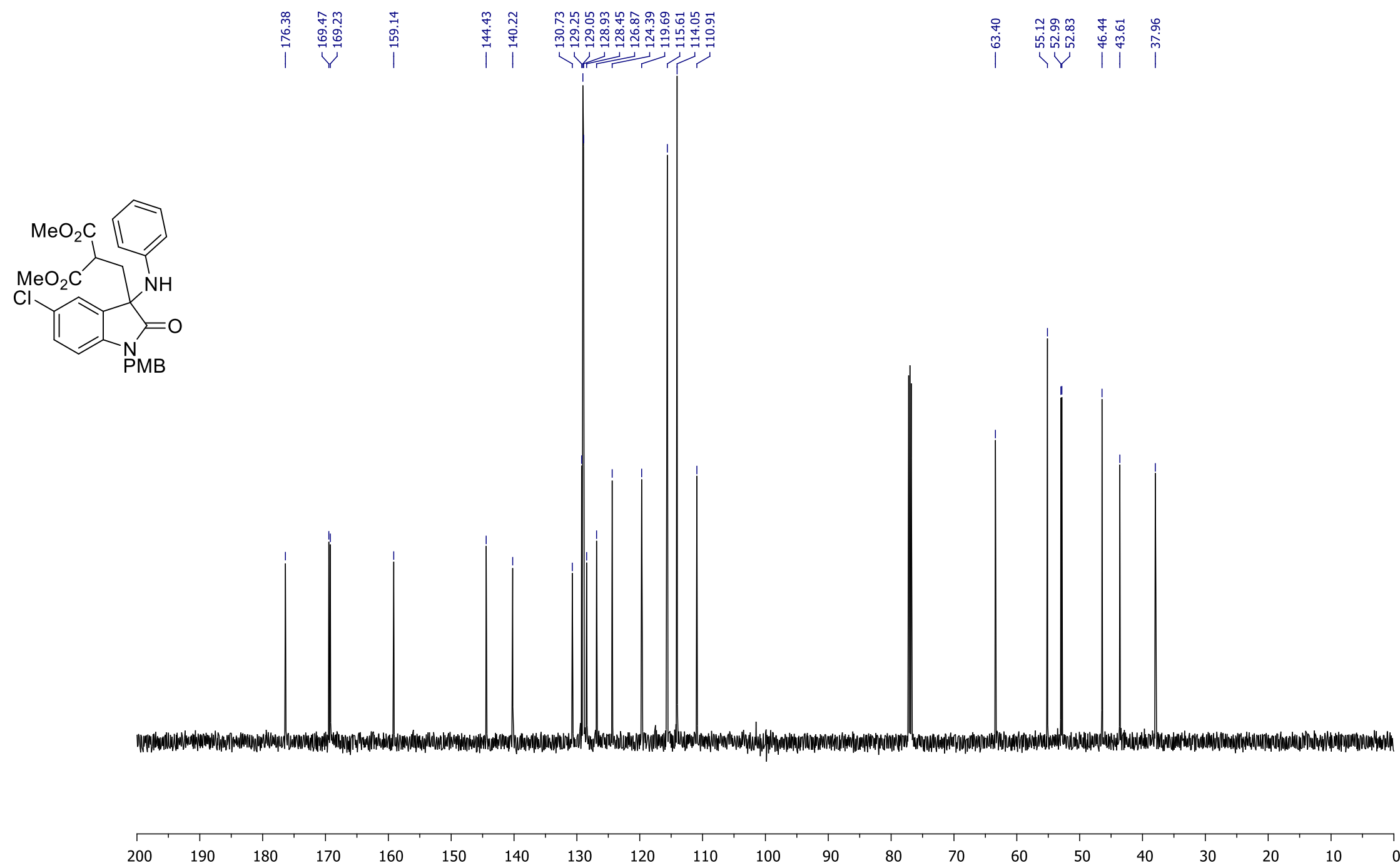

$160 \quad 150 \quad 140$

$130 \quad 120$

110

100

90

$80 \quad 70$

$60 \quad 50$

40

$30 \quad 20$

$10 \quad 0$ 
Dimethyl \{[5-bromo-1-(4-methoxybenzyl)-2-oxo-3-(phenylamino)-2,3-dihydro-1H-indol-3-yl]methyl $\}$ malonate (7f)

${ }^{1} \mathrm{H} \mathrm{NMR}\left(\mathrm{CDCl}_{3}, 600 \mathrm{MHz}\right)$

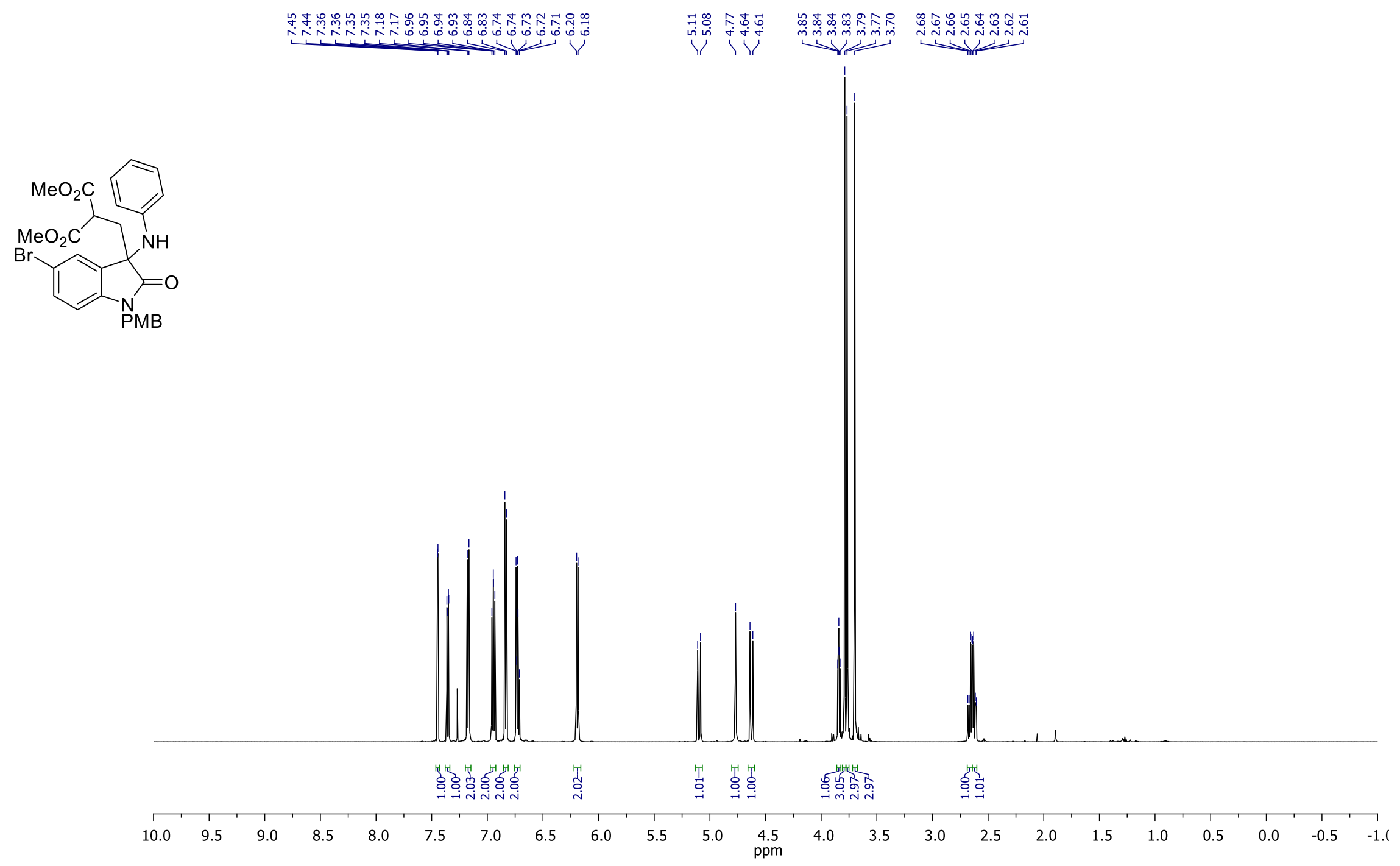


Dimethyl \{[5-bromo-1-(4-methoxybenzyl)-2-oxo-3-(phenylamino)-2,3-dihydro-1H-indol-3-yl]methyl $\}$ malonate (7f)

${ }^{13} \mathrm{C} \mathrm{NMR}\left(\mathrm{CDCl}_{3}, 150 \mathrm{MHz}\right)$

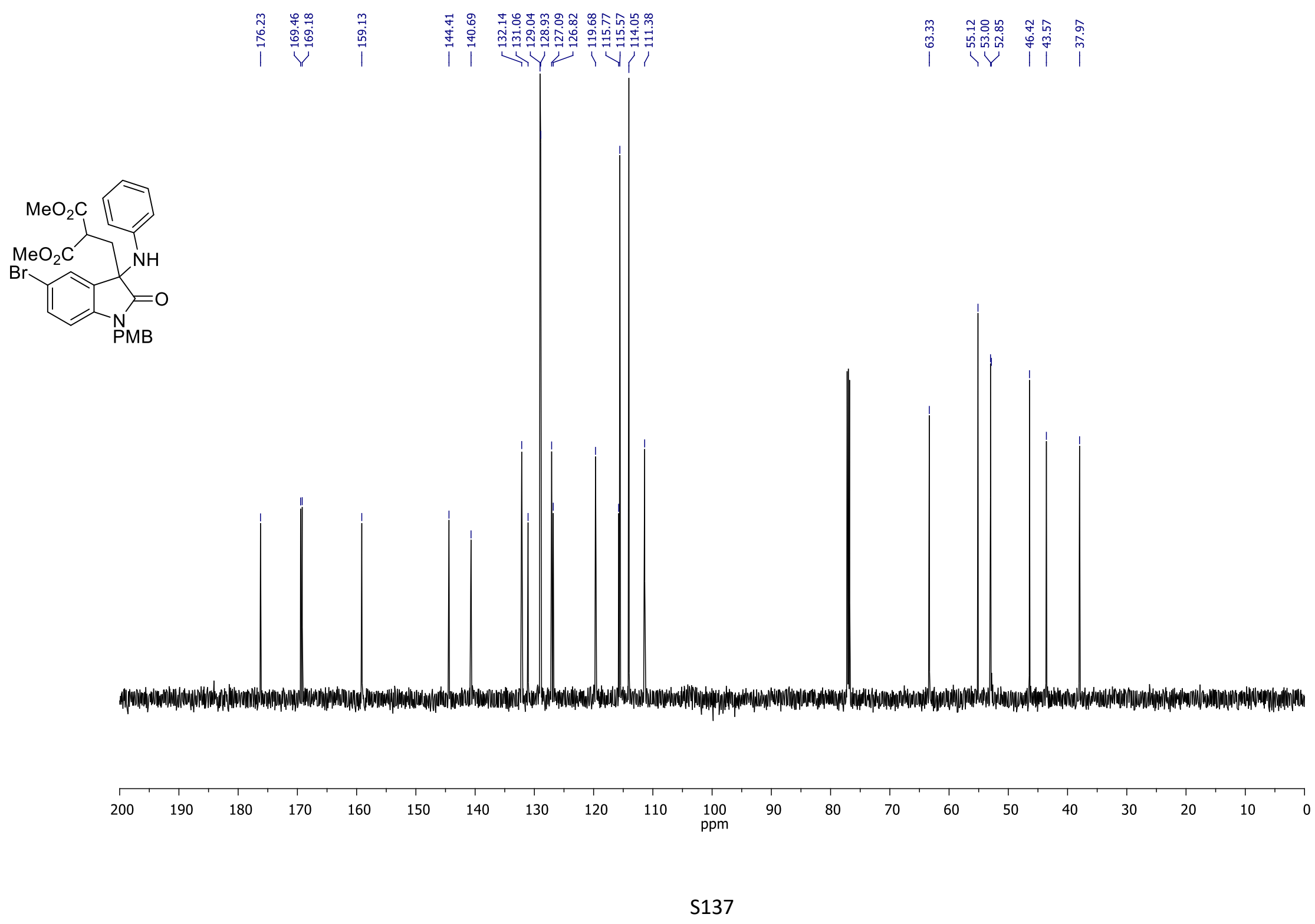


Dimethyl (\{3-[(4-fluorophenyl)amino]-1-(4-methoxybenzyl)-2-oxo-2,3-dihydro-1H-indol-3-yl\}methyl)malonate (7g)

${ }^{1} \mathrm{H} \mathrm{NMR}\left(\mathrm{CDCl}_{3}, 600 \mathrm{MHz}\right)$
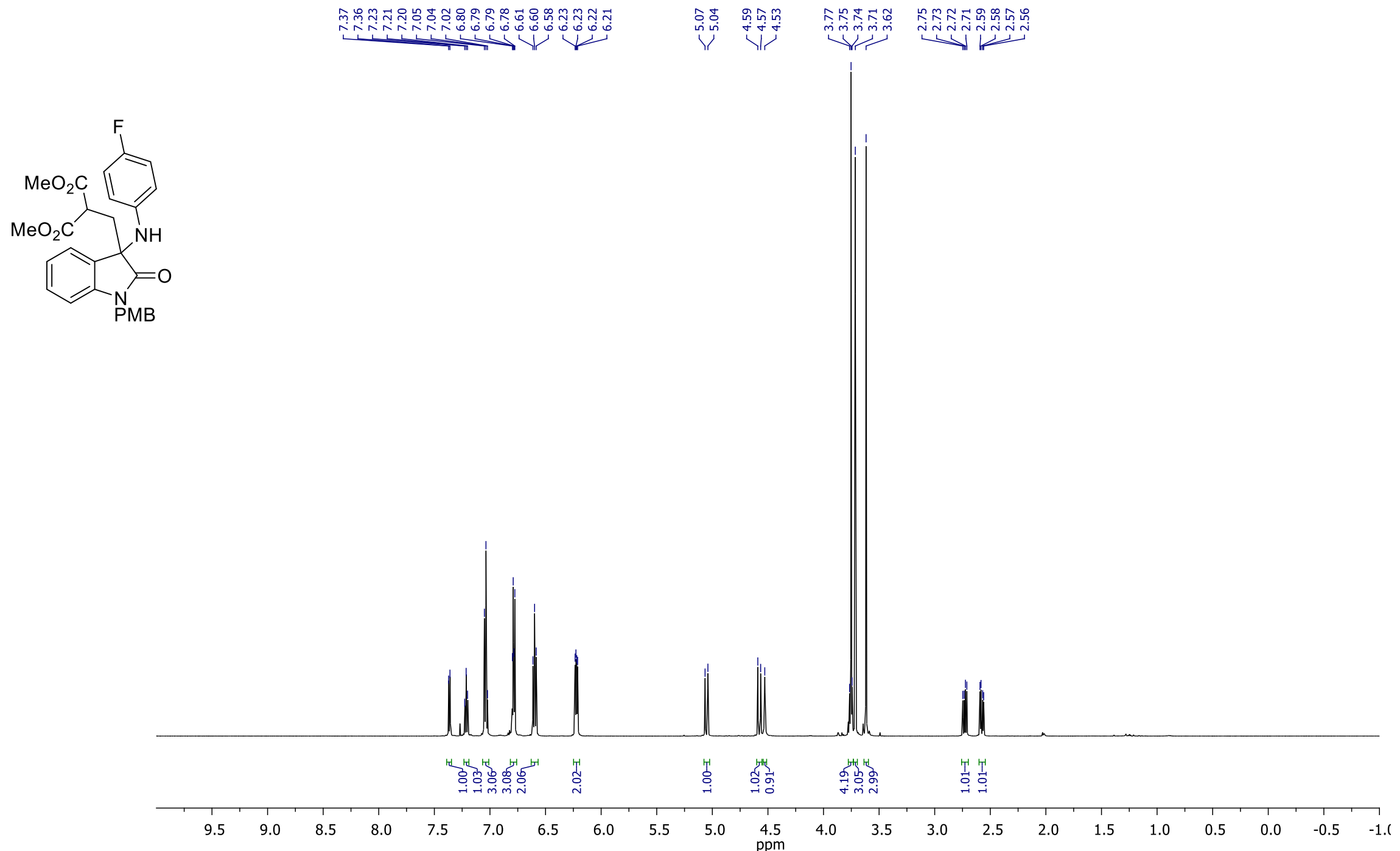
Dimethyl (\{3-[(4-fluorophenyl)amino]-1-(4-methoxybenzyl)-2-oxo-2,3-dihydro-1H-indol-3-yl\}methyl)malonate (7g)

${ }^{13} \mathrm{C} \mathrm{NMR}\left(\mathrm{CDCl}_{3}, 150 \mathrm{MHz}\right)$
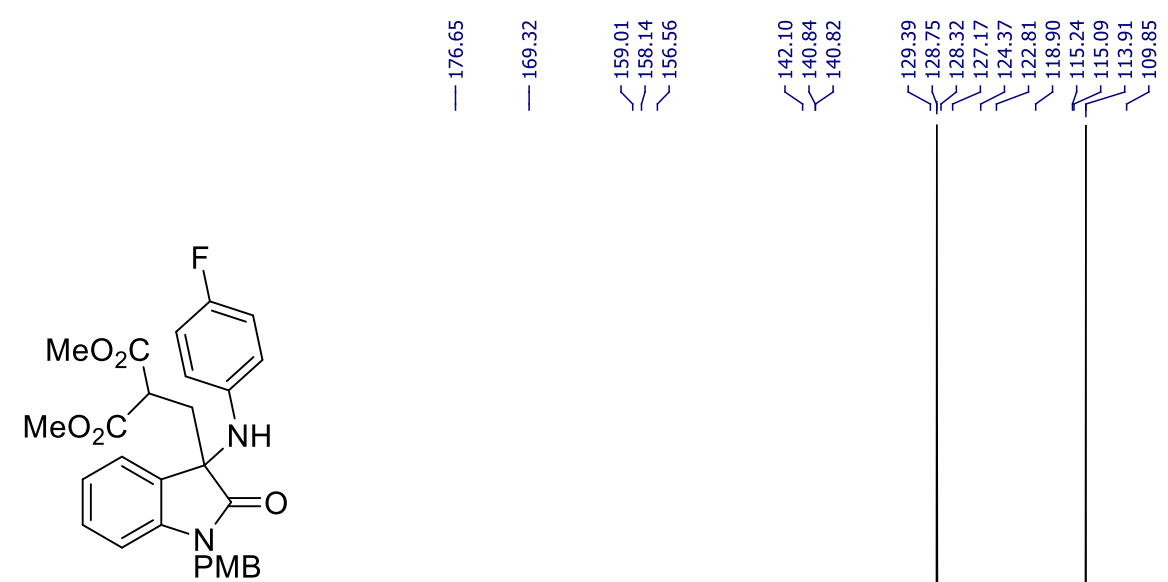

焉

PMB
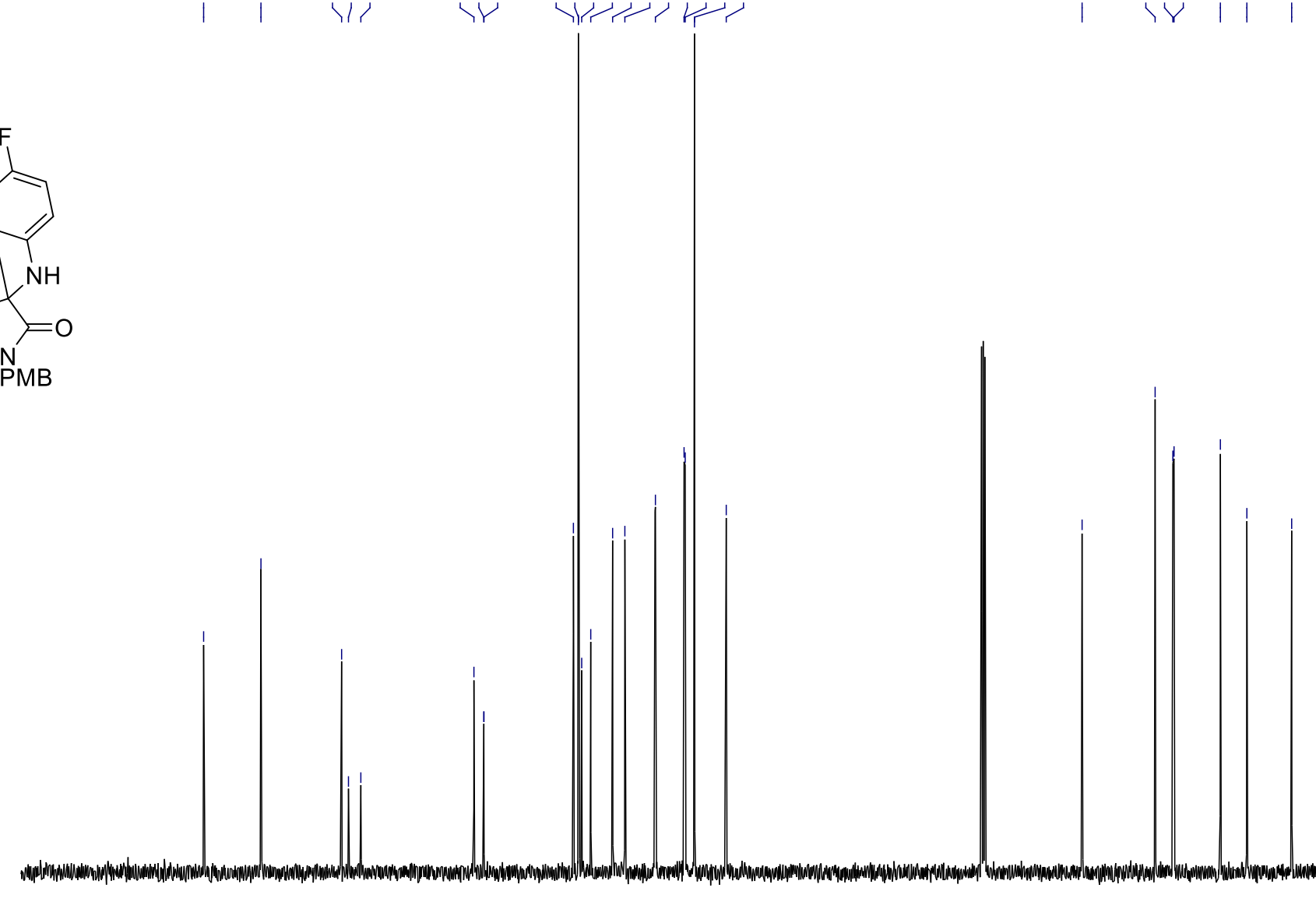

200

180

160

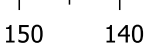

130

110

ppm

90

$80 \quad 70$

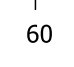

$50 \quad 40$

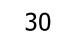

20

$10 \quad 0$ 
Dimethyl (\{3-[(4-chlorophenyl)amino]-1-(4-methoxybenzyl)-2-oxo-2,3-dihydro-1H-indol-3-yl\}methyl)malonate (7h)

${ }^{1} \mathrm{H} \mathrm{NMR}\left(\mathrm{CDCl}_{3}, 600 \mathrm{MHz}\right)$

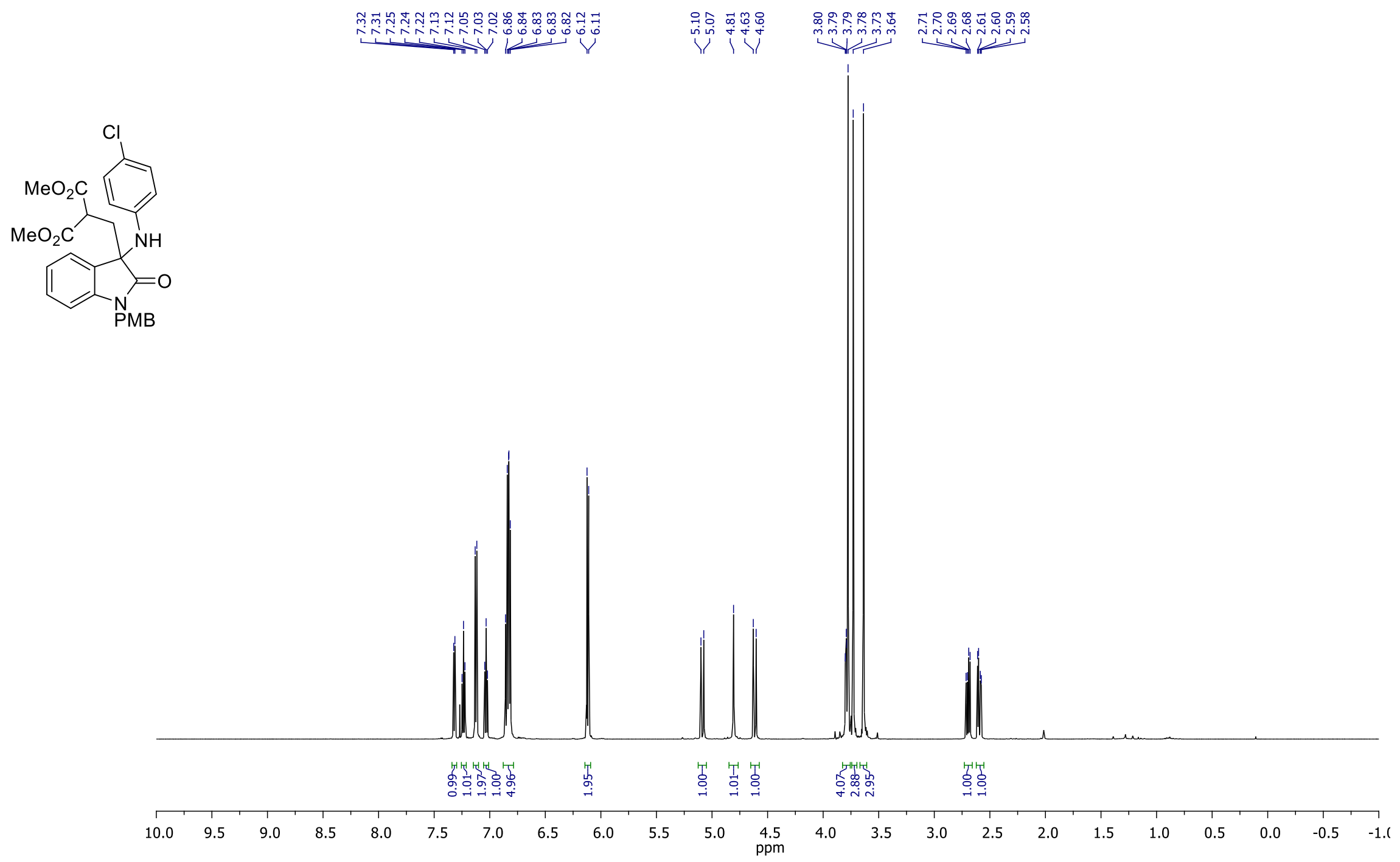


Dimethyl (\{3-[(4-chlorophenyl)amino]-1-(4-methoxybenzyl)-2-oxo-2,3-dihydro-1H-indol-3-yl\}methyl)malonate (7h)

${ }^{13} \mathrm{C} \mathrm{NMR}\left(\mathrm{CDCl}_{3}, 150 \mathrm{MHz}\right)$

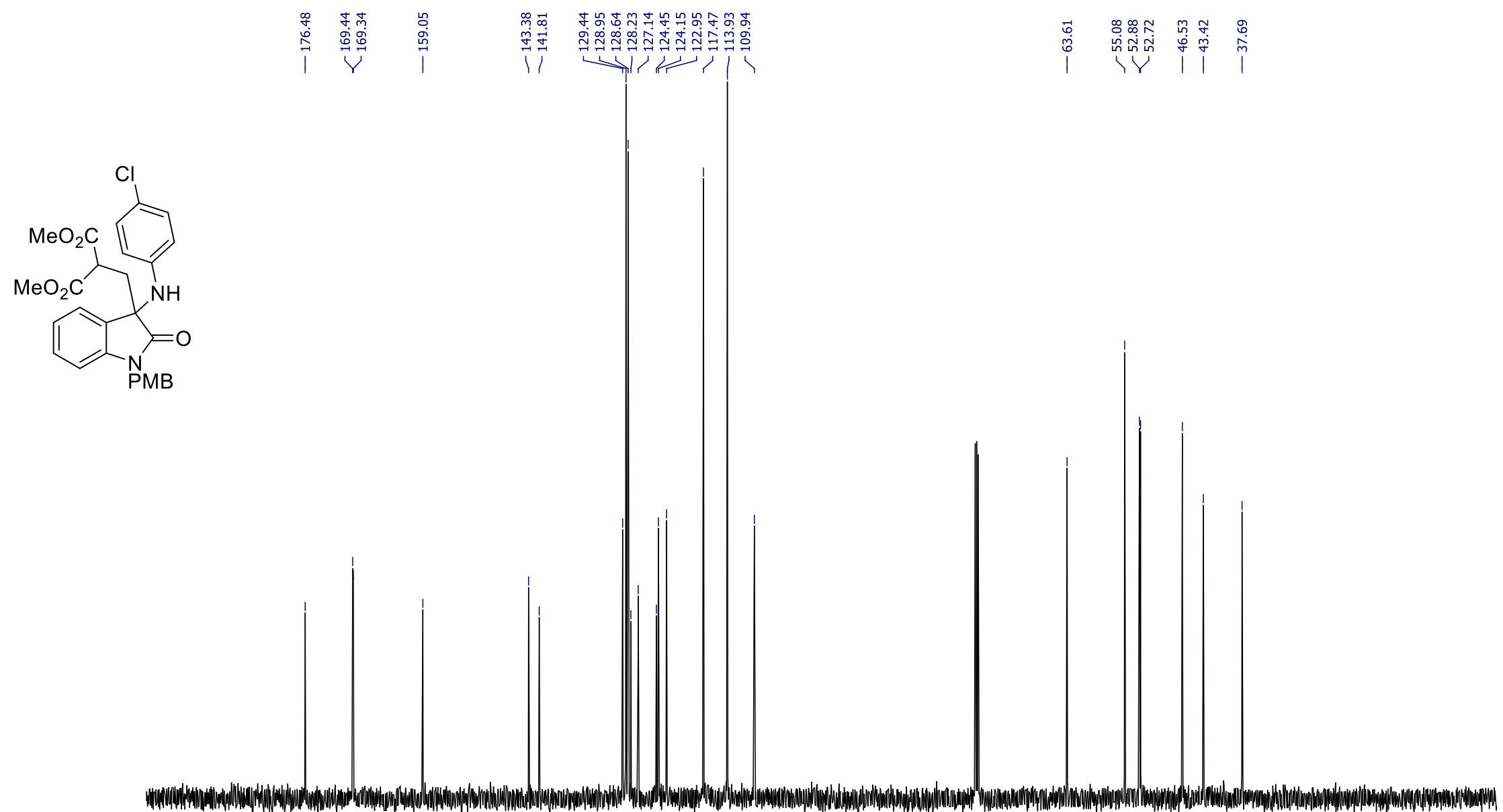

200

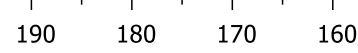

$150 \quad 140$

$130 \quad 120$

110

ppm

90

$80 \quad 70$

60

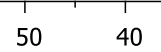

30

20 
Dimethyl (\{3-[(3-chlorophenyl)amino]-1-(4-methoxybenzyl)-2-oxo-2,3-dihydro-1H-indol-3-yl\}methyl)malonate (7i)

${ }^{1} \mathrm{H} \mathrm{NMR}\left(\mathrm{CDCl}_{3}, 600 \mathrm{MHz}\right)$
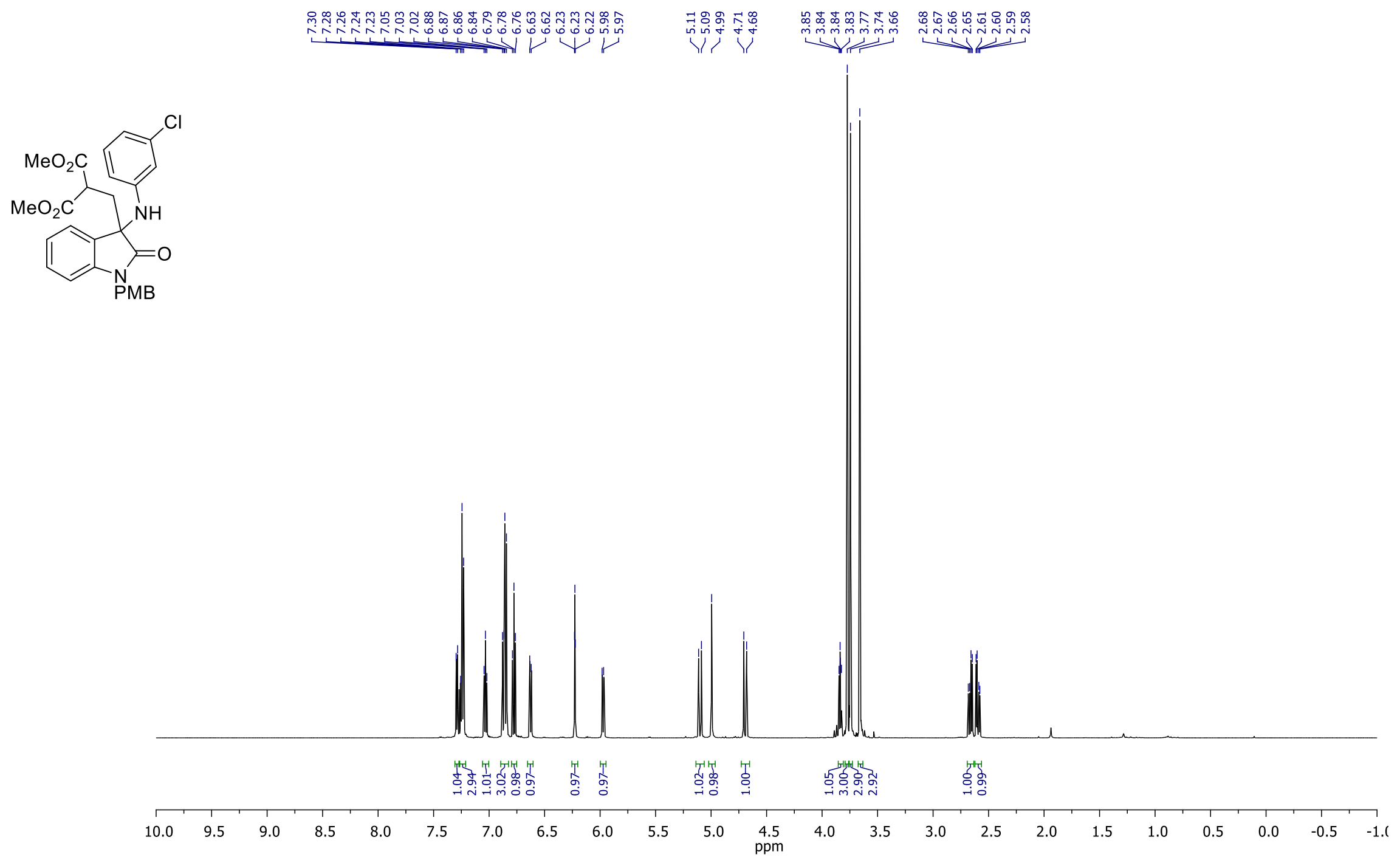
Dimethyl (\{3-[(3-chlorophenyl)amino]-1-(4-methoxybenzyl)-2-oxo-2,3-dihydro-1H-indol-3-yl\}methyl)malonate (7i)

${ }^{13} \mathrm{C} \mathrm{NMR}\left(\mathrm{CDCl}_{3}, 150 \mathrm{MHz}\right)$

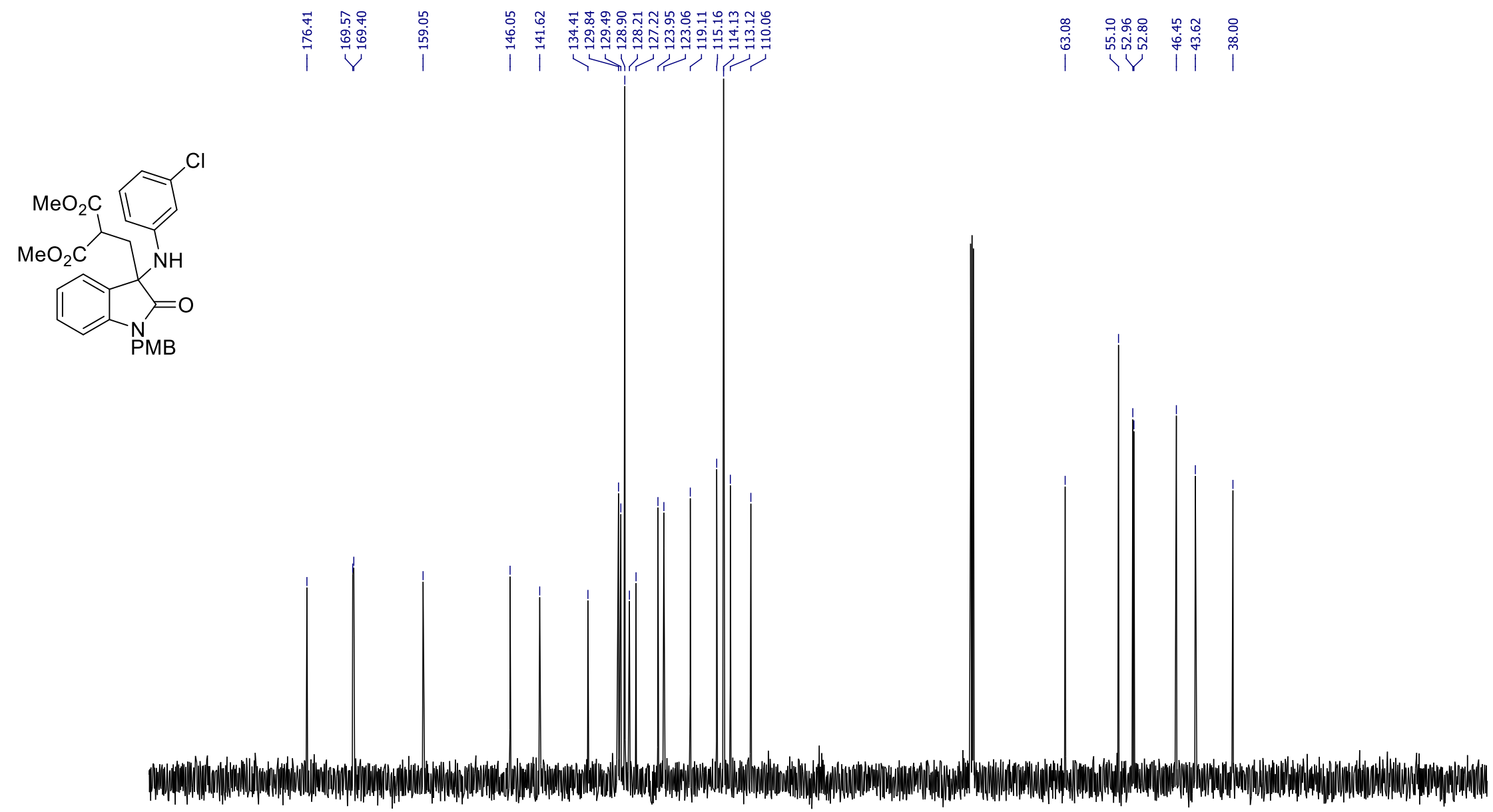

160

$150 \quad 140$

$130 \quad 120$

110

100

90

$80 \quad 70$

60

$50 \quad 40$

30

$20 \quad 10$ 
Dimethyl (\{3-[(4-bromophenyl)amino]-1-(4-methoxybenzyl)-2-oxo-2,3-dihydro-1H-indol-3-yl\}methyl)malonate (7j)

${ }^{1} \mathrm{H} \mathrm{NMR}\left(\mathrm{CDCl}_{3}, 600 \mathrm{MHz}\right)$

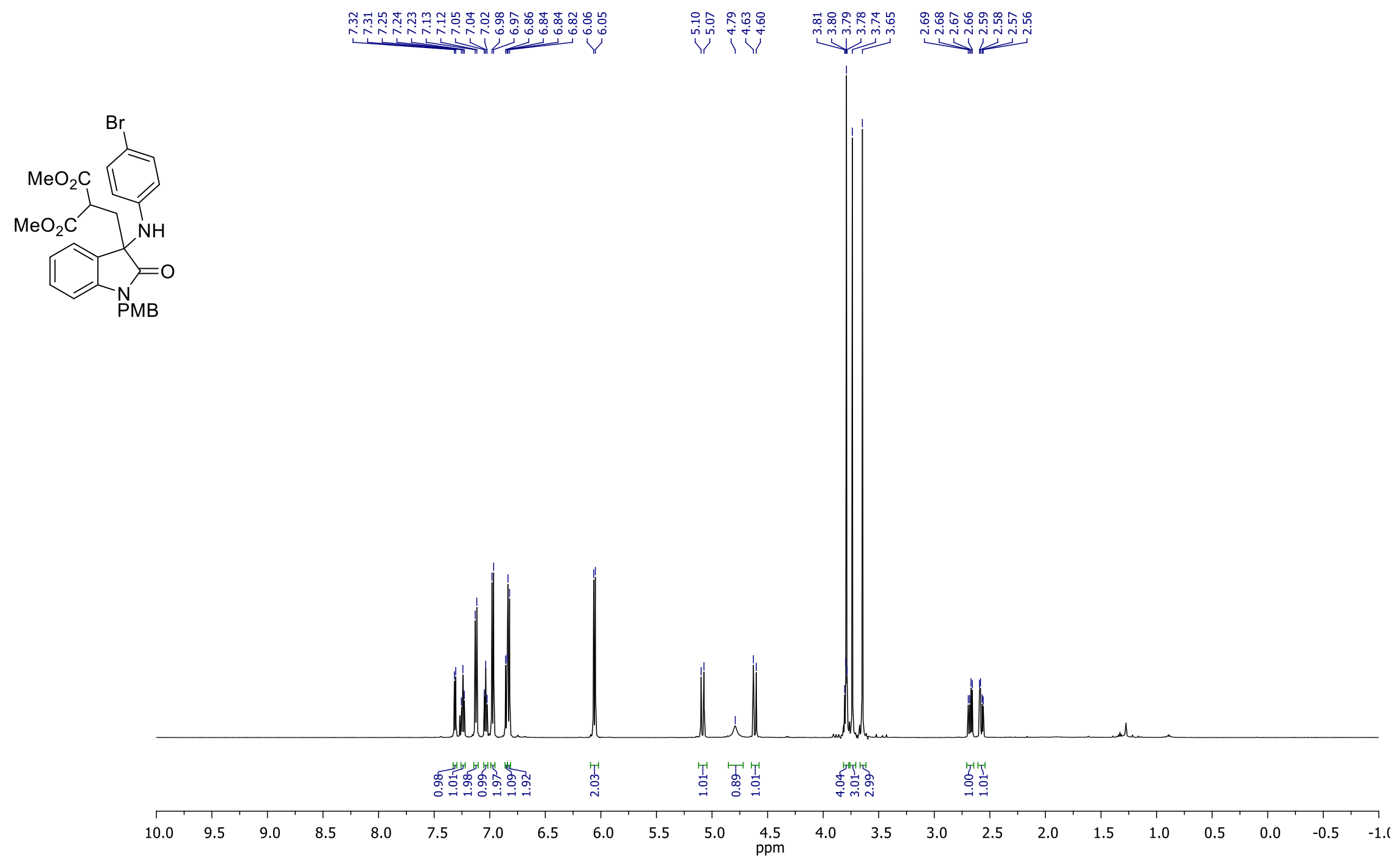


Dimethyl (\{3-[(4-bromophenyl)amino]-1-(4-methoxybenzyl)-2-oxo-2,3-dihydro-1H-indol-3-yl\}methyl)malonate (7j)

${ }^{13} \mathrm{C} \mathrm{NMR}\left(\mathrm{CDCl}_{3}, 150 \mathrm{MHz}\right)$

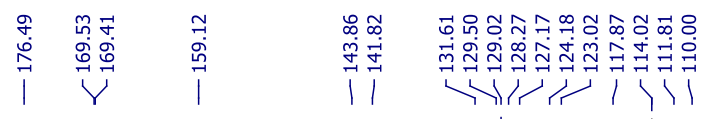

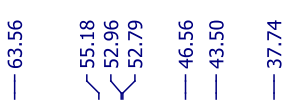
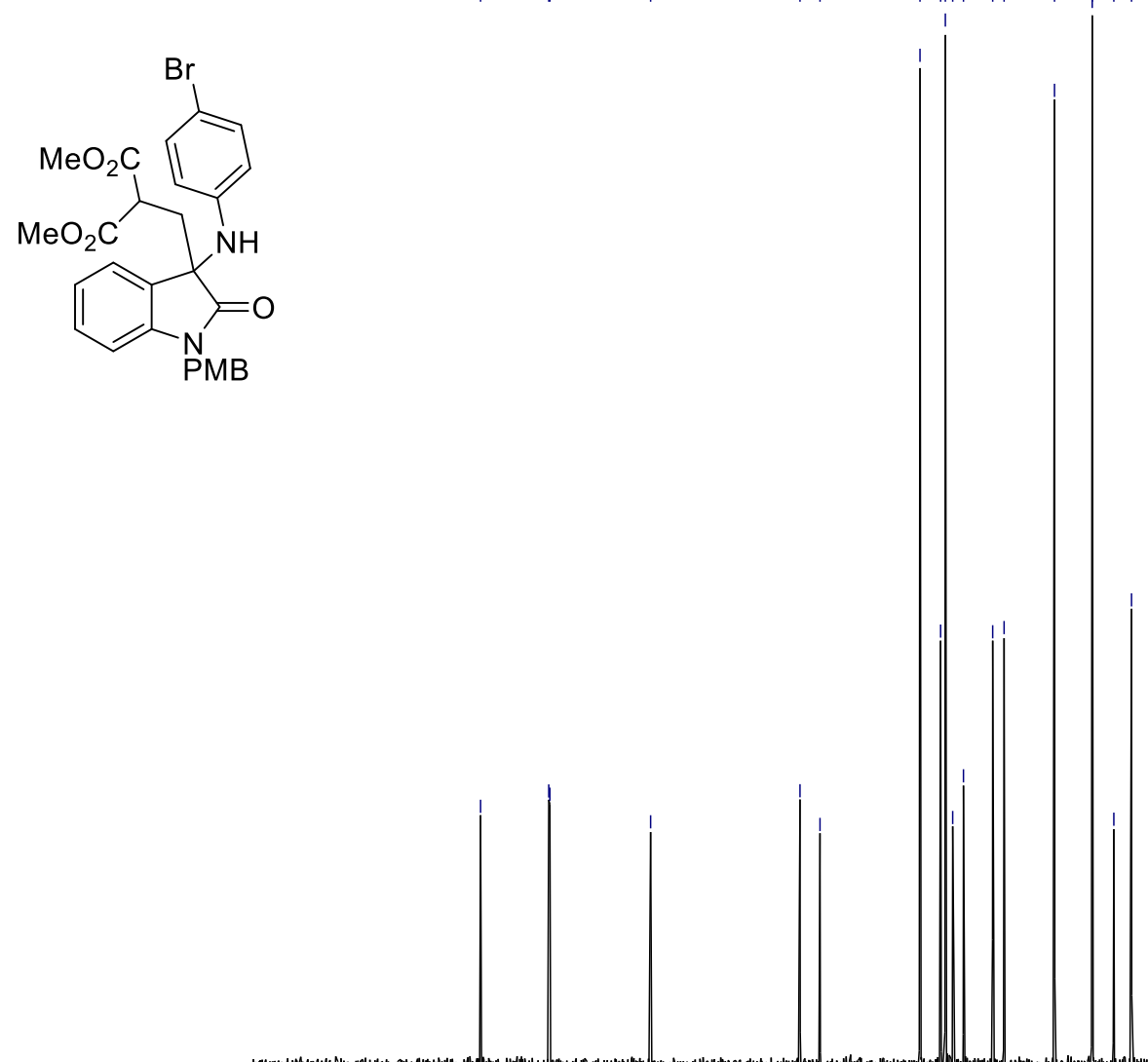

(n)

200

190

180

160

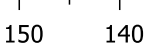

130

11

100
$\mathrm{ppm}$

90

$80 \quad 70$

60

$50 \quad 40$

30

20

$10 \quad 0$ 
Dimethyl (\{3-1-(4-methoxybenzyl)-[(4-methylphenyl)amino]-2-oxo-2,3-dihydro-1H-indol-3-yl\}methyl)malonate (7k)

${ }^{1} \mathrm{H} \mathrm{NMR}\left(\mathrm{CDCl}_{3}, 600 \mathrm{MHz}\right)$

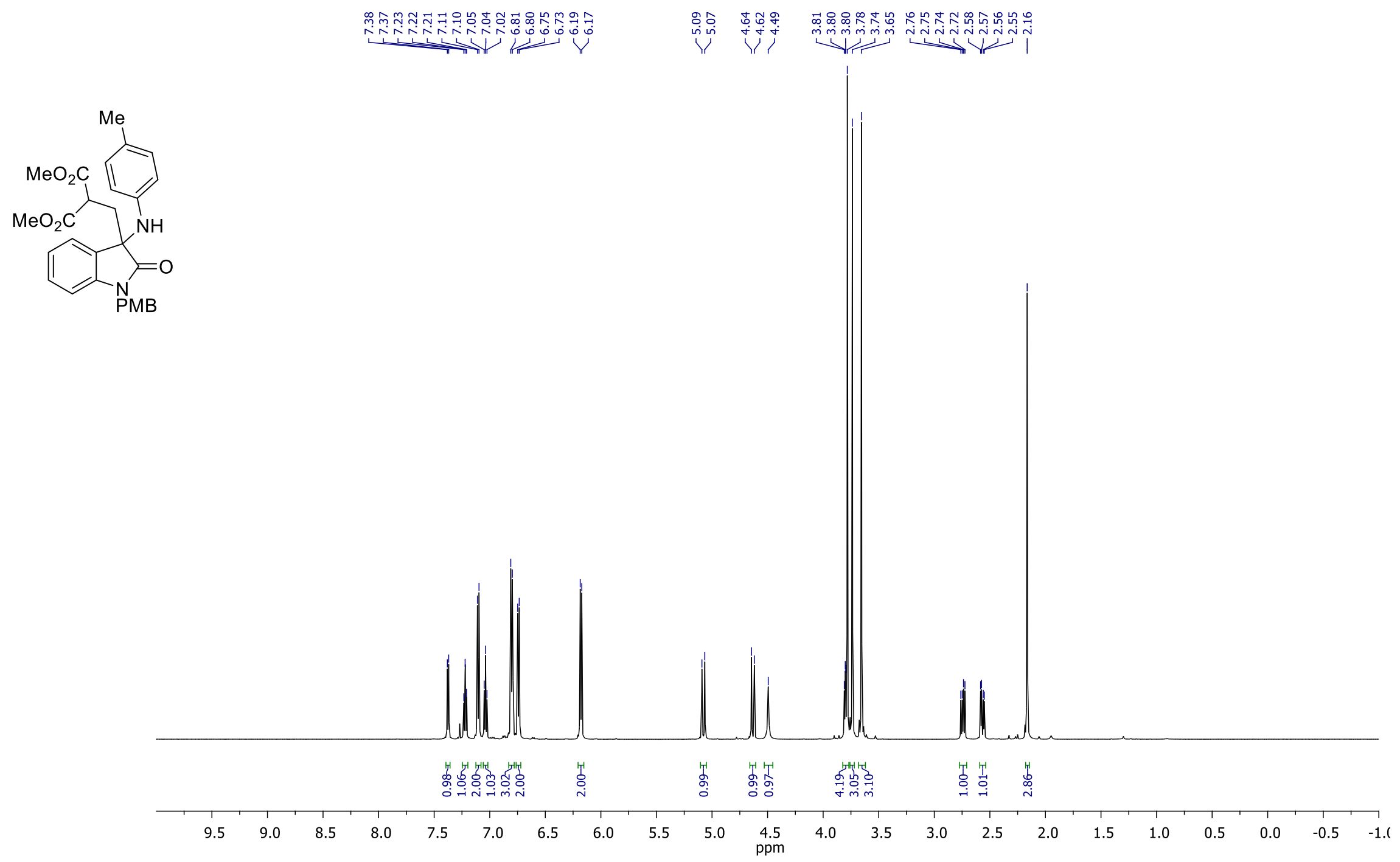


Dimethyl (\{3-1-(4-methoxybenzyl)-[(4-methylphenyl)amino]-2-oxo-2,3-dihydro-1H-indol-3-yl\}methyl)malonate (7k)

${ }^{13} \mathrm{C} \mathrm{NMR}\left(\mathrm{CDCl}_{3}, 150 \mathrm{MHz}\right)$

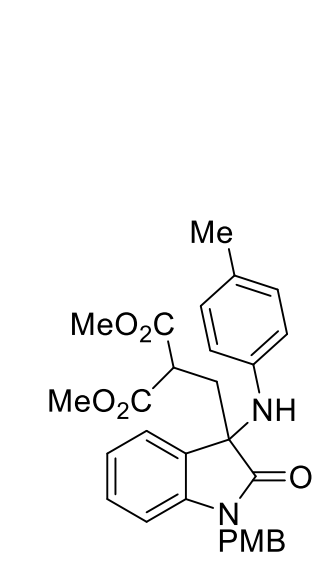

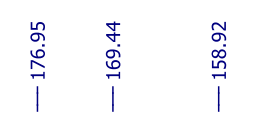

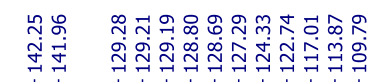

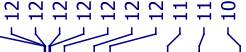

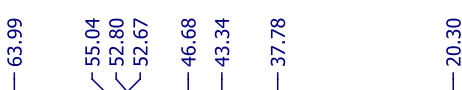

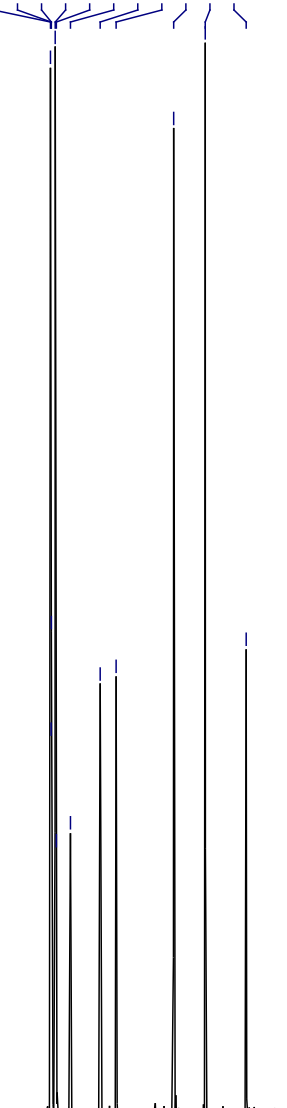

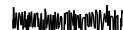

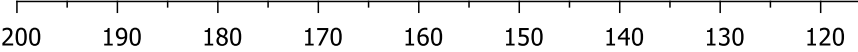

110

ppm

90

$80 \quad 70$

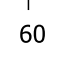

50

40 
Dimethyl (\{3-1-(4-methoxybenzyl)-[(4-methoxyphenyl)amino]-2-oxo-2,3-dihydro-1H-indol-3-yl\}methyl)malonate (7l)

${ }^{1} \mathrm{H} \mathrm{NMR}\left(\mathrm{CDCl}_{3}, 600 \mathrm{MHz}\right)$

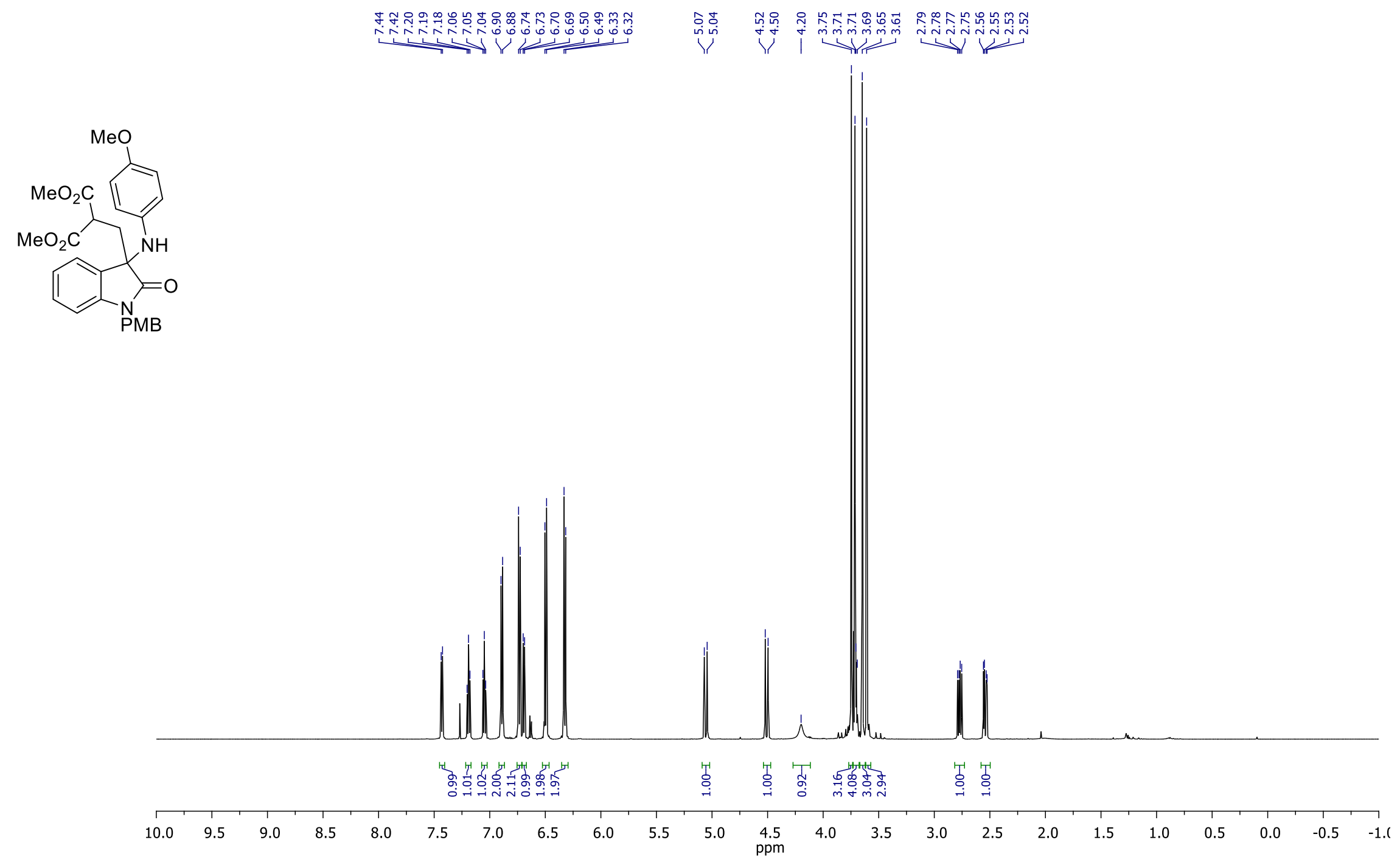


Dimethyl (\{3-1-(4-methoxybenzyl)-[(4-methoxyphenyl)amino]-2-oxo-2,3-dihydro-1H-indol-3-yl\}methyl)malonate (7l)

${ }^{13} \mathrm{C} \mathrm{NMR}\left(\mathrm{CDCl}_{3}, 150 \mathrm{MHz}\right)$

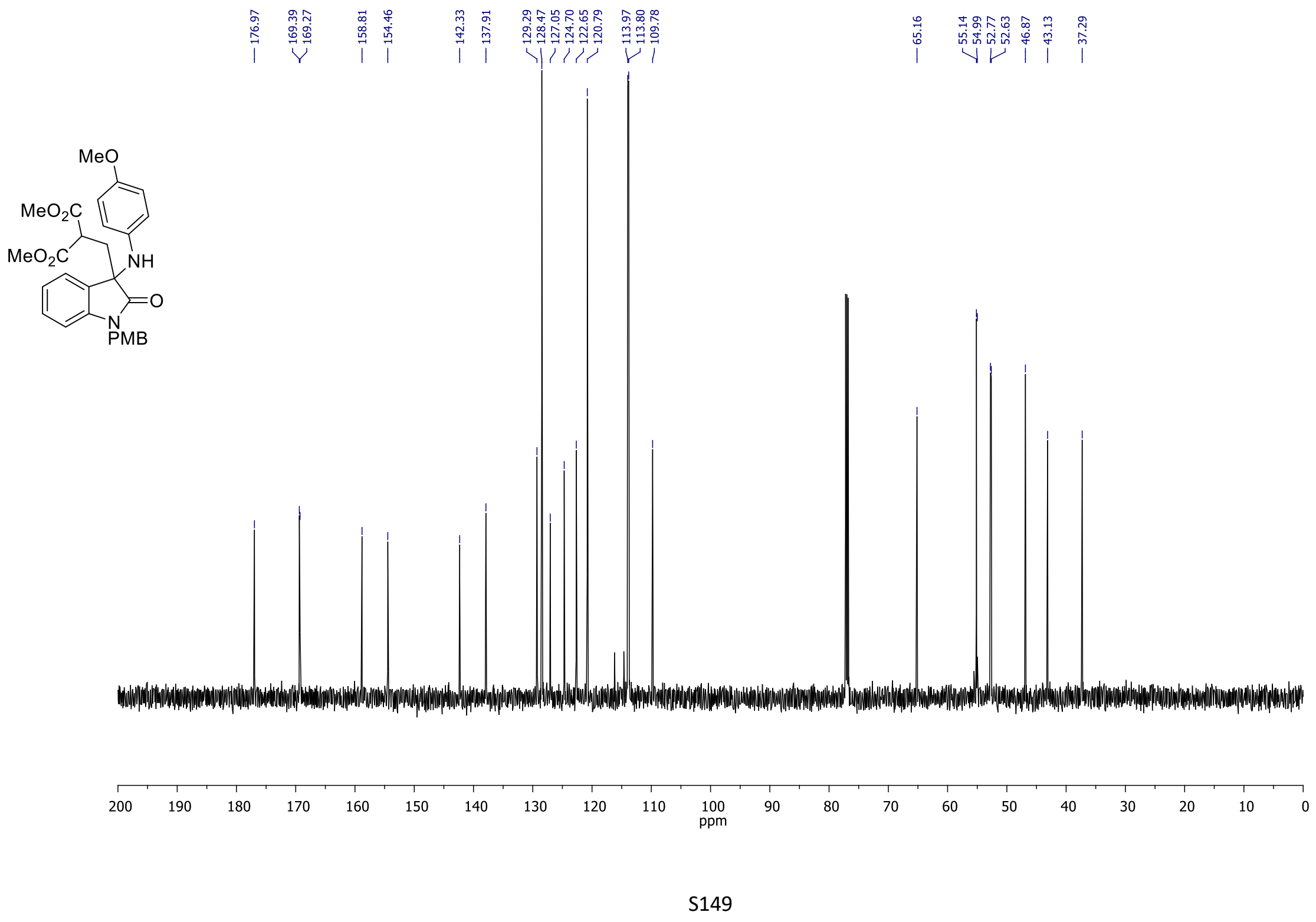


Dimethyl (\{3-1-(4-methoxybenzyl)-[(2-methoxyphenyl)amino]-2-oxo-2,3-dihydro-1H-indol-3-yl $\}$ methyl)malonate (7m)

${ }^{1} \mathrm{H} \mathrm{NMR}\left(\mathrm{CDCl}_{3}, 600 \mathrm{MHz}\right)$

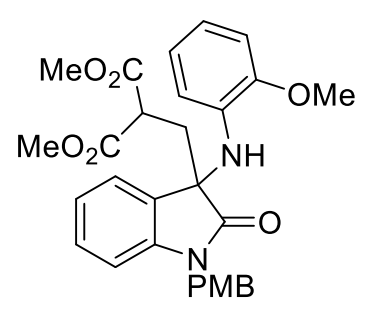

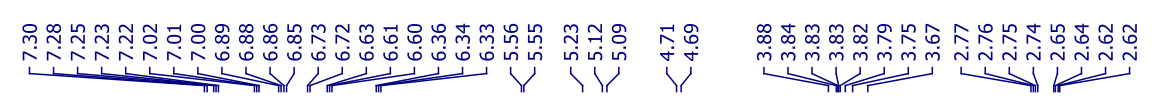
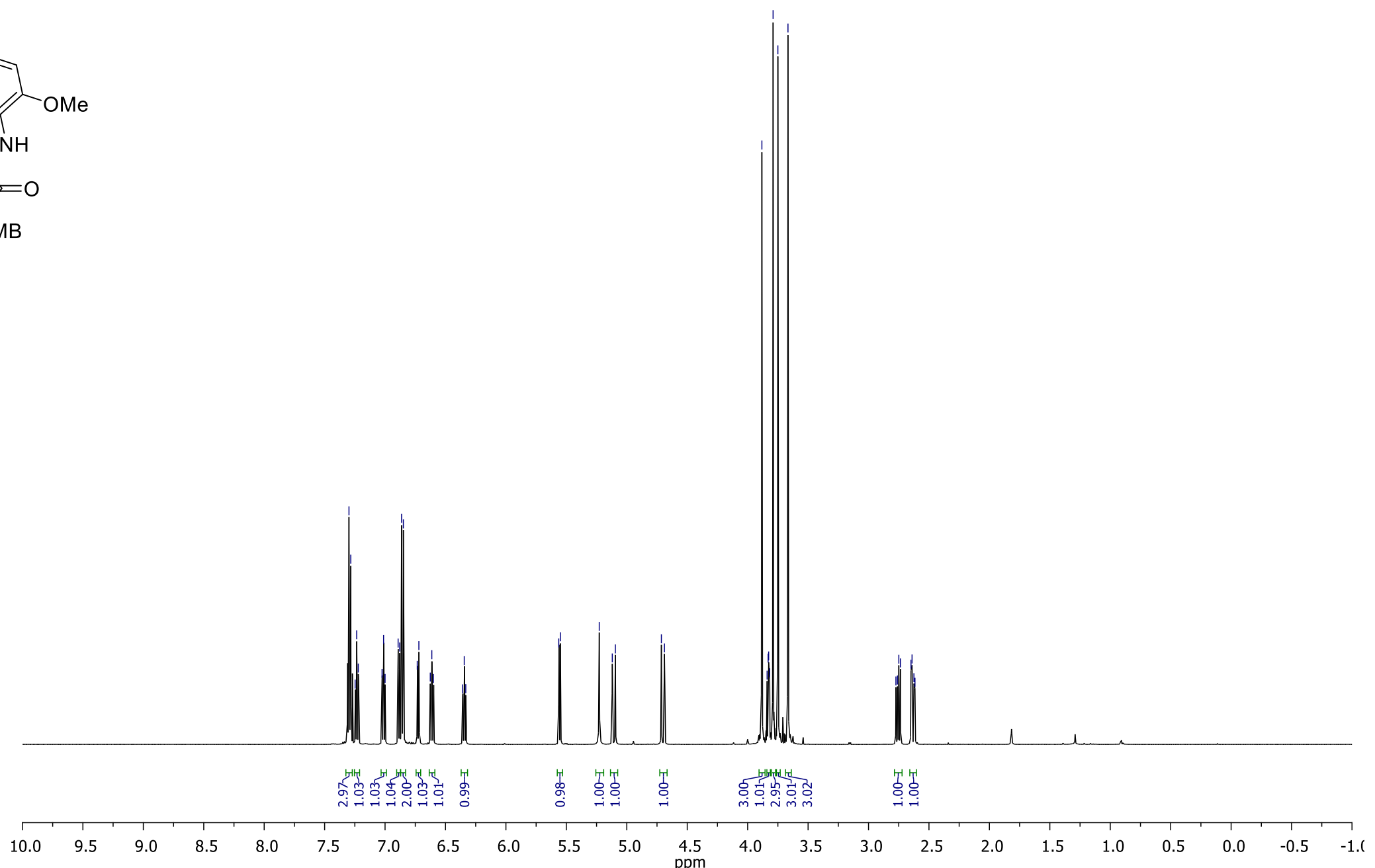
Dimethyl (\{3-1-(4-methoxybenzyl)-[(2-methoxyphenyl)amino]-2-oxo-2,3-dihydro-1H-indol-3-yl $\}$ methyl)malonate (7m)

${ }^{13} \mathrm{C} \mathrm{NMR}\left(\mathrm{CDCl}_{3}, 150 \mathrm{MHz}\right)$

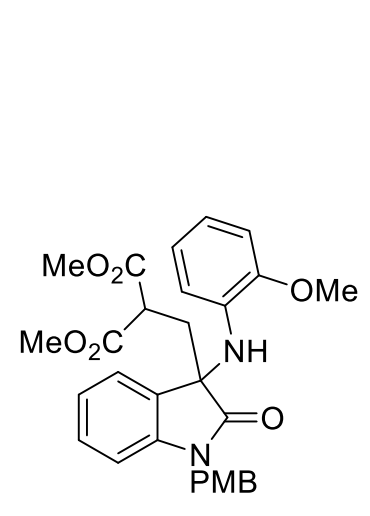

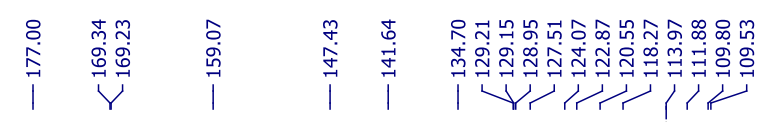

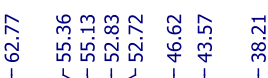


Dimethyl (\{3-1-(4-methoxybenzyl)-[(4-nitrophenyl)amino]-2-oxo-2,3-dihydro-1H-indol-3-yl\}methyl)malonate (7n)

${ }^{1} \mathrm{H} \mathrm{NMR}\left(\mathrm{CDCl}_{3}, 600 \mathrm{MHz}\right)$

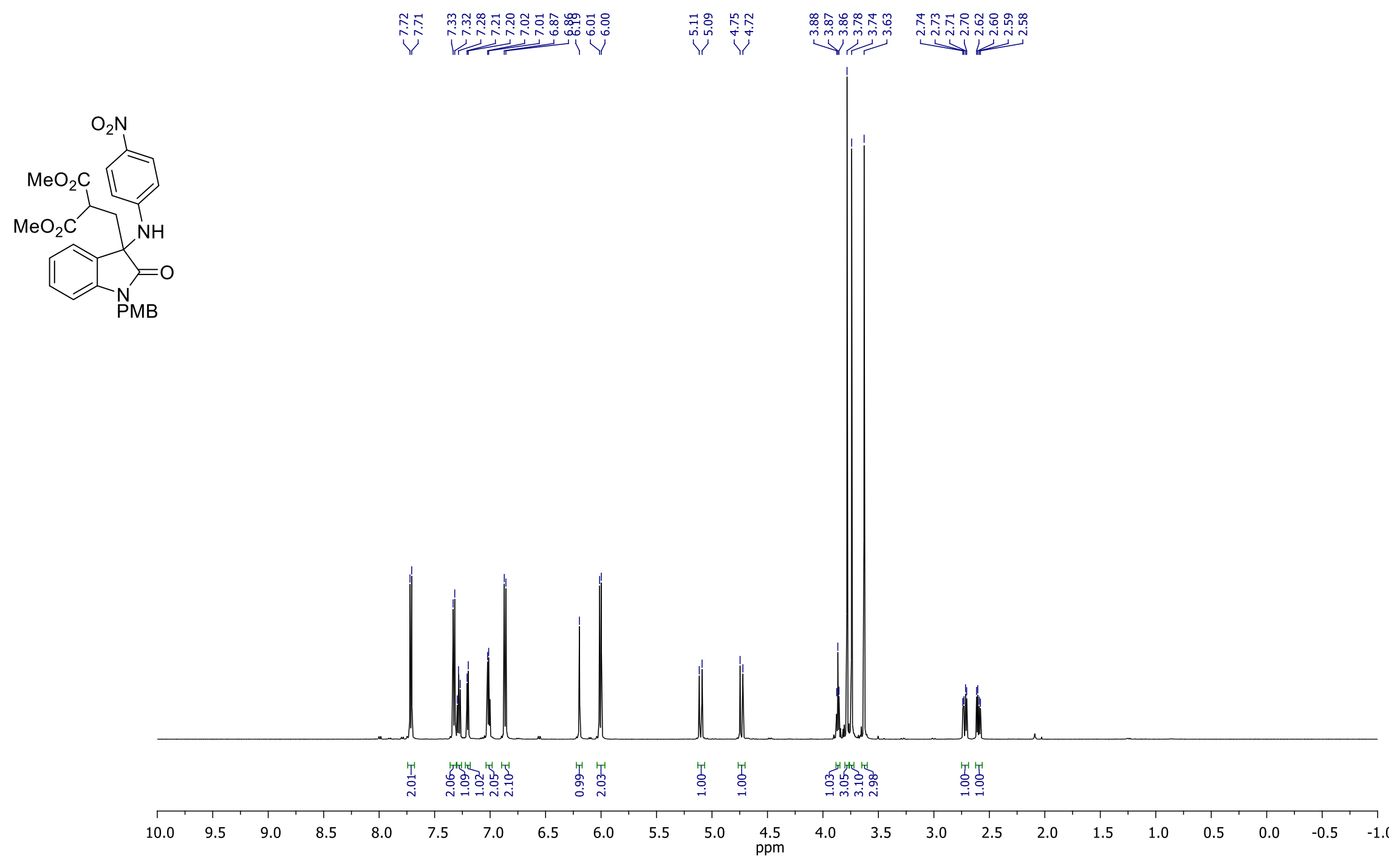


Dimethyl (\{3-1-(4-methoxybenzyl)-[(4-nitrophenyl)amino]-2-oxo-2,3-dihydro-1H-indol-3-yl\}methyl)malonate (7n)

${ }^{13} \mathrm{C} \mathrm{NMR}\left(\mathrm{CDCl}_{3}, 150 \mathrm{MHz}\right)$

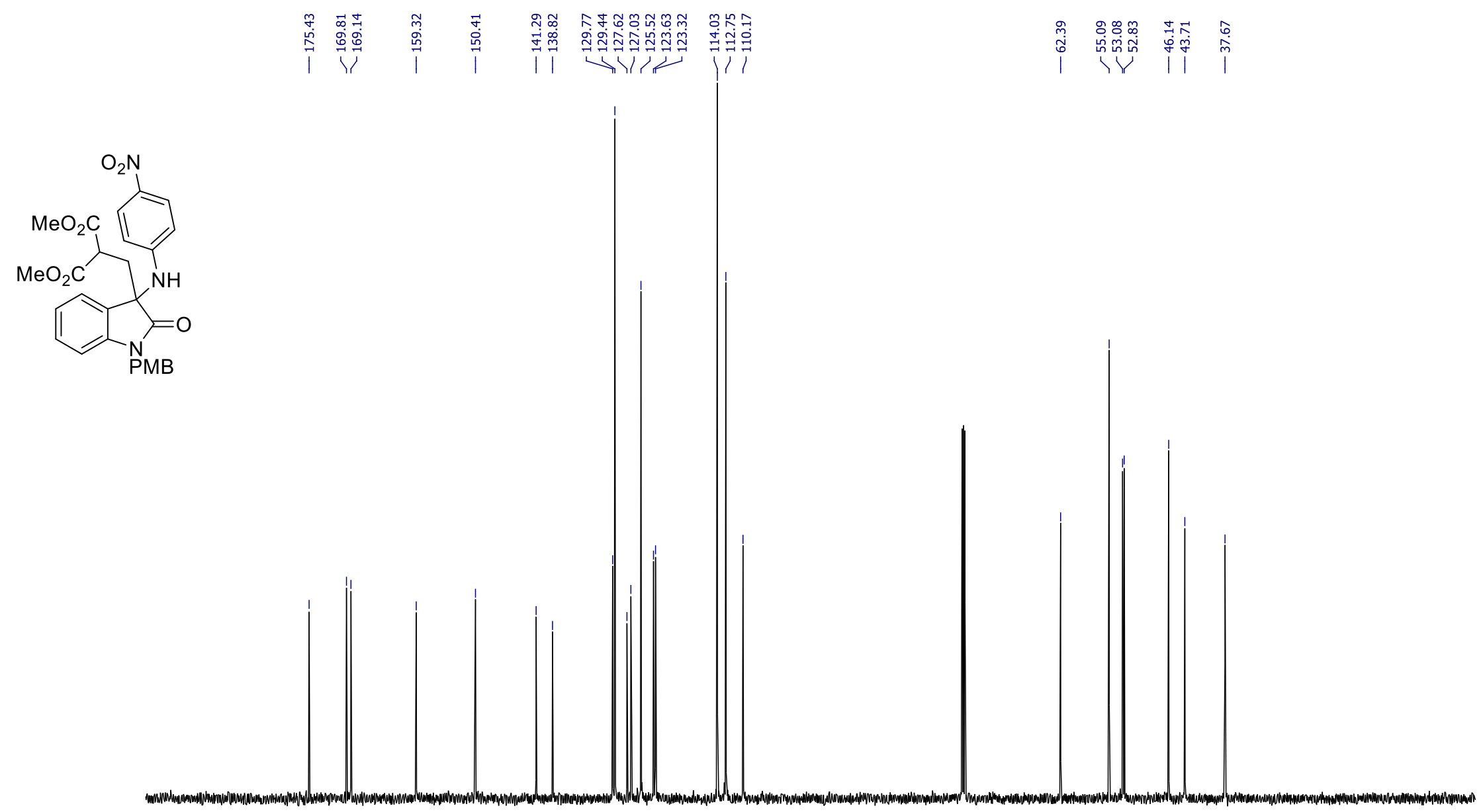

190 
Methyl 1-(4-methoxybenzyl)-2,5' -dioxo-1'-phenyl-1,2-dihydrospiro[indole-3,2'-pyrrolidine]-4' -carboxylate (8a)

${ }^{1} \mathrm{H} \mathrm{NMR}\left(\mathrm{CDCl}_{3}, 600 \mathrm{MHz}\right)$

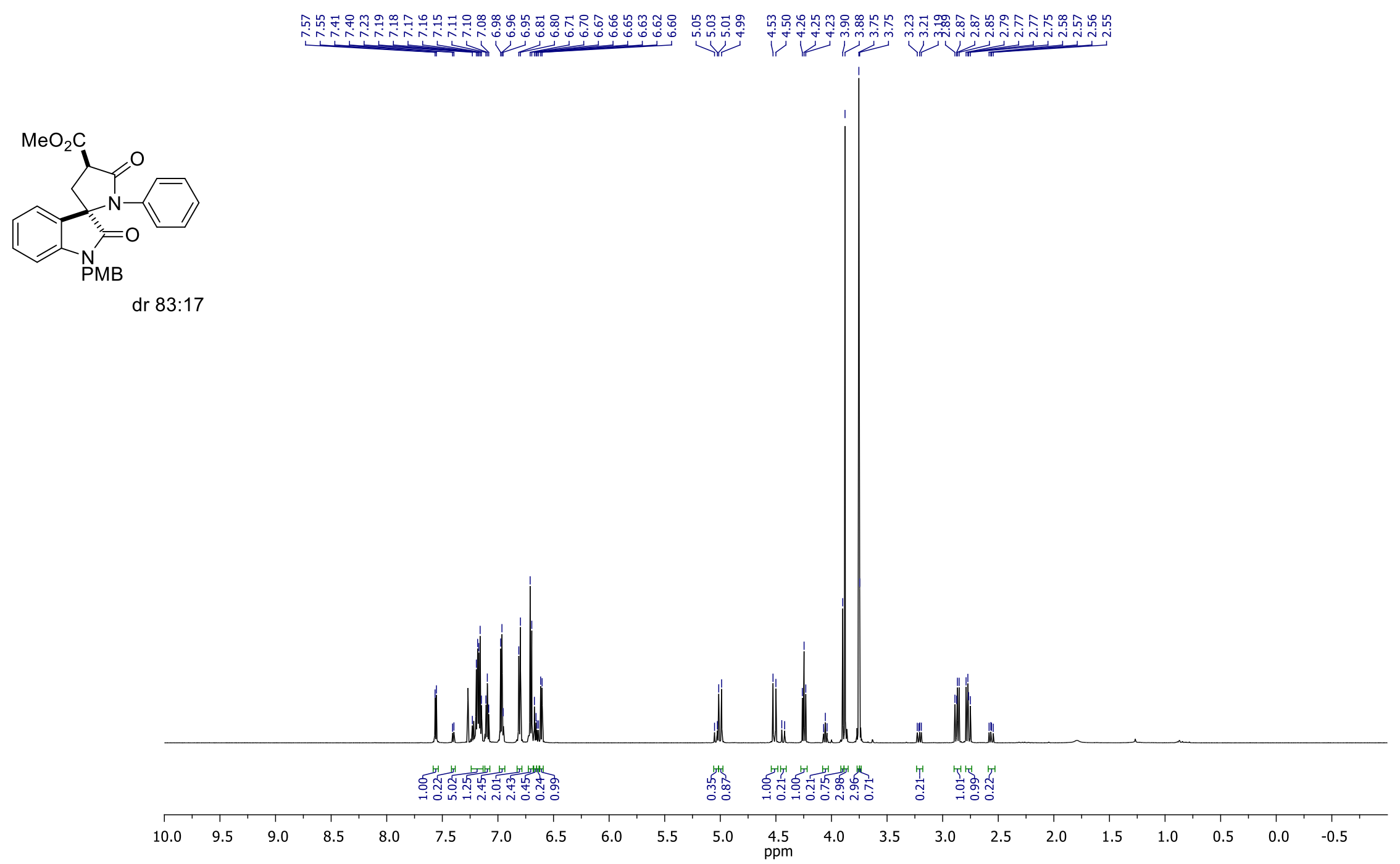


Methyl 1-(4-methoxybenzyl)-2,5' -dioxo-1'-phenyl-1,2-dihydrospiro[indole-3,2' -pyrrolidine]-4' -carboxylate (8a)

${ }^{1} \mathrm{H}-{ }^{1} \mathrm{H}$ NOESY $\left(\mathrm{CDCl}_{3}, 600 \mathrm{MHz}\right)$
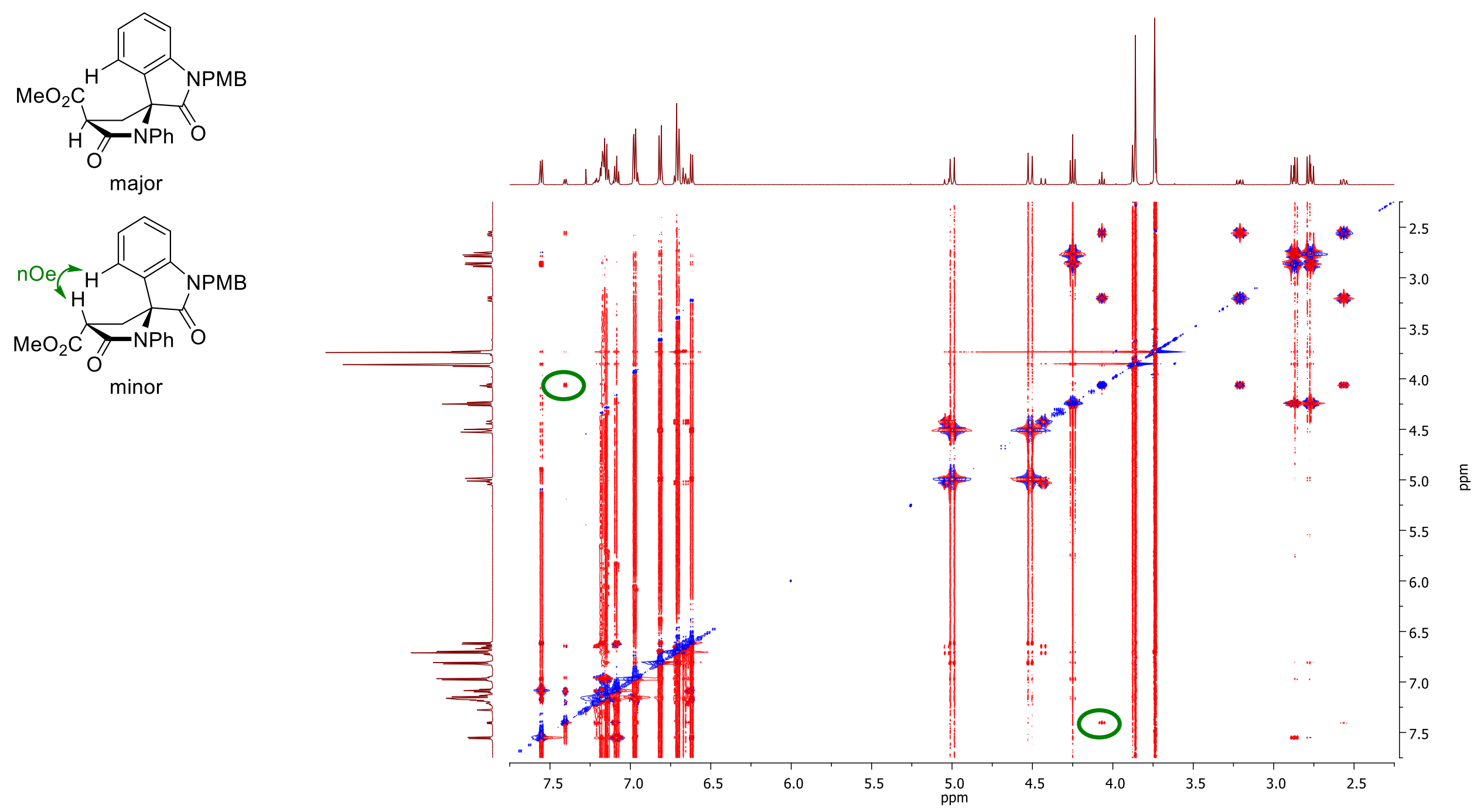
Methyl 1-(4-methoxybenzyl)-2,5'-dioxo-1'-phenyl-1,2-dihydrospiro[indole-3,2' -pyrrolidine]-4' -carboxylate (8a)

${ }^{13} \mathrm{C} \mathrm{NMR}\left(\mathrm{CDCl}_{3}, 150 \mathrm{MHz}\right)$

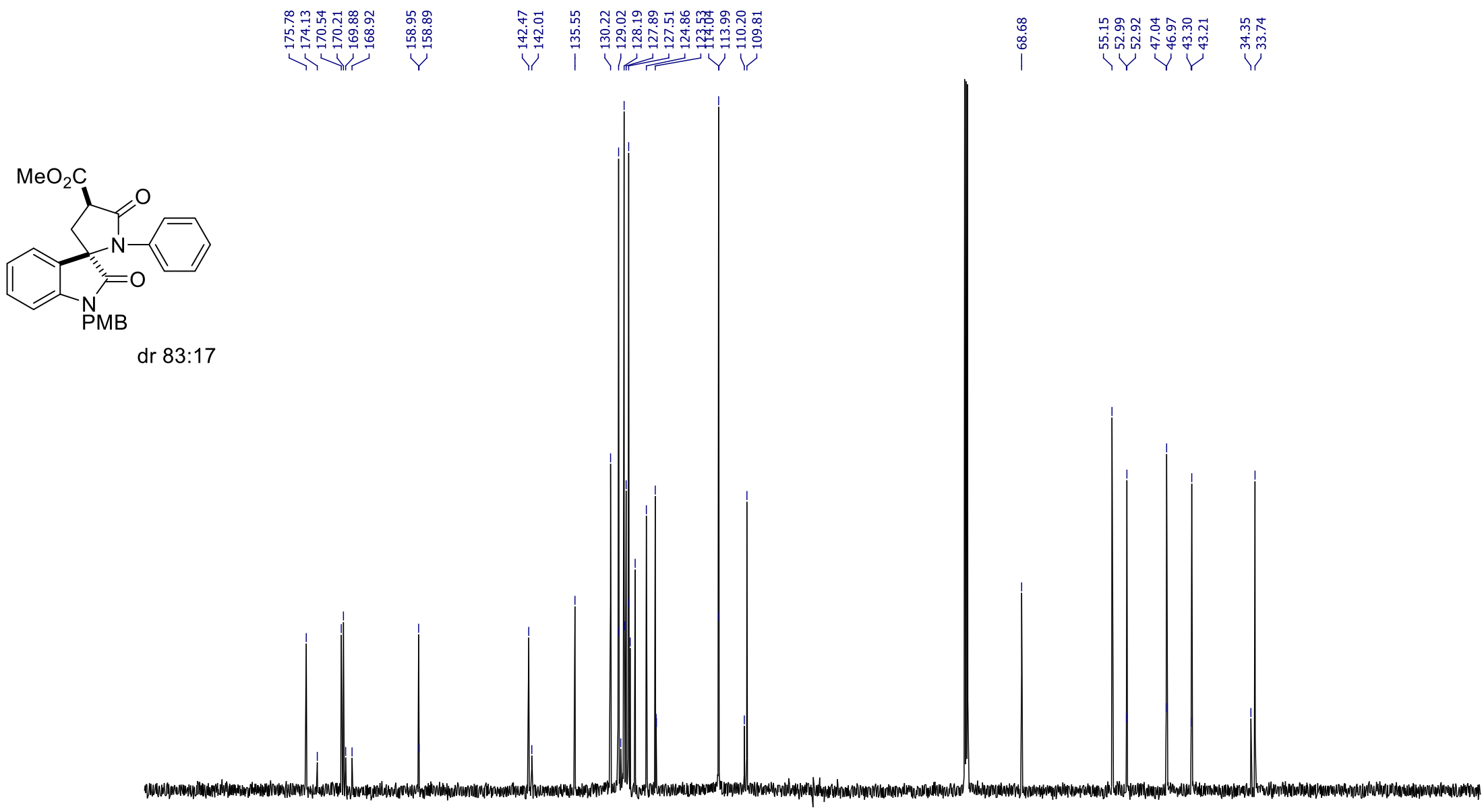

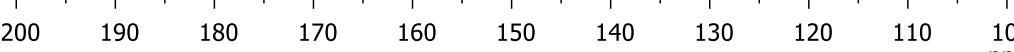


Methyl 1-(4-methoxybenzyl)-5-methyl-2,5'-dioxo-1'-phenyl-1,2-dihydrospiro[indole-3,2' -pyrrolidine]-4'-carboxylate (8b)

${ }^{1} \mathrm{H} \mathrm{NMR}\left(\mathrm{CDCl}_{3}, 600 \mathrm{MHz}\right)$
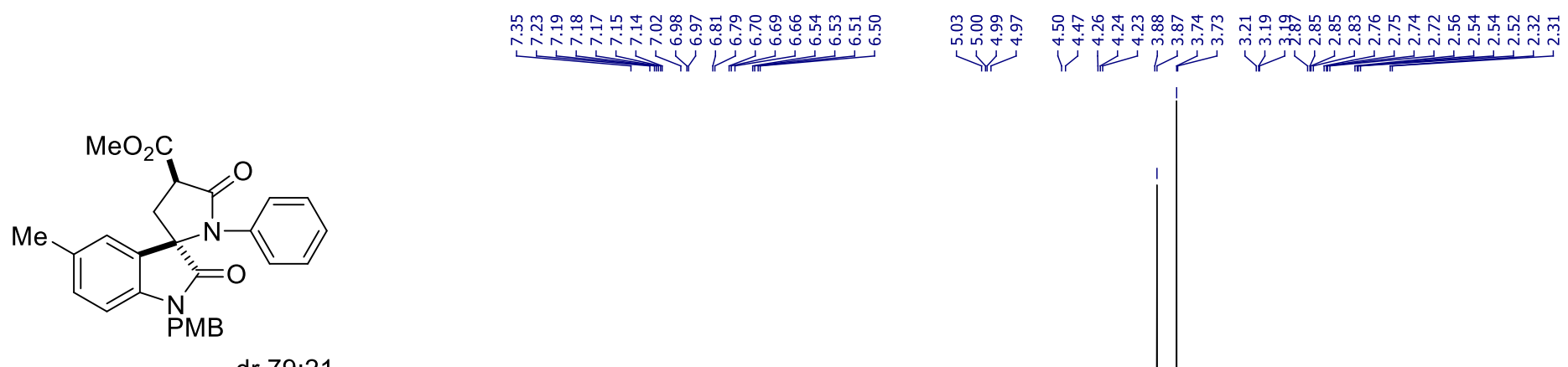

dr 79:21

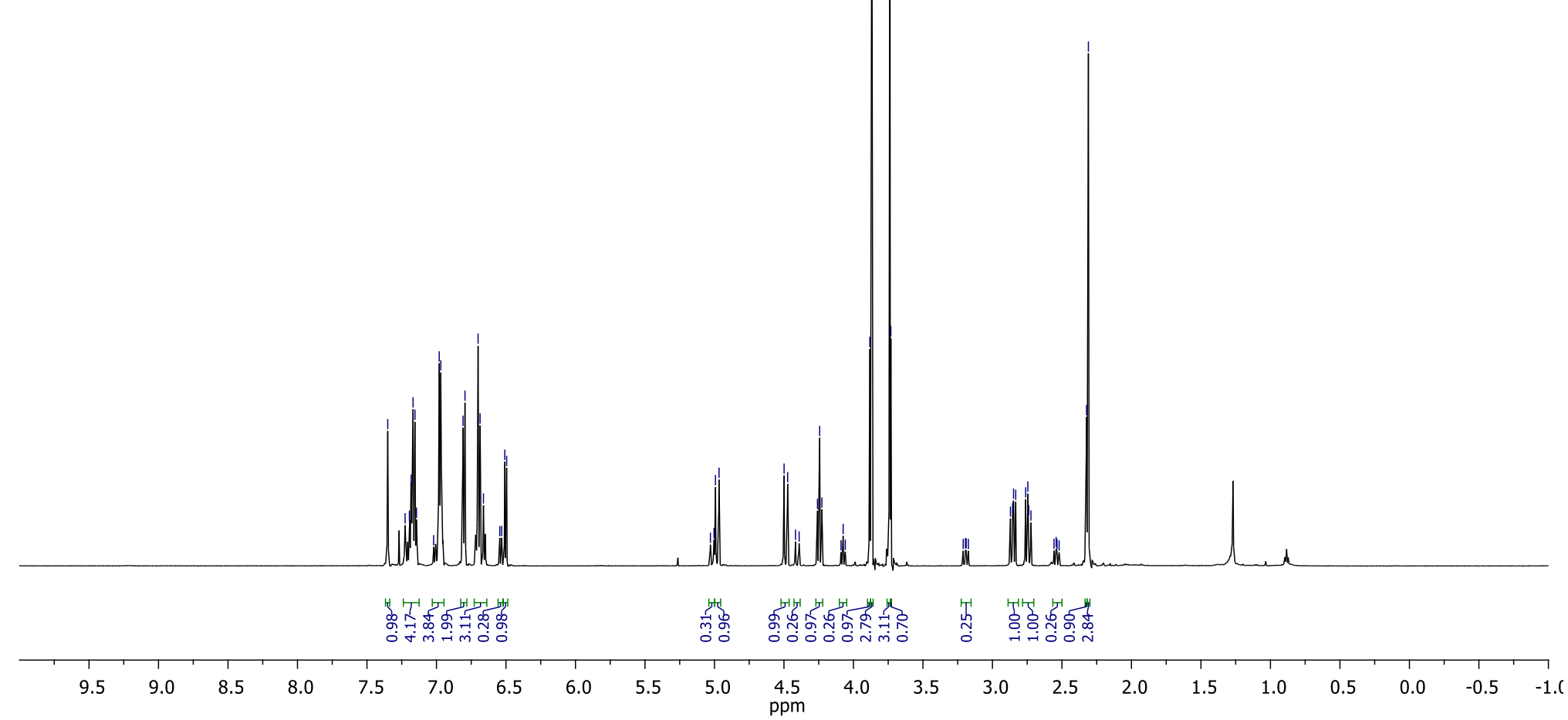


Methyl 1-(4-methoxybenzyl)-5-methyl-2,5'-dioxo-1'-phenyl-1,2-dihydrospiro[indole-3,2' -pyrrolidine]-4'-carboxylate (8b)

${ }^{13} \mathrm{C} \mathrm{NMR}\left(\mathrm{CDCl}_{3}, 150 \mathrm{MHz}\right)$

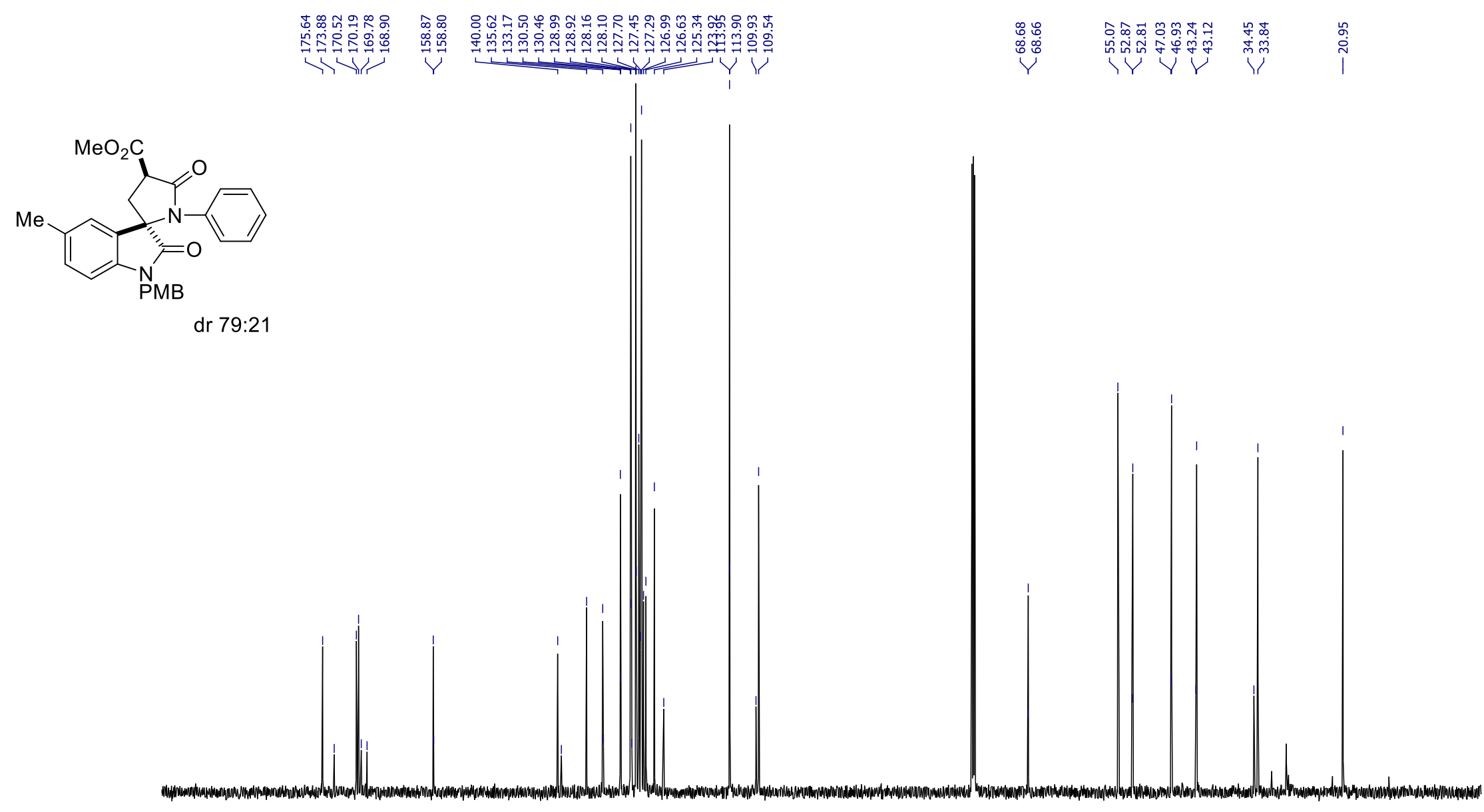

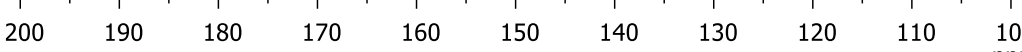


Methyl 1-methyl-2,5' -dioxo-1' -phenyl-1,2-dihydrospiro[indole-3,2' -pyrrolidine]-4' -carboxylate (8c)

${ }^{1} \mathrm{H} \mathrm{NMR}\left(\mathrm{CDCl}_{3}, 600 \mathrm{MHz}\right)$

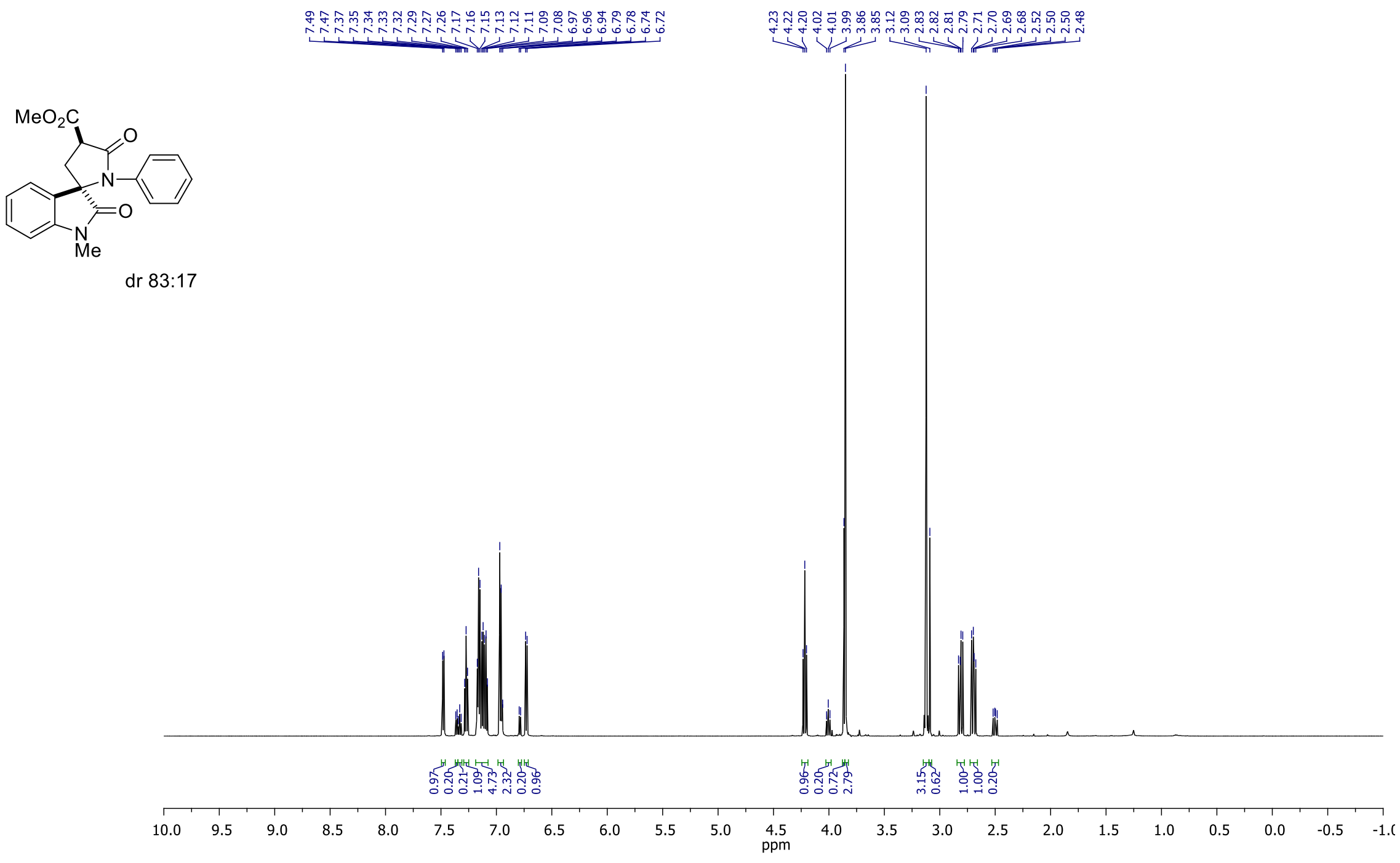


Methyl 1-methyl-2,5' -dioxo-1' -phenyl-1,2-dihydrospiro[indole-3,2' -pyrrolidine]-4' -carboxylate (8c)

${ }^{13} \mathrm{C} \mathrm{NMR}\left(\mathrm{CDCl}_{3}, 150 \mathrm{MHz}\right)$

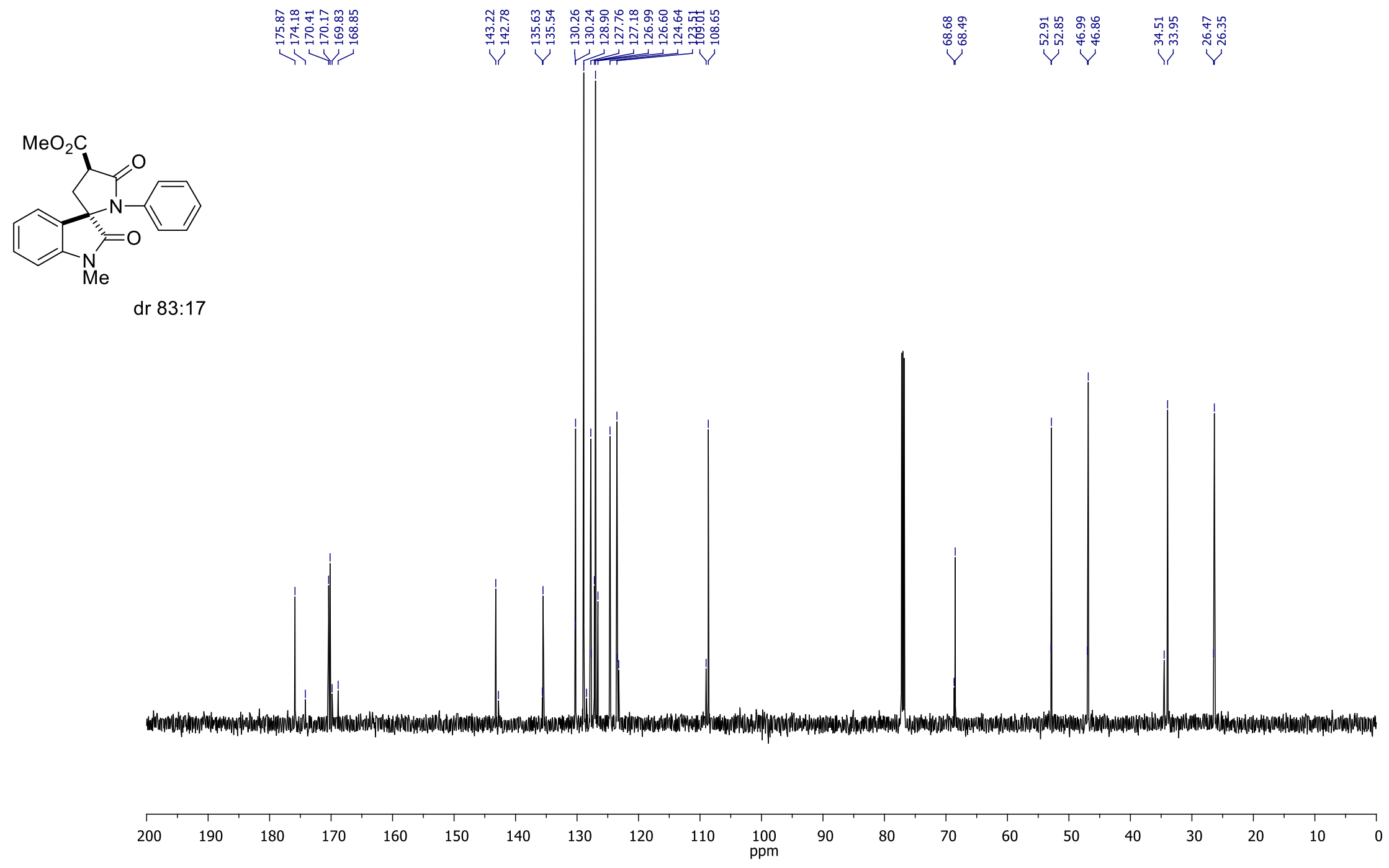


${ }^{1} \mathrm{H} \mathrm{NMR}\left(\mathrm{CDCl}_{3}, 600 \mathrm{MHz}\right)$

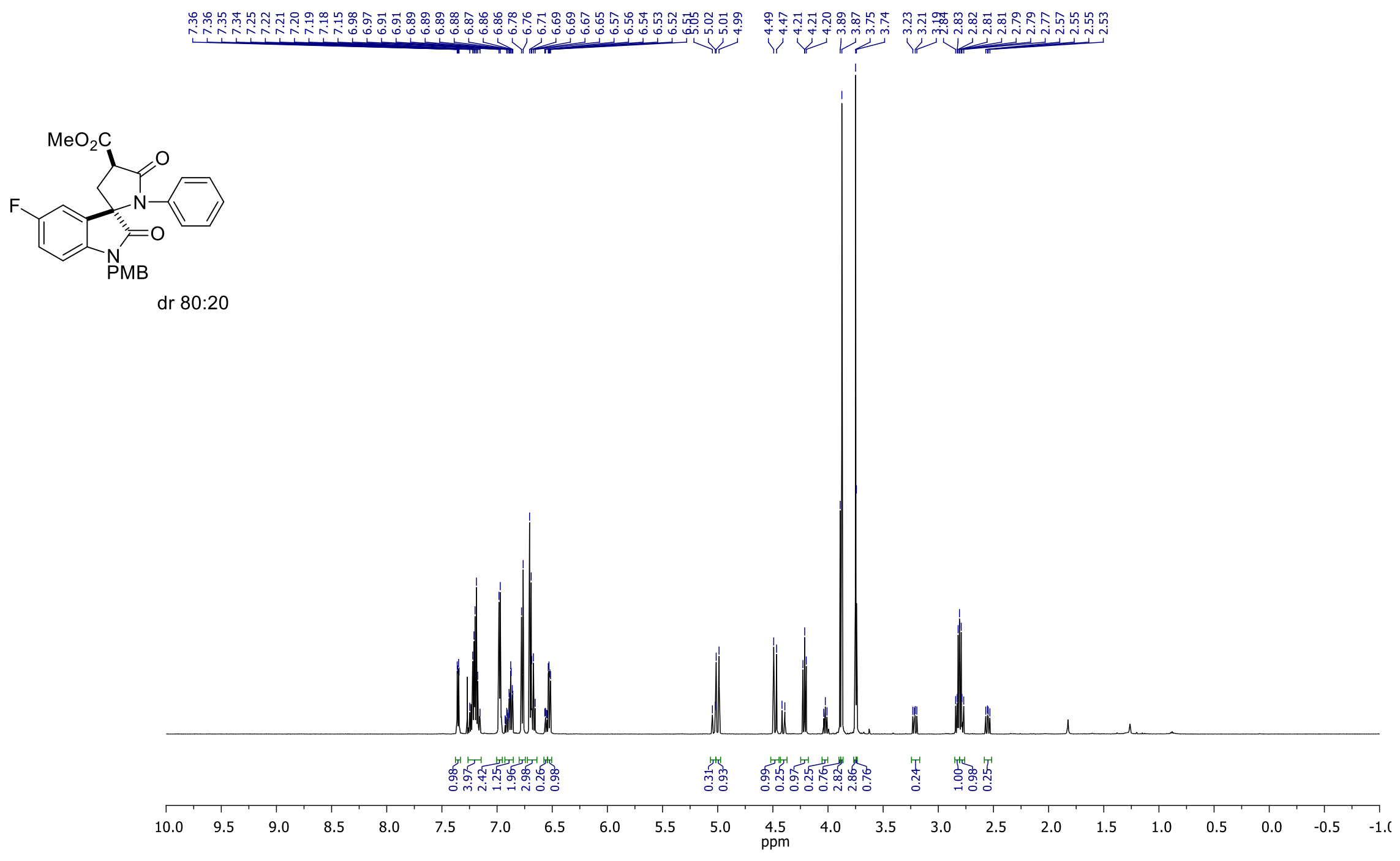


Methyl 5-fluoro-1-(4-methoxybenzyl)-2,5'-dioxo-1'-phenyl-1,2-dihydrospiro[indole-3,2' -pyrrolidine]-4' -carboxylate (8d)

${ }^{13} \mathrm{C} \mathrm{NMR}\left(\mathrm{CDCl}_{3}, 150 \mathrm{MHz}\right)$

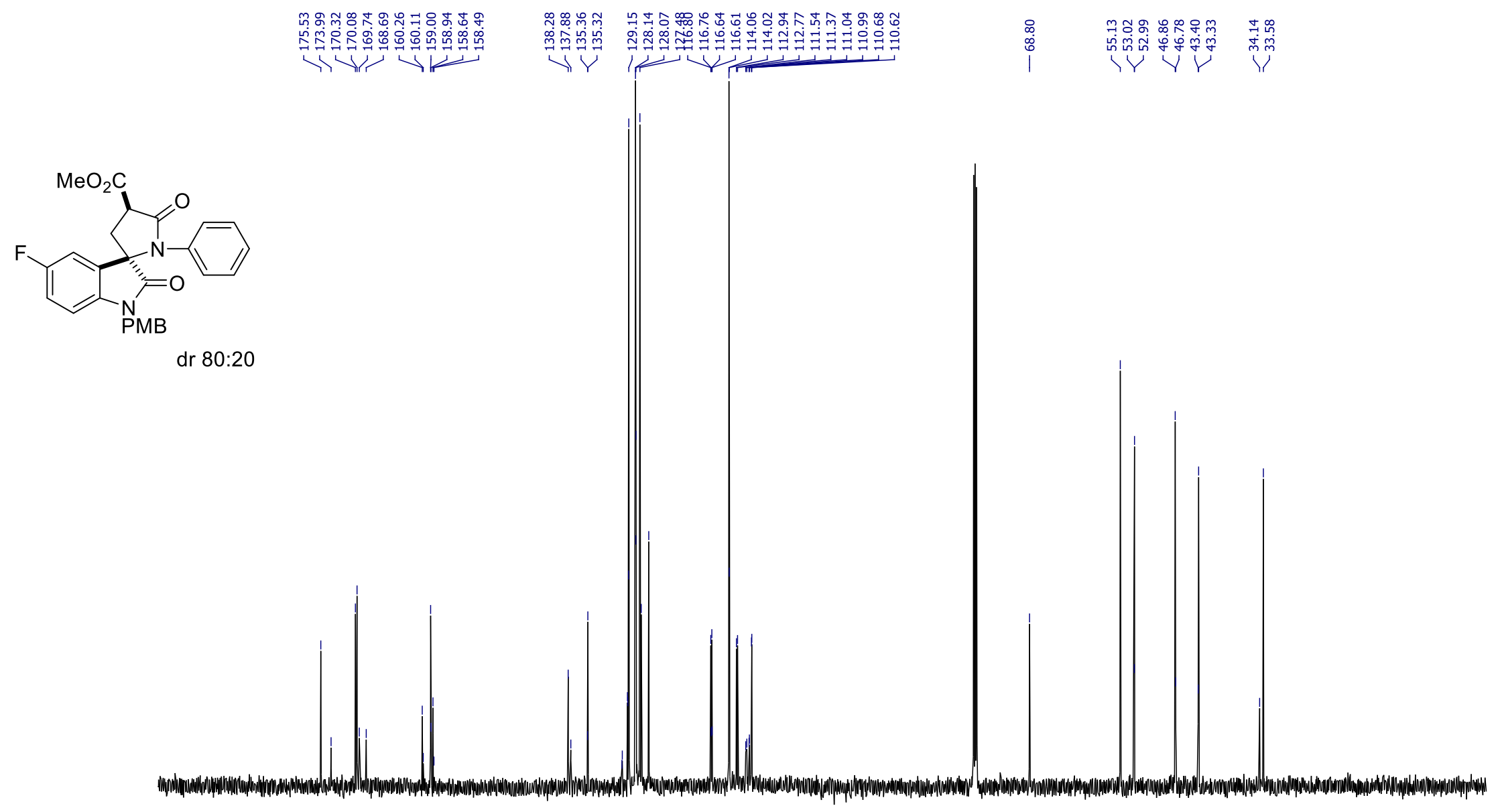

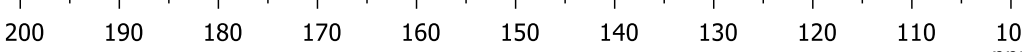


${ }^{1} \mathrm{H} \mathrm{NMR}\left(\mathrm{CDCl}_{3}, 600 \mathrm{MHz}\right)$

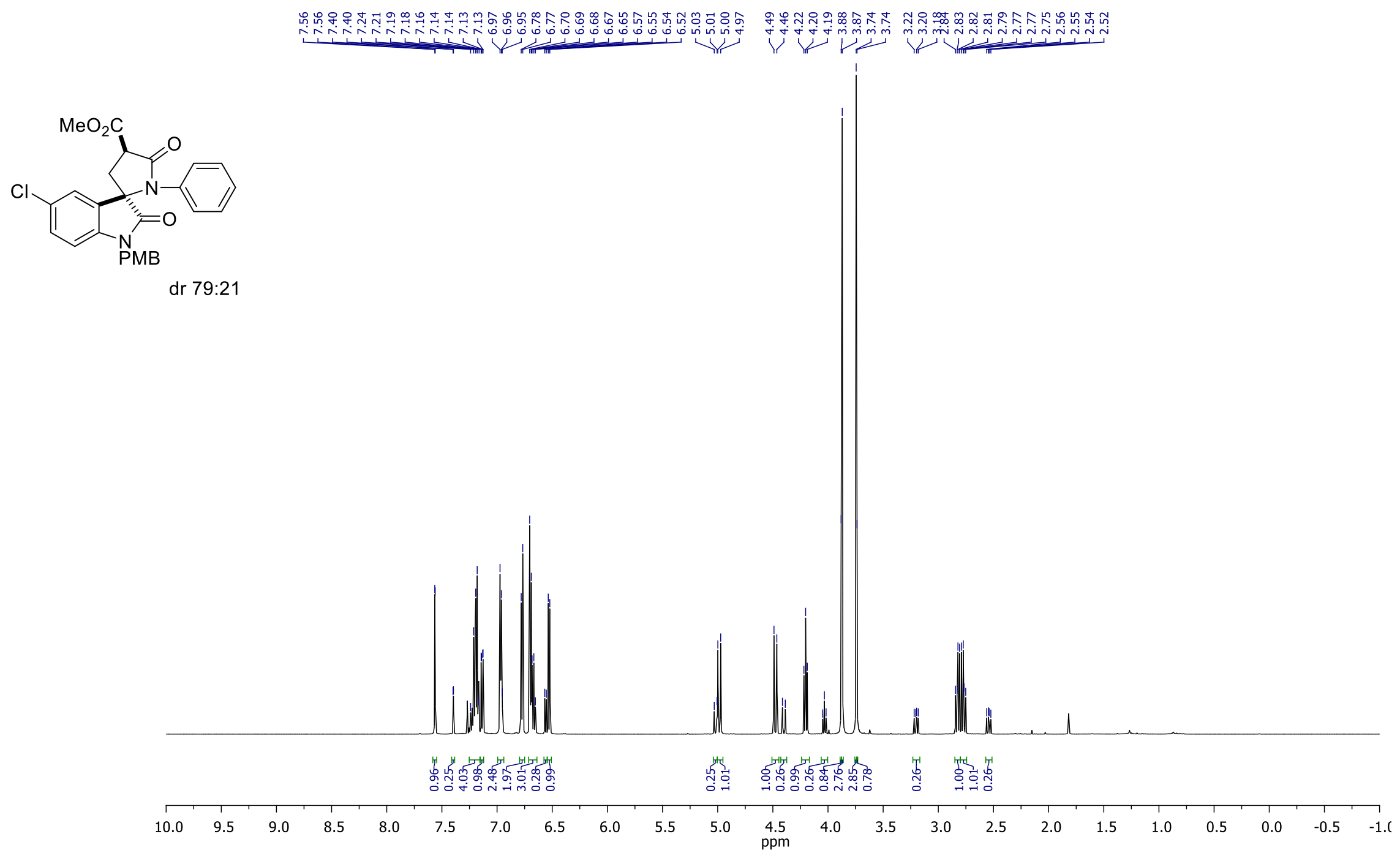


Methyl 5-chloro-1-(4-methoxybenzyl)-2,5'-dioxo-1'-phenyl-1,2-dihydrospiro[indole-3,2' -pyrrolidine]-4' -carboxylate (8e)

${ }^{13} \mathrm{C} \mathrm{NMR}\left(\mathrm{CDCl}_{3}, 150 \mathrm{MHz}\right)$

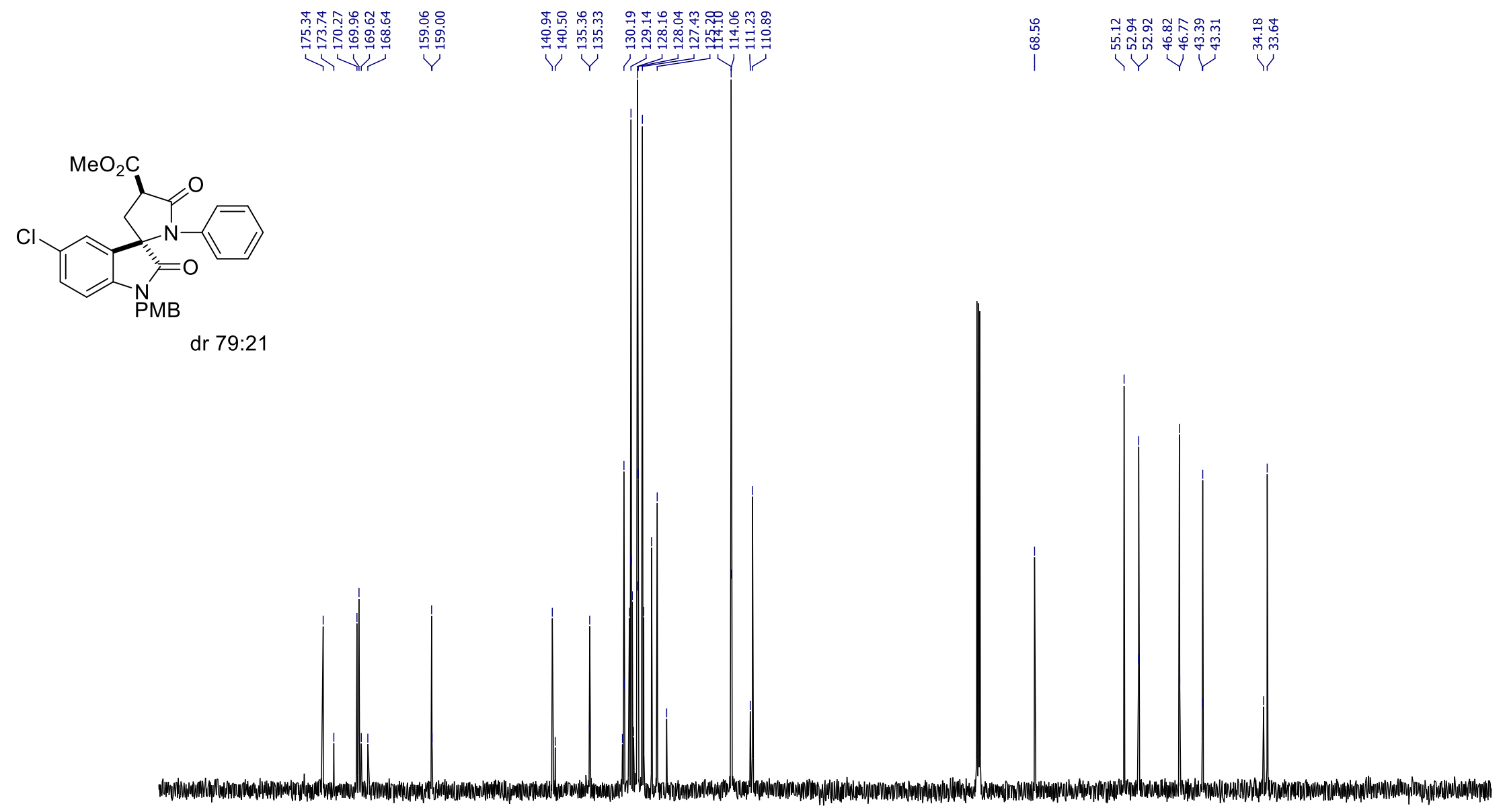

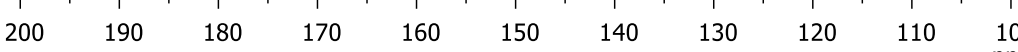


Methyl 5-bromo-1-(4-methoxybenzyl)-2,5' -dioxo-1' -phenyl-1,2-dihydrospiro[indole-3,2' -pyrrolidine]-4' -carboxylate (8f)

${ }^{1} \mathrm{H} \mathrm{NMR}\left(\mathrm{CDCl}_{3}, 600 \mathrm{MHz}\right)$

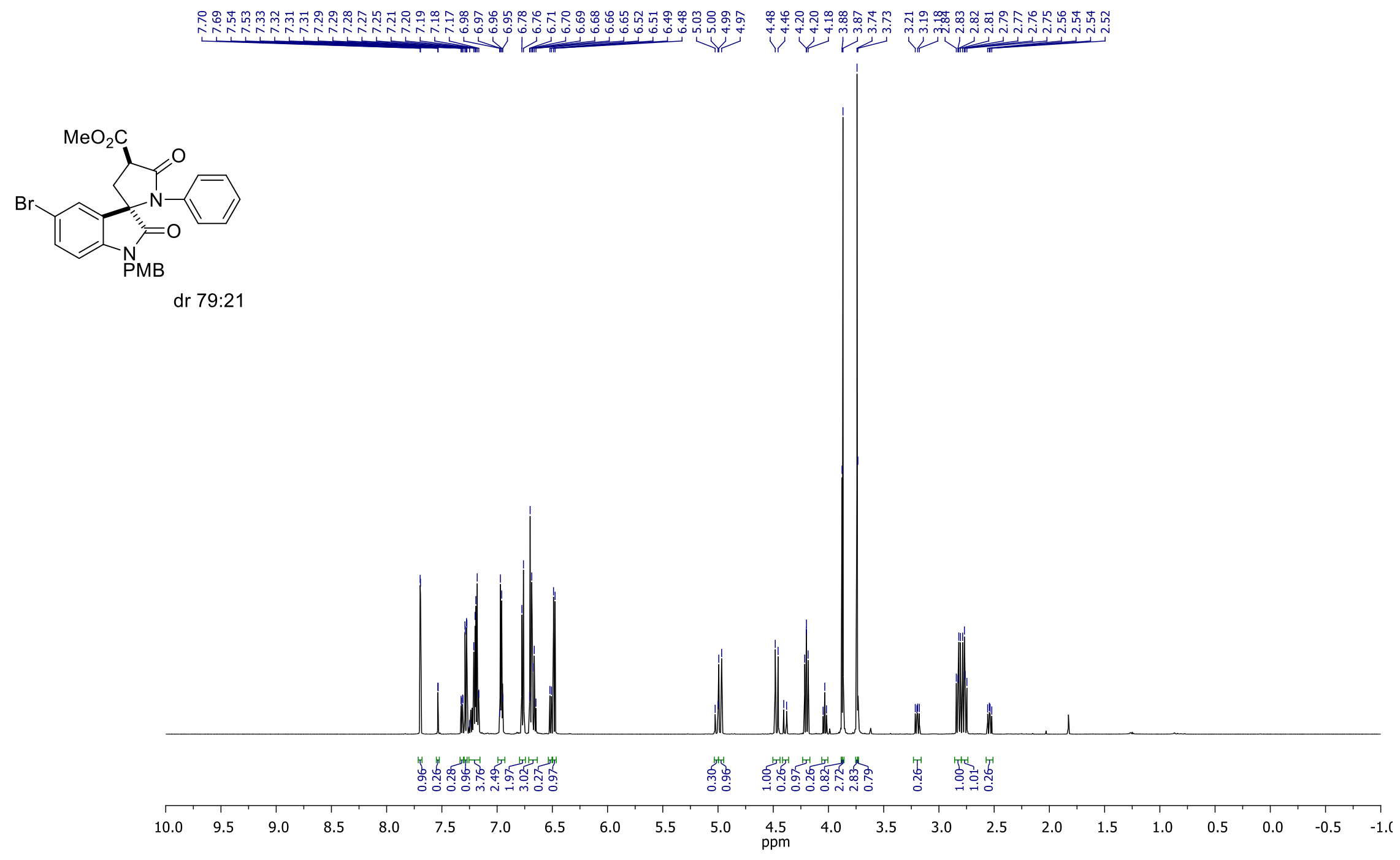


Methyl 5-bromo-1-(4-methoxybenzyl)-2,5' -dioxo-1' -phenyl-1,2-dihydrospiro[indole-3,2' -pyrrolidine]-4' -carboxylate (8f)

${ }^{13} \mathrm{C} \mathrm{NMR}\left(\mathrm{CDCl}_{3}, 150 \mathrm{MHz}\right)$

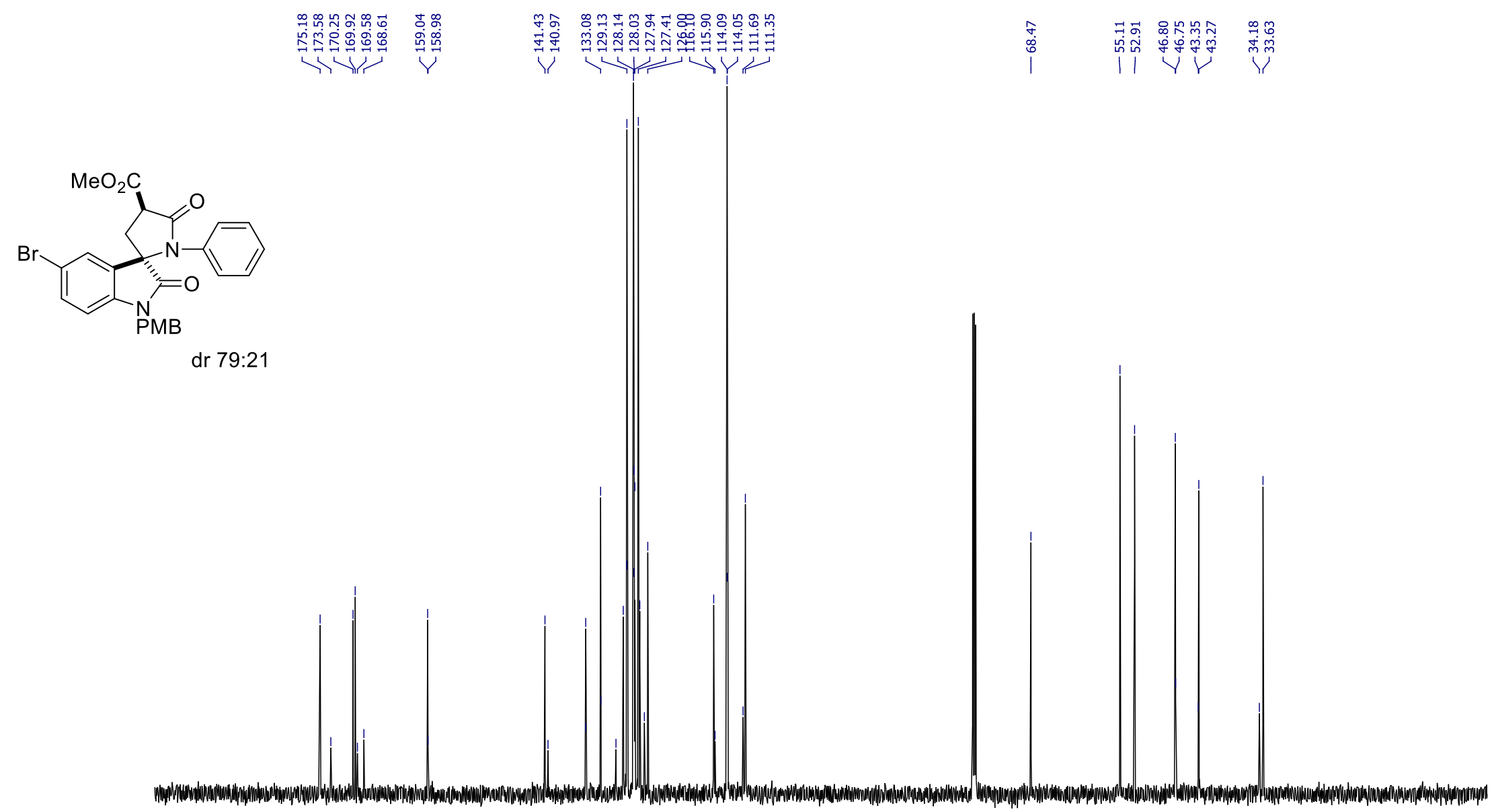

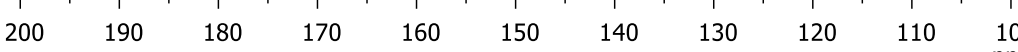


Methyl 1'-(4-fluorophenyl)-1-(4-methoxybenzyl)-2,5'-dioxo-1,2-dihydrospiro[indole-3,2'-pyrrolidine]-4'-carboxylate (8g) ${ }^{1} \mathrm{H} \mathrm{NMR}\left(\mathrm{CDCl}_{3}, 600 \mathrm{MHz}\right)$

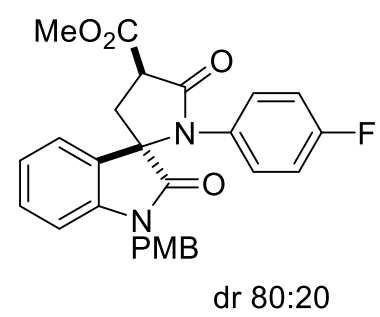

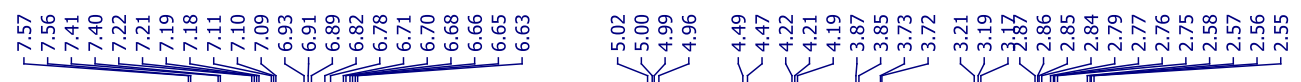

dr 80:20

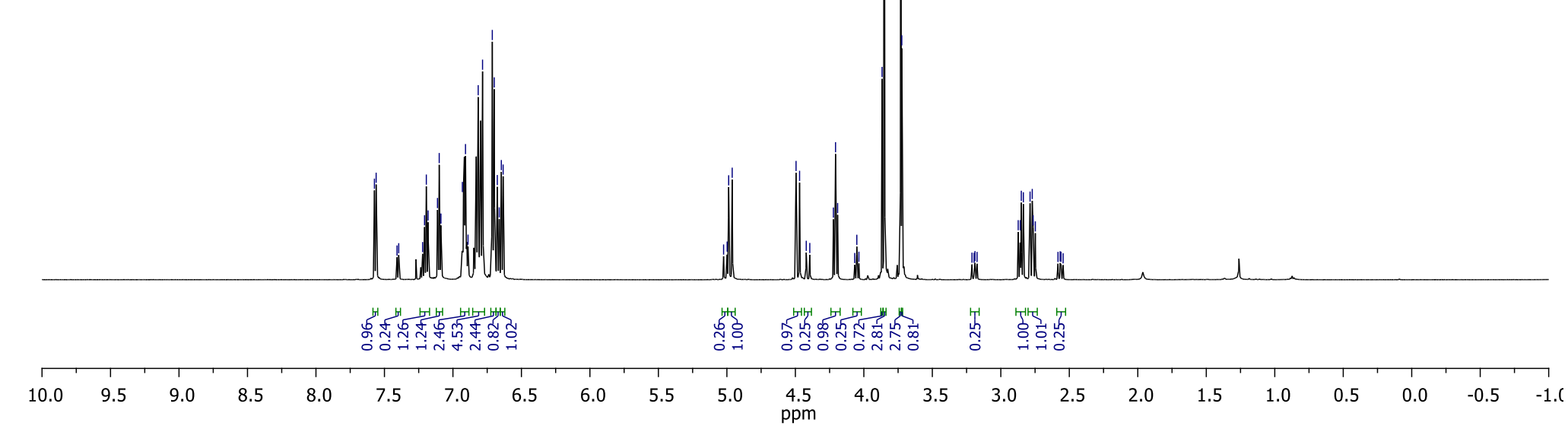


Methyl 1'-(4-fluorophenyl)-1-(4-methoxybenzyl)-2,5'-dioxo-1,2-dihydrospiro[indole-3,2'-pyrrolidine]-4'-carboxylate (8g)

${ }^{13} \mathrm{C} \mathrm{NMR}\left(\mathrm{CDCl}_{3}, 150 \mathrm{MHz}\right)$

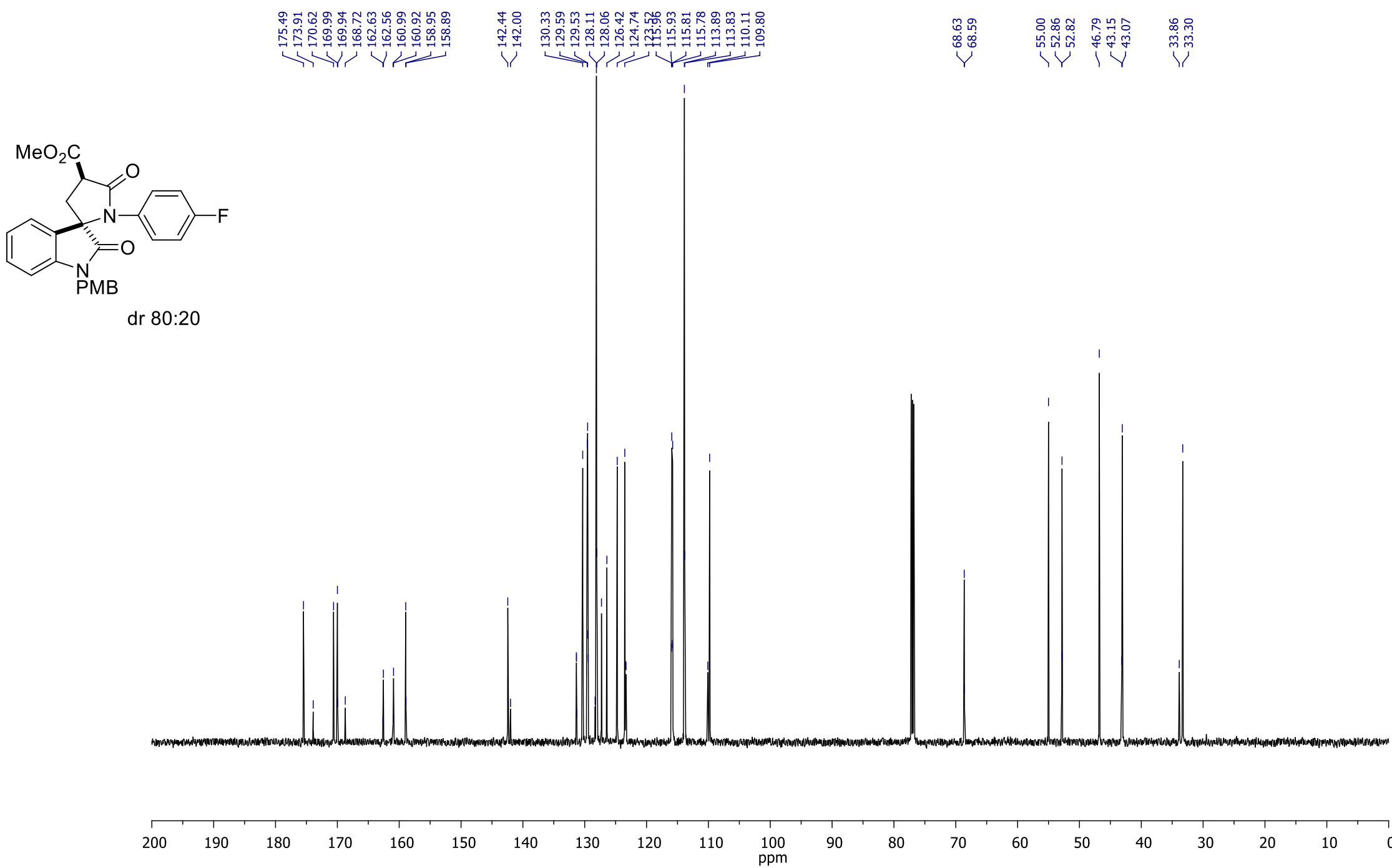


Methyl 1'-(4-chlorophenyl)-1-(4-methoxybenzyl)-2,5'-dioxo-1,2-dihydrospiro[indole-3,2'-pyrrolidine]-4'-carboxylate (8h)

${ }^{1} \mathrm{H} \mathrm{NMR}\left(\mathrm{CDCl}_{3}, 600 \mathrm{MHz}\right)$

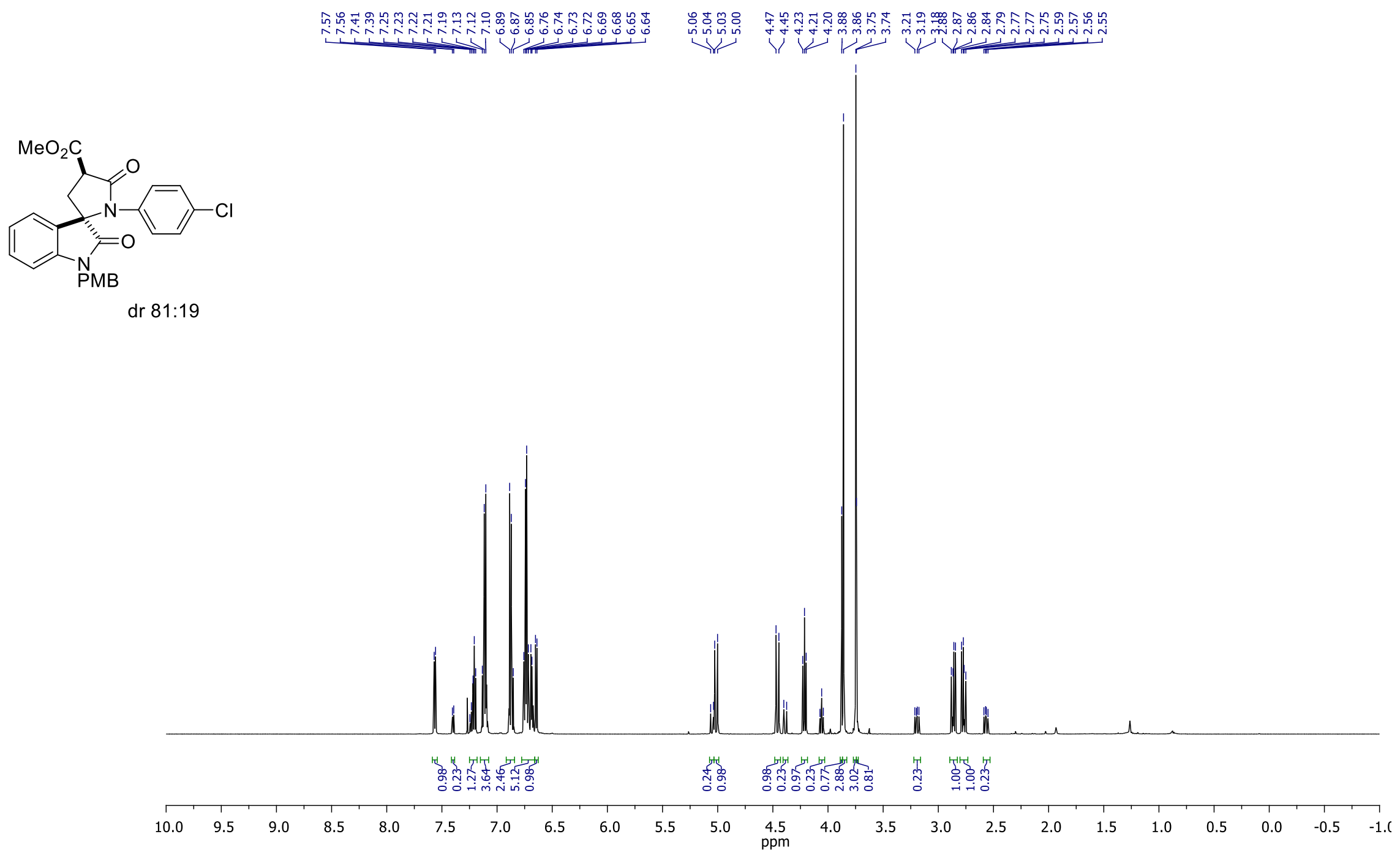


Methyl 1'-(4-chlorophenyl)-1-(4-methoxybenzyl)-2,5'-dioxo-1,2-dihydrospiro[indole-3,2'-pyrrolidine]-4'-carboxylate (8h)

${ }^{13} \mathrm{C} \mathrm{NMR}\left(\mathrm{CDCl}_{3}, 150 \mathrm{MHz}\right)$

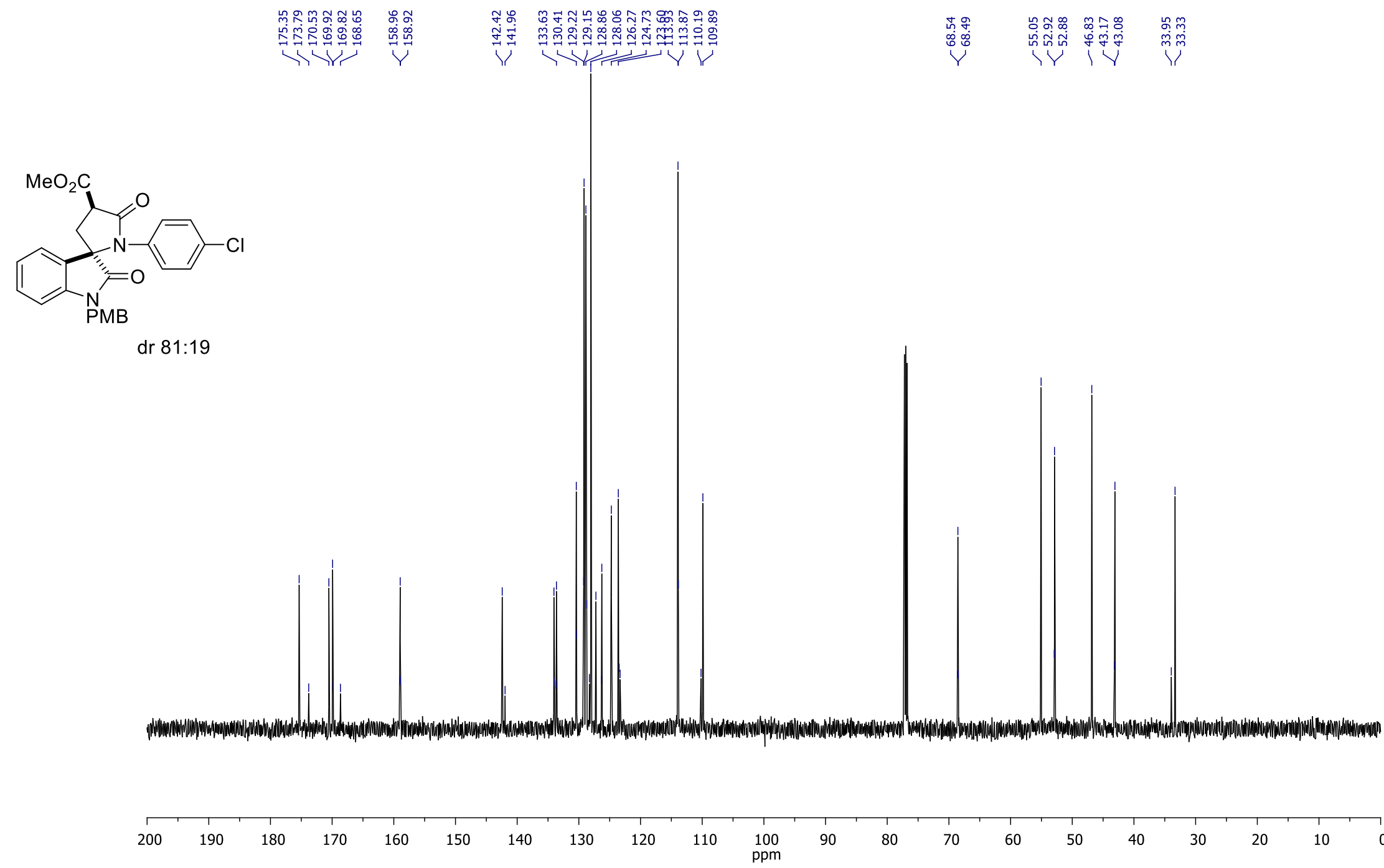


Methyl 1'-(3-chlorophenyl)-1-(4-methoxybenzyl)-2,5'-dioxo-1,2-dihydrospiro[indole-3,2' -pyrrolidine]-4' -carboxylate (8i)

${ }^{1} \mathrm{H} \mathrm{NMR}\left(\mathrm{CDCl}_{3}, 600 \mathrm{MHz}\right)$

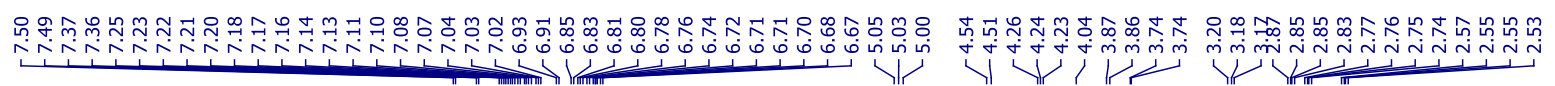

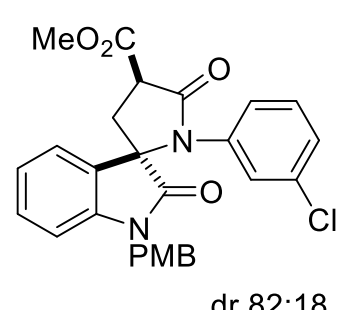

dr 82:18

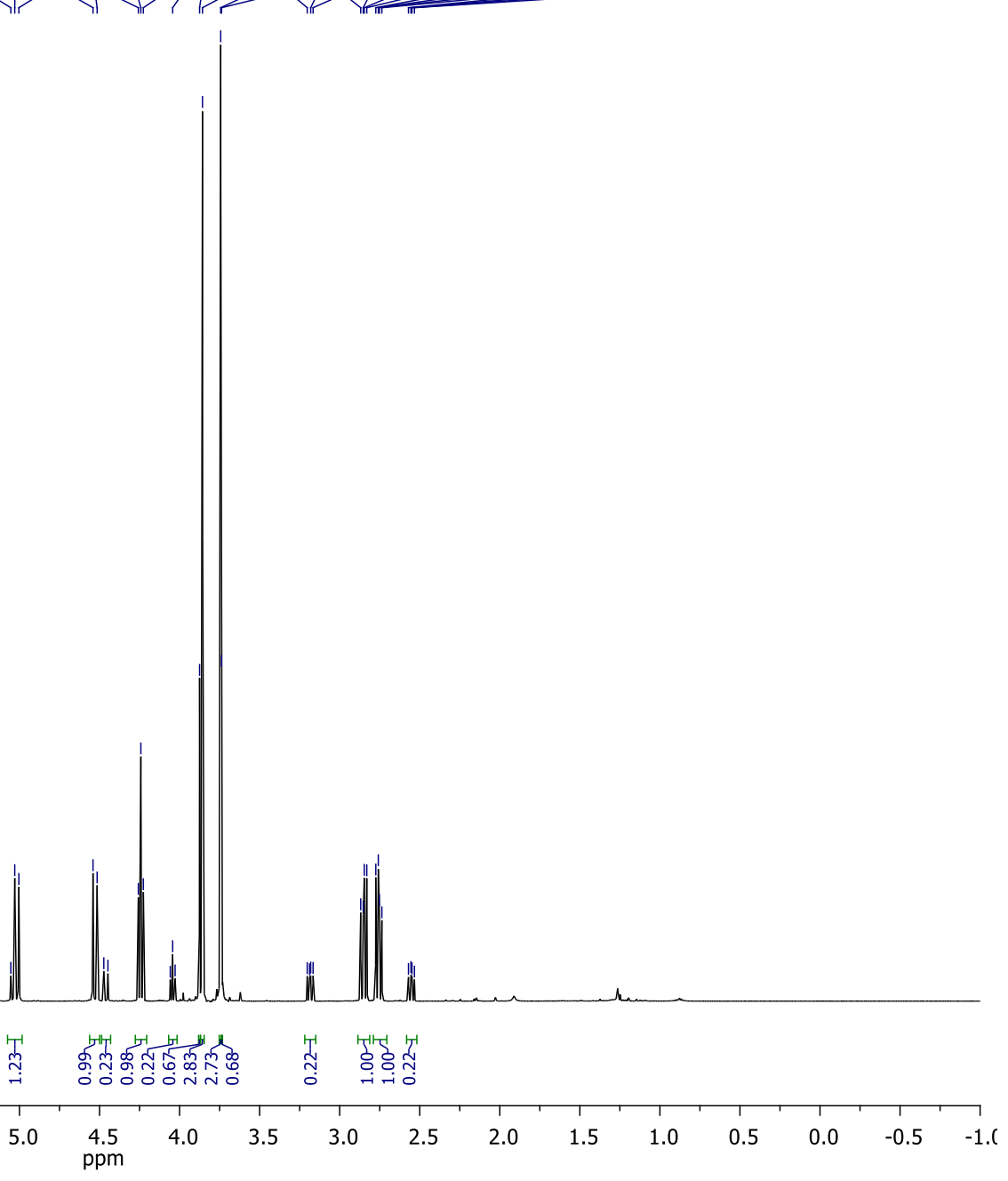


Methyl 1'-(3-chlorophenyl)-1-(4-methoxybenzyl)-2,5'-dioxo-1,2-dihydrospiro[indole-3,2'-pyrrolidine]-4'-carboxylate (8i)

${ }^{13} \mathrm{C} \mathrm{NMR}\left(\mathrm{CDCl}_{3}, 150 \mathrm{MHz}\right)$

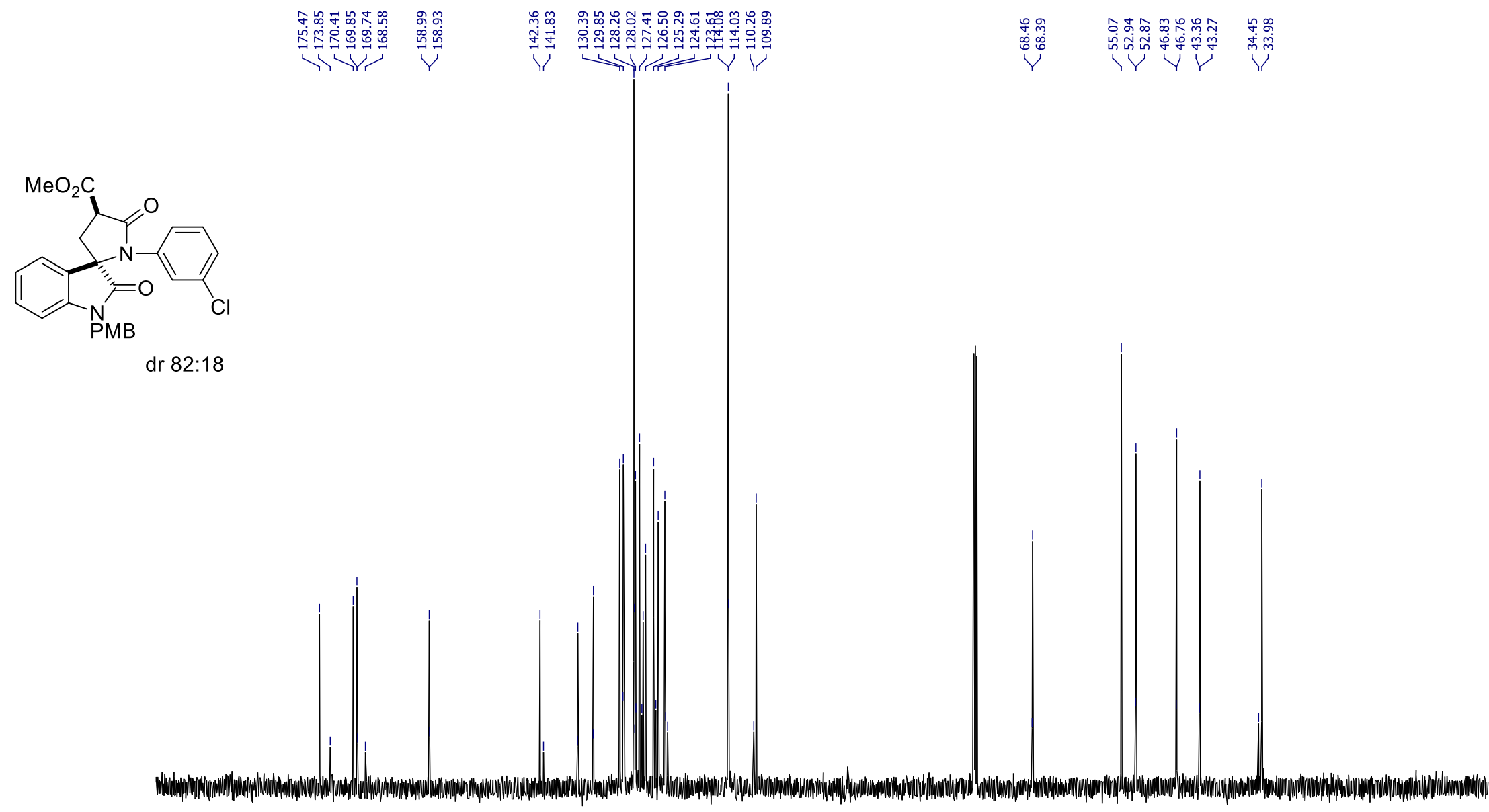

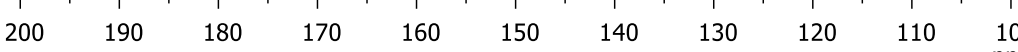


Methyl 1' -(4-bromophenyl)-1-(4-methoxybenzyl)-2,5' -dioxo-1,2-dihydrospiro[indole-3,2' -pyrrolidine]-4' -carboxylate (8j)

${ }^{1} \mathrm{H} \mathrm{NMR}\left(\mathrm{CDCl}_{3}, 600 \mathrm{MHz}\right)$

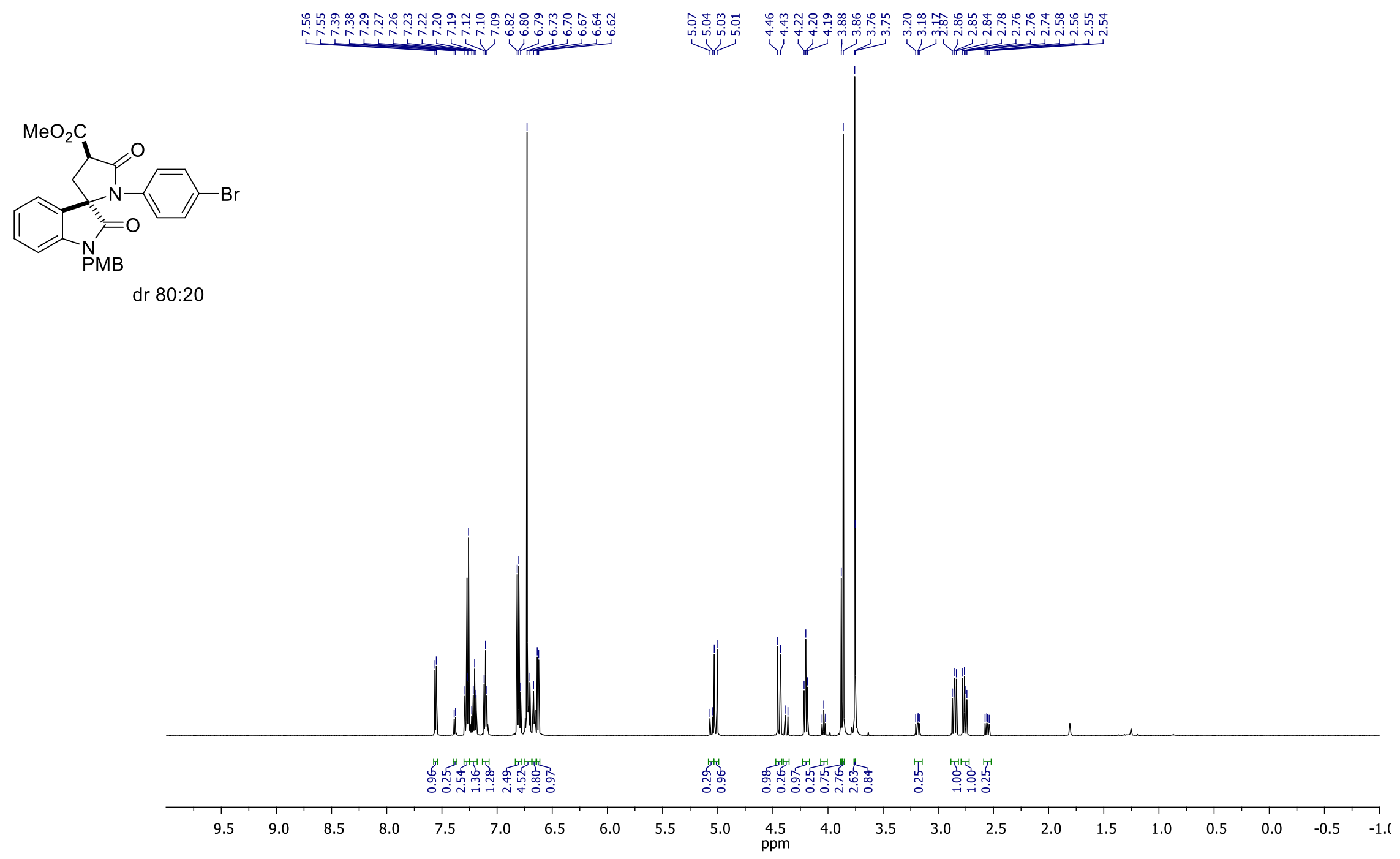


Methyl 1' -(4-bromophenyl)-1-(4-methoxybenzyl)-2,5' -dioxo-1,2-dihydrospiro[indole-3,2' -pyrrolidine]-4' -carboxylate (8j)

${ }^{13} \mathrm{C} \mathrm{NMR}\left(\mathrm{CDCl}_{3}, 150 \mathrm{MHz}\right)$

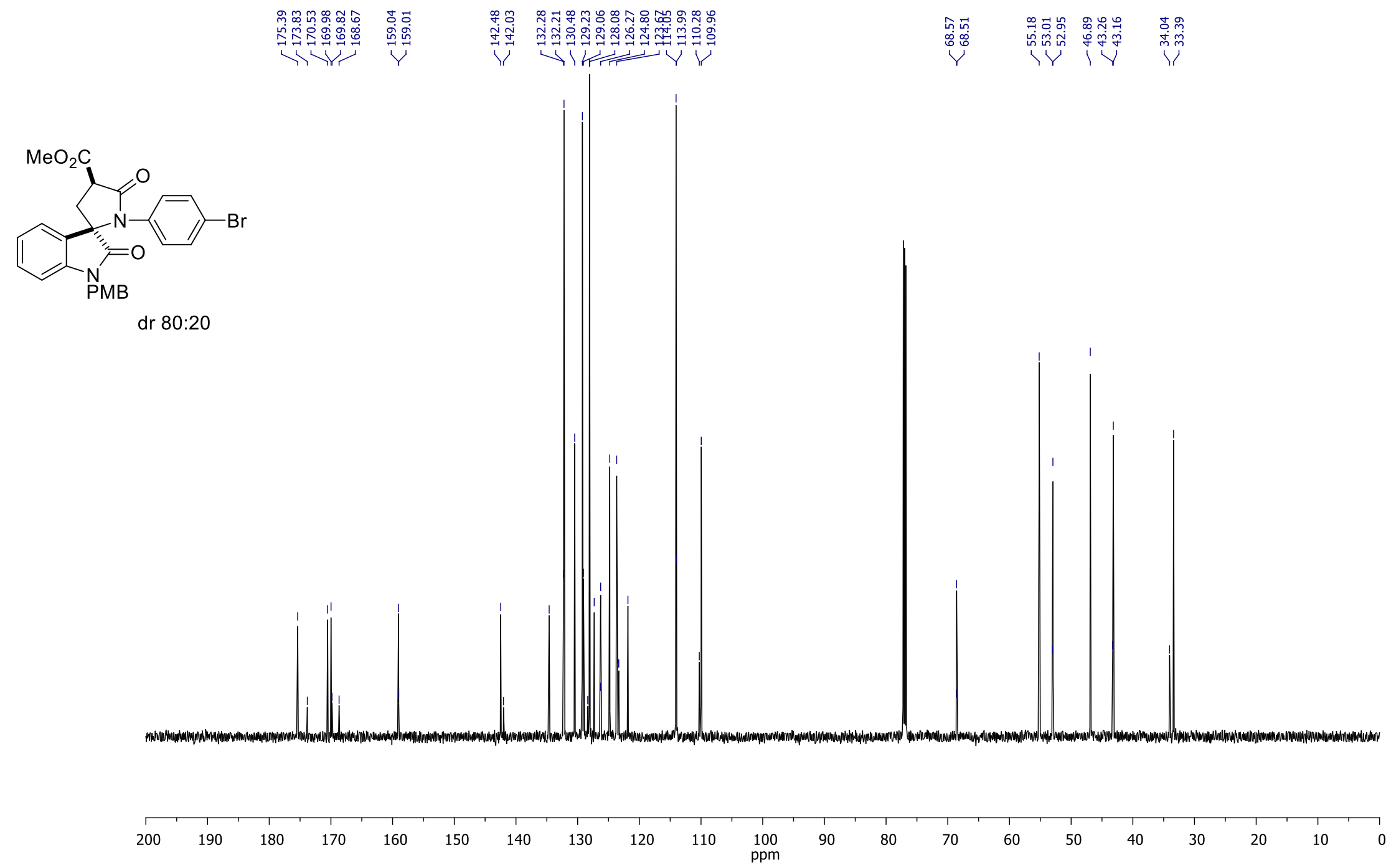


Methyl 1-(4-methoxybenzyl)-1' -(4-methylphenyl)-2,5' -dioxo-1,2-dihydrospiro[indole-3,2'-pyrrolidine]-4'-carboxylate (8k)

${ }^{1} \mathrm{H} \mathrm{NMR}\left(\mathrm{CDCl}_{3}, 600 \mathrm{MHz}\right)$

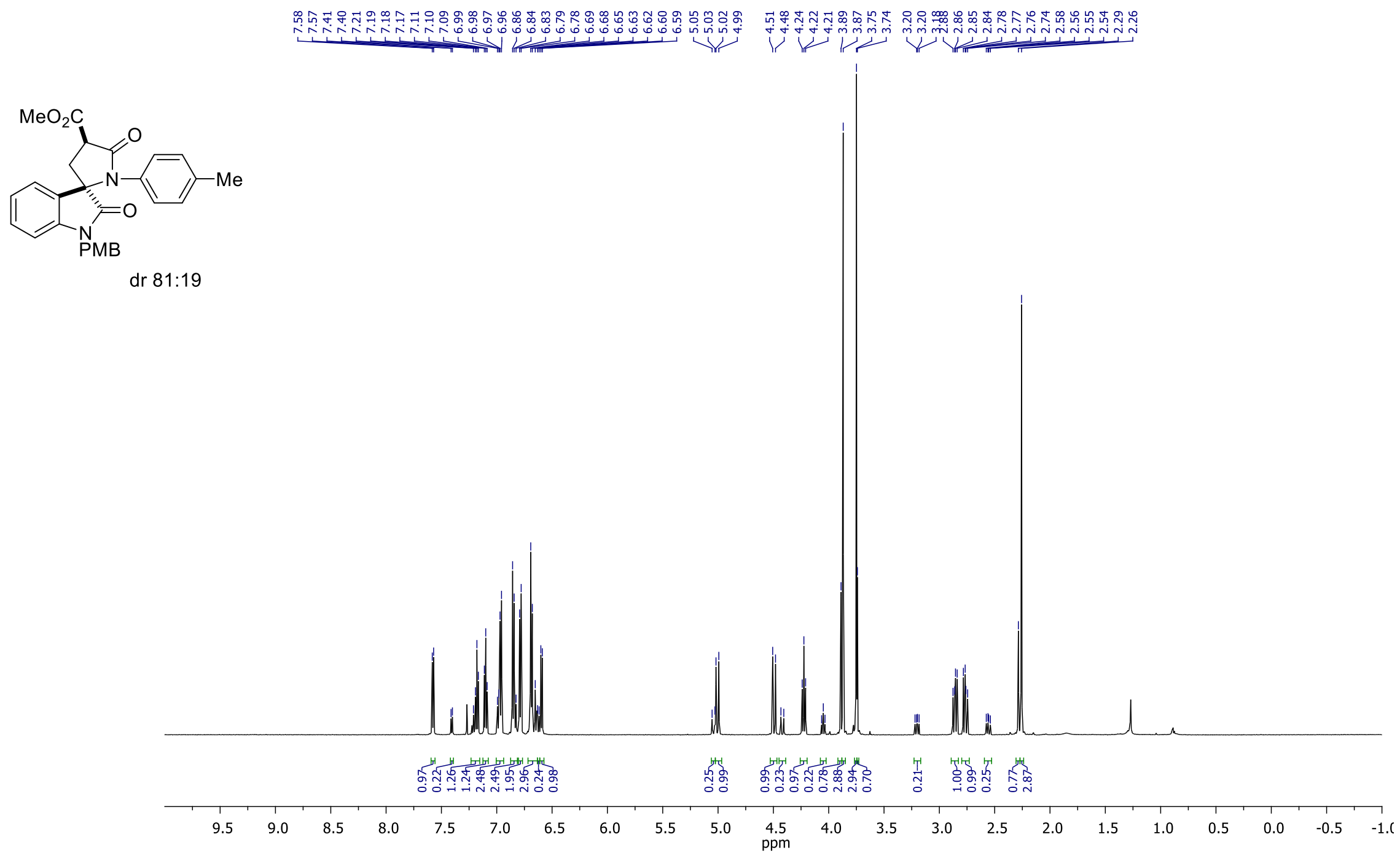


Methyl 1-(4-methoxybenzyl)-1'-(4-methylphenyl)-2,5' -dioxo-1,2-dihydrospiro[indole-3,2'-pyrrolidine]-4' -carboxylate (8k)

${ }^{13} \mathrm{C} \mathrm{NMR}\left(\mathrm{CDCl}_{3}, 150 \mathrm{MHz}\right)$

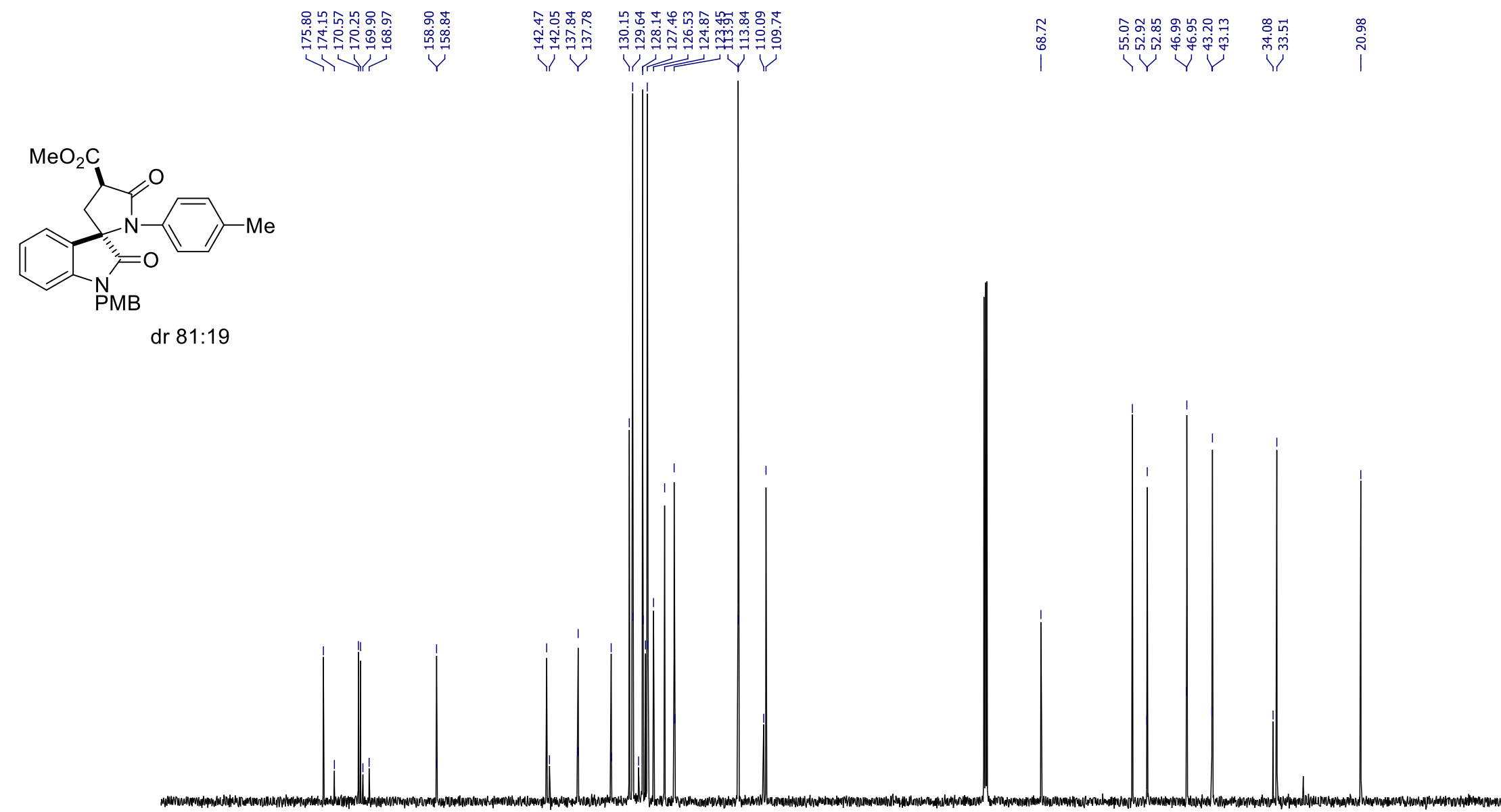

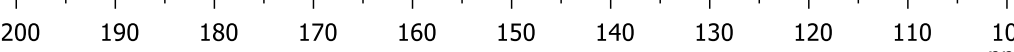


Methyl 1-(4-methoxybenzyl)-1' -(4-methoxyphenyl)-2,5' -dioxo-1,2-dihydrospiro[indole-3,2'-pyrrolidine]-4' -carboxylate (81) ${ }^{1} \mathrm{H}$ NMR $\left(\mathrm{CDCl}_{3}, 600 \mathrm{MHz}\right)$

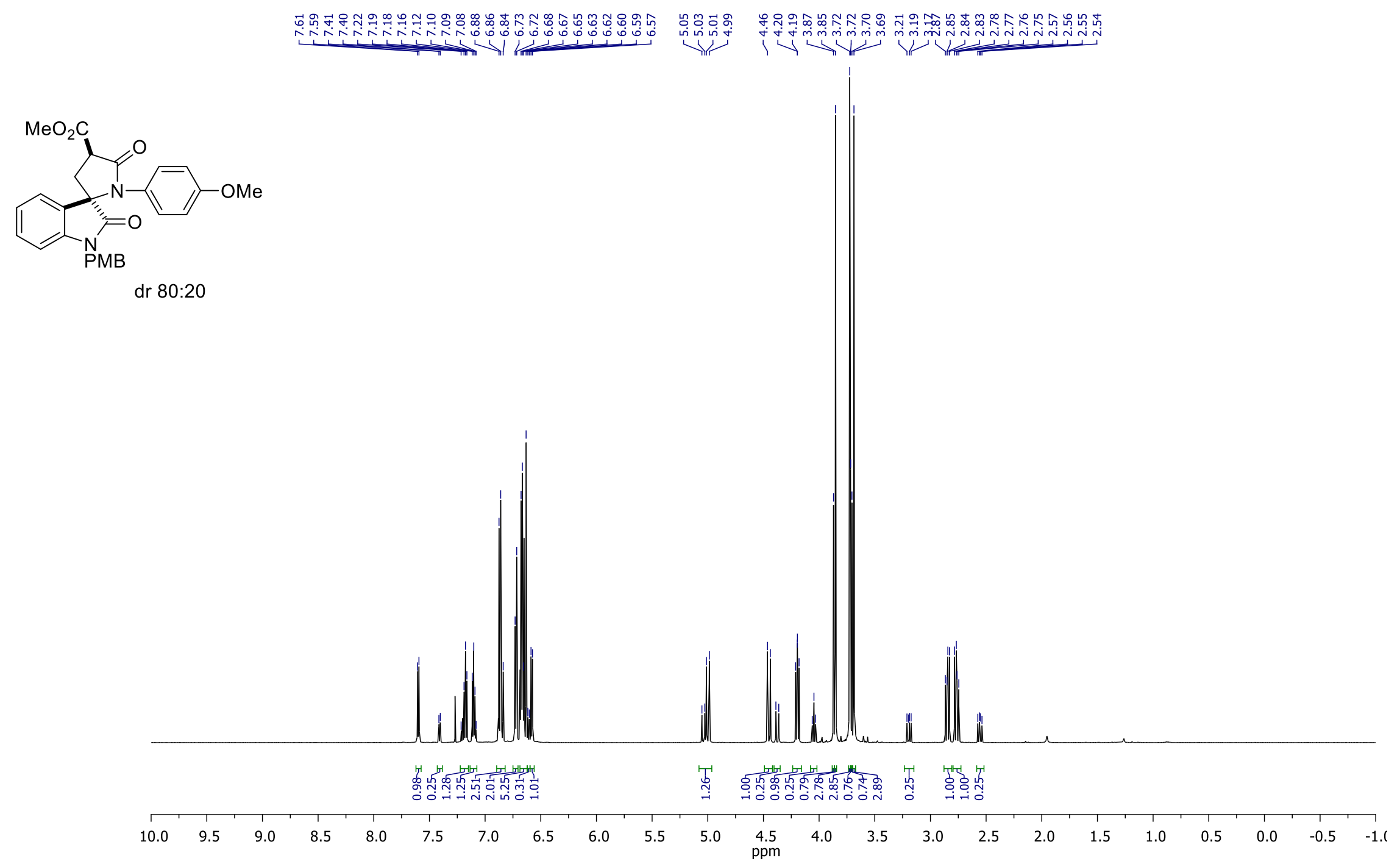


Methyl 1-(4-methoxybenzyl)-1' -(4-methoxyphenyl)-2,5' -dioxo-1,2-dihydrospiro[indole-3,2' -pyrrolidine]-4' -carboxylate (81)

${ }^{13} \mathrm{C} \mathrm{NMR}\left(\mathrm{CDCl}_{3}, 150 \mathrm{MHz}\right)$

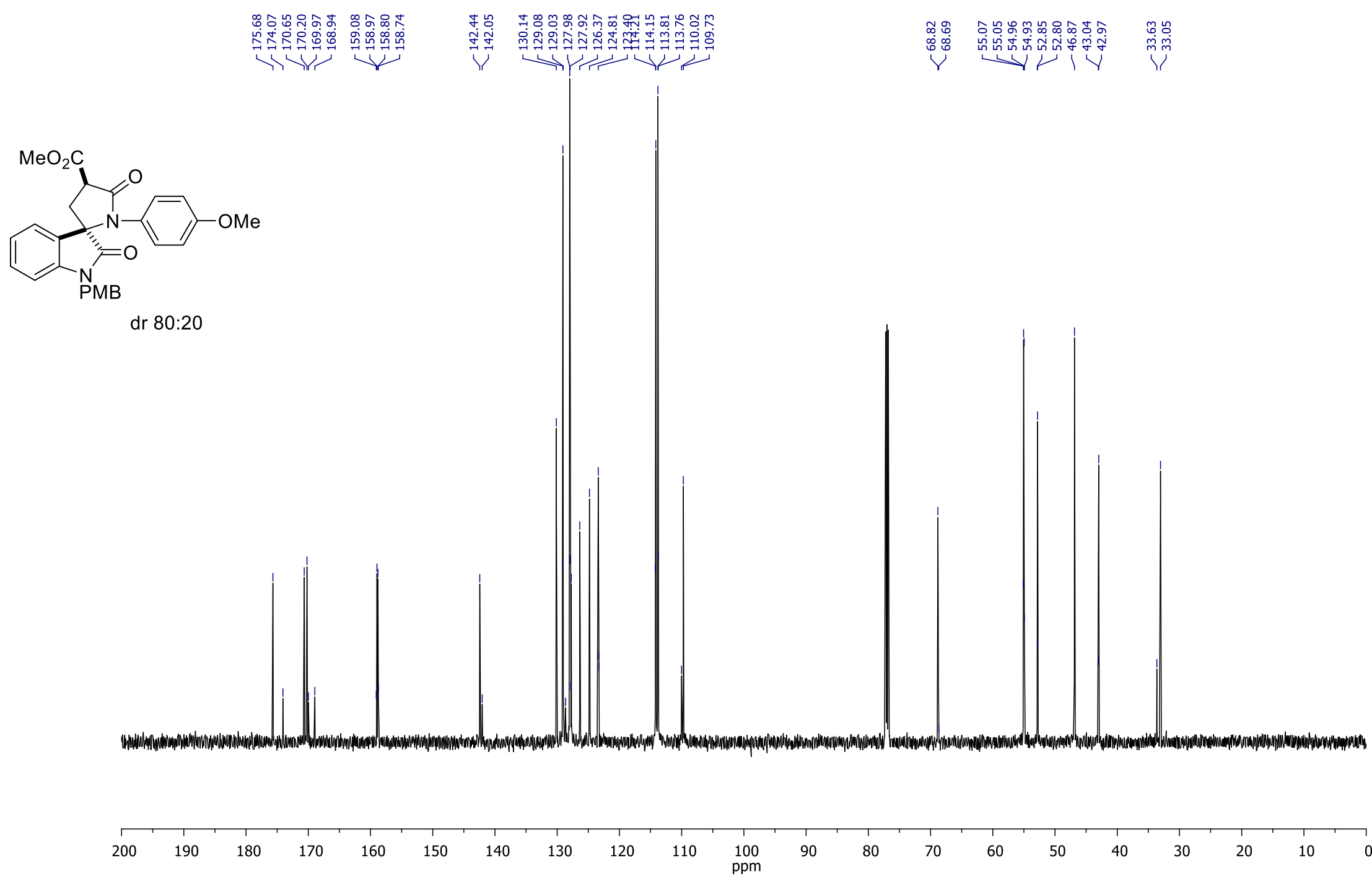


Methyl 1-(4-methoxybenzyl)-1'-(2-methoxyphenyl)-2,5' -dioxo-1,2-dihydrospiro[indole-3,2'-pyrrolidine]-4'-carboxylate (8m)

${ }^{1} \mathrm{H} \mathrm{NMR}\left(\mathrm{CDCl}_{3}, 600 \mathrm{MHz}\right)$

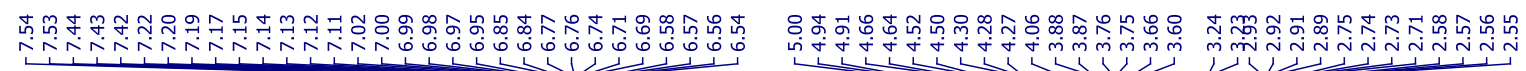
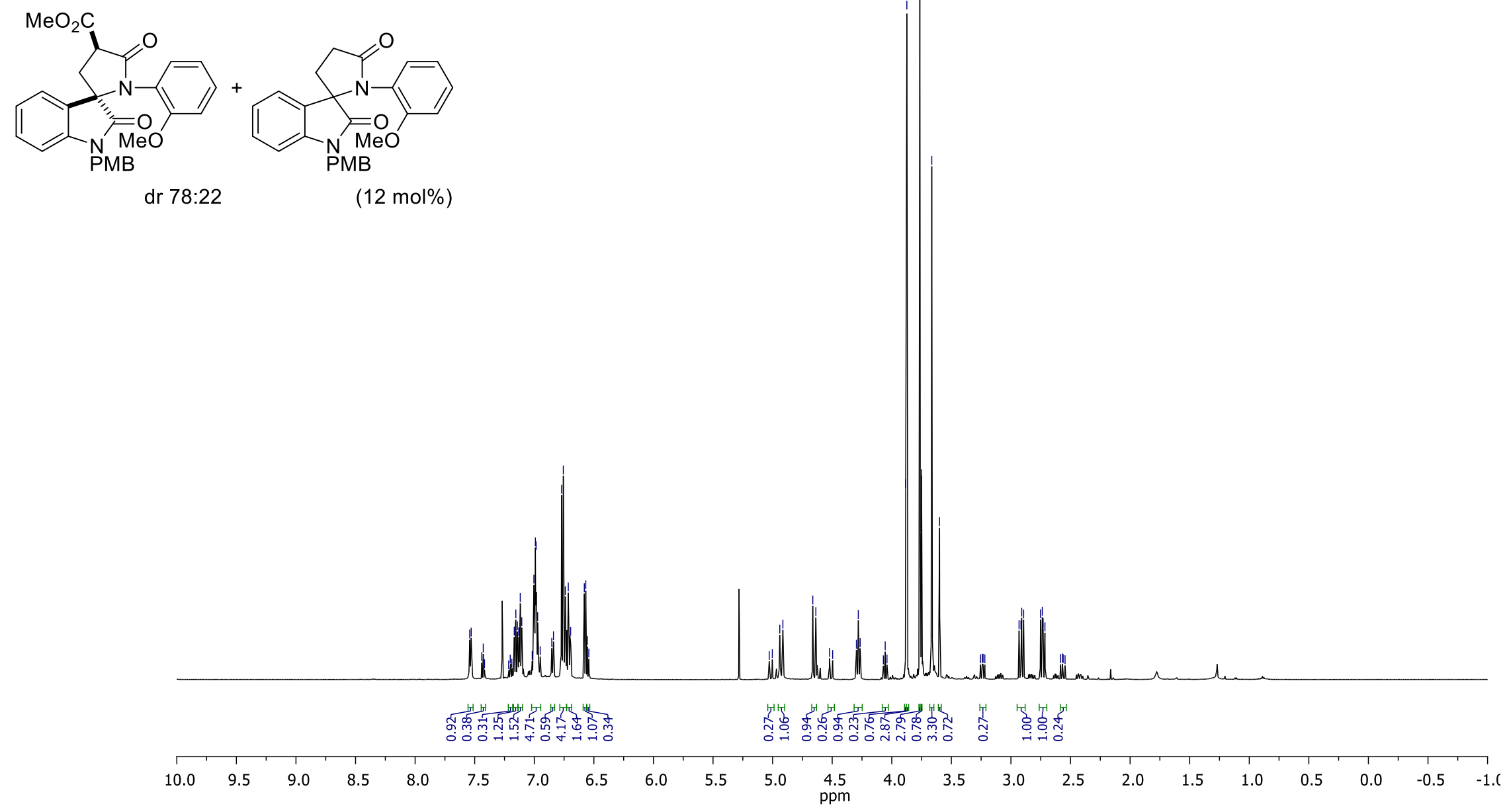
Methyl 1-(4-methoxybenzyl)-1'-(2-methoxyphenyl)-2,5' -dioxo-1,2-dihydrospiro[indole-3,2'-pyrrolidine]-4'-carboxylate (8m)

${ }^{13} \mathrm{C} \mathrm{NMR}\left(\mathrm{CDCl}_{3}, 150 \mathrm{MHz}\right)$

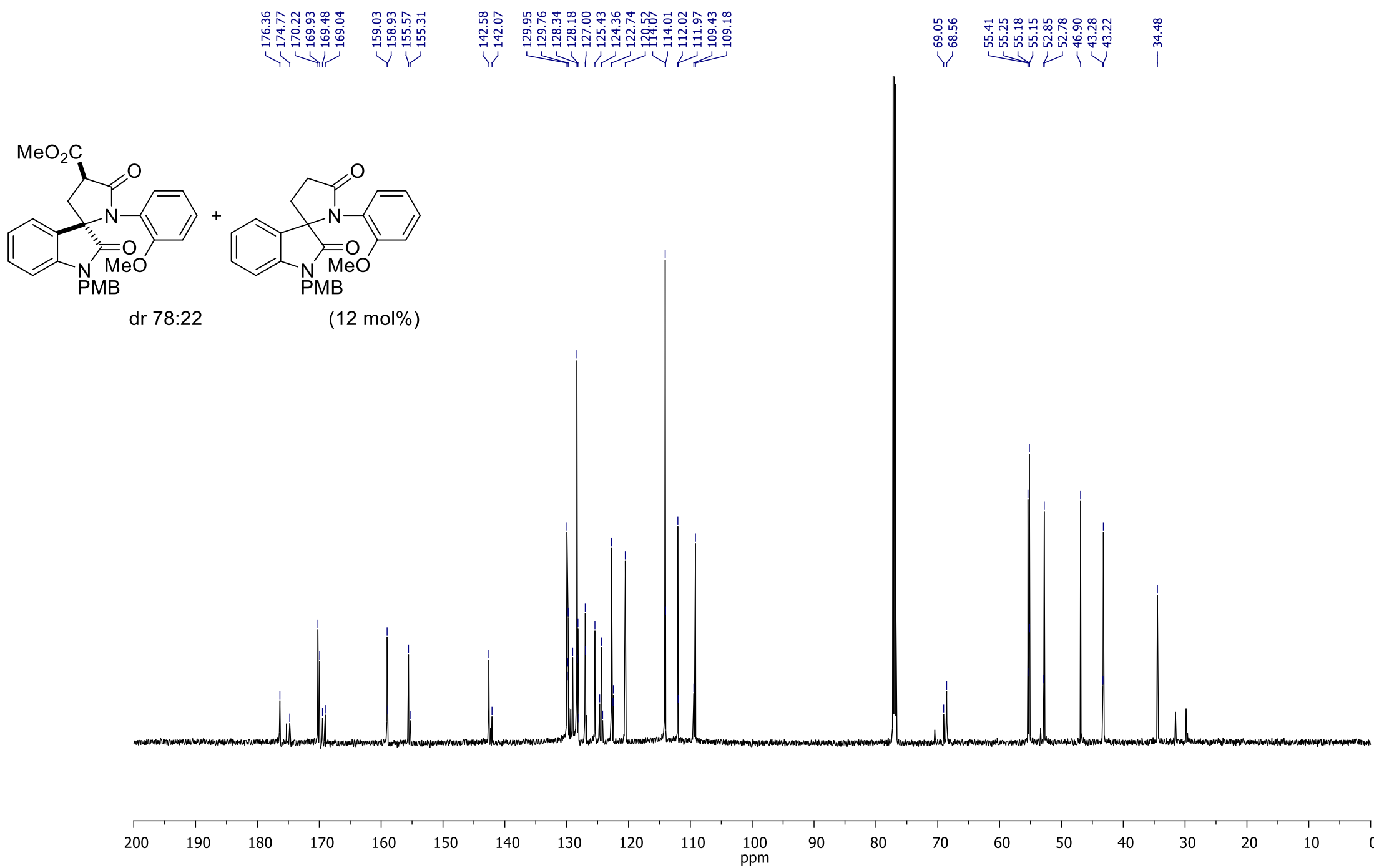


Methyl 1-(4-methoxybenzyl)-1' -(4-nitrophenyl)-2,5' -dioxo-1,2-dihydrospiro[indole-3,2' -pyrrolidine]-4' -carboxylate (8n)

${ }^{1} \mathrm{H} \mathrm{NMR}\left(\mathrm{CDCl}_{3}, 600 \mathrm{MHz}\right)$

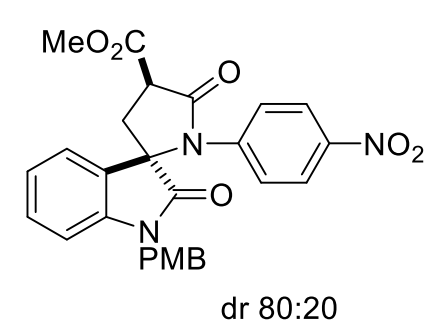

我

dr 80:20

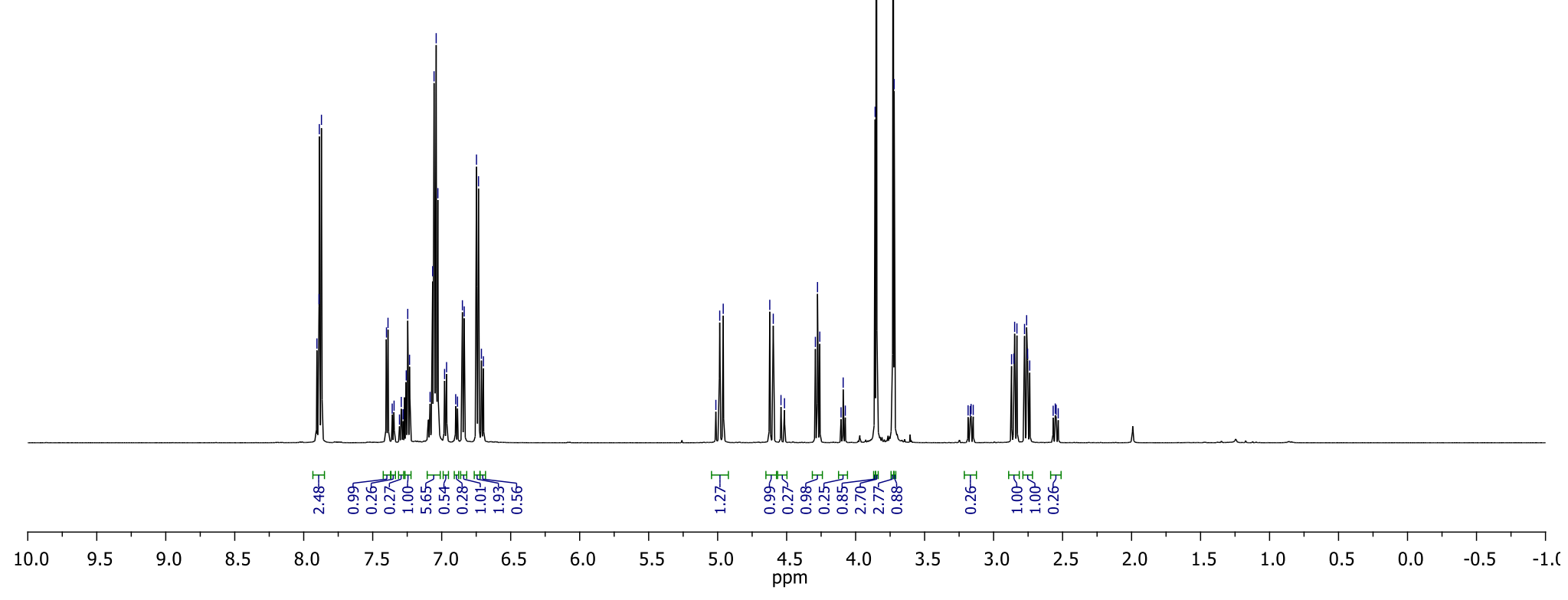


Methyl 1-(4-methoxybenzyl)-1' -(4-nitrophenyl)-2,5' -dioxo-1,2-dihydrospiro[indole-3,2'-pyrrolidine]-4' -carboxylate (8n)

${ }^{13} \mathrm{C} \mathrm{NMR}\left(\mathrm{CDCl}_{3}, 150 \mathrm{MHz}\right)$

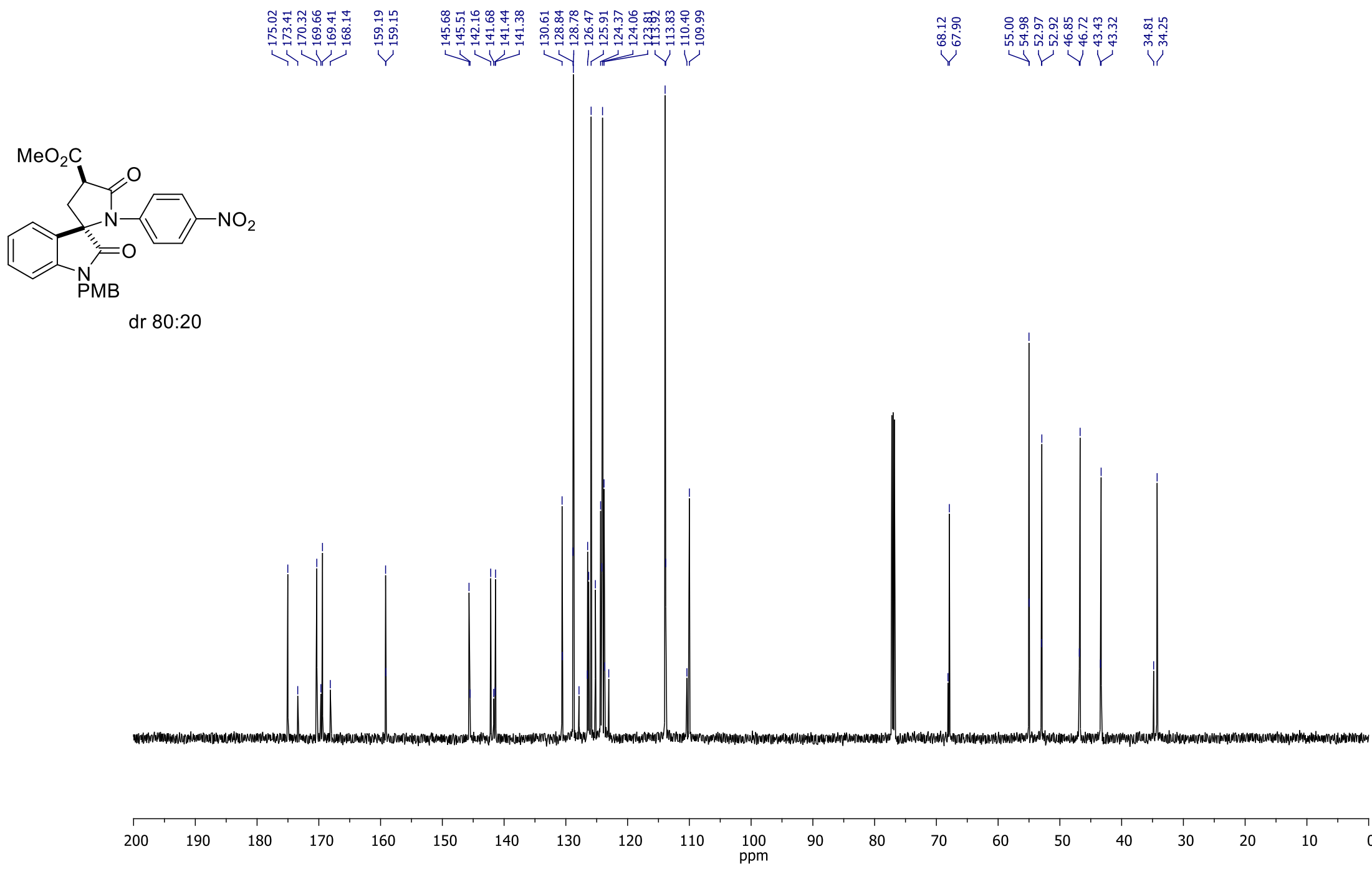


Methyl 2,5'-dioxo-1'-phenyl-1,2-dihydrospiro[indole-3,2' -pyrrolidine]-4' -carboxylate (9a)

${ }^{1} \mathrm{H} \mathrm{NMR}\left(\mathrm{CDCl}_{3}, 600 \mathrm{MHz}\right)$

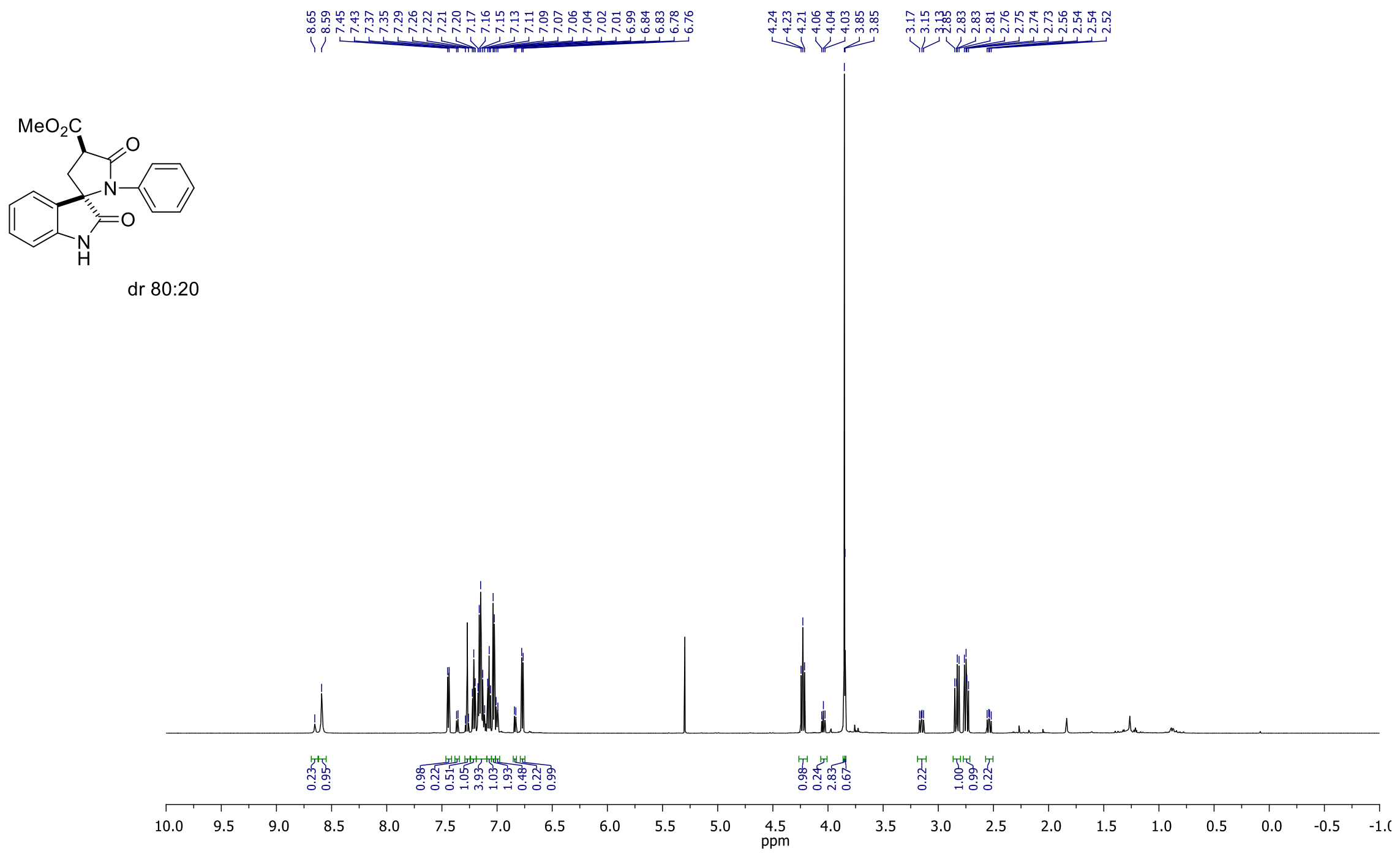


Methyl 2,5'-dioxo-1'-phenyl-1,2-dihydrospiro[indole-3,2' -pyrrolidine]-4' -carboxylate (9a)

${ }^{13} \mathrm{C} \mathrm{NMR}\left(\mathrm{CDCl}_{3}, 150 \mathrm{MHz}\right)$

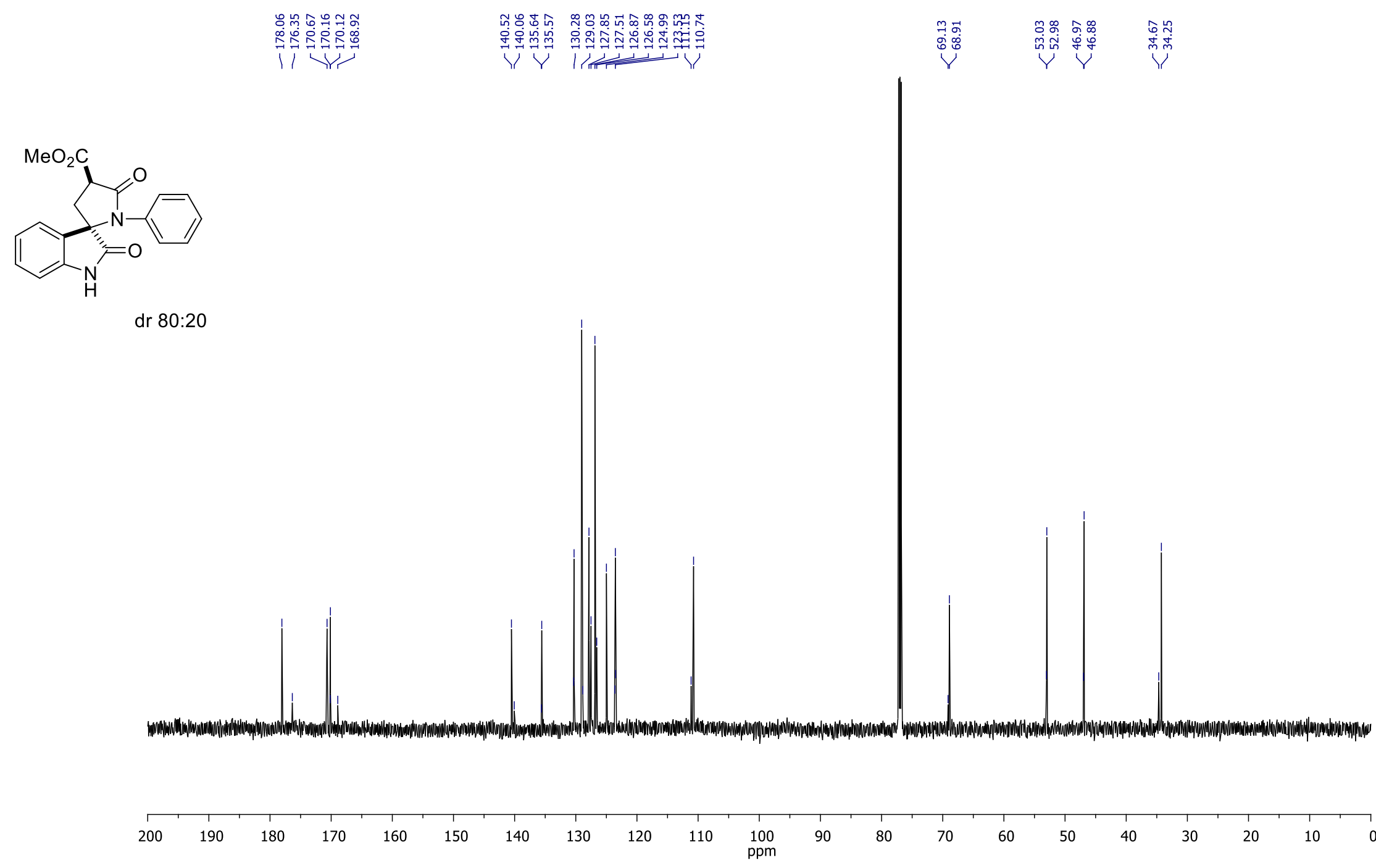


Methyl 5-methyl-2,5'-dioxo-1'-phenyl-1,2-dihydrospiro[indole-3,2'-pyrrolidine]-4' -carboxylate (9b)

${ }^{1} \mathrm{H} \mathrm{NMR}\left(\mathrm{CDCl}_{3}, 600 \mathrm{MHz}\right)$

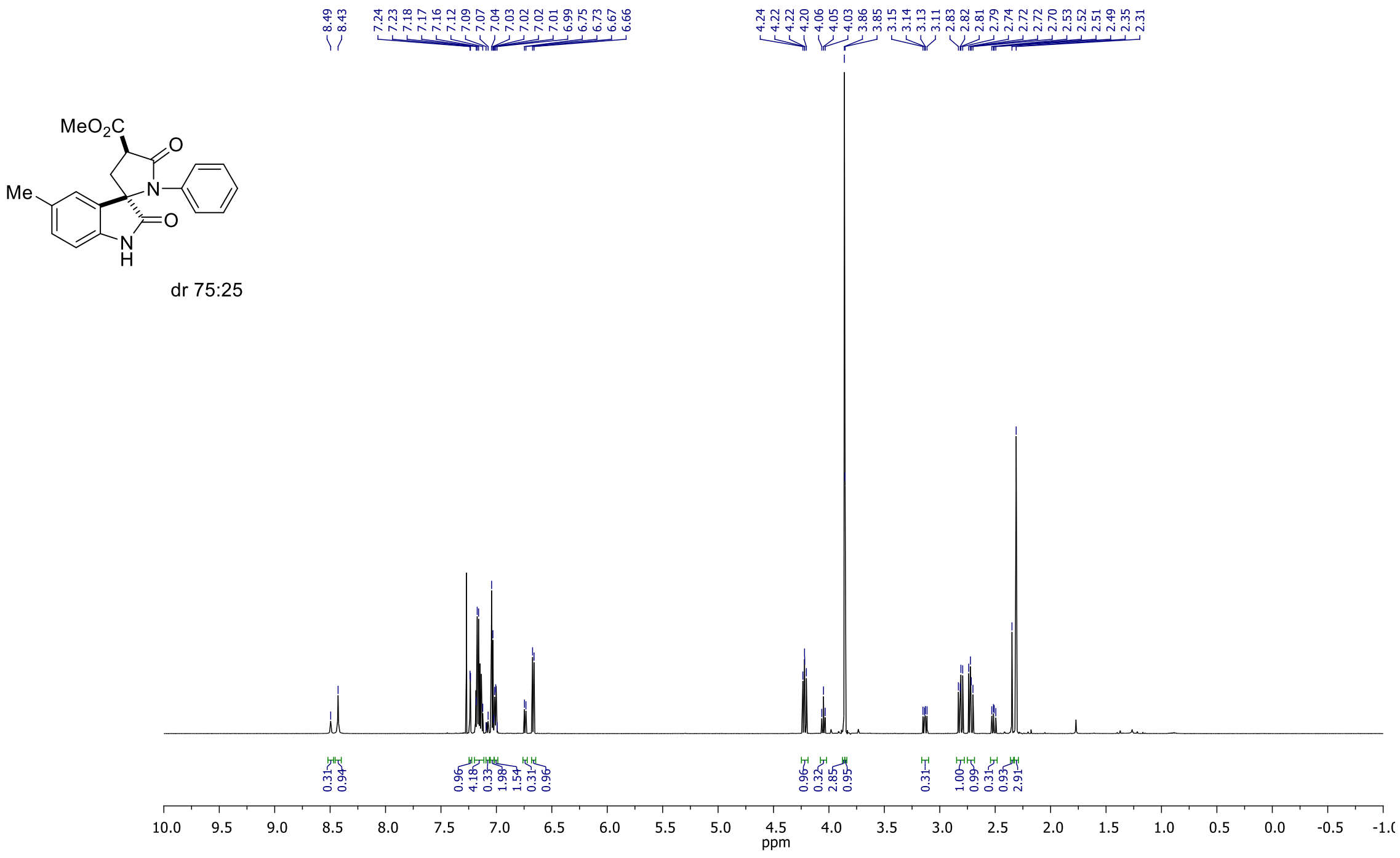


Methyl 5-methyl-2,5'-dioxo-1'-phenyl-1,2-dihydrospiro[indole-3,2'-pyrrolidine]-4' -carboxylate (9b)

${ }^{13} \mathrm{C} \mathrm{NMR}\left(\mathrm{CDCl}_{3}, 150 \mathrm{MHz}\right)$

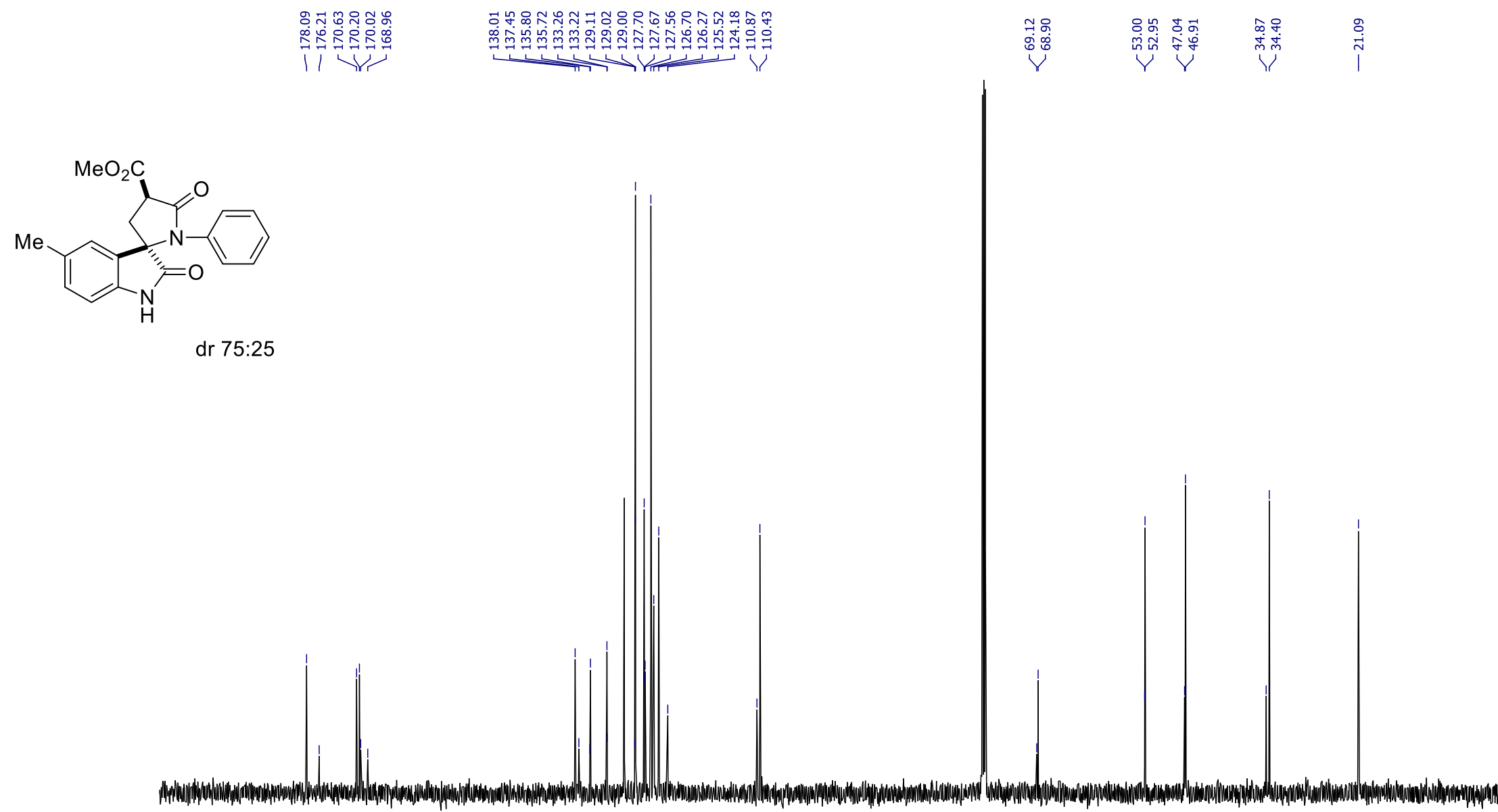

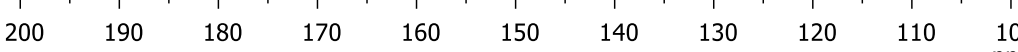


Methyl 5-fluoro-2,5' -dioxo-1'-phenyl-1,2-dihydrospiro[indole-3,2' -pyrrolidine]-4' -carboxylate (9c)

${ }^{1} \mathrm{H} \mathrm{NMR}\left(\mathrm{CDCl}_{3}, 600 \mathrm{MHz}\right)$
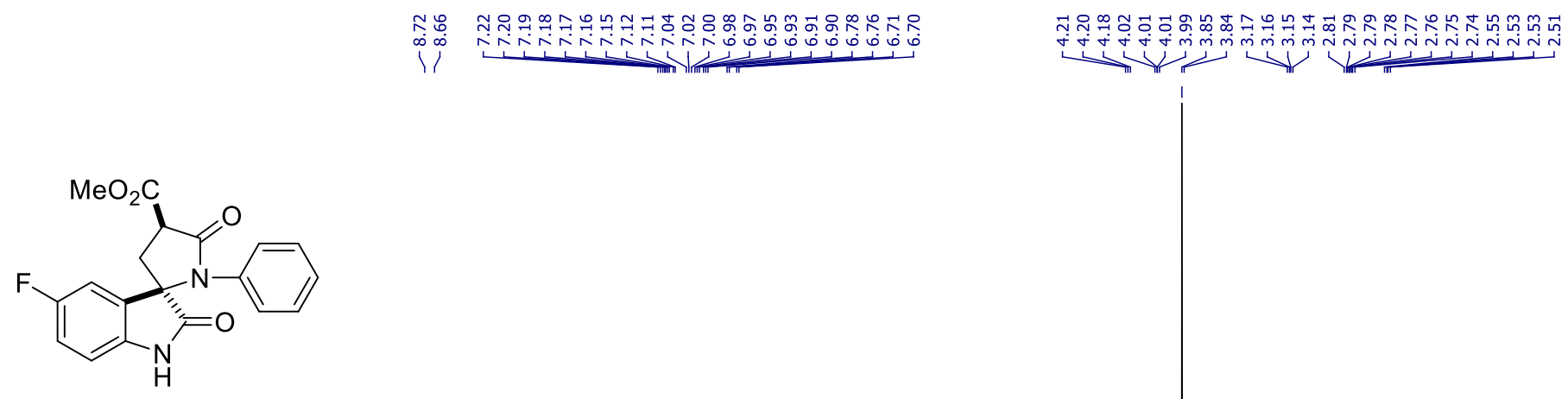

dr 82:18

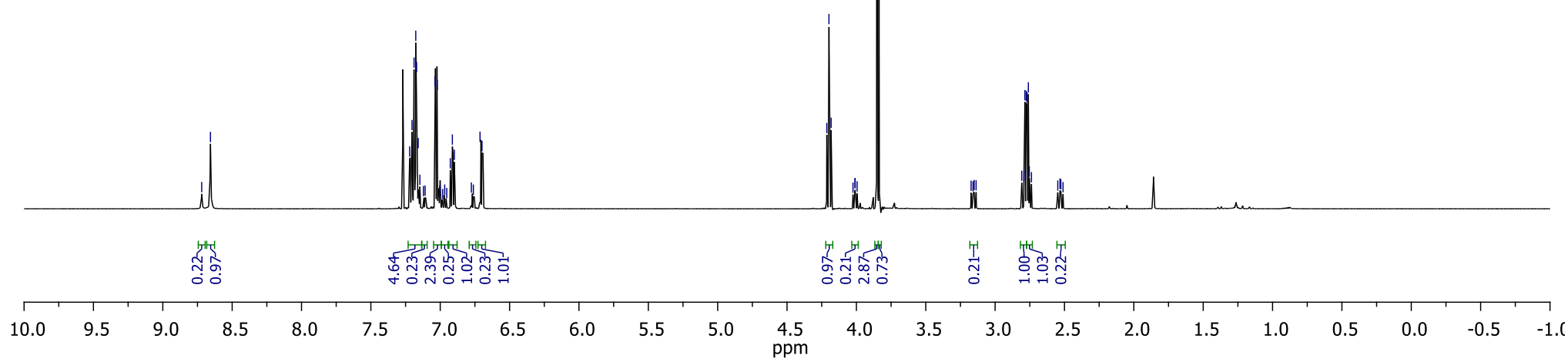


Methyl 5-fluoro-2,5' -dioxo-1'-phenyl-1,2-dihydrospiro[indole-3,2' -pyrrolidine]-4' -carboxylate (9c)

${ }^{13} \mathrm{C} \mathrm{NMR}\left(\mathrm{CDCl}_{3}, 150 \mathrm{MHz}\right)$

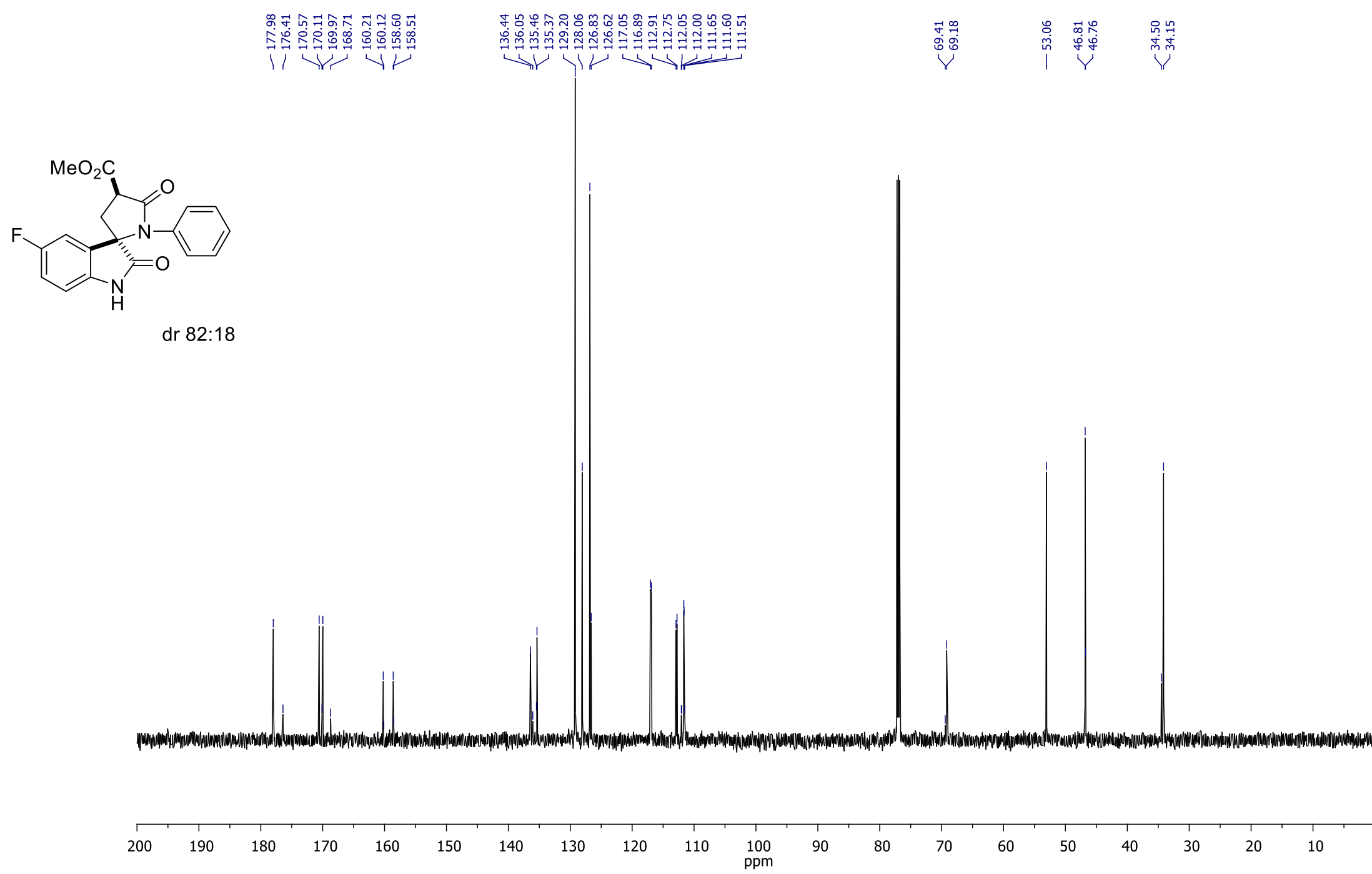


Methyl 5-chloro-2,5' -dioxo-1'-phenyl-1,2-dihydrospiro[indole-3,2' -pyrrolidine]-4' -carboxylate (9d)

${ }^{1} \mathrm{H} \mathrm{NMR}\left(\mathrm{CDCl}_{3}, 600 \mathrm{MHz}\right)$

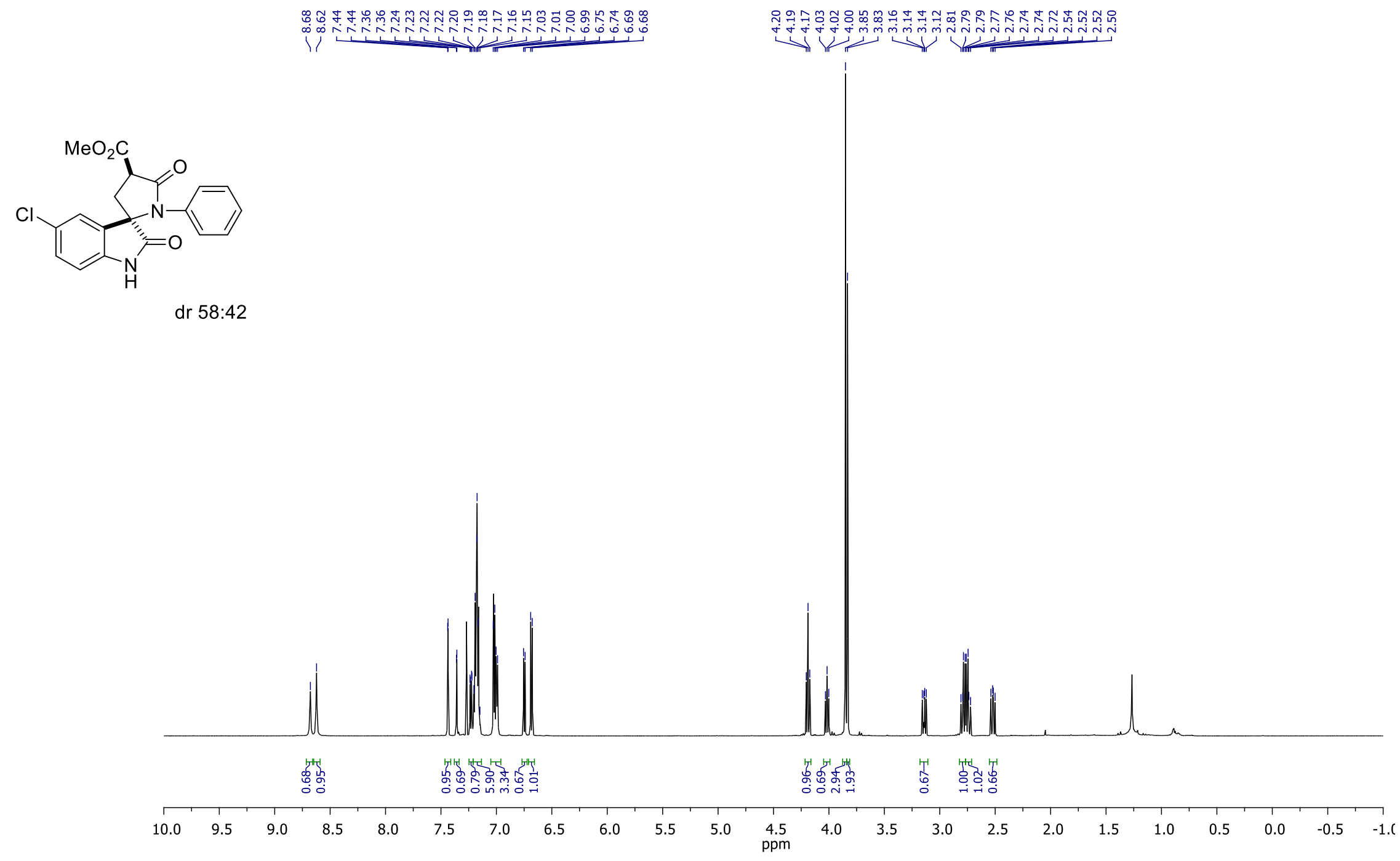


Methyl 5-chloro-2,5'-dioxo-1'-phenyl-1,2-dihydrospiro[indole-3,2' -pyrrolidine]-4' -carboxylate (9d)

${ }^{13} \mathrm{C} \mathrm{NMR}\left(\mathrm{CDCl}_{3}, 150 \mathrm{MHz}\right)$

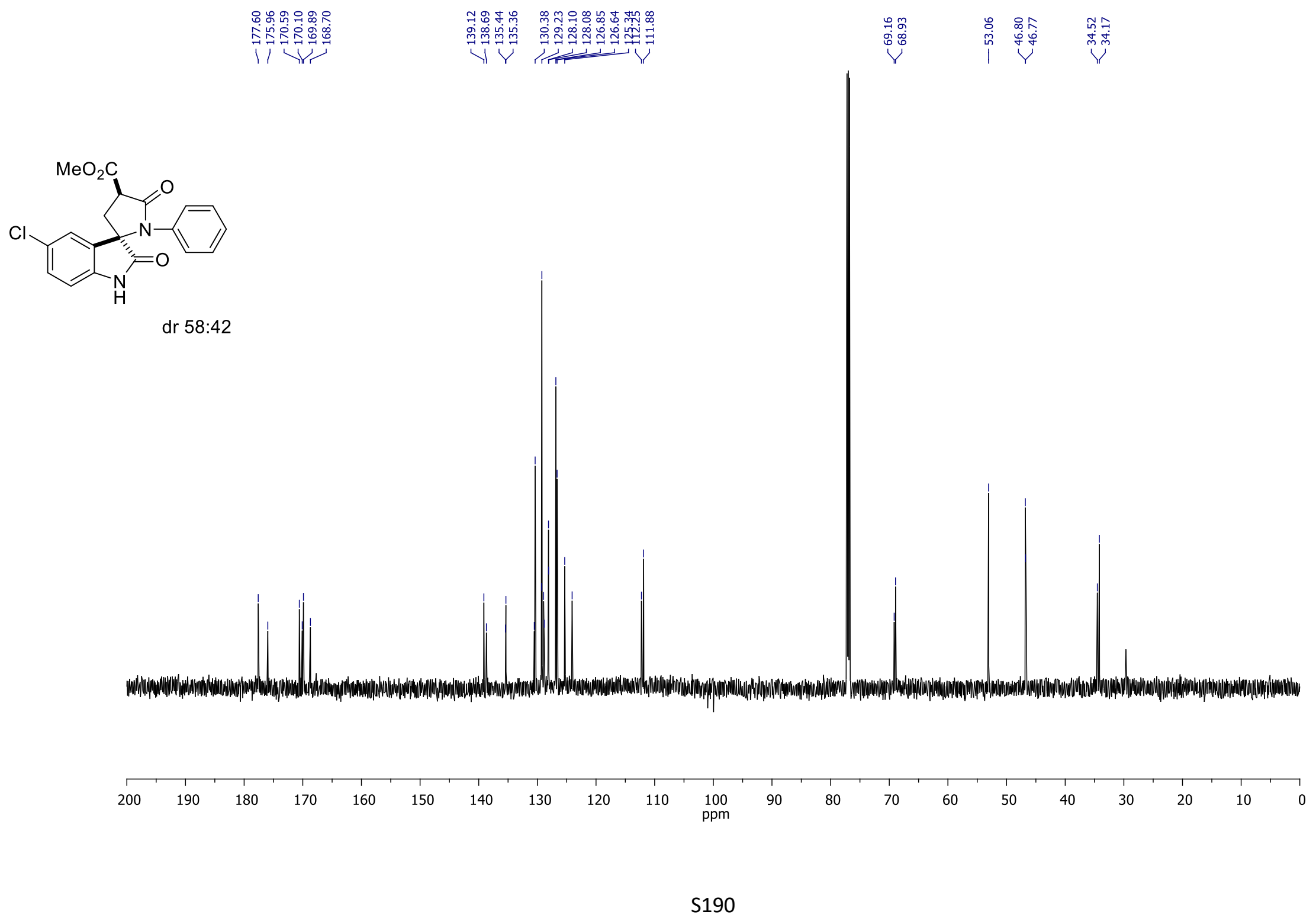


Methyl 5-bromo-2,5' -dioxo-1' -phenyl-1,2-dihydrospiro[indole-3,2'-pyrrolidine]-4' -carboxylate (9e)

${ }^{1} \mathrm{H} \mathrm{NMR}\left(\mathrm{CDCl}_{3}, 600 \mathrm{MHz}\right)$

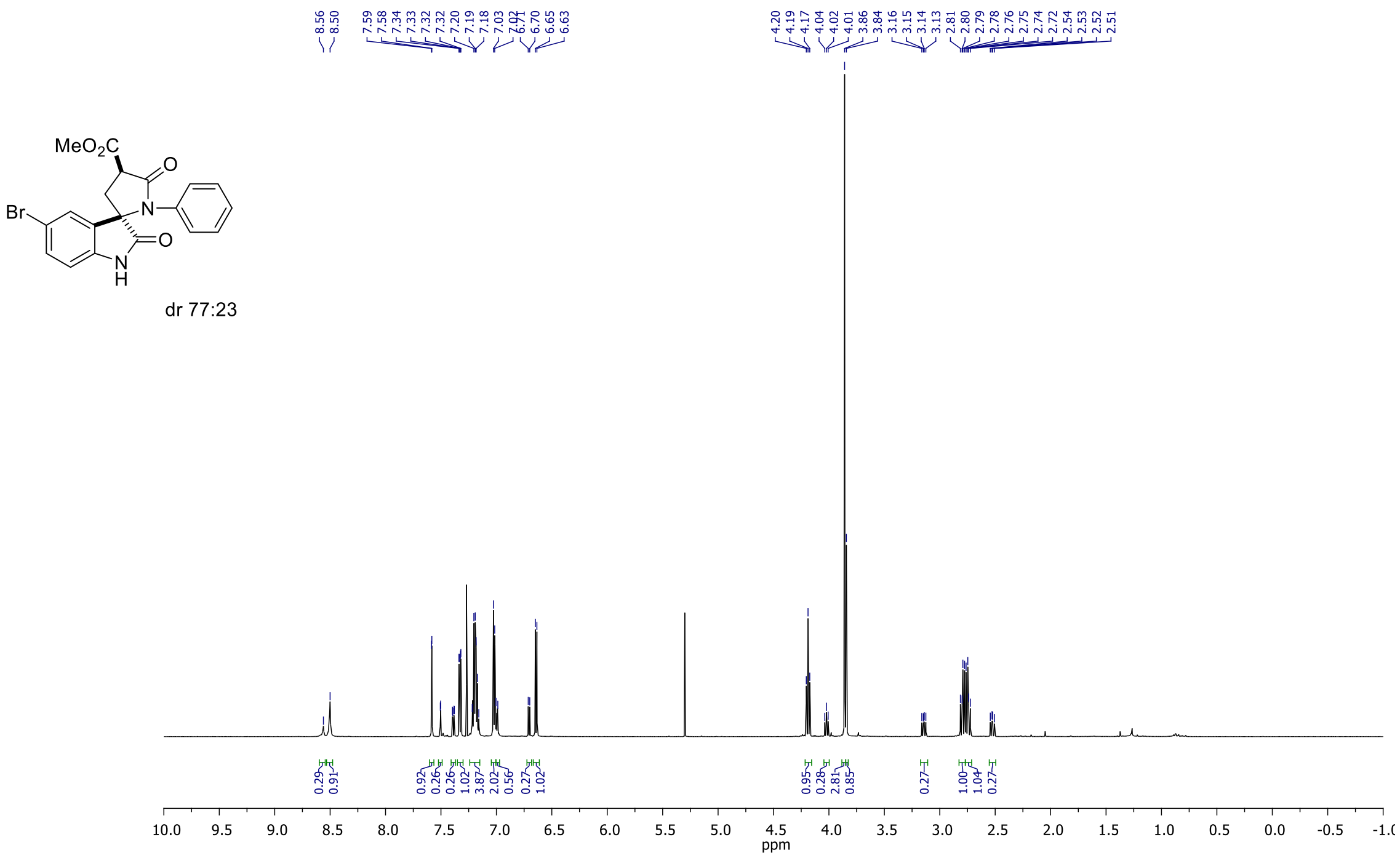


Methyl 5-bromo-2,5' -dioxo-1' -phenyl-1,2-dihydrospiro[indole-3,2'-pyrrolidine]-4' -carboxylate (9e)

${ }^{13} \mathrm{C} \mathrm{NMR}\left(\mathrm{CDCl}_{3}, 150 \mathrm{MHz}\right)$
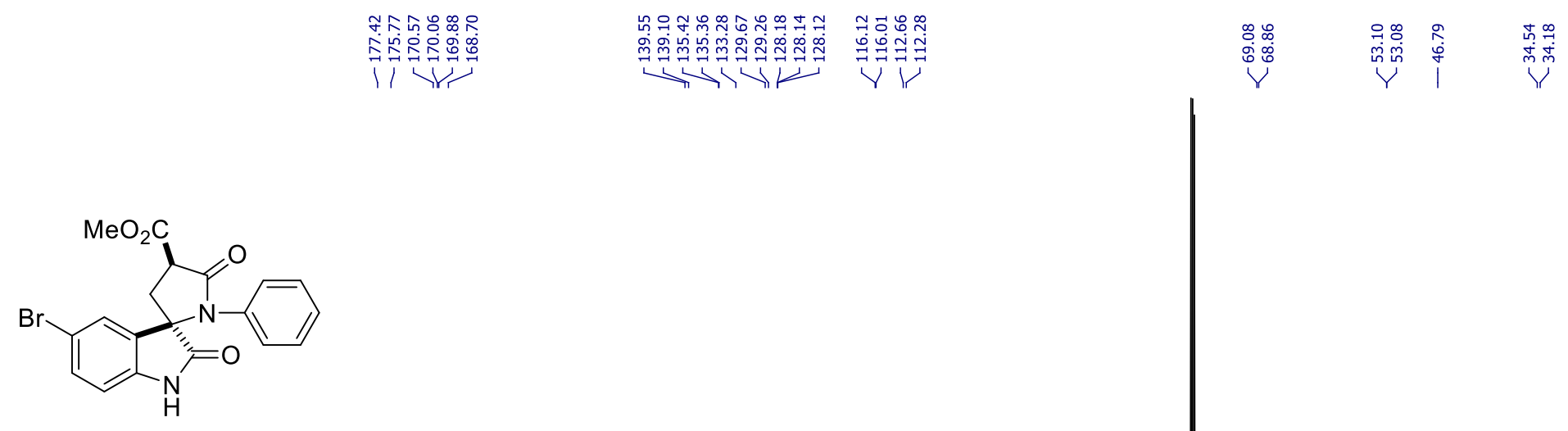

dr $77: 23$

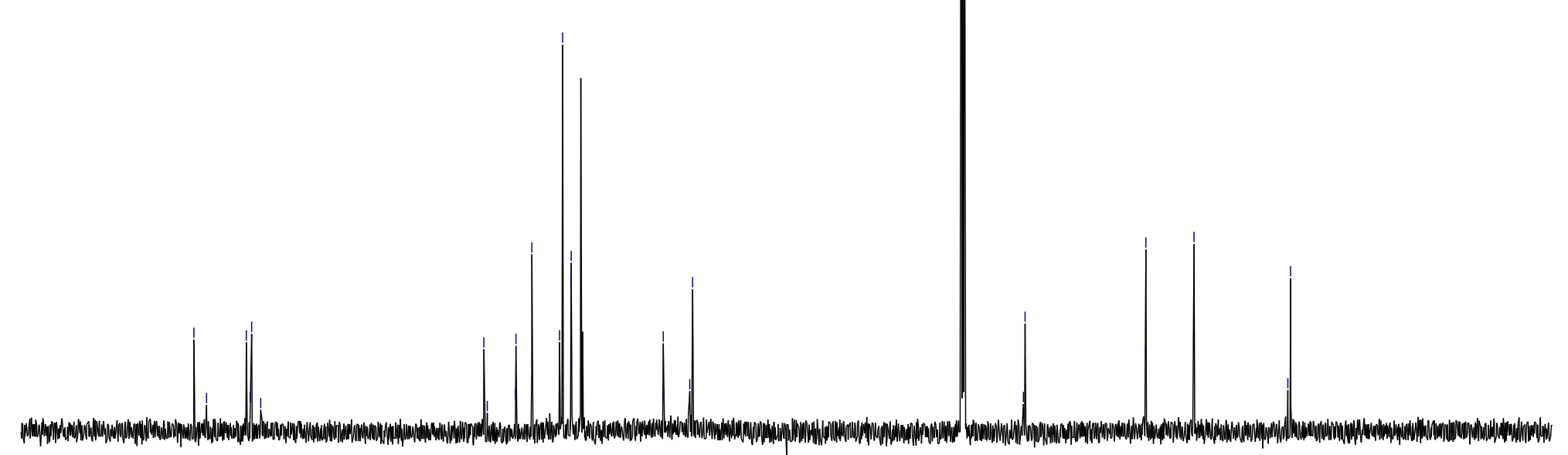

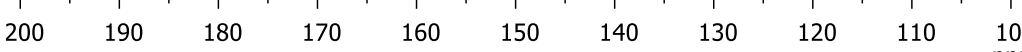
100 
Methyl 1'-(4-fluorophenyl)-2,5' -dioxo-1,2-dihydrospiro[indole-3,2' -pyrrolidine]-4' -carboxylate (9f)

${ }^{1} \mathrm{H} \mathrm{NMR}\left(\mathrm{CDCl}_{3}, 600 \mathrm{MHz}\right)$

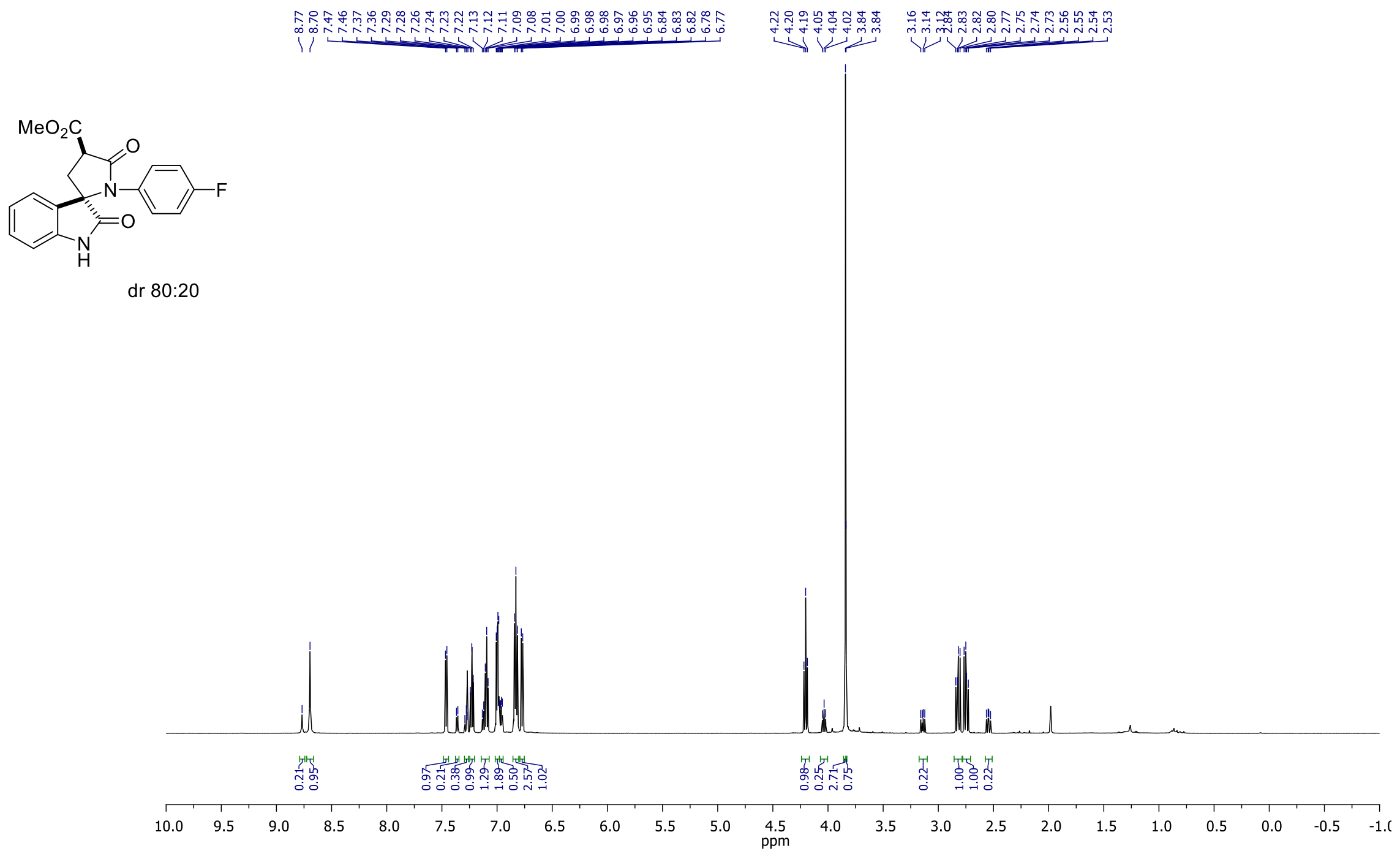


Methyl 1'-(4-fluorophenyl)-2,5' -dioxo-1,2-dihydrospiro[indole-3,2' -pyrrolidine]-4' -carboxylate (9f)

${ }^{13} \mathrm{C} \mathrm{NMR}\left(\mathrm{CDCl}_{3}, 150 \mathrm{MHz}\right)$

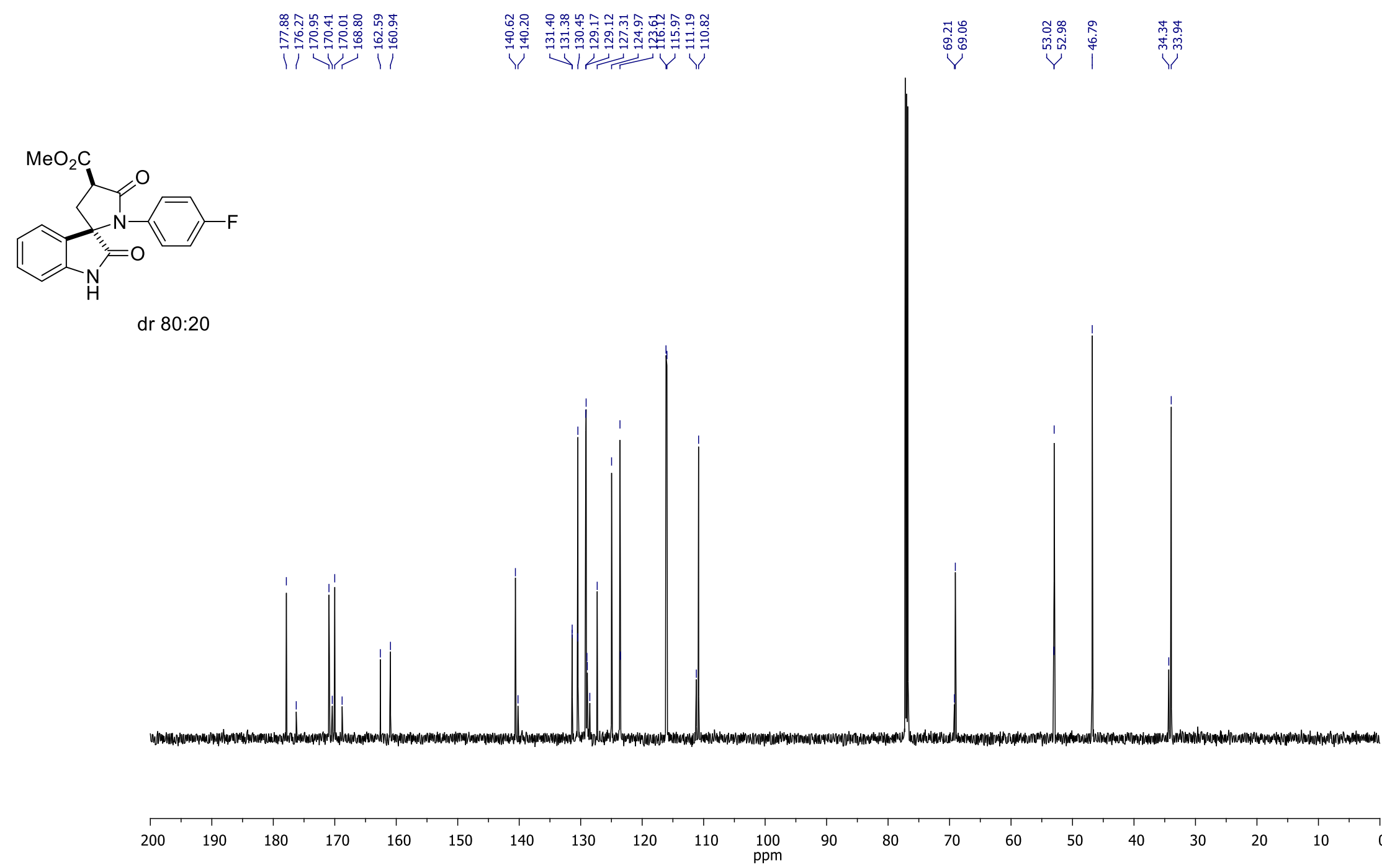


Methyl 1'-(4-chlorophenyl)-2,5' -dioxo-1,2-dihydrospiro[indole-3,2' -pyrrolidine]-4' -carboxylate (9g)

${ }^{1} \mathrm{H} \mathrm{NMR}\left(\mathrm{CDCl}_{3}, 600 \mathrm{MHz}\right)$
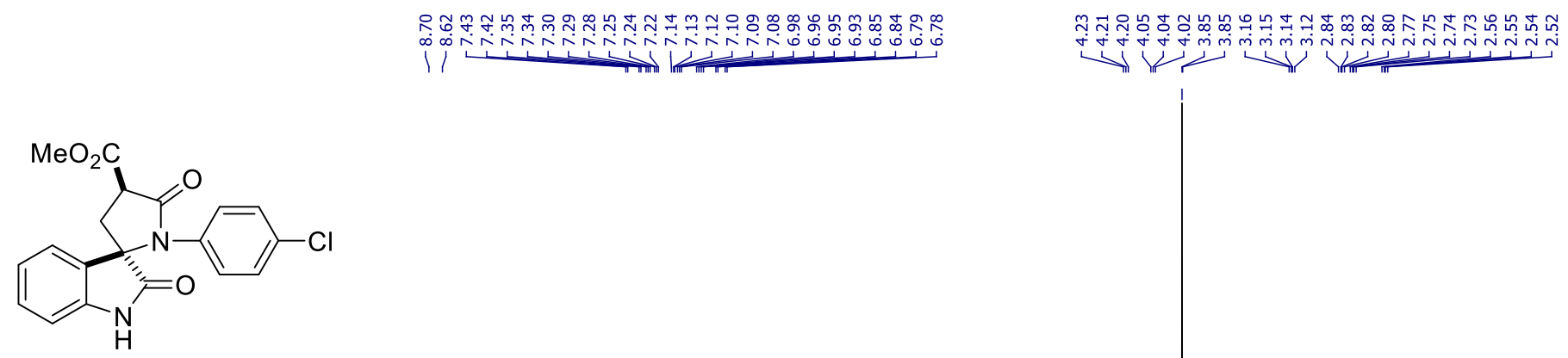

dr $80: 20$

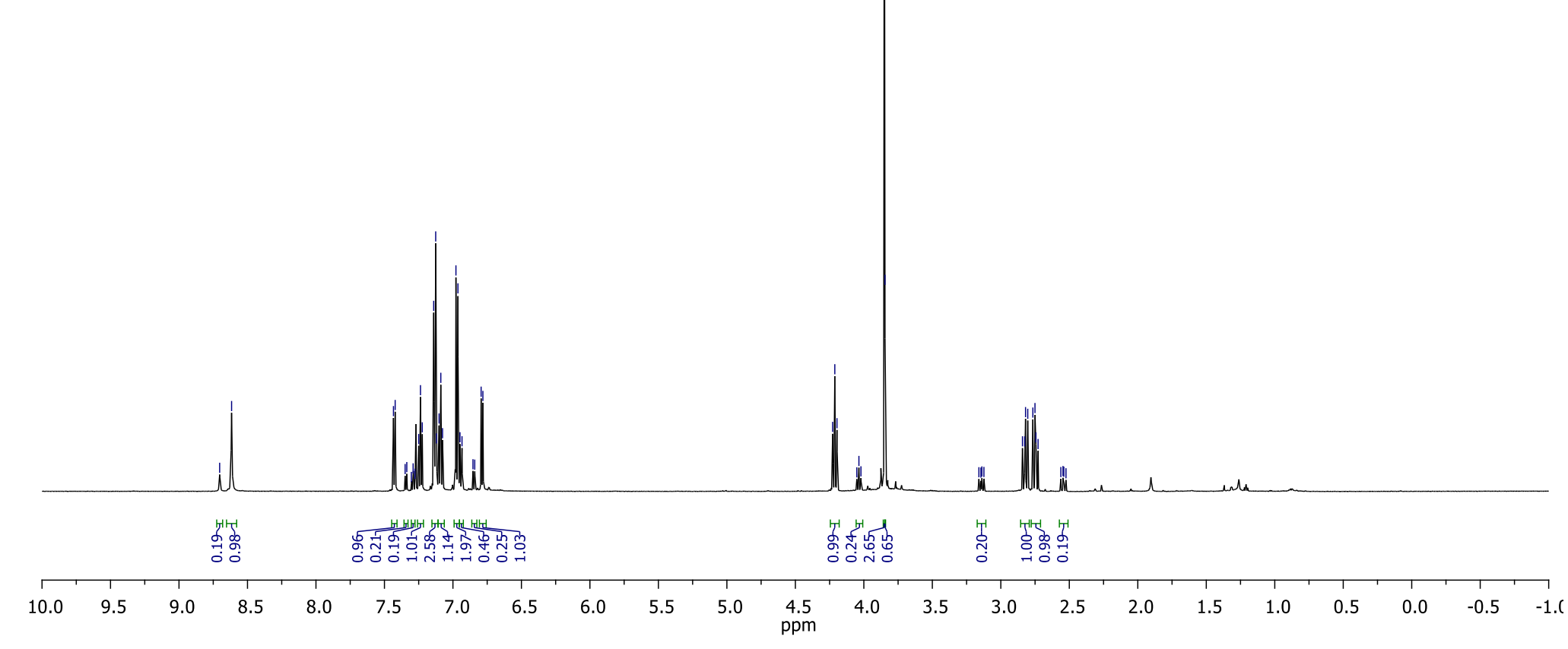


Methyl 1'-(4-chlorophenyl)-2,5' -dioxo-1,2-dihydrospiro[indole-3,2' -pyrrolidine]-4' -carboxylate (9g)

${ }^{13} \mathrm{C} \mathrm{NMR}\left(\mathrm{CDCl}_{3}, 150 \mathrm{MHz}\right)$

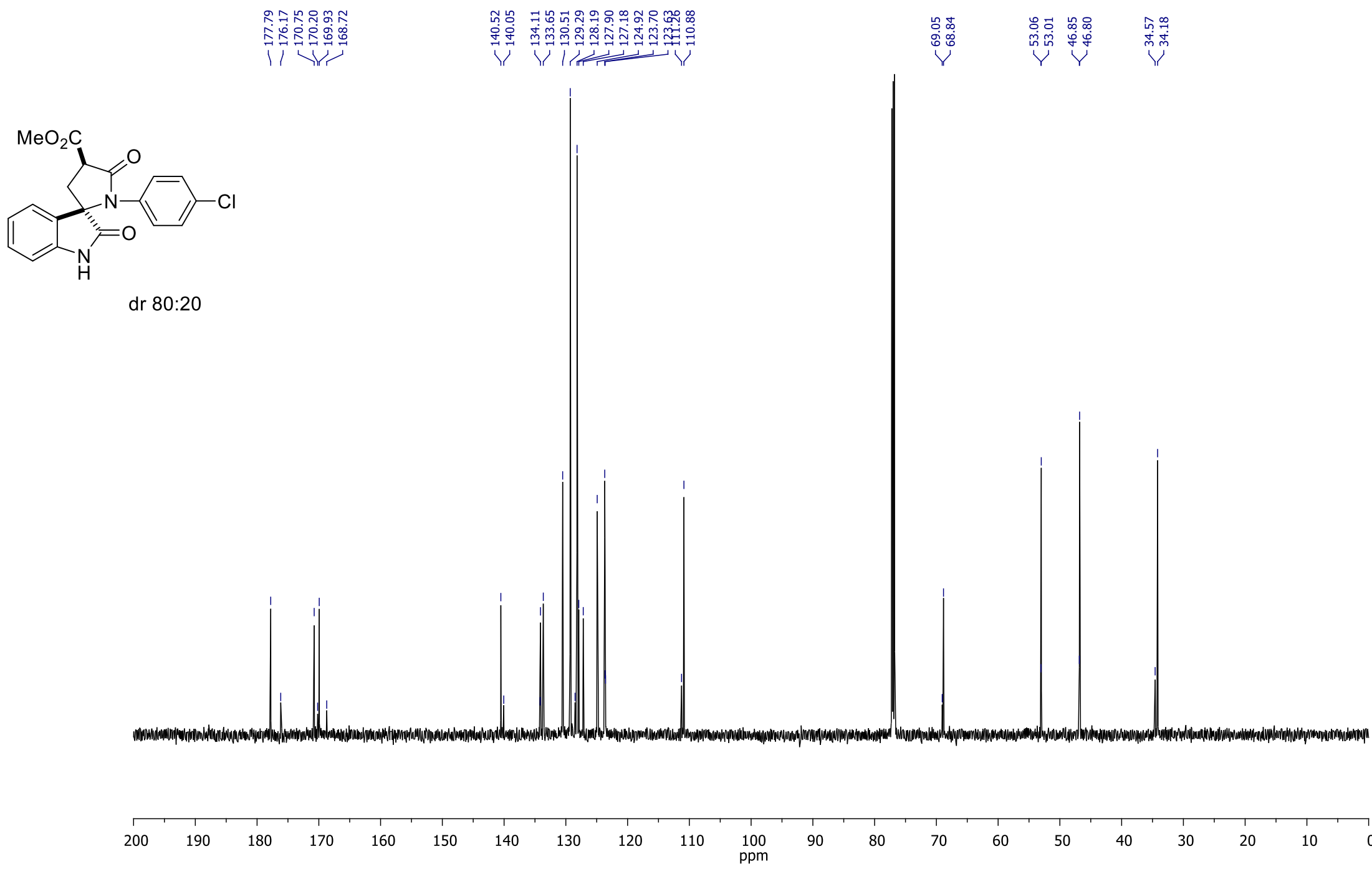


Methyl 1'-(3-chlorophenyl)-2,5' -dioxo-1,2-dihydrospiro[indole-3,2' -pyrrolidine]-4' -carboxylate (9h)

${ }^{1} \mathrm{H} \mathrm{NMR}\left(\mathrm{CDCl}_{3}, 600 \mathrm{MHz}\right)$

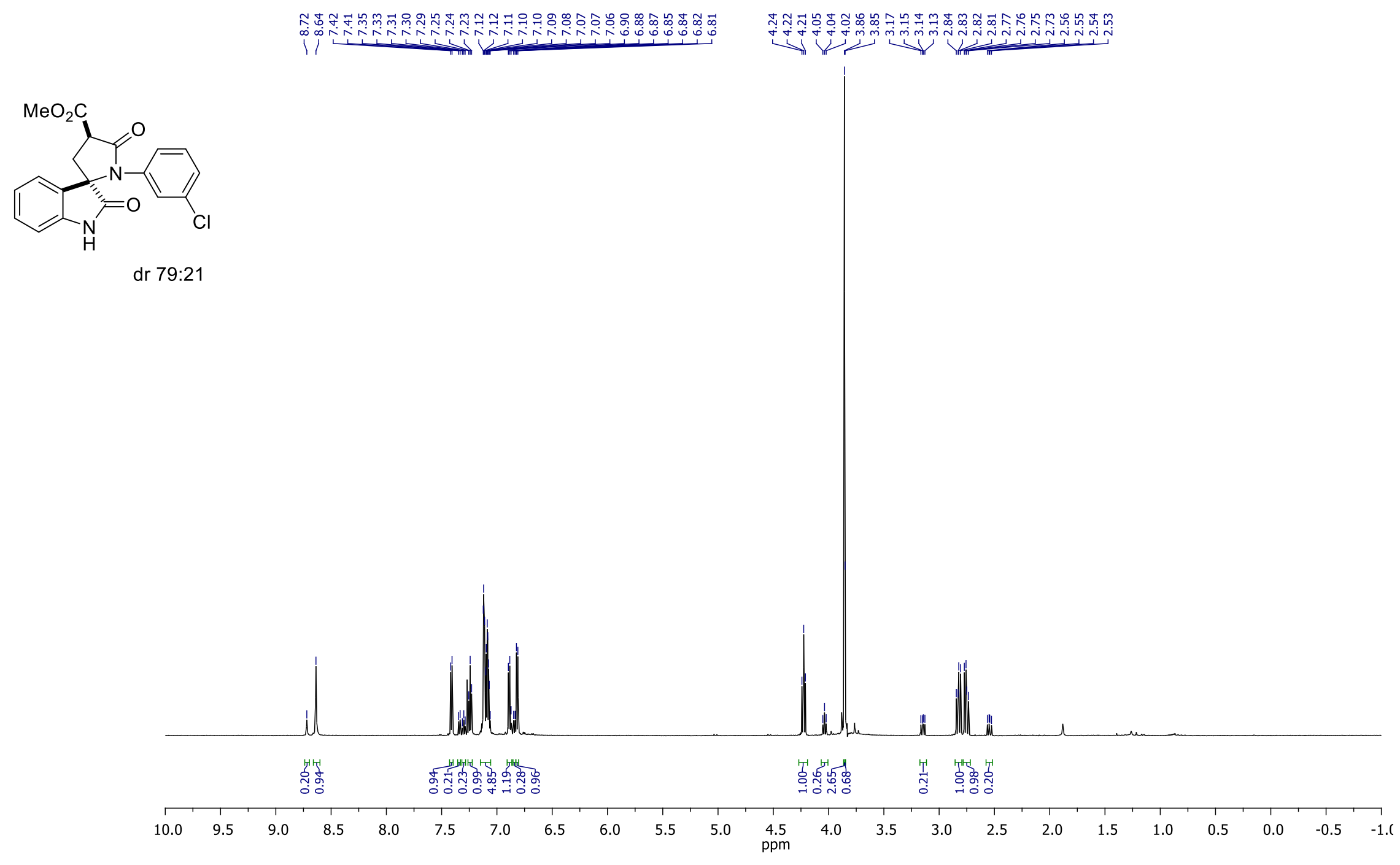


Methyl 1'-(3-chlorophenyl)-2,5' -dioxo-1,2-dihydrospiro[indole-3,2' -pyrrolidine]-4' -carboxylate (9h)

${ }^{13} \mathrm{C} \mathrm{NMR}\left(\mathrm{CDCl}_{3}, 150 \mathrm{MHz}\right)$
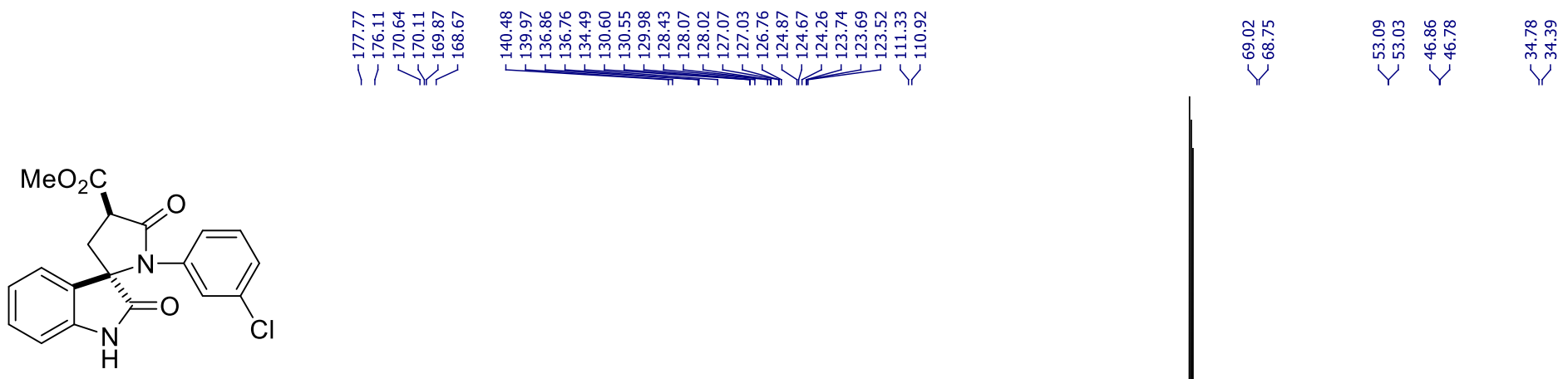

dr 79:21

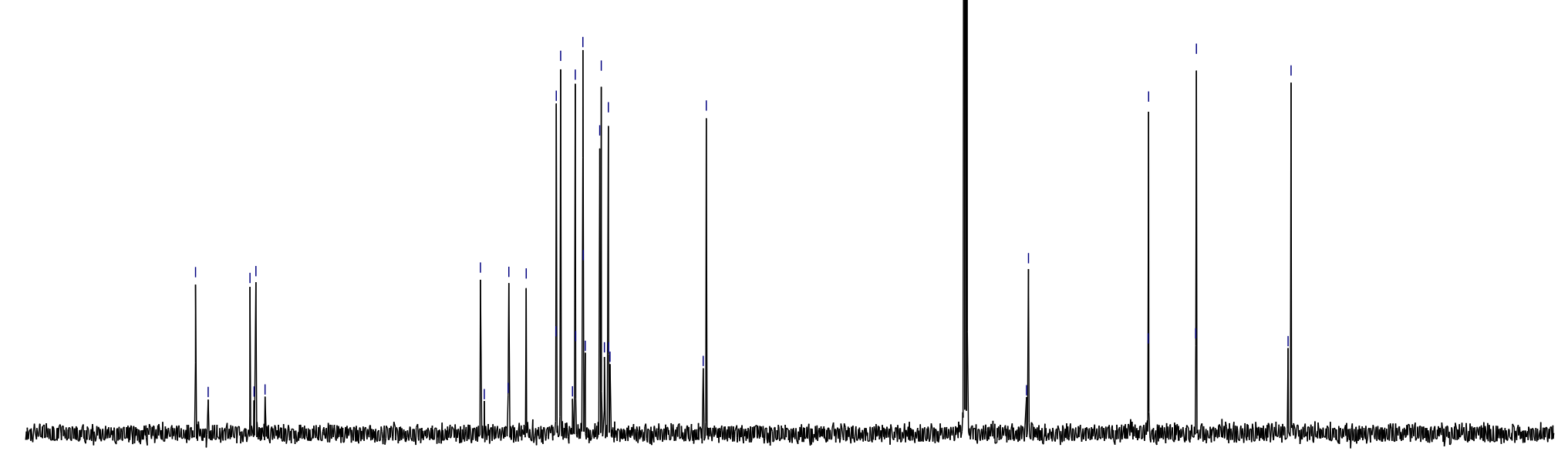

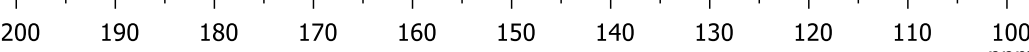

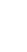


Methyl 1' -(4-bromophenyl)-2,5'-dioxo-1,2-dihydrospiro[indole-3,2' -pyrrolidine]-4' -carboxylate (9i)

${ }^{1} \mathrm{H} \mathrm{NMR}\left(\mathrm{CDCl}_{3}, 600 \mathrm{MHz}\right)$

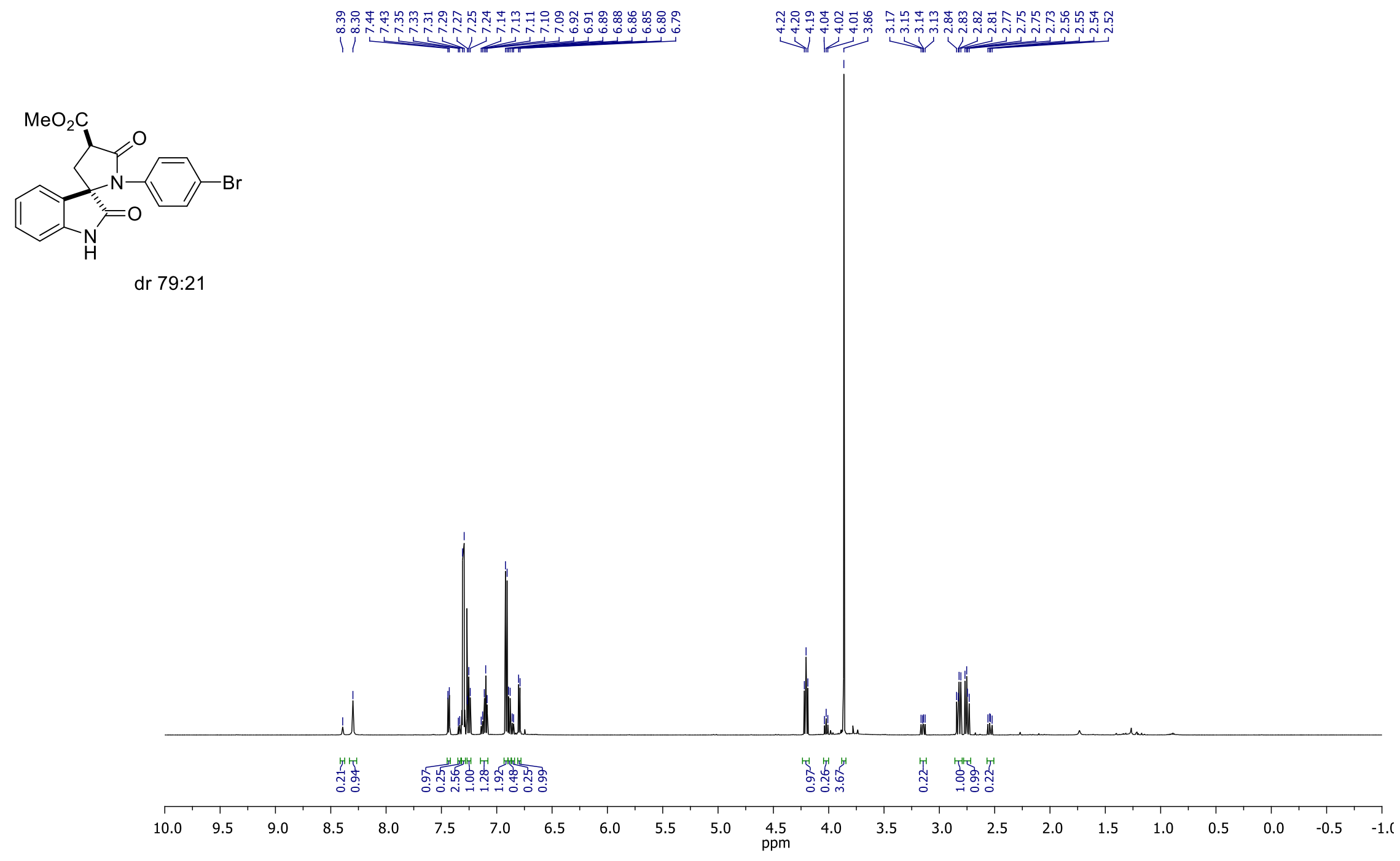


Methyl 1'-(4-bromophenyl)-2,5'-dioxo-1,2-dihydrospiro[indole-3,2' -pyrrolidine]-4' -carboxylate (9i)

${ }^{13} \mathrm{C} \mathrm{NMR}\left(\mathrm{CDCl}_{3}, 150 \mathrm{MHz}\right)$
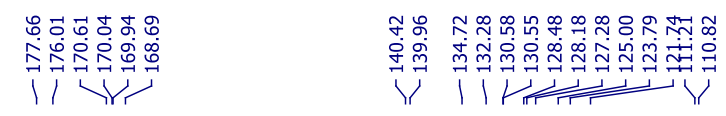

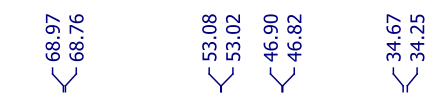

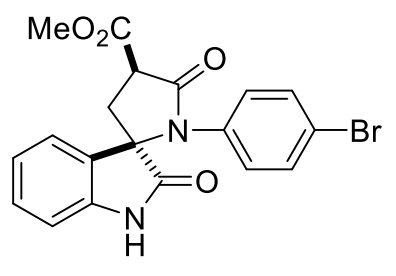

dr 79:21

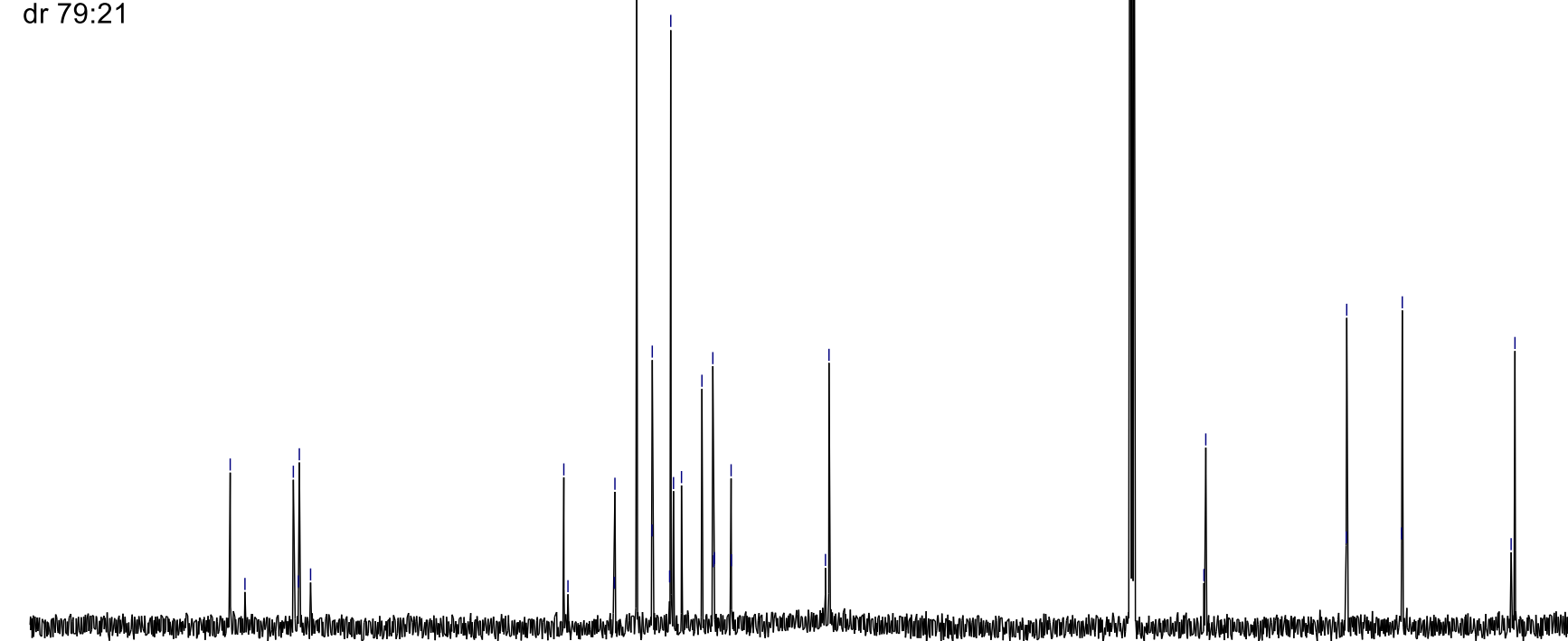

$\begin{array}{lllllllllll}200 & 190 & 180 & 170 & 160 & 150 & 140 & 130 & 120 & 110 & 100\end{array}$ 
Methyl 1'-(4-methylphenyl)-2,5'-dioxo-1,2-dihydrospiro[indole-3,2'-pyrrolidine]-4' -carboxylate (9j)

${ }^{1} \mathrm{H} \mathrm{NMR}\left(\mathrm{CDCl}_{3}, 600 \mathrm{MHz}\right)$

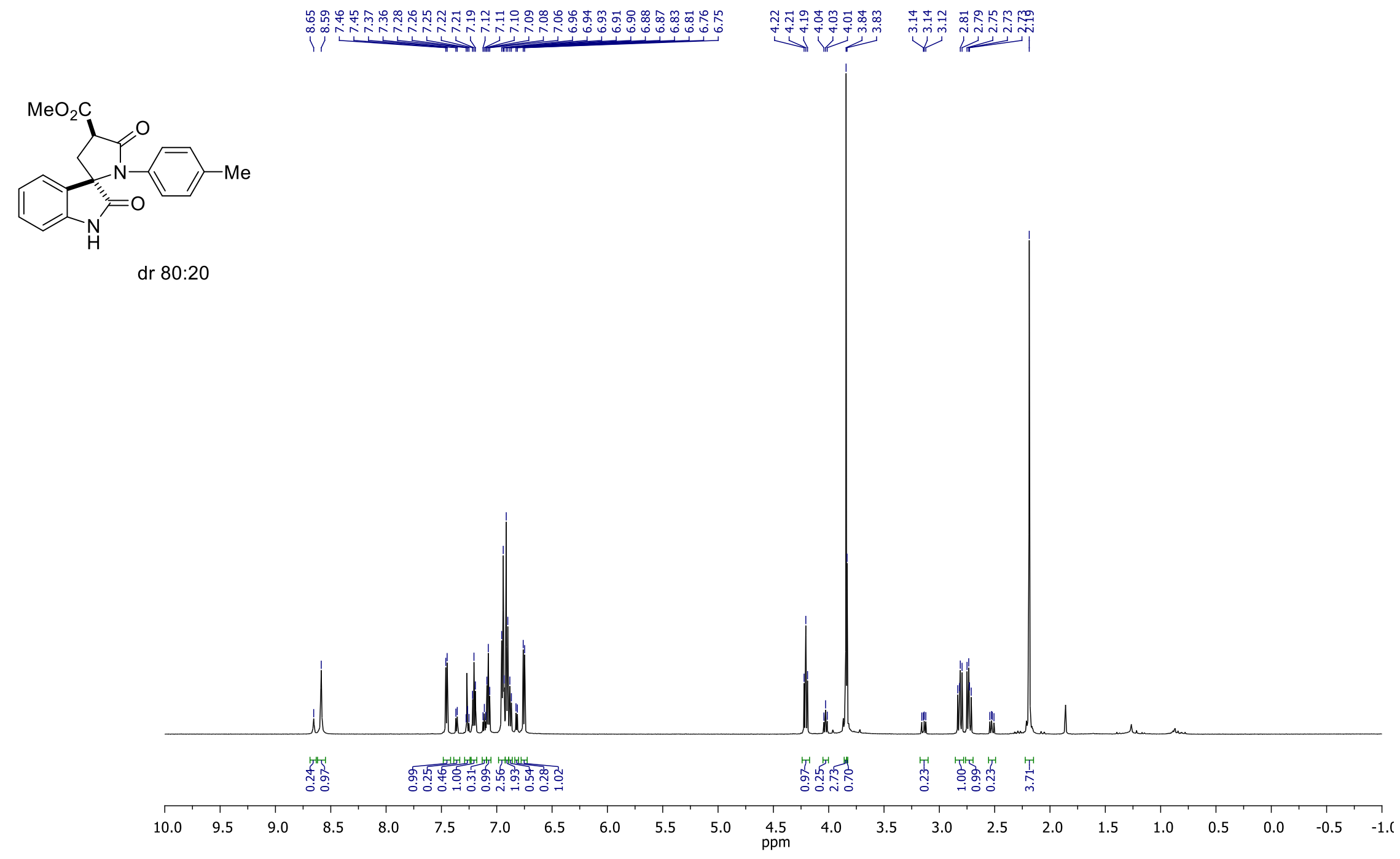


Methyl 1'-(4-methylphenyl)-2,5'-dioxo-1,2-dihydrospiro[indole-3,2'-pyrrolidine]-4' -carboxylate (9j)

${ }^{13} \mathrm{C} \mathrm{NMR}\left(\mathrm{CDCl}_{3}, 150 \mathrm{MHz}\right)$

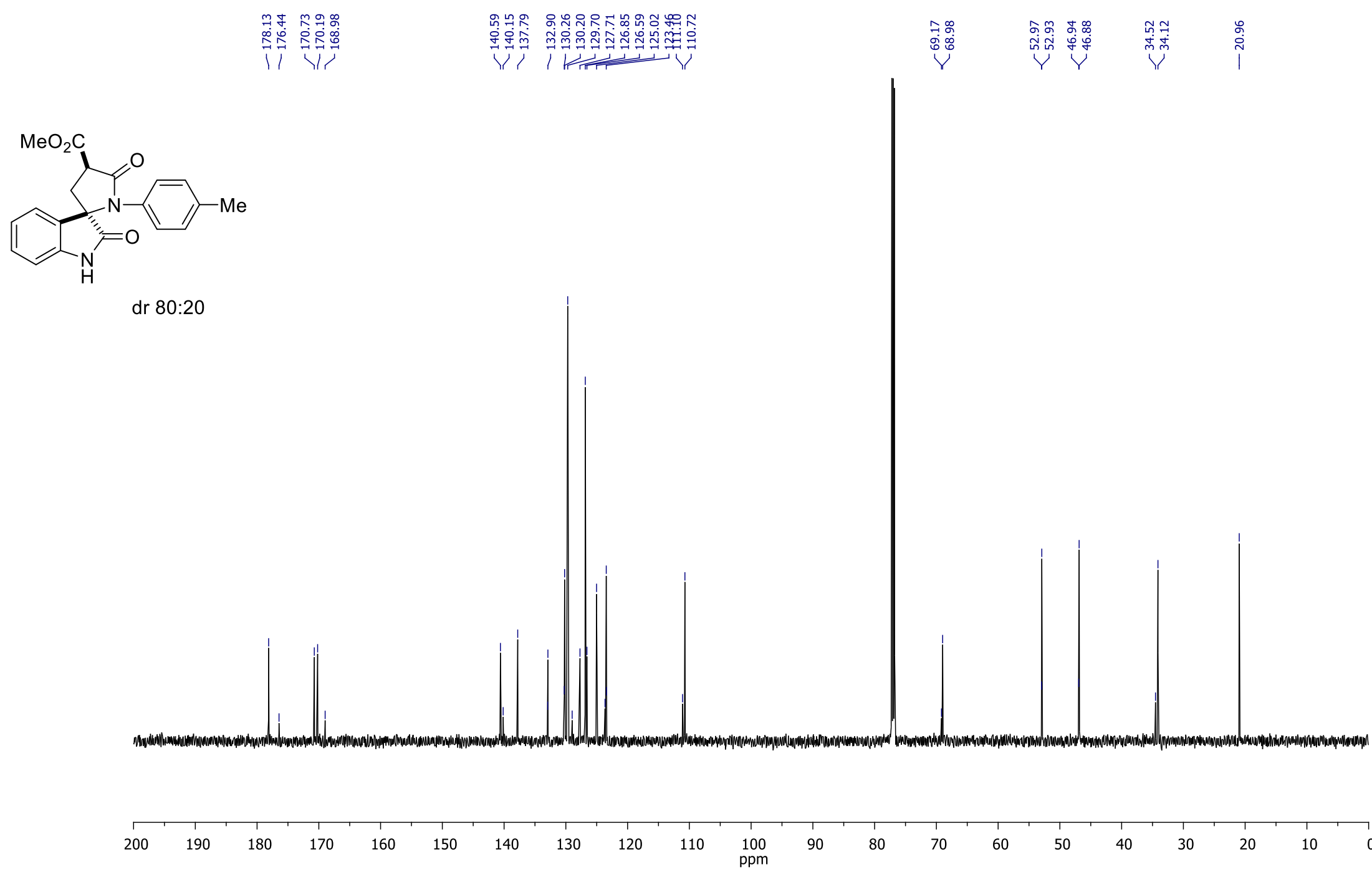


Methyl 1'-(4-methoxyphenyl)-2,5'-dioxo-1,2-dihydrospiro[indole-3,2' -pyrrolidine]-4' -carboxylate (9k)

${ }^{1} \mathrm{H} \mathrm{NMR}\left(\mathrm{CDCl}_{3}, 600 \mathrm{MHz}\right)$

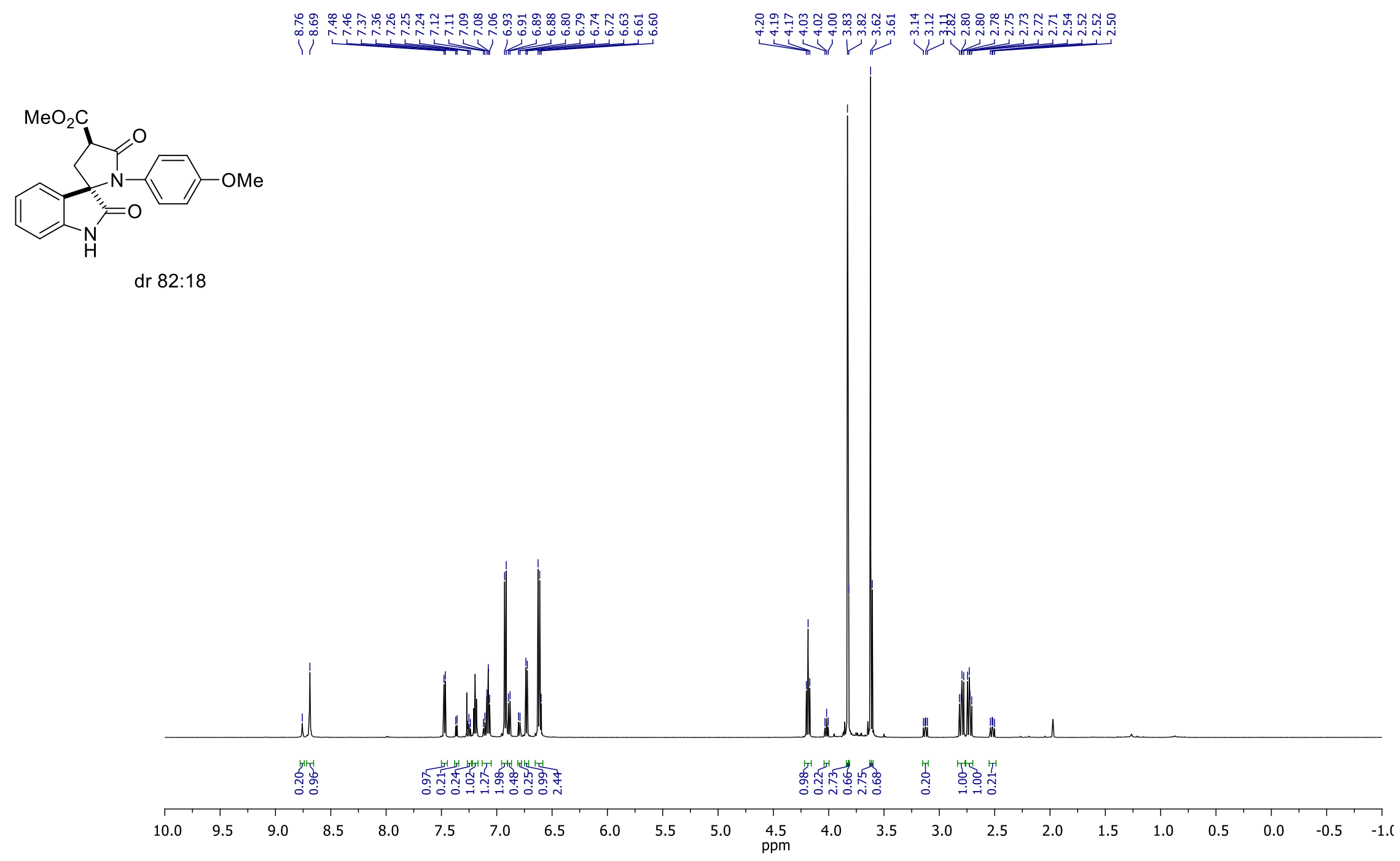


Methyl 1'-(4-methoxyphenyl)-2,5'-dioxo-1,2-dihydrospiro[indole-3,2' -pyrrolidine]-4' -carboxylate (9k)

${ }^{13} \mathrm{C} \mathrm{NMR}\left(\mathrm{CDCl}_{3}, 150 \mathrm{MHz}\right)$

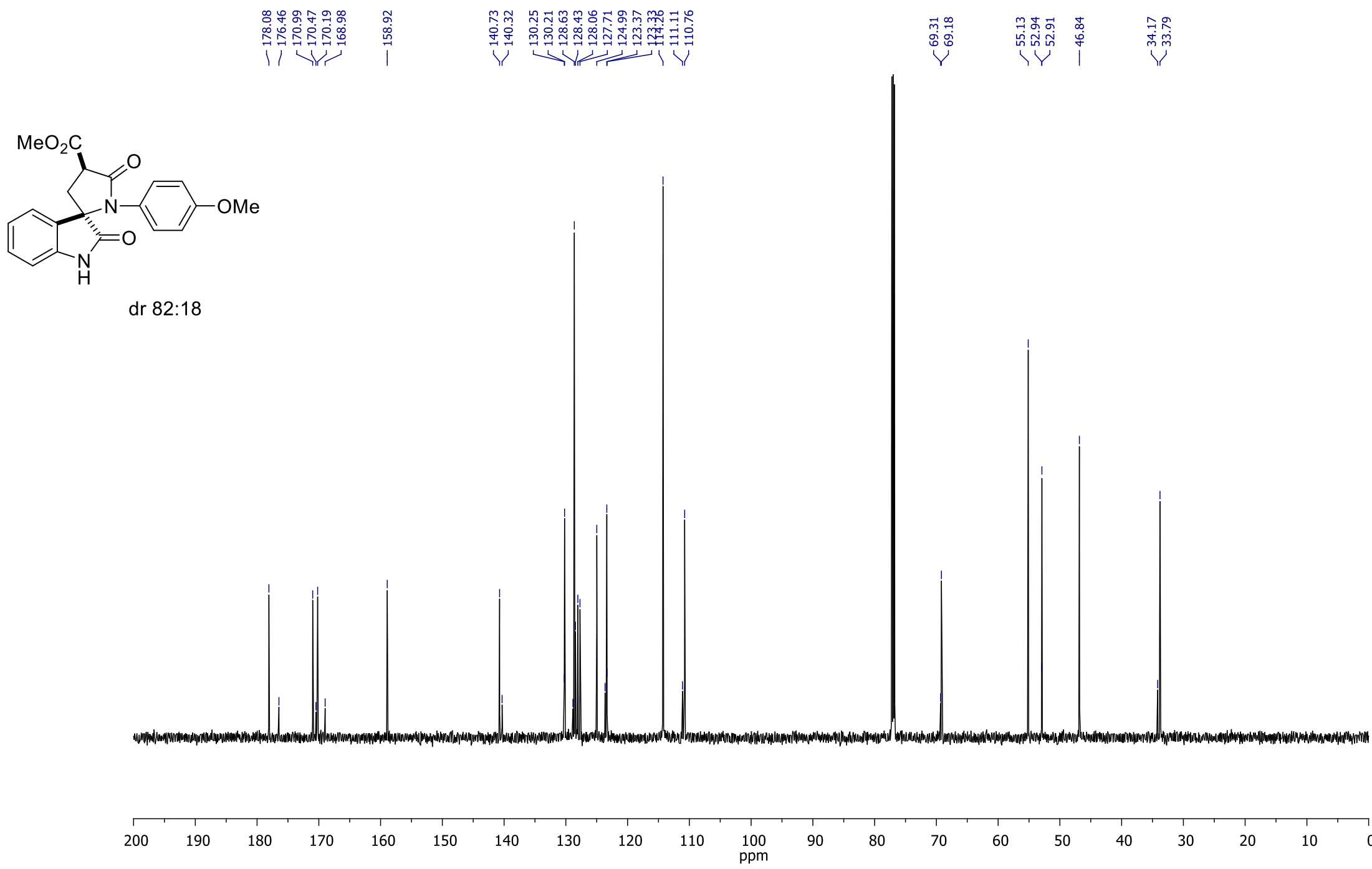


Methyl 1'-(4-nitrophenyl)-2,5' -dioxo-1,2-dihydrospiro[indole-3,2' -pyrrolidine]-4' -carboxylate (9l)

${ }^{1} \mathrm{H} \mathrm{NMR}\left(\mathrm{CDCl}_{3}, 600 \mathrm{MHz}\right)$

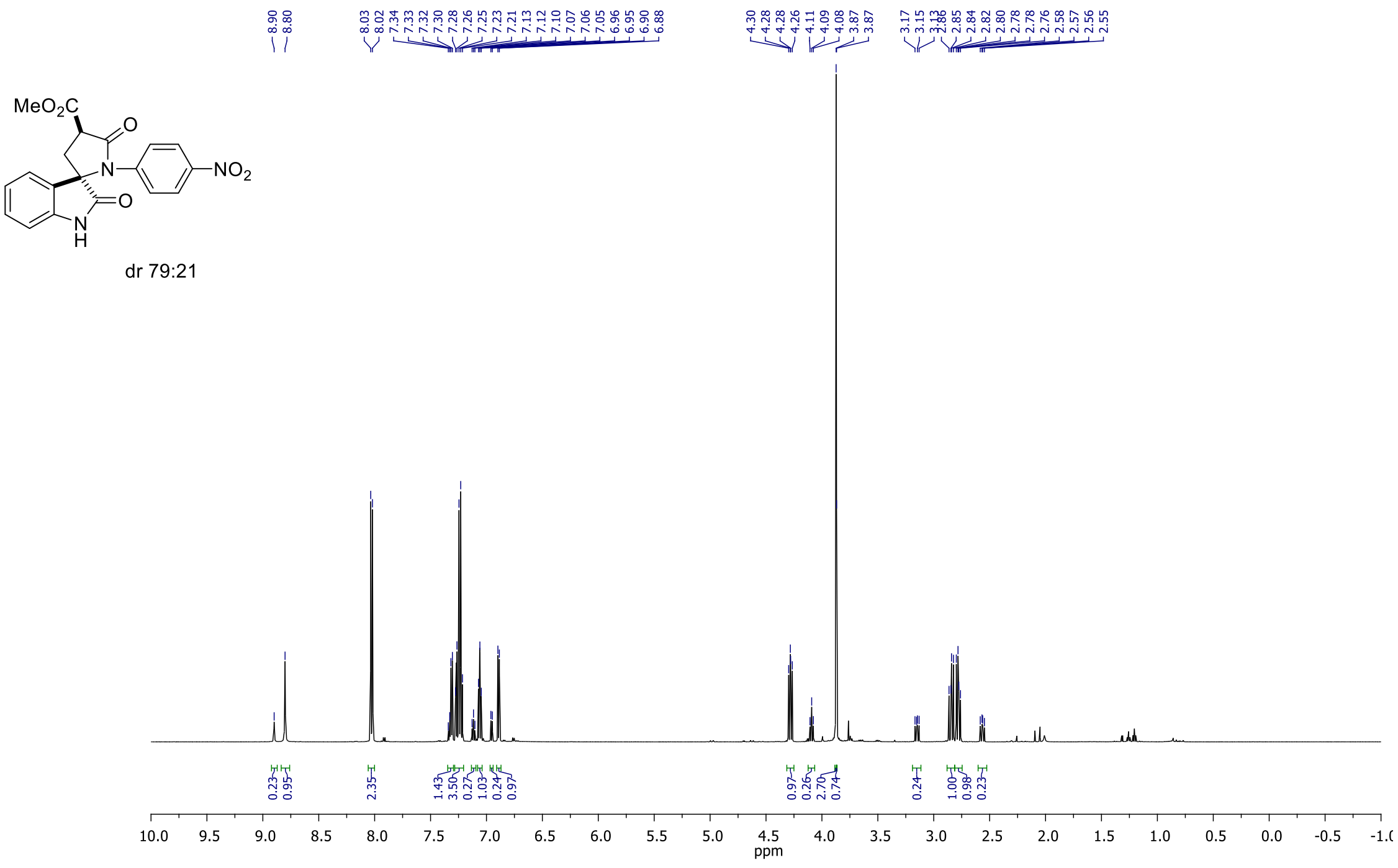


Methyl 1'-(4-nitrophenyl)-2,5' -dioxo-1,2-dihydrospiro[indole-3,2' -pyrrolidine]-4' -carboxylate (9I)

${ }^{13} \mathrm{C} \mathrm{NMR}\left(\mathrm{CDCl}_{3}, 150 \mathrm{MHz}\right)$

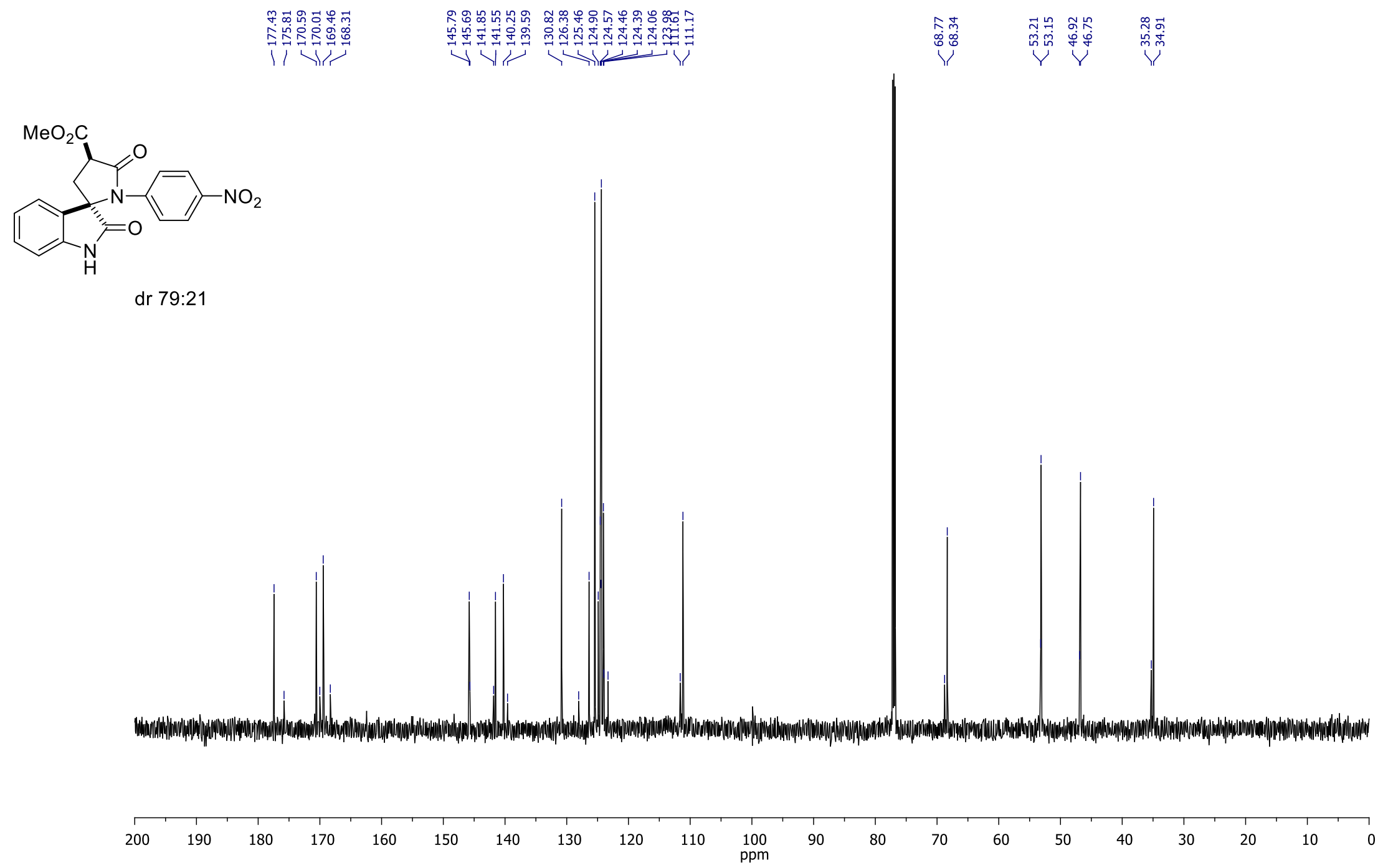


Methyl (1RS,2RS)-2-(benzylcarbamoyl)-1' -(4-methoxybenzyl)-2' -oxo-1', $2^{\prime}$-dihydrospiro[cyclopropane-1,3'-indole]-2-carboxylate (10a) ${ }^{1} \mathrm{H} \mathrm{NMR}\left(\mathrm{CDCl}_{3}, 600 \mathrm{MHz}\right)$
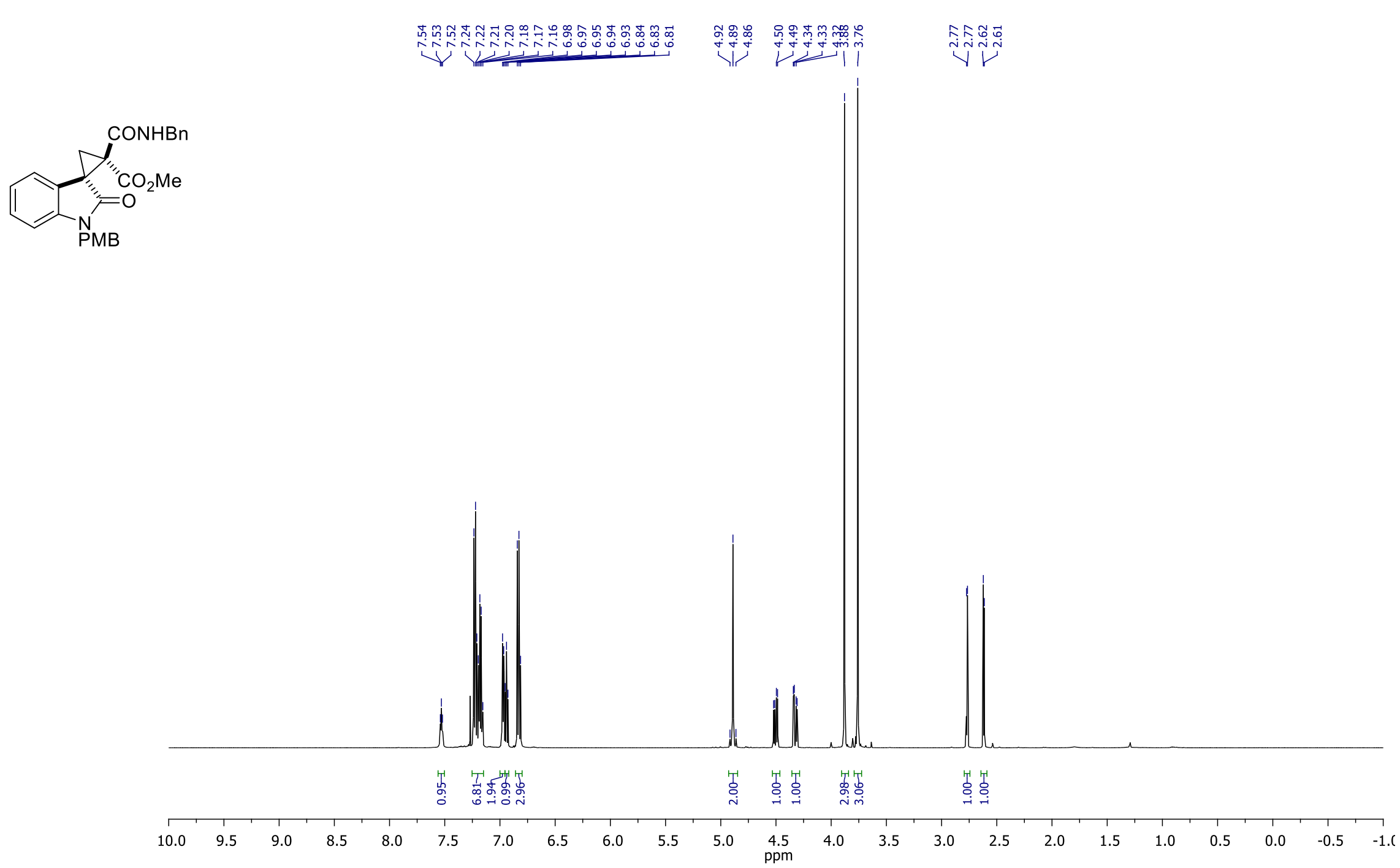
${ }^{1} \mathrm{H}-{ }^{1} \mathrm{H}$ NOESY $\left(\mathrm{CDCl}_{3}, 600 \mathrm{MHz}\right)$

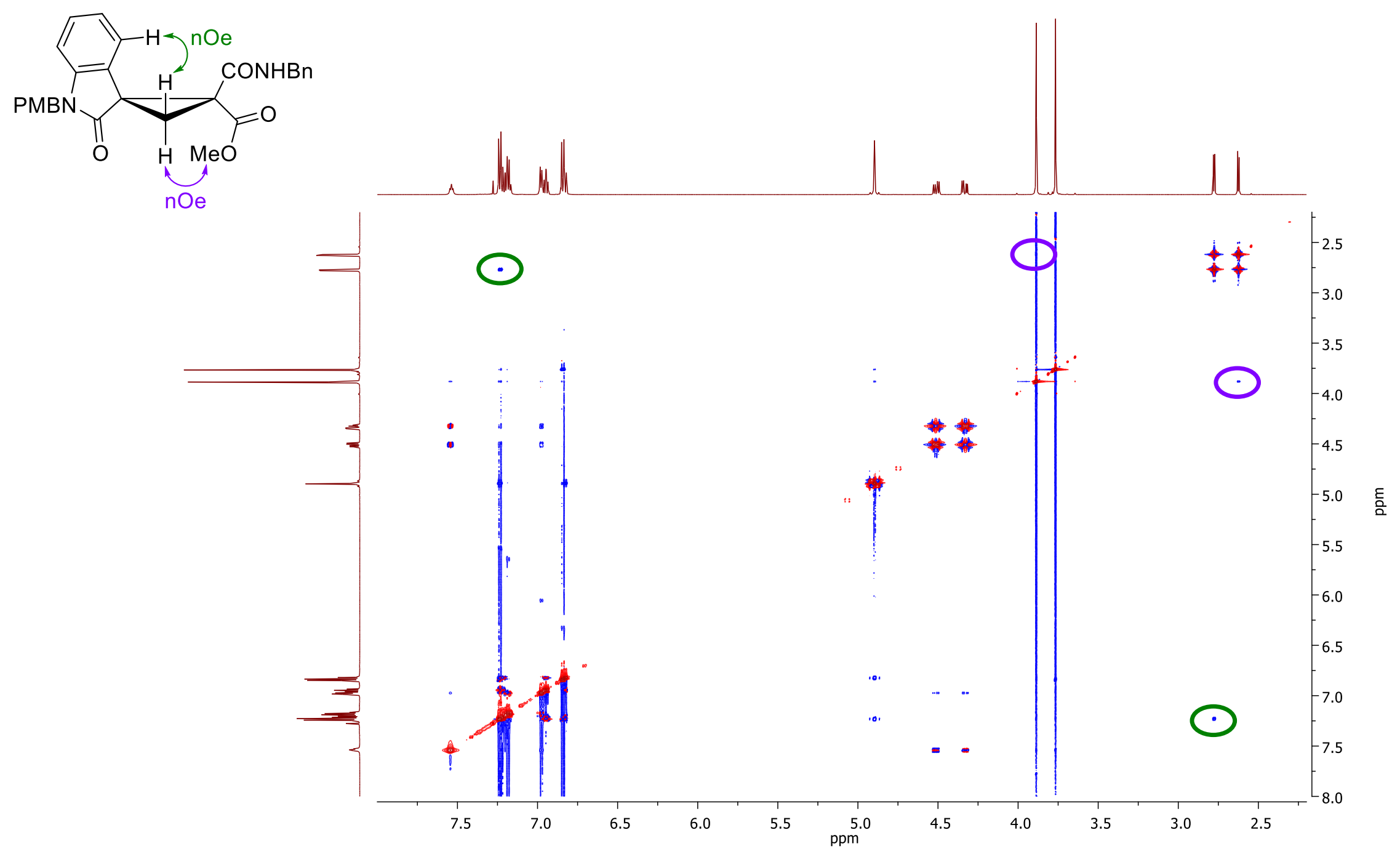


Methyl (1RS,2RS)-2-(benzylcarbamoyl)-1' -(4-methoxybenzyl)-2' -oxo-1' $2^{\prime}$-dihydrospiro[cyclopropane-1,3'-indole]-2-carboxylate (10a)

${ }^{13} \mathrm{C} \mathrm{NMR}\left(\mathrm{CDCl}_{3}, 150 \mathrm{MHz}\right)$

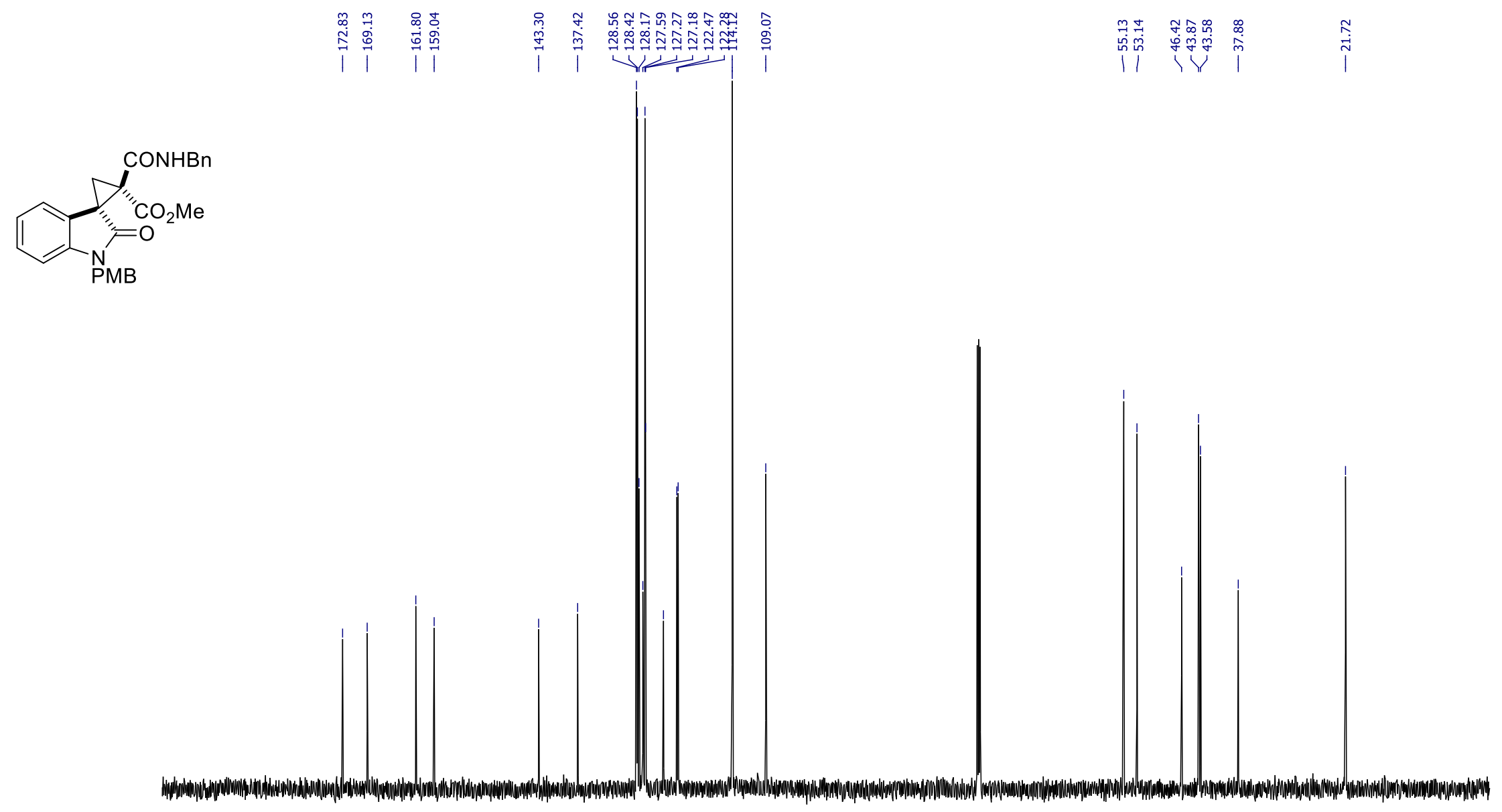

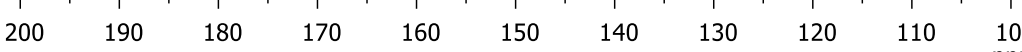

$90 \quad 80$

60

$50 \quad 40$

30

$20 \quad 10 \quad 0$ 
Methyl (1RS,2RS)-2-(butylcarbamoyl)-1'-(4-methoxybenzyl)-2' -oxospiro[cyclopropane-1,3'-indoline]-2-carboxylate (10b)

${ }^{1} \mathrm{H} \mathrm{NMR}\left(\mathrm{CDCl}_{3}, 600 \mathrm{MHz}\right)$

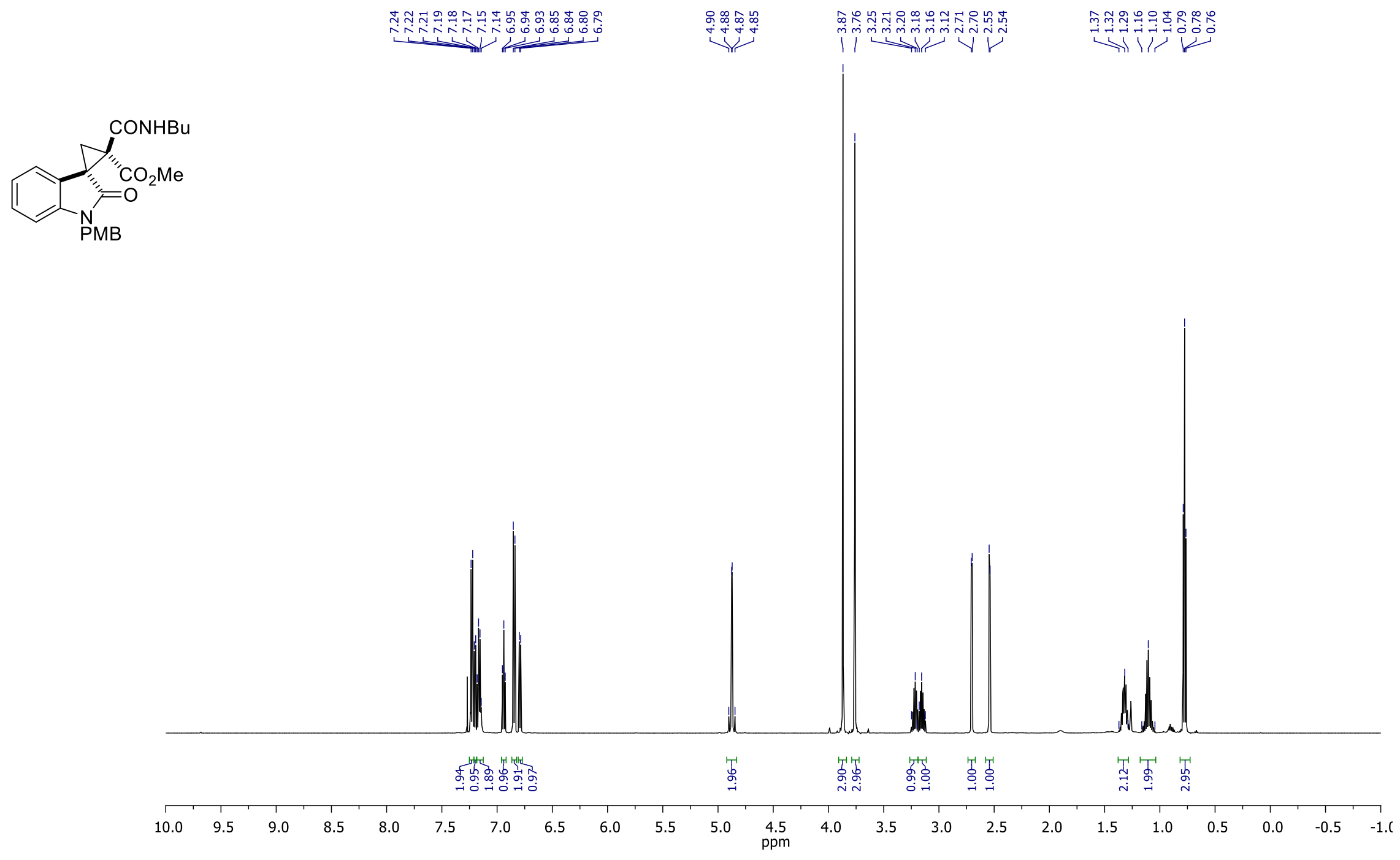


${ }^{1} \mathrm{H}-{ }^{1} \mathrm{H}$ NOESY $\left(\mathrm{CDCl}_{3}, 600 \mathrm{MHz}\right)$

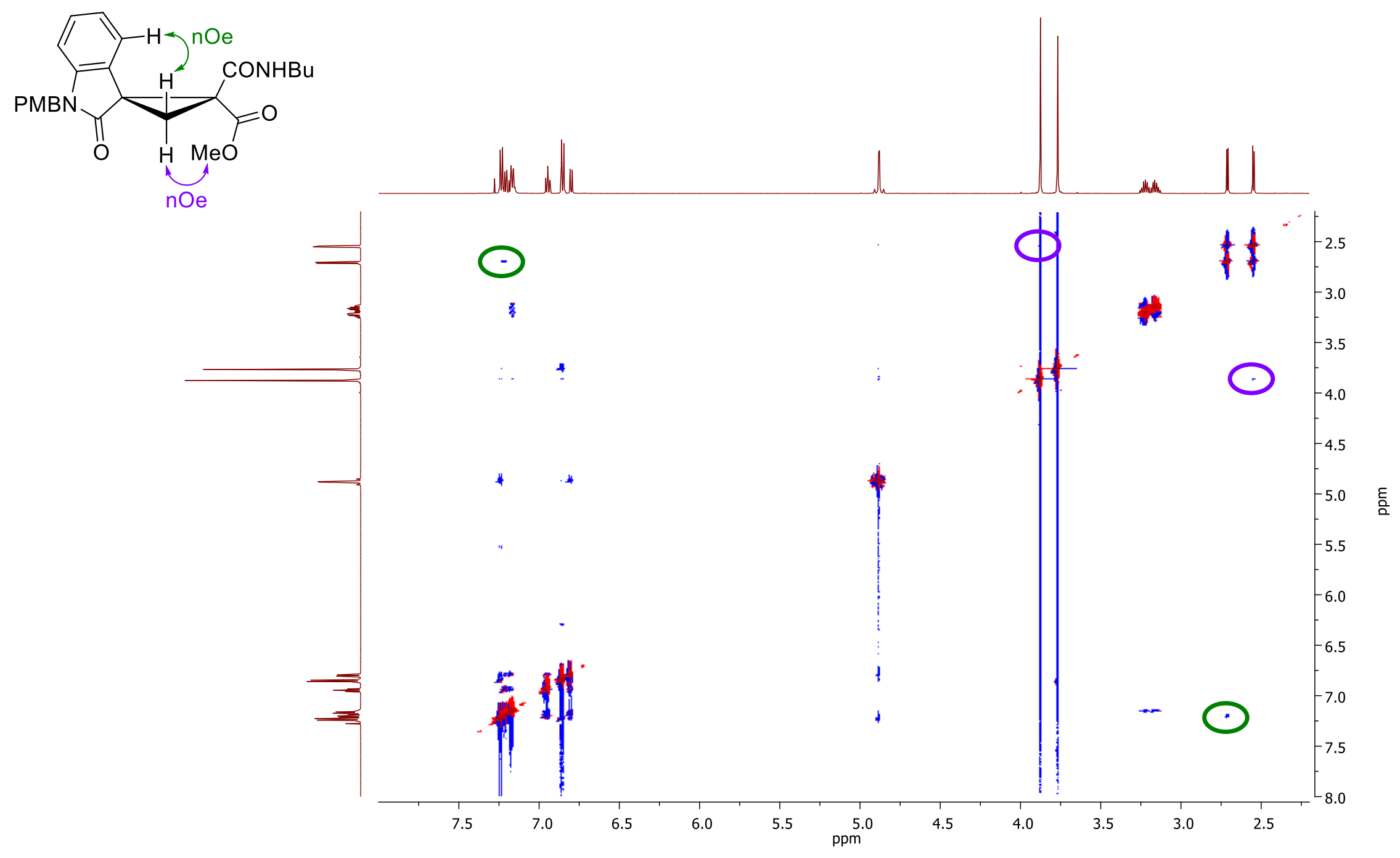


Methyl (1RS,2RS)-2-(butylcarbamoyl)-1'-(4-methoxybenzyl)-2'-oxospiro[cyclopropane-1,3'-indoline]-2-carboxylate (10b)

${ }^{13} \mathrm{C} \mathrm{NMR}\left(\mathrm{CDCl}_{3}, 150 \mathrm{MHz}\right)$

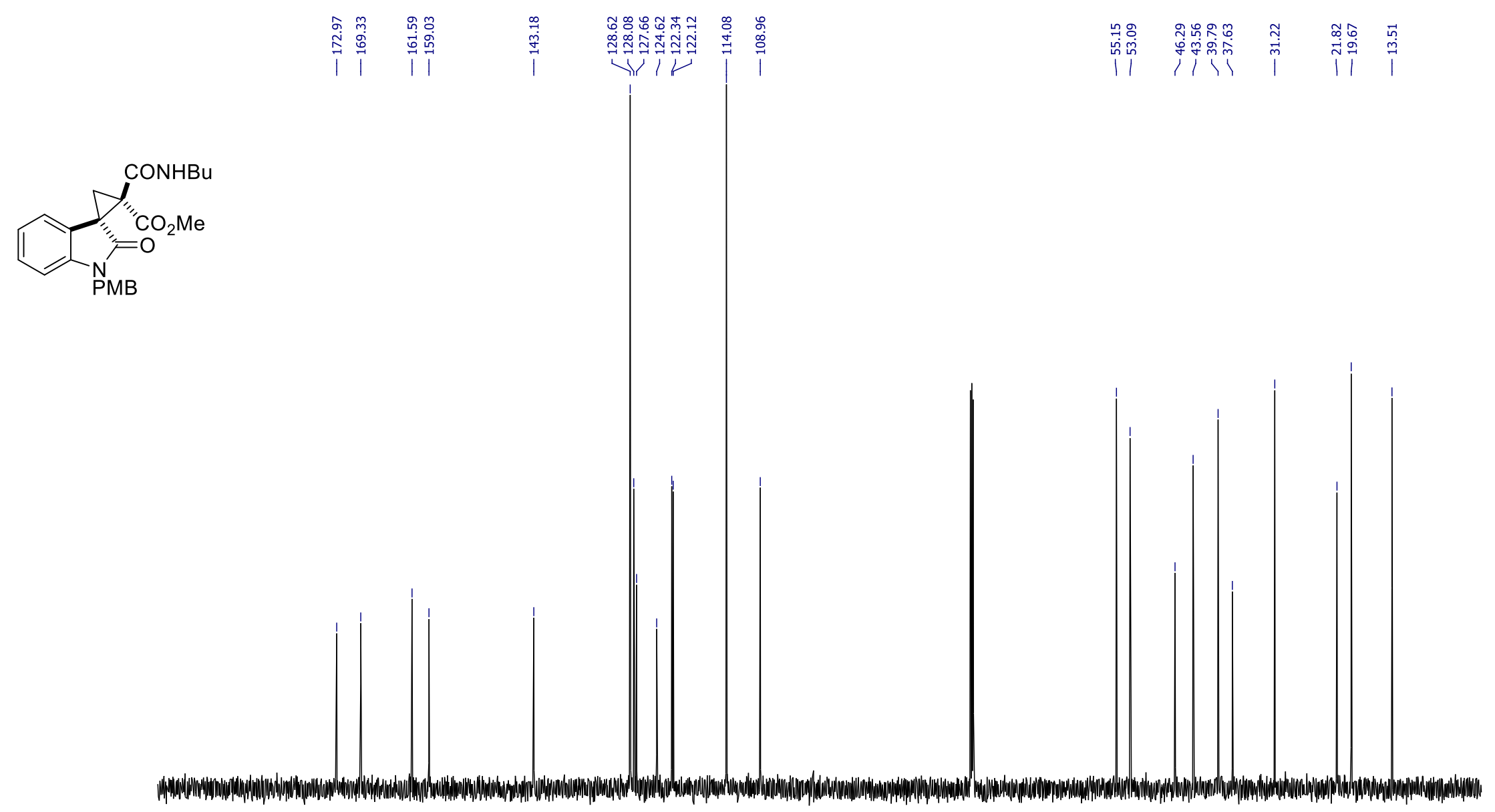

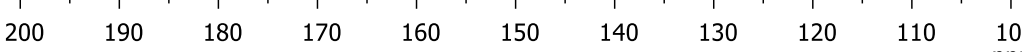

$90 \quad 80$

60

$50 \quad 40$

30

$\begin{array}{lll}10 & 10\end{array}$ 
${ }^{1} \mathrm{H} \mathrm{NMR}\left(\mathrm{CDCl}_{3}, 600 \mathrm{MHz}\right)$
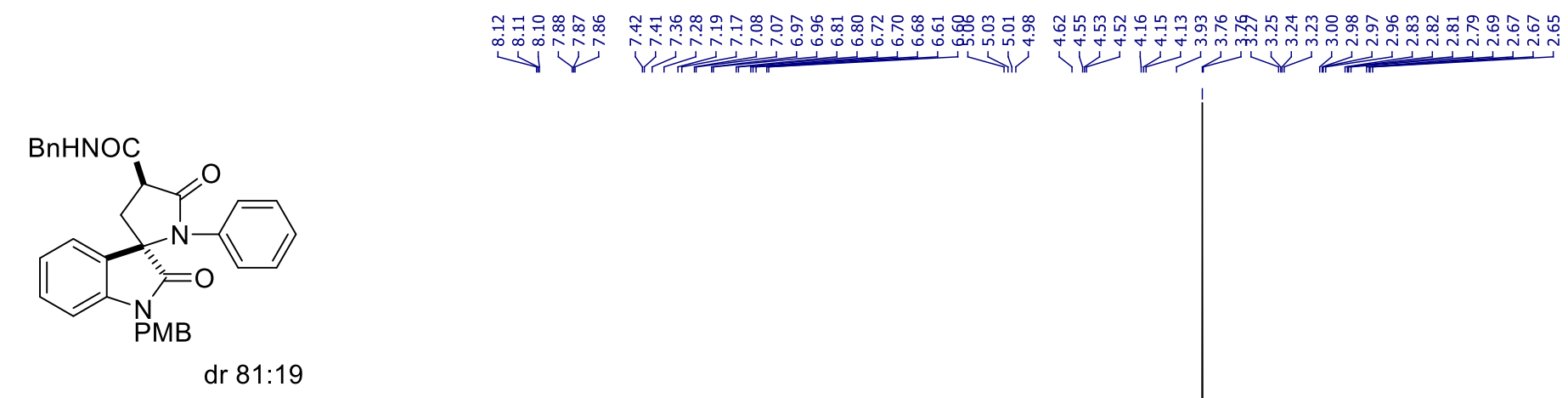

dr 81:19

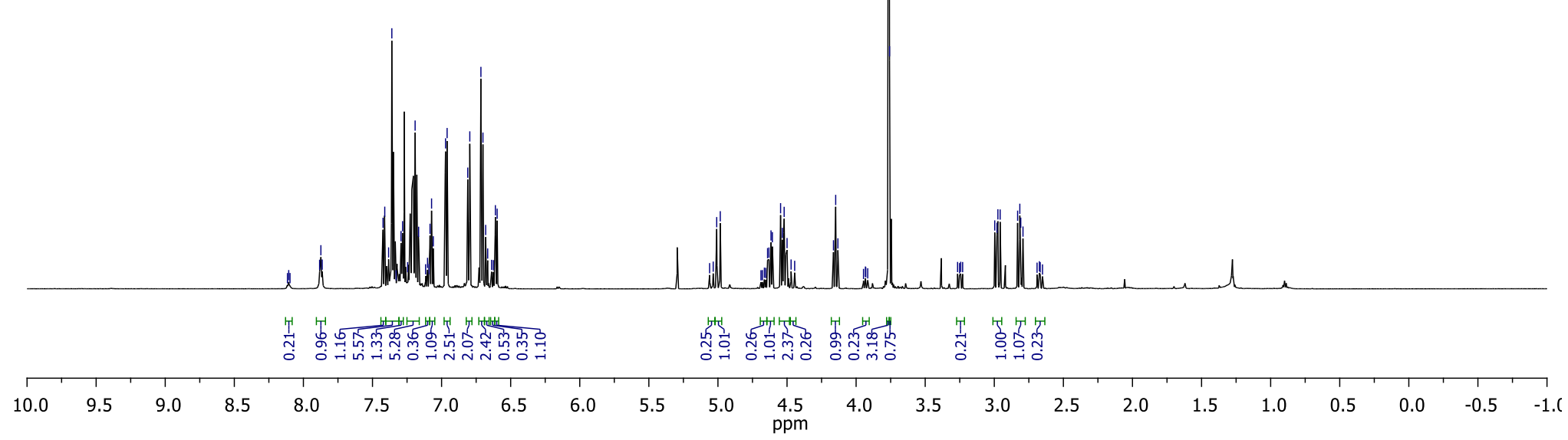


$N$-benzyl-1-(4-methoxybenzyl)-2,5' -dioxo-1' -phenyl-1,2-dihydrospiro[indole-3,2' -pyrrolidine]-4'-carboxamide (11a)

${ }^{13} \mathrm{C} \mathrm{NMR}\left(\mathrm{CDCl}_{3}, 150 \mathrm{MHz}\right)$

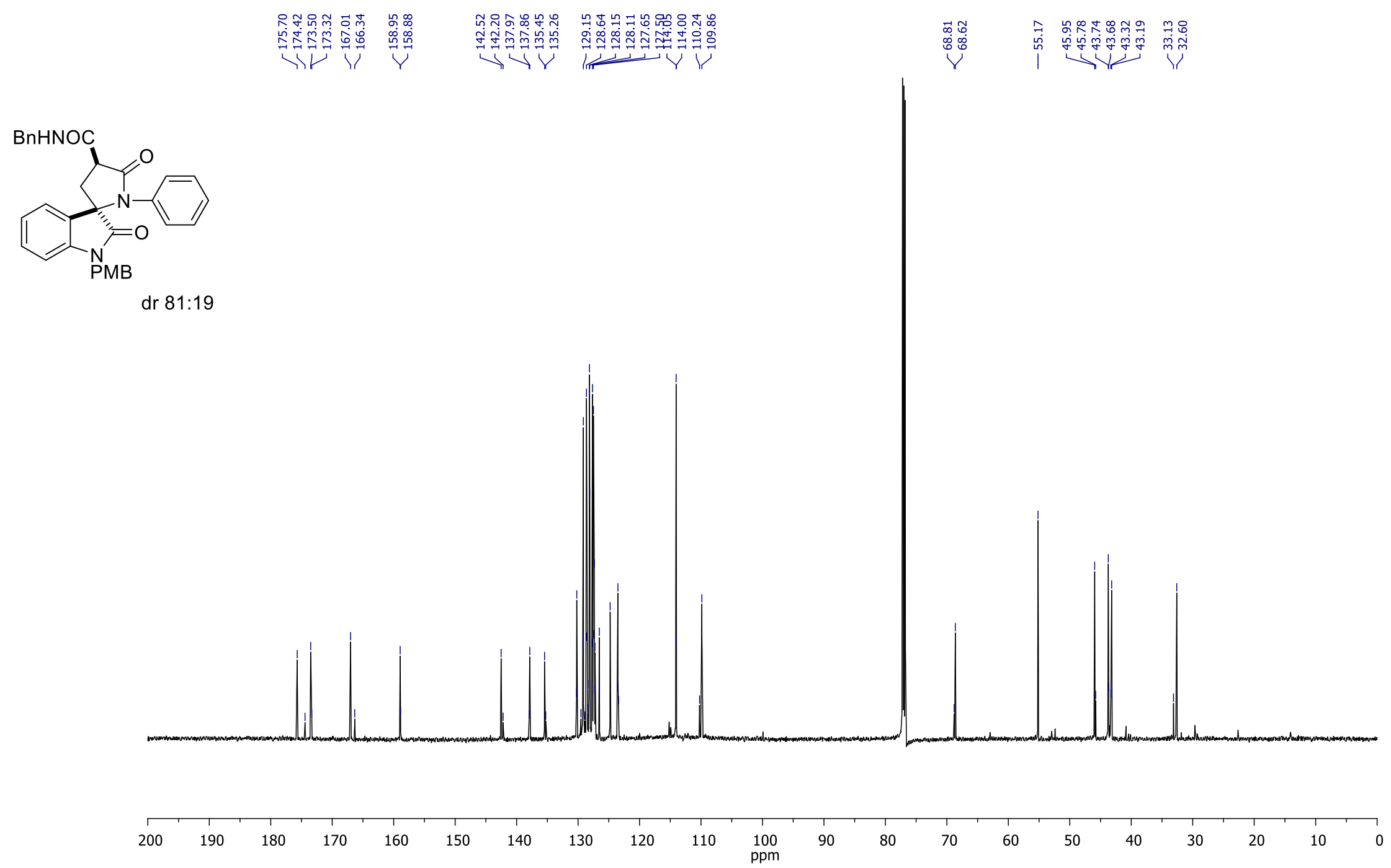


$N$-Benzyl-1-(4-methoxybenzyl)-1'-(4-methoxyphenyl)-2,5'-dioxo-1,2-dihydrospiro[indole-3,2'-pyrrolidine]-4' -carboxamide (11b)

${ }^{1} \mathrm{H} \mathrm{NMR}\left(\mathrm{CDCl}_{3}, 600 \mathrm{MHz}\right)$

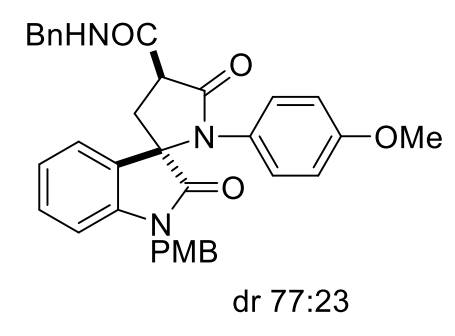

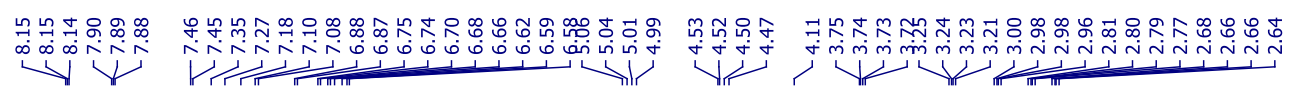

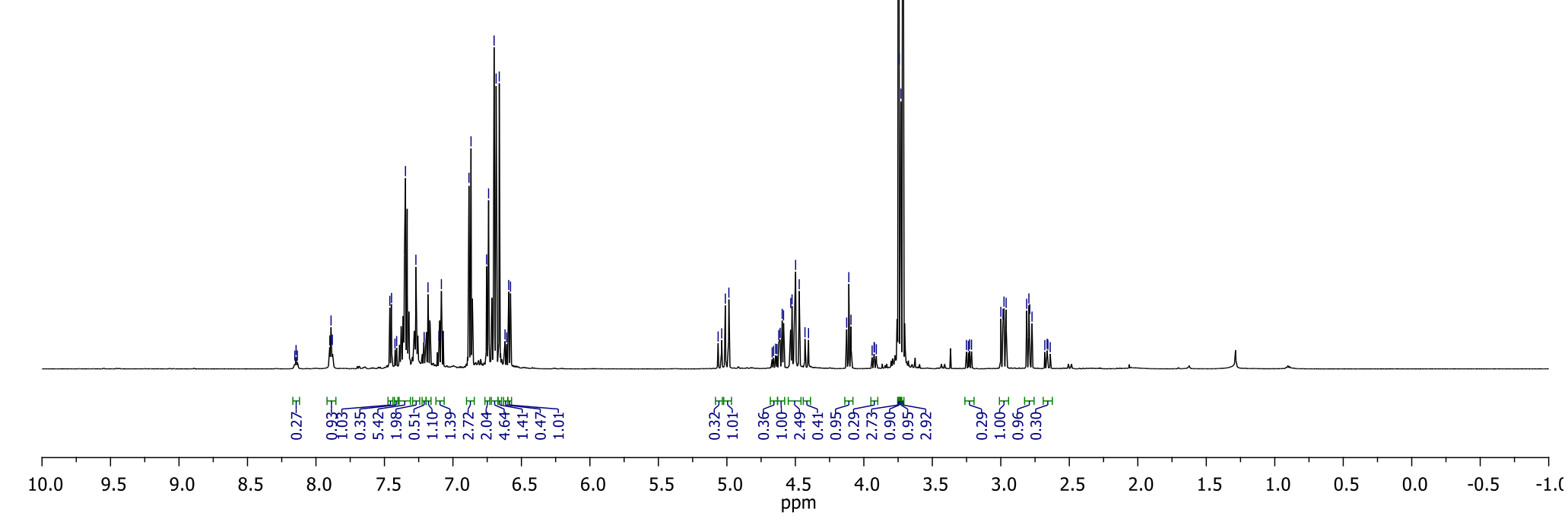


$N$-Benzyl-1-(4-methoxybenzyl)-1'-(4-methoxyphenyl)-2,5'-dioxo-1,2-dihydrospiro[indole-3,2'-pyrrolidine]-4'-carboxamide (11b)

${ }^{13} \mathrm{C} \mathrm{NMR}\left(\mathrm{CDCl}_{3}, 150 \mathrm{MHz}\right)$

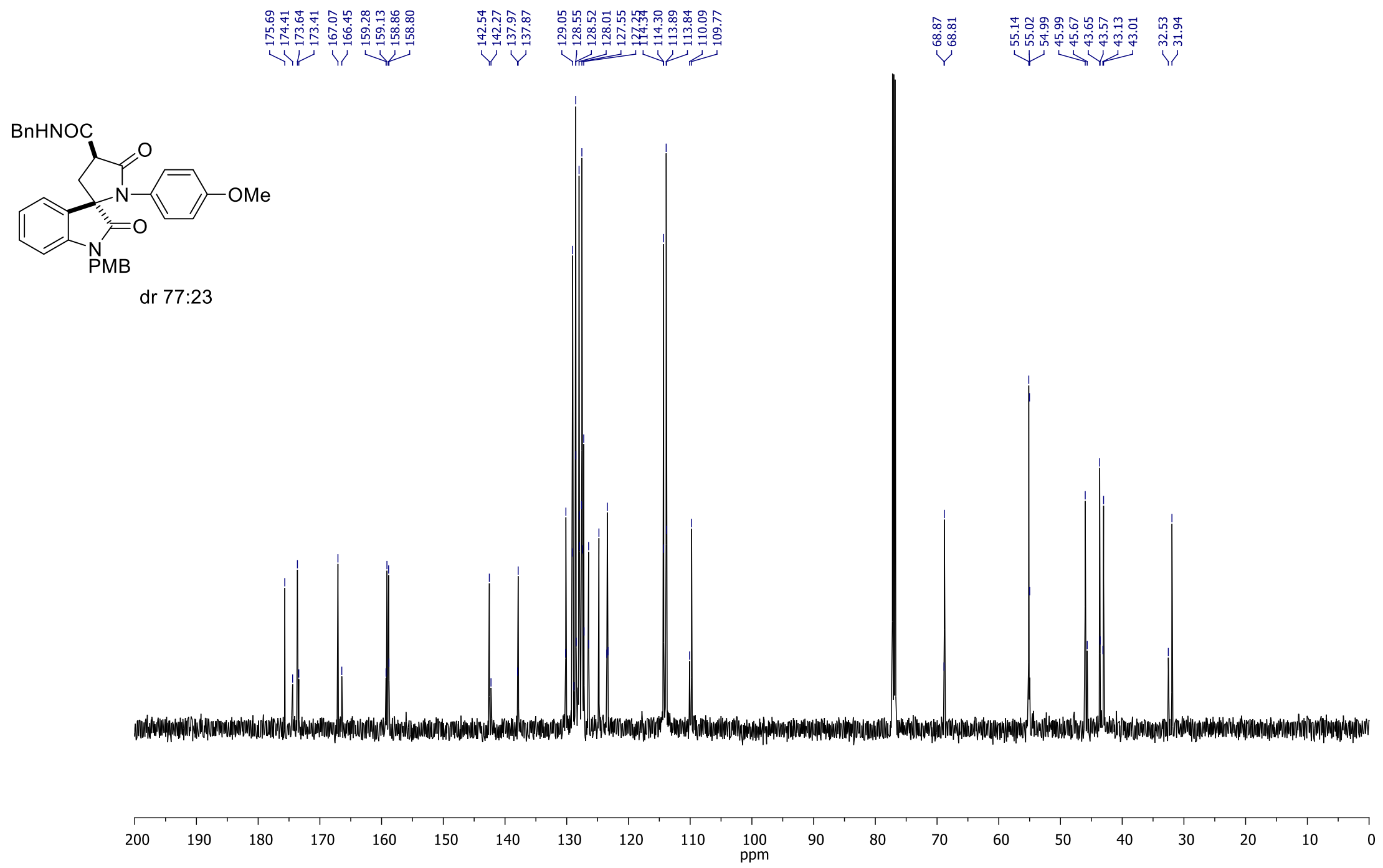


${ }^{1} \mathrm{H} \mathrm{NMR}\left(\mathrm{CDCl}_{3}, 600 \mathrm{MHz}\right)$

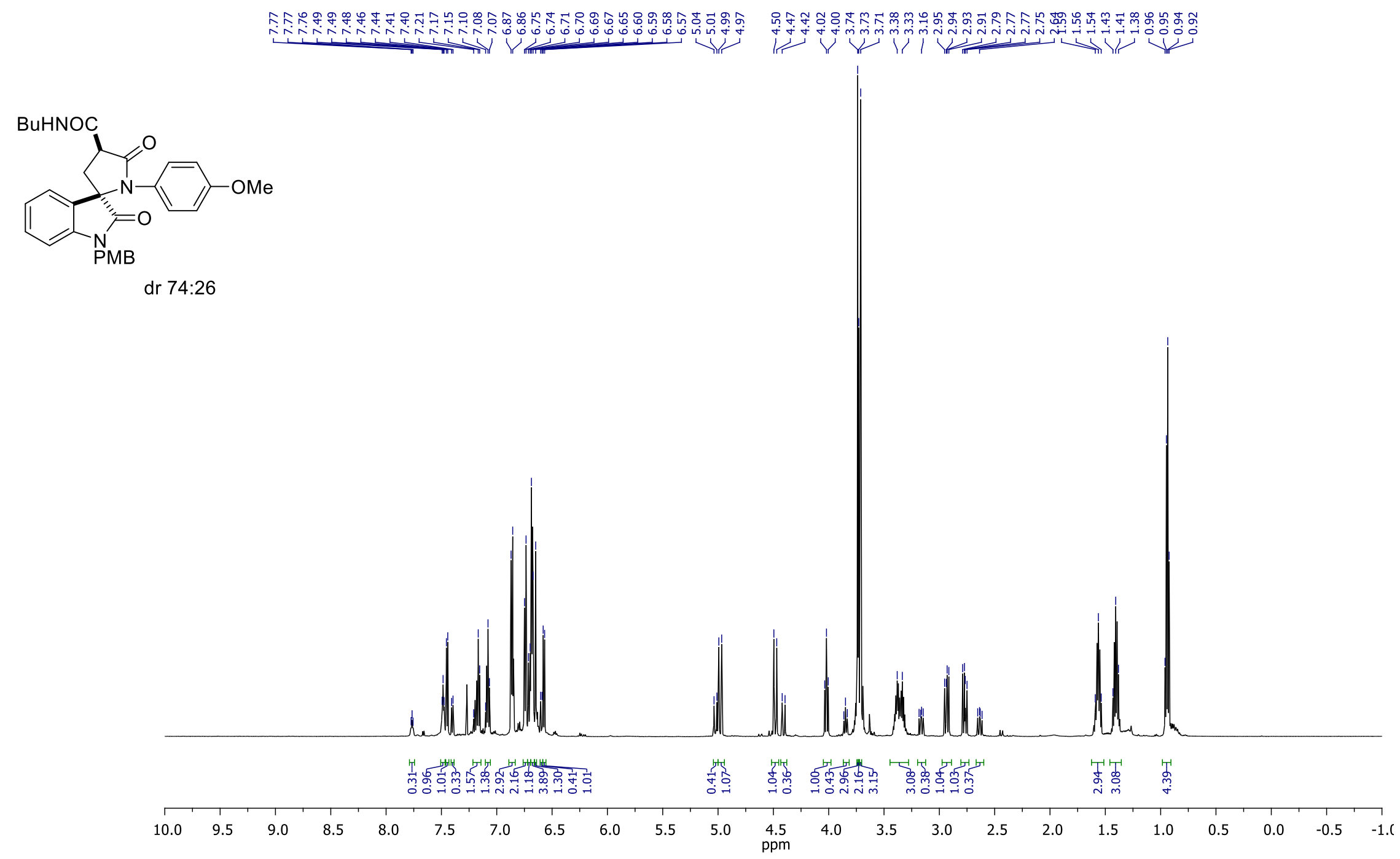


${ }^{13} \mathrm{C} \mathrm{NMR}\left(\mathrm{CDCl}_{3}, 150 \mathrm{MHz}\right)$

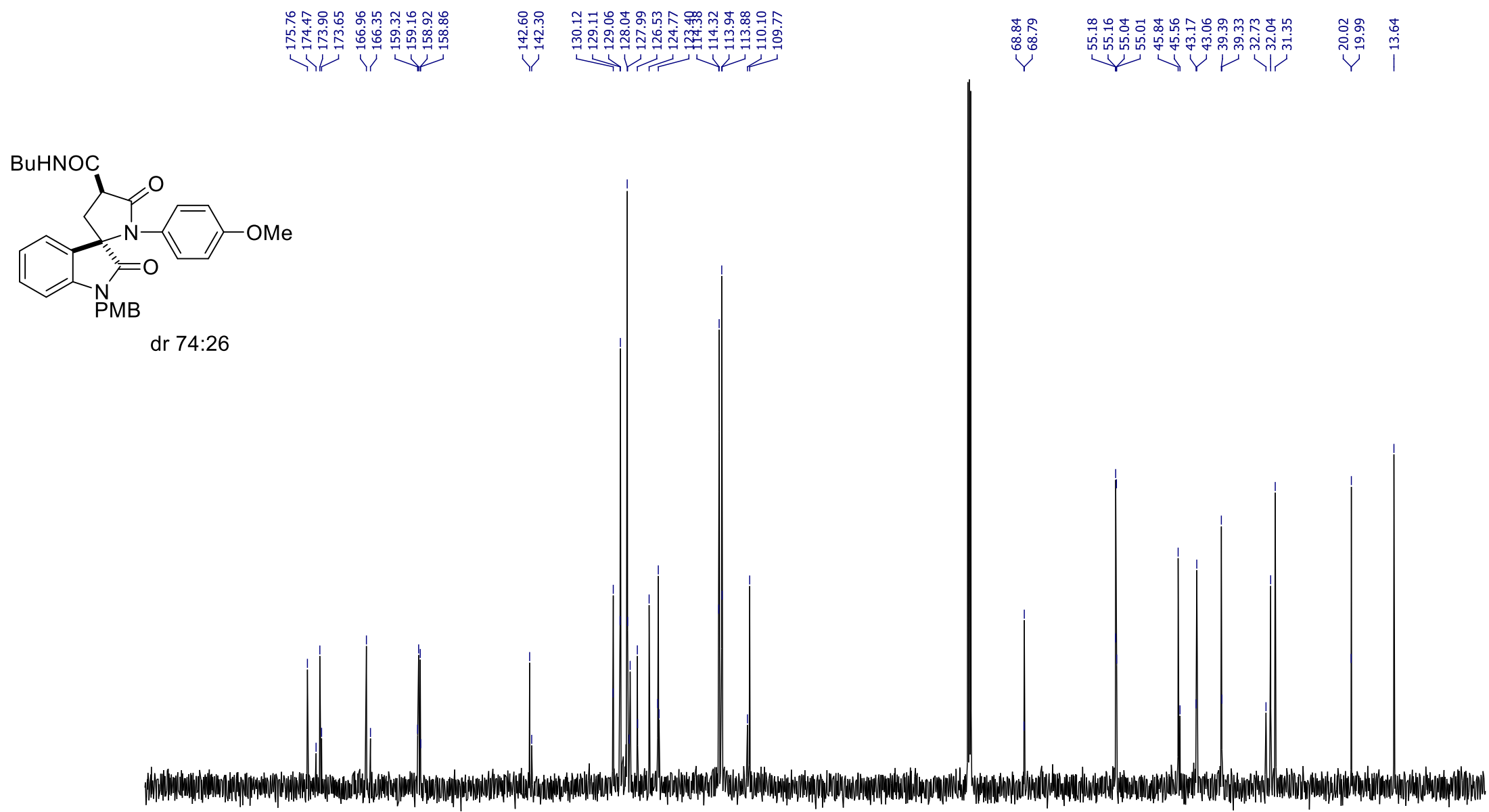

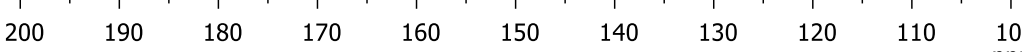

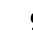

$90 \quad 80$

60

$50 \quad 40$

30

$20 \quad 10 \quad 0$ 